\title{
Mlanagement and Operating Contractor Plan for Transition to the Project Hanford Management Contractor
}

Prepared for the U.S. Department of Energy Offics of Erivironmental Restoration and Waste Manegement

\section{(29) Westinghouse Hanford Company Richilard, Wastington}

Henford Opcratiors 3.J Engivesring Fortraciut for the

II S. Dapartmert of EnerFy inder Contract DE.ACO6-87RL1C930 


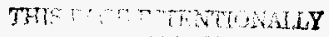
Sink 
LEGAL DISCLAMER

This report was prepared as an account of work sponsored by an agency of the United States Government. Noither the

United States Government nor any ageney thereof, nor any of their amployees, nor any of their contractors, subcontractors ot their ernployees, makes any warranty, express or implied, of assumos any legal liability or responsibility for the accuracy, completeness, or any third party's use or the results of such use of any infomation, apparatus, product, or process disclosed, or represents that its use would not infringe privatoly owned rights. Reference horein to any specific commercial product, process, or service by trade name, trademark, manufacturer, or otherwise, does not necessarily constitute or imply its endorsement, recommendation, or favoring by the United States Government or any ageney thereof or its contractors or subcontractors. The views and opinions of authors expressed herein do not necessarily state or reflect those of the United States Government or any agency thereof.

This report has been reproduced from the best available copy. Availablo in paper copy and microfiche.

Available to the U.S. Department of Energy and its contractors from

Ofilice of Seientific and Technical Information

P.0. Box 62

Oak Ridge, TN 37831

(615) 576-8401

Primed in the Unitud Stones of Ammirica

DISCLM-3.CHP $(1.91)$ 
mis: $\therefore$ a is - avis: 


\title{
Management and Operating Contractor Plan for Transition to the Project Hanford Management Contractor
}

\author{
M\&O Contractor Transition Team \\ Date Published \\ June 1996
}

Prepared for the U.S. Department of Energy Office of Environmental Restoration and Waste Management

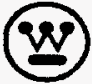

Westinghouse

P.O Box 1970

Hanford Company Richland, Washington

Hanford Operations and Engineering Contractor for the

U.S. Department of Energy under Contract DE-ACO6-87RL10930

Puproend Use of Document This document was prepared for use within the U.S. Department of Enerone and its

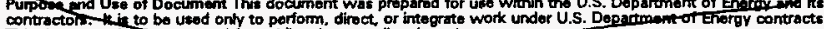
This document is not apprevend for public robase until neviewed.

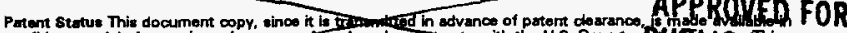
confidence solely for use in pertomapes of work under Dolntecte with the U.S. Departm.

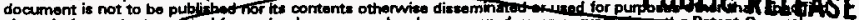

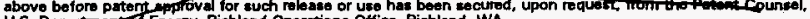
U.S. Departenent 6 F Energy. Pichland Operations Offico, Richland, WA.

Approved for Public Release 
THA : C.. B BLEN 


\section{INFORMATION RELEASE REQUEST \\ PUBLIC DOCUMENT CHANGE/REVISION \\ (Short Form No. 1)}

1. Base Document ID Number: WHC-SP-1181

2. Base Document Title:

Management and Operating Contractor Plan for Transition to the Project Hanford Management Contractor

\section{Change/Revision Number: REVISION 1}

4. Change/Revision Date: $06 / 29 / 96$

5. Unclassified Category:* UC - 2000

6. Budget \& Reporting Code:* B\&R - EM

I verify this change/revision to the base document indicated above:

- Complies with the provisions of WHC-CM-3-4

- Contains no Limited-Use information

- Contains no classified or Applied Technology references

- Does not change the intent or meaning of the base document

- And, the base document itself is approved for public release.

7. Responsible Manager: Jack L. Waite

MSIN: H6-33 Telephone Number:

$372-1772$

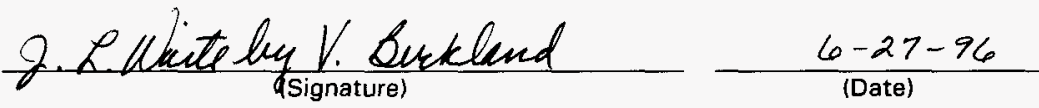

8. Information Release Administration Specialist: * *

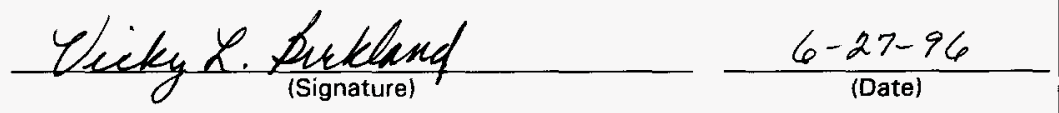

* Required only for full revisions transmitted to OSTI.

* *Required only for full revisions. 


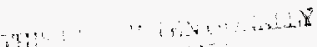

anivis. 


\section{RELEASE AUTHORIZATION}

Document

Number:

Document

Title:
WHC-SP-1181, Rev. 1

Management and Operating contractor Plan for

Transition to the Project Hanford Management

contractor

Release Date: $06 / 27 / 96$

This document was reviewed following the procedures described in WHC-CM-3-4 and is:

APPROVED FOR PUBLIC RELEASE

whC Information Release Administration specialist: 


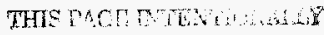
TELT BLANK 


\section{TABLE OF CONTENTS}

1.0 INTRODUCTION . . . . . . . . . . . . . . . . . . .1-1

2.0 PLAN AND IMPLEMENTATION APPROACH $\ldots \ldots \ldots \ldots \ldots \ldots \ldots .2$.

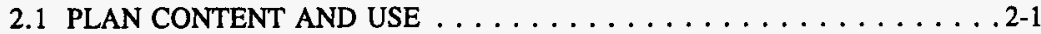

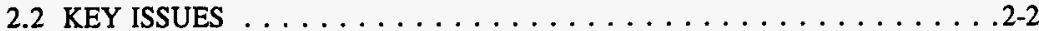

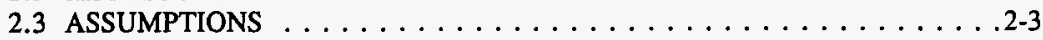

2.4 ACTION TRACKING AND RECORDKEEPING $\ldots \ldots \ldots \ldots \ldots \ldots \ldots . \ldots \ldots$

2.5 COMPLETION VERIFICATION PROCESS $\ldots \ldots \ldots \ldots \ldots \ldots \ldots .2-7$

2.6 TRANSITION LOGISTICS $\ldots \ldots \ldots \ldots \ldots \ldots \ldots \ldots \ldots \ldots . . \ldots \ldots$

2.7 M\&O TRANSITION MANPOWER AND COST ESTIMATE $\ldots \ldots \ldots \ldots 2-8$

2.8 TRANSITION SCHEDULE $\ldots \ldots \ldots \ldots \ldots \ldots \ldots \ldots \ldots \ldots \ldots \ldots \ldots . . \ldots \ldots \ldots$

2.9 ACTION DESCRIPTIONS . . . . . . . . . . . . . . . . . 2-11

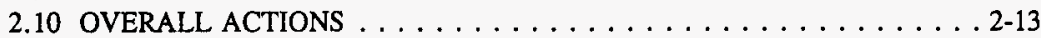

2.11 WHC CONTRACT CLOSEOUT ACTIVITIES $\ldots \ldots \ldots \ldots \ldots . \ldots .14$

3.0 MANAGEMENT AND INTEGRATION $\ldots \ldots \ldots \ldots \ldots \ldots \ldots \ldots \ldots \ldots$ 3-1

3.1 SITE PLANNING AND INTEGRATION . . . . . . . . . . . 3-1

3.1.1 Support to RL Strategic Planning .............. 3-1

3.1.2 Project/Program Planning and Baseline Management . . . . . . . 3-1

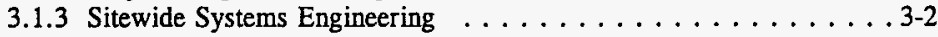

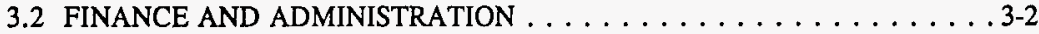

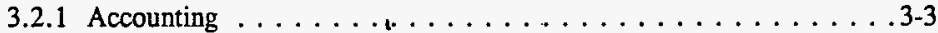

3.2.1.1 Accounts Receivables . . . . . . . . . . . . 3-3

3.2.1.2 Department Integrated Standardized Core Accounting

System (Discas) Interface for Plant and Equipment . . . . . 3-4

3.2.1.3 Transfer of General Ledger Accounts . . . . . . . . 3-4

3.2.1.4 Treasury . . . . . . . . . . . . . . . . 3-4

3.2.1.5 Payroll Activities . . . . . . . . . . . 3-5

3.2.1.6 Tax Filings - City, County, Federal . . . . . . . 3-5

3.2.1.7 Fiscal Year-End - Closeout . . . . . . . . . . 3-5

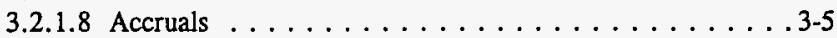

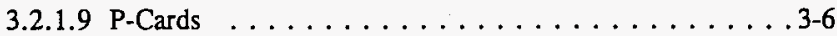

3.2.1.10 Travel . . . . . . . . . . . . . . . . 3-6

3.2.1.11 Work Authorization (Hanford Site) . . . . . . . 3-6

3.2.1.12 Work For Others (Non-Hanford) . . . . . . . . 3-7

3.2.1.13 Pensions . . . . . . . . . . . . . . . 3-7

3.2.1.14 Savings Plans . . . . . . . . . . . . . 3-7

3.2.1.15 Insurance Plans $\ldots \ldots \ldots \ldots \ldots \ldots \ldots . . \ldots \ldots$

3.2 .1 .16 Vacation . . . . . . . . . . . . . . 3-8

3.2.1.17 Nuclear Materials Accounting . . . . . . . . . . 3-8

3.2.2 Planning and Management Control Systems . . . . . . . . . 3-8

3.2.2.1 Environmental Planning Data System . . . . . . . 3-9

3.2.2.2 Financial Data System . . . . . . . . . . . 3-9 


\section{TABLE OF CONTENTS (cont'd)}

3.2.2.3 Management Control System . . . . . . . . . . . 3-10

3.2.2.4 DOE Site and Performance Reporting . . . . . . 3-11

3.2.2.5 Project Analysis \& Reporting System . . . . . . . . 3-11

3.2.2.6 Miscellaneous Planning \& Reporting Databases . . . . 3-11

3.2.2.7 Overhead Rate Structure . . . . . . . . . . 3-11

3.2.2.8 Financial Systems . . . . . . . . . . . 3-12

3.2.2.9 Miscellaneous Financial Administration and Control

Actions . . . . . . . . . . . . . 3-12

3.3 HUMAN RESOURCES . . . . . . . . . . . . . . . . 3-13

3.3.1 Personnel Resources (WHC/BCSR) . . . . . . . . . . 3-14

3.3.1.1 Staffing . . . . . . . . . . . . . . . 3-15

3.3.1.2 Site Access Support Services . . . . . . . . . 3-15

3.3.1.3 Outplacement . . . . . . . . . . . . . . 3-16

3.3.1.4 Personnel Records and Database Administration . . . . 3-17

3.3.2 Personnel Resources (ICF KH) . . . . . . . . . . . . 3-17

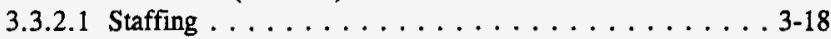

3.3.2.2 Site Access Support Services . . . . . . . . . . . 3-18

3.3.2.3 Outplacement . . . . . . . . . . . . . . . 3-19

3.3.2.4 Personnel Records and Database Administration . . . . 3-19

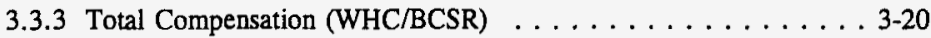

3.3.3.1 Salary Plan . . . . . . . . . . . 3-20

3.3.3.2 Workers Compensation . . . . . . . . . . 3-20

3.3.3.3 Job Classifications and Salary Structure ... . . . . 3-21

3.3.3.4 Performance Appraisals . . . . . . . . . . . . 3-22

3.3.3.5 Benefits Administration . . . . . . . . . . 3-22

3.3.3.6 Return-to-Work . . . . . . . . . . . . . . 3-25

3.3.3.7 Pension and Savings . . . . . . . . . . . 3-25

3.3.3.8 Recognition Programs . . . . . . . . . . . . . 3-26

3.3.3.9 Other . . . . . . . . . . . . . . . 3-26

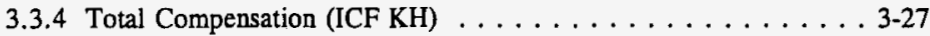

3.3.4.1 Salary Plan . . . . . . . . . . . . . 3-27

3.3.4.2 Other . . . . . . . . . . . . . . . 3-27

3.3.5 Labor Relations (WHC/BCSR) . . . . . . . . . . . 3-27

3.3.5.1 Collective Bargaining Agreements . . . . . . . 3-28

3.3.5.2 Bargaining Unit Leadership/Demographics . . . . . . 3-28

3.3.5.3 Notifications . . . . . . . . . . . . . . . . . 3.29

3.3.5.4 Grievance and Arbitration History/Caseload . . . . 3-29

3.3.6 Labor Relations (ICF KH) . . . . . . . . . . . . . . 3-30

3.3.6.1 Collective Bargaining Agreements . . . . . . 3-30

3.3.6.2 Bargaining Unit Leadership/Demographics . . . . . . 3-31

3.3.6.3 Notifications . . . . . . . . . . . . . . 3-31

3.3.6.4 Grievance and Arbitration History/Caseload ... . . 3-32 


\section{TABLE OF CONTENTS (cont'd)}

3.3.7 Workforce Development and Diversity (WHC/BCSR) . . . . 3-32

3.3.7.1 EEO/AAP . . . . . . . . . . . . . . 3-33

3.3.7.2 Diversity Recognition and Training . . . . . . . . . 3-33

3.3.7.3 Educational Reimbursement Programs . . . . . . . . 3-34

3.3.7.4 Development Training . . . . . . . . . . . . . . 3-34

3.3.7.5 Education Enrichment Programs . . . . . . . . 3-35

3.3.7.6 Graduate Rotational Program . . . . . . . . . . . 3-35

3.3.8 Workforce Development and Diversity (ICF KH) . . . . . . 3-35

3.3.8.1 EEO/AAP . . . . . . . . . . . . 3-35

3.3.8.2 Diversity Recognition and Training . . . . . . . 3-36

3.3.8.3 Education Enrichment Programs . . . . . . . . 3-36

3.3.9 General Operations . . . . . . . . . . . . . . . . . . 3-37

3.3.9.1 Transferring Vacation Hours for Family Medical

Emergencies . . . . . . . . . . . . . . . . . 3-37

3.3.9.2 Employee Work Schedules . . . . . . . . . . . 3-37

3.3.9.3 Salary Continuance During Absences . . . . . . . . . 3-38

3.3.9.4 Vacation and Holidays ... . . . . . . . . 3-38

3.3.9.5 Salaried Nonexempt Workforce Adjustment . . . . . . 3-39

3.3.9.6 Business Management . . . . . . . . . . . . . 3-39

3.3.10 Re-engineering Support . . . . . . . . . . . . . . . . 3-39

3.4 EMPLOYEE CONCERNS . . . . . . . . . . . . . . . . . . 3-40

3.5 ENVIRONMENTAL, SAFETY, QUALITY, AND HEALTH $\ldots \ldots \ldots 3-41$

3.5.1 Safety, Quality, and Health . . . . . . . . . . . . . 3-41

3.5.1.1 Program Management . . . . . . . . . . . 3-41

3.5.1.2 Safety . . . . . . . . . . . . . . 3-43

3.5.1.3 Radiological Control (RadCon) . . . . . . . . . . 3-46

3.5.1.4 Quality Assurance ... . . . . . . . . . . 3-51

3.5.1.5 Compliance Assurance . . . . . . . . . . . . 3-54

3.5.1.6 Facility Evaluation Boards . . . . . . . . . . 3-54

3.5.2 Work Control . . . . . . . . . . . . . . . . . . 3-55

3.5.3 Environmental Compliance . . . . . . . . . . . . . 3-56

3.5.3.1 Hanford Environmental Management Program . . . . . 3-59

3.5.3.2 Reporting . . . . . . . . . . . . 3-66

3.5.3.3 Environmental Requirements Management ... . . . 3-66

3.5.3.4 Non-Tri-Party Agreement Compliance Agreement . . . . 3-66

3.5.3.5 Outsources . . . . . . . . . . . . . . . 3-68

3.5.4 Tri-Party Agreement . . . . . . . . . . . . . . . 3-68

3.5.5 Safety Analysis and Nuclear Engineering . . . . . . . . . . 3-70

3.5.6 Occupational Health Services . . . . . . . . . . . . . 3-71

3.5.7 Centralized Occurrence Reporting . . . . . . . . . . . 3-71 


\section{TABLE OF CONTENTS (cont'd)}

3.6 PROCUREMENT AND MATERIALS MANAGEMENT $\ldots \ldots \ldots \ldots$. . 3-73

3.6.1 Procurement . . . . . . . . . . . . . . . 3-73

3.6.1.1 Open Audit/Surveillance Items . . . . . . . . . . . . 3-74

3.6.1.2 Identify Funding Reserves/Contingency Needs for Close

Out Process . . . . . . . . . . . . . . . 3-74

3.6.1.3 Purchase Order Assignment/Transfer to One or More

New Companies . . . . . . . . . . . . . . 3-74

3.6.1.4 Purchase Orders With Open Concerns . . . . . . . 3-74

3.6.1.5 Purchase Orders in Termination Process . . . . . . . . 3-75

3.6.1.6 Purchase Orders With Open Options . . . . . . . 3-75

3.6.1.7 Open Procurement Actions Beyond Transition . . . . . 3-75

3.6.1.8 Open Solicitations . . . . . . . . . . . . . 3-76

3.6.1.9 Purchase Order Terms and Conditions . . . . . . 3-77

3.6.1.10 Actions with DOE (RL \& HQ) . . . . . . . . 3-77

3.6.1.11 Subcontractor Property Activities . . . . . . . 3-77

3.6.1.12 Software License Agreements . . . . . . . . . 3-78

3.6.1.13 Procurement Reference Material . . . . . . . . . . . 3-78

3.6.1.14 Automation System Information . . . . . . . . . . . 3-78

3.6.1.15 Outreach Activities . . . . . . . . . . . . . 3-79

3.6.2 Property Management . . . . . . . . . . . . . . . . . 3-79

3.6.2.1 Capital and Sensitive Property . . . . . . . . . . 3-79

3.6.2.2 Other Financially Controlled Inventories . . . . . . . 3-80

3.6.2.3 Export Control and High Risk Property . . . . . . . 3-80

3.6.2.4 Activities with DOE (RL \& HQ) . . . . . . . . 3-80

3.6.2.5 Property Reference Material . . . . . . . . . . . . . 3-80

3.6.3 Economic Transfer . . . . . . . . . . . . . . . . . . . 3-81

3.6.3.1 Intellectual Property . . . . . . . . . . . . 3-81

3.6.3.2 Loan agreements, CRADAs and MOUs activities ... 3-81

3.6.3.3 Patent, Copyright, Royalty, and Invention activiti . . . 3-95

3.6.3.4 Asset Conversion Activities . . . . . . . . . . 3-81

3.6.3.5 Activities with DOE (RL \& HQ) $\ldots \ldots \ldots \ldots \ldots$ 3-82

3.6.4 Materials Management . . . . . . . . . . . . . . 3-82

3.6.4.1 P-Card Assignment/Transfer to One or More New

Companies . . . . . . . . . . . . . . . . . 3-82

3.6.4.2 Surplus Sales Assignment/Transfer to One or More

New Companies . . . . . . . . . . . . . 3-83

3.6.4.3 Warehouse Consolidation Plan (gas dock elimination, surplus material move into 1163 and configuration of 1167

into excess store) . . . . . . . . . . . . . . . . 3-83

3.6.4.4 General Services Administration (GSA) Waiver Request 3-83

3.6.4.5 Activities with DOE (RL \& HQ) . . . . . . . 3-83

3.6.4.6 Materials Management Reference Materials . . . . . . 3-83

3.7 RE-ENGINEERING $\ldots \ldots \ldots \ldots \ldots \ldots \ldots \ldots \ldots \ldots \ldots$.8 .84 


\section{TABLE OF CONTENTS (cont'd)}

3.8 TECHNOLOGY MANAGEMENT . . . . . . . . . . . . . . 3-85

3.8.1 National Technology Development Program (EM-50) . . . . . . 3-85

3.8.2 Technology Integration (Implementation), Site Technology

Coordination . . . . . . . . . . . . . . . . . . 3-87

3.9 GENERAL COUNSEL . . . . . . . . . . . . . . . . 3-88

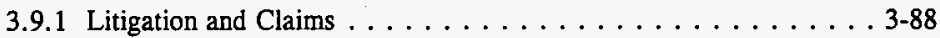

3.9.2 Transition Agreement . . . . . . . . . . . . . 3-88

3.10 INTERNAL AUDIT $\ldots \ldots \ldots \ldots \ldots \ldots \ldots \ldots \ldots \ldots \ldots . \ldots \ldots \ldots$

3.11 WORK FOR OTHERS $\ldots \ldots \ldots \ldots \ldots \ldots \ldots \ldots$. . . . . . . . . . . . . . . . .

3.11.1 Support to EM-40 Headquarters . . . . . . . . . . . . 3-90

3.11.2 Support to EM - Lease and Administration of Contractor Support

Facility . . . . . . . . . . . . . . . 3-90

3.12 OTHER MANAGEMENT AND INTEGRATION ACTIVITIES . . . . . . 3-91

3.12.1 Pollution Prevention/Waste Minimization . . . . . . . 3-91

3.12.2 U.S. Department of Energy Directives . . . . . . . . . 3-92

3.12.3 Hanford PeopleCORE . . . . . . . . . . . . . . . . 3.92

4.0 HANFORD PROJECTS $\ldots \ldots \ldots \ldots \ldots \ldots \ldots \ldots \ldots \ldots . \ldots \ldots$. . . . . . . . . .

4.1 TANK WASTE REMEDIATION SYSTEM $\ldots \ldots \ldots \ldots \ldots \ldots \ldots$. . . . . . .

4.1.1 Management Systems . . . . . . . . . . . . . 4-2

4.1.2 Tank Farm Operations - Evaporator . . . . . . . . . 4-3

4.1.3 Tank Farm Operations - 200 East Tank Farms . . . . . . . . 4-6

4.1.4 Tank Farm Operations - 200 West Tank Farms . . . . . . . . . . 4-9

4.1.5 Safety Issue Resolution/TWRS Engineering . . . . . . . . . 4-12

4.1.6 Waste Characterization . . . . . . . . . . . . . . 4-13

4.1.6.1 Waste Characterization - Technical Planning, Data

Evaluation, and Data Management Activities . . . . . . 4-14

4.1.6.2 Waste Characterization - Operations (Field) Activities . 4-15

4.1.7 Waste Disposal Program . . . . . . . . . . . . . 4-17

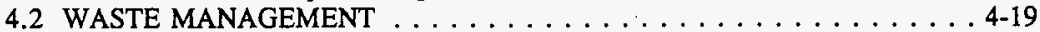

4.2 .1 Solid Waste . . . . . . . . . . . . . . . . 4-19

4.2.2 Liquid Effluents Program . . . . . . . . . . . . 4-25

4.2.2.1 200 Area Treated Effluent Disposal Facility . . . . . 4-26

4.2.2.2 Liquid Effluent Retention Facility . . . . . . . . . 4-29

4.2.2.3 200 Area Effluent Treatment Facility . . . . . . . . 4-31

4.2.2.4 300 Area Treated Effluent Disposal Facility . . . . . 4 4-33

4.2.2.5 340 Waste Handling Facility . . . . . . . . 4-35

4.3 SPENT NUCLEAR FUEL $\ldots \ldots \ldots \ldots \ldots \ldots \ldots \ldots \ldots \ldots . \ldots \ldots$ 4.38 $\ldots \ldots \ldots$

4.3.1 Spent Nuclear Fuel Project Integration . . . . . . . . . . . 4-39

4.3.2 Spent Nuclear Fuel Systems Integration . . . . . . . . . . . . . 4-39 . . . . . . . . . . . . .

4.3.3 K Basins Maintenance and Operations . . . . . . . . . . 4-40

4.3.4 K Basin Material Removal and Clean-Up Project . . . . . . . . . 4-41

4.3.5 Multi-Canister Overpack (MCO) Acquisition . . . . . . . 4-41 


\section{TABLE OF CONTENTS (cont'd)}

4.3.6 Cask/Transportation System . . . . . . . . . . . 4-41

4.3.7 Canister Storage Building . . . . . . . . . . . . 4-42

4.3.8 Fuel Conditioning Processes . . . . . . . . . . . . 4-42

4.3.9 Other Hanford Fuel Retrieval Processing and Storage . . . . . . 4-43

4.4 FACILITY STABILIZATION $\ldots \ldots \ldots \ldots \ldots \ldots \ldots \ldots \ldots \ldots$.43 $\ldots \ldots \ldots$

4.4 .1 PUREX $\ldots \ldots \ldots \ldots \ldots \ldots \ldots \ldots \ldots \ldots \ldots \ldots \ldots \ldots \ldots$

4.4.2 Plutonium Finishing Plant $\ldots \ldots \ldots \ldots \ldots \ldots \ldots$. . . . . . . . . .

4.4 .3 Fast Flux Test Facility . . . . . . . . . . . . . . . . 4-48

4.4.4 B Plant/Waste Encapsulation and Storage Facility . . . . . . . 4-50

4.4.5 300/400 Area Fuel Fabrication Facilities and 400 Area Fuels and Materials Examination Facility ... . . . . . . . . . 4-53

4.4.6 Transfer of 324 and 327 Building PNNL Facilities . . . . . . . 4-55

4.4.7 Materials and Future Transition Programs . . . . . . . . 4-58 4.5 ENVIRONMENTAL RESTORATION (ER) (INTEGRATION) . . . . . 4-60

5.0 INFRASTRUCTURE $\ldots \ldots \ldots \ldots \ldots \ldots \ldots \ldots \ldots \ldots \ldots$. . . . . . . . . . . . .

5.1 INFRASTRUCTURE OPERATIONS AND MAINTENANCE $\ldots \ldots \ldots .5-1$

5.1 .1 Facility Acquisition, Operations, and Maintenance $\ldots \ldots \ldots \ldots$ 5-1

5.1 .2 Utilities . . . . . . . . . . . . . . . . .5-3

5.1.2.1 Steam Plant Operations . . . . . . . . . . . 5-3

5.1.2.2 300 Area Water/Sewer Operations . . . . . . . 5-5

5.1.2.3 200 Area Water/Sewer Operations . . . . . . . 5-6

5.1.2.4 Site Electrical Systems . . . . . . . . . . . . . . 5-7

5.1.3 Transportation Infrastructure and Services . . . . . . . . . 5-9

5.1.4 Fire and Emergency Response . . . . . . . . . . . . 5-11

5.1.4.1 Fire Department Emergency Services ... . . . . 5-11

5.1.4.2 Fire Systems Testing and Emergency Response ... . . 5-11

5.1.4.3 Services Provided to Others ........... . 5-12

5.1.4.4 Other - New RL Work Scope Planned for the HFD . . . 5-12

5.1.4.5 Specialized Training for Emergency Response Fire

Department Personnel . . . . . . . . . . . . . . 5-12

5.1.4.6 Fire Prevention . . . . . . . . . . . . . 5-12

5.1.4.7 Fire Systems Maintenance . . . . . . . . . 5-12

5.1.4.8 Respiratory Protection Equipment Services ... . . . 5 5-12

5.1.4.9 Contracts for Service and Purchase Requisitions that Extend Past October 1, $1996 \ldots \ldots$. . . . . . 5-13

5.1.4.10 Emergency Services Facilities, Buildings, and Capital

Projects for Replacement . . . . . . . . . . . . . 5-13

5.1 .5 Fabrication Shops . . . . . . . . . . . . . . . 5-15

5.1.6 Integrated Pest Management Services . . . . . . . . . . 5-17

5.1.7 Non-Radioactive/Non-Dangerous Waste Handling $\ldots \ldots \ldots .5$ 5-19

5.1 .8 Site Crane \& Rigging Services . . . . . . . . . . . . 5-21

5.1 .9 Landlord Program . . . . . . . . . . . . . 5-22 


\section{TABLE OF CONTENTS (cont'd)}

5.1.10 Resource and Energy Management . . . . . . . . . . . 5-23

5.2 REAL ESTATE AND MATERIAL MANAGEMENT $\ldots \ldots \ldots \ldots$ 5-25

5.2.1 Land-Use Planning and Management $\ldots \ldots \ldots \ldots \ldots \ldots$ 5-25

5.2.2 Calibration and Engineering Laboratories . . . . . . . . . 5-27

5.2.2.1 Engineering Laboratories . . . . . . . . . . . . 5-27

5.2.2.2 Calibration Laboratory (AKA Standards Laboratory) . . 5-28

5.3 INFORMATION AND COMMUNICATION $\ldots \ldots \ldots \ldots \ldots \ldots .5$ 5-28

5.3 .1 Operations and Support . . . . . . . . . . . . . 5-29

5.3.2 Information and Scientific Systems . . . . . . . . . . . . . 5-29

5.3.3 President's Office/Technical Staff/Business Management . . . . 5-30

5.3.4 Documentation and Records Management . . . . . . . . 5-31

5.3.5 Media Management Systems . . . . . . . . . . . 5-32

5.3.6 Telecommunications and Network Engineering . . . . . 5.33

5.3.7 Baseline Summary Outline . . . . . . . . . . . . . 5-34

5.3.8 Hanford Information System Inventory $\ldots \ldots \ldots \ldots \ldots \ldots$. . . . . . .

5.3.9 Data Management and Standards . . . . . . . . . . 5-36

6.0 OTHER CROSSCUTTING SERVICES $\ldots \ldots \ldots \ldots \ldots \ldots \ldots \ldots$. $\ldots \ldots \ldots$

6.1 SAFEGUARDS AND SECURITY $\ldots \ldots \ldots \ldots \ldots \ldots \ldots \ldots \ldots \ldots$ 6-1

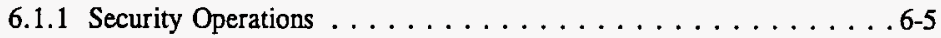

6.1 .2 Protective Forces $\ldots \ldots \ldots \ldots \ldots \ldots \ldots \ldots \ldots$

6.1 .3 Information Security $\ldots \ldots \ldots \ldots \ldots \ldots \ldots \ldots .6 \ldots .6 \ldots$

6.1.4 Personnel Security and Central Badging . . . . . . . . . 6-8

6.1.5 Protection Program Management . . . . . . . . . . . . 6-8

6.1.6 Material Control and Accountability . . . . . . . . . . . . . 6-9

6.1 .7 Technical Security . . . . . . . . . . . . . . . 6-10

6.2 HANFORD ANALYTICAL SERVICES PROGRAM . . . . . . . . . 6-10

6.2.1 Sample Management Office . . . . . . . . . . . 6-11

6.2.2 High Level Analytical Services . . . . . . . . . . . . . . 6-12

6.2.3 Low-Level Analytical Services . . . . . . . . . . . . . . 6-14

6.3 HANFORD SITE TRAFFIC MANAGER . . . . . . . . . . . 6-16

6.3.1 Hanford Site Transportation and Packaging Support . . . . . . 6-17

6.3.2 DOE Headquarters EM Transportation Program Support . . . . 6 6-19

6.3.3 Transportation and Packaging Work For Others Services . . . . 6-20

6.4 ARCHITECT ENGINEER/CONSTRUCTION MANAGEMENT . . . . . 6-20

6.4.1 Architect Engineer Services . . . . . . . . . . . . 6-21

6.4.2 Construction Management Services . . . . . . . . . . 6-24

6.5 CONSTRUCTION PROJECT MANAGEMENT . . . . . . . . . 6-26

6.6 ENVIRONMENTAL MONITORING AND SERVICES $\ldots \ldots \ldots \ldots \ldots 6-27$

6.6.1 Hanford Technical Services . . . . . . . . . . . . 6-27

6.6.1.1 RCRA Operational Monitoring Office . . . . . . . 6-28

6.6 .1 .2 Geosciences . . . . . . . . . . . . 6-28

6.6 .1 .3 Well Services . . . . . . . . . . . . 6-30 
TABLE OF CONTENTS (cont'd)

6.6.1.4 Near Field Monitoring $\ldots \ldots \ldots$. . . . . . . 6-31

6.6.1.5 Geophysics Investigations . . . . . . . . . . . . 6-32

6.6.1.6 Environmental Operations Sampling . . . . . . . . 6-33

6.6.1.7 Special Projects . . . . . . . . . . . . . 6-35

6.6.2 CERCLA Monitoring . . . . . . . . . . . . . 6-35

6.6.3 Groundwater Surveillance Monitoring Project . . . . . . . 6-36

6.7 EXTERNAL/INTERNAL COMMUNICATIONS . . . . . . . . . . . 6-37

6.7.1 Interface with Site Communications/Public Affairs . . . . . . . 6-37

6.7.2 Integration of WHC/ICF KH/BCSR Program Components Into the PHMC Program . . . . . . . . . . . . . . . . 6-37

6.7.3 Tri-Party Agreement Community Relations Plan . . . . . . . . 6-37

6.7.4 Introduce the PHMC Personnel to Hanford's External Publics . . 6-38

6.7.5 Provide Employees With Necessary Information During Transition 6-38

6.7.6 U.S. Department of Energy "Hanford Reach" and "DOE This Month" Communications . . . . . . . . . . . . . . . . 6-39

6.7.7 Hanford Site Emergency Preparedness Communications . . . . . 6-40

6.7.8 WHC/ICF Kaiser/BCSR Farewell Activities . . . . . . . . 6-40

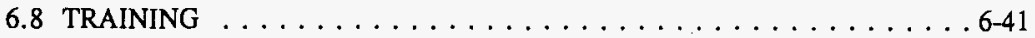

6.8 .1 Training Services . . . . . . . . . . . . 6-41

6.8.2 Hazardous Materials Management and Emergency Response . . 6 6-43

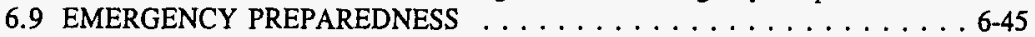

6.9 .1 Emergency Management . . . . . . . . . . . . . 6-45

6.9.2 Radiological Assistance Program . . . . . . . . . . 6-46

6.9.3 ESH Management Plan . . . . . . . . . . . . . . . . . . . . . 6-48

6.10 DIRECT SUPPORT TO RL $\ldots \ldots \ldots \ldots \ldots \ldots \ldots \ldots \ldots .48$ 
WHC-SP-1181 Rev. 1

TABLE OF CONTENTS (cont'd)

\section{FIGURE}

2-1. Project Hanford Management Contractor Summary Transition Schedule . . . . . 2-10

\section{TABLE}

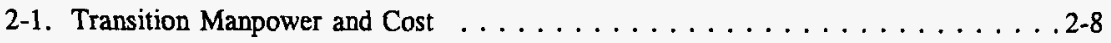




\section{ACRONYMS}

AAP

ACES

ADA

ADS

A-E

ALARA

AOP

APIP

ASME

ASNT

ATP

BAT/AKART

BCC

BCSR

BEMR

BHI

BOE

CAA

CAMB

CAMS

CAP

CDMP

CDR

CERCLA

CFR

CIO

CM

CMM

COE

COMSEC

$\mathrm{COO}$

CSB

CSG

CWA

CWC

D\&D

DCRT

DISCAS

DNFSB

DOE

DOT
Affirmative Action Plan

Access Control Security System

Americans With Disabilities

Activity Data Sheet

Architect-Engineer

As Low As Reasonably Achievable

Air Operating Permit

Assessment Program Implementation Plan

American Society of Mechanical Engineers

American Society for Nondestructive Testing

Acceptance Test Procedure

Best Available Technology/All Known And Reasonable

Treatment

Baseline Change Control

BCS Richland, Inc.

Baseline Environmental Management Report

Bechtel Hanford, Inc.

basis of estimate

Clean Air Act

Corrective Action Management Board

Corrective Action Management System

Cost Account Plan

Completed Decision Making Package

Conceptual Design Report

Comprehensive Environmental Response Compensation and

Liability Act

Code of Federal Regulations

Chief Information Officer

construction management

Central Milestone Module

center of expertise

Communications Security

Conduct of Operations

Canister Storage Building

Central Support Group

Clean Water Act

Central Waste Complex

Decontamination and Decommissioning

double-contained receiver tank

Department Integrated Standardized Core Accounting System

Defense Nuclear Facilities Safety Board

U.S. Department of Energy

Department of Transportation 


\section{ACRONYMS (cont'd)}

$\begin{array}{ll}\text { DQO } & \text { data quality objective } \\ \text { DST } & \text { double-shell tank } \\ \text { DW } & \text { dangerous waste } \\ \text { EA } & \text { environmental assessment } \\ \text { EAP } & \text { Environmental Assurance, Permits and Policy } \\ \text { ECM } & \text { Environmental Compliance Manual } \\ \text { ECN } & \text { Engineering Change Notice } \\ \text { Ecology } & \text { Washington State Department of Ecology } \\ \text { ECP } & \text { Employee Concerns Program } \\ \text { EEO } & \text { Equal Employment Opportunity } \\ \text { EEOC } & \text { Equal Employment Opportunity Commission } \\ \text { EIS } & \text { Environmental Impact Statement } \\ \text { EO } & \text { Executive Order } \\ \text { EL } & \text { Engineering Laboratories } \\ \text { EM } & \text { Environmental Management } \\ \text { EMP } & \text { Effluent Monitoring Plan } \\ \text { EMT } & \text { Emergency Medical Technician } \\ \text { EPA } & \text { Environmental Protection Agency } \\ \text { EPCRA } & \text { Emergency Planning and Community Right-To-Know Act } \\ \text { EPDS } & \text { Environmental Planning Data System } \\ \text { ER } & \text { environmental restoration } \\ \text { ERC } & \text { Environmental Restoration Contractor } \\ \text { ERDF } & \text { Environmental Restoration Disposal Facility } \\ \text { ESH } & \text { Environmental, Safety, and Health } \\ \text { ETF } & \text { Effluent Treatment Facility } \\ \text { ETS } & \text { Environmental Tracking System } \\ \text { EU } & \text { electrical utilities } \\ \text { FDS } & \text { Financial Data System } \\ \text { FEB } & \text { Facility Evaluation Board } \\ \text { FEMP } & \text { Facility Effluent Monitoring Plan } \\ \text { FFCA } & \text { Federal Facilities Compliance Agreement } \\ \text { FFTF } & \text { Fast Flux Test Facility } \\ \text { FMCS } & \text { Federal Mediation and Conciliation Service } \\ \text { FMEF } & \text { Fuels and Materials Examination Facility } \\ \text { FOCI } & \text { Foreign Ownership Control and Influence } \\ \text { FP } & \text { fire protection } \\ \text { FSM } & \text { Fire Systems Maintenance } \\ \text { FTE } & \text { full-time equivalent } \\ \text { FY } & \text { fiscal year } \\ \text { GPF } & \text { general purpose facility } \\ \text { GSA } & \text { General Services Administration } \\ \text { GTR } & \text { Government Transportation Request } \\ \text { GWM } & \text { Groundwater Management } \\ & \\ & \end{array}$




\section{ACRONYMS (cont'd)}

HAMMER

HAMTC

HASP

HATS

HDPE

HEHF

HEIS

HEMP

HFD

HGET

HGIS

HGU

HISI

HLAN

HLW

HR

HRC

HRP

HSRCM

HSIS

HSSA

HTS

HQ

IAEA

IAMIT

ICF $\mathrm{KH}$

IDWA

IH

IMUST

INEL

IP/ER

IPC

IROF

IS

ISS

IWR

JCS

JIC

LERF

LLBG

LLMW

LLW

LP
Hazardous Materials Management and Emergency Response

Hanford Atomic Metal Trades Council

Hanford Analytical Services Program

Hanford Action Tracking System

High-Density Polyethylene

Hanford Environmental Health Foundation

Hanford Environmental Information System

Hanford Environmental Management Program

Hanford Fire Department

Hanford General Employee Training

Hanford Geographic Information System

Hanford Guards Union

Hanford Information System Inventory

Hanford Local Area Network

High-Level Waste

Human Resources

Human Rights Commission

Human Reliability Program

Hanford Site Radiological Control Manual

Hanford Site Integrated Schedule

Hanford Site Stabilization Agreement

Hanford Technical Service

Headquarters

International Atomic Energy Agency

Inter-Agency Management Integration Team

ICF Kaiser Hanford

Inter-Department Work Authorization

Industrial Hygiene

Inactive Miscellaneous Underground Tank

Idaho National Engineering Laboratory

Implementation Plan/Exemption Request

Integration Project Coordinator

Involuntary Reduction of Force

Industrial Safety

Information and Scientific Systems

Inter-Agency Work Request

Job Control System

Joint Information Center

Liquid Effluent Retention Facility

Low-Level Burial Ground

Low-Level Mixed Waste

Low-Level Waste

Landlord Program 
WHC-SP-1181 Rev. 1

ACRONYMS (cont'd)

M\&O

MBA

MC\&A

MCAP

MCO

MCS

MDD

MIP

MMS

MOU

MRP

MSDS

MSA

MT

MVT

MYPP

NDA

NDE

NEPA

NESHAP

NEx

NFPA

NIOSH

NIST

NLRB

NNPP

NOV

NPDES

NRDWSF

NTS

OFCCP

OMP

ONC

OPC

OPEIU

ORPS

ORR

OSHA

P2/WMin

P\&E

PA

PAAA

PAPR
Management and Operating

Material Balance Area

Material Control and Accountability

Material Control and Accountability Plan

Multi-Canister Overpack

Management Control System

Mission Direction Document

Management Integration Plan

Media Management Systems

Memorandum of Understanding

Management Requirements and Procedures

Material Safety Data Sheet

Major System Acquisition

Magnetic Particle Testing

Master Validation Table

Multi-Year Program Plan

Nondestructive Assay

Nondestructive Examination

National Environmental Policy Act of 1969

National Emission Standards for Hazardous Air Pollutants

Non-Exempt

National Fire Protection Association

National Institute of Safety and Health

National Institute of Standards and Technology

National Labor Relations Board

Naval Nuclear Propulsion Program

notice or violation

National Pollutant Discharge Elimination System

Nonradioactive Dangerous Waste Storage Facility

Noncompliance Tracking System

Office of Federal Contract Compliance Program

Operations and Maintenance Program

Occurrence Notification Center

Other Project Costs

Office of Professional Employees International Union

Occurrence Reporting Processing System

Official Readiness Review

Occupational Safety and Health Administration

Pollution Prevention/Waste Minimization

Plant and Equipment

Performance Assessment

Price Anderson Amendment Act

Powered Air Purifying Respirator 


\section{ACRONYMS (cont'd)}

PAR

PCB

PEC

PFP

PHMC

PM

PMCS

PMM

PNNL

POC

PPA

PPM

PRTR

PSAP

PT

PTS

PUREX

QA

QC/NDT

R\&EM

RAP

RCRA

RDA

RD\&D

RDS

REAC/TS

RFP

RFS

RIDS

RL

RLPS

ROC

ROF

RMW

ROM

RT

S\&H

SA\&NE

SAIC

SALDS

SAR

SARP

SAS
Project Analysis and Reporting System

polychlorinated biphenyl

pre-existing condition

Plutonium Finishing Plant

Project Hanford Management Contractor

preventive maintenance

Project Management and Control System

Procurement and Materials Management

Pacific Northwest National Laboratory

Point-of-Contact

Property Protected Area

Protection Program Management

Plutonium Recycle Test Reactor

Personnel Security Assurance Program

Penetrant Testing

Progress Tracking System

Plutonium-Uranium Extraction (Facility)

Quality Assurance

Quality Control and Nondestructive Technicians

Resource and Energy Management

Radiological Assistance Program

Resource Conservation and Recovery Act

Re-Deployment Assistance

Research, Development, and Demonstration

Risk Data Sheet

Radiological Emergency Assistance Control/Training Site

Request For Proposal

Request For Service

Records Inventory and Disposition Schedules

U.S. Department of Energy-Richland Operations Office

RL Property System

Re-Employment Opportunity Center

Reduction of Force

Radioactive Mixed Waste

RCRA Operational Monitoring

Radiographic Testing

Safety and Health

Safety Analysis and Nuclear Engineering

Science Applications International Corporation

State-Approved Land Disposal Site

Safety Analysis Report

Safety Analysis Report for Packaging

Safeguards and Security 


\section{ACRONYMS (cont'd)}

SC\&RS

SCBA

SEP

SFS

SGML

SID

SL

SMO

SMP

SMS

SNF

SNM

S/RIDS

SSPP

SST

STCG

SW

SWD

TBP

TEDF

TEC

TFO

TPA

TPAI

TPC

TRS

TRU

TRUMW

TRUPACT

TRUSAF

TSCA

TSD

TTP

TWRS

$\mathrm{UO}_{3}$

VA

VLDPE

VPP

WAC

WBS

WDOH
Site Crane and Rigging Services

Self-Contained Breathing Apparatus

Safety Evaluation for Packaging

Site Fabrication Service

Standard Generalized Markup Language

Site Infrastructure Division

Standards Laboratory

Sample Management Office

Software Management Plan

Site Management System

Spent Nuclear Fuel

Special Nuclear Material

Standards and Requirements Identification and Documentation

System

Site Support Program Plan

Single-Shell Tank

Site Technology Coordination Group

Solid Waste

Solid Waste Disposal

Tri Butyl Phosphate

Treated Effluent Disposal Facility

Total Estimated Cost

Tank Farm Operations

Tri-Party Agreement

Tri-Party Agreement Integration

Total Project Cost

Training Services

Transuranic

Transuranic Mixed Waste

Transuranic Package Transporter

Transuranic Assay and Storage Facility

Toxic Substance Control Act

Treatment, Storage, and Disposal

Technical Task Plan

Tank Waste Remediation System

Uranium Trioxide

Vulnerability Assessment

Very Low-Density Polyethylene

Voluntary Protection Program

Washington Administrative Code, or

Waste Acceptance Criteria

Work Breakdown Structure

Washington State Department of Health 
WHC-SP-1181 Rev. 1

\section{ACRONYMS (cont'd)}

WEC

WESF

WHC

WIDS

WIPP

WRAP

WSCF
Westinghouse Electric Corporation

Waste Encapsulation and Storage Facility

Westinghouse Hanford Company

Waste Information Data System

Waste Isolation Pilot Plant

Waste Receiving and Processing Module

Waste Sampling and Characterization Facility 


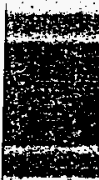




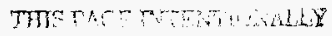
A C BLANK 


\subsection{INTRODUCTION}

This is a major revision to this transition plan to incorporate a change to the Project Hanford Management Contractor (PHMC) contract award date from June 1, 1996, to the latter part of July 1996.

This plan has been developed by Westinghouse Hanford Company (WHC), and its subcontractors ICF Kaiser Hanford (ICF KH) and BCS Richland, Inc. (BCSR), at the direction of the U.S. Department of Energy (DOE), Richland Operations Office (RL). WHC and its subcontractors are hereafter referred to as the Management and Operating (M\&O) Contractor. The plan identifies actions involving the M\&O Contractor that are critical to (1) prepare for a smooth transition to the PHMC, and (2) support and assist the PHMC and RL in achieving transition as planned, with minimal impact to ongoing baseline activities.

The plan is structured around two primary phases. The first is the pre-award phase, which started in mid-February 1996 and is currently scheduled to be completed by July 31 , 1996. The second is the follow-on two-month post-award phase during August and September of 1996, resulting in contract takeover October 1, 1996. Considering the magnitude and complexity of the scope of work being transitioned, completion in two months will require major effort by all parties. To better ensure success, the M\&O Contractor has developed a pre-award phase that is intended to maximize readiness for transition. Priority is given to preparation for facility assessments and processing of personnel, as these areas are determined to be on the critical path for transition. In addition, the M\&O Contractor will put emphasis during the pre-award phase to close out open items prior to contract award, to include grievances, employee concerns, audit findings, compliance issues, etc.

Even though committed to transition, the M\&O Contractor's priority remains with completion of the currently agreed to baseline scope of work. In going from a four-month transition period to a two-month transition period, especially at the end of the fiscal year, some currently planned $M \& O$ Contractor activities will be impacted. It is the intent of the M\&O Contractor to minimize such impacts, but the extent of impacts will be determined on how many of the assumptions and approaches for transition contained within this revised plan are accepted by RL and the PHMC. For example, if the PHMC elects to bring in a large staff to support a 100 percent walk through of the site as part of their pre-existing condition assessment, ongoing operations would be significantly impacted. Based on the assumptions contained in this plan, most impacts should be administrative in nature.

Section 2.0 contains key issues and assumptions, an overall transition schedule, and generic definitions for some of the important repetitive actions. The remaining sections are basically organized around the statement of work contained within the PHMC Request for Proposal (RFP) and provide general descriptions of work, along with pre- and post-award actions to be completed, the date to be completed, and the responsible assignee(s). The preaward actions have been captured in an action tracking system and are being routinely statused until completion. Due to the shortened transition period, a more detailed transition schedule reflecting activities by all parties has been included as Appendix A. In lieu of a 
consolidated transition plan, which is called for in the current request for proposal, the M\&O Contractor recommends, immediately following contract award, that the parties jointly update the schedule and establish a list of actions using the action tracking system.

Pacific Northwest National Laboratory (PNNL), Bechtel Hanford, Inc. (BHI), and Hanford Environmental Health Foundation (HEHF) have continued to be involved in the development of this plan. They have elected to include specific actions on their part to better ensure integration of their activities with those of the PHMC during transition. In addition, and in coordination with RL, selected RL actions have been included to facilitate transition. 

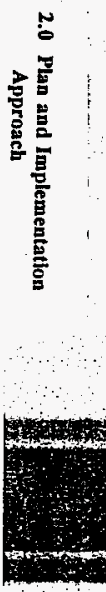


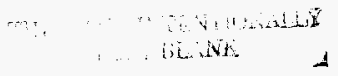




\subsection{PLAN AND IMPLEMENTATION APPROACH}

This plan has been developed by a broad-based M\&O Contractor Transition Team representing all areas of WHC and its subcontractors, ICF KH and BCSR (hereafter referred to as the M\&O Contractor). In addition, close coordination was maintained with and support was provided by the RL multi-contractor team and the RL extended transition team points of contact. The contents of the plan are based on the PHMC RFP (RL 1996a, as amended), and reflect the experience and knowledge of the participating team members to ensure an orderly and timely transition. In addition, transition plans from both prior Hanford transitions and recent transitions at other DOE sites were used in structuring this plan.

The M\&O Contractor transition effort is being managed and executed as a project. A Project Manager has been designated who is directly responsible to the President of WHC for successfully carrying out the M\&O Contractor transition activities necessary for a smooth transition to the PHMC. Each program and functional area has designated a transition team member, who reports directly to the Project Manager for activities governed by this plan.

\subsection{PLAN CONTENT AND USE}

This plan has been organized around the statement of work contained within the PHMC RFP and supports the RL Transition Plan for the PHMC (RL 1996b), dated February 1996, with the exception of the change to the transition period. The plan identifies action items necessary to achieve a smooth transition in two phases. The first phase is the pre-award period (i.e., pre-transition) and the second phase is post-award (i.e., transition). In addition to M\&O Contractor actions, this plan includes actions from other Hanford contractors to ensure integration of their activities with those of the PHMC. Specific actions that are solely the responsibility of the PHMC per the RFP are not identified in this plan, but a more definitive transition schedule has been included as Appendix A, which reflects activities involving all participants.

This plan will continue to govern the execution of the extended pre-award phase. All pre-award actions have been assigned a specific completion date and a responsible individual(s). The pre-award phase includes conduct of those actions that can be completed without knowledge or participation of the PHMC, with the goal to enhance the readiness to initiate transition.

The current RL transition plan shows the development of a consolidated RL, M\&O Contractor, and PHMC transition plan within one month following contract award. Considering a two-month transition period, the M\&O Contractor recommends that a formal consolidated plan not be produced. Instead, the schedule contained in Appendix A should be updated and enhanced as the consolidated transition plan, and additional supporting actions be added to the action tracking system (see Section 2.4). 
This plan does not include actions that are related to closeout of the M\&O contract. A separate $M \& O$ Contractor team will be established to plan and execute this effort.

\subsection{KEY ISSUES}

The following identifies key issues which, if not resolved or clarified, could impact the ability for a smooth transition:

- The initial version of this plan assumed a four-month transition period, which in itself was considered a key issue as far as time available for a smooth transition. To transition in two months will require significant effort and coordination by all parties. The assumptions and conditions associated with a two-month transition are critical, and have therefore been expanded in Section 2.3 and further defined under each program or functional area. To try and accomplish more than proposed in this plan could result in a condition that the PHMC is not ready to takeover on October 1, 1996. DOE must accept that many of the changes in business practices proposed by the PHMC that would have been implemented during a longer transition period, will now be deferred for implementation after transition. In addition, sufficient time or resources will not be available for $100 \%$ assessments and inventories. Therefore, associated liabilities must be adequately addressed for all parties involved.

- Even though the M\&O Contractor is committed to support transition, the priority remains with completion of existing planned workscope and associated commitments in a safe and environmentally sound manner. Even considering the approach proposed in this plan, which is designed to minimize impacts to ongoing work, it is fully expected that conflicts between current commitments and transition activities will occur. RL must be willing to accept such impacts and negotiate equitable changes appropriately. A decision by DOE and/or the PHMC to accomplish more than proposed in this plan during transition would further exacerbate this situation.

- BHI and WHC are currently in the process of trying to resolve landlord responsibility issues that still remain from the previous Environmental Restoration Contractor (ERC) transition. RL has been party to these discussions. These issues need to be resolved prior to PHMC contract award. RL needs to clarify roles of all contractors, including PNNL, delineating who is responsible for what, so they do not affect the transition to the PHMC.

- Transfer of contractor responsibility as co-operator for Resource Conservation and Recovery Act of 1976 (RCRA) permitting documentation requires 90 days advance notification to the Washington State Department of Ecology (Ecology), and submittal of associated and recertified permitting documentation, unless relief is obtained from Ecology. WHC cannot remain on permits after contract 
WHC-SP-1181 Rev. 1

transition. This is consistent with Section $\mathrm{E}$ of the "Agreement on Regulatory Interactions" between RL and WHC, dated August 14, 1995.

\subsection{ASSUMPTIONS}

The following assumptions relate to the key issues described in Section 2.2:

- In addition to those provided within each functional area, the following general assumptions are critical to transitioning in two months:

- All incumbent WHC/BCSR/ICF KH employees, other than the approximately 230 employees occupying management positions as defined in the RFP (i.e., "...those above first-line managerial/supervisory level...") will, in effect, be "mapped" to the PHMC organization. Mapping of employees, to include interviews/offers to management personnel will be completed by September 1, 1996. Involuntary separation notices to managers not employed by the PHMC will be issued no sooner than September 30, 1996.

- Critical PHMC personnel necessary for transition will be on board no later than August 5, 1996 (based on an award by July 31, 1996).

- To allow adequate time to prepare facilities, RL will provide authorization no later than July 1, 1996 for the M\&O Contractor to establish fully equipped office space for the PHMC. Space for up to 200 PHMC personnel will be provided.

- The PHMC will continue to use existing WHC systems, manuals, and forms for finance and accounting, management and control, procurement, property accounting, human resources, etc. Except for those changes mandatory to make them function for the PHMC, all other changes to these systems will be made after the PHMC assumes responsibility for the site activities.

- RL will attempt to minimize external interaction on the site during transition as much as possible, or attempt to integrate such interactions with transition. There are some activities however, that will still occur and will be factored into the planning for transition as they are identified. Activities by the Defense Nuclear Safety Board or the regulators are examples, as the actions taken by these organizations are not always controlled by DOE. As these activities are identified, DOE will notify WHC as soon as possible so that they may be considered and work reprioritized if necessary. WHC will bring such interferences in the transition schedule to the attention of the DOE Transition Manager.

- RL and the PHMC shall conduct their readiness review activities in parallel with transition actions. 
- The bulk of field assessment and inventory activities must be completed by mid September to allow sufficient time for final verification of readiness, corrective actions, and completion of a Transition (or Transfer) Agreement. The PHMC will be ready to initiate field activities by August 12, 1996 to include required training and clearance for access.

- A joint process will be established by August 1, 1996 and implemented by August 6, 1996 to resolve issues and disagreements in real time to ensure progress continues on schedule. The process must be capable of presenting an issue to affected parties within 24 hours of identification, followed by resolution within two days. BHI, HEHF, and PNNL will be party to this process along with $\mathrm{RL}$, the M\&O Contractor, and the PHMC.

- All parties shall establish a defined process for making timely decisions on their part in support of transition. Responsible individuals for making decisions will be clearly identified to all parties.

- For areas where accountability is not fully determined (including, but not limited to, facility pre-existing conditions and property), DOE or the PHMC must assume liability, or continue to support a cognizant WHC team as part of contract closeout following transition.

- Tours and briefings will be limited and focused. Senior PHMC management will be limited to site and major program briefings, and facility discussions and tours will be conducted in parallel and directed at the incoming cognizant facility management personnel.

- 100 percent walkthroughs will not be conducted in support of pre-existing condition assessments. The PHMC shall review evaluation packages provided, appropriately assess areas of uncertainty, and selectively verify the accuracy of the data provided.

- A formal consolidated transition plan will not be prepared as currently called for in the request for proposal. A transition schedule (like Appendix A) with a supporting action list will be used.

- RL shall provide the M\&O Contractor satisfactory relief for scope and commitments that must be impacted in order to accomplish transition. Specifically:

- The M\&O Contractors priority will remain with the current scope and commitments, unless such relief is obtained.

- M\&O Contractor personnel critical to successful completion of current scope and commitments, to include $M \& O$ Contractor transition efforts, will not be secunded by the PHMC for their use during transition. 
- RL shall resolve outstanding landlord responsibility issues between WHC and BHI prior to contract award. In addition, RL will clarify any other remaining scope or responsibility issues among Hanford contractors.

- Decisions concerning the transfer of program scope between existing contractors (BHI, WHC, HEHF, and PNNL) will occur prior to contract award, or will be deferred until after contract takeover. Examples are the transfer of Environmental Management (EM-50) technology tasks or environmental monitoring activities.

- RL shall obtain relief from Ecology concerning the 90-day requirement for advance notification of a signatory change to a RCRA permit.

- RL shall notify the PHMC by August 5, 1996 of the plan to move 324 Building and 327 Building responsibility and associated workscope from PNNL to the PHMC by October 1, 1996.

The following additional assumptions are provided:

- Transition plans submitted with the PHMC proposals will not be made available to the M\&O Transition Team prior to contract award. Following award, the PHMC transition plan for the selected PHMC will be provided to the M\&O Transition Team. Prior versions of the M\&O Transition Plan have been placed in the DOE reading room for access by the proposing teams, and it is assumed that this revision will also be placed in the DOE reading room.

- Prior to contract award, RL will clarify PNNL and PHMC roles with respect to site planning and integration support to RL so that it can be properly addressed in transition.

- On October 1, 1996 all work will be transferred to the PHMC. The M\&O Contractor will continue beyond takeover on activities associated with contract closeout and as provided for by the PHMC.

- Prior to contract award RL will clarify what support to DOE (HQ and RL) and other contractors will continue as part of the PHMC scope.

- Tank Waste Remediation System (TWRS) Privatization will proceed as currently planned through transition.

- All formal transition actions, decisions, and agreements will be made with the PHMC integrating contractor, and not the major subcontractors. The PHMC integrating contractor will assign a transition manager as the primary point-ofcontact (POC). This is not intended to preclude day-to-day discussions, presentations, requests for information, facility inspections, etc., which will occur among all contractors involved. 
- All actions completed per the transition plan will be adequately documented as completed by the assigned responsible individual(s).

- The cost of space and services provided for use by the PHMC will be covered by the PHMC's transition account. This would not apply to turn-around space provided in existing satellite areas, but would apply to services obtained for that space specifically for PHMC use.

- RL shall approve program change requests, which are considered necessary to carry out the M\&O Contractor scope contained in this transition plan. For areas that do not submit change requests, the actions contained within this plan are considered to be included in the authorized scope of the direct or indirect program to which it is charged.

- All materials produced for formal briefings to the PHMC will be coordinated and reviewed with the appropriate RL POC. This does not apply to informal briefings (or discussions) and workshops, recognizing that the RL POC may participate in such activities.

\subsection{ACTION TRACKING AND RECORDKEEPING}

An action tracking system has been established to track the actions contained in this transition plan, and subsequently those developed jointly with the PHMC. The system includes for each action:

- Unique identification number cross-referenced to the appropriate transition plan section

- Program/functional area

- Action description

- Responsible individual(s)

- Planned completion date

- Actual completion date

- Status.

The system has multiple sorting capabilities, and will be the primary tool used in status meetings between the participating organizations.

Updates to actions (including addition of new actions, date changes, and actionee changes) will be made through the action tracking system. This will eliminate the need to 
continually update the plan itself. The plan would only be updated for a major change (i.e., extension of transition period) that would result in many actions having to be revised at one time.

WHC shall maintain a record of transition activities involving the M\&O Contractor. This will include documented completion of actions, transition agreements that are established, and any other pertinent data.

\subsection{COMPLETION VERIFICATION PROCESS}

Selected actions associated with programs and their facilities will require verification of completeness by the responsible RL program. Such actions will include, but are not limited to, property inventories, special nuclear material (SNM) inventories, and assessments of pre-existing conditions. This verification requirement will be integrated into the readiness review conducted during transition.

\subsection{TRANSITION LOGISTICS}

A key element towards a successful transition is ensuring that sufficient and adequate space and services are made available to transition participants in a timely manner. The M\&O Contractor is prepared to assist in this effort. This applies to central space and satellite space within program/project areas, facilities, and functional areas. Specific action items have been identified in this plan for providing logistic support to include:

- Office space (both central and remote)

- Computers and network access

- Telephones

- Government vehicles

- Supplies

- Other services as necessary.

It is important to have as much information as possible in advance of the need date to be responsive and to eliminate any unnecessary costs. Due to the two-month transition, space and equipment will be established prior to contract award for up to 200 PHMC personnel. The M\&O Contractor recommends that RL query the selected PHMC immediately following contract award to obtain any information on their logistical needs, so that additional needs can be provided in a timely manner.

Many actions have been included to identify and locate documentation for use by the PHMC. With exception of critical documentation, it is not intended to make copies to be provided to the PHMC, unless specifically requested. Existing documents may be located in a central area for ease of access. A complete set of "Smart Books" will be provided within the prime central location for the PHMC's use. 


\subsection{M\&O TRANSITION MANPOWER AND COST ESTIMATE}

Table 2-1 identifies M\&O Contractor manpower and cost estimates for carrying out the actions contained in this plan. The estimates cover those activities that will be charged to the programs currently assigned to the M\&O Contractor. They do not include any costs incurred by the M\&O Contractor that, if incurred, would be charged to the PHMC transition account. Such activities would include cost for space and equipment provided to the PHMC Transition Team, or costs associated with modifications to current systems at the request of the PHMC for continued use after contract takeover.

Change packages have been submitted to cover the majority of the estimated costs contained in Table 2-1. Most of these change packages cover both pre-award and post-award activities, whereas some only cover the pre-award phase, and will require supplemental change packages for post-award.

The costs in the table have been updated to reflect the approved and/or submitted change packages. Whereas separate costs were previously shown for pre-award and postaward, only combined costs are shown in this update, as many of the change packages did not differentiate between the two phases. The estimated cost of M\&O Contractor transition efforts has been reduced. This is primarily due to refined estimates being developed through the change process and the reduced scope for the post-award phase recognizing a shorter transition period. Actual costs incurred through May 1996 is approximately $\$ 700 \mathrm{~K}$.

Table 2-1. Transition Manpower and Cost.

\begin{tabular}{|l|c|c|}
\hline \multicolumn{1}{c|}{ Transition Area } & Man-Months & Cost (\$000) \\
\hline Project Management and Administration & 25 & $\$ 150$ \\
\hline Management and Integration & 130 & 900 \\
\hline Hanford Projects & 175 & 1,570 \\
\hline Infrastructure & 75 & 620 \\
\hline Other Crosscutting Services & 90 & 710 \\
\hline \multicolumn{1}{|c|}{ TOTAL } & 495 & $\$ 3,950$ \\
\hline
\end{tabular}




\subsection{TRANSITION SCHEDULE}

Figure 2-1 provides a summary of the current transition schedule, reflecting the major elements of a two-month transition. Appendix A provides a more detailed transition schedule, which is the basis for the actions contained in this plan.

In going from a four-month transition period to a two-month period, the overall scope of transition activities must be reduced. At the same time, many more areas end up on the critical path, which therefore reduces the probability of success. The assumptions contained in Section 2.3 as being key to accomplishing a two-month transition must be adhered to in these critical areas. Critical areas include; 1) inventory and transfer of property, 2) subcontract transfer and termination, 3) transfer of software licenses, 4) personnel placement and transfer, 5) financial closeout/startup, 6) SNM inventory, and 7) pre-existing condition assessment. These areas have been highlighted on the transition schedule contained in Appendix A.

Other areas are not considered critical only because in a two-month transition period, the only viable option is to takeover existing processes and packages (e.g., labor agreements, benefit packages, and existing systems). Such areas will still require close attention and coordination between all applicable parties to ensure they are successfully transferred by October 1, 1996. 


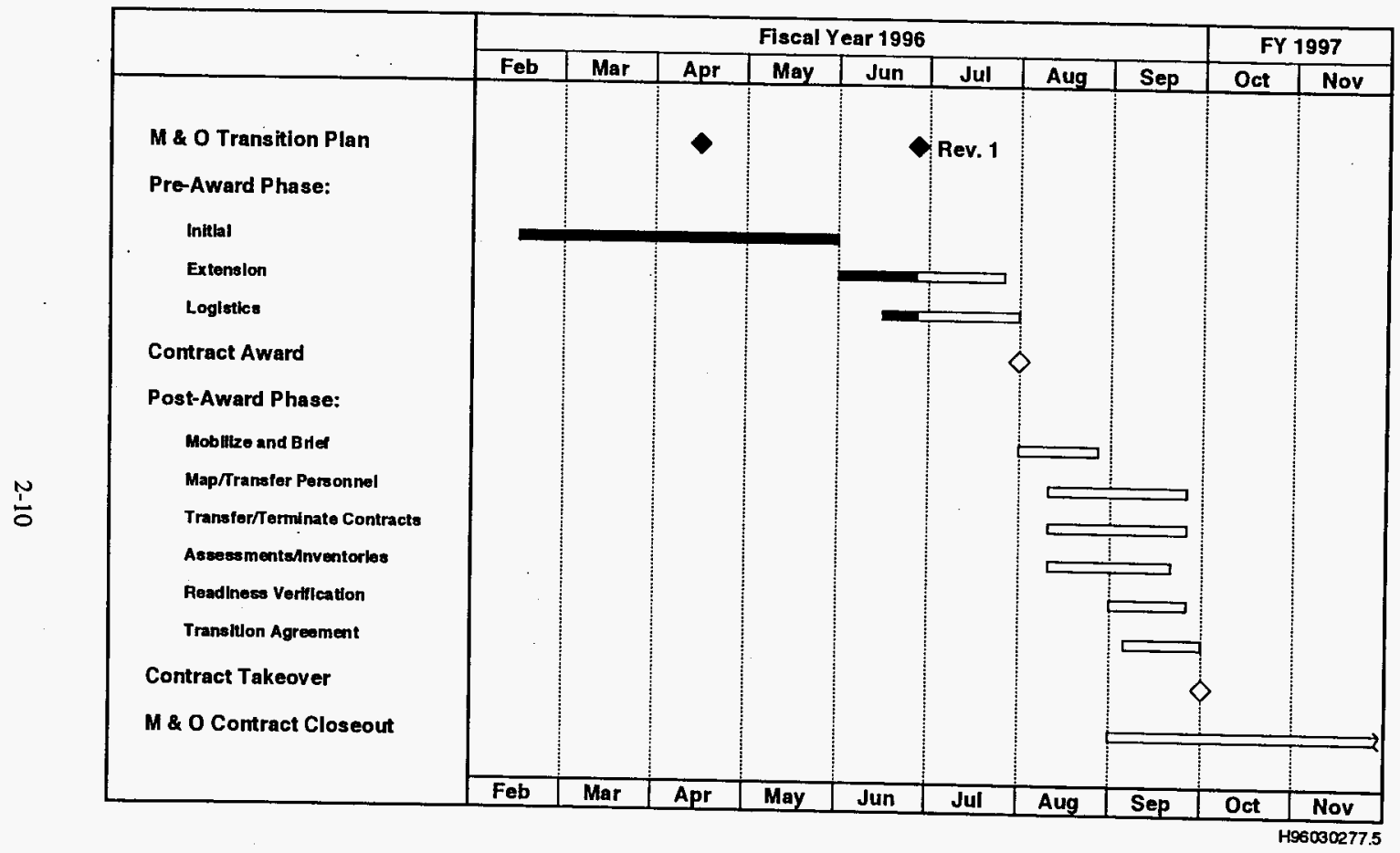

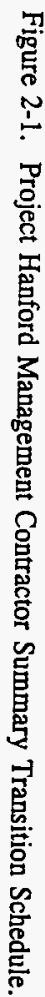




\subsection{ACTION DESCRIPTIONS}

The follow-on sections in this plan identify many actions in support of transition, some of which are repeated for each program, project, or facility. The following provides further definition with respect to the scope and content of these repetitive actions:

- Key Program Documentation

- Key documentation includes, program/project "Smart Book," current multiyear program plans (MYPPs), work breakdown structures (WBS), Program/project Charter, activity data sheets (ADSs), risk data sheets (RDSs), cost account plans (CAPs), basis of estimate (BOE) library, change control log and file, cost and schedule status (i.e., Site Management System [SMS] report), progress tracking system (PTS) report, and current schedules. Includes other administrative plans and procedures. Also includes key technical reports, studies, and plans to include program level QA and safety documentation. For facilities not addressed separately, include applicable key facility documentation and pre-existing condition information.

- Program/Project Briefings

- Describe the mission and scope of the program or project. Provide the overall program status in terms of budget, schedule commitments, and compliance. Introduce key program personnel. Discuss pre-existing conditions not addressed at the facility or operational level. Provide listing of and introduce POCs, address any logistic issues, and discuss schedule and support for tours, pre-existing condition assessments, and conduct of inventories.

- Key Facility or Operations Documentation

- Documentation includes, facility or operations "Smart Book," primary facility drawings and in-process engineering change notices (ECNs); Operations and Maintenance records (e.g., shift operating logs); safety and QA documentation; radiological control (RadCon) documentation; permits and regulatory submittals; environmental monitoring records; key construction project documentation; assessments and surveillance; training records; administrative; operating, and maintenance procedures; facility plans (e.g., building emergency plan, health and safety plan); subcontracts or agreements for outside support; and any other documentation essential to the continued safe operation of the facility. Include pre-existing condition documentation.

\section{- Smart Books}

- In conjunction with assembling key program/project or facility/operations documentation, "Smart Books" will be prepared. The purpose of the "Smart Book" is to summarize key data for use by the PHMC personnel, without making them wade through the large set of documents provided. Standard 
outlines for the "Smart Books" will be provided, and will include facility pictures, briefing material, documentation listings, POCs, subcontract listings and other relevant information.

\section{- Facility or Field Operation Briefings}

- Due to the reduced transition period, facility/operational briefings will normally be informal and primarily involve incoming PHMC personnel who will be directly involved in the operations (e.g., facility manager).

- Describe the facility (or operation) and its operating history. Introduce key facility POCs. Provide relevant budget and schedule information. Discuss all permits relative to the facility or operation. Discuss existing subcontracts and support agreements. Discuss QA, Safety, and RadCon programs. Discuss pre-existing conditions.

- Facility or Operations POC

- POCs should include the facility manager, managers of each organization at the facility, shift operations managers, system cognizant engineers and technical leads, persons in charge of ongoing activities, individuals responsible for contracts or agreements for outside support, and managers of supporting and/or client organizations in other programs.

For other operations or technical services, include key scientists/engineers and consultants.

\section{- Pre-Existing Conditions}

- A checklist for evaluating pre-existing conditions will be provided.

- In addition to other environmental, safety, and health (ESH) issues, preexisting conditions should address issues associated with environmental regulations, Occupational Safety and Health Administration (OSHA), Price Anderson Amendment Act (PAAA), and related DOE orders. Specifically include corrective actions from assessments and surveillances which have not yet been completed; occurrences and permit violations; ongoing regulatory compliance issues; limits on operations; unresolved environmental safety or health issues; conduct of operations ( $\mathrm{COO})$ issues and status of implementation; status of procedures and their relationship to the facility safety basis; and security risk evaluation issues. Include the planned resolution for each. Areas of uncertainty will be documented to ensure they are addressed jointly with the PHMC. Any physical (or walk-through) assessments done in advance of the PHMC will be done to facilitate readiness for transition. 


\subsection{OVERALL ACTIONS}

\begin{tabular}{|c|c|c|}
\hline Pre-Award Actions & $\begin{array}{l}\text { Scheduled } \\
\text { Date }\end{array}$ & Actionee(s) \\
\hline $\begin{array}{l}\text { 1. Establish Action Tracking System and enter pre-award actions for } \\
\text { tracking. }\end{array}$ & $\begin{array}{l}4 / 19 / 96 \\
\text { Completed }\end{array}$ & JL Waite \\
\hline 2. Establish Transition Record file and initiate record keeping. & $\begin{array}{l}5 / 01 / 96 \\
\text { Completed }\end{array}$ & JL Waite \\
\hline $\begin{array}{l}\text { 3. Assemble site listing of key POCs by program/project, facility, or } \\
\text { function. }\end{array}$ & $7 / 15 / 96$ & JL Waite \\
\hline $\begin{array}{l}\text { 4. Identify available central office space for use by the PHMC } \\
\text { Transition Team if needed. }\end{array}$ & $\begin{array}{l}5 / 15 / 96 \\
\text { Completed }\end{array}$ & JL Waite \\
\hline 5. Develop pre-existing conditions checklist. & $\begin{array}{c}4 / 30 / 96 \\
\text { Completed }\end{array}$ & $\begin{array}{l}\text { RA Dutton } \\
\text { RH Engelmann } \\
\text { AR Hawkins }\end{array}$ \\
\hline $\begin{array}{l}\text { 6. Develop standard outlines and content for program level and } \\
\text { project/facility/operations level "Smart Books." }\end{array}$ & $\begin{array}{c}4 / 15 / 96 \\
\text { Completed }\end{array}$ & $\begin{array}{l}\text { JL Waite } \\
\text { CL Nansen }\end{array}$ \\
\hline 7. Prepare a set of location maps for use by the PHMC. & $7 / 22 / 96$ & JL Waite \\
\hline $\begin{array}{l}\text { 8. Prepare a composite listing of inter contractor MOUs among the } \\
\text { M\&O Contractor and PNNL, BHI, and HEHF, and identify which } \\
\text { shouid be teminated verses those which should be modified for } \\
\text { use by the PHMC. }\end{array}$ & $7 / 22 / 96$ & $\begin{array}{l}\text { GW Jackson } \\
\text { MS Hanson (PNNL) } \\
\text { GF Jones (BHI) } \\
\text { JJ Maher III (HEHF) }\end{array}$ \\
\hline $\begin{array}{l}\text { 9. Develop a detailed priority list and schedule for Key } \\
\text { Functions/Program/Project-level briefings. }\end{array}$ & $7 / 15 / 96$ & D Butler \\
\hline $\begin{array}{l}\text { 10. Based on the PEC evaluations, develop and prioritize a list of } \\
\text { proposed walkthroughs for the PHMC. }\end{array}$ & $7 / 22 / 96$ & D Butter \\
\hline $\begin{array}{l}\text { 11. Finalize sitewide information for use by the PHMC on; 1) support } \\
\text { to DOE, and 2) committees, etc. Include in Site "Smart Book." }\end{array}$ & $7 / 22 / 96$ & JL Waite \\
\hline Post-Award Actions & & \\
\hline 1. RL confirm space requirements for PHMC Transition Team. & $8 / 05 / 96$ & LA Marzette (RL) \\
\hline 2. RL confirm any other logistic support to PHMC Transition Team. & $8 / 05 / 96$ & LA Marzette (RL) \\
\hline $\begin{array}{l}\text { 3. PHMC Transition Team central office space available for use to } \\
\text { include furniture, computers, telephones, and other office } \\
\text { equipment. }\end{array}$ & $8 / 05 / 96$ & JL Waite \\
\hline 4. All remaining logistical support provided to the PHMC Team. & $8 / 19 / 96$ & JL Waite \\
\hline 5. Provide site orientation briefing and tour to the PHMC Team. & $8 / 06 / 96-8 / 12 / 96$ & $\begin{array}{l}\text { GW Jackson } \\
\text { J Erickson }\end{array}$ \\
\hline
\end{tabular}




\subsection{WHC CONTRACT CLOSEOUT ACTIVITIES}

A separate WHC team and associated plan will be established to address closeout of the WHC M\&O contract, to include its major subcontractors ICF KH and BCSR. The following transition action is necessary to support closeout:

\begin{tabular}{|c|c|c|}
\hline Post-Award Actions & $\begin{array}{c}\text { Scheduled } \\
\text { Date }\end{array}$ & Actionee(s) \\
\hline $\begin{array}{l}\text { 1. PHMC/WHC/subcontractors Memorandum of Understanding } \\
\text { (MOU) negotiated to allow as needed access to former } \\
\text { employees, data, and facilities for closeout activities. (Will be } \\
\text { included as part of WHC/PHMC/DOE transfer agreement.) }\end{array}$ & $9 / 20 / 96$ & $\begin{array}{l}\text { IM Knoll, Jr. } \\
\text { SR Morgan } \\
\text { CD Maxson } \\
\text { RL Benedetti } \\
\text { WJ Fuller (BCSR) } \\
\text { RL Contracting Officer }\end{array}$ \\
\hline
\end{tabular}




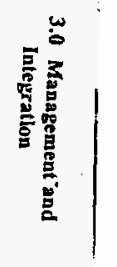


Y T TENTHNULU

1.2. L LLANE

1

$=$ 


\subsection{MANAGEMENT AND INTEGRATION}

\subsection{SITE PLANNING AND INTEGRATION}

Site planning and integration includes RL strategic and mission planning, sitewide systems engineering and integration activities, and the development and maintenance of lifecycle program and project baselines.

\begin{tabular}{|c|c|c|}
\hline Pre-Award Actions & $\begin{array}{l}\text { Scheduled } \\
\text { Date }\end{array}$ & Actionee(s) \\
\hline $\begin{array}{l}\text { 1. Develop a list and assemble key site planning and } \\
\text { integration documentation and information for use by the } \\
\text { PHMC. Include information on databases. }\end{array}$ & $\begin{array}{c}5 / 31 / 96 \\
\text { Completed }\end{array}$ & $\begin{array}{l}\text { ML Grygiel } \\
\text { JK Fish }\end{array}$ \\
\hline $\begin{array}{l}\text { 2. Prepare and submit Site Planning and Integration "Smart } \\
\text { Book." }\end{array}$ & $7 / 15 / 96$ & $\begin{array}{l}\text { ML Grygiel } \\
\text { JL Waite }\end{array}$ \\
\hline Post-Award Actions & & \\
\hline $\begin{array}{l}\text { 1. Provide briefing to PHMC on the overall Hanford Site } \\
\text { Planning and Integration process }\end{array}$ & $\begin{array}{l}8 / 12 / 96 \\
8 / 19 / 96\end{array}$ & $\begin{array}{l}\text { EW Higgins (RL) } \\
\text { MB Triplett (PNNL) } \\
\text { GW Jackson }\end{array}$ \\
\hline
\end{tabular}

\subsubsection{Support to RL Strategic Planning}

This activity is responsible to provide support to RL and PNNL in the development and integration of the strategic level planning documents (Hanford Strategic Thinking and the Mission Direction Document [MDD]), as well as to assist in the integration of program input to the MYPP planning process.

\begin{tabular}{|l|l|l|}
\hline Post-Award Actions & Scheduled & Actionee(s) \\
\hline 1. Provide briefing or discussion with PHMC on M\&O & $8 / 19 / 96-$ & ST Smith \\
Contractor support to the RL strategic planniag effort. & $9 / 02 / 96$ & \\
\hline
\end{tabular}

\subsubsection{Project/Program Planning and Baseline Management}

The current planning process centers around the development of MYPPs for the direct funded programs, and Site Support Program Plans (SSPPs) for indirect and direct support activities. Each program and support area will address their specific plans in other sections of this transition plan. This section addresses the overall planning processes currently in use, and current ongoing planning activities that the PHMC may need to get involved in. 
Site baseline management involves the integration and maintenance of the overall technical, schedule, and cost baseline for the site. The technical baseline is further addressed in Section 3.1.3.

\begin{tabular}{|c|c|c|}
\hline Pre-Aurerd Actions & $\begin{array}{l}\text { Scheduled } \\
\text { Date }\end{array}$ & Actionee(s) \\
\hline $\begin{array}{l}\text { 1. Assemble a set of the most recent MYPPs/SSPPs, and } \\
\text { integrated site baseline data (including HSIS) for use by the } \\
\text { PHMC. }\end{array}$ & $\begin{array}{l}5 / 31 / 96 \\
\text { Completed }\end{array}$ & JK Fish \\
\hline Post-Award Actions & & \\
\hline $\begin{array}{l}\text { 1. Prepare briefing and have available for discussion with the } \\
\text { PHMC on the program planning and integrated site baseline } \\
\text { processes. }\end{array}$ & $8 / 09 / 96$ & JK Fish \\
\hline $\begin{array}{l}\text { 2. Make available to the PHMC the FY } 1997 \text { detailed } \\
\text { execution work plans. }\end{array}$ & $8 / 15 / 96$ & JK Fish \\
\hline
\end{tabular}

\subsubsection{Sitewide Systems Engineering}

This area is responsible for development and maintenance of the site level technical baseline, interface control documentation, operational scenarios to model the performance of the Hanford mission, publication of the site level specification and identification and tracking of technical integration issues. This activity also has responsibility to ensure integration of its products with the strategic level planning documentation being developed by PNNL and to ensure that technical considerations are included in the MYPP planning guidance provided to the program level.

This activity is governed by RLPD and RLID 430.1 and the Hanford Management Plan.

\begin{tabular}{|c|c|c|}
\hline Post-Award Actions & $\begin{array}{l}\text { Scheduled } \\
\text { Date }\end{array}$ & Actionee(s) \\
\hline $\begin{array}{l}\text { 1. Provide briefing or discussion with PHMC on sitewide } \\
\text { system engineering and integration activities. }\end{array}$ & $\begin{array}{l}8 / 19 / 96 \\
9 / 02 / 96\end{array}$ & PA Baynes \\
\hline
\end{tabular}

\subsection{FINANCE AND ADMINISTRATION}

\section{Assumptions}

- In the Treasury area, Tax Link, Viewstart, Dynacom and other electronic data transmission vehicles currently in use will be needed by the PHMC; PHMC and subs will use the same bank to facilitate payment transactions; and the current 
Letter of Credit will remain in use until all outstanding payments issued through September 30, 1996, have cleared.

- In the Travel Area, the current credit card program and other travel-related contracts will be discontinued and the PHMC will need assistance to implement new programs; relocations and travel in progress at September 30, 1996, will be turned over to the PHMC for closeout.

- In the Pension and Savings area, the current plans will be assumed by the PHMC and only minor changes would be required during the two-month transition window.

- In the Payroll area, a centralized payroll is assumed with ongoing operations similar to the current setup; systems modifications would be minimal, and business rules would remain the same for startup; and predecessor/successor rules apply. Personnel assignments must be accomplished by September 1, 1996, in order to facilitate system modifications and data transfers.

- An agreement will be in place with WHC, the PHMC and RL addressing closeout activities required beyond September 30, 1996.

- The PHMC will be available upon contract award to review the provisions of the contract with each team and to address any modifications required to the detailed closeout/startup plans. Such review will be done expeditiously to ensure adequate time to implement.

- The PHMC indirect/overhead liquidation rate structure will be consistent with existing system capabilities, and execution year planning rate assumptions.

- The M\&O Contractor execution year planning will be modified as appropriate after transition.

- Existing financial systems applications will remain in place with only minor changes implemented during transition to accommodate PHMC needs.

\subsubsection{Accounting}

3.2.1.1 Accounts Receivables. The PHMC will be a RL non-integrated contract. Certain ledger accounts will be transferred and carried on RL's books. 


\begin{tabular}{|l|l|l|}
\hline \multicolumn{1}{|c|}{ Post-Award Actions } & \multicolumn{1}{|c|}{$\begin{array}{l}\text { Scheduled } \\
\text { Dafe }\end{array}$} & \multicolumn{1}{|c|}{ Actionee(s) } \\
\hline 1. Identify all receivables by account/company/employee. & $9 / 01 / 96$ & $\begin{array}{l}\text { J Arrnstrong (lead) } \\
\text { D Long } \\
\text { M Howard }\end{array}$ \\
\hline $\begin{array}{l}\text { 2. Write-off and establish reserves where possible. } \\
\text { 3. Identify accounts which are to be transferred to RL. }\end{array}$ & $9 / 01 / 96$ & J Armstrong \\
\hline $\begin{array}{l}\text { 4. Transfer General Ledger receivable accounts to } \\
\text { RL/PHMC/subcontractors. }\end{array}$ & Discas Closing & $\begin{array}{l}\text { J Salancy } \\
\text { RL } \\
\text { PHMC }\end{array}$ \\
\hline
\end{tabular}

\subsubsection{Department Integrated Standardized Core Accounting System (Discas) Interface}

for Plant and Equipment. Establish a DISCAS interface with RL, which provides a monthly update of transactions for Plant and Equipment (P\&E). These affected accounts will no longer reside on the contractor's books.

\begin{tabular}{|c|c|c|}
\hline - Pre-Award Actions & $\begin{array}{l}\text { Scheduled } \\
\text { Date }\end{array}$ & Actionee(s) \\
\hline $\begin{array}{l}\text { 1. Develop a comprehensive plan to identify } P \& E \text { accounts, } \\
\text { transactions, and automate the data transfer to } R L \text {. }\end{array}$ & $\begin{array}{c}6 / 01 / 96 \\
\text { Completed }\end{array}$ & $\begin{array}{l}\text { J Shelt } \\
\text { A Lake }\end{array}$ \\
\hline Post-Award Actions & & \\
\hline $\begin{array}{l}\text { 1. Implement the automated transfer prior to year-end } \\
\text { (if feasible). }\end{array}$ & Discas Closing & $\begin{array}{l}\text { I Shelt } \\
\text { BCSR } \\
\text { A Lake }\end{array}$ \\
\hline
\end{tabular}

3.2.1.3 Transfer of General Ledger Accounts. The PHMC will be a RL non-integrated contractor. As a result, certain general ledger accounts will no longer reside on the contractor's books. These accounts are to be transferred to RL.

\begin{tabular}{|l|c|l|}
\hline \multicolumn{1}{|c|}{ Pre-Award Actions } & $\begin{array}{c}\text { Scheduled } \\
\text { Date }\end{array}$ & Actionee(s) \\
\hline 1. Identify ledger accounts which are to be transferred to RL. & $\begin{array}{c}6 / 01 / 96 \\
\text { Completed }\end{array}$ & $\begin{array}{l}\text { J Salancy } \\
\text { A Lake }\end{array}$ \\
\hline $\begin{array}{l}\text { 2. Identify all issues associated with an early transfer (prior to } \\
\text { FY-end closing) of accounts to RL. }\end{array}$ & $7 / 20 / 96$ & $\begin{array}{l}\text { J Salancy } \\
\text { A Lake }\end{array}$ \\
\hline $\begin{array}{l}\text { Post-Award Actions } \\
\text { 1. Transfer of account balances to RL. }\end{array}$ & by 9/30/96 & $\begin{array}{l}\text { J Salancy } \\
\text { A Lake }\end{array}$ \\
\hline
\end{tabular}

3.2.1.4 Treasury. The current contractor government bank accounts and associated treasury activities will have to be dispositioned and transitioned. Also, a startup plan for the PHMC needs to be developed. The primary efforts will be to ensure treasury operations are current during transition. 
WHC-SP-1181 Rev. 1

\begin{tabular}{|c|c|c|}
\hline \multicolumn{1}{|c|}{ Pre-Award Actions } & $\begin{array}{l}\text { Scheduled } \\
\text { Date }\end{array}$ & Actionee(s) \\
\hline \begin{tabular}{l} 
1. $\begin{array}{l}\text { Develop a detailed closeout/startup plan for all treasury- } \\
\text { related activities. }\end{array} \quad$ Post-Award Actions \\
\hline $\begin{array}{l}\text { 1. Review detailed closeout/startup plan for all treasury-related } \\
\text { activities. }\end{array}$
\end{tabular} & $\begin{array}{l}\text { M Byrd } \\
\text { PHMC }\end{array}$ \\
\hline
\end{tabular}

3.2.1.5 Payroll Activities. A range of payroll issues need to be addressed. These are general pay issues, system issues, tax issues, banking issues, etc.

\begin{tabular}{|l|c|c|}
\hline \multicolumn{1}{|c|}{ Pre-Award Actions } & $\begin{array}{c}\text { Scheduled } \\
\text { Date }\end{array}$ & Actionee(s) \\
\hline $\begin{array}{l}\text { 1. Develop a detailed closeout/startup plan for atl payroll- } \\
\text { related activities. }\end{array}$ & $7 / 15 / 96$ & M Byrd \\
\hline \multicolumn{1}{c|}{ Post-Award Actions } & & \\
\hline $\begin{array}{l}\text { 1. Review detailed closeout/startup plan for all payroll-related } \\
\text { activities with the PHMC and implement. }\end{array}$ & $9 / 30 / 96$ & $\begin{array}{l}\text { M Byrd } \\
\text { PHMC }\end{array}$ \\
\hline
\end{tabular}

3.2.1.6 Tax Filings - City, County, Federal. Tax reports will be required by prior and new site contractors. Develop a plan to assume a successful transition. Some of these may be identified in other parts of the plan.

\begin{tabular}{|l|l|l|}
\hline \multicolumn{1}{|c|}{ Pre-Award Actions } & Scheduled & Actionee(s) \\
\hline $\begin{array}{l}\text { 1. Identify all tax reports and filings which will need to be } \\
\text { made for the M\&O Contractors. }\end{array}$ & $7 / 15 / 96$ & $\begin{array}{l}\text { J Salancy (Lead) } \\
\text { M Hinchee } \\
\text { F Melton }\end{array}$ \\
\hline $\begin{array}{l}\text { 1. Develop a plan to file timely and accurate tax reports on } \\
\text { behalf of the M\&O Contractors. }\end{array}$ & $9 / 01 / 96$ & $\begin{array}{l}\text { J Salancy (Lead) } \\
\text { M Hinchee }\end{array}$ \\
\hline $\begin{array}{l}\text { 2. Prepare necessary documents to assure transition for proper } \\
\text { tax reporting by the PHMC. }\end{array}$ & $9 / 01 / 96$ & $\begin{array}{l}\text { J Salancy } \\
\text { PHMC }\end{array}$ \\
\hline $\begin{array}{l}\text { 3. Determine tax consequences due to billings between Hanford } \\
\text { contractors and subcontractors. }\end{array}$ & $9 / 01 / 96$ & J Salancy \\
\hline
\end{tabular}

3.2.1.7 Fiscal Year-End - Closeout. This section will be addressed as part of consolidated transition planning.

3.2.1.8 Accruals (Accounts payable and other fund liabilities). Forty to sixty million will be accrued for services performed but not paid as of September 30, 1996. They will have to be dispositioned. 


\begin{tabular}{|l|l|l|}
\hline \multicolumn{1}{|c|}{ Pre-Award Actions } & $\begin{array}{l}\text { Scheduled } \\
\text { Date }\end{array}$ & \multicolumn{1}{|c|}{ Actionee(s) } \\
\hline $\begin{array}{l}\text { 1. Develop a plan to effectively transfer the FY 1996 accruals to } \\
\text { the PHMC. }\end{array}$ & $7 / 31 / 96$ & $\begin{array}{l}\text { D Long (lead) } \\
\text { K Harris } \\
\text { C Stice } \\
\text { F Zvonar }\end{array}$ \\
\hline 2. Review Rocky Flats Process (or other applicable sites). & $7 / 31 / 96$ & J Shelt \\
\hline $\begin{array}{l}\text { 3. Identify all areas for accruals (P-cards, services, Intra DOE } \\
\text { Work Orders, claims, etc). }\end{array}$ & $7 / 19 / 96$ & $\begin{array}{l}\text { D Long (lead) } \\
\text { K Harris } \\
\text { C Stice }\end{array}$ \\
\hline
\end{tabular}

\subsubsection{P-Cards}

\begin{tabular}{|c|c|c|}
\hline Pre-Award Actions & $\begin{array}{l}\text { Scheduled } \\
\text { Date }\end{array}$ & Actionee(s) \\
\hline $\begin{array}{l}\text { 1. Develop a plan to close out P-cards under current WHC } \\
\text { contract. }\end{array}$ & $7 / 31 / 96$ & $\begin{array}{l}\text { R Dahlin (lead) } \\
\text { C Mayo }\end{array}$ \\
\hline ४ Postaward Actions & & \\
\hline 1. Develop a plan to implement P-cards for PHMC. & $9 / 01 / 96$ & $\begin{array}{l}\text { R Dahlin (lead) } \\
\text { C Mayo } \\
\text { PHMC }\end{array}$ \\
\hline
\end{tabular}

3.2.1.10 Travel. Develop plans for the closeout, transfer, and disposition of travel related activities.

\begin{tabular}{|c|c|c|}
\hline ४ Pre-Award Actions & $\begin{array}{l}\text { Scheduled } \\
\text { Date }\end{array}$ & Actionee(s) \\
\hline $\begin{array}{l}\text { 1. Develop a detailed closeout/startup plan for all travel- } \\
\text { related activities. }\end{array}$ & $7 / 15 / 96$ & T Beyers \\
\hline Post-Award Actions & & \\
\hline $\begin{array}{l}\text { 1. Review detailed closeout/startup plan for all travel-related } \\
\text { activities with the PHMC and implement. }\end{array}$ & $9 / 30 / 96$ & $\begin{array}{l}\text { T Beyers } \\
\text { PHMC }\end{array}$ \\
\hline
\end{tabular}

3.2.1.11 Work Authorization (Hanford Site). A mechanism is needed for work authorization between the PHMC, subcontractors, and other Hanford contractors. Also, there are related tax impacts due to the contractual arrangements.

\begin{tabular}{|l|c|c|}
\hline \multirow{2}{*}{ Pre-Award Actions } & Scheduled \\
Date & $7 / 31 / 96$ & $\begin{array}{l}\text { K Harris } \\
\text { C Stice }\end{array}$ \\
\hline $\begin{array}{l}\text { 1. Develop a listing of current sources of work to/from at the } \\
\text { Hanford Site. }\end{array}$ & Actionee(s) \\
\hline
\end{tabular}




\begin{tabular}{|l|c|l|}
\hline \multicolumn{1}{|c|}{ Post-Award Actions } & Scheduled & \\
& Date & Actionee(s) \\
\hline 1. Provide a summary of tax impacts. & $8 / 15 / 96$ & J Salancy \\
\hline 2. Develop work authorization mechanism. & $9 / 01 / 96$ & $\begin{array}{l}\text { RL } \\
\text { PHMC }\end{array}$ \\
\hline 3. Communicate and implement for FY 1997 start-up. & $9 / 30 / 96$ & PHMC \\
\hline
\end{tabular}

3.2.1.12 Work For Others (Non-Hanford). There are various mechanisms for the contracting of work performed by the M\&O Contractor. (MPOs, Intra DOE Work Orders, DOE RFSs, IWRs, IDWAs, etc.) See Section 3.11 for preparatory actions associated with work for others.

\begin{tabular}{|c|c|c|}
\hline Pre-Award Actions & $\begin{array}{l}\text { Scheduled } \\
\text { Date }\end{array}$ & Actionee(s) \\
\hline 1. Identify all work for others. & $\begin{array}{c}6 / 01 / 96 \\
\text { Completed }\end{array}$ & $\begin{array}{l}\text { J Knoll } \\
\text { P Felts }\end{array}$ \\
\hline - Post-Award Actions & & ह \\
\hline 1. Closeout or reassign current contractual agreements. & $9 / 30 / 96$ & $\begin{array}{l}\text { J Knoll (lead) } \\
\text { P Felts } \\
\text { R Meyer } \\
\text { AN Lake } \\
\text { PHMC }\end{array}$ \\
\hline
\end{tabular}

3.2.1.13 Pensions. The PHMC will assume the current Pension Plan and related assets and liabilities. The Pension and Savings organization must maintain current operations and work towards assigning responsibility to new contractors(s).

\begin{tabular}{|l|c|c|}
\hline \multicolumn{1}{|c|}{ Pre-Award Actions } & Scheduled & Date \\
\hline $\begin{array}{l}\text { 1. Prepare summary of all pension trust agreements and other } \\
\text { related contracts. }\end{array}$ & $\begin{array}{c}6 / 01 / 96 \\
\text { Completed }\end{array}$ & M Byrd \\
\hline $\begin{array}{l}\text { 2. Develop a detailed closeout/startup plan for all pension } \\
\text { related activities. }\end{array}$ & $7 / 15 / 96$ & M Byrd \\
\hline $\begin{array}{l}\text { 1. Trust agreements, investment management agreements, } \\
\text { service agreements, consultant agreements, third party } \\
\text { agreements, plan documents, summary plan descriptions, } \\
\text { actuarial reports, discrimination testing, annual reports, and } \\
\text { committee minutes should all be reviewed and reassigned. }\end{array}$ & $9 / 30 / 96$ & M Byrd \\
\hline $\begin{array}{l}\text { 2. Review detailed closeout/startup plan for all pension-related } \\
\text { activities with the PHMC and implement. }\end{array}$ & $9 / 30 / 96$ & $\begin{array}{l}\text { M Byrd } \\
\text { PHMC }\end{array}$ \\
\hline
\end{tabular}

3.2.1.14 Savings Plans. The RFP requires bidders to propose a $401 \mathrm{~K}$ plan. A transition to the new plan(s) will have to be performed. 


\begin{tabular}{|c|c|c|}
\hline Pre-Award Actions & $\begin{array}{l}\text { Scheduled } \\
\text { Date }\end{array}$ & Actionee(s) \\
\hline 1. Identify all trust agreements and other related contracts. & $\begin{array}{l}\text { 6/01/96 } \\
\text { Completed }\end{array}$ & M Byrd \\
\hline $\begin{array}{l}\text { 2. Develop a detailed closeout/startup plan for all savings plan- } \\
\text { related activities. }\end{array}$ & $7 / 15 / 96$ & M Byrd \\
\hline औ $\quad$ Post-Award Actions & & \\
\hline $\begin{array}{l}\text { 1. Develop a transition plan to migrate to new } 401 \mathrm{~K} \text { as } \\
\text { proposed by the PHMC. }\end{array}$ & $8 / 05 / 96$ & PHMC \\
\hline $\begin{array}{l}\text { 2. Trust agreements service agreements, consultant agreements, } \\
\text { third party agreements, plan documents, summary plan } \\
\text { descriptions, actuarial reports, discrimination testing, annual } \\
\text { reports, and committee minutes should all be reviewed and } \\
\text { transition plan established. }\end{array}$ & $9 / 30 / 96$ & $\begin{array}{l}\text { M Byrd } \\
\text { PHMC }\end{array}$ \\
\hline $\begin{array}{l}\text { 3. Review detailed closeout/startup plan for all savings plan- } \\
\text { related activities with the PHMC and implement. }\end{array}$ & $9 / 30 / 96$ & $\begin{array}{l}\text { M Byrd } \\
\text { PHMC }\end{array}$ \\
\hline
\end{tabular}

3.2.1.15 Insurance Plans. The RFP requires bidders to propose a health plan. The current plans will be transitioned to the new plans.

\begin{tabular}{|c|c|c|}
\hline Pre-Award Actions & $\begin{array}{l}\text { Scheduled } \\
\text { Date }\end{array}$ & Actionee(s) \\
\hline $\begin{array}{l}\text { 1. Prepare sunmary of all insurance agreements and other } \\
\text { related contracts. }\end{array}$ & $\begin{array}{l}6 / 01 / 96 \\
\text { Completed }\end{array}$ & M Byrd \\
\hline
\end{tabular}

3.2.1.16 Vacation. The current accrued vacations liability for employees will need to be transferred to the PHMC or RL.

\begin{tabular}{|c|c|c|}
\hline Pre-Award Actions & $\begin{array}{l}\text { Scheduled } \\
\text { Date }\end{array}$ & Actionee(s) \\
\hline $\begin{array}{l}\text { Develop a detailed closeout/startup plan to address } \\
\text { transfer of vacations liability to the PHMC or RL. }\end{array}$ & $7 / 15 / 96$ & M Byrd \\
\hline Post-Award Actions & & \\
\hline 1. DOE decision on Vacation Liability Methodology. & $8 / 01 / 96$ & A Lake (RL) \\
\hline $\begin{array}{l}\text { 2. Transfer employees accrued vacation hours and associated } \\
\text { liability to PHMC or RL (to be decided by } R L \text { ). }\end{array}$ & $9 / 30 / 96$ & M Byrd \\
\hline
\end{tabular}

3.2.1.17 Nuclear Materials Accounting. This section will be addressed as part of the consolidated transition plan.

\subsubsection{Planning and Management Control Systems}

The Planning and Management Control Systems describe the processes used to manage the cost, schedule, and technical baselines of work performed by WHC for RL. 


\begin{tabular}{|c|c|c|}
\hline Pre-Award Actions & $\begin{array}{l}\text { Scheduled } \\
\text { Date }\end{array}$ & Actionee(s) \\
\hline $\begin{array}{l}\text { 1. Develop a list and assemble key Planning and Management } \\
\text { Control System Documentation and information for use by } \\
\text { the PHMC. }\end{array}$ & $\begin{array}{l}6 / 01 / 96 \\
\text { Completed }\end{array}$ & PJ Bence \\
\hline Post-Award Actions & & \\
\hline $\begin{array}{l}\text { 1. Provide a briefing to the PHMC on the overall Planning and } \\
\text { Management Control System philosophy for the site. }\end{array}$ & $8 / 16 / 96$ & KD Cameron \\
\hline $\begin{array}{l}\text { 2. Prepare and have available to the PHMC a briefing on } \\
\text { assembled documentation and processes used by the M\&O } \\
\text { Contractor in support of the Planning and Managernent } \\
\text { Control Systems. }\end{array}$ & $9 / 06 / 96$ & PJ Bence \\
\hline
\end{tabular}

\subsubsection{Environmental Planning Data System. The Environmental Planning Data System} (EPDS) includes the following modules: ADS, RDS/Priority List, Central Milestone Module (CMM), Baseline Change Control (BCC), and WBS Master Validation Table (MVT). These modules are used in support of the RL budget submittal process, progress tracking, and baseline control.

\begin{tabular}{|c|c|c|}
\hline $\begin{array}{r}4+8 \\
+3\end{array}$ & $\begin{array}{l}\text { Scheduled } \\
\text { Date }\end{array}$ & Actionee(s) \\
\hline $\begin{array}{l}\text { 1. Assemble a list of key documentation associated with the } \\
\text { EPDS and its associated modules. }\end{array}$ & $\begin{array}{l}5 / 15 / 96 \\
\text { Completed }\end{array}$ & JE Tarcza \\
\hline 2. Verify that the ADS module is up-to-date. & $\begin{array}{l}\text { 6/01/96 } \\
\text { Completed }\end{array}$ & AJ Watts \\
\hline 3. Verify that the CMM module is up-to-date. & $\begin{array}{c}6 / 01 / 96 \\
\text { Completed }\end{array}$ & BE Paul \\
\hline 4. Verify that the BCC module is up-to-date. & $\begin{array}{l}6 / 01 / 96 \\
\text { Completed }\end{array}$ & E Schultz \\
\hline 5. Verify that the RDS/Priority List mocule is up-to-date. & $\begin{array}{c}6 / 01 / 96 \\
\text { Completed }\end{array}$ & BA Reichmuth \\
\hline 6. Verify that the MVT module is up-to-date. & $\begin{array}{l}6 / 01 / 96 \\
\text { Completed }\end{array}$ & BE Paul \\
\hline $\begin{array}{l}\text { 7. Prepare and make available a briefing on the EPDS and its } \\
\text { modules (including the planned transition to the Project } \\
\text { Management and Control System (PMCS). }\end{array}$ & $7 / 19 / 96$ & JE Tarcza \\
\hline
\end{tabular}

\subsubsection{Financial Data System (FDS).}

\begin{tabular}{|l|l|l|}
\hline \multicolumn{1}{|c|}{ Pre-Award Actions } & Scheduled & \multicolumn{1}{|c|}{ Actioneè(s) } \\
\hline 1. Develop a list and assemble key elements of the Financial \\
$\begin{array}{l}\text { Data System's funds and Budget and Performance modules in } \\
\text { support of the WHC M\&O site portion. Other modules } \\
\text { covered in 3.2.2.8. }\end{array}$ & $\begin{array}{c}6 / 01 / 96 \\
\text { Completed }\end{array}$ & PJ Bence \\
\hline
\end{tabular}




\begin{tabular}{|c|c|c|}
\hline Pre-Award Actions & $\begin{array}{c}\text { Scheduled } \\
\text { Date }\end{array}$ & Actionee(s) \\
\hline $\begin{array}{l}\text { 2. Prepare and make available to the PHMC a briefing on } \\
\text { assembled documentation and processes used by the M\&O } \\
\text { contractor. }\end{array}$ & $7 / 26 / 96$ & $\begin{array}{l}\text { PJ Bence } \\
\text { DR Layfield }\end{array}$ \\
\hline
\end{tabular}

\subsection{Funds Module.}

\begin{tabular}{|c|c|c|}
\hline Pre-Award Actions & $\begin{array}{l}\text { Scheduled } \\
\text { Date }\end{array}$ & Actionee(s) \\
\hline $\begin{array}{l}\text { 1. Develop FUNDS reconciliation for funds table to the M\&O } \\
\text { Contractor organizational breakdown structure. }\end{array}$ & $\begin{array}{l}6 / 01 / 96 \\
\text { Completed }\end{array}$ & $\begin{array}{l}\text { CT Hewitt } \\
\text { MK Qualheim }\end{array}$ \\
\hline Post-Award Actions & & \\
\hline $\begin{array}{l}\text { 1. Transfer database in support of the WHC current year } \\
\text { funding/cost profile } \\
\text { - Expense } \\
\text { - Capital } \\
\text { - Special } \\
\text { - RL/HQ. }\end{array}$ & $9 / 06 / 96$ & $\begin{array}{l}\text { CT Hewitt } \\
\text { MK Qualheim } \\
\text { ML Vodney } \\
\text { ML Vodney }\end{array}$ \\
\hline
\end{tabular}

\subsection{Budget Module.}

\begin{tabular}{|l|r|r|r|}
\hline & Post-Award Actions & Scheduled & \\
& & Date & Actionee(s) \\
\hline 1. Provide Budget data files of the approved Program Baseline. & $9 / 27 / 96$ & DR Layfield \\
\hline
\end{tabular}

\subsection{Performance Module.}

\begin{tabular}{|c|c|c|}
\hline Post-Award Actions & $\begin{array}{l}\text { Scheduled } \\
\text { Date }\end{array}$ & Actionee(s) \\
\hline $\begin{array}{l}\text { 1. Make available information and docurnentation on the } \\
\text { processes used by the M\&O Contractor in support of } \\
\text { performance. }\end{array}$ & $8 / 16 / 96$ & $\begin{array}{l}\text { PJ Bence } \\
\text { DR Layfield }\end{array}$ \\
\hline
\end{tabular}

\subsubsection{Management Control System.}

\begin{tabular}{|l|l|l|}
\hline Post-Award Actions & Scheduled & \\
Date & Actionee(s) \\
\hline $\begin{array}{l}\text { 1. Prepare and have available to the PHMC a briefing of the } \\
\text { M\&O Contractor's Management Control System Policy and } \\
\text { Procedures. Make available copies of the pertinent }\end{array}$ & $8 / 09 / 96$ & $\begin{array}{l}\text { PJ Bence } \\
\text { RS Wade }\end{array}$ \\
\hline
\end{tabular}




\subsubsection{DOE Site and Performance Reporting.}

\begin{tabular}{|c|c|c|}
\hline \multicolumn{1}{|c|}{ Pre-Award Actions } & $\begin{array}{c}\text { Scheduled } \\
\text { Date }\end{array}$ & Actionee(s) \\
\hline $\begin{array}{l}\text { 1. Develop a list of the DOE site and performance reporting } \\
\text { systems being utilized by the M\&O Contractor and their } \\
\text { processes. }\end{array}$ & $\begin{array}{c}6 / 01 / 96 \\
\text { Completed }\end{array}$ & C Edwards \\
\hline \multicolumn{1}{|c|}{ Post-Award Actions } & & $8 / 05 / 96$ \\
\hline $\begin{array}{l}\text { 1. Prepare and have available for the PHMC a briefing of the } \\
\text { Program Management and Control System implementation, } \\
\text { systems, impacts to other performance reporting systems } \\
\text { (SMS, PTS, CMM, etc.) }\end{array}$ & C Edwards \\
\hline
\end{tabular}

\subsubsection{Project Analysis \& Reporting System. Project Analysis \& Reporting System} (PARS) is a cost and performance analytical tool designed for ease of use by Cost Account Managers and management. All Hanford Site employees have access. PARS extracts data from the FDS.

\begin{tabular}{|c|c|c|}
\hline Pre-Award Actions & $\begin{array}{l}\text { Scheduled } \\
\text { Date }\end{array}$ & Actionee(s) \\
\hline $\begin{array}{l}\text { 1. Assemble documentation associated with PARS including: } \\
\text { - Sample reports } \\
\text { - System description } \\
\text { - Manuals } \\
\text { - Procedures. }\end{array}$ & $\begin{array}{c}5 / 15 / 96 \\
\text { Cornpleted }\end{array}$ & $\begin{array}{l}\text { RF Blake } \\
\text { RA Marshall }\end{array}$ \\
\hline 2. Verify that the PARS module is up-to-date. & $\begin{array}{l}\text { 6/01/96 } \\
\text { Completed }\end{array}$ & $\begin{array}{l}\text { R Blake } \\
\text { R Marshall }\end{array}$ \\
\hline $\begin{array}{l}\text { 3. Make PARS documentation available for use by the PHMC. } \\
\text { Provide copies upon request. }\end{array}$ & $\begin{array}{l}6 / 10 / 96 \\
\text { Completed }\end{array}$ & $\begin{array}{l}\text { R Blake } \\
\text { R Marshall }\end{array}$ \\
\hline
\end{tabular}

\subsubsection{Miscellaneous Planning \& Reporting Databases.}

\begin{tabular}{|l|c|l|}
\hline \multicolumn{1}{|c|}{ Pre-Award Actions } & \multicolumn{1}{|c|}{$\begin{array}{c}\text { Scheduled } \\
\text { Date }\end{array}$} & Actionee(s) \\
\hline $\begin{array}{l}\text { 1. Develop a list and assemble key Planning and Reporting } \\
\text { Databases utilized by the M\&O Contractor in support of the } \\
\text { site. }\end{array}$ & $\begin{array}{l}6 / 01 / 96 \\
\text { Completed }\end{array}$ & PJ Bence \\
\hline $\begin{array}{l}\text { 2. Make available information regarding miscellaneous } \\
\text { processes in support of the control functions for the M\&O } \\
\text { contractor. }\end{array}$ & $7 / 26 / 96$ & $\begin{array}{l}\text { PJ Bence } \\
\text { CT Hewitt } \\
\text { AA Carter } \\
\text { BA Reichnuth } \\
\text { JK Fish }\end{array}$ \\
\hline
\end{tabular}

3.2.2.7 Overhead Rate Structure. It is assumed the overhead rate structure will change under the PHMC concept. The necessary actions and changes will need to occur to implement the new overhead methodology. 


\begin{tabular}{|c|c|c|}
\hline Pre-Award Actions & $\begin{array}{l}\text { Scheduled } \\
\text { Date }\end{array}$ & Actionee(s) \\
\hline 1. Prepare an overhead/indirect briefing for the PHMC. & $\begin{array}{c}6 / 01 / 96 \\
\text { Completed }\end{array}$ & AA Carter \\
\hline $\begin{array}{l}\text { 2. Develop the overhead/indirect methodology to be used for } \\
\text { FY } 1997 \text {. }\end{array}$ & $7 / 01 / 96$ & AA Carter \\
\hline Post-Award Actions & & \\
\hline $\begin{array}{l}\text { 1. Notify programs/projects of specific overhead assessments for } \\
\text { FY } 1997 .\end{array}$ & $8 / 31 / 96$ & $\begin{array}{l}\text { AA Carter } \\
\text { PHMC }\end{array}$ \\
\hline $\begin{array}{l}\text { 2. Implement the necessary system changes to support the } \\
\text { PHMC overhead methodology. }\end{array}$ & $9 / 28 / 96$ & PC Felts \\
\hline
\end{tabular}

\subsubsection{Financial Systems.}

\begin{tabular}{|c|c|c|}
\hline Pre-Award Actions & $\begin{array}{l}\text { Scheduled } \\
\text { Date }\end{array}$ & Actionee(s) \\
\hline $\begin{array}{l}\text { 1. Prepare an overview of the Financial Systems currently in } \\
\text { use. }\end{array}$ & $\begin{array}{l}\text { 6/01/96 } \\
\text { Completed }\end{array}$ & $\begin{array}{l}\text { P Felis } \\
\text { BCSR }\end{array}$ \\
\hline Post-Award Actions & & \\
\hline $\begin{array}{l}\text { 1.. Perform assessment of financial systems to accommodate a } \\
\text { two-month transition period. }\end{array}$ & $7 / 26 / 96$ & $\begin{array}{l}\text { BCSR } \\
\text { FAC Transition Team }\end{array}$ \\
\hline
\end{tabular}

\subsubsection{Miscellaneous Financial Administration and Control Actions.}

\begin{tabular}{|l|l|l|}
\hline \multicolumn{1}{|c|}{ Pre-Award Actions } & $\begin{array}{l}\text { Scheduled } \\
\text { Date }\end{array}$ & Actionee(s) \\
\hline $\begin{array}{l}\text { 1. Verify "Smart Book" is current, to include update of any } \\
\text { material contained within, and forward to Transition Project } \\
\text { Manager. }\end{array}$ & $7 / 15 / 96$ & RB Agee \\
\hline $\begin{array}{l}\text { 2. Prepare and make available to the PHMC an overview of the } \\
\text { FAC organization and associated transition activities. }\end{array}$ & $7 / 15 / 96$ & DW Messinger \\
\hline $\begin{array}{l}\text { 3. Disposition action items as documented in the HATS } \\
\text { database. Process appropriate closure notices. }\end{array}$ & $7 / 15 / 96$ & $\begin{array}{l}\text { PC Felts } \\
\text { MJ Byrd } \\
\text { LR Hafer }\end{array}$ \\
\hline $\begin{array}{l}\text { 4. Complete an inventory of all software assigned to FAC } \\
\text { employees. Ensure that appropriate update documentation is } \\
\text { processed. }\end{array}$ & $7 / 15 / 96$ & CL Biberstine \\
\hline $\begin{array}{l}\text { 5. Prepare satellite areas for use by the PHMC, to include } \\
\text { services and inclusion of selected key documentation. }\end{array}$ & $7 / 22 / 96$ & $\begin{array}{l}\text { PC Felts } \\
\text { MJ Byrd } \\
\text { LR Hafer } \\
\text { Bldg. Admin. }\end{array}$ \\
\hline
\end{tabular}




\begin{tabular}{|c|c|c|}
\hline Pre-Award Actions & Schedaled & Date \\
\hline $\begin{array}{l}\text { 6. Complete an analysis of existing Financial Systern } \\
\text { applications and document present capabilities and transition } \\
\text { alternatives for the PHMC. }\end{array}$ & $7 / 26 / 96$ & DD Ripplinger \\
\hline Post-Award Actions & & \\
\hline $\begin{array}{l}\text { 1. Support development of an agreement between the PHMC, } \\
\text { RL, and WHC regarding closeout of the WHC contract. }\end{array}$ & $9 / 20 / 96$ & KC Williams \\
\hline
\end{tabular}

\subsection{HUMAN RESOURCES}

Human Resources will ensure continuity in all of its functional areas and fulfill ongoing commitments during contract transition. A comprehensive listing, by function, of key issues, actions, and activities is set forth below. Through this process all essential data will be readily available to carry out a smooth and informed transition of the workforce to the incoming PHMC contractor. Key assumptions made to support a 60-day transition are as follows:

- All incumbent WHC/BCSR/ICF KH employees, other than the approximately 230 employees occupying management positions as defined in the RFP, i.e., "...those above first-line managerial/supervisory level..." , will, in effect, be "mapped" to the PHMC organization. Involuntary separation notices to managers not employed by the PHMC will be issued no sooner than September 30, 1996.

- PHMC will determine the employer-of-record for all transitioning personnel and report assignments for existing HRIS and payroll system processing. This will be accomplished by September 1, 1996, to ensure uninterrupted employee wage payment.

- PHMC will have a one month "selection" window (August) for scheduling and completing management interviews and offers.

- Permanent separation notices will be issued to managers not offered positions of comparable responsibility and they will be provided separation benefits consistent with the current Hanford Site Workforce Restructuring Plan.

- A shortened transition period will impede the PHMC's ability to implement any substantive changes to HR policies, procedures, and practices concurrent with takeover due to likely impacts on employees engaged in activities or coverages of such programs as vacations, holidays, educational assistance, shift schedules, etc. Therefore, most HR, if not all, related policies will likely transition on October 1, 1996. 
- Insufficient time to effectively communicate and/or enroll employees in new insurance programs and other benefits will result in PHMC carryover of existing M\&O Contractor health and welfare plans with coverage continuation until at least January 1, 1997. This would also apply to the M\&O Contractor pension and $401 \mathrm{k}$ plans.

- With BHI enrollment in all M\&O Contractor benefit plans, administered by WHC, service continuation for its nearly 800 employees will be required until such time that alternative administration or new plan introduction can be accomplished.

- Worker's Compensation and all other statutory benefits will continue as they are administered presently and, therefore, would be carried over by the PHMC at transition.

- The PHMC in all likelihood cannot bargain new labor contracts in the compressed 60-day transition period, given two union contracts (HAMTC and Hanford Guards Union (HGU) and the historical bargaining time line of six to ten months. Therefore, to avoid progress of work disruptions and any potential for a union strike, the PHMC will, most likely, become signatory to present M\&O Contractor labor agreements or sign memorandums of understanding to continue present contract terms and conditions in full force until replacement agreements are reached and signed.

- The PHMC will attain signatory status or sign memoranda within the M\&O Contractor 60-day window leading to termination of WHC labor contracts with HAMTC and HGU on September 30, 1996.

- The above approach will likely apply to the ICF KH labor agreement with the OPEIU, covering approximately 50 clerical employees.

- ICF KH will conduct negotiations for a new QC/NDT agreement, based on the August 31, 1996, current contract expiration date. Contract covers approximately ten employees. PHMC may, as appropriate, seek input to the bargaining process and contract assignment.

- Privacy of corporate and personnel records must be assured. Personnel and payroll records for identified M\&O Contractor executives will be segregated and access limited.

\subsubsection{Personnel Resources (WHC/BCSR)}

Develop actions to prepare, describe, and deliver essential information and records regarding staffing, site access support, outplacement, personnel records and database management. 


\subsubsection{Staffing.}

\begin{tabular}{|c|c|c|}
\hline Pre-Award Actions & $\begin{array}{l}\text { Scheduled } \\
\text { Date }\end{array}$ & Actionee(s) \\
\hline $\begin{array}{l}\text { 1. Establish selection process and appropriately formatted pre- } \\
\text { interview information on the background and experience of } \\
\text { incumbent employees. }\end{array}$ & $\begin{array}{c}5 / 15 / 96 \\
\text { Completed }\end{array}$ & $\begin{array}{l}\text { RW Holleran } \\
\text { DE Hansen }\end{array}$ \\
\hline $\begin{array}{l}\text { 2. Develop a hiring process flow chart for all categories of } \\
\text { employees. }\end{array}$ & $\begin{array}{c}6 / 01 / 96 \\
\text { Completed }\end{array}$ & $\begin{array}{l}\text { RW Holleran } \\
\text { DE Hansen }\end{array}$ \\
\hline $\begin{array}{l}\text { 3. Develop procedure for placement options for all incumbent } \\
\text { employees, e.g. selected; not selected; offer refused; WARN, } \\
\text { etc. }\end{array}$ & $\begin{array}{l}6 / 01 / 96 \\
\text { Completed }\end{array}$ & $\begin{array}{l}\text { RW Holleran } \\
\text { DE Hansen }\end{array}$ \\
\hline $\begin{array}{l}\text { 4. Develop appropriate reports depicting status of all } \\
\text { staffing/placement activities. }\end{array}$ & $\begin{array}{c}\text { 6/01/96 } \\
\text { Completed }\end{array}$ & $\begin{array}{l}\text { RW Holleran } \\
\text { DE Hansen }\end{array}$ \\
\hline $\begin{array}{l}\text { 5. Update the contractual site-wide bargaining unit recall lists } \\
\text { and non-exempt/non-bargaining recall list. }\end{array}$ & $\begin{array}{c}\text { 6/01/96 } \\
\text { Completed }\end{array}$ & $\begin{array}{l}\text { RW Holleran } \\
\text { DE Hansen }\end{array}$ \\
\hline $\begin{array}{l}\text { 6. Update the Re-Deployment Assistance (RDA) list of non- } \\
\text { bargaining employees requiring job re-assignments. }\end{array}$ & $\begin{array}{c}\text { 6/01/96 } \\
\text { Completed }\end{array}$ & $\begin{array}{l}\text { RW Holleran } \\
\text { DE Hansen }\end{array}$ \\
\hline 7. Prepare stafing plans, status, and rational for the PHMC. & $\begin{array}{c}6 / 01 / 96 \\
\text { Completed }\end{array}$ & $\begin{array}{l}\text { RW Holleran } \\
\text { DE Hansen }\end{array}$ \\
\hline - $\quad$ Post-Award Actions & & \\
\hline $\begin{array}{l}\text { 1. Recommend to PHMC use of a centralized clearinghouse to } \\
\text { manage placements during transition. Coordinate employment } \\
\text { interview schedules, determine offer letter requirements and } \\
\text { maintain tracking system for interviews, offers, accepts and } \\
\text { declines. }\end{array}$ & $8 / 05 / 96$ & $\begin{array}{l}\text { RW Holleran } \\
\text { DE Hansen }\end{array}$ \\
\hline $\begin{array}{l}\text { 2. Provide PHMC with WHC flow chart depicting } \\
\text { staffing/placement processes. }\end{array}$ & $8 / 05 / 96$ & $\begin{array}{l}\text { RW Holleran } \\
\text { DE Hansen }\end{array}$ \\
\hline $\begin{array}{l}\text { 3. Provide PHMC with procedure identified by WHC for } \\
\text { disposition of all non-transitioning incumbent employees. }\end{array}$ & $8 / 15 / 96$ & $\begin{array}{l}\text { RW Holleran } \\
\text { DE Hansen }\end{array}$ \\
\hline $\begin{array}{l}\text { 4. Provide PHMC with reports, as appropriate, depicting pre- } \\
\text { turnover status of all staffing/placement activities. }\end{array}$ & $8 / 15 / 96$ & $\begin{array}{l}\text { RW Holleran } \\
\text { DE Hansen }\end{array}$ \\
\hline $\begin{array}{l}\text { 5. Provide PHMC with sitewide bargaining unit recall lists and } \\
\text { salaried non-exempt downgrade and recall lists. }\end{array}$ & $10 / 01 / 96$ & $\begin{array}{l}\text { RW Holleran } \\
\text { DE Hansen }\end{array}$ \\
\hline 6. Provide appropriate information on excess employees. & $10 / 01 / 96$ & $\begin{array}{l}\text { RW Holleran } \\
\text { DE Hansen }\end{array}$ \\
\hline 7. Provide staffing plans, status, and rational to the PHMC. & $10 / 01 / 96$ & $\begin{array}{l}\text { RW Holleran } \\
\text { DE Hansen }\end{array}$ \\
\hline
\end{tabular}

\subsubsection{Site Access Support Services.}

\begin{tabular}{|c|c|c|}
\hline \multicolumn{1}{|c|}{ Pre-Award Actions } & $\begin{array}{c}\text { Scheduled } \\
\text { Date }\end{array}$ & Actionee(s) \\
\hline $\begin{array}{l}\text { 1. Develop description of Pre-Employment Investigations } \\
\text { process flow. }\end{array}$ & $\begin{array}{c}6 / 01 / 96 \\
\text { Completed }\end{array}$ & ER Jackson \\
\hline
\end{tabular}




\begin{tabular}{|c|c|c|}
\hline Pre-Award Actions & $\begin{array}{l}\text { Scheduled } \\
\text { Date }\end{array}$ & Actionee(s) \\
\hline $\begin{array}{l}\text { 2. Develop description of Human Reliability Program (HRP) } \\
\text { processes including Personnel Security Assurance Program } \\
\text { (PSAP), U.S. Department of Transportation (DOT), and all } \\
\text { other substance abuse testing programs. }\end{array}$ & $\begin{array}{l}\text { 6/01/96 } \\
\text { Completed }\end{array}$ & $\begin{array}{l}\text { ER Jackson } \\
\text { BD Corder }\end{array}$ \\
\hline $\begin{array}{l}\text { 3. Develop description of Foreign National Visitors assignments } \\
\text { process, including usage statistics. }\end{array}$ & $\begin{array}{c}\text { 6/01/96 } \\
\text { Completed } \\
\end{array}$ & ER Jackson \\
\hline \multicolumn{3}{|l|}{ Post-Award Actions } \\
\hline 1. Provide process flow of Pre-Employment Investigations. & 9/01/96 & ER Jackson \\
\hline $\begin{array}{l}\text { 2. Provide description of Human Reliability Program (HRP) } \\
\text { processes including PSAP, DOT, and all other substances } \\
\text { abuse testing programs. }\end{array}$ & $9 / 01 / 96$ & $\begin{array}{l}\text { ER Jackson } \\
\text { BD Corder }\end{array}$ \\
\hline $\begin{array}{l}\text { 3. Provide Foreign National Visitors assignments process, } \\
\text { including usage statistics. }\end{array}$ & 9/01/96 & ER Jackson \\
\hline
\end{tabular}

\subsubsection{Outplacement.}

\begin{tabular}{|c|c|c|}
\hline Pre-Award Actions & $\begin{array}{c}\text { Scheduled } \\
\text { Date }\end{array}$ & Actionee(s) \\
\hline $\begin{array}{l}\text { 1. Contact General Counsel for determination of ownership of } \\
\text { "former employee" Re-Employment Opportunity Center } \\
\text { (ROC) files and usage database. }\end{array}$ & $\begin{array}{l}5 / 15 / 96 \\
\text { Completed }\end{array}$ & CH Smith \\
\hline $\begin{array}{l}\text { 2. Collect copies of Hanford Workforce Restructuring Plan and } \\
\text { the ROC brochure. }\end{array}$ & $\begin{array}{c}6 / 01 / 96 \\
\text { Completed }\end{array}$ & $\mathrm{CH}$ Smith \\
\hline $\begin{array}{l}\text { 3. Prepare a list of all former employees with } 3161 \text { Plan hiring } \\
\text { preference. }\end{array}$ & $\begin{array}{l}6 / 01 / 96 \\
\text { Completed }\end{array}$ & CH Smith \\
\hline $\begin{array}{l}\text { 4. Prepare guidance worksheets and coordinate applicable } \\
\text { resources for preparation of resumes, or the like, prior to } \\
\text { employees interviewing with the PHMC contractor. }\end{array}$ & $\begin{array}{l}\text { 6/01/96 } \\
\text { Completed }\end{array}$ & $\begin{array}{l}\text { CH Smith } \\
\text { CA Locke }\end{array}$ \\
\hline $\begin{array}{l}\text { 5. Develop a recommendation for the PHMC contractor to } \\
\text { support continuation of outplacement services aiready in } \\
\text { progress. }\end{array}$ & $\begin{array}{l}6 / 01 / 96 \\
\text { Completed }\end{array}$ & CH Smith \\
\hline Post-Award Actions & & 18 \\
\hline $\begin{array}{l}\text { 1. Coordinate with PHMC contractor to ensure job search, } \\
\text { interviewing and transition resources for employees not } \\
\text { receiving or declining employment offers. }\end{array}$ & $8 / 05 / 96$ & CH Smith \\
\hline $\begin{array}{l}\text { 2. Provide to the PHMC contractor copies of Hanford } \\
\text { Workforce Restructuring Plan and the ROC brochure. }\end{array}$ & $8 / 05 / 96$ & CH Smith \\
\hline $\begin{array}{l}\text { 3. Provide a list of all former employees with } 3161 \text { Plan hiring } \\
\text { preference. }\end{array}$ & $8 / 05 / 96$ & CH Smith \\
\hline $\begin{array}{l}\text { 4. Provide guidance worksheets and coordinate applicable } \\
\text { resources for preparation of resumes, or the like, prior to } \\
\text { employees interviewing with the PHMC contractor. }\end{array}$ & $8 / 05 / 96$ & $\begin{array}{l}\text { CH Smith } \\
\text { CA Locke }\end{array}$ \\
\hline
\end{tabular}




\begin{tabular}{|l|c|c|}
\hline Post-Award Actions & Scheduled & Actionee(s) \\
\hline $\begin{array}{l}\text { 5. Provide recommendation for the PHMC contractor to support } \\
\text { continuation of outplacement services already in progress. }\end{array}$ & $8 / 15 / 96$ & CH Srnith \\
\hline
\end{tabular}

\subsubsection{Personnel Records and Database Administration.}

\begin{tabular}{|c|c|c|}
\hline Pre-Award Actions & $\begin{array}{l}\text { Scheduled } \\
\text { Date }\end{array}$ & Actionee(s) \\
\hline $\begin{array}{l}\text { 1. Determine, in conjunction with General Counsel, extent of } \\
\text { employee records and data to be transferred to PHMC. }\end{array}$ & $\begin{array}{l}6 / 01 / 96 \\
\text { Completed }\end{array}$ & $\begin{array}{l}\text { CA Locke } \\
\text { CK MacLeod }\end{array}$ \\
\hline $\begin{array}{l}\text { 2. Review readiness to provide PHMC appropriate employee } \\
\text { demographic data, by organization, such as rame, position, } \\
\text { classification, employment stanus and service date. }\end{array}$ & $\begin{array}{l}\text { 6/01/96 } \\
\text { Completed }\end{array}$ & CA Locke \\
\hline 3. Plan a demonstration of PeoplesOFT capabilities for PHMC. & $\begin{array}{l}6 / 01 / 96 \\
\text { Completed }\end{array}$ & CA Locke \\
\hline $\begin{array}{l}\text { 4. Prepare update for system modification priorities and } \\
\text { troubleshooting activities to the PeopleSoft database. }\end{array}$ & $\begin{array}{c}6 / 01 / 96 \\
\text { Completed }\end{array}$ & CA Locke \\
\hline 5. Develop detailed organization charts/lists. & $\begin{array}{l}\text { 6/01/96 } \\
\text { Completed }\end{array}$ & CA Locke \\
\hline $\begin{array}{l}\text { 6. Develop listing of computer hardware/software for Human } \\
\text { Resources department personnel. }\end{array}$ & $\begin{array}{l}\text { 6/01/96 } \\
\text { Completed }\end{array}$ & CA Locke \\
\hline Post-Award Actions & & \\
\hline $\begin{array}{l}\text { 1. Provide personnel data and final lists transferring incumbent } \\
\text { employees to PHMC. }\end{array}$ & $10 / 01 / 96$ & CA Locke \\
\hline $\begin{array}{l}\text { 2. Coordinate with PHMC to determine method for transferring } \\
\text { required employee personnel information. }\end{array}$ & $9 / 01 / 96$ & CA Locke \\
\hline $\begin{array}{l}\text { 3. Obtain appropriate personnel information releases from } \\
\text { employees. }\end{array}$ & $10 / 01 / 96$ & CA Locke \\
\hline 4. Demonstrate PeopleSOFT to PHMC contractor. & $10 / 01 / 96$ & CA Locke \\
\hline 5. Provide appropriate organization charts/lists to PHMC. & $8 / 05 / 96$ & CA Locke \\
\hline $\begin{array}{l}\text { 6. Provide a listing of computer hardware/software for Human } \\
\text { Resources department personnel. }\end{array}$ & $10 / 01 / 96$ & CA Locke \\
\hline 7. Initiate status change forms for employees. & $10 / 01 / 96$ & CA Loke \\
\hline
\end{tabular}

\subsubsection{Personnel Resources (ICF KH)}

Develop actions, where activity is unique or not provided by WHC, to prepare, describe, and deliver essential information and records regarding staffing, site access support, outplacement, personnel records, and database management. ICF KH will coordinate with WHC to avoid duplication in briefings or submission of multiple formats. 


\subsubsection{Staffing.}

\begin{tabular}{|c|c|c|}
\hline Pre-Award Actions & $\begin{array}{l}\text { Scheduled } \\
\text { Date }\end{array}$ & Actionee(s) \\
\hline $\begin{array}{l}\text { 1. Establish selection process and appropriately formatted pre- } \\
\text { interview information on the background and experience of } \\
\text { incumbent employees. Coordinate with WHC, as } \\
\text { appropriate, to avoid multiple formats. }\end{array}$ & $\begin{array}{c}5 / 15 / 96 \\
\text { Completed }\end{array}$ & $\begin{array}{l}\text { VC Hodgin } \\
\text { CG Larsen }\end{array}$ \\
\hline $\begin{array}{l}\text { 2. Develop a hiring process flow chart for all categories of } \\
\text { employees. }\end{array}$ & $\begin{array}{c}\text { 6/01/96 } \\
\text { Completed }\end{array}$ & $\begin{array}{l}\text { VC Hodgin } \\
\text { CG Larsen }\end{array}$ \\
\hline $\begin{array}{l}\text { 3. Develop procedure for placement options for all incumbent } \\
\text { employees, e.g., selected; not selected; offer refused; } \\
\text { WARN, etc. }\end{array}$ & $\begin{array}{l}\text { 6/01/96 } \\
\text { Completed }\end{array}$ & $\begin{array}{l}\text { VC Hodgin } \\
\text { CG Larsen }\end{array}$ \\
\hline $\begin{array}{l}\text { 4. Develop appropriate reports depicting status of all } \\
\text { staffing/placement activities. }\end{array}$ & $\begin{array}{c}6 / 01 / 96 \\
\text { Completed }\end{array}$ & $\begin{array}{l}\text { VC Hodgin } \\
\text { CG Larsen }\end{array}$ \\
\hline $\begin{array}{l}\text { 5. Update the contractual sitewide bargaining unit recall lists } \\
\text { and non-exempt/non-bargaining recall list. }\end{array}$ & $\begin{array}{c}6 / 01 / 96 \\
\text { Completed }\end{array}$ & $\begin{array}{l}\text { VC Hodgin } \\
\text { CG Larsen }\end{array}$ \\
\hline $\begin{array}{l}\text { 6. Update the Re-Deployment Assistance (RDA) list of non- } \\
\text { bargaining employees requiring job re-assignments. }\end{array}$ & $\begin{array}{c}6 / 01 / 96 \\
\text { Completed }\end{array}$ & $\begin{array}{l}\text { VC Hodgin } \\
\text { JJ Chumn }\end{array}$ \\
\hline 7. Prepare staffing plans, status, and rational for the PHMC. & $\begin{array}{c}\text { 6/01/96 } \\
\text { Completed }\end{array}$ & $\begin{array}{l}\text { VC Hodgin } \\
\text { CG Larsen }\end{array}$ \\
\hline Post-Award Actions & & \\
\hline $\begin{array}{l}\text { 1. Recommend to PHMC use of a centralized clearing house to } \\
\text { manage placements during transition. Coordinate } \\
\text { employment interview schedules, determine offer letter } \\
\text { requirements and maintain tracking system for interviews, } \\
\text { offers, accepts and declines. }\end{array}$ & $8 / 05 / 96$ & $\begin{array}{l}\text { VC Hodgin } \\
\text { CG Larsen }\end{array}$ \\
\hline $\begin{array}{l}\text { 2. Provide PHMC with flow chart depicting staffing/placement } \\
\text { processes. }\end{array}$ & $8 / 05 / 96$ & $\begin{array}{l}\text { VC Hodgin } \\
\text { CG Larsen }\end{array}$ \\
\hline $\begin{array}{l}\text { 3. Provide PHMC with procedure identified for disposition of } \\
\text { all incumbent employees. }\end{array}$ & $8 / 15 / 96$ & $\begin{array}{l}\text { VC Hodgin } \\
\text { CG Larsen }\end{array}$ \\
\hline $\begin{array}{l}\text { 4. Provide PHMC with reports, as appropriate, depicting status } \\
\text { of all staffing/placement activities. }\end{array}$ & $8 / 15 / 96$ & $\begin{array}{l}\text { VC Hodgin } \\
\text { CG Larsen } \\
\text { JJ Chunn }\end{array}$ \\
\hline 5. Provide appropriate information on excess employees. & $10 / 01 / 96$ & $\begin{array}{l}\text { VC Hodgin } \\
\text { JJ Chunn }\end{array}$ \\
\hline 6. Provide staffing plans, status, and rational to the PHMC. & $10 / 01 / 96$ & $\begin{array}{l}\text { VC Hodgin } \\
\text { CG Larsen }\end{array}$ \\
\hline
\end{tabular}

\subsubsection{Site Access Support Services}

\begin{tabular}{|c|c|c|}
\hline Pre-Award Actions & $\begin{array}{l}\text { Scheduled } \\
\text { Date }\end{array}$ & Actionee(s) \\
\hline $\begin{array}{l}\text { 1. Develop description of Pre-Employment Investigations } \\
\text { process flow. }\end{array}$ & $\begin{array}{l}6 / 01 / 96 \\
\text { Completed }\end{array}$ & CG Larsen \\
\hline
\end{tabular}




\begin{tabular}{|c|c|c|}
\hline Post-Award Actions & $\begin{array}{c}\text { Scheduled } \\
\text { Date }\end{array}$ & Actionee(s) \\
\hline 1. Provide process flow of Pre-Employment Investigations. & 9/01/96 & CG Larsen \\
\hline
\end{tabular}

\subsubsection{Outplacement.}

\begin{tabular}{|c|c|c|}
\hline Pre-Ayard Actions & $\begin{array}{c}\text { Scheduled } \\
\text { Date }\end{array}$ & Actionee(s) \\
\hline $\begin{array}{l}\text { 1. Prepare a list of all former employees with } 3161 \text { Plan hiring } \\
\text { preference. }\end{array}$ & $\begin{array}{c}6 / 01 / 96 \\
\text { Completed }\end{array}$ & CG Larsen \\
\hline Post-Award Actions & & \\
\hline $\begin{array}{l}\text { 1. Provide a list of all former employees with } 3161 \text { Plan hiring } \\
\text { preference. }\end{array}$ & $8 / 05 / 96$ & CG Larsen \\
\hline
\end{tabular}

\subsubsection{Personnel Records and Database Administration.}

\begin{tabular}{|c|c|c|}
\hline Pre-Award Actions & Scheduled & Actionee(s) \\
\hline $\begin{array}{l}\text { 1. Determine, in conjunction with General Counsel, extent of } \\
\text { employee records and data to be transferred to PHMC. }\end{array}$ & $\begin{array}{l}\text { 6/01/96 } \\
\text { Completed }\end{array}$ & $\begin{array}{l}\text { VC Hodgin } \\
\text { Olsen }\end{array}$ \\
\hline $\begin{array}{l}\text { 2. Review readiness to provide PHMC appropriate employee } \\
\text { demographic data, by organization, such as name, position, } \\
\text { classification, employment status and service date. }\end{array}$ & $\begin{array}{l}6 / 01 / 96 \\
\text { Completed }\end{array}$ & $\begin{array}{l}\text { VC Hodgin } \\
\text { CG Larsen }\end{array}$ \\
\hline 3. Develop detailed organization charts/lists. & $\begin{array}{c}6 / 01 / 96 \\
\text { Completed } \\
\end{array}$ & $\begin{array}{l}\text { VC Hodgin } \\
\text { CG Larsen } \\
\end{array}$ \\
\hline $\begin{array}{l}\text { 4. Develop listing of computer hardware/software for Human } \\
\text { Resources department personnel. }\end{array}$ & $\begin{array}{c}6 / 01 / 96 \\
\text { Completed }\end{array}$ & CG Larsen \\
\hline \multicolumn{3}{|l|}{ Post-Award Actions } \\
\hline $\begin{array}{l}\text { 1. Provide personnel data and final lists transferring incumbent } \\
\text { employees to PHMC. }\end{array}$ & $10 / 01 / 96$ & $\begin{array}{l}\text { VC Hodgin } \\
\text { CG Larsen }\end{array}$ \\
\hline 2. Initiate status change forms for employees. & $10 / 01 / 96$ & CG Larsen \\
\hline $\begin{array}{l}\text { 3. Obtain appropriate personnel information releases from } \\
\text { employees. }\end{array}$ & $10 / 01 / 96$ & $\begin{array}{l}\text { VC Hodgin } \\
\text { CG Larsen }\end{array}$ \\
\hline 4. Provide appropriate organization charts/tists to PHMC. & $10 / 01 / 96$ & $\begin{array}{l}\text { VC Hodgin } \\
\text { CG Larsen }\end{array}$ \\
\hline $\begin{array}{l}\text { 5. Provide a listing of computer hardware/software for Human } \\
\text { Resources department personnel. }\end{array}$ & $10 / 01 / 96$ & CG Larsen \\
\hline
\end{tabular}




\subsubsection{Total Compensation (WHC/BCSR)}

Determine, develop, and prepare appropriate data regarding the salary plan, worker's compensation, job classifications, salary structures, and other compensation programs. Perform the same requirements as they relate to benefit plan documentation, plan coverages and status, return-to-work, pension, savings, recognition programs and the like.

\subsubsection{Salary Plan.}

\begin{tabular}{|c|c|c|}
\hline Pre-Award Actions & $\begin{array}{l}\text { Scheduled } \\
\text { Date }\end{array}$ & Actionee(s) \\
\hline $\begin{array}{l}\text { 1. Prepare a list of calendar year salary plan actions scheduled } \\
\text { for October, November, and December } 1996 .\end{array}$ & $\begin{array}{l}\text { 6/01/96 } \\
\text { Completed }\end{array}$ & $\begin{array}{l}\text { FA Powers } \\
\text { H Lacher }\end{array}$ \\
\hline $\begin{array}{l}\text { 2. Determine ownership of prior year salary increase plans and } \\
\text { disposition accordingly. }\end{array}$ & $\begin{array}{c}6 / 01 / 96 \\
\text { Completed }\end{array}$ & $\begin{array}{l}\text { FA Powers } \\
\text { H Lacher }\end{array}$ \\
\hline $\begin{array}{l}\text { 3. Determine distribution of remaining salary increase funds } \\
\text { which are usually distributed as adjustments in the } \\
\text { November/December time frame. Estimate available funds } \\
\text { and recommend a distribution method. }\end{array}$ & $\begin{array}{l}6 / 01 / 96 \\
\text { Completed }\end{array}$ & $\begin{array}{l}\text { FA Powers } \\
\text { H Lacher }\end{array}$ \\
\hline \multicolumn{3}{|l|}{ Post-Award Actions } \\
\hline $\begin{array}{l}\text { 1. Status PHMC contractor regarding salary actions for } \\
\text { October, November, and December } 1996 \text { for those } \\
\text { employees accepting offers. }\end{array}$ & $10 / 01 / 96$ & $\begin{array}{l}\text { FA Powers } \\
\text { H Lacher }\end{array}$ \\
\hline $\begin{array}{l}\text { 2. Review salary planning process and provide PHMC } \\
\text { contractor a list of prior year salary increase plans as } \\
\text { appropriate. }\end{array}$ & $10 / 01 / 96$ & $\begin{array}{l}\text { FA Powers } \\
\text { H Lacher }\end{array}$ \\
\hline $\begin{array}{l}\text { 3. Coordinate with PHMC to determine distribution of } \\
\text { remaining salary increase funds which are usually distributed } \\
\text { as adjustments in the November/December time frame. } \\
\text { Estimate available funds and recommend a distribution } \\
\text { method. }\end{array}$ & $10 / 01 / 96$ & $\begin{array}{l}\text { FA Powers } \\
\text { H Lacher }\end{array}$ \\
\hline
\end{tabular}

\subsubsection{Workers Compensation.}

\begin{tabular}{|c|c|c|}
\hline Pre-Award Actions & $\begin{array}{c}\text { Scheduled } \\
\text { Date }\end{array}$ & Actionee(s) \\
\hline $\begin{array}{l}\text { 1. Develop a computerized report to compile a preliminary } \\
\text { listing of Workers Compensation liabilities including names, } \\
\text { provisions under which they left, staus including liabilities } \\
\text { associated with all potential outcomes (ICF KH, BCSR, and } \\
\text { WHC). }\end{array}$ & $\begin{array}{c}6 / 01 / 96 \\
\text { Completed }\end{array}$ & $\begin{array}{l}\text { FA Powers } \\
\text { H Lacher }\end{array}$ \\
\hline Post-Award Actions & & \\
\hline $\begin{array}{l}\text { 1. Provide the PHMC contractor a complete preliminary list of } \\
\text { employees on Workers Compensation by company (ICF KH, } \\
\text { BCSR, and WHC). }\end{array}$ & $8 / 05 / 96$ & $\begin{array}{l}\text { FA Powers } \\
\text { H Lacher }\end{array}$ \\
\hline
\end{tabular}




\begin{tabular}{|c|c|c|}
\hline Post-Award Actions & $\begin{array}{c}\text { Scheduled } \\
\text { Date }\end{array}$ & Actionee(s) \\
\hline $\begin{array}{l}\text { 2. Generate a final report for turnover of Workers } \\
\text { Compensation liabilities including names, provisions under } \\
\text { which they left, and the liabilities associated with all potential } \\
\text { outcomes. }\end{array}$ & $10 / 01 / 96$ & $\begin{array}{l}\text { FA Powers } \\
\text { H Lacher }\end{array}$ \\
\hline
\end{tabular}

\subsubsection{Job Classifications and Salary Structure.}

\begin{tabular}{|c|c|c|}
\hline Pre-Award Actions & $\begin{array}{l}\text { Scheduled } \\
\text { Date }\end{array}$ & Actionee(s) \\
\hline $\begin{array}{l}\text { 1. Prepare a complete list of employees by name, organization, } \\
\text { title, grade and actual salary. }\end{array}$ & $\begin{array}{l}\text { 6/01/96 } \\
\text { Completed }\end{array}$ & $\begin{array}{l}\text { FA Powers } \\
\text { H Lacher }\end{array}$ \\
\hline $\begin{array}{l}\text { 2. Prepare an analysis of the broadbanding salary structure pilot } \\
\text { and recommendation regarding future implementation. }\end{array}$ & $\begin{array}{l}6 / 01 / 96 \\
\text { Completed }\end{array}$ & $\begin{array}{l}\text { FA Powers } \\
\text { H Lacher }\end{array}$ \\
\hline 3. Prepare a complete listing of all jobs by title and grade. & $\begin{array}{l}6 / 01 / 96 \\
\text { Completed }\end{array}$ & $\begin{array}{l}\text { FA Powers } \\
\text { H Lacher }\end{array}$ \\
\hline $\begin{array}{l}\text { 4. Generate a report which compiles a preliminary listing of all } \\
\text { employees who qualify for certification pay or stipends and } \\
\text { the criteria under which these are paid. }\end{array}$ & $\begin{array}{l}\text { 6/01/96 } \\
\text { Completed }\end{array}$ & $\begin{array}{l}\text { FA Powers } \\
\text { H Lacher }\end{array}$ \\
\hline $\begin{array}{l}\text { 5. Develop a list of bargaining unit, salaried non-exempt and } \\
\text { exempt salary structures. }\end{array}$ & $\begin{array}{l}6 / 01 / 96 \\
\text { Completed }\end{array}$ & $\begin{array}{l}\text { FA Powers } \\
\text { H Lacher }\end{array}$ \\
\hline $\begin{array}{l}\text { 6. Prepare copies of all compensation policies including } \\
\text { temporary upgrades, appointments of fellow/advisory } \\
\text { engineers, and signature authority as well as a detailed } \\
\text { description of any other practices. }\end{array}$ & $\begin{array}{l}6 / 01 / 96 \\
\text { Completed }\end{array}$ & $\begin{array}{l}\text { FA Powers } \\
\text { H Lacher }\end{array}$ \\
\hline Post-Award Actions & & \\
\hline 1. Provide a complete listing of all jobs by title and grade. & $8 / 05 / 96$ & $\begin{array}{l}\text { FA Powers } \\
\text { H Lacher }\end{array}$ \\
\hline $\begin{array}{l}\text { 2. Provide an analysis of the broadbanding pilot and } \\
\text { recommendation regarding future implementation. }\end{array}$ & $9 / 01 / 96$ & $\begin{array}{l}\text { FA Powers } \\
\text { H Lacher }\end{array}$ \\
\hline $\begin{array}{l}\text { 3. Generate and transmit a final tumover report listing all } \\
\text { PHMC contractor accepted employees by name, organization. } \\
\text { title, grade and acnual salary. }\end{array}$ & $10 / 01 / 96$ & $\begin{array}{l}\text { FA Powers } \\
\text { H Lacher }\end{array}$ \\
\hline $\begin{array}{l}\text { 4. Generate a final turnover report listing all employees who } \\
\text { qualify for certification pay or stipends. }\end{array}$ & $10 / 01 / 96$ & $\begin{array}{l}\text { FA Powers } \\
\text { H Lacher }\end{array}$ \\
\hline $\begin{array}{l}\text { 5. Provide a list of bargaining unit, salaried non-exempt and } \\
\text { exempt salary structures. }\end{array}$ & $10 / 01 / 96$ & $\begin{array}{l}\text { FA Powers } \\
\text { H Lacher }\end{array}$ \\
\hline $\begin{array}{l}\text { 6. Provide copies of all compensation policies including } \\
\text { temporary upgrades, appointments of fellow/advisory } \\
\text { engineers, and signature authority as well as a detailed } \\
\text { description of any other practices. }\end{array}$ & $10 / 01 / 96$ & $\begin{array}{l}\text { FA Powers } \\
\text { H Lacher }\end{array}$ \\
\hline
\end{tabular}




\subsubsection{Performance Appraisals.}

\begin{tabular}{|c|c|c|}
\hline \multicolumn{1}{|c|}{ Pre-Award Actions } & $\begin{array}{l}\text { Scheduled } \\
\text { Date }\end{array}$ & Actionee(s) \\
\hline $\begin{array}{l}\text { 1. Complete an analysis of the pilot of The Employee Appraiser } \\
\text { sofware and prepare a recommendation regarding future } \\
\text { implementation. }\end{array}$ & $\begin{array}{c}6 / 01 / 96 \\
\text { Completed }\end{array}$ & $\begin{array}{l}\text { FA Powers } \\
\text { H Lacher }\end{array}$ \\
\hline $\begin{array}{l}\text { 1. Post-Award Actions } \\
\text { Appraiser software and make a recommendation regarding } \\
\text { future implementation. }\end{array}$ & $9 / 01 / 96$ & $\begin{array}{l}\text { FA Powers } \\
\text { H Lacher }\end{array}$ \\
\hline
\end{tabular}

\subsubsection{Benefits Administration.}

\section{Plan Documentation}

\begin{tabular}{|c|c|c|}
\hline Pre-Award Actions & $\begin{array}{l}\text { Scheduled } \\
\text { Date }\end{array}$ & Actionee(s) \\
\hline $\begin{array}{l}\text { 1. Identify and gather copies of all formal plan documents, } \\
\text { summary plan descriptions, and administrative contracts with } \\
\text { third parties. Review these for language pertaining to } \\
\text { sponsor change or plan termination. }\end{array}$ & $\begin{array}{l}6 / 01 / 96 \\
\text { Completed }\end{array}$ & $\begin{array}{l}\text { FA Powers } \\
\text { JR Sorensen }\end{array}$ \\
\hline $\begin{array}{l}\text { 2. Document practices and procedures not otherwise } \\
\text { documented (e.g., not in MRPs, etc.) and all known } \\
\text { commitments made by previous contractors. }\end{array}$ & $\begin{array}{c}6 / 01 / 96 \\
\text { Completed }\end{array}$ & $\begin{array}{l}\text { FA Powers } \\
\text { JR Sorensen }\end{array}$ \\
\hline $\begin{array}{l}\text { 3. To the extent they are available, collect copies of all } \\
\text { predecessor plan documents and summary plan descriptions } \\
\text { and a listing of the people to whom they apply (also covered } \\
\text { separately under the specific heading). }\end{array}$ & $\begin{array}{c}6 / 01 / 96 \\
\text { Completed }\end{array}$ & $\begin{array}{l}\text { FA Powers } \\
\text { JR Sorensen }\end{array}$ \\
\hline Post-Award Actions & & \\
\hline $\begin{array}{l}\text { 1. Provide, as appropriate, to PHMC practices and procedures } \\
\text { not otherwise documented (e.g., not in MRPs, etc.) and all } \\
\text { known commitments made by previous contractors. }\end{array}$ & $8 / 05 / 96$ & $\begin{array}{l}\text { FA Powers } \\
\text { JR Sorensen }\end{array}$ \\
\hline $\begin{array}{l}\text { 2. Provide appropriate copies, to the extent they are available, } \\
\text { of all predecessor plan documents and summary plan } \\
\text { descriptions and a listing of the people to whom they apply } \\
\text { (also covered separately under the specific heading). }\end{array}$ & $8 / 05 / 96$ & $\begin{array}{l}\text { FA Powers } \\
\text { JR Sorensen }\end{array}$ \\
\hline $\begin{array}{l}\text { 3. Notify third-party vendors/administrators of all benefit plans } \\
\text { of the upcoming contract changeover and arrange with them } \\
\text { and the PHMC contractor, for transfer, modification or } \\
\text { termination of agreements between them and PHMC. }\end{array}$ & $8 / 05 / 96$ & $\begin{array}{l}\text { FA Powers } \\
\text { JR Sorensen }\end{array}$ \\
\hline
\end{tabular}




\section{Plan Coverage and Status}

\begin{tabular}{|c|c|c|}
\hline Pre-Award Actions : & $\begin{array}{l}\text { Scheduled } \\
\text { Date }\end{array}$ & Actionee(s) \\
\hline $\begin{array}{l}\text { 1. Develop a report which compiles a preliminary listing of } \\
\text { long-term disability participants including identification of } \\
\text { specific provisions and the funding sources which apply to } \\
\text { each individual or group of individuals. Will include } \\
\text { commitments for medical, life or continued pension } \\
\text { accruals. Must ensure a link between these and any future } \\
\text { plans. }\end{array}$ & $\begin{array}{c}6 / 01 / 96 \\
\text { Completed }\end{array}$ & $\begin{array}{l}\text { FA Powers } \\
\text { JR Sorensen }\end{array}$ \\
\hline $\begin{array}{l}\text { 2. Develop a report which compiles a preliminary listing of all } \\
\text { plan liabilities and cornmitments, verify assumption of same } \\
\text { by the PHMC contractor, as appropriate. }\end{array}$ & $\begin{array}{l}6 / 01 / 96 \\
\text { Completed }\end{array}$ & $\begin{array}{l}\text { FA Powers } \\
\text { JR Sorensen }\end{array}$ \\
\hline $\begin{array}{l}\text { 3. Develop a report which compiles a listing of short-term } \\
\text { disability participants including salary continuance status, } \\
\text { payment amount being received, the obligation to return } \\
\text { them to their same position, and the potential liability if } \\
\text { they go to long-term disability after } 6 \text { months. }\end{array}$ & $\begin{array}{l}\text { 6/01/96 } \\
\text { Completed }\end{array}$ & $\begin{array}{l}\text { FA Powers } \\
\text { JR Sorensen }\end{array}$ \\
\hline $\begin{array}{l}\text { 4. Identify any other obligations (e.g., sick leave accrual from } \\
\text { BCSR) carried over from predecessor contractors. }\end{array}$ & $\begin{array}{c}6 / 01 / 96 \\
\text { Completed }\end{array}$ & $\begin{array}{l}\text { FA Powers } \\
\text { JR Sorensen }\end{array}$ \\
\hline $\begin{array}{l}\text { 5. Develop a health care plan report which identifies } \\
\text { participants by plan (including vision and dental), current } \\
\text { eligibility and enrollment status, and contribution amounts } \\
\text { by company. Break down into activities (several groups } \\
\text { within this), retired (several groups within this), } \\
\text { terminations, COBRA, leaves of absence, etc. }\end{array}$ & $\begin{array}{c}\text { 6/01/96 } \\
\text { Completed }\end{array}$ & $\begin{array}{l}\text { FA Powers } \\
\text { JR Sorensen }\end{array}$ \\
\hline 6. Complete an eligibility review for all plan participants. & $\begin{array}{c}\text { 6/01/96 } \\
\text { Completed }\end{array}$ & $\begin{array}{l}\text { FA Powers } \\
\text { JR Sorensen }\end{array}$ \\
\hline $\begin{array}{l}\text { 7. Develop a computerized report which compiles a } \\
\text { preliminary list of plan participants receiving ternination } \\
\text { benefits, their current status, and dates when coverage level } \\
\text { or contribution rates will change. }\end{array}$ & $\begin{array}{c}\text { 6/01/96 } \\
\text { Completed }\end{array}$ & $\begin{array}{l}\text { FA Powers } \\
\text { JR Sorensen }\end{array}$ \\
\hline $\begin{array}{l}\text { 8. Develop a report which compiles a preliminary listing of } \\
\text { employees who are enrolled in Personal Accident insurance } \\
\text { for employee and spouse, including contribution amounts. }\end{array}$ & $\begin{array}{l}\text { 6/01/96 } \\
\text { Completed }\end{array}$ & $\begin{array}{l}\text { FA Powers } \\
\text { JR Sorensen }\end{array}$ \\
\hline \multicolumn{3}{|l|}{ Pre-Award Actions } \\
\hline $\begin{array}{l}\text { 9. Develop a report which compiles a prelininary listing of all } \\
\text { employees who are on family, educational, military or } \\
\text { personal leave. Include any benefits coverages they have, } \\
\text { expected return dates and obligations to place them in a } \\
\text { position upon return. }\end{array}$ & $\begin{array}{c}6 / 01 / 96 \\
\text { Completed }\end{array}$ & $\begin{array}{l}\text { FA Powers } \\
\text { JR Sorensen }\end{array}$ \\
\hline $\begin{array}{l}\text { 10. Develop a report which compiles a preliminary listing of } \\
\text { life insurance obligations including all participants eligibie } \\
\text { for life insurance by carrier, the amounts, beneficiaries, } \\
\text { dependents covered, and the amount of participant } \\
\text { contributions. }\end{array}$ & $\begin{array}{l}\text { 6/01/96 } \\
\text { Completed }\end{array}$ & $\begin{array}{l}\text { FA Powers } \\
\text { JR Sorensen }\end{array}$ \\
\hline
\end{tabular}




\begin{tabular}{|c|c|c|}
\hline Pre-Award Actions & $\begin{array}{l}\text { Scheduled } \\
\text { Date }\end{array}$ & Actionee(s) \\
\hline $\begin{array}{l}\text { 11. Develop report of Income Protection Plan participants; } \\
\text { active employees, as well as those receiving benefits from } \\
\text { the plan. }\end{array}$ & $\begin{array}{l}6 / 01 / 96 \\
\text { Completed }\end{array}$ & $\begin{array}{l}\text { FA Powers } \\
\text { JR Sorensen }\end{array}$ \\
\hline Post-Award Actions & & \\
\hline $\begin{array}{l}\text { 1. Provide the PHMC a report listing short-term disability } \\
\text { participants including salary continuance status, payment } \\
\text { amount being received, the obligation to return them to } \\
\text { their same position, and the potential liability if they go to } \\
\text { long-term disability after } 6 \text { months. }\end{array}$ & $8 / 05 / 96$ & $\begin{array}{l}\text { FA Powers } \\
\text { JR Sorensen }\end{array}$ \\
\hline $\begin{array}{l}\text { 2. Arrange for assumption or transition of Flexible Spending } \\
\text { Accounts for the remainder of the calendar year. }\end{array}$ & $8 / 05 / 96$ & \begin{tabular}{|l|l} 
FA Powers \\
JR Sorensen \\
\end{tabular} \\
\hline $\begin{array}{l}\text { 3. Determine whether WHC will continue to provide payroll } \\
\text { deduction for Group Universal Life insurance coverage. }\end{array}$ & $8 / 05 / 96$ & $\begin{array}{l}\text { FA Powers } \\
\text { JR Sorensen }\end{array}$ \\
\hline $\begin{array}{l}\text { 4. Generate and provide a final turnover report for the PHMC } \\
\text { listing all long-term disability participants including } \\
\text { identification of specific plan provisions and the funding } \\
\text { sources which apply to each. Include commitments for } \\
\text { medical, life, or continued pension accruals. }\end{array}$ & $10 / 01 / 96$ & $\begin{array}{l}\text { FA Powers } \\
\text { JR Sorensen }\end{array}$ \\
\hline $\begin{array}{l}\text { 5. Generate a final PHMC turnover report listing all plan } \\
\text { liabilities and commitments. }\end{array}$ & $10 / 01 / 96$ & \begin{tabular}{|l|l} 
FA Powers \\
JR Sorensen \\
\end{tabular} \\
\hline $\begin{array}{l}\text { 6. Generate a final turnover report listing short-term disability } \\
\text { participants including salary continuance status, payment } \\
\text { amount being received, the obligation to return them to } \\
\text { their same position, and the potential liability if they go to } \\
\text { long-term disability. }\end{array}$ & $10 / 01 / 96$ & $\begin{array}{l}\text { FA Powers } \\
\text { JR Sorensen }\end{array}$ \\
\hline $\begin{array}{l}\text { 7. Generate a final turnover health care plan report which } \\
\text { identifies participants by plan (including vision and dental), } \\
\text { current eligibility and enrollment status, and contribution } \\
\text { amounts by company. Break down into actives (several } \\
\text { groups within this), retired (several groups within this), } \\
\text { terminations, COBRA, leaves of absence, etc. }\end{array}$ & $10 / 01 / 96$ & $\begin{array}{l}\text { FA Powers } \\
\text { JR Sorensen }\end{array}$ \\
\hline $\begin{array}{l}\text { 8. Generate a final turnover list of plan participants receiving } \\
\text { termination benefits, their current status, and dates when } \\
\text { coverage level or contribution rates will change. }\end{array}$ & $10 / 01 / 96$ & $\begin{array}{l}\text { FA Powers } \\
\text { JR Sorensen }\end{array}$ \\
\hline $\begin{array}{l}\text { 9. Generate a final turnover list of employees who are } \\
\text { enroiled in Personal Accident insurance for employee and } \\
\text { spouse, including contribution amounts. }\end{array}$ & $10 / 01 / 96$ & $\begin{array}{l}\text { FA Powers } \\
\text { JR Sorensen }\end{array}$ \\
\hline $\begin{array}{l}\text { 10. Generate a final murnover list of all employees who are on } \\
\text { family, educationas, military or personal leave. Include any } \\
\text { benefit coverages they have, expected return dates and } \\
\text { obligations, if any, to place them in a position upon return. }\end{array}$ & $10 / 01 / 96$ & $\begin{array}{l}\text { FA Powers } \\
\text { JR Sorensen }\end{array}$ \\
\hline $\begin{array}{l}\text { 11. Generate a final turnover report listing life insurance } \\
\text { obligations including all participants eligible for life } \\
\text { insurance by carrier, the amounts, dependents covered, and } \\
\text { the amount of participant contributions. }\end{array}$ & $10 / 01 / 96$ & $\begin{array}{l}\text { FA Powers } \\
\text { JR Sorensen }\end{array}$ \\
\hline
\end{tabular}




\begin{tabular}{|c|c|c|c|}
\hline & Post-Award Actions & $\begin{array}{c}\text { Scheduled } \\
\text { Date }\end{array}$ & Actionee(s) \\
\hline 12. & $\begin{array}{l}\text { Generate a final turnover list of employees who are } \\
\text { enrolled in the Income Protection Plan. Include a list of } \\
\text { former employees who are receiving benefits from the } \\
\text { plan. }\end{array}$ & $10 / 01 / 96$ & $\begin{array}{l}\text { FA Powers } \\
\text { JR Sorensen }\end{array}$ \\
\hline 13. & $\begin{array}{l}\text { Provide notification to those individuals who have benefits } \\
\text { (i.e., COBRA, leave of absence, and retiree status) of any } \\
\text { plan changes affecting coverage. }\end{array}$ & $10 / 01 / 96$ & $\begin{array}{l}\text { FA Powers } \\
\text { JR Sorensen }\end{array}$ \\
\hline
\end{tabular}

\subsubsection{Return-to-Work.}

\begin{tabular}{|l|l|l|}
\hline \multicolumn{1}{|c|}{ Pre-Award Actions } & Scheduled & \multicolumn{1}{|c|}{ Actionee(s) } \\
\hline $\begin{array}{l}\text { 1. Document the return-to-work process including all applicable } \\
\text { MRPs and other appropriate policies/ practices. }\end{array}$ & $\begin{array}{l}6 / 01 / 96 \\
\text { Completed }\end{array}$ & $\begin{array}{l}\text { FA Powers } \\
\text { JR Sorensen }\end{array}$ \\
\hline $\begin{array}{l}\text { 2. Prepare a list of any employees being tracked under the } \\
\text { Workability program expected return date and obligations, if } \\
\text { any, for a position upon return. }\end{array}$ & $\begin{array}{c}6 / 01 / 96 \\
\text { Completed }\end{array}$ & $\begin{array}{l}\text { FA Powers } \\
\text { JR Sorensen }\end{array}$ \\
\hline $\begin{array}{l}\text { Post-Award Actions } \\
\text { 1. Provide the PHMC documented relationships between the } \\
\text { return-to-work coordinator, HEHF, and Workability. }\end{array}$ & $8 / 05 / 96$ & $\begin{array}{l}\text { FA Powers } \\
\text { JR Sorensen }\end{array}$ \\
\hline $\begin{array}{l}\text { 2. Prepare and transmit a final turnover report listing any } \\
\text { employees under the managed disability program expected } \\
\text { return date and obligation, if any, for a position upon return. }\end{array}$ & $10 / 01 / 96$ & $\begin{array}{l}\text { FA Powers } \\
\text { JR Sorensen }\end{array}$ \\
\hline
\end{tabular}

\subsubsection{Pension and Savings.}

\begin{tabular}{|c|c|c|}
\hline Pre-Award Actions & $\begin{array}{l}\text { Scheduled } \\
\text { Date }\end{array}$ & Actionee(s) \\
\hline $\begin{array}{l}\text { 1. Develop a report which compiles a preliminary listing of all } \\
\text { vested terminated employees and the accrued pension } \\
\text { liability. }\end{array}$ & $\begin{array}{l}6 / 01 / 96 \\
\text { Completed }\end{array}$ & $\begin{array}{l}\text { MJ Byrd } \\
\text { FA Powers } \\
\text { JR Sorensen }\end{array}$ \\
\hline $\begin{array}{l}\text { 2. Develop a report which identifies all accive employees and } \\
\text { their accrued pension benefit. }\end{array}$ & $\begin{array}{l}6 / 01 / 96 \\
\text { Completed }\end{array}$ & $\begin{array}{l}\text { MJ Byrd } \\
\text { FA Powers } \\
\text { JR Sorensen }\end{array}$ \\
\hline $\begin{array}{l}\text { 3. Prepare a list of current participants in the Savings Plan } \\
\text { including deductions, year-to-date contributions, outstanding } \\
\text { loans and the company match commitment. }\end{array}$ & $\begin{array}{l}\text { 6/01/96 } \\
\text { Completed }\end{array}$ & $\begin{array}{l}\text { FA Powers } \\
\text { JR Sorensen }\end{array}$ \\
\hline Post-Award Actions & & \\
\hline $\begin{array}{l}\text { 1. Provide documentation on the membership and role of the } \\
\text { Pension and Savings Committee and arrange for transfer of } \\
\text { responsibilities to the PHMC contractor. Include a complete } \\
\text { accounting of all plan funds. }\end{array}$ & $9 / 01 / 96$ & $\begin{array}{l}\text { M Byrd } \\
\text { FA Powers } \\
\text { JR Sorensen }\end{array}$ \\
\hline $\begin{array}{l}\text { 2. Determine with PHMC, continuation of liaison to the } \\
\text { Retirees Association. }\end{array}$ & $9 / 01 / 96$ & $\begin{array}{l}\text { FA Powers } \\
\text { JR Sorensen }\end{array}$ \\
\hline
\end{tabular}




\begin{tabular}{|ll|c|l|}
\hline \multicolumn{1}{|c|}{ Post-Award Actions } & $\begin{array}{c}\text { Scheduled } \\
\text { Date }\end{array}$ & \multicolumn{1}{|c|}{ Actionee(s) } \\
\hline 3. $\quad \begin{array}{l}\text { Generate a final turnover list of all active employees and } \\
\text { their accrued pension benefit as of 9/30/96. }\end{array}$ & $9 / 01 / 96$ & $\begin{array}{l}\text { MJ Byrd } \\
\text { FA Powers } \\
\text { JR Sorensen }\end{array}$ \\
\hline 4. $\quad \begin{array}{l}\text { Generate a final turnover list of all current retirees (and } \\
\text { dependents if applicable), the plan and provisions under } \\
\text { which they retired, the medical and life insurance they } \\
\text { have, and the contribution amounts being paid. }\end{array}$ & $10 / 01 / 96$ & $\begin{array}{l}\text { FA Powers } \\
\text { JR Sorensen }\end{array}$ \\
\hline 5. $\begin{array}{l}\text { Generate a final turnover list of all vested terminated } \\
\text { employees and the accrued pension liability. }\end{array}$ & $10 / 01 / 96$ & $\begin{array}{l}\text { FA Powers } \\
\text { JR Sorensen }\end{array}$ \\
\hline 6. $\begin{array}{l}\text { If the PHMC offers a savings plan, provide list of current } \\
\text { participants including deductions, year-to-date } \\
\text { contributions, outstanding loans and the company match } \\
\text { commitment. }\end{array}$ & $10 / 01 / 96$ & $\begin{array}{l}\text { FA Powers } \\
\text { JR Sorensen }\end{array}$ \\
\hline
\end{tabular}

\subsubsection{Recognition Programs.}

\begin{tabular}{|l|c|l|}
\hline \multicolumn{1}{|c|}{ Pre-Award Actions } & $\begin{array}{c}\text { Scheduled } \\
\text { Date }\end{array}$ & Actionee(s) \\
\hline $\begin{array}{l}\text { 1. Prepare a description of current employee recognition and } \\
\text { service award programs and budget. Coordinate with PHMC } \\
\text { to deternine compatibility with their programs and adapt } \\
1997 \text { eligibility list for transitioned employees. }\end{array}$ & $\begin{array}{l}6 / 01 / 96 \\
\text { Completed }\end{array}$ & $\begin{array}{l}\text { FA Powers } \\
\text { JR Sorensen }\end{array}$ \\
\hline $\begin{array}{l}\text { 1. Provide description of current employee recognition and } \\
\text { service award programs including budget. Generate a list of } \\
\text { employees who will be eligible for service awards in } \\
\text { FY } 1997 \text { by company and level of award. }\end{array}$ & $9 / 01 / 96$ & FA Powers \\
\hline
\end{tabular}

\subsubsection{Other.}

\begin{tabular}{|l|c|c|}
\hline \multicolumn{1}{|c|}{ Post-Award Actions } & \multicolumn{1}{|c|}{$\begin{array}{l}\text { Scheduled } \\
\text { Date }\end{array}$} & Actionee(s) \\
\hline $\begin{array}{l}\text { 1. Identify the status and cost of the Hanford Family Care } \\
\text { Services contract with Working Solutions, Inc. Arrange for } \\
\text { termination or continuation of the contract. }\end{array}$ & $8 / 15 / 96$ & $\begin{array}{l}\text { FA Powers } \\
\text { JR Sorensen }\end{array}$ \\
\hline $\begin{array}{l}\text { 2. Provide details of current Employee Assistance Program } \\
\text { services. }\end{array}$ & $8 / 15 / 96$ & $\begin{array}{l}\text { FA Powers } \\
\text { JR Sorensen }\end{array}$ \\
\hline $\begin{array}{l}\text { 3. Determine whether the PHMC contractor will allow BHI to } \\
\text { continue purchasing benefit plans. Provide funding } \\
\text { arrangement information. }\end{array}$ & $8 / 15 / 96$ & $\begin{array}{l}\text { FA Powers } \\
\text { JR Sorensen }\end{array}$ \\
\hline
\end{tabular}




\subsubsection{Total Compensation (ICF KH)}

\subsubsection{Salary Plan.}

\begin{tabular}{|c|c|c|}
\hline Pre-Award Actions & $\begin{array}{c}\text { Scheduled } \\
\text { Date } \\
\end{array}$ & Actionee(s) \\
\hline $\begin{array}{l}\text { 1. Prepare a list of calendar year salary plan actions scheduled } \\
\text { for October, November, and December } 1996 .\end{array}$ & $\begin{array}{c}6 / 01 / 96 \\
\text { Completed }\end{array}$ & BJ Smetana \\
\hline $\begin{array}{l}\text { 2. Determine ownership of prior year salary increase plans and } \\
\text { disposition accordingly. }\end{array}$ & $\begin{array}{l}6 / 01 / 96 \\
\text { Completed }\end{array}$ & BJ Smetana \\
\hline $\begin{array}{l}\text { 3. Determine distribution of remaining salary increase funds } \\
\text { which are usually distributed as adjustments in the } \\
\text { November/December time frame. Estimate available funds } \\
\text { and recommend a distribution method. }\end{array}$ & $\begin{array}{l}\text { 6/01/96 } \\
\text { Completed }\end{array}$ & BJ Smetana \\
\hline Post-Award Actions & & \\
\hline $\begin{array}{l}\text { 1. Status PHMC contractor regarding salary actions for } \\
\text { October, November, and December } 1996 \text { for those } \\
\text { employees accepting offers. }\end{array}$ & $10 / 01 / 96$ & BJ Smetana \\
\hline $\begin{array}{l}\text { 2. Review salary planning process and provide PHMC } \\
\text { contractor a list of prior year salary increase plans as } \\
\text { appropriate. }\end{array}$ & $10 / 01 / 96$ & BI Smetana \\
\hline $\begin{array}{l}\text { 3. Coordinate with PHMC contractor to determine distribution } \\
\text { of remaining salary increase funds which are usually } \\
\text { distributed as adjustments in the November/December time } \\
\text { frame. Estimate available and recommend a distribution } \\
\text { method. }\end{array}$ & $10 / 01 / 96$ & BJ Smetana \\
\hline
\end{tabular}

\subsubsection{Other.}

\begin{tabular}{|l|c|c|}
\hline \multicolumn{1}{|c|}{ Post-Award Actions } & $\begin{array}{c}\text { Scheduled } \\
\text { Date }\end{array}$ & Actionee(s) \\
\hline $\begin{array}{l}\text { 1. Identify the stanus and cost of the Hanford Family Care } \\
\text { Services contract with Working Solutions, Inc. Arrange for } \\
\text { termination or continuation of the contract. }\end{array}$ & $8 / 05 / 96$ & JJ Chunn \\
\hline $\begin{array}{l}\text { 2. Provide details of current Employee Assistance Program } \\
\text { services. }\end{array}$ & $8 / 15 / 96$ & JJ Chunn \\
\hline
\end{tabular}

\subsubsection{Labor Relations (WHC/BCSR)}

Determine, develop, and prepare appropriate data and information regarding the bargaining unit workforce, bargaining agents, collective bargaining agreements, union officials, grievance and arbitration history and caseloads. Further, address the labor relations climate and Federal agency notification requirements. 


\subsubsection{Collective Bargaining Agreements.}

\begin{tabular}{|c|c|c|}
\hline Pre-Award Actions & $\begin{array}{l}\text { Scheduled } \\
\text { Date }\end{array}$ & Actionee(s) \\
\hline $\begin{array}{l}\text { 1. Prepare summary of the collective bargaining agreements } \\
\text { with the Hanford Atomic Metal Trades Council (HAMTC) } \\
\text { and Hanford Guards Union (HGU) describing future } \\
\text { contractual obligations for actions such as negotiations, } \\
\text { general wage increases, etc. }\end{array}$ & $\begin{array}{l}5 / 01 / 96 \\
\text { Completed }\end{array}$ & $\begin{array}{l}\text { BD Corder } \\
\text { GF Saskowsky }\end{array}$ \\
\hline $\begin{array}{l}\text { 2. HAMTC Appendix " } A \text { " bargaining issues: Prepare a } \\
\text { summary and status of any ongoing negotiations with } \\
\text { affiliated local unions. }\end{array}$ & $\begin{array}{l}5 / 01 / 96 \\
\text { Completed }\end{array}$ & $\begin{array}{l}\text { BD Corder } \\
\text { GF Saskowsky }\end{array}$ \\
\hline $\begin{array}{l}\text { 3. Prepare copies of current labor agreements and other letters } \\
\text { and memorandums of understanding. }\end{array}$ & $\begin{array}{l}5 / 01 / 96 \\
\text { Completed }\end{array}$ & $\begin{array}{l}\text { BD Corder } \\
\text { GF Saskowsky }\end{array}$ \\
\hline $\begin{array}{l}\text { 4. Assemble necessary information to support ongoing } \\
\text { transitional consultation with the PHMC on collective } \\
\text { bargaining issues, historical past practices, and negotiations } \\
\text { strategy. }\end{array}$ & $\begin{array}{l}5 / 01 / 96 \\
\text { Completed }\end{array}$ & $\begin{array}{l}\text { BD Corder } \\
\text { GF Saskowsky }\end{array}$ \\
\hline \multicolumn{3}{|l|}{ Post-Award Actions } \\
\hline $\begin{array}{l}\text { 1. Brief PHMC on collective bargaining agreements with } \\
\text { HAMTC and HGU describing future contractual obligations } \\
\text { for actions such as negotiations, general wage increases, etc. }\end{array}$ & $8 / 05 / 96$ & $\begin{array}{l}\text { BD Corder } \\
\text { GF Saskowsky }\end{array}$ \\
\hline $\begin{array}{l}\text { 2. Provide PHMC contractor with details and status of HAMTC } \\
\text { Appendix "A" bargaining issues, including a summary and } \\
\text { status of any ongoing negotiations with affiliated local unions. }\end{array}$ & $8 / 05 / 96$ & $\begin{array}{l}\text { BD Corder } \\
\text { GF Saskowsky }\end{array}$ \\
\hline $\begin{array}{l}\text { 3. Provide copies of current labor agreements and other letters } \\
\text { and memotandums of understanding. }\end{array}$ & $8 / 05 / 96$ & $\begin{array}{l}\text { BD Corder } \\
\text { GF Saskowsky }\end{array}$ \\
\hline $\begin{array}{l}\text { 4. Provide ongoing transitional consultation with the PHMC on } \\
\text { collective bargaining issues, historical past practices, and } \\
\text { negotiations strategy. }\end{array}$ & $8 / 05 / 96$ & $\begin{array}{l}\text { BD Corder } \\
\text { GF Saskowsky }\end{array}$ \\
\hline
\end{tabular}

\subsubsection{Bargaining Unit Leadership/Demographics.}

\begin{tabular}{|c|c|c|}
\hline Pre-Award Actions & $\begin{array}{l}\text { Scheduled } \\
\text { Date }\end{array}$ & Actionee(s) \\
\hline $\begin{array}{l}\text { 1. Develop appropriate charts demonstrating union leadership } \\
\text { structure for HAMTC and HGU including business agents, } \\
\text { officers, affiliated locals, project chief stewards and the } \\
\text { steward structure in general. }\end{array}$ & $\begin{array}{l}5 / 01 / 96 \\
\text { Completed }\end{array}$ & $\begin{array}{l}\text { BD Corder } \\
\text { GF Saskowsky }\end{array}$ \\
\hline $\begin{array}{l}\text { 2. Summarize the HAMTC and HGU workforce population by } \\
\text { years of service, classification and location (areas). }\end{array}$ & $\begin{array}{l}\text { 6/01/96 } \\
\text { Completed }\end{array}$ & $\begin{array}{l}\text { BD Corder } \\
\text { GF Saskowsky }\end{array}$ \\
\hline Post-Award Actions & & \\
\hline $\begin{array}{l}\text { 1. Provide PHMC contractor copies of charts demonstrating } \\
\text { union leadership structure for HAMTC and HGU including } \\
\text { business agents, officers, affiliated locals, project chief } \\
\text { stewards and the steward structure in general. }\end{array}$ & $8 / 05 / 96$ & $\begin{array}{l}\text { BD Corder } \\
\text { GF Saskowsky }\end{array}$ \\
\hline
\end{tabular}




\begin{tabular}{|l|l|l|}
\hline \multicolumn{1}{|c|}{ Post-Award Actions } & $\begin{array}{l}\text { Scheduled } \\
\text { Date }\end{array}$ & Actionee(s) \\
\hline 2. $\begin{array}{l}\text { Brief PHMC on workforce demographics and provide } \\
\text { summary listings covering the HAMTC and HGU } \\
\begin{array}{l}\text { workforce population by years of service, classification and } \\
\text { location (areas). }\end{array}\end{array}$ & $\begin{array}{l}\text { B/05/96 Corder } \\
\text { GF Saskowsky }\end{array}$ \\
\hline 3. $\begin{array}{l}\text { Provide PHMC a listing of numbers and names of } \\
\text { bargaining unit employees on termination status with recall } \\
\text { rights by classification. }\end{array}$ & $9 / 01 / 96$ & $\begin{array}{l}\text { BD Corder } \\
\text { GF Saskowsky }\end{array}$ \\
\hline
\end{tabular}

\subsubsection{Notifications.}

\begin{tabular}{|c|c|c|}
\hline 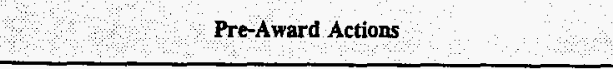 & $\begin{array}{l}\text { Scheduled } \\
\text { Date }\end{array}$ & Actionee(s) \\
\hline $\begin{array}{l}\text { 1. Prepare notification to Federal Mediation and Conciliation } \\
\text { Service (FMCS) and the unions that the collective bargaining } \\
\text { agreements with HAMTC and HGU are terminating effective } \\
10 / 01 / 96 \text {. }\end{array}$ & $\begin{array}{l}\text { 6/01/96 } \\
\text { Completed }\end{array}$ & BD Corder \\
\hline Post-Award Actions & & \\
\hline $\begin{array}{l}\text { 1. Notify the FMCS that the collective bargaining agreements } \\
\text { with HAMTC and HGU are terminating effective } 10 / 01 / 96 \text {. }\end{array}$ & $8 / 05 / 96$ & BD Corder \\
\hline $\begin{array}{l}\text { 2. Notify the unions that the collective bargaining agreements } \\
\text { between WHC and HAMTC/HGU will terminate effective } \\
10 / 01 / 96 \text {. }\end{array}$ & $8 / 05 / 96$ & BD Corder \\
\hline
\end{tabular}

\subsubsection{Grievance and Arbitration History/Caseload.}

\begin{tabular}{|c|c|c|}
\hline ra & $\begin{array}{l}\text { Scheduled } \\
\text { Date }\end{array}$ & Actionee(s) \\
\hline $\begin{array}{l}\text { 1. Prepare summaries and analysis of arbitration cases and } \\
\text { grievances filed by calendar year from January } 1,1993 \\
\text { through present time. Analysis to include summary by issue } \\
\text { and outcome of arbitration. }\end{array}$ & $\begin{array}{l}5 / 31 / 96 \\
\text { Completed }\end{array}$ & $\begin{array}{l}\text { BD Corder } \\
\text { GF Saskowsky }\end{array}$ \\
\hline $\begin{array}{l}\text { 2. Prepare summaries of any open NLRB charges and past cases } \\
\text { of major significance to the Hanford Site. }\end{array}$ & $\begin{array}{l}6 / 01 / 96 \\
\text { Completed }\end{array}$ & $\begin{array}{l}\text { BD Corder } \\
\text { GF Saskowsky }\end{array}$ \\
\hline $\begin{array}{l}\text { 3. Determine, with General Counsel review, ownership of in } \\
\text { process grievances and arbitrations at nrmover. }\end{array}$ & $\begin{array}{c}6 / 01 / 96 \\
\text { Completed }\end{array}$ & $\begin{array}{l}\text { BD Corder } \\
\text { GF Saskowsky }\end{array}$ \\
\hline Post-Award Actions & & \\
\hline $\begin{array}{l}\text { 1. Provide a summary analysis of arbitration cases and } \\
\text { grievances filed by calendar year from January } 1,1993 \text {, } \\
\text { through present time. Analysis to include summary by issue } \\
\text { and outcome of arbitration. }\end{array}$ & $9 / 01 / 96$ & $\begin{array}{l}\text { BD Corder } \\
\text { GF Saskowsky }\end{array}$ \\
\hline $\begin{array}{l}\text { 2. Provide summaries of any open National Labor Relations } \\
\text { Board (NLRB) charges and past cases of major significance } \\
\text { to the Hanford Site. }\end{array}$ & $10 / 01 / 96$ & $\begin{array}{l}\text { BD Corder } \\
\text { GF Saskowsky }\end{array}$ \\
\hline
\end{tabular}




\begin{tabular}{|l|l|l|}
\hline \multicolumn{1}{|c|}{ Post-Award Actions } & Scheduled & \multicolumn{1}{c|}{ Actionee(s) } \\
\hline 3. $\begin{array}{l}\text { Provide listing of General Counsel reviewed grievances in } \\
\text { process or pending/scheduled for arbitration including } \\
\text { subject, local union and date of arbitration if available. } \\
\text { Also, arbitration cases pending award. }\end{array}$ & $10 / 01 / 96$ & $\begin{array}{l}\text { BD Corder } \\
\text { GF Saskowsky }\end{array}$ \\
\hline
\end{tabular}

\subsubsection{Labor Relations (ICF KH)}

ICF KH Labor Relations will assist WHC Labor Relations in transferring HAMTC information.

The following collective bargaining agreements are unique to ICF $\mathrm{KH}$ and information listed below, as applicable, will be provided for each as indicated:

- Hanford Site Stabilization Agreement (HSSA)

- Office and Professional Employees International Union (OPEIU)

- Quality Control and Nondestructive Technicians (QC/NDT).

\subsubsection{Collective Bargaining Agreements.}

\begin{tabular}{|c|c|c|}
\hline Pre-Award Actions & $\begin{array}{l}\text { Scheduled } \\
\text { Date }\end{array}$ & Actionee(s) \\
\hline $\begin{array}{l}\text { 1. Prepare summary of the collective bargaining agreements } \\
\text { describing further contractual obligations for actions such as } \\
\text { negotiations, general wage increases, etc. }\end{array}$ & $\begin{array}{c}5 / 01 / 96 \\
\text { Completed }\end{array}$ & JA Carter \\
\hline 2. Prepare a summary and status of any ongoing negotiations. & $\begin{array}{l}5 / 01 / 96 \\
\text { Completed }\end{array}$ & JA Carter \\
\hline $\begin{array}{l}\text { 3. Prepare copies of current labor agreements and other letters } \\
\text { and MOUs. }\end{array}$ & $\begin{array}{c}5 / 01 / 96 \\
\text { Completed }\end{array}$ & JA Carter \\
\hline $\begin{array}{l}\text { 4. Assemble necessary information to support ongoing } \\
\text { transitional consultation with the PHMC on collective } \\
\text { bargaining issues, historical past practices, and negotiations } \\
\text { strategy. }\end{array}$ & $\begin{array}{c}5 / 01 / 96 \\
\text { Completed }\end{array}$ & JA Carter \\
\hline $\begin{array}{l}\text { 5. Prepare information necessary for continuing consultation to } \\
\text { the PHMC with regard to the application and administration } \\
\text { of the HSSA and the transitioning of the existing construction } \\
\text { workforce to the PHMC. }\end{array}$ & $\begin{array}{l}\text { 6/01/96 } \\
\text { Completed }\end{array}$ & JA Carter \\
\hline Post-Award Actions & & \\
\hline $\begin{array}{l}\text { 1. Brief PHMC on collective bargaining agreements describing } \\
\text { further contractual obligations for actions such as } \\
\text { negotiations, general wage increases, etc. }\end{array}$ & $8 / 05 / 96$ & JA Carter \\
\hline $\begin{array}{l}\text { 2. Provide new contractor with details and status of bargaining } \\
\text { issues, including a summary and status of any ongoing } \\
\text { negotiations with unions. }\end{array}$ & $8 / 05 / 96$ & JA Carter \\
\hline
\end{tabular}




\begin{tabular}{|l|c|c|}
\hline \multicolumn{1}{|c|}{ Post-Award Actions } & $\begin{array}{c}\text { Scheduled } \\
\text { Date }\end{array}$ & Actionee(s) \\
\hline $\begin{array}{l}\text { 3. Provide copies of current labor agreements and other letters } \\
\text { and MOUs. }\end{array}$ & $8 / 05 / 96$ & JA Carter \\
\hline $\begin{array}{l}\text { 4. Provide ongoing transitional consultation with the PHMC on } \\
\text { collective bargaining issues, historical past practices, and } \\
\text { negotiations strategy. }\end{array}$ & $8 / 05 / 96$ & JA Carter \\
\hline $\begin{array}{l}\text { 5. Provide continuing consultation to the PHMC with regard to } \\
\text { the application and administration of the HSS A and the } \\
\text { transitioning of the existing construction workforce to the } \\
\text { PHMC. }\end{array}$ & $9 / 30 / 96$ & JA Carter \\
\hline
\end{tabular}

\subsubsection{Bargaining Unit Leadership/Demographics.}

\begin{tabular}{|c|c|c|}
\hline Pre-Award Actions & $\begin{array}{l}\text { Scheduled } \\
\text { Date }\end{array}$ & Actionee(s) \\
\hline $\begin{array}{l}\text { 1. Develop appropriate charts demonstrating union leadership } \\
\text { structure including business agents, officers, affiliated locals, } \\
\text { project chief stewards, and the steward structure in general. }\end{array}$ & $\begin{array}{l}6 / 01 / 96 \\
\text { Completed }\end{array}$ & JA Carter \\
\hline $\begin{array}{l}\text { 2. Summarize the bargaining workforce population by years of } \\
\text { service, classification, and location (areas). }\end{array}$ & $\begin{array}{l}\text { 6/01/96 } \\
\text { Completed }\end{array}$ & IA Cant \\
\hline Post-Award Actions & & \\
\hline $\begin{array}{l}\text { 1. Provide new contractor copies of charts demonstrating union } \\
\text { leadership structure for bargaining units, including business } \\
\text { agents, officers, affiliated locals, project chief stewards, and } \\
\text { the steward structure in general. }\end{array}$ & $8 / 05 / 96$ & JA Carter \\
\hline $\begin{array}{l}\text { 2. Brief PHMC on workforce demographics and provide } \\
\text { summary listings covering the bargaining unit workforce } \\
\text { populations by years of service, classification, and location } \\
\text { (areas). }\end{array}$ & $8 / 05 / 96$ & JA Carter \\
\hline $\begin{array}{l}\text { 3. Provide PHMC a listing of numbers of bargaining unit } \\
\text { employees terminated with recall rights by classification. }\end{array}$ & $9 / 01 / 96$ & JA Carter \\
\hline
\end{tabular}

\subsubsection{Notifications.}

\begin{tabular}{|c|c|c|}
\hline Pre-Award Actions & $\begin{array}{l}\text { Scheduled } \\
\text { Date }\end{array}$ & Actionee(s) \\
\hline $\begin{array}{l}\text { 1. Prepare notification to FMCS and the unions that the } \\
\text { collective bargaining agreements with HAMTC and HGU are } \\
\text { terminating effective 10/01/96. }\end{array}$ & $\begin{array}{l}\text { 6/01/96 } \\
\text { Completed }\end{array}$ & JA Carter \\
\hline Post-Award Actions & & \\
\hline $\begin{array}{l}\text { 1. Prepare notification to FMCS and the union that the } \\
\text { collective bargaining agreements are terminating effective } \\
10 / 01 / 96 \text {. }\end{array}$ & $8 / 05 / 96$ & JA Carter \\
\hline
\end{tabular}




\begin{tabular}{|l|c|c|}
\hline \multicolumn{1}{|c|}{ Post-Award Actions } & Scheduled & Actionee(s) \\
\hline $\begin{array}{l}\text { 2. Notify the FMCS that the collective bargaining agreements } \\
\text { are terminating effective 10/01/96. }\end{array}$ & $8 / 05 / 96$ & JA Carter \\
\hline $\begin{array}{l}\text { 3. Notify the unions that the collective bargaining agreements } \\
\text { between them and ICF } \text { KH will terminate effective 10/01/96. }\end{array}$ & $8 / 05 / 96$ & JA Carter \\
\hline
\end{tabular}

\subsubsection{Grievance and Arbitration History/Caseload.}

\begin{tabular}{|l|c|l|}
\hline \multicolumn{1}{|c|}{ Pre-Award Actions } & $\begin{array}{c}\text { Scheduled } \\
\text { Date }\end{array}$ & Actionee(s) \\
\hline $\begin{array}{l}\text { 1. Prepare a summary and analysis of arbitration cases and } \\
\text { grievances filed by calendar year from January 1, 1993, } \\
\text { through present time. Analysis to inciude summary by issue } \\
\text { and outcome of arbitration. }\end{array}$ & $\begin{array}{c}5 / 31 / 96 \\
\text { Completed }\end{array}$ & JA Carter \\
\hline $\begin{array}{l}\text { 2. Prepare a summary of any open NLRB charges and past } \\
\text { cases of major significance to the Hanford Site. }\end{array}$ & $\begin{array}{c}6 / 01 / 96 \\
\text { Completed }\end{array}$ & JA Carter \\
\hline $\begin{array}{l}\text { 3. Determine, with General Counsel review, ownership of in- } \\
\text { process grievances and arbitrations at turnover. }\end{array}$ & $\begin{array}{c}6 / 01 / 96 \\
\text { Completed }\end{array}$ & JA Carter \\
\hline $\begin{array}{l}\text { Post-Award Actions } \\
\text { 1. Provide a summary analysis of arbitration cases and } \\
\text { grievances filed by calendar year from January 1, 1993, } \\
\text { through present time. Analysis to include summary by issue } \\
\text { and outcome of arbitration. }\end{array}$ & $9 / 01 / 96$ & JA Carter \\
\hline $\begin{array}{l}\text { 2. Provide a summary of any open NLRB charges and past } \\
\text { cases of major significance to the Hanford Site. }\end{array}$ & $10 / 01 / 96$ & JA Carter \\
\hline $\begin{array}{l}\text { 3. Provide a list of General Counsel-reviewed grievances in } \\
\text { process or pending/scheduled for arbitration including } \\
\text { subject, local union, and date of arbitration if available. Also } \\
\text { arbitration cases pending award. }\end{array}$ & $10 / 01 / 96$ & JA Carter \\
\hline
\end{tabular}

\subsubsection{Workforce Development and Diversity (WHC/BCSR)}

Determine, develop, and prepare appropriate data regarding Equal Employment Opportunity (EEO), Affirmative Action Plan (AAP) activities and Americans with Disabilities Act (ADA) compliance, personal/professional development, education reimbursement, and community outreach/educational enrichment programs. 


\subsubsection{EEO/AAP.}

\begin{tabular}{|c|c|c|}
\hline Pre-Award Actions & $\begin{array}{c}\text { Scheduled } \\
\text { Date } \\
\end{array}$ & Actionee(s) \\
\hline $\begin{array}{l}\text { 1. Coordinate with Personnel Resources to compile all pertinent data } \\
\text { for preparation of a } 1997 \text { AAP with special emphasis on applicant } \\
\text { tracking records, promotions and placement statistics, and labor } \\
\text { market data. }\end{array}$ & $\begin{array}{c}6 / 01 / 96 \\
\text { Completed }\end{array}$ & $\begin{array}{l}\text { CA Marrero } \\
\text { RW Holleran }\end{array}$ \\
\hline $\begin{array}{l}\text { 2. Prepare a briefing and provide copies of the current AAP } \\
\text { including a statistical profile reflecting female and minority } \\
\text { representation by category/classification in the workforce. }\end{array}$ & $\begin{array}{c}6 / 01 / 96 \\
\text { Completed }\end{array}$ & $\begin{array}{l}\text { G Mitchell } \\
\text { CK Macleod }\end{array}$ \\
\hline $\begin{array}{l}\text { 3. Conduct a comprehensive review of internal and external } \\
\text { complaints and cases by agency, and status for General Counsel } \\
\text { determination of ownership and protocol for PHMC contractor } \\
\text { briefing. }\end{array}$ & $\begin{array}{c}6 / 01 / 96 \\
\text { Completed }\end{array}$ & $\begin{array}{l}\text { CA Marrero } \\
\text { G Mitchell }\end{array}$ \\
\hline $\begin{array}{l}\text { 4. Compile a list of external oversight agency contacts (Human } \\
\text { Rights Commission [HRC], Equal Employment Opportunity } \\
\text { Commission [EEOC], Office of Federal Contract Compliance } \\
\text { Program [OFCCP], etc.) in Region X. }\end{array}$ & $\begin{array}{c}6 / 01 / 96 \\
\text { Completed }\end{array}$ & $\begin{array}{l}\text { CA Marrero } \\
\text { G Mitchell }\end{array}$ \\
\hline \multicolumn{3}{|l|}{ Post-Award Actions } \\
\hline $\begin{array}{l}\text { 1. Provide recommendations and input to the PHMC related to } \\
\text { specific data, goal setting and preparation strategy to develop } \\
\text { and implement a } 1997 \mathrm{AAP} \text {. }\end{array}$ & $9 / 01 / 96$ & $\begin{array}{l}\text { CA Marrero } \\
\text { G Mitchell }\end{array}$ \\
\hline $\begin{array}{l}\text { 2. Provide PHMC a briefing and provide copies of the current } \\
\text { AAP including a statistical profile reflecting female and } \\
\text { minority representation by category/classification in the } \\
\text { workforce. }\end{array}$ & $9 / 01 / 96$ & $\begin{array}{l}\text { G Mitchell } \\
\text { CK Macleod }\end{array}$ \\
\hline $\begin{array}{l}\text { 3. Brief PHMC contractor on internal and external complaints and } \\
\text { cases by agency and status based on General Counsel } \\
\text { determination of ownership and protocol. }\end{array}$ & $9 / 01 / 96$ & $\begin{array}{l}\text { CA Marrero } \\
\text { G Mitchell }\end{array}$ \\
\hline $\begin{array}{l}\text { 4. Review agency contacts listings with PHMC contractor } \\
\text { including a briefing on relationships and protocol. Provide } \\
\text { listing of external oversight agency contacts (HRC, EEOC, } \\
\text { OFCCP, etc.) in Region X. }\end{array}$ & $9 / 01 / 96$ & $\begin{array}{l}\text { CA Marrero } \\
\text { G Mitchell }\end{array}$ \\
\hline
\end{tabular}

\subsubsection{Diversity Recognition and Training.}

\begin{tabular}{|c|c|c|}
\hline ग 4 Pre-Award Actions & $\begin{array}{c}\text { Scheduled } \\
\text { Date }\end{array}$ & Actionee(s) \\
\hline $\begin{array}{l}\text { 1. Develop a briefing document including current activities in } \\
\text { support of nationally recognized diversity events (Black History } \\
\text { Month, Women's History Month, etc.) and a timeline/calendar of } \\
\text { observances. }\end{array}$ & $\begin{array}{c}6 / 01 / 96 \\
\text { Completed }\end{array}$ & $\begin{array}{l}\text { CA Marrero } \\
\text { G Mitchell }\end{array}$ \\
\hline $\begin{array}{l}\text { 2. Prepare a listing and brief description of training programs and } \\
\text { requirements in areas related to Title VII, i.e., "EEO } 2000^{n} \text { and } \\
\text { "Sexual Harassment in the Workplace." }\end{array}$ & $\begin{array}{c}6 / 01 / 96 \\
\text { Completed }\end{array}$ & $\begin{array}{l}\text { CA Marrero } \\
\text { G Mitchell }\end{array}$ \\
\hline
\end{tabular}




\begin{tabular}{|l|c|l|}
\hline \multicolumn{1}{|c|}{ Post-Award Actions } & Scheduled & Actionee(s) \\
\hline $\begin{array}{l}\text { 1. Brief PHMC on diversity recognition events and activities } \\
\text { including a timeline/calendar of observances. Determine } \\
\text { continuation of approach or an alternative. }\end{array}$ & $9 / 01 / 96$ & $\begin{array}{l}\text { CA Marrero } \\
\text { G Mitchell }\end{array}$ \\
\hline $\begin{array}{l}\text { 2. Provide PHMC contractor a listing and brief description of } \\
\text { training programs and requirements in areas related to Title VI, } \\
\text { i.e., "EEO 2000" and "Sexual Harassment in the Workplace." }\end{array}$ & $9 / 01 / 96$ & $\begin{array}{l}\text { CA Marrero } \\
\text { G Mitchell }\end{array}$ \\
\hline
\end{tabular}

\subsubsection{Educational Reimbursement Programs.}

\begin{tabular}{|c|c|c|}
\hline Pre-Award Actions & $\begin{array}{l}\text { Scheduled } \\
\text { Date }\end{array}$ & Actionee(s) \\
\hline $\begin{array}{l}\text { 1. Develop a briefing packet including copies of existing policy, } \\
\text { participation statistics by contractor and costs related to the } \\
\text { Educational Reimbursement Program for active employees. }\end{array}$ & $\begin{array}{c}\text { 6/01/96 } \\
\text { Completed }\end{array}$ & $\begin{array}{l}\text { CA Marrero } \\
\text { KR Banta }\end{array}$ \\
\hline $\begin{array}{l}\text { 2. Prepare a briefing including statistical and cost information for } \\
\text { the Educational Reimbursement Program coverage of terminated } \\
\text { employees eligible for benefits described in the Hanford } \\
\text { Workforce Restructuring Plan ( } 3161 \mathrm{Plan}) \text {. }\end{array}$ & $\begin{array}{l}\text { 6/01/96 } \\
\text { Completed }\end{array}$ & $\begin{array}{l}\text { CA Marrero } \\
\text { KR Banta }\end{array}$ \\
\hline Post-Award Actions & & \\
\hline $\begin{array}{l}\text { 1. Brief PHMC on existing poiicy, participation statistics by } \\
\text { contractor and costs related to the Educational Reimbursement } \\
\text { Program for active employees and provide copies of policy, } \\
\text { forms and other guidelines. Deternine if the same or alternate } \\
\text { program will be used prior to Fall college enroliments. }\end{array}$ & $8 / 15 / 96$ & $\begin{array}{l}\text { CA Martero } \\
\text { KR Banta }\end{array}$ \\
\hline $\begin{array}{l}\text { 2. Brief PHMC on statistical and cost information for the } \\
\text { Educational Reimbursernent Program coverage of terminated } \\
\text { employees eligible for benefits described in the Hanford } \\
\text { Workforce Restructuring Plan ( } 3161 \text { Plans). }\end{array}$ & $8 / 15 / 96$ & $\begin{array}{l}\text { CA Marrero } \\
\text { KR Banta }\end{array}$ \\
\hline
\end{tabular}

\subsubsection{Development Training}

\begin{tabular}{|l|c|l|}
\hline Seheduled & Date & Actionee(s) \\
\hline $\begin{array}{l}\text { 1. Develop a briefing packet that includes information on } \\
\text { development training courses, delivery methods and } \\
\text { processes. }\end{array}$ & $\begin{array}{c}5 / 31 / 96 \\
\text { Completed }\end{array}$ & $\begin{array}{l}\text { CA Marrero } \\
\text { KR Banta }\end{array}$ \\
\hline $\begin{array}{l}\text { 1. Provide PHMC briefing on training courses, delivery } \\
\text { methods and processes. }\end{array}$ & $8 / 15 / 96$ & $\begin{array}{l}\text { CA Marrero } \\
\text { KR Banta }\end{array}$ \\
\hline
\end{tabular}




\subsubsection{Education Enrichment Programs.}

\begin{tabular}{|c|c|c|}
\hline Pre-Award Actions & $\begin{array}{c}\text { Seheduled } \\
\text { Date }\end{array}$ & Actionee(s) \\
\hline $\begin{array}{l}\text { 1. Prepare a comprehensive briefing and supporting } \\
\text { documentation covering strategic education partnerships, } \\
\text { university/college and } \mathrm{K}-12 \text { educarion involvement. }\end{array}$ & $\begin{array}{c}\text { 6/01/96 } \\
\text { Completed }\end{array}$ & $\begin{array}{l}\text { CA Marrero } \\
\text { DG Carter } \\
\text { JE Bongers (BCSR) }\end{array}$ \\
\hline $\begin{array}{l}\text { 2. Prepare information covering student development and } \\
\text { internship activities at pre-college (school-to-work initiative), } \\
\text { college (Partnership for Environmental Technology } \\
\text { Education) and university (Associated Westem Universities) } \\
\text { levels. }\end{array}$ & $\begin{array}{l}\text { 6/01/96 } \\
\text { Completed }\end{array}$ & $\begin{array}{l}\text { CA Marrero } \\
\text { DG Carter }\end{array}$ \\
\hline Post-Award Actions & & \\
\hline $\begin{array}{l}\text { 1. Provide PHMC with a comprehensive briefing and supporting } \\
\text { documentation covering strategic education partnerships, } \\
\text { university/college and } \mathrm{K}-12 \text { eduction involvement. } \\
\text { Determine path forward with PHMC contractor. }\end{array}$ & $8 / 15 / 96$ & $\begin{array}{l}\text { CA Marrero } \\
\text { DG Carter } \\
\text { JE Bongers }\end{array}$ \\
\hline $\begin{array}{l}\text { 2. Brief new contractor and provide information covering } \\
\text { snudent development and internship activities at pre-college } \\
\text { (school-to-work initjative), college (Partnership for } \\
\text { Environmental Technology Education) and university } \\
\text { (Associated Western Universities) levels. }\end{array}$ & $8 / 15 / 96$ & $\begin{array}{l}\text { CA Marrero } \\
\text { DG Carter }\end{array}$ \\
\hline
\end{tabular}

\subsubsection{Graduate Rotational Program.}

\begin{tabular}{|c|c|c|}
\hline Pre-Award Actions & $\begin{array}{c}\text { Scheduled } \\
\text { Date }\end{array}$ & Actionee(s) \\
\hline $\begin{array}{l}\text { 1. Prepare a briefing on the Graduate Rotational Program for } \\
\text { strategic recruitunent of technical undergraduates for the } \\
\text { purpose of hiring them in rotational work experiences at } \\
\text { Hanford. }\end{array}$ & $\begin{array}{c}6 / 01 / 96 \\
\text { Completed }\end{array}$ & $\begin{array}{l}\text { G Mitchell } \\
\text { DE Hansen }\end{array}$ \\
\hline Post-Award Actions & & \\
\hline $\begin{array}{l}\text { 1. Brief the PHMC on the Graduate Rotational Program for } \\
\text { strategic recruitment of technical undergraduates for the } \\
\text { purpose of hiring them in rotational work experiences at } \\
\text { Hanford. Determine interest in continuing the program. }\end{array}$ & $8 / 15 / 96$ & $\begin{array}{l}\text { G Mitchell } \\
\text { DE Hansen }\end{array}$ \\
\hline
\end{tabular}

\subsubsection{Workforce Development and Diversity (ICF KH)}

\subsubsection{EEO/AAP.}

\begin{tabular}{|l|l|l|}
\hline Pre-Award Actions & Scheduled & \\
Date & Actionee(s) \\
\hline $\begin{array}{l}\text { 1. Prepare a briefing and provide copies of the current AAP } \\
\text { including a statistical profile reflecting female and minority } \\
\text { representation by category/classification in the workforce. }\end{array}$ & $\begin{array}{c}6 / 01 / 96 \\
\text { Completed }\end{array}$ & $\begin{array}{l}\text { VC Hodgin } \\
\text { Lewis }\end{array}$ \\
\hline
\end{tabular}




\begin{tabular}{|l|c|l|}
\hline \multicolumn{1}{|c|}{ Pre-Award Actions } & $\begin{array}{c}\text { Scheduled } \\
\text { Date }\end{array}$ & \multicolumn{1}{|c|}{ Actionee(s) } \\
\hline $\begin{array}{l}\text { 2. Conduct a comprehensive review of internal and external } \\
\text { complaints and cases by agency and stans for General Counsel } \\
\text { determination of ownership and protocol for PHMC contractor } \\
\text { briefing. }\end{array}$ & $\begin{array}{c}6 / 01 / 96 \\
\text { Completed }\end{array}$ & $\begin{array}{l}\text { VC Hodgin } \\
\text { Lewis }\end{array}$ \\
\hline $\begin{array}{l}\text { 3. Compile a list of external oversight agency contacts (HRC, } \\
\text { EEOC, OFCCP, etc.) in Region X. }\end{array}$ & $\begin{array}{c}6 / 01 / 96 \\
\text { Completed }\end{array}$ & $\begin{array}{l}\text { VC Hodgin } \\
\text { Lewis }\end{array}$ \\
\hline $\begin{array}{l}\text { Post-Award Actions } \\
\text { 1. Provide PHMC a briefing and provide copies of the current } \\
\text { AAP including a statistical profile reflecting female and minority } \\
\text { representation by category/classification in the workforce. }\end{array}$ & $9 / 01 / 96$ & $\begin{array}{l}\text { VC Hodgin } \\
\text { Lewis }\end{array}$ \\
\hline $\begin{array}{l}\text { 2. Brief PHMC contractor on internal and external complaints and } \\
\text { cases by agency and status based on General Counsel } \\
\text { determination of ownership and protocol. }\end{array}$ & $9 / 01 / 96$ & $\begin{array}{l}\text { VC Hodgin } \\
\text { Lewis }\end{array}$ \\
\hline $\begin{array}{l}\text { 3. Review agency contacts listings with PHMC contractor } \\
\text { including a briefing on relationships and protocol. Provide } \\
\text { listing of external oversight agency contacts (HRC, EEOC, } \\
\text { OFCCP, etc.) in Region X. }\end{array}$ & $9 / 01 / 96$ & $\begin{array}{l}\text { VC Hodgin } \\
\text { Lewis }\end{array}$ \\
\hline
\end{tabular}

\subsubsection{Diversity Recognition and Training.}

\begin{tabular}{|l|c|l|}
\hline \multicolumn{1}{|c|}{ Pre-Award Actions } & $\begin{array}{c}\text { Scheduled } \\
\text { Date }\end{array}$ & Actionee(s) \\
\hline $\begin{array}{l}\text { 1. Develop a briefing document including current activities in } \\
\text { support of nationally recognized diversity events (Black } \\
\text { History Month, Women's History Month, etc.) and a } \\
\text { timeline/calendar of observances. }\end{array}$ & $\begin{array}{c}6 / 01 / 96 \\
\text { Completed }\end{array}$ & $\begin{array}{l}\text { VC Hodgin } \\
\text { Lewis }\end{array}$ \\
\hline $\begin{array}{l}\text { 1. Briest-Award Actions } \\
\text { including a timeline/calendar of observances. Determine } \\
\text { continuation of approach or an alternative. }\end{array}$ & $9 / 01 / 96$ & $\begin{array}{l}\text { VC Hodgin } \\
\text { Lewis }\end{array}$ \\
\hline
\end{tabular}

\subsubsection{Education Enrichment Programs.}

\begin{tabular}{|l|c|c|}
\hline \multicolumn{1}{|c|}{ Pre-Award Actions } & Scheduled & Actionee(s) \\
\hline $\begin{array}{l}\text { 1. Prepare information covering student development and } \\
\text { internship activities at pre-college (school-to-work initiative), } \\
\text { college (Partnership for Environmental Technology } \\
\text { Education) and university (Associated Western Universities) } \\
\text { levels. }\end{array}$ & $\begin{array}{c}6 / 01 / 96 \\
\text { Completed }\end{array}$ & JJ Chunn \\
\hline
\end{tabular}




\begin{tabular}{|c|c|c|}
\hline Post-Award Actions & $\begin{array}{c}\text { Scheduled } \\
\text { Date }\end{array}$ & Actionee(s) \\
\hline $\begin{array}{l}\text { 1. Brief new contractor and provide information covering } \\
\text { student development and internship activities at pre-college } \\
\text { (school-to-work initiative), college (Partnership for } \\
\text { Environmental Technology Education) and university } \\
\text { (Associated Western Universities) levels. }\end{array}$ & $8 / 15 / 96$ & JJ Chunn \\
\hline
\end{tabular}

\subsubsection{General Operations}

Determine, develop, and prepare appropriate data regarding employee relations, special programs, general policies, business management and the like.

\subsubsection{Transferring Vacation Hours for Family Medical Emergencies.}

\begin{tabular}{|c|c|c|}
\hline Pre-Award Actions & $\begin{array}{l}\text { Scheduled } \\
\text { Date }\end{array}$ & Actionee(s) \\
\hline $\begin{array}{l}\text { 1. Prepare a briefing on the voluntary transfer of vacation from } \\
\text { one employee to another due to family medical emergencies } \\
\text { including historical usage statistics. }\end{array}$ & $\begin{array}{c}\text { 6/01/96 } \\
\text { Completed }\end{array}$ & JT Eckert \\
\hline $\begin{array}{l}\text { 2. Develop a current participation status report reflecting } \\
\text { donors, recipients and committed vacation hours. }\end{array}$ & $\begin{array}{c}6 / 01 / 96 \\
\text { Completed } \\
\end{array}$ & JT Eckert \\
\hline Post-Award Actions & & \\
\hline $\begin{array}{l}\text { 1. Brief PHMC on the voluntary transfer of vacation from } \\
\text { one employee to another due to family medical } \\
\text { emergencies including historical usage statistics. }\end{array}$ & $8 / 05 / 96$ & JT Eckert \\
\hline $\begin{array}{l}\text { 2. Provide current participation status teport reflecting donor, } \\
\text { recipients and committed vacation hours. Coordinate with } \\
\text { PHMC to determine continuation of the program or an } \\
\text { approach to process participants likely to carry forward } \\
\text { past contract turnover. }\end{array}$ & $8 / 05 / 96$ & JT Eckert \\
\hline
\end{tabular}

\subsubsection{Employee Work Schedules.}

\begin{tabular}{|l|c|c|}
\hline \multicolumn{1}{|c|}{ Pre-Award Actions } & $\begin{array}{c}\text { Scheduled } \\
\text { Date }\end{array}$ & Actionee(s) \\
\hline $\begin{array}{l}\text { 1. Develop a briefing on non-bargaining unit standard work } \\
\text { schedules, 8 X 9's, 12-hour shifts and assignment to } \\
\text { nonstandard work schedules. }\end{array}$ & $\begin{array}{c}6 / 01 / 96 \\
\text { Completed }\end{array}$ & MM Hawkins \\
\hline $\begin{array}{l}\text { 2. Prepare copies of work schedules, policy staternents and } \\
\text { statistical data identifying employees assigned to } 8 \text { X 9's and } \\
\text { 12-hour shifts. }\end{array}$ & $\begin{array}{c}6 / 01 / 96 \\
\text { Completed }\end{array}$ & IM Hawkins \\
\hline
\end{tabular}




\begin{tabular}{|l|c|l|}
\hline \multicolumn{1}{|c|}{ Post-Award Actions } & Scheduled & Date \\
\hline $\begin{array}{l}\text { 1. Brief PHMC on non-bargaining unit standard work } \\
\text { schedules, 8 X 9's, 12-hour shifts and assignment to } \\
\text { nonstandard work schedules. }\end{array}$ & $9 / 01 / 96$ & IM Hawkins \\
\hline $\begin{array}{l}\text { 2. Provide copies of work schedules, policy statements and } \\
\text { statistical data identifying employees assigned to 8X 9's and } \\
\text { 12-hour shifts. }\end{array}$ & $9 / 01 / 96$ & MM Hawkins \\
\hline
\end{tabular}

\subsubsection{Salary Continuance During Absences.}

\begin{tabular}{|l|c|c|}
\hline \multicolumn{1}{|c|}{ Pre-Award Actions } & $\begin{array}{c}\text { Scheduled } \\
\text { Date }\end{array}$ & Actionee(s) \\
\hline $\begin{array}{l}\text { 1. Develop briefing on the requirements and guidelines for the } \\
\text { continuation of salary payments during periods of employee } \\
\text { absence. Cover inclusion, by reference, in collective } \\
\text { bargaining agreements. }\end{array}$ & $\begin{array}{c}6 / 01 / 96 \\
\text { Completed }\end{array}$ & MK Strankman \\
\hline$\quad$ Post-Award Actions & $9 / 01 / 96$ & MK Strankman \\
\hline $\begin{array}{l}\text { 1. Brief PHMC on the requirements and guidelines for the } \\
\text { continuation of salary payments during periods of employee } \\
\text { absence. Cover inclusion, by reference, in collective } \\
\text { bargaining agreements. }\end{array}$ & \\
\hline
\end{tabular}

\subsubsection{Vacation and Holidays.}

\begin{tabular}{|c|c|c|}
\hline Pre-Award Actions & $\begin{array}{c}\text { Scheduled } \\
\text { Date }\end{array}$ & Actionee(s) \\
\hline $\begin{array}{l}\text { 1. Prepare a briefing on the accrual method of vacation } \\
\text { accumulation and the holiday schedule including qualification } \\
\text { criteria. Cover inclusion in collective bargaining agreements. }\end{array}$ & $\begin{array}{c}6 / 01 / 96 \\
\text { Completed }\end{array}$ & FA Blowe \\
\hline $\begin{array}{l}\text { 2. Develop a preliminary accrued vacation liability report and } \\
\text { identify scheduled holidays through the balance of the } \\
\text { calendar year. }\end{array}$ & $\begin{array}{c}6 / 01 / 96 \\
\text { Completed }\end{array}$ & FA Blowe \\
\hline Post-Award Actions & & \\
\hline $\begin{array}{l}\text { 1. Brief PHMC on the accrual method of vacation accumulation } \\
\text { and the holiday schedule including qualification criteria. } \\
\text { Cover inclusion in collective bargaining agreements. }\end{array}$ & $9 / 01 / 96$ & FA Blowe \\
\hline $\begin{array}{l}\text { 2. Review preliminary accrued vacation liability report with } \\
\text { PHMC and determine vacation policy to be used after } \\
\text { turnover. Establish amount of accrued vacation transferable } \\
\text { to provide a basis for pre-turnover vacation payout. }\end{array}$ & $9 / 01 / 96$ & FA Blowe \\
\hline
\end{tabular}




\subsubsection{Salaried Nonexempt Workforce Adjustment.}

\begin{tabular}{|c|c|c|}
\hline$\quad$ Pre-Award Actions & $\begin{array}{c}\text { Scheduled } \\
\text { Date }\end{array}$ & Actionee(s) \\
\hline $\begin{array}{l}\text { 1. Prepare a briefing on responsibility and requirements for } \\
\text { adjustments in the salaried nonexempt workforce, including } \\
\text { transfer, promotion, and reduction of force (ROF). }\end{array}$ & $\begin{array}{c}6 / 01 / 96 \\
\text { Completed }\end{array} \quad$ Post-Award Actions & KA Hill \\
\hline $\begin{array}{l}\text { 1. Brief the PHMC on responsibility and requirements for } \\
\text { adjustments in the salaried nonexempt workforce, including } \\
\text { transfer, promotion, and ROF. }\end{array}$ & $9 / 01 / 96$ & KA Hill \\
\hline
\end{tabular}

\subsubsection{Business Management.}

\begin{tabular}{|c|c|c|}
\hline \multicolumn{1}{|c|}{ Pre-Award Actions } & $\begin{array}{c}\text { Scheduled } \\
\text { Date }\end{array}$ & Actionee(s) \\
\hline $\begin{array}{l}\text { 1. Develop the FY 1997 SSPP including supporting budgets, } \\
\text { workscope definition and milestone commitments. }\end{array}$ & $\begin{array}{c}5 / 01 / 96 \\
\text { Completed }\end{array}$ & $\begin{array}{l}\text { HA Sieber } \\
\text { RC Boldt }\end{array}$ \\
\hline Post-Award Actions & & \\
\hline $\begin{array}{l}\text { 1. Coordinate with PHMC to obtain input to the FY 1997 SSPP } \\
\text { including supporting budgets, workscope definition and } \\
\text { milestone commitments. }\end{array}$ & $8 / 15 / 96$ & $\begin{array}{l}\text { HA Sieber } \\
\text { RC Boldt }\end{array}$ \\
\hline
\end{tabular}

\subsubsection{Re-engineering Support}

Determine, develop, and prepare appropriate data and information regarding pilot programs and processes dealing with the human resources aspects of re-engineering initiatives.

\begin{tabular}{|c|c|c|}
\hline $\begin{array}{l}\text { Q } \\
\text { Pre-Award Actions }\end{array}$ & $\begin{array}{l}\text { Scheduled } \\
\text { Date }\end{array}$ & Actionee(s) \\
\hline $\begin{array}{l}\text { 1. Prepare a briefing that will identify divisions, departments or } \\
\text { facilities where re-engineered processes have been implemented } \\
\text { utilizing piloted human resources programs and procedures for such } \\
\text { activities as staffing, compensation, performance assessment and } \\
\text { training. Include information on results or expected outcomes of } \\
\text { pilots. }\end{array}$ & $\begin{array}{c}5 / 01 / 96 \\
\text { Completed }\end{array}$ & JF Oswald \\
\hline $\begin{array}{l}\text { 2. Assemble information and data specific to in-place, self-directed } \\
\text { work teams including names, classifications, roles and work } \\
\text { locations to minimize disruption to in-place or planned re- } \\
\text { engineering initiatives. }\end{array}$ & $\begin{array}{l}5 / 01 / 96 \\
\text { Completed }\end{array}$ & JF Oswald \\
\hline
\end{tabular}




\begin{tabular}{|c|c|c|}
\hline Post-Award Actions & $\begin{array}{c}\text { Scheduled } \\
\text { Date }\end{array}$ & Actionee(s) \\
\hline $\begin{array}{l}\text { 1. Provide PHMC briefing regarding divisions, departments or } \\
\text { facilities where re-engineered processes have been implemented } \\
\text { utilizing piloted human resources programs and procedures for such } \\
\text { activities as staffing, compensation, performance assessment and } \\
\text { training. Coordinate with PHMC to continue and/or modify } \\
\text { approach, based on results or expected outcomes of pilots. }\end{array}$ & $8 / 15 / 96$ & JF Oswald \\
\hline $\begin{array}{l}\text { 2. Provide PHMC information and data specific to in-place self- } \\
\text { directed work teams including names, classifications, roles and } \\
\text { work locations to minimize disruption to in-place or planned re- } \\
\text { engineering initiatives. Recommend an approach and discuss } \\
\text { feasibility of intact transition to PHMC. }\end{array}$ & $8 / 15 / 96$ & JF Oswald \\
\hline
\end{tabular}

\subsection{EMPLOYEE CONCERNS}

The Employee Concerns Program (ECP) responds to and tracks to closure concerns raised by WHC/BCSR employees.

\begin{tabular}{|l|c|l|}
\hline \multicolumn{1}{|c|}{ Pre-Awrard Actions } & Scheduled & Date \\
\hline $\begin{array}{l}\text { 1. Determine how to disposition and/or resolve employee } \\
\text { concerns that remain open at end of WHC contract (9/30/96). } \\
\text { Will need input/guidance from General Counsel's Office. }\end{array}$ & $5 / 31 / 96$ & MG Dickinson \\
\hline $\begin{array}{l}\text { 2. Determine disposition of closed employee concerns files; } \\
\text { computerized database; and logbooks. Will need } \\
\text { input/guidance from General Counsel's Office. }\end{array}$ & $5 / 31 / 96$ & MG Dickinson \\
\hline $\begin{array}{l}\text { 1. Transfer, if appropriate, information necessary to } \\
\text { resolve/complete those concerns that remain open at the time } \\
\text { start of the PHMC contract. }\end{array}$ & $9 / 30 / 96$ & MG Dickinson \\
\hline $\begin{array}{l}\text { 2. Transfer, as determined appropriate, employee concerns files } \\
\text { and information. }\end{array}$ & $9 / 30 / 96$ & MG Dickinson \\
\hline
\end{tabular}




\subsection{ENVIRONMENTAL, SAFETY, QUALITY, AND HEALTH}

\section{Assumptions}

- The PHMC will use existing Radiation Protection Services records and systems.

- The PHMC senior/executive management will attain the minimum (as defined by RL) qualifications necessary to assume responsibility for emergency response by October 1, 1996. These qualifications may include completion of one emergency response exercise prior to takeover. If management cannot accomplish this training in the available time, the PHMC will define what measures will be taken to cover the period until management can be adequateiy trained.

- Current procedures define safety responsibilities with respect to our existing organization structure. The PHMC will map existing responsibilities against any new organization structure put in place by October 1, 1996. This mapping will be adequate to assure that safety responsibilities are both cleat and fully covered. Newly assigned PHMC managers and staff will be trained to the procedural requirements of their positions.

\subsubsection{Safety, Quality, and Health}

\subsubsection{Program Management.}

\subsection{Budget.}

\begin{tabular}{|c|c|c|}
\hline \multicolumn{1}{|c|}{ Pre-Award Actions } & $\begin{array}{c}\text { Scheduled } \\
\text { Date }\end{array}$ & \multicolumn{1}{|c|}{ Actionee(s) } \\
\hline 1. Assemble current budget documentation (SSPP). & $\begin{array}{c}5 / 31 / 96 \\
\text { Completed }\end{array}$ & SJ Richardson \\
\hline Post-Award Actions & & \\
\hline 1. Provide status of overall organization budget. & $\begin{array}{c}8 / 02 / 96- \\
9 / 02 / 96\end{array}$ & JK Pettey \\
\hline 2. Provide briefing on S\&H management planning process and & $8 / 12 / 96-$ & JK Pettey \\
status (EH planning process, including RDS and ADS). & $9 / 02 / 96$ & \\
\hline 3. Provide budget documentation. & $8 / 05 / 96$ & JK Pettey \\
\hline
\end{tabular}

\subsection{Administration.}

\begin{tabular}{|c|c|c|}
\hline Pre-Award Actions & $\begin{array}{c}\text { Scheduled } \\
\text { Date }\end{array}$ & Actionee(s) \\
\hline $\begin{array}{l}\text { 1. Document status of customer commitments against current } \\
\text { program plan. }\end{array}$ & $\begin{array}{l}5 / 31 / 96 \\
\text { Completed }\end{array}$ & SJ Richardson \\
\hline
\end{tabular}




\begin{tabular}{|l|c|l|}
\hline \multicolumn{1}{|c|}{ Post-Award Actions } & Scheduled & \multicolumn{1}{|c|}{ Actionee(s) } \\
\hline 1. Provide organization charts, staff directory. & $8 / 05 / 96$ & VB Moore \\
\hline 2. Provide training status. & $9 / 02 / 96$ & VB Moore \\
\hline
\end{tabular}

\subsection{Corrective Action Management System (CAMS).}

\begin{tabular}{|l|c|l|}
\hline \multicolumn{1}{|c|}{ Pre-Award Actions } & $\begin{array}{c}\text { Scheduled } \\
\text { Date }\end{array}$ & \multicolumn{1}{|c|}{ Actionee(s) } \\
\hline $\begin{array}{l}\text { 1. Assemble documentation on CAMS (WHC-CM-1-4) and } \\
\text { Corrective Action Management Board (CAMB). }\end{array}$ & $\begin{array}{c}5 / 31 / 96 \\
\text { Completed }\end{array}$ & RB Balthazor \\
\hline $\begin{array}{l}\text { 2. Document current policy status and status of outstanding } \\
\text { CAMB actions. Post-Award Actions }\end{array}$ & $\begin{array}{c}5 / 31 / 96 \\
\text { Completed }\end{array}$ & RB Balthazor \\
\hline $\begin{array}{l}\text { 1. Provide briefing on CAMS. } \\
\text { 2. Provide CAMS documentation. }\end{array}$ & $8 / 12 / 96-$ & RB Balthazor \\
\hline
\end{tabular}

3.5.1.1.4 Hanford Action Tracking System. The Hanford Action Tracking System (HATS) provides a mechanism to monitor, status, and trend corrective actions at Hanford. The re-engineering process designated HATS as the preferred official corrective action system, and can also handle non-corrective action items. Actions are tracked from initiation through closure. HATS is designed to cover a multi-contractor environment, providing a global perspective of corrective action activities. HATS is being considered for use in documenting pre-existing conditions.

\begin{tabular}{|l|c|l|}
\hline \multicolumn{1}{|c|}{ Pre-Award Actions } & $\begin{array}{c}\text { Scheduled } \\
\text { Date }\end{array}$ & \multicolumn{1}{c|}{ Actionee(s) } \\
\hline $\begin{array}{l}\text { 1. Publish a HATS/CAM Process Guide to define business rules } \\
\text { and user responsibilities. }\end{array}$ & $\begin{array}{c}5 / 01 / 96 \\
\text { Completed }\end{array}$ & $\begin{array}{l}\text { MK Britton } \\
\text { PM Knoll, Ir. }\end{array}$ \\
\hline \multicolumn{1}{c|}{ Post-Award Actions } & & \\
\hline $\begin{array}{l}\text { 1. Provide the HATS Business Process User's Guide and a list } \\
\text { of open items that require closure. }\end{array}$ & $8 / 05 / 96$ & $\begin{array}{l}\text { MK Britton } \\
\text { JM Knoll, Jr. }\end{array}$ \\
\hline $\begin{array}{l}\text { 2. Conduct a presentation and a demonstration of the HATS } \\
\text { functionality. (Include relationship to CICS) }\end{array}$ & $9 / 01 / 96$ & $\begin{array}{l}\text { MK Britton } \\
\text { JM Knoll, Jr. }\end{array}$ \\
\hline $\begin{array}{l}\text { 3. Provide training and documentation to ensure a smooth } \\
\text { transition of HATS administrative responsibilities and } \\
\text { requirements. }\end{array}$ & $9 / 30 / 96$ & $\begin{array}{l}\text { MK Britton } \\
\text { JM Knoll, Jr. }\end{array}$ \\
\hline
\end{tabular}




\subsection{Points of Contact.}

\begin{tabular}{|c|c|c|}
\hline Post-Award Actions & $\begin{array}{l}\text { Scheduled } \\
\text { Date }\end{array}$ & Actionee(s) \\
\hline 1. Provide Safety, Quality and Health points of contact list. & $8 / 05 / 96$ & VB Moore \\
\hline
\end{tabular}

\subsubsection{Safety.}

\subsection{Administration.}

\begin{tabular}{|c|c|c|}
\hline . 1.4 PreAward Actions & $\begin{array}{l}\text { Schediled } \\
\text { Date }\end{array}$ & Actionee(s) \\
\hline 1. Assemble current safety plans, policies and procedures. & $\begin{array}{l}5 / 31 / 96 \\
\text { Completed }\end{array}$ & GR Franz \\
\hline $\begin{array}{l}\text { 2. Assemble documentation for Industrial Safety (IS) and Fire } \\
\text { Protection (FP) only: This relates to the assembly of all } \\
\text { WHC programmatic (levels } 1 \text { and 2) policies, standards, and } \\
\text { procedures relating to IS and FP, as well as all company } \\
\text { level safety program plans, goals, objectives, and milestones. }\end{array}$ & $\begin{array}{c}5 / 31 / 96 \\
\text { Completed }\end{array}$ & JR Bell \\
\hline 3. Verify contents of safety resource library. & $\begin{array}{c}5 / 31 / 96 \\
\text { Completed }\end{array}$ & JR Bell \\
\hline Post-Award Actions & & \\
\hline $\begin{array}{l}\text { 1. Brief PHMC on medical services provided by HEHF } \\
\text { (program and budget status). }\end{array}$ & $\begin{array}{l}8 / 12 / 96 \\
9 / 02 / 96\end{array}$ & JJ Maher III \\
\hline $\begin{array}{l}\text { 2. Brief PHMC on medical services provided by HEHF (budget } \\
\text { status). }\end{array}$ & $\begin{array}{l}8 / 12 / 96 \\
9 / 02 / 96\end{array}$ & $\begin{array}{l}\text { JE Best } \\
\text { RO Volk }\end{array}$ \\
\hline 3. Transfer medical surveillance enrollment records. & $9 / 29 / 96$ & $\begin{array}{l}\text { MK Britton } \\
\text { JJ Maher II }\end{array}$ \\
\hline 4. Transfer safety resource library. & $9 / 29 / 96$ & L Rogers \\
\hline 5. Brief PHMC on safety awareness program. & $8 / 19 / 96$ & L Rogers \\
\hline
\end{tabular}

\subsection{Accident Investigation and OSHA Injury Records.}

\begin{tabular}{|l|c|c|}
\hline \multicolumn{1}{|c|}{ Pre-Award Actions } & $\begin{array}{c}\text { Scheduled } \\
\text { Date }\end{array}$ & Actionee(s) \\
\hline $\begin{array}{l}\text { 1. Assembly data on open accident investigations: Files are } \\
\text { kept by the Accident Investigator working the case. A report } \\
\text { can be developed listing all open cases at any point in time. }\end{array}$ & $\begin{array}{c}6 / 01 / 96 \\
\text { Completed }\end{array}$ & L Rogers \\
\hline $\begin{array}{l}\text { 2. Prepare current statistical data. A complete, current } \\
\text { statistical data report can be done in a single person day from } \\
\text { notice to proceed. }\end{array}$ & $\begin{array}{c}6 / 01 / 96 \\
\text { Completed }\end{array}$ & L Rogers \\
\hline
\end{tabular}




\begin{tabular}{|l|c|c|}
\hline \multicolumn{1}{|c|}{ Post-Award Actions } & $\begin{array}{c}\text { Scheduled } \\
\text { Date }\end{array}$ & Actionee(s) \\
\hline $\begin{array}{l}\text { 1. Transfer accident files and data: this is assumed to include } \\
\text { only giving the new contractor the file keys and passwords. } \\
\text { Includes auditable OSHA records, including logs, and OSHA } \\
\text { database. }\end{array}$ & $9 / 29 / 96$ & L Rogers \\
\hline
\end{tabular}

\subsection{Regulatory Implementation}

\begin{tabular}{|c|c|c|}
\hline Pre-Award Actions & $\begin{array}{l}\text { Scheduled } \\
\text { Date. }\end{array}$ & Actionee(s) \\
\hline $\begin{array}{l}\text { 1. One page summary (DSI, Internal Memo, etc.) providing a } \\
\text { brief overview of the status on issuing and/or developing } \\
\text { Implementation Plans (IPs)/Exemption Requests (ERs) for the } \\
\text { balance of the Phase I Rules. }\end{array}$ & $\begin{array}{c}\text { 6/01/96 } \\
\text { Completed }\end{array}$ & DM Busche \\
\hline Post-Award Actions & & 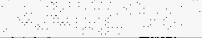 \\
\hline $\begin{array}{l}\text { 1. Summary report detailing the status of issuing Rules and/or } \\
\text { preparing IP/ERs for each WHC managed Nuclear Facility } \\
\text { (e.g., positioning, negotiated w/regulator, compliance } \\
\text { baselines, validation staws, etc.) }\end{array}$ & 9/02/96 & DM Busche \\
\hline 2. Project Files (for each rule and each facility). & $9 / 02 / 96$ & DM Busche \\
\hline 3. Document repository for IPs/ERs prepared. & $9 / 20 / 96$ & DM Busche \\
\hline
\end{tabular}

\subsection{Subcontractor/Procurement Oversight.}

\begin{tabular}{|c|c|c|}
\hline Pre-Award Actions & $\begin{array}{l}\text { Scheduled } \\
\text { Date }\end{array}$ & Actionee(s) \\
\hline $\begin{array}{l}\text { 1. Document status of ongoing subcontractor/procurement } \\
\text { oversight. }\end{array}$ & $\begin{array}{c}6 / 01 / 96 \\
\text { Completed }\end{array}$ & $\begin{array}{l}\text { DL Bloom } \\
\text { BG Baker } \\
\text { RD Melbihess } \\
\end{array}$ \\
\hline Post-Award Actions & $\%$ & \\
\hline $\begin{array}{l}\text { 1. Transfer subcontractor/procurement oversight status } \\
\text { information. }\end{array}$ & $9 / 02 / 96$ & $\begin{array}{l}\text { DL Bloom } \\
\text { BG Baker } \\
\text { RD Melbihess }\end{array}$ \\
\hline
\end{tabular}

\subsection{Industrial Hygiene and Safety (including IH Equipment Lab)}

\begin{tabular}{|c|c|c|}
\hline Pre-Award Actions & $\begin{array}{l}\text { Scheduled } \\
\text { Date }\end{array}$ & Actionee(s) \\
\hline $\begin{array}{l}\text { Verify equipment list (2); verify calibration status (3) of IH } \\
\text { Equipment Laboratory (HHEL). Note: These actions relate } \\
\text { only to exposure monitoring equipment directly controlled } \\
\text { by IHEL. IHEL maintains exposure monitoring equipment } \\
\text { for WHC and ICF KH. }\end{array}$ & $\begin{array}{c}6 / 01 / 96 \\
\text { Completed }\end{array}$ & GR Franz \\
\hline
\end{tabular}




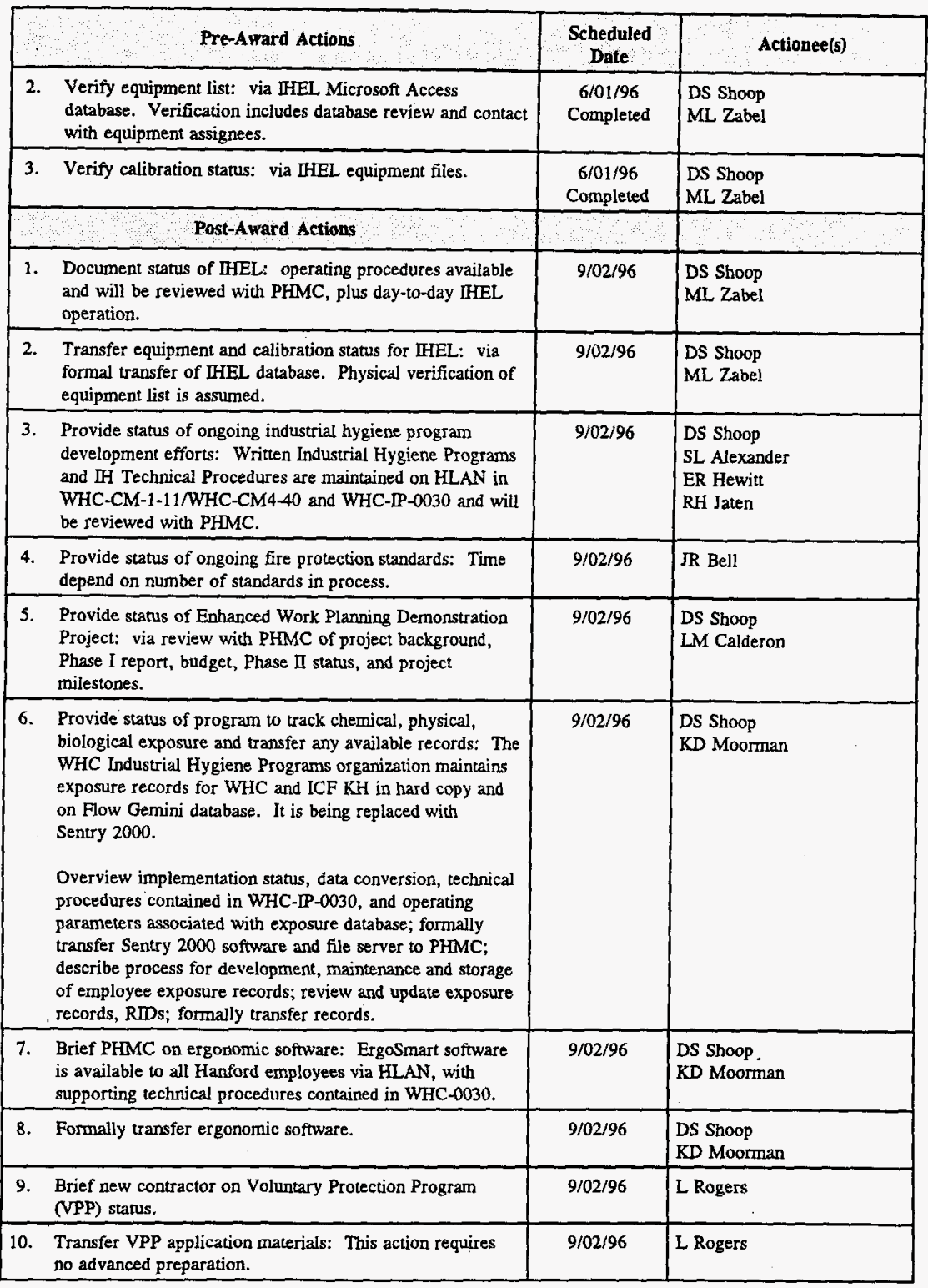




\subsection{Fire Safety}

\begin{tabular}{|l|c|c|}
\hline \multicolumn{1}{|c|}{ Pre-Award Actions } & $\begin{array}{c}\text { Scheduled } \\
\text { Date }\end{array}$ & Actionee(s) \\
\hline 1. Document status of ongoing fire safety oversight. & $\begin{array}{c}6 / 01 / 96 \\
\text { Completed }\end{array}$ & JR Bell \\
\hline \multicolumn{1}{|c|}{ Post-Award Actions } & & \\
\hline $\begin{array}{l}\text { 1. Transfer fire and property damage records: we keep no such } \\
\text { official records on behalf of the company, other than the } \\
\text { annual surnmary reports which are in office files. Action: } \\
\text { turn over all office files. }\end{array}$ & $9 / 02 / 96$ & JR Bell \\
\hline \begin{tabular}{l} 
2. Transfer fire safety oversight status information. \\
\hline
\end{tabular} & $9 / 02 / 96$ & JR Bell \\
\hline
\end{tabular}

\subsubsection{Radiological Control (RadCon).}

\subsection{Administration.}

\begin{tabular}{|c|c|c|}
\hline Pre-Award Actions & $\begin{array}{c}\text { Scheduled } \\
\text { Date }\end{array}$ & Actionee(s) \\
\hline $\begin{array}{l}\text { 1. Prepare summary package outlining RadCon Central Support } \\
\text { Group (CSG), Center of Expertise (COE) and Site Dosimetry } \\
\text { Services } \\
\text { - Include program and budget fomulation (both direct and } \\
\text { indirect activities. }\end{array}$ & $\begin{array}{c}6 / 01 / 96 \\
\text { Completed }\end{array}$ & DJ Newland \\
\hline Post-Award Actions & & \\
\hline $\begin{array}{l}\text { 1. Brief PHMC on current status of CSG/COE activities and } \\
\text { Site Dosimetry Services } \\
\text { Provide program and budget status detail (SSPP, ADS, } \\
\text { RDS). }\end{array}$ & $9 / 02 / 96$ & DJ Newland \\
\hline $\begin{array}{l}\text { 2. Discuss with PHMC transition actions associated to } \\
\text { decentraled RadCon Organization } \\
\text { - HPT transfer to the line } \\
\text { - Funding transitions } \\
\text { - Bargaining Unit support }\end{array}$ & $9 / 02 / 96$ & DJ Newland \\
\hline $\begin{array}{l}\text { 3. Provide PHMC status of outstanding customer commitments } \\
\text { (RadCon Path Forward) } \\
\text { - Sitewide RadCon Action Plans } \\
\text { - Facility Specific Action Plans }\end{array}$ & $9 / 02 / 96$ & DJ Newland \\
\hline
\end{tabular}

\subsection{Centralized RadCon CSG and COE.}

\begin{tabular}{|c|c|c|}
\hline Pre-Award Actions & $\begin{array}{c}\text { Scheduled } \\
\text { Date }\end{array}$ & Actionee(s) \\
\hline $\begin{array}{l}\text { 1. Prepare to brief PHMC on centralized RadCon support } \\
\text { activities and sitewide implementation roles } \\
\text { - Technical Radiological Engineering support. }\end{array}$ & $\begin{array}{c}6 / 01 / 96 \\
\text { Completed }\end{array}$ & PB Chadly \\
\hline
\end{tabular}




\begin{tabular}{|l|c|c|}
\hline \multicolumn{1}{|c|}{ Post-Award Actions } & Scheduled & Date \\
\hline $\begin{array}{l}\text { 1. Assemble CSG and COE completed decision making } \\
\text { packages (CDMP) for transfer. }\end{array}$ & $9 / 02 / 96$ & PB Chadly \\
\hline $\begin{array}{l}\text { 2. Provide PHMC with CSG and COE sitewide infrastructure } \\
\text { and requirements impiementation process } \\
-\quad \text { Address sitewide requirements consistency issues. }\end{array}$ & $9 / 02 / 96$ & PB Chadly \\
\hline $\begin{array}{l}\text { 3. Provide PHMC overview/status of Hanford Site Radiological } \\
\text { Control Manual (HRSCM) support services. }\end{array}$ & $9 / 02 / 96$ & PB Chadly \\
\hline $\begin{array}{l}\text { 4. Brief PHMC on external contractor procured services/support } \\
\text { status. }\end{array}$ & $9 / 02 / 96$ & PB Chadly \\
\hline
\end{tabular}

3.5.1.3.3 Site Personnel Dosimetry Services. Note: If the PHMC decides not to utilize the sitewide radiation protection services, the other Hanford contractors and DOE-RL will need to develop new strategies to obtain/maintain these services. Therefore, a separate transition plan dealing with all Hanford contractors and RL will need to be developed.

\begin{tabular}{|c|c|c|}
\hline Pre-Award Actions: & $\begin{array}{l}\text { Scheduled } \\
\text { Date }\end{array}$ & Actionee(s) \\
\hline $\begin{array}{l}\text { 1. Prepare to brief PHMC on all sitewide radiation protection } \\
\text { services currently provided by PNNL } \\
\text { - In-vivo counting (whole-body, lung, organ, skeletal) } \\
\text { - In-vitro bioassay program } \\
\text { - External dosineter processing/reporting } \\
\text { - External expert contracting services/oversight } \\
\text { - Historical and current sadiation exposure and RadCon } \\
\text { program records project } \\
\text { - RadCon instrumentation calibration, maintenance and } \\
\text { testing programs } \\
\text { - Sitewide radiation protection committee structure, charters } \\
\text { and membership }\end{array}$ & $\begin{array}{l}\text { 6/01/96 } \\
\text { Completed }\end{array}$ & $\begin{array}{l}\text { LR McKay } \\
\text { ME Hevland (PNNL) }\end{array}$ \\
\hline Post-Award Actions & & \\
\hline $\begin{array}{l}\text { 1. Assemble auditable personnel dosimetry record } \\
\text { documentation. }\end{array}$ & $9 / 29 / 96$ & $\begin{array}{l}\text { LR McKay } \\
\text { ME Hevland (PNNL) }\end{array}$ \\
\hline $\begin{array}{l}\text { 2. Brief PHMC on internal dosimetry program } \\
\text { - Prepare/transfer routine internal dosimetry records } \\
\text { - Provide emergency response evaluation results } \\
\text { - Transfer employee "home location" logistics database. }\end{array}$ & $9 / 29 / 96$ & $\begin{array}{l}\text { LR McKay } \\
\text { ME Hevland (PNNL) }\end{array}$ \\
\hline $\begin{array}{l}\text { 3. Brief PHAC on external dosimetry program } \\
\text { - Prepare/transfer routine external dosimetry records } \\
\text { - Provide dose reconstruction and exposure evaluation data } \\
\text { - Transfer worker restrictions data. }\end{array}$ & $9 / 29 / 96$ & $\begin{array}{l}\text { LR McKay } \\
\text { ME Hevland (PNNL) }\end{array}$ \\
\hline $\begin{array}{l}\text { 4. Brief PHMC on subcontractor dosimetry program } \\
\text { - Provide dosimetry interface matrix for plants/projects } \\
\text { - Prepare/transfer subcontractor dosimetry records. }\end{array}$ & $9 / 29 / 96$ & $\begin{array}{l}\text { LR McKay } \\
\text { ME Hevland (PNNL) }\end{array}$ \\
\hline 5. Transfer personnel dosimetry records database and software. & $9 / 29 / 96$ & $\begin{array}{l}\text { LR McKay } \\
\text { ME Hevland (PNNL) }\end{array}$ \\
\hline $\begin{array}{l}\text { 6. Transfer Dosimetry and Medical Services Manual } \\
\text { (WHC-CM-4-16). }\end{array}$ & $9 / 29 / 96$ & $\begin{array}{l}\text { LR McKay } \\
\text { ME Hevland (PNNL) }\end{array}$ \\
\hline
\end{tabular}




\subsection{RadCon Technical Standards.}

\begin{tabular}{|c|c|c|}
\hline Pre-Award Actions & $\begin{array}{l}\text { Scheduled } \\
\text { Date }\end{array}$ & Actionee(s) \\
\hline $\begin{array}{l}\text { 1. Prepare to brief PHMC on established minimum RadCon } \\
\text { technical standards and documents } \\
\text { - Applied Radiological Controls (WHC-CM-4-14) } \\
\text { - Dosimetry and Medical Services Manual } \\
\text { (WHC-CM-4-16) } \\
\text { - Health Physics Procedures (WHC-IP-0718) } \\
\text { - Access Control Entry System User Manual } \\
\text { (WHC-IP-0993) } \\
\text { - WHC Occupational As Low As Reasonably Achievable } \\
\text { (ALARA) Program } \\
\text { (WHC-IP-1043). }\end{array}$ & $\begin{array}{c}\text { 6/01/96 } \\
\text { Completed }\end{array}$ & PB Chadly \\
\hline - $\quad$ Post-Award Actions & & \\
\hline $\begin{array}{l}\text { 1. Assemble/transfer current RadCon Path Forward plan, } \\
\text { standards, policies and implementing procedures } \\
\text { - Validate current RadCon standards } \\
\text { - Assemble/status outstanding field change requests } \\
\text { - Provide central and facility specific copies to new } \\
\text { contractor. }\end{array}$ & $9 / 02 / 96$ & PB Chaḋly \\
\hline
\end{tabular}

\subsection{Fixed/Portable Radiological Monitoring Equipment.}

\begin{tabular}{|c|c|c|}
\hline \multicolumn{1}{|c|}{ Post-Award Actions } & Scheduled & Actionee(s) \\
\hline $\begin{array}{l}\text { 1. Document current status of RadCon monitoring } \\
\text { equipment/Lab } \\
\text { - Verify current RadCon instrumentation and equipment list } \\
\text { - Verify calibration standards and records } \\
\text { - Provide current technical standards, procurement data, } \\
\text { technical basis and supporting data for instruments }\end{array}$ & $9 / 29 / 96$ & LR McKay \\
- Transfer equipment, calibration standards, and records. & & \\
\hline 2. Provide PHMC status of ongoing RadCon equipment \\
decentralization and prepare for transfer \\
- Pooled instrument calibration and maintenance \\
- Interface with Hanford Instrument Evaluation Committee.
\end{tabular}




\subsection{RadCon Technical Compliance/Documentation.}

\begin{tabular}{|c|c|c|}
\hline Post-Award Actions & $\begin{array}{c}\text { Scheduled } \\
\text { Date }\end{array}$ & Actionee(s) \\
\hline $\begin{array}{l}\text { 1. Assemble supporting data for compliance actions } \\
\text { (10 Code of Federal Regulations [CFR] } 835, \mathrm{RCM} \text {, etc.) } \\
\text { - Document status of compliance actions in progress } \\
\text { - Transfer RadCon RPP and S/RID compliance data } \\
\text { - Transfer RadCon implementation activity database } \\
\text { - Provide COE CDMP closure packages, compliance plans } \\
\text { and schedules on implementation actions } \\
\text { - Provide Interpretive Authority Technical Equivalency } \\
\text { Determination (TED) data demonstrating compliance. }\end{array}$ & $9 / 02 / 96$ & PB Chadly \\
\hline
\end{tabular}

\subsection{Radiological Access Control Entry System (ACES).}

\begin{tabular}{|l|l|l|}
\hline \multicolumn{1}{|c|}{ Pre-Award Actions } & $\begin{array}{c}\text { Scheduled } \\
\text { Date }\end{array}$ & \multicolumn{1}{c|}{ Actionee(s) } \\
\hline 1. Verify accuracy of ACES database. & $\begin{array}{c}6 / 01 / 96 \\
\text { Completed }\end{array}$ & LR McKay \\
\hline \multicolumn{1}{c|}{ Post-Award Actions } & & \\
\hline $\begin{array}{l}\text { 1. Brief/train PHMC on employee/visitor ACES operation. } \\
\text { hardware licensing agreements } \\
\text { - ACES training standards/records } \\
\text { ACES password database. }\end{array}$ & $9 / 02 / 96$ & LR McKay \\
\hline $\begin{array}{l}\text { 3. Transfer Access Control Entry System User Manual } \\
\text { (WHC-LP-0993). }\end{array}$ & $9 / 02 / 96$ & LR McKay \\
\hline
\end{tabular}

\subsection{Occupational ALARA Program.}

\begin{tabular}{|c|c|c|}
\hline Pre-Award Actions & $\begin{array}{l}\text { Scheduled } \\
\text { Date }\end{array}$ & Actionee(s) \\
\hline $\begin{array}{l}\text { 1. Prepare to brief PHMC on existing occupational ALARA } \\
\text { program } \\
\text { - Facility and site infrastructure } \\
\text { - Centralized Radiological Engineering support } \\
\text { - Centralized Contamination Control Improvement Program } \\
\text { - Radiological Emergency Assistance Center/Training Site } \\
\text { (REAC/TS). }\end{array}$ & $\begin{array}{c}6 / 01 / 96 \\
\text { Completed }\end{array}$ & PB Chadly \\
\hline Post-Award Actions & & \\
\hline 1. Assemble ALARA Program documentation and current plans. & $9 / 02 / 96$ & PB Chadly \\
\hline $\begin{array}{l}\text { 2. Transfer current program documentation, record reports, and } \\
\text { supplemental training plans } \\
\text { - WHC Occupational ALARA Program (WHC-IP-1043) } \\
\text { - Optimization methodology and training materials. }\end{array}$ & $9 / 02 / 96$ & PB Chadly \\
\hline 3. Transfer REAC and equipment. & $9 / 29 / 96$ & PB Chadly \\
\hline
\end{tabular}




\subsection{RadCon COE Internal Assessment Program.}

\begin{tabular}{|c|c|c|}
\hline Pre-Award Actions & $\begin{array}{l}\text { Scheduled } \\
\text { Date }\end{array}$ & Actionee(s) \\
\hline $\begin{array}{l}\text { 1. Prepare to brief PHMC on current COE assessment program } \\
\text { and support provided to the Facility Evaluation Board (FEB). }\end{array}$ & $\begin{array}{c}6 / 01 / 96 \\
\text { Completed }\end{array}$ & PB Chadly \\
\hline Post-Award Actions & 0 & : \\
\hline 1. Document status of ongoing sitewide RadCon assessments. & $9 / 02 / 96$ & PB Chadly \\
\hline $\begin{array}{l}\text { 2. Transfer assessment status tracking and trending program and } \\
\text { associated records. }\end{array}$ & $9 / 02 / 96$ & PB Chadly \\
\hline $\begin{array}{l}\text { 3. Transfer all formal interpretations (both facility and site } \\
\text { level) documentation on RadCon requirements. }\end{array}$ & $9 / 02 / 96$ & PB Chadly \\
\hline $\begin{array}{l}\text { 4. Provide company level RadCon S/RID review and approval } \\
\text { data. }\end{array}$ & $9 / 02 / 96$ & PB Chadly \\
\hline
\end{tabular}

\subsection{RadCon Site Training.}

\begin{tabular}{|l|c|c|}
\hline \multicolumn{1}{|c|}{ Pre-Award Actions } & $\begin{array}{c}\text { Scheduled } \\
\text { Date }\end{array}$ & \multicolumn{1}{|c|}{ Actionee(s) } \\
\hline 1. Prepare to brief PHMC onsite RadCon training program. & $\begin{array}{c}6 / 01 / 96 \\
\text { Completed }\end{array}$ & PB Chadly \\
\hline $\begin{array}{l}\text { 1. Provide PHMC with training lesson plans, review/approval } \\
\text { data, and training effectiveness evaluations. }\end{array}$ & $9 / 02 / 96$ & PB Chadly \\
\hline $\begin{array}{l}\text { 2. Provide PHMC current status on RadCon Technician and } \\
\text { recertification. }\end{array}$ & $9 / 02 / 96$ & PB Chadly \\
\hline 3. Transfer associated radiological training records. & $9 / 02 / 96$ & PB Chadly \\
\hline
\end{tabular}

\subsection{RadCon Sitewide Project/Sub-Contractor Support.}

\begin{tabular}{|c|c|c|}
\hline Pre-Award Actions & $\begin{array}{c}\text { Scheduled } \\
\text { Date }\end{array}$ & Actionee(s) \\
\hline $\begin{array}{l}\text { 1. Prepare to brief PHMC on existing RadCon construction } \\
\text { projects, landlord, vehicle maintenance, laundry facility, } \\
\text { RCRA, shipping/receiving, HPT supplemental crew and } \\
\text { diversified subcontractor support activities } \\
\text { - ICF Kaiser } \\
\text { - INS } \\
\text { - PNNL } \\
\text { - IRM } \\
\text { - BCSR, as appropriate. }\end{array}$ & $\begin{array}{c}\text { 6/01/96 } \\
\text { Completed }\end{array}$ & RL Watts \\
\hline Post-Award Actions & & \\
\hline 1. Transfer existing contracts to PHMC. & 9/02/96 & RL Watts \\
\hline
\end{tabular}




\subsubsection{Quality Assurance (QA).}

\subsection{General Organization.}

\begin{tabular}{|l|l|l|}
\hline Post-Award Actions & $\begin{array}{c}\text { Scheduled } \\
\text { Date }\end{array}$ & Actionee(s) \\
\hline 1. Provide organization charts and charters. & $8 / 05 / 96$ & DG Farwick \\
\hline 2. Provide organization points of contact list and staff directory. & $8105 / 96$ & DG Farwick \\
\hline
\end{tabular}

\subsection{QA Program.}

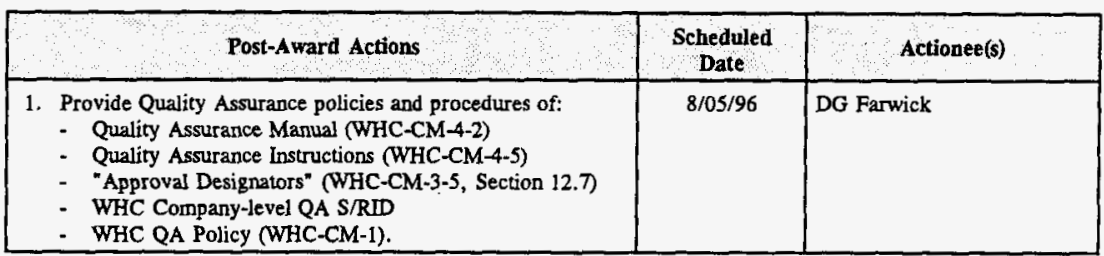

\subsection{10 Code of Federal Regulations (CFR) 830.120 Regulatory}

\section{Compliance Status.}

\begin{tabular}{|l|l|l|}
\hline \multicolumn{1}{|c|}{ Post-Award Actions } & Scheduled \\
Date & Actionee(s) \\
\hline $\begin{array}{l}\text { 1. Provide Regulatory Compliance status of Noncompliance } \\
\text { Notification Reports reported to-date/history. }\end{array}$ & $9 / 02 / 96$ & RN Sherman \\
\hline $\begin{array}{l}\text { 2. Summary of pending investigations/issues. } \\
\text { - Summary Report with background; specific rule } \\
\text { noncompliance; and status of investigation, including root } \\
\text { cause and corrective action(s). } \\
\text { NoTE: Assume one investigation/issue is in progress and } \\
\text { adjust time needed to complete summary report based on } \\
\text { the number of open events. }\end{array}$ & $9 / 02 / 96$ & RN Sherman \\
\hline $\begin{array}{l}\text { 3. Surnmary of outstanding corrective action commitments } \\
\text { Summary report of oper commitments and corrective } \\
\text { actions with identififition of source of commitment. }\end{array}$ & $9 / 02 / 96$ & RN Sherman \\
\hline
\end{tabular}

\subsection{WHC Management Assessment Program.}

\begin{tabular}{|l|l|l|}
\hline Post-Award Actions & Scheduled & Actionee(s) \\
\hline 1. Provide annual 1995 Management Assessment Report. & $9 / 02 / 96$ & DG Farwick \\
\hline $\begin{array}{l}\text { 2. Provide copy of APIP and summarize current implementation } \\
\text { status. }\end{array}$ & $9 / 02 / 96$ & DG Farwick \\
\hline
\end{tabular}




\subsection{Suspect/Counterfeit Materials Program.}

\begin{tabular}{|l|c|c|}
\hline \multicolumn{1}{|c|}{ Pre-Awrd Actions } & $\begin{array}{c}\text { Scheduled } \\
\text { Date }\end{array}$ & \multicolumn{1}{c|}{ Actionee(s) } \\
\hline 1. Update investigations in process. & $\begin{array}{c}6 / 01 / 96 \\
\text { Compieted }\end{array}$ & JN Nansen \\
\hline \multicolumn{1}{|c|}{ Post-Award Actions } & & \\
\hline 1. Brief PHMC on program history and current status. & $9 / 02 / 96$ & JN Nansen \\
\hline 2. Brief PHMC on training program. & $9 / 02 / 96$ & RD Moerman \\
\hline 3. Provide copy of calendar year (CY) 1995 annual report. & $9 / 02 / 96$ & CR Hoover \\
\hline
\end{tabular}

\subsection{Quality Assurance Oversight/Assessment Activities.}

\begin{tabular}{|c|c|c|}
\hline \multicolumn{1}{|c|}{ Post-Award Actions } & Scheduled & Date \\
\hline $\begin{array}{l}\text { 1. Provide copy and status of CY 1996 oversight/assessment } \\
\text { schedule. }\end{array}$ & $9 / 02 / 96$ & AY Cooper \\
\hline 2. Provide FY 1996 Annual QA Management Assessment Plan. & $9 / 02 / 96$ & DG Farwick \\
\hline
\end{tabular}

\subsection{Natural Phenomena Hazards Mitigation.}

\begin{tabular}{|c|c|c|}
\hline Pre-Award Actions & $\begin{array}{l}\text { Scheduled } \\
\text { Date }\end{array}$ & Actionee(s) \\
\hline $\begin{array}{l}\text { 1. Provide a status report of the ongoing program to implement } \\
\text { DOE Order } 5480.28 \text {, NPH Mitigation. }\end{array}$ & $\begin{array}{l}6 / 01 / 96 \\
\text { Cormpleted }\end{array}$ & TJ Conrads \\
\hline $\begin{array}{l}\text { 2. Provide copies of SP-1174, "WHC Structural Analysis and } \\
\text { Design Criteria." }\end{array}$ & $\begin{array}{c}6 / 01 / 96 \\
\text { Completed }\end{array}$ & TJ Conrads \\
\hline Post-Award Actions & & \\
\hline 1. Provide copy of implementation documents issued to-date. & $9 / 02 / 96$ & TJ Conrads \\
\hline 2. Provide summary status report of order implementation. & $9 / 02 / 96$ & TJ Conrads \\
\hline
\end{tabular}

\subsection{Codes and Standards Compliance Activities.}

\begin{tabular}{|c|c|c|}
\hline \multicolumn{1}{|c|}{ Pre-Award Actions } & $\begin{array}{l}\text { Scheduled } \\
\text { Date }\end{array}$ & \multicolumn{1}{|c|}{ Actionee(s) } \\
\hline $\begin{array}{l}\text { 1. Update list of WHC S/RIDs and outstanding commitments: } \\
\text { copies of company S/RIDs should be provided by the } \\
\text { responsible interpretative authorities. }\end{array}$ & $\begin{array}{c}6 / 01 / 96 \\
\text { Completed }\end{array}$ & $\begin{array}{l}\text { MD Jackson } \\
\text { PS Shoop } \\
\text { JR Bell }\end{array}$ \\
\hline $\begin{array}{l}\text { 1. Provide historical background and summary of DNFSB } \\
\text { recommendations related to S/RD program. }\end{array}$ & $9 / 02 / 96$ & MD Jackson \\
\hline 2. Provide copy of WHC S/RID program procedures & $9 / 02 / 96$ & MD Jackson \\
\hline
\end{tabular}




\subsection{Nuclear Safety Regulatory Compliance.}

\begin{tabular}{|l|l|l|}
\hline \multicolumn{1}{|c|}{ Post-Award Actions } & Scheduled & Actionee(s) \\
\hline 1. Copy of Nuclear Safety Rule Compliance (WHC-IP-1112). & $9 / 02 / 96$ & TA Demitruk \\
\hline 2. Copy of milestone transmittal letter and basis for facility list. & $9 / 02 / 96$ & TA Demitruk \\
\hline $\begin{array}{l}\text { 3. Provide status of RL Docket file: } \\
\text { - Listing of document types submitted }\end{array}$ & $9 / 02 / 96$ & TA Demitruk \\
- Summary overview report \\
- Computer operator desk instructions/users manual
\end{tabular}

\subsection{Price Anderson Amendment Act.}

\begin{tabular}{|c|c|c|}
\hline Post-Award Actions & $\begin{array}{c}\text { Scheduled } \\
\text { Date }\end{array}$ & Actionee(s) \\
\hline $\begin{array}{l}\text { 1. Brief PHMC on status of Nuclear Safety Rules and } \\
\text { Implementation Plans for Hanford nuclear facilities } \\
\text { - Presentation/briefing to include details of reports and, } \\
\text { status, and areas needing specific attention (i.e., regulator } \\
\text { negotiated positions, legally enforceable due dates, etc.). }\end{array}$ & $9 / 02 / 96$ & DM Lucoff \\
\hline $\begin{array}{l}\text { 2. Brief PHMC on statos of pending Nuclear Safety Rules and } \\
\text { future implementation requirements. } \\
\text { - Presentation/briefing topics: ditails of reports and files, } \\
\text { status, areas needing specific attention (1.e., regulator } \\
\text { negotiated positions, legally enforceable due dates, etc.). }\end{array}$ & $9 / 02 / 96$ & DM Lucoff \\
\hline $\begin{array}{l}\text { 3. Brief PHMC on training requirements for personnel involved } \\
\text { with PAAA rule implernentation and non-compliance } \\
\text { reporting. Provide training as necessary. } \\
\text { - Presentation/briefing topics: reports and files, status, areas } \\
\text { needing specific attention (i.e., regulator negotiated } \\
\text { positions, legally enforceable due dates, etc.). }\end{array}$ & $9 / 02 / 96$ & $\begin{array}{l}\text { DM Busche } \\
\text { RN Sherman }\end{array}$ \\
\hline
\end{tabular}

\subsection{Regulatory Implementation.}

\begin{tabular}{|c|c|c|}
\hline Pre-Award Actions & $\begin{array}{l}\text { Scheduled } \\
\text { Date }\end{array}$ & Actionee(s) \\
\hline $\begin{array}{l}\text { 1. Summarize pending self-reporting activities. } \\
\text { (Assume one investigation/issue is in progress and adjust time } \\
\text { needed to complete summary report based on the number of } \\
\text { open events.) }\end{array}$ & $\begin{array}{l}6 / 01 / 96 \\
\text { Completed }\end{array}$ & RN Sherman \\
\hline $\begin{array}{l}\text { 2. Summarize pending/open corrective actions associated with } \\
\text { NNRs. (Assume one investigation/issue is in progress and } \\
\text { adjust time needed to complete summary report based on the } \\
\text { number of open events.) }\end{array}$ & $\begin{array}{l}\text { 6/01/96 } \\
\text { Completed }\end{array}$ & RN Sherman \\
\hline Post-Award Actions & & \\
\hline $\begin{array}{l}\text { 1. Provide current status of minor noncompliances identified in } \\
\text { the WHC internal reporting database. }\end{array}$ & $9 / 02 / 96$ & RN Sherman \\
\hline
\end{tabular}




\begin{tabular}{|l|l|l|}
\hline Post-Award Actions & Scheduled & Actionee(s) \\
\hline \multicolumn{1}{c|}{$\begin{array}{l}\text { Provide copies of summary reports provided to DOE on a } \\
\text { quarterly basis describing minor noncompliances added to the } \\
\text { local database Reference existing WBS } \\
\text { Designator } 6.7 .2 .5 .2 .3 .06 .\end{array}$} & $9 / 02 / 96$ & RN Sherman \\
\hline
\end{tabular}

\subsection{DNFSB Coordination/Site General Status.}

\begin{tabular}{|l|c|c|}
\hline \multicolumn{1}{|c|}{ Pre-Award Actions } & $\begin{array}{c}\text { Scheduled } \\
\text { Date }\end{array}$ & Actionee(s) \\
\hline $\begin{array}{l}\text { 1. Prepare package for briefing PHMC on status of DNFSB } \\
\text { activities affecting WHC/Hanford. }\end{array}$ & $\begin{array}{c}6 / 01 / 96 \\
\text { Completed }\end{array}$ & JW Hales \\
\hline \multicolumn{1}{|c|}{ Post-Award Actions } & & \\
\hline $\begin{array}{l}\text { 1. Provide copies of all active DNFSB recommendations. } \\
\text { DNFSB comnitments for past six months. }\end{array}$ & $9 / 02 / 96$ & $\begin{array}{l}\text { JW Hales } \\
\text { SA Baver }\end{array}$ \\
\hline $\begin{array}{l}\text { 2. Provide copies of monthly status reports on WHC/Hanford } \\
\text { 3rief PHMC on status of DNFSB activities and near-term } \\
\text { commitments/deliverables for WHC/Hanford facilities. }\end{array}$ & $9 / 02 / 96$ & JW Hales \\
\hline
\end{tabular}

3.5:1.5 Compliance Assurance. All records of the Compliance Assurance organization will be dispersed to records management and to the Facility Evaluation Board prior to contract transition.

3.5.1.6 Facility Evaluation Boards. Paragraph 2.11, Performance Assessment, in Section J, Appendix F, of the RFP states: "Describe the process that the contractor shall use to establish an organization reporting directly to the President of the company and independent of other line management to conduct performance-based assessments of facilities and major projects. The assessments will cover, as a minimum, operations, maintenance, environmental compliance, industrial safety, radiological controls, and training for all Contractor major contractors and subcontractors engaged in Hanford Projects, Programs, and Facility Operations. Other areas will be covered as deemed necessary by the contractor to assure safe, effective, and efficient operation or project management using the criteria of the DOE approved Authorization basis, SAR, Hanford procedures, S/RID's, etc., (or if not yet approved, the implementing DOE Orders.) This organization shall work directly with the Performance Assessment Division of DOE-RL ES\&H organization for coordination and oversight."

The WHC Facility Evaluation Organization, a product of the reengineering effort, is being developed to fully satisfy these requirements with a proven methodology based on almost two years of successful experiences at Savannah River. Facility Evaluation is managed by a Director, reporting to the WHC President and Executive Vice President. Evaluations are conducted by multi-disciplinary teams called Facility Evaluation Boards selected for their experience and technical expertise. Information to be provided to the PHMC will include: 
- Supporting information on FEB activities including:

- FEB background information -- reengineering team

- FEB organization

- FEB charters and authorizing procedures on internal assessments

- FEB internal procedures and assessment performance objectives and criteria

- Qualifications of the FEB team members, if requested

- Results of 1996 evaluations including the Integrated Audits and Appraisals and FEB assessments

- FEB schedule (draft) for FY 1997

- Preliminary FY 1997 plans and budgets for independent assessment of facilities and projects

\begin{tabular}{|l|c|l|}
\hline \multicolumn{1}{|c|}{ Pre-Award Actions } & $\begin{array}{c}\text { Scheduled } \\
\text { Date }\end{array}$ & Actionee(s) \\
\hline $\begin{array}{l}\text { 1. Prepare briefing and supporting information on the facility } \\
\text { evaluation boards. Include concepts, organizations, internal } \\
\text { procedures, and assessment performance objectives. }\end{array}$ & $\begin{array}{c}6 / 01 / 96 \\
\text { Completed }\end{array}$ & JR Knight \\
\hline $\begin{array}{l}\text { 2. Verify, and upds:e as necessary, "Smart Book, " to include } \\
\text { information developed in item \#1 above. Subnit copy of } \\
\text { "Smart Book" to transition team. }\end{array}$ & $7 / 15 / 96$ & JR Kright \\
\hline Post-Award Actions & $8 / 05 / 96$ & JR Knight \\
\hline 1. Provide PHMC FEB information (see text above). & $8 / 12 / 96-$ & JR Knight \\
\hline 2. Brief the PHMC on the Facility Evaluation Board. & $8 / 30 / 96$ & \\
\hline
\end{tabular}

\subsubsection{Work Control}

Operations and Maintenance Programs (OMP) coordinates, at the company level, programs that support work control within the company. OMP oversees the COO and maintenance, and provide "services" such as performance monitoring, procedure development, statistical analysis and MIP development. We also oversee the Job Control System which is used by all facilities for documenting work control. Numerous management "self-assessments" are coordinated by OMP and provide current status of our facilities in these areas. All of these functions will transition in some form to the PHMC contractor. OMP will coordinate this transition.

\begin{tabular}{|c|c|c|}
\hline Pre-Award Actions & $\begin{array}{c}\text { Scheduled } \\
\text { Date }\end{array}$ & Actionee(s) \\
\hline 1. Identify WHC Directives which govern ConOps. & $\begin{array}{c}4 / 15 / 96 \\
\text { Completed }\end{array}$ & CP Arnes \\
\hline
\end{tabular}




\begin{tabular}{|c|c|c|}
\hline Pre-Award Actions & $\begin{array}{l}\text { Scheduled } \\
\text { Date }\end{array}$ & Actionee(s) \\
\hline $\begin{array}{l}\text { 2. Outline Assessment Status for All Facilities and prepare } \\
\text { briefing. }\end{array}$ & $\begin{array}{l}5 / 01 / 96 \\
\text { Completed }\end{array}$ & CP Ames \\
\hline 3. Prepare Brief on CMMS Issues. & $\begin{array}{l}5 / 01 / 96 \\
\text { Completed }\end{array}$ & ER Hamm \\
\hline 4. Prepare Brief on Budget Issues. & $\begin{array}{c}5 / 01 / 96 \\
\text { Completed } \\
\end{array}$ & JC Bickford \\
\hline 5. Prepare Brief on Configuration Management Initiative. & $\begin{array}{l}5 / 01 / 96 \\
\text { Completed }\end{array}$ & ER Hamm \\
\hline 6. Prepare Brief on Lesson Learned Program. & $\begin{array}{c}5 / 01 / 96 \\
\text { Completed }\end{array}$ & JC Bickford \\
\hline 7. Prepare Brief on current Performance Indicators. & $\begin{array}{c}5 / 01 / 96 \\
\text { Completed }\end{array}$ & SS Prevette \\
\hline \multicolumn{3}{|l|}{ Post-Award Actions } \\
\hline 1. Provide copies of WHC directives in use. & $8 / 10 / 96$ & RD Raaz \\
\hline 2. Present briefings as requested by PHMC. & $\begin{array}{l}8 / 12 / 96- \\
9 / 02 / 96\end{array}$ & RD Raaz \\
\hline
\end{tabular}

\subsubsection{Environmental Compliance}

The Environmental Services organization is involved (directly or indirectly) with the preparation of documents, reporting, and negotiations concerning environmental compliance within the M\&O Contractor. Programs and facilities have identified actions involving program and facility specific environmental information, including assemblage of key existing documentation, preparation of briefings, documenting a listing of key knowledgeable individuals as POCs, and identifying specific permits. The following actions are established to address sitewide environmental compliance and permitting actions conducted by Environmental Services.

A key transition issue for Environmental Services involves transfer of Resource Conservation and Recovery Act documentation responsibility from WHC to the PHMC. Key assumptions for this transfer are:

- Environmental permit transfer will involve transfer of the following Resource Conservation and Recovery Act (RCRA) documentation: (1) one RCRA Part A, consisting of one Form 1 and 33 Form 3 documents (one for each treatment, storage, and/or disposal [TSD] unit), each of which is individually certified; (2) one RCRA Part B permit application, including documentation for one TSD unit included in the Hanford Facility RCRA Permit, and application documentation for 10 TSD units not yet included in the final status Hanford Facility RCRA Permit; (3) the Hanford Facility RCRA Permit; and (4) one RCRA Research, Development, and Demonstration (RD\&D) Permit covering a single TSD unit. 
- It is assumed that Ecology will cooperate with RL and the PHMC in meeting the intent of the 90-day advance notification requirement for transfer of the RCRA permit documentation. It is assumed that, prior to takeover, the PHMC must recertify the Part A Form 1 and Form 3 documents, Part B permit application documentation for the one TSD unit included in the Hanford Facility RCRA Permit, and RD\&D permit application documentation for the one TSD unit included in the RD\&D Permit.

- It is assumed that an agreement will be reached among Ecology, DOE-RL, WHC, and the PHMC that the Part B permit application documentation for the 10 TSD units currently in progress, but not yet included in the Hanford Facility RCRA Permit, will not require recertification by the PHMC prior to takeover. Recertification by DOE-RL and the PHMC will be done when each TSD unit specific Part B application documentation is finalized and submitted in accordance with the comment-resolution workshop process and the schedule included in the Hanford Facility RCRA Permit. This approach is consistent with the stepwise permitting process outlined in the Tri-Party Agreement. As this schedule extends over a 5-year period, agreement must be reached between DOE-RL and the PHMC regarding assumption of liability upon termination of the WHC contract.

\begin{tabular}{|c|c|c|}
\hline Pre-Award Actions & $\begin{array}{l}\text { Scheduled } \\
\text { Date } \\
\end{array}$ & Actionee(s) \\
\hline $\begin{array}{l}\text { 1. Develop a list of key sitewide environmental compliance } \\
\text { and permitting documentation. (Provide to RL POC for } \\
\text { review). Prepare and distribute guidance to actionees on } \\
\text { material to be included in "Smart Books" and briefings. }\end{array}$ & $\begin{array}{l}5 / 15 / 96 \\
\text { Completed }\end{array}$ & RH Engelmann \\
\hline $\begin{array}{l}\text { 2. Assemble key documentation, or identify its location, for } \\
\text { use by the PHMC. Includes development of a "Smart } \\
\text { Book" to include information developed below. }\end{array}$ & $\begin{array}{c}\text { 6/01/96 } \\
\text { Completed } \\
\text { "Smart Book" } \\
\text { Only } \\
\text { See item \#8 }\end{array}$ & $\begin{array}{l}\text { RH Engelmann } \\
\text { SM Price } \\
\text { JJ Luke } \\
\text { LP Diediker } \\
\text { GC Cummins } \\
\text { RC Brune } \\
\text { JO Skolrud } \\
\text { EM Greager } \\
\text { DJ Carrell } \\
\end{array}$ \\
\hline $\begin{array}{l}\text { 3. Identify briefings and prepare briefing material to address } \\
\text { sitewide environmental activities for presentation to the } \\
\text { PHMC. Topics to be covered should include sitewide } \\
\text { environmental reports, sitewide RCRA permitting, central } \\
\text { regulatory analyses and requirements activities, etc. } \\
\text { (coordinate number and content of briefings with RL } \\
\text { POC). }\end{array}$ & REPLACED & . \\
\hline 4. Develop a list of key M\&O Contractor POCs. & $\begin{array}{l}\text { 6/01/96 } \\
\text { Completed }\end{array}$ & RH Engelmann \\
\hline $\begin{array}{l}\text { 5. Develop a list and description of Environmental Services } \\
\text { subcontracts. Include any Task Orders still planned to be } \\
\text { executed prior to contract takeover. }\end{array}$ & $\begin{array}{l}\text { 6/01/96 } \\
\text { Completed }\end{array}$ & RG Upchurch \\
\hline $\begin{array}{l}\text { 6. Verify the accuracy of property assignments within the } \\
\text { Environmental Services organization. }\end{array}$ & $\begin{array}{l}6 / 01 / 96 \\
\text { Completed }\end{array}$ & SM Cooley \\
\hline
\end{tabular}




\begin{tabular}{|c|c|c|c|}
\hline & Pre-Award Actions & $\begin{array}{l}\text { Scheduled } \\
\text { Date }\end{array}$ & Actionee(s) \\
\hline & $\begin{array}{l}\text { Identify space and equipment within the Environmental } \\
\text { Services area for use by the PHMC, if needed. }\end{array}$ & $\begin{array}{l}\text { 6/01/96 } \\
\text { Completed }\end{array}$ & SM Cooley \\
\hline 8. & $\begin{array}{l}\text { Finalize/submit (to EAP POC, WHC Contract Transition } \\
\text { Project) "Smart Book." Incorporate items } 9 \& 10 \text { into } \\
\text { update. }\end{array}$ & $7 / 15 / 96$ & $\begin{array}{l}\text { WT Dixon } \\
\text { RH Engelmann } \\
\text { JR Robertson } \\
\text { RC Brunke } \\
\text { GC Curnmins } \\
\text { SM Price } \\
\text { WE Toebe } \\
\text { JO Skolrud } \\
\text { RC Bownan } \\
\text { EM Greager } \\
\text { LP Diediker } \\
\text { JJ Luke } \\
\end{array}$ \\
\hline 9. & $\begin{array}{l}\text { Assemble key documentation, or identify (in "Smart } \\
\text { Book") its location, for use by the PHMC. }\end{array}$ & $7 / 15 / 96$ & $\begin{array}{l}\text { WT Dixon } \\
\text { RH Engelmann } \\
\text { JR Robertson } \\
\text { RC Brunke } \\
\text { GC Cummins } \\
\text { SM Price } \\
\text { WE Toebe } \\
\text { JO Skolrud } \\
\text { RC Bowmnan } \\
\text { EM Greager } \\
\text { LP Diediker } \\
\text { JJ Luke }\end{array}$ \\
\hline 10. & $\begin{array}{l}\text { Complete briefing material to address sitewide } \\
\text { environmental activities for presentation to the PHMC. } \\
\text { (Coordinate content with EAP POC). Incorporate into } \\
\text { updated "Smart Book." Environmental Services activities } \\
\text { to be addressed (include status of activities). } \\
\text { - HEMP program management } \\
\text { - Regulatory reporting } \\
\text { - RCRA permitting } \\
\text { - Air \& water permitting, including air operating permit, } \\
\text { FF-01 permit and NPDES permits } \\
\text { - POC services for regulatory inspection } \\
\text { - Regulatory inspection files, NOV database } \\
\text { - RCRA permit general inspections } \\
\text { - NEPA support } \\
\text { - Effluent reporting, effluent monitoring and facility } \\
\text { effluent monitoring plans } \\
\text { - Environmental requirements management } \\
\text { - NESHAPS FFCA } \\
\text { - Outsourced work } \\
\text { - Work for others }\end{array}$ & $7 / 15 / 96$ & $\begin{array}{l}\text { WT Dixon } \\
\text { RH Engelmann } \\
\text { JR Robertson } \\
\text { RC Brunke } \\
\text { GC Cummins } \\
\text { SM Price } \\
\text { WE Toebe } \\
\text { JO Skolrud } \\
\text { RC Bowman } \\
\text { EM Greager } \\
\text { LP Diediker } \\
\text { II Luke }\end{array}$ \\
\hline 11. & Space and office equipment available to PHMC. & $7 / 22 / 96$ & $\begin{array}{l}\text { RH Engelmann } \\
\text { SM Cooley }\end{array}$ \\
\hline 12. & $\begin{array}{l}\text { Verify pre-existing condition checklist and update if } \\
\text { needed. Submit changes to HATS, Contract Transition } \\
\text { Project office, etc. }\end{array}$ & $7 / 22 / 96$ & $\begin{array}{l}\text { WT Dixon } \\
\text { LP Diediker }\end{array}$ \\
\hline
\end{tabular}




\begin{tabular}{|c|c|c|}
\hline Post-Award Actions & $\begin{array}{c}\text { Scheduled } \\
\text { Date }\end{array}$ & Actionee(s) \\
\hline $\begin{array}{l}\text { 1. Provide the PHMC access to the key documentation and } \\
\text { the "Smart Book." }\end{array}$ & $8 / 01 / 96$ & $\begin{array}{l}\text { WT Dixon } \\
\text { RH Engelmann } \\
\text { MT Jansky } \\
\end{array}$ \\
\hline $\begin{array}{l}\text { 2. Provide updated status of ongoing Environmental Services } \\
\text { activities to PHMC. }\end{array}$ & $9 / 24 / 96$ & $\begin{array}{l}\text { WT Dixon } \\
\text { Leadership Team }\end{array}$ \\
\hline $\begin{array}{l}\text { 3. Provide environmental briefing(s) to PHMC (see pre- } \\
\text { award item } \# 9 \text { above). }\end{array}$ & by $9 / 30 / 96$ & $\begin{array}{l}\text { WT Dixon } \\
\text { Leaderstip Team }\end{array}$ \\
\hline 4. Perform necessary property inventory with PHMC. & $9 / 30 / 96$ & SM Cooley \\
\hline $\begin{array}{l}\text { 5. Turn over Environmental Services working files and } \\
\text { activities responsibilities to PHMC. }\end{array}$ & $10 / 01 / 96$ & $\begin{array}{l}\text { WT Dixon } \\
\text { Leadership Team }\end{array}$ \\
\hline
\end{tabular}

3.5.3.1 through 3.5.3.6 as follows, provide further information and specific actions pertaining to the activities listed above in Pre-Award Action 9 (Prepare briefing material...).

3.5.3.1 Hanford Environmental Management Program. The Hanford Environmental Management Program (HEMP) was established in 1986 to create and implement a structured approach toward achieving sitewide environmental compliance. The purpose of the HEMP is to provide guidance and support across WHC mission areas to ensure facilities/programs achieve compliance with environmental requirements and regulatory agreements. This program also provides RL with a mechanism to coordinate specific environmental activities between multiple contractors. For sitewide reports and permits, WHC coordinates the activities of all Hanford Site contractors as directed by RL. WHC also provides a focal point for regulator access and technical data requests, and sitewide compliance resolution.

\begin{tabular}{|l|c|c|}
\hline Pre-Award Actions & $\begin{array}{c}\text { Scheduled } \\
\text { Date }\end{array}$ & Actionee(s) \\
\hline $\begin{array}{l}\text { 1. Compile key program documents (e.g., FY 1996 MYPP, } \\
\text { ADS, RDS), and prepare briefing. }\end{array}$ & REPLACED & \\
\hline
\end{tabular}

3.5.3.1.1 Regulatory Reporting. WHC coordinates the preparation of 11 sitewide regulatory reports for $\mathrm{RL}$ that will require continued input from the contractor team. These reports are the Emergency Planning and Community Right-To-Know Act (EPCRA) Section 311 Material Safety Data Sheet List Upgrades (four quarterly reports), EPCRA Section 312 Tier Two Emergency and Hazardous Chemical Inventory, EPCRA 313 Toxic Chemical Release Inventory, Annual Polychlorinated Biphenyl (PCB) Document Log, Annual PCB Report, Annual Dangerous Waste Report, Land Disposal Restrictions Status Report (Tri-Party Agreement Milestone M-26-01), and RCRA 3016 Inventory of Federal Agency Hazardous Waste Facilities (on even numbered calendar years).

\begin{tabular}{|c|c|c|}
\hline Post-Award Actions & $\begin{array}{c}\text { Scheduled } \\
\text { Date }\end{array}$ & \multicolumn{1}{c|}{ Actionee(s) } \\
\hline $\begin{array}{l}\text { 1. Establish PHMC subcontractor POCs for each of the } \\
\text { specified sitewide regulatory reports by project or facility. }\end{array}$ & $8 / 01 / 96-9 / 15 / 96$ & $\begin{array}{l}\text { JO Skolrud } \\
\text { PHMC }\end{array}$ \\
\hline
\end{tabular}




\begin{tabular}{|l|l|l|}
\hline \multicolumn{1}{|c|}{ Post-Award Actions } & \multicolumn{1}{|c|}{$\begin{array}{c}\text { Scheduled } \\
\text { Date }\end{array}$} & Actionee(s) \\
\hline $\begin{array}{l}\text { 2. Identify PHMC subcontractor certification authority for } \\
\text { specified sitewide regulatory reports, as required by project } \\
\text { or facitity. }\end{array}$ & $8 / 01 / 96-9 / 15 / 96$ & $\begin{array}{l}\text { JO Skolrud } \\
\text { PHMC }\end{array}$ \\
\hline $\begin{array}{l}\text { 3. Establish and maintain a network of subcontractor field } \\
\text { reporting representatives from each facility, or project, as } \\
\text { needed to assure timely completion of specified reports. }\end{array}$ & $8 / 01 / 96-9 / 15 / 96$ & $\begin{array}{l}\text { JO Skolrud } \\
\text { PHMC }\end{array}$ \\
\hline $\begin{array}{l}\text { 4. Provide specialized training and database system access } \\
\text { authorization for new PHMC subcontractor database } \\
\text { administrators and reporting representatives to allow access to } \\
\text { needed data files and data transmittal capabilities. }\end{array}$ & $8 / 01 / 96-9 / 15 / 96$ & $\begin{array}{l}\text { JO Skolrud } \\
\text { PHMC }\end{array}$ \\
\hline
\end{tabular}

3.5.3.1.2 Sitewide RCRA Permitting. The Hanford Facility is a single RCRA facility consisting of 66 treatment, storage, and/or disposal (TSD) units. Nine of these units have been officially clean closed in accordance with the Washington Administrative Code (WAC). WHC has management responsibility for 33 of the remaining 57 units, with other Hanford Site contractors having management responsibilities for the remaining units. The units managed by WHC must be transitioned to the PHMC. The RCRA permitting documentation involved in this transition includes the: (1) Hanford Facility RCRA Permit, (2) Hanford Facility Part A Permit Application (which includes one Form 1 and a Form 3 for each TSD unit), (3) Hanford Facility Dangerous Waste Part B Permit Application, and (4) Closure Plans.

Transfer of WHC responsibility as co-operator for RCRA permitting documentation requires 90 days advance notification to Ecology, and submittal of the associated, recertified permitting documentation, unless this requirement is waived by Ecology. Assuming an October 1, 1996, takeover date, processing of all RCRA permitting documentation by July 1 , 1996, is not possible based on a August 1, 1996, contract award date. If circumstances preclude the PHMC from assuming co-operator responsibilities for RCRA permitting documentation by October 1, 1996, WHC will exercise Section E of the Agreement on Regulatory Interactions between RL and WHC dated August 14, 1995. This memorandum of agreement states that "...in the event of a change in contract scope or termination or expiration of the Contract, $\mathrm{RL}$ will require the successor contractor to accept transfer of all permits for which WHC is a permittee or signatory, or in the alternative, RL will accept such responsibility, subject to the provisions of Contract, and WHC shall be relieved of liability and responsibility for permit violations where the operative facts occur after the effective date of the change in scope, termination, or expiration of this contract." The WHC will pursue a documented agreement with Ecology that Ecology will: (1) waive the 90-day requirement for submittal of recertified permitting documentation, and (2) agree that WHC will be removed from the RCRA permitting documentation as co-operator as of October 1 , 1996, leaving RL as the owner/operator if the PHMC is not yet ready to assume co-operator responsibilities. 


\begin{tabular}{|l|c|l|}
\hline \multicolumn{1}{|c|}{ Pre-Award Actions } & Scheduled & \multicolumn{1}{|c|}{ Actionee(s) } \\
\hline $\begin{array}{l}\text { 1. Complete negotiation among RL, WHC, and Ecology } \\
\text { regarding the 90-day RCRA permitting documentation } \\
\text { submittal requirements and clarifying assumptions of } \\
\text { co-operator responsibilities. }\end{array}$ & $6 / 28 / 96$ & $\begin{array}{l}\text { SM Price } \\
\text { C Clark }\end{array}$ \\
\hline
\end{tabular}

Further details on the plan for accomplishing transition of RCRA permitting documentation is provided below:

\subsection{Hanford Facility RCRA Permit. The Hanford Facility RCRA Permit} (RCRA Permit) is currently in two portions. The Dangerous Waste Portion (DW Portion), was issued by Ecology to RL (Owner/Operator), WHC (Co-operator), BHI (Co-operator), and PNNL (Co-operator), effective September 28, 1994, through September 27, 2004. The Hazardous and Solid Waste Amendments Portion, was issued by the U.S. Environmental Protection Agency (EPA) to RL (Owner/Operator) only effective September 28, 1994, through September 27, 2004. Due to the number of Hanford Site TSD units, a stepwise permitting process is being used. Any TSD units not yet included in the RCRA Permit (DW Portion) will be incorporated in a future, annual permit modification. Until incorporated, TSD units will continue to operate under interim status.

Permit Condition I.A.2. (DW Portion) states that WHC is identified as a Permittee for activities subject to the Conditions of this Permit where its agents, employees, or subcontractors have operational and/or management responsibilities and control. The Permittee for the DW Portion will need to be transferred from WHC to the PHMC.

Permit Condition I.E.14. (DW Portion) states that the Permit may be transferred to a new co-operator in accordance with the provisions of WAC. 173-303-830(2).

WAC 173-303-830(2)(b) allows that changes in the ownership or operational control of a facility may be made as a Class 1 modification (requires notification) with prior written approval of Ecology. The new owner or operator must submit a revised permit application no later than 90 days prior to the scheduled change.

Because Permit Condition II.H.3. (DW Portion) states that the permittees are exempt from the requirements of WAC 173-303-620 (Financial Requirements) no action to transfer or demonstrate compliance with financial requirements is required.

\begin{tabular}{|l|c|c|}
\hline \multicolumn{1}{|c|}{ Pre-Award Actions } & Scheduled & Actionee(s) \\
\hline $\begin{array}{l}\text { 1. Submit Class 1 modification notifying Ecology of a change in } \\
\text { the permittee from WHC to the PHMC. (A determination } \\
\text { must be made with RL, and agreed to by Ecology, as to the } \\
\text { permitree[s] [Co-operator (s)] to transfer RCRA Permit } \\
\text { responsibilities to the PHMC by October 1, 1996. If } \\
\text { Ecology does not approve the submitted Class 1 modification, } \\
\text { RL would accept sole responsibility per MOA.) }\end{array}$ & $7 / 10 / 96$ & SM Price \\
\hline
\end{tabular}




\begin{tabular}{|l|l|l|}
\hline Post-Award Actions & Scheduled & \multicolumn{1}{|c|}{ Actionee(s) } \\
\hline 1. Support the PHMC's certification and resubmittal to Ecology & $8 / 01 / 06-$ & SM Price \\
of the following RCRA permitting documentation: (1) one & $10 / 01 / 96$ & PHMC \\
Part A permit application (Part A), Form 1 and 33 Part A, & & \\
Form 3s; (2) Part B permit application docurnentation for & & \\
$\sim$ 10 TSD units; (3) Hanford Facility RCRA Permit; and (4) & & \\
one Research, Development, and Demonstration (RD\&D) & & \\
Permit. Transfer management responsibilities for TSD unit \\
closures to the PHMC.
\end{tabular}

Further information pertaining to the post-award action above is provided below and in 3.5.3.1.4 through 3.5.3.1.6.

An Ecology-initiated Class 3 modification of the RCRA Permit also is scheduled for 1996. Currently the schedule calls for: (1) all information from WHC to be certified and formally delivered to Ecology by end of July 1996, (2) the 45-day Public Comment Period to commence mid-August 1996, (3) a permit decision to be issued in late September 1996, and, (4) the permit to be in effect in early December 1996. Three WHC-managed TSD units and a revised inspection plan for 616 NRDWSF are expected to be added to the RCRA Permit by the modification.

A Research, Development, and Demonstration (RD\&D) Permit for the Waste Water Pilot Plant (WWPP) was issued by the EPA, separate from the Hanford Facility RCRA Permit. This permit was issued to RL (owner/operator) and WHC (co-operator) and also will need to be transferred to the PHMC.

3.5.3.1.4 Hanford Facility Part A Permit Application. There is one Form 1 and 33 Hanford Facility Dangerous Waste Part A Permit Application Form $3 s$ that must be addressed. Washington Administrative Code 173-303-805(7)(a)(iv) requires these Part A forms to be resubmitted (certified) by the PHMC to Ecology no later than 90 days prior to the scheduled change in management control. Assuming the change becomes effective on October 1, 1996, the Part A forms must be resubmitted no later than July 1, 1996, unless the agreement identified in Section 3.5.3.1.2 is obtained.

\begin{tabular}{|l|c|l|}
\hline \multicolumn{1}{|c|}{ Pre-Award Actions } & Scheduled & \multicolumn{1}{|c|}{ Actionee(s) } \\
\hline $\begin{array}{l}\text { 1. Perform a WHC review of the Part A, Form 1 and the } \\
\text { Part A, Form 3 permit applications (managed by WHC) } \\
\text { against their respective TSD unit to determine trueness, } \\
\text { accuracy, and completeness. }\end{array}$ & Completed & SM Price \\
& & $\begin{array}{l}\text { D Allison } \\
\text { MJ Hall } \\
\text { RD Pierce } \\
\text { DL Flyckt } \\
\end{array}$ \\
& & $\begin{array}{l}\text { RW Szelmeczka } \\
\text { GJ LeBaron } \\
\text { TA Dillhoff } \\
\text { DJ McBride }\end{array}$ \\
& & SE Killoy \\
& & DE Rasmussen \\
\hline
\end{tabular}


3.5.3.1.5 Hanford Facility Dangerous Waste Part B Permit Application. Of the 33 units for which WHC retains responsibility, 1 unit has been granted final status, 10 units are slated to receive final status, and the remaining units will be closed as discussed above.

WAC 173-303-830(2)(b) requires that the PHMC submit revised Part B permit applications (certified) for the 10 units seeking final status to Ecology no later than 90 days prior to the scheduled change in management control. In light of the Hanford Facility permitting strategy identified in Section 3.5.3.1.2 and the enormous effort resubmittal of the Part B permit applications represents, an agreement must be achieved between all parties concerned (e.g., WHC, RL, the PHMC, and Ecology). This agreement must address the following:

- Relieve the PHMC of the need to submit revised Part B permit applications no later than 90 days prior to the scheduled date for assuming management responsibility.

- Identify permit application submittal dates supportive of the permit modification schedule contained in the Hanford Facility RCRA Permit.

- Remove WHC from the RCRA Part B permit applications as co-operator as of October 1, 1996.

- Reach agreement among the parties regarding assumptions of liability upon termination of the WHC contractor.

Operational control of the sole final status TSD unit (616 NRDWSF) that WHC has management responsibility for, shall be transferred in accordance with Condition I.E.14. of the Hanford Facility RCRA Permit (see Section 3.5.3.1.3.).

3.5.3.1.6 Closure Plans. Currently, there are 14 interim status TSD units that are not operating and will be closed under RCRA. Eight of these TSD units for which WHC has management responsibility have already been certified clean closed and revised Part A, Form 3 permit applications submitted to Ecology. Closure plans have been or will be developed for the remaining 6 units and submitted to Ecology for approval and for eventual inclusion in the Hanford Facility RCRA Permit. The Part A, Form 3 permit applications for the remaining TSD units undergoing closure assigned to the PHMC will have to be recertified (see Section 3.5.3.1.4).

3.5.3.1.7 Sitewide Air and Water Permitting. There are two categories of Clean Air Act (CAA) and Clean Water Act of 1977 (CWA) related permits onsite; facility specific and sitewide. All CAA and CWA permits are owned by RL. That is, neither WHC nor any other contractor is a co-signer to a permit application and the permits have been issued to RL. Therefore, there is no requirement to sign over any CAA or CWA permit, or permit application, to the PHMC. PHMC will need to verify that CWA and CAA permit conditions are being met. 


\section{CAA Permits}

Facility specific CAA permits are issued by the Washington State Departments of Health (WDOH), Ecology, and/or the EPA to RL under a regulatory order of approval with activity-specific conditions and/or operating limitations. The conditions/limitations are incorporated into the "Hanford Site Air Operating Permit (AOP) Application," (DOE/RL-95-07) initially submitted from RL to Ecology on May 26, 1995. Each facility owner is responsible for meeting the conditions of its permit(s).

The current sitewide CAA license, FF-01, is with the DOH. Once the Hanford Site AOP Application (DOE/RL-95-07) is finalized as a site AOP, expected in November 1997. the FF-01 license will be canceled. Until issuance of the permit, frequent/periodic updates to the application ensures its currentness and allows for the continued operation onsite of all points that emit regulated constituents to atmosphere.

\section{CWA Permits}

All CWA permits are owned by RL. There are two types of CWA related permits: National Pollutant Discharge Elimination System (NPDES) and WAC 173-216. NPDES permits allow for discharges to the Columbia River. WAC 173-216 permits allow for discharges to the soil column.

NPDES Permit WA-000374-3 was issued to RL on December 7, 1981, and is a sitewide permit. The permit was originally issued to cover the discharge from eight outfalls on the Hanford Site. Because of shutdown and deactivation activities there are now only four active outfalls covered by the permit. Those outfalls are 003 and 004 in the $100 \mathrm{~K}$ Area, N-Springs in the $100 \mathrm{~N}$ Area, and 013 in the 300 Area. Outfall 013 services a PNNL facility. BHI monitors N-Springs. The 003 and 004 Outfalls service facilities that will transfer to the PHMC.

NPDES Permit WA-002591-7 was issued to RL on October 31, 1994, for discharges from the 300 Area Treated Effluent Disposal Facility (see the Liquid Effluent Program section).

NPDES Permit WA-R-00-000F was issued to RL on August 27, 1992. It permits the discharge of stormwater runoff from industrial points on the Hanford Site to the Columbia River.

WAC 173-216 permits have been issued for the 200 Area Effluent Treatment Facility (ETF), the 200 Area Treated Effluent Disposal Facility (TEDF), and those discharges identified in Consent Order DE 91NM-177 (216 Consent Order). The construction of septic systems are permitted with the Department of Health. Management of potable water is conducted in accordance with Department of Health regulations.

\subsection{Regulator Inspection Support.}

Regulatory Inspection POC--WHC currently acts as the initial point of contact for all environmental regulatory inspections of the Hanford Site. It is assumed that the PHMC will 
be performing this role as well. Pertinent information associated with these inspections will be maintained by the PHMC in a central inspection file.

\begin{tabular}{|c|c|c|}
\hline Pre-Award Actions & $\begin{array}{l}\text { Scheduled } \\
\text { Date }\end{array}$ & Actionee(s) \\
\hline $\begin{array}{l}\text { 1. Complete the new standardized access requirements system } \\
\text { for regulatory agency inspectors. }\end{array}$ & $\begin{array}{c}6 / 01 / 96 \\
\text { Completed }\end{array}$ & SA Szendre \\
\hline $\begin{array}{l}\text { 2. Complete protocol with PNNL for Iegulatory agency } \\
\text { inspection point of contact responsibilities. }\end{array}$ & $\begin{array}{l}6 / 15 / 96 \\
\text { Completed }\end{array}$ & SA Szendre \\
\hline $\begin{array}{l}\text { 3. Complete protocol with the ERC Team for regulatory agency } \\
\text { inspection point of contact responsibilities. }\end{array}$ & $\begin{array}{l}6 / 15 / 96 \\
\text { Completed }\end{array}$ & SA Szendre \\
\hline
\end{tabular}

RCRA Facility Permit Inspection--Condition II.O of the Hanford Site RCRA Permit includes the requirement for annual inspections of the 100, 200 East, 200 West, 300, 400, and 1100 Areas, along with inspections of the banks of the Columbia River twice per year.

Notices of Violation (NOV) Database and Reporting--A database of NOV and enforcement actions is maintained to meet RL reporting requirements and certain permitting requirements associated with permit expansions under interim status. This database includes sitewide information and will be the responsibility of the PHMC.

\begin{tabular}{|l|l|l|}
\hline Post-Award Actions & Scheduled & Actionee(s) \\
\hline $\begin{array}{l}\text { 1. Complete as many NOV corrective actions as possible and } \\
\text { close as many of the open items on the NOV database as } \\
\text { possible. }\end{array}$ & $9 / 30 / 96$ & $\begin{array}{l}\text { SA Szendre } \\
\text { affected ECOs }\end{array}$ \\
\hline
\end{tabular}

3.5.3.1.9 National Environmental Policy Act of 1969 (NEPA) Compliance. WHC screens proposed activities for NEPA compliance requirements, and prepares documents (environmental assessments [EA], categorical exclusions, Environmental Impact Statement [EIS] technical support documents) used by RL in the NEPA process.

Copies of all Hanford EISs and EAs and related records are maintained centrally. Copies of categorical exclusions for M\&O Contractor managed activities are also held centrally.

HEMP also funds Hanford Site NEPA training support, and input to sitewide planning efforts and procedures.

3.5.3.1.10 Crosscutting Noncompliances. In July 1995, RL and its contractors identified all known potential non-permitted treatment, storage, and disposal activities at Hanford, and all potential environmental noncompliant conditions. There were identified 160 individual potential noncompliant conditions on the Hanford Site. Of these, 66 are facility-specific and the remaining 94 involve, or crosscut, more than one RL Program or facility. 
WHC-SP-1181 Rev. 1

\begin{tabular}{|l|c|c|}
\hline \multicolumn{1}{|c|}{ Post-Award Actions } & Scheduled & \multicolumn{1}{|c|}{ Actionee(s) } \\
\hline $\begin{array}{l}\text { 1. Efforts will be made to formally resolve all known } \\
\text { noncompliances prior to contract takeover. }\end{array}$ & $9 / 30 / 96$ & $\begin{array}{l}\text { RH Engelmann } \\
\text { LM Ditmer }\end{array}$ \\
\hline
\end{tabular}

\subsubsection{Reporting.}

3.5.3.2.1 Effluent Reporting. The PHMC will be required by Federal, Washington State and RL requirements to monitor and report on Hanford Site effluents. Reports required:

- Monthly NPDES reporting to Ecology, Washington State Department of Health, and EPA

- Discharge Information System (EIS/ODIS) report to INEL

- Environmental releases report to RL

- Radionuclide Air Emissions Report to EPA and Washington State Department of Health

- Biannual/PTRAEU Report to Washington State Department of Health

- Monthly Excess Emission Report on Baghouse Bypass notifications to Ecology

- Annual Air Emissions Inventory Update to Ecology (to include the report pursuant to WAC 173-400-105, "Annual Inventory of Non-Radiological Airborne Emissions")

\subsection{Effluent Monitoring Plan and Facility Effluent Monitoring Plan}

Reporting. The PHMC will be required to provide updating on existing EMP and Facility Effluent Monitoring Plans (FEMPs) as required by DOE Orders (will be per 10 CFR 834). These are on a three-year cycle with the next due date in November 1997.

\subsubsection{Environmental Requirements Management. WHC provides regulatory} analysis/interpretation/consultation to RL and projects, as requested, to resolve environmental issues. This support covers the applicability of several environmental areas (e.g., RCRA, CAA, CWA) to help RL and projects adequately plan for future missions as they become identified. Additionally, a proactive approach is pursued by WHC in providing comments to positively influence proposed Federal, state, and local rulemakings in the interest of RL and the Hanford Site.

\subsubsection{Non-Tri-Party Agreement Compliance Agreement.}

3.5.3.4.1 NESHAPS Federal Facility Compliance Agreement. A Federal Facility Compliance Agreement (FFCA) was signed on February 7, 1994, by RL and EPA, Region 10. Milestones in the FFCA bring all stack sampling systems on the Hanford Site into compliance with requirements in 40 CFR 61 Subpart H. WHC is bringing its stacks into 
compliance. WHC has met or exceeded all of it's milestones. Because the FFCA agreement is between RL and EPA, the new contractor/s will be subject to the FFCA and will be required to meet remaining milestones.

3.5.3.4.2 216 Consent Order. Ecology and DOE negotiated the 216 Consent Order, formally known as Consent Order No. DE 91NM-177 in 1991. The purpose of the consent order is to control the discharge of liquid effluents on the Hanford Reservation in accordance with applicable state water quality criteria and establish a schedule for permits to be secured for effluent streams discharged at the Hanford Site. Milestones for three of the following types of liquid effluent streams were established; Phase I streams, Phase II streams and miscellaneous streams. Most of the milestones for the Phase I and Phase II liquid effluent streams are complete. Milestones for the miscellaneous streams included submitting an inventory of disposal sites, submitting a plan to identify and evaluate all miscellaneous streams and submitting a plan and schedule for disposition and regulatory compliance for all remaining miscellaneous streams. The plans and schedule were submitted as required and is currently being implemented onsite. To be further addressed in actions contained in Section 4.2.2, Liquid Effluents Program.

3.5.3.4.3 Heart of America Settlement. As per agreement in the Heart of America Northwest, et al. vs Westinghouse, et al., WHC agreed to notify Heart of America in instances where "For releases where immediate written notification is provided to regulatory agencies, Defendants, or one Defendant, as appropriate, shall provide a copy of any written notification provided under the statutes and regulation cited above to Plaintiffs by facsimile within one business day following such notification." Procedures for notifying Heart of America, in such instances as noted above, have been incorporated into the WHC Environmental Compliance Manual (ECM) 7-5. Heart of America and the offsite environmental regulatory agency are notified by WHC External Communications of a release within twenty-four hours of the event. See WHC-CM-7-5, Environmental Compliance, Page 5 of 53. To be further addressed in actions contained in Section 4.1, TWRS.

\subsection{RL/EPA Region 10 Compliance Agreement for Trench 94 (PCBs).} Region 10 of EPA and RL, are parties to this agreement which was entered into under the authority of Executive Order (E.O.) 12088. This agreement was being entered into to bring burial ground 218-E-12B, Trench 94, into compliance with the Toxic Substance Control Act (TSCA). Trench 94 is that area set aside for disposal of submarine reactor compartments in the 200 East Area 218-E-12B Burial Ground.

Full compliance with Section 15 of TSCA would be attained by EPA's granting of approval pursuant to TSCA, to the RL to operate a chemical waste landfill at Trench 94 , for the sole purpose of landfilling submarine reactor compartments containing PCB-contaminated components (with low level of radioactivity). To be further addressed in actions contained in Section 4.2.1, Solid Waste.

3.5.3.4.5 DOE/NNPP/EPA Federal Facility Compliance Agreement on Storage of PCBs. Agreement was tentatively reached between DOE, U.S. Navy Naval Nuclear Propulsion Program (NNPP), and EPA on storage of PCB articles and containers of waste beyond the one year TSCA limit. This agreement has been signed by DOE and NNPP and is currently in EPA possession for signature. 
The agreement requires Annual Status Report detailing specific information including covered PCB wastes, justification for continued storage beyond one year, and a summary of the compliance status and steps taken to mitigate storage deficiencies.

The agreement specifies M\&O Contractors as "co-operators" of DOE sites. Transfer to PHMC is necessary. To be further addressed in actions contained in Section 4.2.1, Solid Waste.

\subsubsection{Outsources.}

3.5.3.5.1 SAIC/CH2M Hill Subcontract. Significant subcontract ${ }^{1}$ support for environmental compliance issues is provided to WHC and ICF KH through two multi-million dollar, multi-year, task order Purchase Orders managed by Environmental Services:

- MTB-SVV-315924 (now MTB-SVV-452401) issued to Science Applications International Corporation (SAIC) with Hart-Crowser and Foster Wheeler as subcontractors.

- MRS-SVV-296951 (now TRS-SVV-416662) issued to CH2M Hill with IT and Environmental Issues as subcontractors.

3.5.3.5.2 Work for Others. Currently, WHC is conducting limited permitting and closure plan work for BHI and PNNL.

\begin{tabular}{|l|c|c|}
\hline \multicolumn{1}{|c|}{ Post-Award Actions } & $\begin{array}{c}\text { Scheduled } \\
\text { Date }\end{array}$ & Actionee(s) \\
\hline $\begin{array}{l}\text { 1. Negotiate transfer of work from WHC with BHI, PNNL and } \\
\text { PHMC. }\end{array}$ & $9 / 15 / 96$ & SM Price \\
\hline
\end{tabular}

\subsubsection{Tri-Party Agreement}

The Tri-Party Agreement is a legal binding document signed by DOE, EPA, and Ecology. The Tri-Party Agreement establishes a Hanford Sitewide RCRA/CERCLA compliance agreement comprised of programmatic milestones and completion dates for waste management and environmental restoration (ER) activities spanning the time frame of 1989 through 2028. WHC Tri-Party Agreement Integration (TPAI) organization has the lead responsibility for providing integration management for the successful completion of all TriParty Agreement programmatic milestones for all site contractors.

Some of the activities performed by TPAI in supporting RL include: maintaining TriParty Agreement change control systems, implement amendments to the Tri-Party Agreement document, conduct monthly IAMIT meetings for RL, EPA, and Ecology, conduct monthly

WHC Procurement will work with the new contractor to transition existing contracts. 
Tri-Party Agreement Training Course, provide status of Tri-Party Agreement milestones, and maintain the Tri-Party Agreement Handbook.

\begin{tabular}{|c|c|c|}
\hline Pre-Award Actions & $\begin{array}{c}\text { Scheduled } \\
\text { Date } \\
\end{array}$ & Actionee(s) \\
\hline $\begin{array}{l}\text { 1. Assemble copies of the Environmental Tracking Systern } \\
\text { (ETS) reports and have available to provide status of Tri- } \\
\text { Party Agreement milestones and identify responsible } \\
\text { personnel. }\end{array}$ & $\begin{array}{l}6 / 01 / 96 \\
\text { Completed }\end{array}$ & R Morrison \\
\hline $\begin{array}{l}\text { 2. Assemble copies of the current Tri-Party Agreement } \\
\text { document available for PHMC review. }\end{array}$ & $\begin{array}{l}6 / 01 / 96 \\
\text { Completed }\end{array}$ & R Morrison \\
\hline $\begin{array}{l}\text { 3. Develop list of unresolved Tri-Party Agreement Disputes, } \\
\text { open change requests, on going and anticipated regulator } \\
\text { negotiations and unresolved Tri-Party Agreement document } \\
\text { issues. }\end{array}$ & $\begin{array}{l}5 / 17 / 96 \\
\text { Completed }\end{array}$ & R Morrison \\
\hline $\begin{array}{l}\text { 4. Assemble copies of latest LAMIT meeting minutes to } \\
\text { identify current issues of discussion between RL, EPA and } \\
\text { Ecology. }\end{array}$ & $\begin{array}{l}5 / 17 / 96 \\
\text { Completed }\end{array}$ & F Calapristi \\
\hline $\begin{array}{l}\text { 5. Assembly status of current and future Tri-Party Agreement } \\
\text { negotiations. }\end{array}$ & $\begin{array}{l}5 / 17 / 95 \\
\text { Completed }\end{array}$ & R Morrison \\
\hline $\begin{array}{l}\text { 6. Develop list of missed Tri-Party Agreement milestones for } \\
\text { consideration of potential liabilities. }\end{array}$ & $\begin{array}{l}5 / 17 / 96 \\
\text { Completed }\end{array}$ & $L$ Arnold \\
\hline Post-Award Actions & & $\cdots$ \\
\hline $\begin{array}{l}\text { 1. Provide personnel and assignment list for the Tri-Party } \\
\text { Agreement Integration organization. }\end{array}$ & $8 / 09 / 96$ & L Arnold \\
\hline $\begin{array}{l}\text { 2. Have copies of the Tri-Party Agreement document, with } \\
\text { latest changes, available for PHMC review. }\end{array}$ & $8 / 09 / 96$ & R Morrison \\
\hline $\begin{array}{l}\text { 3. Provide list of unresolved Tri-Party Agreement Disputes, } \\
\text { open change requests, ongoing/anticipated regulator } \\
\text { negotiations and unresolved Tri-Party Agreement document } \\
\text { issues. }\end{array}$ & $8 / 09 / 96$ & R Morrison \\
\hline $\begin{array}{l}\text { 4. Have copy of Tri-Party Agreement Handbook available for } \\
\text { PHMC review. }\end{array}$ & $8 / 09 / 96$ & F Calapristi \\
\hline $\begin{array}{l}\text { 5. Have copies of the ETS reports available to provide } \\
\text { June 1, 1996, status of Tri-Party Agreement milestones and } \\
\text { identify responsible personnel. }\end{array}$ & $8 / 16 / 96$ & R Morrison \\
\hline $\begin{array}{l}\text { 6. Arrange introductory meeting between RL, EPA, Ecology } \\
\text { and PHMC. }\end{array}$ & $8 / 27 / 96$ & L Arnold \\
\hline $\begin{array}{l}\text { 7. Provide status of current and future Tri-Party Agreement } \\
\text { negotiations. }\end{array}$ & $8 / 16 / 96$ & R Morrison \\
\hline 8. Schedule Tri-Party Agreement Training course for PHMC. & $8 / 23 / 96$ & F Calapristi \\
\hline $\begin{array}{l}\text { 9. Provide copies of July IAMIT approved meeting minutes to } \\
\text { identify current issues of discussion between RL, EPA, and } \\
\text { Ecology. }\end{array}$ & $8 / 31 / 96$ & F Calapristi \\
\hline
\end{tabular}




\begin{tabular}{|l|c|c|}
\hline \multicolumn{1}{|c|}{ Post-Award Actions } & $\begin{array}{c}\text { Schedpled } \\
\text { Date }\end{array}$ & Actionee(s) \\
\hline $\begin{array}{l}\text { 10. Deliver all Tri-Party Agreement training course materials } \\
\text { (files, records, overheads, handouts) to PHMC management } \\
\text { so they may continue to provide training as needed. }\end{array}$ & $8 / 31 / 96$ & R Moerman \\
\hline $\begin{array}{l}\text { 11. Maintain "Information Locator Service" required by } \\
\text { M-35-07. }\end{array}$ & $10 / 01 / 96$ & PHMC \\
\hline
\end{tabular}

\subsubsection{Safety Analysis and Nuclear Engineering}

The Safety Analysis and Nuclear Engineering (SA\&NE) organization provides the nuclear and radiological expertise required to achieve the safe management, operations, handling and disposition of nuclear facilities and radioactive materials on the Hanford Site. This organization's expertise provides the safety authorization basis, environmental performance assessments, nuclear fuel characterization, and criticality and shielding analyses in support of nuclear design and operational safety.

SA\&NE functions as an integral part of major projects, thereby providing cost competitive expertise based on decades of site-specific knowledge utilizing a balanced mix of experience and skills.

SA\&NE is currently providing or has recently completed deliverables for site projects and programs including TWRS, SNF, Transition Facilities, Solid Waste, and Special Initiatives.

\begin{tabular}{|c|c|c|}
\hline a & $\begin{array}{l}\text { Scheduled } \\
\text { Date }\end{array}$ & Actionee(s) \\
\hline $\begin{array}{l}\text { 1. Assemble key program/project documentation, and identify } \\
\text { its location, for use by the PHMC. }\end{array}$ & $7 / 15 / 96$ & A Ramble \\
\hline $\begin{array}{l}\text { 2. Identify number of briefings, and prepare organizational } \\
\text { briefing(s) for presentation to the PHMC. }\end{array}$ & $\begin{array}{l}5 / 15 / 96 \\
\text { Completed }\end{array}$ & A Ramble \\
\hline $\begin{array}{l}\text { 3. Develop a listing of key program/project individuals as } \\
\text { POCs. }\end{array}$ & $\begin{array}{l}\text { 6/01/96 } \\
\text { Completed }\end{array}$ & A Ramble \\
\hline $\begin{array}{l}\text { 4. Develop listing of program project subcontracts. Include any } \\
\text { subcontracts still planned to be executed prior to contract } \\
\text { takeover. }\end{array}$ & $\begin{array}{l}\text { 6/01/96 } \\
\text { Completed }\end{array}$ & A Ramble \\
\hline Post-Award Actions & & $\because$ \\
\hline $\begin{array}{l}\text { 1. Provide any documentation or information requested by the } \\
\text { PHMC, if available, that was not identified during the pre- } \\
\text { award phase. }\end{array}$ & $8 / 30 / 96$ & A Ramble \\
\hline 2. Provide organizational briefing to the PHMC. & $\begin{array}{l}8 / 12 / 96 \\
8 / 30 / 96\end{array}$ & A Ramble \\
\hline 3. Provide program/projects briefing to the PHMC. & $\begin{array}{l}8 / 12 / 96- \\
8 / 30 / 96\end{array}$ & A Ramble \\
\hline
\end{tabular}




\subsubsection{Occupational Health Services}

Occupational Health Services are provided to the Hanford Site by the HEHF. The Contractor shall obtain for itself and require of all subcontractors performing work on the Hanford Site the following services from HEHF: occupational medical evaluations including return to work evaluations and work restriction reviews, medical surveillance evaluations, occupational primary care, health care centers/first aid, work conditioning, case management, work site health programs including blood-borne pathogens and immunizations, behavioral health services including employee assistance programs, Material Safety Data Sheet (MSDS) services, and health information services such as medical records and medical scheduling.

\section{Key Assumption}

- PeopleCore System will be carried forward and maintained by the PHMC.

Note: PeopleCore is a sitewide central database which consolidates information about people from diverse automated and non-automated systems. PeopleCore provides single point of entry for data collection, maintenance, and dissemination of information for all Hanford Site contractors. It is the basis for all of HEHF's medical system personnel rosters, and automatically updates these rosters on a daily basis.

\begin{tabular}{|l|l|l|}
\hline \multicolumn{1}{|c|}{ Post-Award Actions } & Scheduled & Actionee(s) \\
\hline $\begin{array}{l}\text { 1. The PHMC shall coordinate with HEHF and reach agreement } \\
\text { regarding service requirements and delivery, snd coordination } \\
\text { and integration between the Contractor's safery and health } \\
\text { organization(s) and HEHF including data gathering and } \\
\text { sharing. The agreement should emphasize a comprehensive } \\
\text { public health approach as being integral to a well run and } \\
\text { safety program, and address cost and resource effectiveness. } \\
\text { (This agreement shall be subject to approval and validation } \\
\text { by RL.) }\end{array}$ & $9 / 01 / 96$ & JJ Maher III \\
R Volk
\end{tabular}

\subsubsection{Centralized Occurrence Reporting}

The Occurrence Notification Center (ONC) is currently managed and staffed by WHC. The group provides centralized infrastructure support to the Hanford Site Occurrence Reporting Program. This support includes software and hardware maintenance, training, tracking, occurrence report distribution to include input into the Occurrence Report Processing System (ORPS). The ONC also provides ancillary services to Emergency 
Preparedness by way of operation and maintenance of communications and notification systems for declared emergencies.

\begin{tabular}{|c|c|c|}
\hline Pre-Award Actions & $\begin{array}{l}\text { Scheduled } \\
\text { Date }\end{array}$ & Actionee(s) \\
\hline $\begin{array}{l}\text { 1. Assemble key program (or project) documentation, or } \\
\text { identify its location, for use by the PHMC. Includes } \\
\text { development of a program/project "Smart Book," including } \\
\text { listings and descriptions developed above. }\end{array}$ & $\begin{array}{l}\text { 6/01/96 } \\
\text { Completed } \\
\text { "Smart Book" } \\
\text { only } \\
\text { See item \#9 }\end{array}$ & DJ Connell \\
\hline 2. Develop an ONC POC List: & $\begin{array}{c}6 / 01 / 96 \\
\text { Completed }\end{array}$ & DJ Connell \\
\hline $\begin{array}{l}\text { 3. Prepare a presentation which covers the work scope, staffing, } \\
\text { and budget of the ONC. }\end{array}$ & $\begin{array}{c}\text { 6/01/96 } \\
\text { Completed }\end{array}$ & DJ Connell \\
\hline $\begin{array}{l}\text { 4. Develop listing and description of program (or project) } \\
\text { databases, including facility (or operations) databases. }\end{array}$ & $\begin{array}{c}6 / 01 / 96 \\
\text { Completed }\end{array}$ & DJ Connell \\
\hline $\begin{array}{l}\text { 5. Develop a listing of special agreement services provided to } \\
\text { RL, other DOE sites, and other entities. }\end{array}$ & $\begin{array}{c}6 / 01 / 96 \\
\text { Completed }\end{array}$ & DJ Connell \\
\hline $\begin{array}{l}\text { 6. Develop a list of current work order services or other form } \\
\text { of agreement to provide work, or obtain work, for other } \\
\text { organizations, on and offsite. }\end{array}$ & $\begin{array}{l}6 / 01 / 96 \\
\text { Completed }\end{array}$ & DJ Connell \\
\hline $\begin{array}{l}\text { 7. Verify the accuracy of property assignments at the } \\
\text { program/project organizational level and correct any } \\
\text { deficiencies. }\end{array}$ & $\begin{array}{l}6 / 01 / 96 \\
\text { Completed }\end{array}$ & DJ Connell \\
\hline 8. Determine pre-existing conditions. & $\begin{array}{c}6 / 15 / 96 \\
\text { Completed }\end{array}$ & DJ Connell \\
\hline 9. Update/submit "Smart Book"/assemble key documents. & $7 / 15 / 96$ & DJ Connell \\
\hline Post-Award Actions & & \\
\hline $\begin{array}{l}\text { 1. Provide the PHMC access to the key program (or project) } \\
\text { documentation, any program level generated pre-existing } \\
\text { conditions information, listing of POCs, and listing of } \\
\text { subcontracts. Provide copies upon request. }\end{array}$ & $8 / 05 / 96$ & DJ Connell \\
\hline $\begin{array}{l}\text { 2. Determine occurrence reporting relationships and } \\
\text { responsibilities of the PHMC and subcontractors. }\end{array}$ & $8 / 15 / 96$ & DJ Connell \\
\hline $\begin{array}{l}\text { 3. Identify needed changes to authority files, ORPS designators, } \\
\text { procedures, and training. }\end{array}$ & $9 / 01 / 96$ & DJ Connell \\
\hline $\begin{array}{l}\text { 4. Complete changes to authority files, ORPS designators, and } \\
\text { procedures. }\end{array}$ & $9 / 15 / 96$ & DJ Connell \\
\hline 5. Implement changes to occurrence report process. & $9 / 30 / 96$ & DJ Connell \\
\hline $\begin{array}{l}\text { 6. Perform a joint program/project level property and equipment } \\
\text { inventory with PHMC personnel. }\end{array}$ & by $9 / 16 / 96$ & DJ Connell \\
\hline $\begin{array}{l}\text { 7. Revise authority files, ORPS designators, and train new } \\
\text { personnel. }\end{array}$ & $9 / 30 / 96$ & DJ Connell \\
\hline $\begin{array}{l}\text { 8. Identify data crossover for retrofit ORPS data with new } \\
\text { ORPS designators. }\end{array}$ & $9 / 30 / 96$ & DJ Connell \\
\hline
\end{tabular}




\subsection{PROCUREMENT AND MATERIALS MANAGEMENT}

\section{General Assumptions}

- The PHMC will assume existing PMM systems, manuals, and forms for use following transition and will defer initiating efforts to implement new or revised processes.

- The present PMM organization structure and manpower level is not changed during the two month transition period.

- RL will coordinate with all external organizations to eliminate PMM external interaction, inspections, and reviews during the transition period.

\subsubsection{Procurement}

\section{Procurement Specific Assumptions}

- Ongoing procurement activities may be seriously impacted during the two-month transition effort. Therefore, only emergency and selective high priority requisition may be processed during this period.

- RL will maintain a significant contingent reserve account to fund terminated and disputed orders.

\begin{tabular}{|c|c|c|}
\hline $\begin{array}{r}+.+3 \\
+2\end{array}$ & $\begin{array}{l}\text { Scheduled } \\
\text { Date }\end{array}$ & Actionee(s) \\
\hline 1. Assemble current organizational data (charts and charters). & $\begin{array}{c}5 / 31 / 96 \\
\text { Completed }\end{array}$ & JJ Jones \\
\hline 2. List of property assigned to PMM. & $\begin{array}{l}5 / 31 / 96 \\
\text { Completed }\end{array}$ & R Dahlin \\
\hline 3. Status of re-engineering initiatives and actions. & $\begin{array}{l}\$ / 31 / 96 \\
\text { Completed }\end{array}$ & RJ Meyer \\
\hline $\begin{array}{l}\text { 4. Assemble copy of all current reports provided to external } \\
\text { customers. }\end{array}$ & $\begin{array}{l}5 / 31 / 96 \\
\text { Completed }\end{array}$ & E Beavers \\
\hline 5. Provide overview of financial situation and work plans. & $\begin{array}{c}5 / 31 / 96 \\
\text { Completed }\end{array}$ & J Stewart \\
\hline Post-Award Actions & 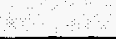 & \\
\hline $\begin{array}{l}\text { 1. Resolve any open issues on PMM department property as time } \\
\text { permits. }\end{array}$ & $9 / 30 / 96$ & R Dahlin \\
\hline $\begin{array}{l}\text { 2. Provide update on organization data, re-engineering, financial } \\
\text { status, and work plans. }\end{array}$ & $8 / 09 / 96$ & RJ Meyer \\
\hline
\end{tabular}




\subsubsection{Open Audit/Surveillance Items.}

\begin{tabular}{|l|l|l|}
\hline \multicolumn{1}{|c|}{ Pre-Award Actions } & $\begin{array}{c}\text { Scheduled } \\
\text { Date }\end{array}$ & \multicolumn{1}{|c|}{ Actionee(s) } \\
\hline 1. Identify and status all open items. & $\begin{array}{c}5 / 31 / 96 \\
\text { Completed }\end{array}$ & $\begin{array}{l}\text { E Higginbothom } \\
\text { B Hubbard }\end{array}$ \\
\hline Post-Award Actions & & \\
\hline $\begin{array}{l}\text { 1. Complete as many audit/surveillance items as possible by } \\
\text { turn over as time permits. }\end{array}$ & $9 / 30 / 96$ & $\begin{array}{l}\text { E Higginbothom } \\
\text { B Hubbard }\end{array}$ \\
\hline \begin{tabular}{l} 
2. Transfer action to appropriate closeout offices. \\
\hline
\end{tabular} & $9 / 30 / 96$ & $\begin{array}{l}\text { E Higginbothom } \\
\text { B Hubbard }\end{array}$ \\
\hline
\end{tabular}

\subsubsection{Identify Funding Reserves/Contingency Needs for Close Out Process.}

\begin{tabular}{|c|c|c|}
\hline Pre-Award Actions & $\begin{array}{l}\text { Scheduled } \\
\text { Date }\end{array}$ & Actionee(s) \\
\hline $\begin{array}{l}\text { 1. Compile listing of orders and funding necessary of purchase } \\
\text { orders in the close out process. }\end{array}$ & $\begin{array}{c}5 / 31 / 96 \\
\text { Completed }\end{array}$ & $\begin{array}{l}\text { E Higginbothom } \\
\text { B Hubbard }\end{array}$ \\
\hline Post-Award Actions & & \\
\hline 1. Update funding reserves/contingency needs for close outs. & $8 / 09 / 96$ & $\begin{array}{l}\text { E Higginbothom } \\
\text { B Hubbard }\end{array}$ \\
\hline 2. Transfer action to appropriate close out offices. & $9 / 30 / 96$ & $\begin{array}{l}\text { E Higginbothom } \\
\text { B Hubbard }\end{array}$ \\
\hline
\end{tabular}

\subsubsection{Purchase Order Assignment/Transfer to One or More New Companies.}

\begin{tabular}{|l|l|l|l|l|}
\hline Pre-Award Actions & Scheduled & Actionee(s) \\
\hline 1. Develop purchase order assignment letter. & $\begin{array}{c}5 / 31 / 96 \\
\text { Cornpleted }\end{array}$ & $\begin{array}{l}\text { M Taylor } \\
\text { S Ace }\end{array}$ \\
\hline
\end{tabular}

\subsubsection{Purchase Orders With Open Concerns.}

\begin{tabular}{|c|c|c|}
\hline Pre-Award Actions & $\begin{array}{l}\text { Scheduled } \\
\text { Date }\end{array}$ & Actionee(s) \\
\hline $\begin{array}{l}\text { 1. Identify all purchase orders with protests, claims or any other } \\
\text { open legal issue. }\end{array}$ & $\begin{array}{l}5 / 31 / 96 \\
\text { Completed }\end{array}$ & $\begin{array}{l}\text { R Hadley } \\
\text { J Snith } \\
\text { B Hubbard } \\
\text { R Dutton }\end{array}$ \\
\hline Post-Award Actions & $\because$ & \\
\hline $\begin{array}{l}\text { 1. Update list of purchase orders with protests, claims or any } \\
\text { other open legal issue. }\end{array}$ & $8 / 09 / 96$ & $\begin{array}{l}\text { R Hadley } \\
\text { J Smith } \\
\text { B Hubbard } \\
\text { R Dutton }\end{array}$ \\
\hline 2. PHMC to identify orders to be transferred to PHMC. & $9 / 01 / 96$ & PHMC \\
\hline
\end{tabular}




\begin{tabular}{|c|c|c|}
\hline Post-Award Actions & $\begin{array}{l}\text { Scheduled } \\
\text { Date }\end{array}$ & Actionee(s) \\
\hline 3. WHC/ICF KH issue assignment letters. & $\begin{array}{l}9 / 10 / 96- \\
9 / 30 / 96\end{array}$ & $\begin{array}{l}\text { R Hadley } \\
\text { J Smith } \\
\text { B Hubbard } \\
\end{array}$ \\
\hline $\begin{array}{l}\text { 4. WHC } 1 \mathrm{CF} \mathrm{KH} \text { issue termination notices to orders not being } \\
\text { assigned to PHMC. }\end{array}$ & $\begin{array}{l}9 / 10 / 96- \\
9 / 30 / 96\end{array}$ & $\begin{array}{l}\text { R Hadley } \\
\text { J Smith } \\
\text { B Hubbard } \\
\end{array}$ \\
\hline 5. Transfer all unassigned orders to respective closeout offices. & $9 / 30 / 96$ & $\begin{array}{l}\text { R Hadley } \\
\text { J Smith } \\
\text { B Hubbard }\end{array}$ \\
\hline
\end{tabular}

\subsubsection{Purchase Orders in Termination Process.}

\begin{tabular}{|c|c|c|}
\hline 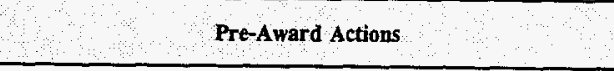 & $\begin{array}{c}\text { Scheduled } \\
\text { Date }\end{array}$ & Actionee(s) \\
\hline $\begin{array}{l}\text { 1. Identify all purchase orders in termination (convenience or } \\
\text { default) process. }\end{array}$ & $\begin{array}{c}5 / 31 / 96 \\
\text { Completed } \\
\text { Completed } \\
\end{array}$ & $\begin{array}{l}\text { R Hadley } \\
\text { J Smith } \\
\text { B Hubbard } \\
\end{array}$ \\
\hline . $\quad$ Post-Award Actions & & \\
\hline $\begin{array}{l}\text { 1. Update list of purchase orders in termination (convenience or } \\
\text { default) process. }\end{array}$ & $8 / 09 / 96$ & $\begin{array}{l}\text { R Hadley } \\
\text { J Smith } \\
\text { B Hubbard } \\
\end{array}$ \\
\hline 2. Transfer action to appropriate contractor closeout offices. & $9 / 30 / 96$ & $\begin{array}{l}\text { R Hadley } \\
\text { J Smith } \\
\text { B Hubbard } \\
\text { R Dutton }\end{array}$ \\
\hline
\end{tabular}

\subsubsection{Purchase Orders With Open Options.}

\begin{tabular}{|c|c|c|}
\hline S $\quad$ Pre-Award Actions & $\begin{array}{c}\text { Scheduled } \\
\text { Date }\end{array}$ & Actionee(s) \\
\hline $\begin{array}{l}\text { 1. Identify all purchase orders with options that could be } \\
\text { exercised for services after September } 30,1996 \text {. }\end{array}$ & $\begin{array}{l}5 / 31 / 96 \\
\text { Completed }\end{array}$ & $\begin{array}{l}\text { R Hadley } \\
\text { J Smith } \\
\text { B Hubbard }\end{array}$ \\
\hline Post-Award Actions & & \\
\hline $\begin{array}{l}\text { 1. Update list of purchase orders with options that could be } \\
\text { exercised for services after September } 30,1996 \text {. }\end{array}$ & $8 / 09 / 96$ & $\begin{array}{l}\text { R Hadiey } \\
\text { J Smith } \\
\text { B Hubbard }\end{array}$ \\
\hline
\end{tabular}

\subsubsection{Open Procurement Actions Beyond Transition.}

\begin{tabular}{|l|c|c|}
\hline \multicolumn{1}{|c|}{ Pre-Award Actions } & $\begin{array}{c}\text { Scheduled } \\
\text { Date }\end{array}$ & Actionee(s) \\
\hline $\begin{array}{l}\text { 1. List all purchase orders that will be open beyond } \\
\text { September 30, 1996. }\end{array}$ & $\begin{array}{l}5 / 31 / 96 \\
\text { Completed }\end{array}$ & $\begin{array}{l}\text { R Hadley } \\
\text { J Smith } \\
\text { B Hubbard }\end{array}$ \\
\hline
\end{tabular}




\begin{tabular}{|c|c|c|}
\hline PreAward Actions & $\begin{array}{l}\text { Scheduled } \\
\text { Date }\end{array}$ & Actionee(s) \\
\hline $\begin{array}{l}\text { 2. List all Memorandum orders with other DOE sites that will } \\
\text { be open beyond September } 30,1996 \text {. }\end{array}$ & $\begin{array}{l}5 / 31 / 96 \\
\text { Completed }\end{array}$ & $\begin{array}{l}\text { R Hadley } \\
\text { J Smith } \\
\text { B Hubbard }\end{array}$ \\
\hline $\begin{array}{l}\text { 3. List all undefinitized purchase orders in work as of contract } \\
\text { award. }\end{array}$ & $\begin{array}{l}5 / 31 / 96 \\
\text { Completed }\end{array}$ & $\begin{array}{l}\text { R Hadley } \\
\text { J Smith } \\
\text { B Hubbard }\end{array}$ \\
\hline $\begin{array}{l}\text { 4. List all Interwork Agreements with WEC and Boeing } \\
\text { subsidiaries that will be open beyond September } 30,1996 \text {. }\end{array}$ & $\begin{array}{c}5 / 31 / 96 \\
\text { Completed }\end{array}$ & $\begin{array}{l}\text { R Hadley } \\
\text { J Smith }\end{array}$ \\
\hline $\begin{array}{l}\text { 5. List all Interworks agreements with ICF KH subsidiaries that } \\
\text { will be open beyond September } 30,1996 \text {. }\end{array}$ & $\begin{array}{l}5 / 31 / 96 \\
\text { Completed }\end{array}$ & C Maxson \\
\hline $\begin{array}{l}\text { 6. List all special agreements (travel agency, air lines/car } \\
\text { rentals, head hunters, etc.) that will be open beyond } \\
\text { September } 30,1996 \text {. }\end{array}$ & $\begin{array}{l}5 / 31 / 96 \\
\text { Completed }\end{array}$ & $\begin{array}{l}\text { R Hadley } \\
\text { J Smith } \\
\text { C Maxson }\end{array}$ \\
\hline Post-Award Actions & & \\
\hline 1. Update all action lists in section 3.6.1.7 (A) above. & $8 / 09 / 96$ & $\begin{array}{l}\text { R Hadley } \\
\text { J Smith } \\
\text { B Hubbard }\end{array}$ \\
\hline 2. PHMC to identify orders to be transferred to PHMC. & $9 / 01 / 96$ & PHMC \\
\hline 3. WHC/CF KH issue assignment letters. & $\begin{array}{l}9 / 10 / 96- \\
9 / 30 / 96\end{array}$ & $\begin{array}{l}\text { R Hadley } \\
\text { J Smith } \\
\text { B Hubbard }\end{array}$ \\
\hline $\begin{array}{l}\text { 4. WHC/ICF KH issue termination notices to orders not being } \\
\text { assigned to PHMC. }\end{array}$ & $\begin{array}{l}9 / 10 / 96- \\
9 / 30 / 96\end{array}$ & $\begin{array}{l}\text { R Hadley } \\
\text { J Smith } \\
\text { B Hubbard }\end{array}$ \\
\hline 5. Transfer all unassigned orders to respective closeout offices. & 9/30/96 & $\begin{array}{l}\text { R Hadley } \\
\text { J Smith } \\
\text { B Hubbard }\end{array}$ \\
\hline
\end{tabular}

\subsubsection{Open Solicitations.}

\begin{tabular}{|c|c|c|}
\hline \multicolumn{1}{|c|}{ Pre-Award Actions } & $\begin{array}{l}\text { Scheduled } \\
\text { Date }\end{array}$ & \multicolumn{1}{|c|}{ Actionee(s) } \\
\hline 1. List of all solicitations and awards in process at award time. & $\begin{array}{c}7 / 15 / 96 \\
\text { Completed }\end{array}$ & $\begin{array}{l}\text { R Hadley } \\
\text { J Smith } \\
\text { T Gasperino }\end{array}$ \\
\hline $\begin{array}{l}\text { Post-Award Actions } \\
\text { September 30, 1996. }\end{array}$ & $8 / 09 / 96$ & $\begin{array}{l}\text { R Hadley } \\
\text { J Smith } \\
\text { B Hubbard }\end{array}$ \\
\hline 2. PHMC to identify solicitations to be transferred to PHMC. & $9 / 01 / 96$ & PHMC \\
\hline
\end{tabular}


WHC-SP-1181 Rev. 1

\begin{tabular}{|c|c|c|}
\hline Post-Award Actions & $\begin{array}{l}\text { Scheduled } \\
\text { Date }\end{array}$ & Actionee(s) \\
\hline 3. WHC/ICF KH issue transfer/assignment letters. & $\begin{array}{l}9 / 10 / 96- \\
9 / 30 / 96\end{array}$ & $\begin{array}{l}\text { R Hadley } \\
\text { I Smith } \\
\text { B Hubbard }\end{array}$ \\
\hline $\begin{array}{l}\text { 4. WHC/CF } \mathrm{KH} \text { issue cancellation notices on solicitations not } \\
\text { being assigned to PHMC. }\end{array}$ & $\begin{array}{l}9 / 10 / 96- \\
9 / 30 / 96\end{array}$ & $\begin{array}{l}\text { R Hadley } \\
\text { J Smith } \\
\text { B Hubbard }\end{array}$ \\
\hline
\end{tabular}

\subsubsection{Purchase Order Terms and Conditions.}

\begin{tabular}{|c|c|c|}
\hline $\begin{array}{ll}4+\infty \\
\end{array}$ & $\begin{array}{l}\text { Scheduled } \\
\text { Date }\end{array}$ & Actionee(s) \\
\hline $\begin{array}{l}\text { 1. Current set of terms and conditions incorporated into purchase } \\
\text { orders. }\end{array}$ & $\begin{array}{c}5 / 31 / 96 \\
\text { Completed }\end{array}$ & $\begin{array}{l}\text { R Hadiey } \\
\text { J Smith } \\
\text { T Gasperino } \\
\end{array}$ \\
\hline Post-Award Actions & & \\
\hline $\begin{array}{l}\text { 1. PHMC to develop and obtain RL approval of Terms and } \\
\text { Conditions to be used in orders after } 9 / 30 / 96 \text {. }\end{array}$ & $9 / 30 / 96$ & PHMC \\
\hline
\end{tabular}

\subsubsection{Actions with DOE (RL \& HQ).}

\begin{tabular}{|c|c|c|}
\hline Pre-Award Actions & $\begin{array}{l}\text { Scheduled } \\
\text { Date }\end{array}$ & Actionee(s) \\
\hline $\begin{array}{l}\text { 1. Provide a listing of procurement actions/initiatives with DOE } \\
\text { (RL \& HQ). }\end{array}$ & $\begin{array}{c}5 / 31 / 96 \\
\text { Completed }\end{array}$ & $\begin{array}{l}\text { R Hadley } \\
\text { J Smith } \\
\text { C Maxson }\end{array}$ \\
\hline \multicolumn{3}{|l|}{ Post-Award Actions } \\
\hline $\begin{array}{l}\text { 1. Update listing of procurement actions/initiatives with DOE } \\
\text { (RL \& HQ). }\end{array}$ & $8 / 09 / 96$ & $\begin{array}{l}\text { R Hadiey } \\
\text { M Taylor } \\
\text { J Smith } \\
\text { C Maxson } \\
\text { E Higginbotham }\end{array}$ \\
\hline
\end{tabular}

\subsubsection{Subcontractor Property Activities.}

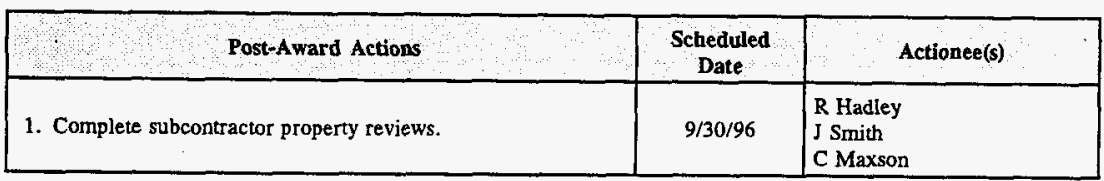




\subsubsection{Software License Agreements.}

\section{Specific Assumption}

- PHMC is not responsible for sitewide licenses nor for the licenses of DOE or other DOE contractors.

\begin{tabular}{|c|c|c|}
\hline Pre-Award Actions & $\begin{array}{l}\text { Scheduled } \\
\text { Date }\end{array}$ & Actionee(s) \\
\hline 1. List all software licenses. & $\begin{array}{c}5 / 31 / 96 \\
\text { Completed }\end{array}$ & $\begin{array}{l}\text { R Hadley } \\
\text { B Hubbard }\end{array}$ \\
\hline 2. Develop software license transfer agreement. & $\begin{array}{l}5 / 31 / 96 \\
\text { Completed }\end{array}$ & $\begin{array}{l}\text { R Hadley } \\
\text { B Hubbard } \\
\text { R Dutton }\end{array}$ \\
\hline \multicolumn{3}{|l|}{ Post-Award Actions } \\
\hline 1. Update software license list. & $8 / 09 / 96$ & $\begin{array}{l}\text { R Hadley } \\
\text { B Hubbard }\end{array}$ \\
\hline 2. PHMC to identify licenses to be transferred to PHMC. & $9 / 01 / 96$ & PHMC \\
\hline 3. WHC issue assignment letters. & $\begin{array}{l}9 / 10 / 96- \\
9 / 30 / 96\end{array}$ & $\begin{array}{l}\text { R Hadley } \\
\text { B Hubbard } \\
\end{array}$ \\
\hline $\begin{array}{l}\text { 4. WHC issue cancellation notices to licenses not being assigned } \\
\text { to PHMC. }\end{array}$ & $\begin{array}{l}9 / 10 / 96- \\
9 / 30 / 96 \\
\end{array}$ & $\begin{array}{l}\text { R Hadley } \\
\text { B Hubbard } \\
\end{array}$ \\
\hline $\begin{array}{l}\text { 5. Negotiate any necessary modifications to sitewide license } \\
\text { agreements. }\end{array}$ & $9 / 30 / 96$ & $\begin{array}{l}\text { PHMC } \\
\text { Other Site Contractors }\end{array}$ \\
\hline $\begin{array}{l}\text { 6. Transfer to WHC closeout office to negotiate and implement } \\
\text { license cancellation/closeout requirements for all licenses not } \\
\text { transferred to PHMC or other DOE contractors. }\end{array}$ & $9 / 30 / 96$ & $\begin{array}{l}\text { R Hadley } \\
\text { B Hubbard }\end{array}$ \\
\hline
\end{tabular}

\subsubsection{Procurement Reference Material.}

\begin{tabular}{|c|c|c|}
\hline Pre-Award Actions & $\begin{array}{l}\text { Scheduled } \\
\text { Date }\end{array}$ & Actionee(s) \\
\hline $\begin{array}{l}\text { 1. Assemble extra set of all current terms and conditions, } \\
\text { manuals, procedures, forms and work place instructions for } \\
\text { procurement functions. }\end{array}$ & $\begin{array}{c}5 / 31 / 96 \\
\text { Completed }\end{array}$ & $\begin{array}{l}\text { M Taylor } \\
\text { B Hubbard } \\
\text { T Gasperino }\end{array}$ \\
\hline $\begin{array}{l}\text { 2. Develop a list of forms that include references or logos that } \\
\text { may need to be changed. }\end{array}$ & $\begin{array}{c}5 / 31 / 96 \\
\text { Completed }\end{array}$ & $\begin{array}{l}\text { M Taylor } \\
\text { B Hubbard }\end{array}$ \\
\hline
\end{tabular}

\subsubsection{Automation System Information.}

\begin{tabular}{|c|c|c|}
\hline Pre-Award Actions & $\begin{array}{l}\text { Scheduled } \\
\text { Date }\end{array}$ & Actionee(s) \\
\hline 1. List of all automated systems with interface details. & $\begin{array}{c}5 / 31 / 96 \\
\text { Completed }\end{array}$ & $\begin{array}{l}\text { M Taylor } \\
\text { S Ace }\end{array}$ \\
\hline $\begin{array}{l}\text { 2. Status of all major system modifications or requirements } \\
\text { changes in process. }\end{array}$ & $\begin{array}{c}5 / 31 / 96 \\
\text { Completed }\end{array}$ & $\begin{array}{l}\text { M Taylor } \\
\text { S Ace }\end{array}$ \\
\hline
\end{tabular}




\begin{tabular}{|l|c|c|}
\hline \multicolumn{1}{|c|}{ Post-Award Actions } & Scheduled & \\
\hline $\begin{array}{l}\text { 1. Update status of all major system modifications or } \\
\text { requirements in process. }\end{array}$ & $8 / 09 / 96$ & $\begin{array}{l}\text { M Taylor } \\
\text { S Ace }\end{array}$ \\
\hline
\end{tabular}

\subsubsection{Outreach Activities.}

\begin{tabular}{|c|c|c|}
\hline Pre-Award Actions & $\begin{array}{l}\text { Scheduled } \\
\text { Date }\end{array}$ & Actionee(s) \\
\hline $\begin{array}{l}\text { 1. Develop summary and assemble listing of all Outreach } \\
\text { commitments of WHC. }\end{array}$ & $\begin{array}{c}5 / 31 / 96 \\
\text { Completed }\end{array}$ & C Crain \\
\hline
\end{tabular}

\subsubsection{Property Management}

\section{Property Assumptions}

- DOE and PHMC concur with the graded inventory approach which is a combination of statistical and 100 percent physical inventories depending upon dollar value and type of property. This approach was previously submitted to RL.

- Convenience storage inventories will not be inventoried as part of the transition effort.

- WHC and DOE will process LDDRs in a timely manner during the transition process.

- Able to dedicate all twelve bar code readers to the effort.

- Able to have twelve government vehicles dedicated to this effort for the full two months.

\subsubsection{Capital and Sensitive Property.}

\begin{tabular}{|l|c|l|}
\hline \multicolumn{1}{|c|}{ Pre-Award Actions } & $\begin{array}{c}\text { Scheduled } \\
\text { Date }\end{array}$ & Actionee(s) \\
\hline $\begin{array}{l}\text { 1. Provide a current inventory of all capital and sensitive } \\
\text { property for WHC/BCSR and ICF Kaiser operations. }\end{array}$ & $\begin{array}{c}5 / 31 / 96 \\
\text { Completed }\end{array}$ & R Dahlin \\
\hline $\begin{array}{l}\text { 1. WHC provide an updated inventory listing with strategy for } \\
\text { graded approach. }\end{array}$ & $8 / 06 / 96$ & R Dahlin \\
\hline 2. PHMC concurs with WHC strategy for graded approach. & $8 / 09 / 96$ & PHMC \\
\hline 3. WHC and PHMC appoint/hire inventory team members. & $8 / 15 / 96$ & $\begin{array}{l}\text { R Dahlin } \\
\text { PHMC }\end{array}$ \\
\hline
\end{tabular}




\begin{tabular}{|c|c|c|}
\hline Post-Award Actions & $\begin{array}{l}\text { Scheduled } \\
\text { Date }\end{array}$ & Actionee(s) \\
\hline 4. Training and equipment inventory teams. & $8 / 16 / 96$ & $\begin{array}{l}\text { R Dahlin } \\
\text { PHMC }\end{array}$ \\
\hline 5. Conduct field inventories. & $\begin{array}{l}8 / 19 / 96- \\
9 / 24 / 96 \\
\end{array}$ & $\begin{array}{l}\text { R Dahlin } \\
\text { PHMC }\end{array}$ \\
\hline 6. Process LDDRs and update inventory database. & $\begin{array}{l}8 / 26 / 96 \\
9 / 29 / 96\end{array}$ & R Dahlin \\
\hline 7. Transfer ownership officially to new contractor(s). & $9 / 30 / 96$ & R Dahlin \\
\hline
\end{tabular}

\subsubsection{Other Financially Controlled Inventories.}

\begin{tabular}{|c|c|c|}
\hline as & $\begin{array}{c}\text { Scheduled } \\
\text { Date }\end{array}$ & Actionee(s) \\
\hline $\begin{array}{l}\text { 1. Provide a current inventory of all capital and sensitive } \\
\text { property for WHC/BCSR and ICF KH operations. }\end{array}$ & $\begin{array}{l}5 / 31 / 96 \\
\text { Completed }\end{array}$ & R Dahlin \\
\hline Post-Award Actions & & \\
\hline $\begin{array}{l}\text { 1. Complete all other inventories of financially controlled } \\
\text { property and reconcile. }\end{array}$ & $9 / 30 / 96$ & R Dahlin \\
\hline 2.. Transfer ownership officially to new contractor(s). & $9 / 30 / 96$ & R Dahlin \\
\hline
\end{tabular}

\subsubsection{Export Control and High Risk Property.}

\begin{tabular}{|c|c|c|}
\hline Post-Award Actions & $\begin{array}{c}\text { Scheduled } \\
\text { Date }\end{array}$ & Actionee(s) \\
\hline $\begin{array}{l}\text { 1. Provide listing of current inventory of all export control and } \\
\text { high risk surplus/excess property in disposal cycle. }\end{array}$ & $8 / 09 / 96$ & R Dahlin \\
\hline
\end{tabular}

\subsubsection{Activities with DOE (RL \& HQ).}

\begin{tabular}{|c|c|c|}
\hline Pre-Award Actions & $\begin{array}{c}\text { Scheduled } \\
\text { Date }\end{array}$ & Actionee(s) \\
\hline 1. List of actions/initiatives with DOE. & $\begin{array}{c}5 / 31 / 96 \\
\text { Completed }\end{array}$ & R Dahlin \\
\hline Post-Award Actions & & \\
\hline 1. Update list of actions/initiatives with DOE. & $8 / 09 / 96$ & R Dahlin \\
\hline
\end{tabular}

\subsubsection{Property Reference Material.}

\begin{tabular}{|l|c|c|}
\hline Pre-Award Actions & $\begin{array}{c}\text { Scheduled } \\
\text { Date }\end{array}$ & Actionee(s) \\
\hline 1. Assemble set of manuals, procedures, instructions. & $\begin{array}{c}5 / 31 / 96 \\
\text { Completed }\end{array}$ & R Dahlin \\
\hline
\end{tabular}




\subsubsection{Economic Transfer}

\subsubsection{Intellectual Property.}

\begin{tabular}{|c|c|c|}
\hline Pre-Award Actions & $\begin{array}{l}\text { Scheduled } \\
\text { Date }\end{array}$ & Actionee(s) \\
\hline $\begin{array}{l}\text { 1. Determine WEC right and interest in WHC developed } \\
\text { intellectual property. }\end{array}$ & $\begin{array}{c}5 / 31 / 96 \\
\text { Completed }\end{array}$ & $\begin{array}{l}\text { B Silva } \\
\text { R Dutton }\end{array}$ \\
\hline 2. List of all intellectual properties. & $\begin{array}{c}5 / 31 / 96 \\
\text { Completed } \\
\end{array}$ & B Silva \\
\hline ४. $\downarrow$ Post-Award Actions & 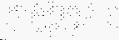 & \\
\hline 1. Transfer/preserve WEC rights as is appropriate. & $9 / 30 / 96$ & $\begin{array}{l}\text { B Silva } \\
\text { R Dutton }\end{array}$ \\
\hline
\end{tabular}

\subsubsection{Loan agreements, CRADAs and MOUs activities.}

\begin{tabular}{|l|l|l|}
\hline \multicolumn{1}{|c|}{ Pre-Award Actions } & Scheduled \\
Date & Actionee(s) \\
\hline $\begin{array}{l}\text { 1. Complete reviews and proviote listing of loan agreements, } \\
\text { CRADAs and MOUs, etc. that will be open beyond } \\
\text { September 30, 1996. }\end{array}$ & $\begin{array}{c}5 / 31 / 96 \\
\text { Completed }\end{array}$ & $\begin{array}{l}\text { B Silva } \\
\text { R Dutton }\end{array}$ \\
\hline Post-Award Actions & & \\
\hline $\begin{array}{l}\text { 1. Transfer existing agreements such as licenses, CRADAs, } \\
\text { Patents, Copy Rights, Trademarks, Invention Disclosures. }\end{array}$ & $9 / 30 / 96$ & $\begin{array}{l}\text { B Silva } \\
\text { R Dution }\end{array}$ \\
\hline
\end{tabular}

\subsubsection{Patent, Copyright, Royalty, and Invention activities.}

\begin{tabular}{|l|l|l|}
\hline \multicolumn{1}{|c|}{ Pre-Award Actions } & $\begin{array}{c}\text { Scheduled } \\
\text { Date }\end{array}$ & Actionee(s) \\
\hline $\begin{array}{l}\text { 1. Complete review and provide listing of all patent, copyright, } \\
\text { royalty and invention activity that will be open as of } \\
\text { September 30, 1996. }\end{array}$ & $\begin{array}{c}\text { 5/31/96 } \\
\text { Completed }\end{array}$ & $\begin{array}{l}\text { B Silva } \\
\text { R Dutton }\end{array}$ \\
\hline Post-A ward Actions & $9 / 30 / 96$ & $\begin{array}{l}\text { B Silva } \\
\text { R Dutton }\end{array}$ \\
\hline \begin{tabular}{l} 
1. Transfer activities as is appropriate to new contractor(s). \\
\hline
\end{tabular}
\end{tabular}

\subsubsection{Asset Conversion Activities.}

\begin{tabular}{|r|c|c|}
\hline \multicolumn{1}{|r|}{\begin{tabular}{r|r|} 
Pre-Award Actions \\
1. Provide status of open asset conversion projects.
\end{tabular}} & $\begin{array}{c}\text { Scheduled } \\
\text { Date }\end{array}$ & Actionee(s) \\
\hline Post-Award Actions & $\begin{array}{c}5 / 31 / 96 \\
\text { Completed }\end{array}$ & B Silva \\
\hline 1. Update status of open asset conversion projects. & & \\
\hline
\end{tabular}




\begin{tabular}{|c|c|c|}
\hline Post-Award Actions & $\begin{array}{c}\text { Scheduled } \\
\text { Date }\end{array}$ & \multicolumn{1}{c|}{ Actionee(s) } \\
\hline 2. Transfer activities as is appropriate to new contractor(s). & $9 / 30 / 96$ & B Silva \\
\hline
\end{tabular}

\subsubsection{Activities with DOE (RL \& HQ).}

\begin{tabular}{|c|c|c|}
\hline Pre-Award Actions & $\begin{array}{l}\text { Scheduled } \\
\text { Date }\end{array}$ & Actionee(s) \\
\hline 1. Compile list of actions/initiatives with DOE. & $\begin{array}{c}5 / 31 / 96 \\
\text { Completed }\end{array}$ & B Silva \\
\hline Post-Award Actions & & $\therefore$ \\
\hline 1. Update list of actions/initiatives with DOE. & $8 / 09 / 96$ & B Silva \\
\hline
\end{tabular}

\subsubsection{Materials Management}

\section{Materials Management Specific Assumptions}

- Some Materials Management efforts will have to be curtailed during the twomonth transition. Expected impact will be closure of the excess yard, suspension of the gift and community redeployment efforts and special delivery requirements such as the year-end computer change out.

\subsubsection{P-Card Assignment/Transfer to One or More New Companies.}

\begin{tabular}{|c|c|c|}
\hline Pre-Award Actions & $\begin{array}{l}\text { Scheduled } \\
\quad \text { Date }\end{array}$ & Actionee(s) \\
\hline 1. Develop assignment clause or letter. & $\begin{array}{l}5 / 31 / 96 \\
\text { Completed }\end{array}$ & R Dahlin \\
\hline Post-Award Actions & & $\because$ \\
\hline $\begin{array}{l}\text { 1. Provide a listing of current card holders and outstanding } \\
\text { transaction balance per organization. }\end{array}$ & $8 / 09 / 96$ & R Dahlin \\
\hline $\begin{array}{l}\text { 2. Determine P-card split and financial liability splits between new } \\
\text { companies. }\end{array}$ & $9 / 01 / 96$ & PHMC \\
\hline 3. Implement transfer. & $9 / 30 / 96$ & $\begin{array}{l}\text { PHMC } \\
\text { R Dahlin } \\
\text { C Mayo }\end{array}$ \\
\hline
\end{tabular}




\subsubsection{Surplus Sales Assignment/Transfer to One or More New Companies.}

\begin{tabular}{|l|c|l|}
\hline \multicolumn{1}{|c|}{ Pre-Award Actions } & $\begin{array}{c}\text { Scheduled } \\
\text { Date }\end{array}$ & \multicolumn{1}{c|}{ Actionee(s) } \\
\hline 1. Develop assignment clause or letter. & $\begin{array}{c}5 / 31 / 96 \\
\text { Completed }\end{array}$ & R Dahlin \\
\hline \multicolumn{1}{|c|}{ Post-Award Actions } & $8 / 09 / 96$ & R Dahlin \\
\hline $\begin{array}{l}\text { 1. Provide listing of current sales agreements and transaction } \\
\text { status. }\end{array}$ & $9 / 01 / 96$ & PHMC \\
\hline $\begin{array}{l}\text { 2. Determine which agreement to continue and financial liability } \\
\text { splits between companies. }\end{array}$ & $9 / 30 / 96$ & R Dahlin \\
\hline 3. Implement transfer. & & \\
\hline
\end{tabular}

3.6.4.3 Warehouse Consolidation Plan (gas dock elimination, surplus material move into 1163 and configuration of 1167 into excess store).

\begin{tabular}{|l|c|c|}
\hline \multicolumn{1}{|c|}{ Pre-Award Actions } & $\begin{array}{c}\text { Scheduled } \\
\text { Date }\end{array}$ & Actionee(s) \\
\hline 1. Complete strategy plan. & $\begin{array}{c}5 / 31 / 96 \\
\text { Completed }\end{array}$ & R Dahlin \\
\hline
\end{tabular}

3.6.4.4 General Services Administration (GSA) Waiver Request.

DELETED.

\subsubsection{Activities with DOE (RL \& HQ).}

\begin{tabular}{|l|c|c|}
\hline \multicolumn{1}{|c|}{ Pre-Award Actions } & $\begin{array}{c}\text { Scheduled } \\
\text { Date }\end{array}$ & Actionee(s) \\
\hline 1. List of actions/initiatives with DOE. & $\begin{array}{c}5 / 31 / 96 \\
\text { Completed }\end{array}$ & R Dahlin \\
\hline Post-Award Actions & & \\
\hline 1. Update list of issues/initiatives with DOE. & $9 / 30 / 96$ & R Dahlin \\
\hline
\end{tabular}

\subsubsection{Materials Management Reference Materials.}

\begin{tabular}{|r|c|c|}
\hline Pre-Award Actions & $\begin{array}{c}\text { Scheduled } \\
\text { Date }\end{array}$ & Actionee(s) \\
\hline 1. Assemble set of manuais, procedures, forms and instructions. & $\begin{array}{c}5 / 31 / 96 \\
\text { Completed }\end{array}$ & R Dahlin \\
\hline
\end{tabular}


WHC-SP-1181 Rev. 1

\subsection{RE-ENGINEERING}

\section{Assumptions}

- Re-engineering of the Hanford Site will continue through the award and transition periods.

- Re-engineered facilities and processes will be "contractor proof" in that they will maintain their re-engineered structure and redesigned work processes by the

. PHMC.

Re-engineering is a fundamental rethinking and redesign of the business processes to achieve dramatic improvements in safety, performance, speed, service and cost. At Hanford, WHC, ICF KH, and BCSR, re-engineering has been or is being undertaken in three major areas: .

- Support and administration areas that provide services to virtually all components of the company.

Examples: Finance, Communications, Infrastructure, Procurement, Contracts, General Counsel, Information Technology, etc.

- Plants/Facilities: PUREX, Fast Flux Test Facility (FFTF), PFP, Solid Waste, T-Plant, B-Plant, SNF, TWRS (West Tanks, East Tanks, Evaporator, Characterization).

- Requirements Management: Safety, Quality, Environmental, S/Rids, RadCon, Emergency Preparedness, etc. To date, re-engineering has been implemented at one plant, PUREX, and is in progress at two other facilities, B-Plant and West Tank Farms.

A draft schedule shows that during calendar year 1996, a total of seven facilities/plants will be re-engineered. Plant re-engineering includes information technology redesign (evaluate JCS work control, project scheduling, etc.), evaluating and defining the culture and the values, redesign of the Management Systems including the performance metrics, defining a new and flatter organization (designing cross functional work teams), developing streamlined work processes, and defining new job descriptions including skills and responsibilities.

In the Support and Administration area, re-engineering has played a major role in setting up a CIO function, streamlining the supply chain function, reducing the size and number of computing resources, simplifying and standardizing department overhead costing, restructuring of the finance organization into a teaming arrangement, aligning infrastructure services to commercial practices, providing communications planning and support for reengineering, alignment of the Human Resources with the re-engineered functions and improving the planning, integration and control process. 
In the Requirements Management area, COEs are being set up in four major areas (RadCon, Environmental, Safety, Quality). These centers are staffed on a part-time basis with Plant people to ensure Plants play a major upfront ownership role in the requirements arena with a corresponding major downsizing of centralized organizations.

\begin{tabular}{|l|c|l|}
\hline \multicolumn{1}{|c|}{ Post-Award Actions } & $\begin{array}{c}\text { Scheduled } \\
\text { Date }\end{array}$ & Actionee(s) \\
\hline $\begin{array}{l}\text { 1. Provide detailed re-tngineering presentation including path } \\
\text { forward. }\end{array}$ & $\begin{array}{c}8 / 15 / 96- \\
8 / 30 / 96\end{array}$ & RJ Bliss \\
\hline $\begin{array}{l}\text { 2. Provide lists and location of all re-engineering documents and } \\
\text { files. }\end{array}$ & $8 / 15 / 96$ & RG Slocum \\
\hline
\end{tabular}

\subsection{TECHNOLOGY MANAGEMENT}

The M\&O Contractor participation in the National Program is twofold; (1) participates in performing technology development tasks that are near or at the implementation phase and (2) performs an integration role between providers of technology and the potential end users (projects) by functioning as part of and in support of the Site Technology Coordination Group (STCG).

\subsubsection{National Technology Development Program (EM-50)}

The EM-50 Program is an applied technology program with a strong focus on implementing technology. The WHC role is primarily implementation accomplished by direct involvement in the final phases of technology development (Engineering Development and Implementation). This involvement is limited to those technologies that the National Program views WHC to be the most appropriate contractor to complete the final phases of an assigned technology. As many as 22 other contractors are also involved in similar efforts at their sites requiring WHC and other Site contractors to interface broadly with the technology community to ensure technology is appropriately considered. Section 3.8.2, "Technology

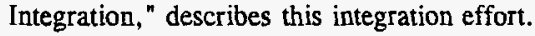

The EM-50 Program at Hanford is managed by a joint contractor team with representation from BHI, PNNL, and WHC. The team interfaces routinely with the RL Technology Program Officer of the Science and Technology Programs Division.

In addition to WHC participation on the joint contractor management team, WHC manages tasks defined by Technical Task Plans (TTPs) with a combined budget of $\$ 7.6 \mathrm{M}$.

The tasks are managed by a WHC Program Office with a budget allocated from each TTP. Participation on the joint contractor team is direct funded by RL providing funding for administrative and management support to the joint team. These functions are defined in a RL EM-50 Program Management Plan. 


\begin{tabular}{|c|c|c|}
\hline Pre-Award Actions & $\begin{array}{c}\text { Scheduled } \\
\text { Date }\end{array}$ & Actionee(s) \\
\hline $\begin{array}{l}\text { 1. Using the PHMC RFP as a basis, review each currently } \\
\text { assigned technology project with RL and determine if it fits } \\
\text { the role of the PHMC as described in Section C. }\end{array}$ & $\begin{array}{c}4 / 19 / 96 \\
\text { Completed }\end{array}$ & JD Berger \\
\hline $\begin{array}{l}\text { 2. Determine the appropriate course of action, assisted by the } \\
\mathrm{RL} \text { Technology Program Officer, for disposition of } \\
\text { technology projects that do not fit the defined PHMC role. }\end{array}$ & $\begin{array}{l}5 / 03 / 96 \\
\text { Completed }\end{array}$ & JD Berger \\
\hline $\begin{array}{l}\text { 3. Assemble key program (or project) documentation, or } \\
\text { identify its location, for use by the PHMC. Includes } \\
\text { development of a program/project "Smart Book," including } \\
\text { listings and descriptions developed below. }\end{array}$ & $\begin{array}{c}\text { 6/01/96 } \\
\text { Completed } \\
\text { "Smart Book" } \\
\text { only } \\
\text { See item } \# 10\end{array}$ & JD Berger \\
\hline $\begin{array}{l}\text { 4. Identify number of briefings, and prepare program (or } \\
\text { project) briefing(s) for presentation to PHMC (coordinate } \\
\text { number and content with RL POC). }\end{array}$ & $7 / 15 / 96$ & JD Berger \\
\hline $\begin{array}{l}\text { 5. Develop a listing of key program (or project) individuals as } \\
\text { POCs, including facility and operations personnel. }\end{array}$ & $\begin{array}{l}6 / 01 / 96 \\
\text { Completed }\end{array}$ & JD Berger \\
\hline $\begin{array}{l}\text { 6. Develop listing and description of program (or project) } \\
\text { subcontracts, including faciitity (or operations) subcontracts. } \\
\text { Include any subcontracts still planned to be executed prior to } \\
\text { contract takeover. }\end{array}$ & $\begin{array}{l}\text { 6/01/96 } \\
\text { Completed }\end{array}$ & JD Berger \\
\hline $\begin{array}{l}\text { 7. Develop listing and description of program (or project) } \\
\text { databases, including facility (or operations) databases. }\end{array}$ & $\begin{array}{c}\text { 6/01/96 } \\
\text { Completed }\end{array}$ & JD Berger \\
\hline $\begin{array}{l}\text { 8. Identify space and equipment within program (or project) } \\
\text { area and facilities for use by the PHMC. }\end{array}$ & $7 / 15 / 96$ & JD Berger \\
\hline $\begin{array}{l}\text { 9. Establish a schedule for the actions defined above in } \\
\text { dispositioning technology projects that do not fit the role of } \\
\text { the PHMC. }\end{array}$ & $7 / 15 / 96$ & JD Berger \\
\hline 10. Update/submit "Smart Book"/assemble key documents. & $7 / 15 / 96$ & JD Berger \\
\hline Post-Award Actions & & \\
\hline $\begin{array}{l}\text { 1. Provide the PHMC access to the key program (or project) } \\
\text { documentation, any program level generated pre-existing } \\
\text { conditions information, listing of POCs, and listing of } \\
\text { subcontracts. Provide copies upon request. }\end{array}$ & $8 / 05 / 96$ & JD Berger \\
\hline $\begin{array}{l}\text { 2. Program/project and facility space and services available for } \\
\text { use by the PHMC. }\end{array}$ & $8 / 05 / 96$ & JD Berger \\
\hline $\begin{array}{l}\text { 3. Provide a review briefing to the PHMC, or the designated } \\
\text { subcontractor, covering pertinent aspects of the technology } \\
\text { projects being transferred. }\end{array}$ & $\begin{array}{l}8 / 12 / 96- \\
8 / 30 / 96\end{array}$ & JD Berger \\
\hline $\begin{array}{l}\text { 4. Host a tour of each technology project to familiarize the } \\
\text { PHMC with each project including current cost, scope and } \\
\text { schedule. }\end{array}$ & $\begin{array}{l}8 / 12 / 96- \\
8 / 30 / 96\end{array}$ & JD Berger \\
\hline $\begin{array}{l}\text { 5. Discuss the concept of the joint contractor technology } \\
\text { management team including the role of the PHMC. } \\
\text { Introduce PHMC personnel to the individuals making up the } \\
\text { leam and to the Program Management Plan that describes } \\
\text { the activities the team is responsible for. }\end{array}$ & $9 / 15 / 96$ & JD Berger \\
\hline
\end{tabular}




\subsubsection{Technology Integration (Implementation), Site Technology Coordination Group Support (STCG)}

The Technology Integration role functions as part of the joint $\mathrm{RL}$ contractor team and provides a major benefit to the contractor making decisions on end use of technology. The personnel filling this role facilitate a technology user organization connection with the providers of technology (EM-50 and others) and the projects that can potentially benefit. It is a "brokering" or facilitation role where candidate technologies are identified and "providers" are connected to "users." This effort functions as part of the STCG and is further facilitated by a direct tie to the Site Technical Integration effort and the Systems Engineering approach.

\begin{tabular}{|l|c|l|}
\hline \multicolumn{1}{|c|}{ Pre-Award Actions } & $\begin{array}{c}\text { Scheduled } \\
\text { Date }\end{array}$ & Actionee(s) \\
\hline $\begin{array}{l}\text { 1. Assemble key program (or project) documentation, or } \\
\text { identify its location, for use by the PHMC. Includes } \\
\text { development of a program/project "Smart Book," including } \\
\text { listings and descriptions developed below. }\end{array}$ & $\begin{array}{c}6 / 01 / 96 \\
\text { Completed } \\
\text { "Smart Book" } \\
\text { only } \\
\text { See item \# }\end{array}$ & JD Berger \\
\hline $\begin{array}{l}\text { 2. Identify number of briefings, and prepare program (or } \\
\text { project) briefing(s) for presentation to PHMC. }\end{array}$ & $7 / 15 / 96$ & JD Berger \\
\hline $\begin{array}{l}\text { 3. Develop a listing of key program (or project) individuals as } \\
\text { POCs, including facility and operations personnel. }\end{array}$ & $\begin{array}{c}6 / 01 / 96 \\
\text { Completed }\end{array}$ & JD Berger \\
\hline $\begin{array}{l}\text { 4. Identify space and equipment within program (or project) } \\
\text { area and facilities for use by the PHMC. }\end{array}$ & $7 / 15 / 96$ & JD Berger \\
\hline 5. Update/submit "Smart Book"/assemble key documents. & $7 / 15 / 96$ & JD Berger \\
\hline $\begin{array}{l}\text { Post-Award Actions } \\
\text { 1. Provide the PHMC access to the key program (or project) } \\
\text { documentation, any program level generated pre-existing } \\
\text { conditions information, listing of POCs, and listing of } \\
\text { subcontracts. Provide copies upon request. }\end{array}$ & $8 / 05 / 96$ & JD Berger \\
\hline $\begin{array}{l}\text { 2. Program/project and facility space and services available for } \\
\text { use by the PHMC. }\end{array}$ & $8 / 05 / 96$ & JD Berger \\
\hline $\begin{array}{l}\text { 3. Provide program/project briefing(s) to the PHMC. } \\
\text { 4. Provide program/project level tour to the PHMC. }\end{array}$ & $\begin{array}{l}8 / 12 / 96- \\
8 / 30 / 96\end{array}$ & JD Berger \\
\hline
\end{tabular}




\subsection{GENERAL COUNSEL}

The General Counsel area includes Contract Administration and Legal matter for WHC. This section will outline the transitional requirements for both sections of the Office of General Counsel. The completion of a comprehensive Transition Agreement which will cover the transfer of WHC's obligations and liabilities to the PHMC or RL, and which rights to access records, information, and former employees in the defense of any retained litigation or in the event of post-transition litigation and/or claims, is considered the critical item that must be accomplished prior the completion of the transition process.

\subsubsection{Litigation and Claims}

\begin{tabular}{|l|l|l|}
\hline \multicolumn{1}{|c|}{ Post-Award Actions } & $\begin{array}{c}\text { Scheduled } \\
\text { Date }\end{array}$ & Actionee(s) \\
\hline $\begin{array}{l}\text { 1. Prepare summary of status of litigation matters, } \\
\text { administration hearings, arbitrations and subcontractor claims } \\
\text { and a recommendation for transfer of some or ail of these } \\
\text { matter to PHMC. Provide summary and recommendation to } \\
\text { RL Office of Chief Counsel. }\end{array}$ & $8 / 15 / 96$ & RA Dutton \\
\hline $\begin{array}{l}\text { 2. Negotiate transfer of some or all of the litigation, } \\
\text { administrative hearing, arbitrations and subcontractor claims } \\
\text { to PHMC including transfer of files and associated documents } \\
\text { and evidence. }\end{array}$ & $9 / 15 / 96$ & RA Dutton \\
\hline
\end{tabular}

\subsubsection{Transition Agreement}

\begin{tabular}{|c|c|c|}
\hline Post-Award Actions & $\begin{array}{c}\text { Scheduled } \\
\text { Date }\end{array}$ & Actionee(s) \\
\hline $\begin{array}{l}\text { 1. Conduct discussion on scope and terms of Transition } \\
\text { Agreement with PHMC contractor counsel and RL Office of } \\
\text { Chief Counsel and others as may be necessary. }\end{array}$ & $8 / 28 / 96$ & RA Dutton \\
\hline $\begin{array}{l}\text { 2. Prepare a draft of Transition Agreement and circulate for } \\
\text { comment. }\end{array}$ & $9 / 12 / 96$ & RA Dutton \\
\hline $\begin{array}{l}\text { 3. Assemble all documents to be incorporated into Transition } \\
\text { Agreernent. }\end{array}$ & $9 / 26 / 96$ & $\begin{array}{l}\text { RA Dutton } \\
\text { JK Knoll }\end{array}$ \\
\hline 4. Negotiate, Finalize and Execute Agreement. & $9 / 30 / 96$ & $\begin{array}{l}\text { RA Dutton } \\
\text { PHMC } \\
\text { DOE }\end{array}$ \\
\hline
\end{tabular}




\subsection{INTERNAL AUDIT}

\begin{tabular}{|l|c|c|}
\hline \multicolumn{1}{|c|}{ Pre-Award Actions } & $\begin{array}{c}\text { Schednle } \\
\text { Date }\end{array}$ & Actionee(s) \\
\hline $\begin{array}{l}\text { 1. Assemble current organizational data (charts and charters). } \\
\text { Completed }\end{array}$ & L/31/96 Greenberg \\
\hline 2. Prepare FY 1997 Audit Plan and obtain RL approval. & $7 / 15 / 96$ & LH Greenberg \\
\hline $\begin{array}{l}\text { 3. Identify and stans all open audit findings. } \\
\text { Post-Award Actions }\end{array}$ & $\begin{array}{c}5 / 31 / 96 \\
\text { Completed }\end{array}$ & LH Greenberg \\
\hline $\begin{array}{l}\text { 1. Brief PHMC on overview of Internal Audit functions } \\
\text { - Audit organizational status } \\
\text { - M\&O Contractor \& OlG Cooperative Audit Strategy } \\
\text { - Open audit findings } \\
- \text { FY 1997 Audit Plan. }\end{array}$ & $8 / 12-8 / 30 / 96$ & LH Greenberg \\
\hline 2. Complete FY 1996 Audit Plan. & & \\
\hline 3. Transfer actions to PHMC. & $9 / 30 / 96$ & LH Greenberg \\
\hline
\end{tabular}

\subsection{WORK FOR OTHERS}

Work For Others is established and meets the requirements as called for in Clauses $\mathrm{H}-17$ and $\mathrm{H}-18$ of the Model Contract. There are over 161 active orders with DOE, other government entities (local, state, and Federal), U.S. commercial and foreign businesses. These active orders have a total value of more than $\$ 99.0 \mathrm{M}$ and provide cost savings by helping lower overhead costs.

\begin{tabular}{|l|c|l|}
\hline \multicolumn{1}{|c|}{ Pre-Award Actions } & $\begin{array}{c}\text { Scheduled } \\
\text { Date }\end{array}$ & \multicolumn{1}{|c|}{ Actionee(s) } \\
\hline $\begin{array}{l}\text { 1. Complete/finalize desk procedures to include transition } \\
\text { actions. }\end{array}$ & $\begin{array}{c}5 / 03 / 96 \\
\text { Completed }\end{array}$ & $\begin{array}{l}\text { AT Broady } \\
\text { I.M.Knoll }\end{array}$ \\
\hline $\begin{array}{l}\text { 2. Update and complete active listing of WFO orders. } \\
\text { Completed }\end{array}$ & $\begin{array}{l}\text { AT Broady } \\
\text { JM Knoll }\end{array}$ \\
\hline $\begin{array}{l}\text { 3. Develop sponsor address listing and draft assignment } \\
\text { notification to sponsors. }\end{array}$ & $\begin{array}{c}5 / 17 / 96 \\
\text { Completed }\end{array}$ & $\begin{array}{l}\text { AT Broady } \\
\text { JM Knoll }\end{array}$ \\
\hline $\begin{array}{l}\text { 4. Review how transitions could impact assigned WFo } \\
\text { personnel to determine potential adverse affects. }\end{array}$ & $\begin{array}{c}5 / 03 / 96 \\
\text { Completed }\end{array}$ & $\begin{array}{l}\text { AT Broady } \\
\text { JM Knoll }\end{array}$ \\
\hline $\begin{array}{l}\text { 5. Prepare Briefing and orientation for newly assigned } \\
\text { personnel. }\end{array}$ & $\begin{array}{c}5 / 24 / 96 \\
\text { Completed }\end{array}$ & $\begin{array}{l}\text { AT Broady } \\
\text { JM Knoll }\end{array}$ \\
\hline $\begin{array}{l}\text { Post-Award Actions } \\
\text { 1. Transmit assignment notifications to sponsors. }\end{array}$ & $8 / 30 / 96$ & $\begin{array}{l}\text { AT Broady } \\
\text { JM Knoll }\end{array}$ \\
\hline 2. Review and revise procedures to include new contractor. & $9 / 05 / 96$ & $\begin{array}{l}\text { AT Broady } \\
\text { SM Knoll }\end{array}$ \\
\hline
\end{tabular}




\begin{tabular}{|l|c|l|}
\hline \multicolumn{1}{|c|}{ Post-Award Actions } & $\begin{array}{c}\text { Scheduled } \\
\text { Date }\end{array}$ & \multicolumn{1}{|c|}{ Actionee(s) } \\
\hline $\begin{array}{l}\text { 3. Transfer all WFO files and listings to newly assigned } \\
\text { personnel. }\end{array}$ & $9 / 30 / 96$ & $\begin{array}{l}\text { AT Broady } \\
\text { MM Knoll }\end{array}$ \\
\hline
\end{tabular}

\subsubsection{Support to EM-40 Headquarters}

Technical support is provided to the DOE HQ EM-40 Program in the areas of management systems development, regulatory analysis, programmatic objective implementation, and environmental compliance review. The support provided by the $M \& O$ Contractor is supplemented with additional technical expertise, when required, utilizing existing contracts. Funding for this effort comes directly from HQ via an HQ ADS. Reporting is directly to HQ with information copies to RL EM-40 Program Manager.

\begin{tabular}{|c|c|c|}
\hline Pre-Award Actions & $\begin{array}{c}\text { Scheduled } \\
\text { Date }\end{array}$ & Actionee(s) \\
\hline $\begin{array}{l}\text { 1. Discuss description of the out year (FY } 1997 \text { plus) } \\
\text { expectation for this effort with HQ. }\end{array}$ & $\begin{array}{l}6 / 01 / 96 \\
\text { Completed }\end{array}$ & ID Berger \\
\hline $\begin{array}{l}\text { 2. Assemble key program (or project) documentation, or } \\
\text { identify its location, for use by the PHMC. Includes } \\
\text { development of a program/project "Smart Book," including } \\
\text { listings and descriptions developed below. }\end{array}$ & $\begin{array}{c}\text { 6/01/96 } \\
\text { Completed } \\
\text { "Smart Book" } \\
\text { only } \\
\text { See item \#5 }\end{array}$ & JD Berger \\
\hline $\begin{array}{l}\text { 3. Identify number of briefings, and prepare program (or } \\
\text { project) briefing(s) for presentation to PHMC. }\end{array}$ & $7 / 15 / 96$ & JD Berger \\
\hline $\begin{array}{l}\text { 4. Develop a listing of key program (or project) individuals as } \\
\text { POCs, including facility and operations personnel. }\end{array}$ & $\begin{array}{l}6 / 01 / 96 \\
\text { Completed }\end{array}$ & JD Berger \\
\hline 5. Update/submit "Smart Book"/assemble key documents. & $7 / 15 / 96$ & JD Berger \\
\hline \multicolumn{3}{|l|}{ Post-Award Actions } \\
\hline $\begin{array}{l}\text { 1. Provide the PHMC access to the key program (or project) } \\
\text { documentation, any program level generated pre-existing } \\
\text { conditions information, listing of POCs, and listing of } \\
\text { subcontracts. Provide copies upon request. }\end{array}$ & $8 / 05 / 96$ & JD Berger \\
\hline $\begin{array}{l}\text { 2. Provide year-to-date reports to the PHMC documenting work } \\
\text { performed. Brief the PHMC on all aspect of this work. }\end{array}$ & $\begin{array}{l}\text { 8/19/96- } \\
9 / 02 / 96 \\
\end{array}$ & ID Berger \\
\hline $\begin{array}{l}\text { 3. Meet with key individuals, both contractor and DOE, to } \\
\text { receive briefings on work status and furure expectations. }\end{array}$ & $\begin{array}{l}8 / 19 / 96- \\
9 / 02 / 96\end{array}$ & JD Berger \\
\hline
\end{tabular}

\subsubsection{Support to EM - Lease and Administration of Contractor Support Facility}

DELETED (Note: DOE-HQ to terminate lease on support facility in FY 1996.) 


\subsection{OTHER MANAGEMENT AND INTEGRATION ACTIVITIES}

\subsubsection{Pollution Prevention/Waste Minimization}

The Pollution Prevention/Waste Minimization (P2/WMin) Program is implemented to meet Federal, State, and DOE regulations, policies, and reporting requirements. WHC provides planning, management, reporting, and oversight support for all $\mathrm{P} 2 / \mathrm{WM}$ in on the Hanford Site. The activities include P2/WMin sitewide coordination, planning, reporting, training, awareness, assessments, incentives, maintenance of tracking systems, toxics reduction, recycling and affirmative procurement programs. A Center of Excellence has been formed within WHC, at RL direction, to provide a streamlined, low-cost enabler of $\mathrm{P} 2 /$ WMin activities across the site. Its purpose is to enable contractor/project generators to actively seek and implement initiatives to eliminate waste generation.

The P2/WMin Program established at Hanford is seen as a leader in the DOE complex and received 4 of the 10 national awards in 1995, as best in class. P2/WMin is DOE's preferred approach to EM because of its many cost and environmental benefits. In FY 1995, the Program documented a total of $\$ 26.4 \mathrm{M}$ in pollution prevention savings, and avoided generation of over 4.3 million kilograms of sanitary waste, 206,000 kilograms of hazardous waste, 32,000 cubic meters of radioactive waste, as well expenditure of 296,000 kilowatt hours of energy.

The P2/WMin FY 1996 MYPP provides baseline requirements, milestones, and regulatory drivers. P2/WMin Program meets the requirements of the RFP DE-RP06-06RL13200, Sections B.1.b (Page C-15) and F (Page C-33).

\begin{tabular}{|c|c|c|}
\hline Pre-Award Actions & $\begin{array}{l}\text { Scheduted } \\
\text { Date }\end{array}$ & Actionee(s) \\
\hline $\begin{array}{l}\text { 1. Develop site guide to WHC, ICF KH, BCSR, PNNL and } \\
\text { ERC P2/WMin documents/records and reports. }\end{array}$ & $\begin{array}{c}5 / 15 / 96 \\
\text { Completed }\end{array}$ & $\begin{array}{l}\text { JM Knoli, Jr. } \\
\text { JR Kirkendall }\end{array}$ \\
\hline $\begin{array}{l}\text { 2. Prepare a briefing on overall requirements/drivers/funding on } \\
\text { P2/WMin. }\end{array}$ & $\begin{array}{l}5 / 24 / 96 \\
\text { Completed }\end{array}$ & $\begin{array}{l}\text { JM Knoll, Jr. } \\
\text { JR Kirkendall }\end{array}$ \\
\hline Post-Award Actions & & \\
\hline $\begin{array}{l}\text { 1. Prepare and present a management overview of the Pollution } \\
\text { Prevention/Waste Minimization Program to the PHMC. }\end{array}$ & $\begin{array}{l}8 / 08 / 96- \\
8 / 19 / 96\end{array}$ & $\begin{array}{l}\text { JM Knoll, Jr. } \\
\text { JR Kirkendall }\end{array}$ \\
\hline $\begin{array}{l}\text { 2. Prepare and present a comprehensive briefing to the PHMC } \\
\text { on the Pollution Prevention/Waste Minimization Program, } \\
\text { including overall requirements/drivers, points of contact, } \\
\text { documentation guide, Pollution Prevention Opportunity } \\
\text { Assessment (P20A) process, program implementation and } \\
\text { waste disposal chargeback pilot implementation. }\end{array}$ & $\begin{array}{l}8 / 12 / 96- \\
8 / 30 / 96\end{array}$ & $\begin{array}{l}\text { JM Knoll, Jr. } \\
\text { JR Kirkendall }\end{array}$ \\
\hline
\end{tabular}




\subsubsection{U.S. Department of Energy Directives}

The current RFP clause H.14 covers the model contract requirements for Laws, Regulations, and DOE Directives. In turn, this clause describes how the new contractor will be expected to comply/integrate the DOE Directives. The listing of DOE Directives for compliance is contained in Section J, Appendix C of the RFP. There currently exists a process/system that receives the directives from the Contracting Officer, identifies the organization responsible for implementation, maintains the company DOE assigned database and works with organizations in preparing and submitting waivers requests to DOE.

\begin{tabular}{|c|c|c|}
\hline Pre-Award Actions & $\begin{array}{l}\text { Scheduled } \\
\text { Date }\end{array}$ & Actionee(s) \\
\hline $\begin{array}{l}\text { 1. Compare current listing of Directives that are for WHC } \\
\text { compliance with the listing in the RFP and identify a listing } \\
\text { of differences. }\end{array}$ & $\begin{array}{l}5 / 31 / 96 \\
\text { Completed }\end{array}$ & $\begin{array}{l}\text { AT Broady } \\
\text { JM Knoll, Jr. }\end{array}$ \\
\hline $\begin{array}{l}\text { 2. Complete listing of active waivers (deviations/exemptions), } \\
\text { and pending waivers. }\end{array}$ & $\begin{array}{c}5 / 17 / 96 \\
\text { Completed } \\
\end{array}$ & \begin{tabular}{|l|} 
AT Broady \\
JM Knoll, Jr.
\end{tabular} \\
\hline $\begin{array}{l}\text { 3. Using listing developed under item } 1 \text { above and the } \\
\text { information regarding organizations in the RFP identify } \\
\text { proposed organizations that will be responsible for unassigned } \\
\text { directives. }\end{array}$ & $7 / 10 / 96$ & $\begin{array}{l}\text { AT Broady } \\
\text { SM Knoll, Jr.. }\end{array}$ \\
\hline 4. Revise, as required, the Directives Desk Procedures. & $7 / 10 / 96$ & $\begin{array}{l}\text { AT Broady } \\
\text { M Knoll, Jr. }\end{array}$ \\
\hline \multicolumn{3}{|l|}{ Post-Award Actions } \\
\hline $\begin{array}{l}\text { 1. Conduct briefing to newly assigned personnel on files and } \\
\text { listings pertaining to the administration of the Directives } \\
\text { including delta developed under No. } 1 \text { of Pre-Award actions. }\end{array}$ & $9 / 20 / 96$ & $\begin{array}{l}\text { AT Broady } \\
\text { JM Knoll, Jr. }\end{array}$ \\
\hline $\begin{array}{l}\text { 2. Transfer all completed files and listings pertaining to } \\
\text { Directives. }\end{array}$ & 9/30/96 & $\begin{array}{l}\text { AT Broady } \\
\text { JM Knoll, Jr. }\end{array}$ \\
\hline $\begin{array}{l}\text { 3. Conduct training on the Directives Desk Procedures and } \\
\text { operation and maintenance of database. }\end{array}$ & $9 / 20 / 96$ & $\begin{array}{l}\text { AT Broady } \\
\text { JM Knoll, Jr. }\end{array}$ \\
\hline
\end{tabular}

\subsubsection{Hanford PeopleCORE}

Hanford PeopleCORE is a sitewide central database that collects information about Hanford RL and contractor people. It is the official source for distributing the information to over 100 key applications (e.g., site phone directory, medical records). Hanford PeopleCORE provides a single point for data collection, maintenance, and dissemination of information for all Hanford contractors and services. 
WHC-SP-1181 Rev. 1

\begin{tabular}{|c|c|c|}
\hline Pre-Award Actions & $\begin{array}{l}\text { Scheduled } \\
\text { Date }\end{array}$ & Actionee(s) \\
\hline 1. Publish a Hanford PeopleCORE Contractor's User's Guide. & $\begin{array}{l}5 / 29 / 96 \\
\text { Completed }\end{array}$ & $\begin{array}{l}\text { MK Britton } \\
\text { MM Knoll, Jr. }\end{array}$ \\
\hline Post-Award Actions & & \\
\hline $\begin{array}{l}\text { 1. Enter new PHMC employees into PeopleCORE as soon as } \\
\text { they are identified. }\end{array}$ & $\begin{array}{l}8 / 01 / 96- \\
8 / 09 / 96\end{array}$ & $\begin{array}{l}\text { MK Britton } \\
\text { MM Knoll, Jr. }\end{array}$ \\
\hline $\begin{array}{l}\text { 2. Brief all new contractors and major subcontractors on } \\
\text { Hanford Site's PeopleCORE system and the site's utilization, } \\
\text { including feeder requirements to Hanford systems. }\end{array}$ & $\begin{array}{l}8 / 12 / 96- \\
8 / 30 / 96\end{array}$ & $\begin{array}{l}\text { MK Brition } \\
\text { JM Knoll, Ir. }\end{array}$ \\
\hline $\begin{array}{l}\text { 3. Identify issues, level of support, resources necessary to } \\
\text { develop data interfaces and processes for new contractor } \\
\text { people information. }\end{array}$ & $8 / 15 / 96$ & $\begin{array}{l}\text { MK Britton } \\
\text { JM Knoll, Ir. }\end{array}$ \\
\hline $\begin{array}{l}\text { 4. Provide training and documentation to ensure a smooth } \\
\text { transition of Hanford PeopleCORE administrative } \\
\text { responsibilities and requirements. }\end{array}$ & $9 / 30 / 96$ & $\begin{array}{l}\text { MK Britton } \\
\text { JM Knoll, Ir. }\end{array}$ \\
\hline
\end{tabular}


WHC-SP-1181 Rev. 1

This page intentionally left blank. 


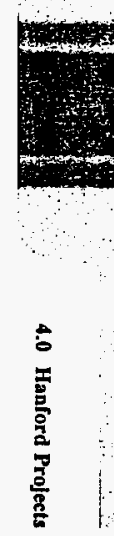




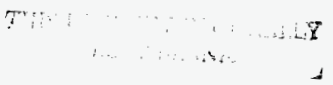




\subsection{HANFORD PROJECTS}

\subsection{TANK WASTE REMEDIATION SYSTEM}

TWRS manages the waste tank farms and the present and future tank waste disposal facilities. TWRS manages the operation of facilities associated with the receipt, containment, storage, and handling of liquid high-level wastes (HLWs) at Hanford, excluding some of those facilities generating the waste. In addition, TWRS develops and executes a program for the characterization, retrieval, pretreatment, and treatment for disposal of single-shell tank (SST) and double-shell tank (DST) wastes.

\begin{tabular}{|c|c|c|}
\hline Pre-Award Actions & $\begin{array}{l}\text { Scheduled } \\
\text { Date }\end{array}$ & Actionee(s) \\
\hline $\begin{array}{l}\text { 1. In outline form for all facilities/operations to follow, } \\
\text { define the appropriate contents and level of detail to be } \\
\text { included in facility/operations briefings to the PHMC. } \\
\text { (Review with RL POC.) }\end{array}$ & $\begin{array}{l}5 / 01 / 96 \\
\text { Completed }\end{array}$ & DL Lenseigne \\
\hline $\begin{array}{l}\text { 2. Assemble key program (or project) documentation, or } \\
\text { identify its location, for use by the PHMC. Includes } \\
\text { development of a program "Smart Book," including } \\
\text { listings and descriptions provided from Sections } 4.1 .1 \\
\text { through 4.1.7. }\end{array}$ & $\begin{array}{l}\text { 6/01/96 } \\
\text { Completed "Smart } \\
\text { Book" only } \\
\text { See item } \# 5\end{array}$ & DL Lenseigne \\
\hline $\begin{array}{l}\text { 3. Identify number of briefings, and prepare program (or } \\
\text { project) briefing(s) for presentation to PHMC. } \\
\text { (Coordinate number and content with RL POC.) }\end{array}$ & $7 / 15 / 96$ & DL Lenseigne \\
\hline $\begin{array}{l}\text { 4. Identify space and equipment within program (or project) } \\
\text { area and facilities for use by the PHMC. }\end{array}$ & $\begin{array}{l}6 / 01 / 96 \\
\text { Completed }\end{array}$ & DL Lenseigne \\
\hline 5. Update/submit "Smart Book"/assemble key documents. & $7 / 15 / 96$ & DL Lenseigne \\
\hline \multicolumn{3}{|l|}{ Post-Award Actions } \\
\hline $\begin{array}{l}\text { 1. PHMC identify space and equipment needs within } \\
\text { program/project area and facilities. }\end{array}$ & $8 / 09 / 96$ & PHMC \\
\hline $\begin{array}{l}\text { 2. Provide the PHMC access to the key program (or project) } \\
\text { documentation, any program level generated pre-existing } \\
\text { conditions information, listing of POCs, and listing of } \\
\text { subcontracts. Provide copies upon request. }\end{array}$ & $8 / 05 / 96$ & DL Lenseigne \\
\hline $\begin{array}{l}\text { 3. Provide program/project and facility space and services } \\
\text { for use by the PHMC. }\end{array}$ & $8 / 05 / 96$ & DL Lenseigne \\
\hline 4. Provide program/project briefing(s) to the PHMC. & $8 / 12 / 96-8 / 19 / 96$ & RD Wojtasek \\
\hline 5. Provide program/project level tour to the PHMC. & $8 / 15 / 96-9 / 01 / 96$ & RD Wojtasek \\
\hline
\end{tabular}




\subsubsection{Management Systems}

Manage the overall TWRS Program within funding levels provided by DOE to ensure that WHC business goals and objectives are achieved. Provide program management and other administrative functions required to identify and develop future missions, submit programmatic and financial reports to $\mathrm{RL}$, and control budgets and other assigned resources.

\begin{tabular}{|c|c|c|}
\hline Pre-Award Actions & $\begin{array}{l}\text { Scheduled } \\
\text { Date } \\
\end{array}$ & Actionee(s) \\
\hline $\begin{array}{l}\text { 1. Identify key documents. Provide listing to } \\
\text { program/project level for consolidation and RL review. }\end{array}$ & $\begin{array}{l}5 / 01 / 96 \\
\text { Completed }\end{array}$ & WJ Schlauder \\
\hline $\begin{array}{l}\text { 2. Develop a listing of key program/project individuals as } \\
\text { POCs. Input to consolidated program listing. }\end{array}$ & $\begin{array}{c}5 / 15 / 96 \\
\text { Completed }\end{array}$ & WJ Schlauder \\
\hline $\begin{array}{l}\text { 3. Develop a listing and description of subcontracts } \\
\text { supporting the program/project and input to } \\
\text { consolidated program/project listing. Include any } \\
\text { subcontracts planned to be executed prior to contract } \\
\text { takeover. }\end{array}$ & $\begin{array}{l}5 / 15 / 96 \\
\text { Completed }\end{array}$ & DL Lenseigne \\
\hline $\begin{array}{l}\text { 4. Develop a listing and description of databases. Provide } \\
\text { list to program/project level for consolidation. }\end{array}$ & $\begin{array}{c}5 / 15 / 96 \\
\text { Completed }\end{array}$ & $\begin{array}{l}\text { CE Wilson III } \\
\text { JD Thomson }\end{array}$ \\
\hline $\begin{array}{l}\text { 5. Identify number of briefings, and prepare briefing(s) } \\
\text { for the PHMC. (Coordinate number and content with } \\
\text { RL POC.) }\end{array}$ & $7 / 15 / 96$ & WJ Schlauder \\
\hline $\begin{array}{l}\text { 6. Assemble key documentation, or identify its location, } \\
\text { for use by the PHMC. Includes preparation of a } \\
\text { "Smart Book," to include information developed in the } \\
\text { following actions. }\end{array}$ & $\begin{array}{l}6 / 01 / 96 \\
\text { Completed "Smart } \\
\text { Book" only } \\
\text { See item } \# 12 \\
\end{array}$ & WJ Schlauder \\
\hline $\begin{array}{l}\text { 7. Develop a list of open action items and commitments, } \\
\text { including starus (Environmental, Tri-Party Agreement, } \\
\text { DNFSB as examples). Provide to RL POC for review. }\end{array}$ & $\begin{array}{l}\text { 6/01/96 } \\
\text { Completed }\end{array}$ & WJ Schlauder \\
\hline $\begin{array}{l}\text { 8. Develop a list of current work order services or other } \\
\text { form of agreement to provide work for other } \\
\text { organizations, on and offsite. }\end{array}$ & $\begin{array}{c}6 / 01 / 96 \\
\text { Completed }\end{array}$ & WJ Schlauder \\
\hline $\begin{array}{l}\text { 9. Develop a list of current work order services or other } \\
\text { form of agreement to obtain work from other } \\
\text { organizations, on and offsite. }\end{array}$ & $\begin{array}{l}6 / 01 / 96 \\
\text { Completed }\end{array}$ & WJ Schlauder \\
\hline $\begin{array}{l}\text { 10. Verify the accuracy of property assignments for the } \\
\text { facility (or operations) and correct any deficiencies. }\end{array}$ & $\begin{array}{l}6 / 01 / 96 \\
\text { Completed }\end{array}$ & WJ Schlauder \\
\hline $\begin{array}{l}\text { 11. Identify space and equipment within program/project } \\
\text { area and facilities for use by the PHMC. }\end{array}$ & $\begin{array}{l}6 / 01 / 96 \\
\text { Completed }\end{array}$ & WJ Schlauder \\
\hline 12. Update/submit "Smart Book"/assemble key documents. & $7 / 15 / 96$ & WJ Schlauder \\
\hline \multicolumn{3}{|l|}{ Post-Award Actions: } \\
\hline $\begin{array}{l}\text { 1. Provide the PHMC access to key documentation, pre- } \\
\text { existing condition information, commitment data, } \\
\text { inventory data, etc. (as established in the pre-award } \\
\text { phase). Provide copies upon request. }\end{array}$ & $8 / 05 / 96$ & WJ Schlauder \\
\hline
\end{tabular}




\begin{tabular}{|l|c|l|}
\hline \multicolumn{1}{|c|}{ Post-Award Actions } & Scheduled & \multicolumn{1}{|c|}{ Actionee(s) } \\
\hline $\begin{array}{l}\text { 2. Provide any additional documentation or information } \\
\text { requested by the PHMC, if available, that were not } \\
\text { identified during the pre-award phase. }\end{array}$ & by $9 / 01 / 96$ & WJ Schlauder \\
\hline 3. Provide briefing(s) to the PHMC. & $8 / 12 / 96-9 / 02 / 96$ & WJ Schlauder \\
\hline 4. Provide a tour to the PHMC. & $9 / 01 / 96-9 / 15 / 96$ & WJ Schlauder \\
\hline $\begin{array}{l}\text { 5. Provide any recurring reports that the PHMC would } \\
\text { like to receive during transition. }\end{array}$ & by $9 / 15 / 96$ & WJ Schlauder \\
\hline $\begin{array}{l}\text { 6. Provide an updated status of ongoing work prior to } \\
\text { contract turnover. }\end{array}$ & by $9 / 30 / 96$ & WJ Schlauder \\
\hline
\end{tabular}

\subsubsection{Tank Farm Operations - Evaporator}

Ensure environmentally sound operations of the 242-A Evaporator Facility, including all auxiliary equipment associated with handling tank waste for evaporative waste volume reduction. Perform functions required for evaporation of water from dilute waste and support to facilities and processes. These functions include preventative and corrective maintenance, facility concentration operations including waste transfer to feed tanks, surveillance monitoring, health physics activities, industrial hygiene and safety functions, and engineering and analysis. Responsible for managing and controlling upgrades to facilities and infrastructure and enhancing the safety of facility operations.

Responsible for the following facilities:

1. 242-A Evaporator Facility

2. 241-AP Tank Farm

3. 241-AW Tank Farm

4. Liquid Effluent Retention Facility (operation only).

\begin{tabular}{|ll|c|l|}
\hline \multicolumn{1}{|c|}{ Pre-Award Actions } & $\begin{array}{c}\text { Scheduled } \\
\text { Date }\end{array}$ & \multicolumn{1}{c|}{ Actionee(s) } \\
\hline 1. & $\begin{array}{l}\text { Develop and validate a comprehensive list of facilities } \\
\text { owned \& operated by the Evaporator Project. }\end{array}$ & $\begin{array}{c}3 / 29 / 96 \\
\text { Completed }\end{array}$ & AK Gardner \\
\hline 2. & $\begin{array}{l}\text { Identify key documents for the facility or operations. } \\
\text { Provide listing to program/project level for } \\
\text { consolidation and RL review. }\end{array}$ & $\begin{array}{c}5 / 01 / 96 \\
\text { Completed }\end{array}$ & $\begin{array}{l}\text { MD Stratton (Evaporator) } \\
\text { LA Garner (Environtnental) } \\
\text { LE Simmons (Training) }\end{array}$ \\
\hline 3. & $\begin{array}{l}\text { Develop a comprehensive list of open-ended } \\
\text { compliance issues (Htanford Action Tracking System } \\
\text { [HATS] as an example). Provide to RL POC for } \\
\text { review. }\end{array}$ & $\begin{array}{l}5 / 01 / 96 \\
\text { Completed }\end{array}$ & $\begin{array}{l}\text { CL Day-Phalen (HATS) } \\
\text { LA Garner (Environmental) } \\
\text { LM Calderon (Safety) } \\
\text { CL Caldwell (RadCon) } \\
\text { J Weber (QA) } \\
\text { MD Stratton (CONOPs) } \\
\text { EJ Austin (S/RIDs Phase 1) }\end{array}$ \\
\hline
\end{tabular}




\begin{tabular}{|c|c|c|c|}
\hline & Pre-Award Actions & $\begin{array}{l}\text { Scheduled } \\
\text { Date }\end{array}$ & Actionee(s) \\
\hline 4. & $\begin{array}{l}\text { Identify POCs for the facility or operations and input to } \\
\text { consolidated program/project listing. }\end{array}$ & $\begin{array}{c}5 / 15 / 96 \\
\text { Completed }\end{array}$ & MD Stratton \\
\hline 5. & $\begin{array}{l}\text { Develop a listing and description of subcontracts } \\
\text { supporting the facility or operation and input to } \\
\text { consolidated program/project listing. Include any } \\
\text { subcontracts planned to be executed prior to contract } \\
\text { takeover. }\end{array}$ & $\begin{array}{l}5 / 15 / 96 \\
\text { Completed }\end{array}$ & RA Finke \\
\hline 6. & $\begin{array}{l}\text { Develop a listing and description of facility or } \\
\text { operations databases. Provide list to program/project } \\
\text { level for consolidation. }\end{array}$ & $\begin{array}{l}5 / 15 / 96 \\
\text { Completed }\end{array}$ & RB Bass \\
\hline 7. & $\begin{array}{l}\text { Identify number of briefings, and prepare facility or } \\
\text { operations briefing(s) for the PHMC. (Coordinate } \\
\text { number and content with RL POC.) }\end{array}$ & $7 / 15 / 96$ & MD Stratton \\
\hline 8. & $\begin{array}{l}\text { Assemble key facility or operations documentation, or } \\
\text { identify its location, for use by the PHMC. Includes } \\
\text { preparation of a facility or operations "Smart Book," to } \\
\text { include information develop in the following actions. }\end{array}$ & $\begin{array}{l}6 / 01 / 96 \\
\text { Completed } \\
\text { "Smart Book" } \\
\text { only } \\
\text { See item } \# 20\end{array}$ & $\begin{array}{l}\text { AK Gardner } \\
\text { RD Gustavson }\end{array}$ \\
\hline 9. & $\begin{array}{l}\text { Using the pre-existing checklist, compile pre-existing } \\
\text { condition information on the facility and/or major } \\
\text { equipment. Conduct, or schedule as a separate post- } \\
\text { award action(s), any assessments required to address all } \\
\text { relevant items on the checklist. Provide completed } \\
\text { checklist to RL POC for review. }\end{array}$ & $\begin{array}{c}6 / 15 / 96 \\
\text { Completed }\end{array}$ & $\begin{array}{l}\text { MD Stratton } \\
\text { RD Gustavson } \\
\text { PR Gunter (Environmental) } \\
\text { LM Calderon (Safety) } \\
\text { CL Caldwell (RadCon) } \\
\text { J Weber (QA) } \\
\text { MD Stratton (CONOPs) } \\
\text { EJ Austin (S/RIDs Phase 1) }\end{array}$ \\
\hline 10. & $\begin{array}{l}\text { Develop a list of open action items and commitments, } \\
\text { inciuding status (Environmental, Tri-Party Agreement, } \\
\text { DNFSB, Secretarial Safety Initiatives, etc.). Provide to } \\
\text { RL POC for review. }\end{array}$ & $\begin{array}{c}\text { 6/01/96 } \\
\text { Completed }\end{array}$ & $\begin{array}{l}\text { RL Nelson } \\
\text { LA Garner (Environmental) }\end{array}$ \\
\hline 11. & $\begin{array}{l}\text { Identify current inventories of feed for the facility, and } \\
\text { materials to support continued operation (i.e., essential } \\
\text { materials, tools and equipment). }\end{array}$ & $7 / 15 / 96$ & $\begin{array}{l}\text { RJ Nicklas (EQ/MAT) } \\
\text { WB Barton (Waste Feed) }\end{array}$ \\
\hline 12. & $\begin{array}{l}\text { Develop a list of facility or operation-specific } \\
\text { mandatory training and qualification requirements for } \\
\text { employees. }\end{array}$ & $\begin{array}{l}\text { 6/01/96 } \\
\text { Completed }\end{array}$ & LE Simmons \\
\hline 13.' & $\begin{array}{l}\text { Develop a listing of information which constitutes the } \\
\text { "authorization basis" for the facility. }\end{array}$ & $\begin{array}{c}6 / 01 / 96 \\
\text { Completed }\end{array}$ & $\begin{array}{l}\text { ML Cowen } \\
\text { JJ Klos }\end{array}$ \\
\hline 14. & $\begin{array}{l}\text { Develop a description of ongoing and planned } \\
\text { construction projects. }\end{array}$ & $\begin{array}{c}\text { 6/01/96 } \\
\text { Completed }\end{array}$ & RD Gustavson \\
\hline 15. & $\begin{array}{l}\text { Develop a list of current work order services or other } \\
\text { form of agreement to provide work for other } \\
\text { organizations, on and offsite. }\end{array}$ & $\begin{array}{c}\text { 6/01/96 } \\
\text { Completed }\end{array}$ & EM Northrop \\
\hline 16. & $\begin{array}{l}\text { Develop a list of current work order services or other } \\
\text { form of agreement to obtain work from other } \\
\text { organizations, on and offsite. }\end{array}$ & $\begin{array}{c}\text { 6/01/96 } \\
\text { Completed }\end{array}$ & EM Northrop \\
\hline
\end{tabular}




\begin{tabular}{|c|c|c|c|}
\hline & Pre-Award Actions & $\begin{array}{l}\text { Scheduled } \\
\text { Date }\end{array}$ & Actionee(s) \\
\hline & $\begin{array}{l}\text { Develop a list of permits governing the facility or } \\
\text { operation, and provide a description of each as to } \\
\text { where and how it applies. }\end{array}$ & $\begin{array}{l}\text { 6/01/96 } \\
\text { Completed }\end{array}$ & LA Garner \\
\hline 18. & $\begin{array}{l}\text { Verify the accuracy of property assignments for the } \\
\text { facility or operations and correct any deficiencies. }\end{array}$ & $\begin{array}{l}6 / 01 / 96 \\
\text { Completed }\end{array}$ & $\begin{array}{l}\text { CL Day-Phalen (Portable } \\
\text { capital equipment \& software } \\
\text { accountability) } \\
\text { AK Gardner (other) } \\
\end{array}$ \\
\hline 19. & $\begin{array}{l}\text { Identify space and equipment within program/project } \\
\text { area and facilities for use by the PHMC. }\end{array}$ & $\begin{array}{c}6 / 01 / 96 \\
\text { Completed }\end{array}$ & RD Gustavson \\
\hline 20. & Update/submit "Smart Book"/assernble key documents. & $7 / 15 / 96$ & $\begin{array}{l}\text { AK Gardner } \\
\text { RD Gustavson } \\
\end{array}$ \\
\hline 21. & $\begin{array}{l}\text { Verify pre-existing condition data is current and } \\
\text { supplement as necessary. }\end{array}$ & $7 / 22 / 96$ & $\begin{array}{l}\text { RD Gustavson } \\
\text { MD Stratton }\end{array}$ \\
\hline & Ensure building assignments are correct in RLPS. & $7 / 22 / 96$ & $\begin{array}{l}\text { RD Gustavson } \\
\text { MD Stratton } \\
\end{array}$ \\
\hline \multicolumn{4}{|c|}{ Post-Award Actions } \\
\hline & $\begin{array}{l}\text { Provide the PHMC access to key documentation, pre- } \\
\text { existing condition information, commitment data, } \\
\text { inventory data, etc. (as established in the pre-award } \\
\text { phase). Provide copies upon request. }\end{array}$ & $8 / 05 / 96$ & $\begin{array}{l}\text { RD Gustavson } \\
\text { MD Stratton }\end{array}$ \\
\hline 2. & $\begin{array}{l}\text { Provide any additional documentation or information } \\
\text { requested by the PHMC, if available, that were not } \\
\text { identified during the pre-award phase. }\end{array}$ & by $9 / 01 / 96$ & $\begin{array}{l}\text { RD Gustavson } \\
\text { MD Stratton }\end{array}$ \\
\hline & Provide facility or operations briefing(s) to the PHMC. & $8 / 12 / 96-9 / 02 / 96$ & $\begin{array}{l}\text { RD Gustavson } \\
\text { MD Stratton }\end{array}$ \\
\hline & Provide a facility or operations tour to the PHMC. & $8 / 12 / 96-9 / 02 / 96$ & $\begin{array}{l}\text { RD Gustavson } \\
\text { MD Stratton } \\
\end{array}$ \\
\hline & $\begin{array}{l}\text { Provide recurring reports that the PHMC would like to } \\
\text { receive during transition. }\end{array}$ & by $9 / 15 / 96$ & $\begin{array}{l}\text { RD Gustavson } \\
\text { MD Stratton } \\
\end{array}$ \\
\hline & Provide necessary training to PHMC personnel. & $8 / 12 / 96-9 / 3 / 96$ & $\begin{array}{l}\text { RD Gustavson } \\
\text { MD Stratton }\end{array}$ \\
\hline & $\begin{array}{l}\text { Assist the PHMC in the conduct of a Pre-Existing } \\
\text { Condition Assessment }\end{array}$ & $8 / 12 / 96-9 / 16 / 96$ & $\begin{array}{l}\text { RD Gustavson } \\
\text { MD Stratton }\end{array}$ \\
\hline & $\begin{array}{l}\text { Perform a joint property and equipment inventory with } \\
\text { the PHMC. }\end{array}$ & by $9 / 16 / 96$ & $\begin{array}{l}\text { RD Gustavson } \\
\text { MD Stratton } \\
\end{array}$ \\
\hline & $\begin{array}{l}\text { Provide an updated status of ongoing work prior to } \\
\text { contract turnover. }\end{array}$ & by $9 / 30 / 96$ & $\begin{array}{l}\text { RD Gustavson } \\
\text { MD Stratton }\end{array}$ \\
\hline & Provide a categorized inventory of wastes. & by $9 / 30 / 96$ & $\begin{array}{l}\text { RD Gustavson } \\
\text { MD Stratton }\end{array}$ \\
\hline
\end{tabular}




\subsubsection{Tank Farm Operations - 200 East Tank Farms}

Ensure environmentally sound operations of the 200 East tank farms, including all facilities and auxiliary equipment associated with handling and storing tank waste. Manage the 200 East Tank Farm Transition Project to establish the SST farms (200 East) into a controlled, clean, and stable configuration in a timely and cost-effective manner. Conduct all activities pertaining to the operation of a permitted TSD facility within the boundary of the current Interim Safety Basis and environmental permits in a manner that ensures compliance with all applicable Federal, state, and local environmental regulations. Perform all support functions required for routine surveillance, operation, and maintenance of the 200 East Area Transition Project facilities to safely monitor status and control of the underground storage of waste. These functions include preventative and corrective maintenance, safe facility operations, waste transfer, onsite acceptance of wastes for tank storage, surveillance monitoring, health physics activities, industrial hygiene and safety functions, engineering and analysis, managing and controlling upgrades to facilities and infrastructure, and preparation for facility turnover to the Waste Disposal Program.

List of major facilities:

- 241-A, -C, -B, -BX, -BY, -AN, -AX, -AZ, and -AY Tank Farms

- 204-AR Unloading Facility

- 209-E Critical Mass Laboratory

- 209-EA 90-Day Storage

- 2400E Dry Material Handling Facility

- 244-AR, Sludge Vault Storage and Processing Facility

- 272-AW TFO Support Facility

- 216-B-62, 216-B-63, 216-A-08, 216-B-55, 216-A-30, 216-A-37-2, 216-A-45 Cribs

- 216-B-3 Expansion Ponds

- 244-BX Vault

- 244-CR Vault

- 244-A Lift Station

- 216-E-16-101 PSW Grout Vault

- 243-G Grout Processing Facility

- Cross-Site Transfer Line (East of 241-EW Vent Station)

- 274-AW Office Building

- 278-AW Document Storage Area

- Miscellaneous transfer piping and units ancillary to Tank Farm facilities

- 14 Inactive Miscellaneous Underground Storage Tanks (IMUSTs).

\begin{tabular}{|l|c|c|c|}
\hline \multicolumn{1}{|c|}{ Pre-Award Actions } & Scheduled & Actionee(s) \\
\hline 1. Produce and verify facility ownership listing. & Date & $\begin{array}{c}3 / 29 / 96 \\
\text { Completed }\end{array}$ & P Hinojosa \\
\hline
\end{tabular}




\begin{tabular}{|c|c|c|}
\hline Pre-Award Actions & $\begin{array}{l}\text { Scheduled } \\
\text { Date }\end{array}$ & Actionee(s) \\
\hline $\begin{array}{l}\text { 2. Identify key documents for the facility or } \\
\text { operations. Provide listing to program/project } \\
\text { level for consolidation and } R L \text { review. }\end{array}$ & $\begin{array}{l}5 / 01 / 96 \\
\text { Completed }\end{array}$ & $\begin{array}{l}\text { P Honojosa (East) } \\
\text { LA Garner (Environmental) } \\
\text { LE Simmons (Training) }\end{array}$ \\
\hline $\begin{array}{l}\text { 3. Develop a comprehensive list of open-ended } \\
\text { compliance issues (HATS as an example). Provide } \\
\text { to RL POC for review. }\end{array}$ & $\begin{array}{l}5 / 01 / 96 \\
\text { Completed }\end{array}$ & $\begin{array}{l}\text { CL Day-Phalen (HATS) } \\
\text { LA Garner (Environmental) } \\
\text { LM Calderon (Safety) } \\
\text { CL Caldwell (RadCon) } \\
\text { J Weber (QA) } \\
\text { P Hinojosa (CONOPs) } \\
\text { EF Austin (S/RIDs Phase 1) }\end{array}$ \\
\hline $\begin{array}{l}\text { 4. Identify POCs for the facility or operations and } \\
\text { input to consolidated program/project listing. }\end{array}$ & $\begin{array}{l}5 / 15 / 96 \\
\text { Completed }\end{array}$ & P Hinojosa \\
\hline $\begin{array}{l}\text { 5evelop a listing and description of subcontracts } \\
\text { supporting the facility or operation and input to } \\
\text { consolidated program/project listing. Include any } \\
\text { subcontracts planned to be executed prior to } \\
\text { contract takeover. }\end{array}$ & $\begin{array}{l}5 / 15 / 96 \\
\text { Completed }\end{array}$ & RA Finke \\
\hline $\begin{array}{l}\text { 6. Develop a listing and description of facility or } \\
\text { operations databases. Provide list to } \\
\text { program/project level for consolidation. }\end{array}$ & $\begin{array}{l}5 / 15 / 96 \\
\text { Completed }\end{array}$ & RB Bass \\
\hline $\begin{array}{l}\text { 7. Identify number of briefings, and prepare facility } \\
\text { or operations briefing(s) for the PHMC. } \\
\text { (Coordinate number and content with RL POC.) }\end{array}$ & $7 / 15 / 96$ & P Hinojosa \\
\hline $\begin{array}{l}\text { 8. Assemble key facility or operations documentation, } \\
\text { or identify its location, for use by the PHMC. } \\
\text { Includes preparation of a facility or operations } \\
\text { "Smart Book," to include information developed in } \\
\text { the following actions. }\end{array}$ & $\begin{array}{l}6 / 01 / 96 \\
\text { Completed "Smart } \\
\text { Book" only } \\
\text { See item } \# 21\end{array}$ & P Hinojosa \\
\hline $\begin{array}{l}\text { 9. Using the pre-existing checklist, compile pre- } \\
\text { existing condition information on the facility and/or } \\
\text { major equipment. Conduct, or schedule as a } \\
\text { separate post-award action(s), any assessments } \\
\text { required to address all relevant items on the } \\
\text { checklist. Provide completed checklist to RL POC } \\
\text { for review. }\end{array}$ & $\begin{array}{l}6 / 15 / 96 \\
\text { Completed }\end{array}$ & $\begin{array}{l}\text { P Hinojosa } \\
\text { RD Gustavson } \\
\text { JF Brown (Environmental) } \\
\text { LM Calderon (Safety) } \\
\text { CL Caldwell (RadCon) } \\
\text { J Weber (QA) } \\
\text { EI Austin (S/RIDS Phase D) } \\
\end{array}$ \\
\hline $\begin{array}{l}\text { 10. Develop a list of open action items and } \\
\text { cormmitments, including status (Environmental, Tri- } \\
\text { Party Agreement, DNFSB, Secretarial Safety } \\
\text { Initiatives, etc.). Provide to RL POC for review. }\end{array}$ & $\begin{array}{l}6 / 01 / 96 \\
\text { Completed }\end{array}$ & $\begin{array}{l}\text { RL Nelson } \\
\text { LA Garner (Environmental) }\end{array}$ \\
\hline $\begin{array}{l}\text { 11. Identify current inventories of feed for the facility, } \\
\text { and materials to support continued operation (i.e., } \\
\text { essential materials, tools and equipment). }\end{array}$ & $7 / 15 / 96$ & $\begin{array}{l}\text { P Hinojosa (Mat/Equip) } \\
\text { WB Barton (Waste) }\end{array}$ \\
\hline $\begin{array}{l}\text { 12. Develop a list of facility or operation-specific } \\
\text { mandatory training and qualification requirements } \\
\text { for employees. }\end{array}$ & $\begin{array}{l}6 / 01 / 96 \\
\text { Completed }\end{array}$ & LE Simmons \\
\hline $\begin{array}{l}\text { 13. Develop a listing of information which constitutes } \\
\text { the "authorization basis" for the facility. }\end{array}$ & $\begin{array}{l}\text { 6/01/96 } \\
\text { Completed }\end{array}$ & $\begin{array}{l}\text { ML Cowen } \\
\text { JJ Klos }\end{array}$ \\
\hline
\end{tabular}




\begin{tabular}{|c|c|c|}
\hline Pre-Award Actions & $\begin{array}{l}\text { Scheduled } \\
\text { Date } \\
\end{array}$ & Actionee(s) \\
\hline $\begin{array}{l}\text { 14. Develop a description of ongoing and planned } \\
\text { construction projects. }\end{array}$ & $\begin{array}{l}6 / 01 / 96 \\
\text { Completed }\end{array}$ & RD Gustavson \\
\hline $\begin{array}{l}\text { 15. Develop a listing of special agreement services } \\
\text { provided to RL, other DOE sites, and other } \\
\text { entities. }\end{array}$ & $\begin{array}{l}\text { 6/01/96 } \\
\text { Completed }\end{array}$ & $\begin{array}{l}\text { P Hinojosa (Vadose zone, } \\
\text { Purgewater storage, LERF } \\
\text { Operation, etc.) }\end{array}$ \\
\hline $\begin{array}{l}\text { 16. Develop a list of current work order services or } \\
\text { other form of agreement to provide work for other } \\
\text { organizations, on and offsite. }\end{array}$ & $\begin{array}{l}6 / 01 / 96 \\
\text { Completed }\end{array}$ & MI Yeager \\
\hline $\begin{array}{l}\text { 17. Develop a list of current work order services or } \\
\text { other form of agreement to obtain work from other } \\
\text { organizations, on and offsite. }\end{array}$ & $\begin{array}{l}\text { 6/01/96 } \\
\text { Completed }\end{array}$ & MI Yeager \\
\hline $\begin{array}{l}\text { 18. Develop a list of permits governing the facility or } \\
\text { operation, and provide a description of each as to } \\
\text { where and how it applies. }\end{array}$ & $\begin{array}{c}\text { 6/01/96 } \\
\text { Completed }\end{array}$ & LA Garner \\
\hline $\begin{array}{l}\text { 19. Verify the accuracy of property assignments for the } \\
\text { facility or operations and correct any deficiencies. }\end{array}$ & $\begin{array}{c}\text { 6/01/96 } \\
\text { Completed }\end{array}$ & $\begin{array}{l}\text { CL Day-Phalen (Portable capital } \\
\text { equipment \& software } \\
\text { accountability) } \\
\text { P Hinojosa (other) }\end{array}$ \\
\hline $\begin{array}{l}\text { 20. Identify space and equipment within } \\
\text { program/project area and facilities for use by the } \\
\text { PHMC. }\end{array}$ & $\begin{array}{l}\text { 6/01/96 } \\
\text { Completed }\end{array}$ & RD Gustavson \\
\hline $\begin{array}{l}\text { 21. Update/submit "Smart Book"/assemble key } \\
\text { documents. }\end{array}$ & $7 / 15 / 96$ & P Hinojosa \\
\hline $\begin{array}{l}\text { 22. Verify pre-existing condition data is current and } \\
\text { supplement as necessary. }\end{array}$ & $7 / 22 / 96$ & $\begin{array}{l}\text { RD Gustavson } \\
\text { P Hinojosa }\end{array}$ \\
\hline 23. Ensure building assignments are correct in RLPS. & $7 / 22 / 96$ & $\begin{array}{l}\text { RD Gustavson } \\
\text { P Honojosa } \\
\end{array}$ \\
\hline Post-Award Actions & & \\
\hline $\begin{array}{l}\text { 1. Provide the PHMC access to key documentation, } \\
\text { pre-existing condition information, commitment } \\
\text { data, inventory data, etc. (as established in the pre- } \\
\text { award phase). Provide copies upon request. }\end{array}$ & $8 / 05 / 96$ & $\begin{array}{l}\text { RD Gustavson } \\
\text { P Hinojosa }\end{array}$ \\
\hline $\begin{array}{l}\text { 2. Provide any additional documentation or } \\
\text { information requested by the PHMC, if available, } \\
\text { that were not identified during the pre-award } \\
\text { phase. }\end{array}$ & by $9 / 01 / 96$ & $\begin{array}{l}\text { RD Gustavson } \\
\text { P Hinojosa }\end{array}$ \\
\hline $\begin{array}{l}\text { 3. Provide facility or operations briefing(s) to the } \\
\text { PHMC. }\end{array}$ & $8 / 12 / 96-9 / 02 / 96$ & $\begin{array}{l}\text { RD Gustavson } \\
\text { P Hinojosa }\end{array}$ \\
\hline 4. Provide a facility or operations tour to the PHMC. & $8 / 12 / 96-9 / 02 / 96$ & $\begin{array}{l}\text { RD Gustavson } \\
\text { P Hinojosa }\end{array}$ \\
\hline $\begin{array}{l}\text { 5. Provide any recurring reports that the PHMC } \\
\text { would like to receive during transition. }\end{array}$ & by $9 / 15 / 96$ & $\begin{array}{l}\text { RD Gustavson } \\
\text { P Hinojosa }\end{array}$ \\
\hline 6. Provide necessary training to PHMC personnel. & $8 / 12 / 96-9 / 23 / 96$ & $\begin{array}{l}\text { RD Gustavson } \\
\text { P Hinojosa }\end{array}$ \\
\hline
\end{tabular}


WHC-SP-1181 Rev. 1

\begin{tabular}{|c|c|c|c|}
\hline & Post-Award Actions & $\begin{array}{l}\text { Scheduled } \\
\text { Date }\end{array}$ & Actionce(s) \\
\hline & $\begin{array}{l}\text { Assist the PHMC in the conduct of a Pre-Existing } \\
\text { Condition Assessment }\end{array}$ & $8 / 12 / 96-9 / 16 / 96$ & $\begin{array}{l}\text { RD Gustavson } \\
\text { P Hinojosa }\end{array}$ \\
\hline 8. & $\begin{array}{l}\text { Provide an updated status of ongoing work prior to } \\
\text { contract nurnover. }\end{array}$ & by $9 / 30 / 96$ & $\begin{array}{l}\text { RD Gustavson } \\
\text { P Hinojosa }\end{array}$ \\
\hline 9. & $\begin{array}{l}\text { Perform a joint property and equipment inventory } \\
\text { with the PHMC. }\end{array}$ & by $9 / 16 / 96$ & $\begin{array}{l}\text { RD Gustavson } \\
\text { P Hinojosa }\end{array}$ \\
\hline 10. & Provide a categorized inventory of wastes. & by $9 / 30 / 96$ & $\begin{array}{l}\text { RD Gustavson } \\
\text { P Hinojosa }\end{array}$ \\
\hline
\end{tabular}

\subsubsection{Tank Farm Operations - 200 West Tank Farms}

Ensure environmentally sound operations of the 200 West tank farms, including all facilities and auxiliary equipment associated with handling and storing tank waste. Manage the 200 West Tank Farm Transition Project to establish the SST farms (200 West) into a controlled, clean, and stable configuration in a timely and cost-effective manner. Conduct all activities pertaining to the operation of a permitted TSD facility within the boundary of the current Interim Safety Basis and environmental permits in a manner that ensures compliance with all applicable Federal, state, and local environmental regulations. Perform all support functions required for routine surveillance, operation, and maintenance of the 200 West Area Transition Project facilities to safely monitor status and control of the underground storage of waste. These functions include preventative and corrective maintenance, facility operations including waste transfer, onsite acceptance of wastes for tank storage, surveillance monitoring, health physics activities, industrial hygiene and safety functions, and engineering and analysis. Responsible for managing and controlling upgrades to facilities and infrastructure, enhancing the safety of facility operations, and preparing for the eventual facility turnover to the Waste Disposal Program.

List of major facilities:

- 207-T Retention Basins

- 241-S, -SX, -SY, -T, -TX, -TY, -U Tank Farms

- 216-S-25, 216-S-26, 216-U-16, 216-U-17, 216-W-LC, 216-Z-20 Cribs

- 216-T-1, 216-T-4-2, 216-U-14 Ditches

- 216-Z-21 Pond

- 242-S and 242-T Evaporator Facilities

- 244-S, -U, -TX DCRT Vaults

- 241-TX-302C Catch Tank

- 241-S-302 Catch Tank

- 2727-W, and -WA Sodium Storage Facility

- 272-WA TFO Support Facility

- 278-WA Document Control Center 
- 241-EW Vent Station

- Cross-Site Transfer Lines (West of and including the 241-EW Vent Station)

- 22 IMUSTs

- Miscellaneous transfer piping and units ancillary to tank farm facilities.

\begin{tabular}{|c|c|c|}
\hline Pre-Award Actions & $\begin{array}{l}\text { Scheduled } \\
\text { Date }\end{array}$ & Actionee(s) \\
\hline $\begin{array}{l}\text { 1. Develop and validate a comprehensive list of facilities } \\
\text { owned \& operated by West Tank Farm Transition } \\
\text { Project. }\end{array}$ & $\begin{array}{l}3 / 29 / 96 \\
\text { Completed }\end{array}$ & LC Mercado \\
\hline $\begin{array}{l}\text { 2. Identify key documents for the facility or operations. } \\
\text { Provide listing to program/project level for } \\
\text { consolidation and } R L \text { review. }\end{array}$ & $\begin{array}{l}5 / 01 / 96 \\
\text { Completed }\end{array}$ & $\begin{array}{l}\text { LC Mercado (West) } \\
\text { LA Garner (Environmental) } \\
\text { LE Simmons (Training) }\end{array}$ \\
\hline $\begin{array}{l}\text { 3. Develop a comprehensive list of open-ended } \\
\text { compliance issues (HATS as an example). Provide to } \\
\text { RL POC for review. }\end{array}$ & $\begin{array}{l}5 / 01 / 96 \\
\text { Completed }\end{array}$ & $\begin{array}{l}\text { CL Day-Phalen (HATS) } \\
\text { LA Garner (Environmental) } \\
\text { LM Calderon (Safety) } \\
\text { SL Bump (RadCon) } \\
\text { J Weber (QA) } \\
\text { LC.Mercado (CONOPs) } \\
\text { EJ Austin (S/RIDs Phase 1) }\end{array}$ \\
\hline $\begin{array}{l}\text { 4. Identify POCs for the facility or operations and input to } \\
\text { consolidated program/project listing. }\end{array}$ & $\begin{array}{c}5 / 15 / 96 \\
\text { Completed }\end{array}$ & LC Mercado \\
\hline $\begin{array}{l}\text { Develop a listing and description of subcontracts } \\
\text { supporting the facility or operation and input to } \\
\text { consolidated program/project listing. Include any } \\
\text { subcontracts planned to be executed prior to contract } \\
\text { takeover. }\end{array}$ & $\begin{array}{c}5 / 15 / 96 \\
\text { Completed }\end{array}$ & RA Finke \\
\hline $\begin{array}{l}\text { 6. Develop a listing and description of facility or } \\
\text { operations databases. Provide list to program/project } \\
\text { level for consolidation. }\end{array}$ & $\begin{array}{l}5 / 15 / 96 \\
\text { Completed }\end{array}$ & RB Bass \\
\hline $\begin{array}{l}\text { 7. Identify number of briefings, and prepare facility (or } \\
\text { operations) briefing(s) for the PHMC. (Coordinate } \\
\text { number and content with } R L \text { POC.) }\end{array}$ & $7 / 15 / 96$ & LC Mercado \\
\hline $\begin{array}{l}\text { 8. Assemble key facility or operations documentation, or } \\
\text { identify its location, for use by the PHMC. Includes } \\
\text { preparation of a facility or operations "Smart Book," to } \\
\text { include information develop in the following actions. }\end{array}$ & $\begin{array}{c}\text { 6/01/96 } \\
\text { Completed } \\
\text { "Smart Book" } \\
\text { only } \\
\text { See item } \# 20 \\
\end{array}$ & $\begin{array}{l}\text { LC Mercado } \\
\text { RD Gustavson }\end{array}$ \\
\hline $\begin{array}{l}\text { 9. Using the pre-existing checklist, compile pre-existing } \\
\text { condition information on the facility and/or major } \\
\text { equipment. Conduct, or schedule as a separate post- } \\
\text { award action(s), any assessments required to address all } \\
\text { relevant items on the checklist. Provide completed } \\
\text { checklist to RL POC for review. }\end{array}$ & $\begin{array}{c}6 / 15 / 96 \\
\text { Completed }\end{array}$ & $\begin{array}{l}\text { LC Mercado } \\
\text { RD Gustavson } \\
\text { MM Barnett (Environmental) } \\
\text { LA Calderon (Safety) } \\
\text { SL Bump (RadCon) } \\
\text { J Weber (QA) } \\
\text { EJ Austin (S/RID Phase D) }\end{array}$ \\
\hline $\begin{array}{l}\text { 10. Develop a list of open action items and commitments, } \\
\text { including status (Environmental, Tri-Party Agreement, } \\
\text { DNFSB, Secretarial Safety Initiatives, etc.). Provide } \\
\text { to RL POC for review. }\end{array}$ & $\begin{array}{c}\text { 6/01/96 } \\
\text { Completed }\end{array}$ & $\begin{array}{l}\text { RL Nelson } \\
\text { LA Garner (Environmental) }\end{array}$ \\
\hline
\end{tabular}




\begin{tabular}{|c|c|c|c|}
\hline 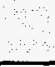 & Pre-Award Actions & $\begin{array}{l}\text { Scheduled } \\
\text { Date }\end{array}$ & Actionee(s) \\
\hline 11. & $\begin{array}{l}\text { Identify current inventories of feed for the facility, and } \\
\text { materials to support continued operation (i.e., essential } \\
\text { materials, tools and equipment). }\end{array}$ & $7 / 15 / 96$ & $\begin{array}{l}\text { WB Barton (Waste) } \\
\text { LC Mercado (Mat/Equip) }\end{array}$ \\
\hline 12. & $\begin{array}{l}\text { Develop a list of facility or operation-specific } \\
\text { mandatory training and qualification requirements for } \\
\text { employees. }\end{array}$ & $\begin{array}{l}\text { 6/01/96 } \\
\text { Completed }\end{array}$ & LE Simmons \\
\hline 13. & $\begin{array}{l}\text { Develop a listing of information which constitutes the } \\
\text { "authorization basis" for the facility. }\end{array}$ & $\begin{array}{c}\text { 6/01/96 } \\
\text { Completed }\end{array}$ & $\begin{array}{l}\text { ML Cowen } \\
\text { JJ Klos }\end{array}$ \\
\hline 14. & $\begin{array}{l}\text { Develop a description of ongoing and planned } \\
\text { construction projects. }\end{array}$ & $\begin{array}{c}\text { 6/01/96 } \\
\text { Completed }\end{array}$ & RD Gustavson \\
\hline 15. & $\begin{array}{l}\text { Develop a list of current work order services or other } \\
\text { form of agreement to provide work for other } \\
\text { organizations, on and offsite. }\end{array}$ & $\begin{array}{c}\text { 6/01/96 } \\
\text { Completed }\end{array}$ & JS Thomas \\
\hline 16. & $\begin{array}{l}\text { Develop a list of current work order services or other } \\
\text { form of agreement to obtain work from other } \\
\text { organizations, on and offsite. }\end{array}$ & $\begin{array}{l}\text { 6/01/96 } \\
\text { Completed }\end{array}$ & JS Thomas \\
\hline 17. & $\begin{array}{l}\text { Develop a list of permits governing the facility or } \\
\text { operation, and provide a description of each as to } \\
\text { where and how it applies. }\end{array}$ & $\begin{array}{l}\text { 6/01/96 } \\
\text { Completed }\end{array}$ & LA Garner \\
\hline 18. & $\begin{array}{l}\text { Verify the accuracy of property assignments for the } \\
\text { facility or operations and correct any deficiencies. }\end{array}$ & $\begin{array}{c}\text { 6/01/96 } \\
\text { Completed }\end{array}$ & $\begin{array}{l}\text { CL Day-Phalen (portable capital } \\
\text { equipment \& software } \\
\text { accountability) } \\
\text { LC Mercado (other) }\end{array}$ \\
\hline 19. & $\begin{array}{l}\text { Identify space and equipment within program/project } \\
\text { area and facilities for use by the PHMC. }\end{array}$ & $\begin{array}{c}6 / 01 / 96 \\
\text { Completed }\end{array}$ & RD Gustavson \\
\hline 20. & Update/submit "Smart Book"/assemble key documents. & $7 / 15 / 96$ & $\begin{array}{l}\text { LC Mercado } \\
\text { RD Gustavson }\end{array}$ \\
\hline & $\begin{array}{l}\text { Verify pre-existing condition data is current and } \\
\text { supplement as necessary. }\end{array}$ & $7 / 22 / 96$ & $\begin{array}{l}\text { RD Gustavson } \\
\text { LC Mercado }\end{array}$ \\
\hline 22. & Ensure building assignments are correct in RLPS. & $7 / 22 / 96$ & $\begin{array}{l}\text { RD Gustavson } \\
\text { LC Mercado }\end{array}$ \\
\hline & Post-Award Actions & & \\
\hline 1. & $\begin{array}{l}\text { Provide the PHMC access to key documentation, pre- } \\
\text { existing condition information, commitment data, } \\
\text { inventory data, etc. (as established in the pre-award } \\
\text { phase). Provide copies upon request. }\end{array}$ & $8 / 05 / 96$ & $\begin{array}{l}\text { RD Gustavson } \\
\text { LC Mercado }\end{array}$ \\
\hline 2. & $\begin{array}{l}\text { Provide any additional documentation or information } \\
\text { requested by the PHMC, if available, that were not } \\
\text { identified during the pre-award phase. }\end{array}$ & by $9 / 01 / 96$ & $\begin{array}{l}\text { RD Gustavson } \\
\text { LC Mercado }\end{array}$ \\
\hline 3. & Provide facility or operations briefing(s) to the PHMC. & $\begin{array}{l}8 / 12 / 96- \\
9 / 02 / 96\end{array}$ & $\begin{array}{l}\text { RD Gustavson } \\
\text { LC Mercado } \\
\end{array}$ \\
\hline 4. & Provide a facility or operations tour to the PHMC. & $\begin{array}{l}8 / 12 / 96 \\
9 / 02 / 96\end{array}$ & $\begin{array}{l}\text { RD Gustavson } \\
\text { LC Mercado }\end{array}$ \\
\hline
\end{tabular}




\begin{tabular}{|ll|c|l|}
\hline \multicolumn{1}{|c|}{ Post-Award Actions } & $\begin{array}{c}\text { Scheduled } \\
\text { Date }\end{array}$ & \multicolumn{1}{|c|}{ Actionee(s) } \\
\hline 5. & $\begin{array}{l}\text { Provide any recurring reports that the PHMC would } \\
\text { like to receive during transition. }\end{array}$ & by $9 / 15 / 96$ & $\begin{array}{l}\text { RD Gustavson } \\
\text { LC Mercado }\end{array}$ \\
\hline 6. & Provide necessary training to PHMC personnel. & $\begin{array}{l}8 / 12 / 96- \\
9 / 23 / 96\end{array}$ & $\begin{array}{l}\text { RD Gustavson } \\
\text { LC Mercado }\end{array}$ \\
\hline 7. & $\begin{array}{l}\text { Assist the PHMC in the conduct of a Pre-Existing } \\
\text { Condition Assessment. }\end{array}$ & $\begin{array}{l}8 / 12 / 96- \\
9 / 16 / 96\end{array}$ & $\begin{array}{l}\text { RD Gustavson } \\
\text { LC Mercado }\end{array}$ \\
\hline 8. & $\begin{array}{l}\text { Perform a joint property and equipment inventory with } \\
\text { the PHMC. }\end{array}$ & by 9/16/96 & $\begin{array}{l}\text { RD Gustavson } \\
\text { LC Mercado }\end{array}$ \\
\hline 9. & $\begin{array}{l}\text { Provide an updated status of ongoing work prior to } \\
\text { contract turnover. }\end{array}$ & by 9/30/96 & $\begin{array}{l}\text { RD Gustavson } \\
\text { LC Mercado }\end{array}$ \\
\hline 10. & Provide a categorized inventory of wastes. & by 9/30/96 & $\begin{array}{l}\text { RD Gustavson } \\
\text { LC Mercado }\end{array}$ \\
\hline
\end{tabular}

\subsubsection{Safety Issue Resolution/TWRS Engineering}

The TWRS Chief Engineer is the TWRS Design Authority and has overall responsibility for establishing, implementing, and maintaining the TWRS technical baseline, the TWRS Authorization Basis and SMS and provides safety analysis, licensing, process engineering, and related technical services to TWRS facility and project organizations, using systems engineering processes, tools, and methodologies.

\begin{tabular}{|c|c|c|}
\hline Pre-Award Actions & $\begin{array}{l}\text { Scheduled } \\
\text { Date }\end{array}$ & Actionee(s) \\
\hline $\begin{array}{l}\text { 1. Identify key documents. Provide listing to } \\
\text { program/project level for consolidation and RL } \\
\text { review. }\end{array}$ & $\begin{array}{l}5 / 01 / 96 \\
\text { Completed }\end{array}$ & DJ Berryman \\
\hline $\begin{array}{l}\text { 2. Identify POCs and input to the consolidated } \\
\text { program/project listing. }\end{array}$ & $\begin{array}{c}5 / 15 / 96 \\
\text { Completed }\end{array}$ & DJ Berryman \\
\hline $\begin{array}{l}\text { 3. Develop a listing and description of subcontracts } \\
\text { supporting the facility (or operation) and input to } \\
\text { consolidated program/project listing. Include any } \\
\text { subcontracts planned to be executed prior to contract } \\
\text { takeover. }\end{array}$ & $\begin{array}{l}5 / 15 / 96 \\
\text { Completed }\end{array}$ & DJ Berryman \\
\hline $\begin{array}{l}\text { 4. Develop a listing and description of databases. } \\
\text { Provide list to program/project level for consolidation. }\end{array}$ & $\begin{array}{c}5 / 15 / 96 \\
\text { Completed }\end{array}$ & $\begin{array}{l}\text { RJ Cash } \\
\text { SR Long }\end{array}$ \\
\hline $\begin{array}{l}\text { 5. Identify number of briefings, and prepare briefing(s) } \\
\text { for the PHMC. (Coordinate number and content with } \\
\text { RL POC.) }\end{array}$ & $7 / 15 / 96$ & AM Umek \\
\hline $\begin{array}{l}\text { 6. Assemble key documentation, or identify its location, } \\
\text { for use by the PHMC. Includes preparation of a } \\
\text { "Smart Book," which will include information } \\
\text { developed in the following actions. }\end{array}$ & $\begin{array}{l}\text { 6/01/96 } \\
\text { Completed "Smart } \\
\text { Book" only } \\
\text { See item } \# 13\end{array}$ & DI Berryman \\
\hline
\end{tabular}




\begin{tabular}{|c|c|c|}
\hline Pre-Award Actions & $\begin{array}{r}\text { Scheduled } \\
\text { Date }\end{array}$ & Actionee(s) \\
\hline $\begin{array}{l}\text { 7. Develop a list of open action items and commitments, } \\
\text { including status (EnvironnentaI, Tri-Party Agreement, } \\
\text { DNFSB as examples). Provide to RL POC for } \\
\text { review. }\end{array}$ & $\begin{array}{l}\text { 6/01/96 } \\
\text { Completed }\end{array}$ & SR Long \\
\hline $\begin{array}{l}\text { 8. Develop a listing of information which constitutes the } \\
\text { "authorization basis" for the facility. }\end{array}$ & $\begin{array}{c}\text { 6/01/96 } \\
\text { Completed }\end{array}$ & $\begin{array}{l}\text { M Cowen } \\
\text { JJ Klos }\end{array}$ \\
\hline $\begin{array}{l}\text { 9. Develop a list of current work order services or other } \\
\text { forn of agreement to provide work for other } \\
\text { organizations, on and offsite. }\end{array}$ & $\begin{array}{c}6 / 01 / 96 \\
\text { Completed }\end{array}$ & $\begin{array}{l}\text { TL Denton } \\
\text { JP Sloughter }\end{array}$ \\
\hline $\begin{array}{l}\text { 10. Develop a list of current work order services or other } \\
\text { form of agreement to obtain work from other } \\
\text { organizations, on and offsite. }\end{array}$ & $\begin{array}{l}6 / 01 / 96 \\
\text { Completed }\end{array}$ & $\begin{array}{l}\text { DJ Berryman } \\
\text { DA Raap } \\
\text { TL Denton } \\
\end{array}$ \\
\hline $\begin{array}{l}\text { 11. Verify the accuracy of property assignments for the } \\
\text { facility (or operations) and correct any deficiencies. }\end{array}$ & $\begin{array}{c}6 / 01 / 96 \\
\text { Completed }\end{array}$ & LA Robinson \\
\hline $\begin{array}{l}\text { 12. Identify space and equipment within program/project } \\
\text { area and facilities for use by the PHMC. }\end{array}$ & $\begin{array}{c}\text { 6/01/96 } \\
\text { Completed } \\
\end{array}$ & DJ Berryman \\
\hline $\begin{array}{l}\text { 13. Update/submit "Smart Book"/assemble key } \\
\text { documents. }\end{array}$ & $7 / 15 / 96$ & DJ Berryman \\
\hline \multicolumn{3}{|l|}{ Post-Award Actions } \\
\hline $\begin{array}{l}\text { 1. Provide the PHMC access to key documentation, pre- } \\
\text { existing condition information, commitment data, } \\
\text { inventory data, etc. (as established in the pre-award } \\
\text { phase). Provide copies upon request. }\end{array}$ & $8 / 05 / 96$ & $\begin{array}{l}\text { TC Varljen } \\
\text { SR Long }\end{array}$ \\
\hline $\begin{array}{l}\text { 2. Provide any additional documentation or information } \\
\text { requested by the PHMC, if available, that were not } \\
\text { identified during the pre-award phase. }\end{array}$ & by $9 / 01 / 96$ & $\begin{array}{l}\text { IC Varljen } \\
\text { SR Long }\end{array}$ \\
\hline 3. Provide briefing(s) to the PHMC. & $8 / 12 / 96-9 / 02 / 96$ & AM Umek \\
\hline $\begin{array}{l}\text { 4. Provide any recurring reports that the PHMC would } \\
\text { like to receive during transition. }\end{array}$ & by $9 / 15 / 96$ & $\begin{array}{l}\text { TC Varljen } \\
\text { SR Long } \\
\end{array}$ \\
\hline $\begin{array}{l}\text { 5. Perform a joint property and equipment inventory with } \\
\text { the PHMC. }\end{array}$ & by $9 / 16 / 96$ & LA Robinson \\
\hline $\begin{array}{l}\text { 6. Provide an updated status of ongoing work prior to } \\
\text { contract turnover. }\end{array}$ & by $9 / 30 / 96$ & $\begin{array}{l}\text { TC Varljen } \\
\text { SR Long }\end{array}$ \\
\hline
\end{tabular}

\subsubsection{Waste Characterization}

Manage the tank waste characterization project to ensure that data necessary for the continued safe operation of the tank farms and the eventual disposal of the waste are attained in a timely and cost-effective manner. This includes identifying data needs, identifying technical bases for data acquisition and use, deploying equipment, maintaining equipment, acquiring samples, analyzing samples, evaluating data, maintaining Characterization databases and publishing Tank Characterization Reports. Characterization Project also 
ensures timely completion of the Tri-Party Agreement characterization milestones for tank characterization and execution of the DNFSB 93-5 Implementation Plan.

\subsubsection{Waste Characterization - Technical Planning, Data Evaluation, and Data Management Activities.}

\begin{tabular}{|c|c|c|}
\hline Pre-Award Actions & $\begin{array}{l}\text { Scheduled } \\
\text { Date }\end{array}$ & Actionee(s) \\
\hline $\begin{array}{l}\text { 1. Identify key documents. Provide listing to } \\
\text { program/project level for consolidation and RL } \\
\text { review. }\end{array}$ & $\begin{array}{l}5 / 01 / 96 \\
\text { Completed }\end{array}$ & DJ McCain \\
\hline $\begin{array}{l}\text { 2. Develop a comprehensive list of openended } \\
\text { compliance issues (HATS as an example). Provide to } \\
\text { RL POC for review. }\end{array}$ & $\begin{array}{c}5 / 01 / 96 \\
\text { Completed }\end{array}$ & LD Pengington \\
\hline $\begin{array}{l}\text { 3. Identify POCs and input to consolidated } \\
\text { program/project listing. }\end{array}$ & $\begin{array}{c}5 / 15 / 96 \\
\text { Completed } \\
\end{array}$ & DI MoCain \\
\hline $\begin{array}{l}\text { 4. Develop a listing and description of subcontracts } \\
\text { supporting the program/project or facility (or } \\
\text { operation) and input to consolidated program/project } \\
\text { listing. Include any subcontracts planned to be } \\
\text { executed prior to contract takeover. }\end{array}$ & $\begin{array}{l}5 / 15 / 96 \\
\text { Completed }\end{array}$ & GA Hanson \\
\hline $\begin{array}{l}\text { 5. Develop a listing and description of databases. } \\
\text { Provide list to program/project level for consolidation. }\end{array}$ & $\begin{array}{c}5 / 15 / 96 \\
\text { Completed }\end{array}$ & JB Schaffer \\
\hline $\begin{array}{l}\text { 6. Identify number of briefings, and prepare briefing(s) } \\
\text { for the PHMC. (Coordinate number and content with } \\
\text { RL POC.) }\end{array}$ & $7 / 15 / 96$ & SJ Eberlein \\
\hline $\begin{array}{l}\text { 7. Assemble key facility documentation, or identify its } \\
\text { location, for use by the PHMC. Includes preparation } \\
\text { of a "Smart Book, "to include information develop in } \\
\text { the following actions. }\end{array}$ & $\begin{array}{c}6 / 01 / 96 \\
\text { Completed } \\
\text { "Smart Book" only } \\
\text { See item } \# 15 \\
\end{array}$ & AE Young \\
\hline $\begin{array}{l}\text { 8. Develop a list of open action items and commitments, } \\
\text { including status (Environmental, Tri-Party Agreement, } \\
\text { DNFSB as examples). Provide to RL POC for } \\
\text { review. }\end{array}$ & $\begin{array}{l}\text { 6/01/96 } \\
\text { Completed }\end{array}$ & LD Pennington \\
\hline $\begin{array}{l}\text { 9. Develop a list of facility (or operation)-specific } \\
\text { mandatory training and qualification requirements for } \\
\text { employees. }\end{array}$ & $\begin{array}{l}\text { 6/01/96 } \\
\text { Completed }\end{array}$ & DJ McCain \\
\hline $\begin{array}{l}\text { 10. Develop a listing of special agreement services } \\
\text { provided to } R L \text {, other DOE sites, and other entities. }\end{array}$ & $\begin{array}{c}6 / 01 / 96 \\
\text { Completed }\end{array}$ & GA Hanson \\
\hline $\begin{array}{l}\text { 11. Develop a list of current work order services or other } \\
\text { form of agreement to provide work for other } \\
\text { organizations, on and offsite. }\end{array}$ & $\begin{array}{l}\text { 6/01/96 } \\
\text { Completed }\end{array}$ & GA Hanson \\
\hline $\begin{array}{l}\text { 12. Develop a list of current work order services or other } \\
\text { form of agreement to obtain work from other } \\
\text { organizations, on and offsite. }\end{array}$ & $\begin{array}{c}6 / 01 / 96 \\
\text { Completed }\end{array}$ & GA Hanson \\
\hline $\begin{array}{l}\text { 13. Verify the accuracy of property assignments for the } \\
\text { facility (or operations) and correct any deficiencies. }\end{array}$ & $\begin{array}{c}6 / 01 / 96 \\
\text { Completed } \\
\end{array}$ & YR Cantu \\
\hline $\begin{array}{l}\text { 14. Identify space and equipment within program/project } \\
\text { area and facilities for use by the PHMC. }\end{array}$ & $\begin{array}{c}6 / 01 / 96 \\
\text { Completed }\end{array}$ & DJ McCain \\
\hline
\end{tabular}




\begin{tabular}{|c|c|c|}
\hline Pre-Award Actions & $\begin{array}{l}\text { Scheduled } \\
\text { Date }\end{array}$ & Actionee(s) \\
\hline $\begin{array}{l}\text { 15. Update/submit "Smart Book"/assemble key } \\
\text { documents. }\end{array}$ & $7 / 15 / 96$ & AE Young \\
\hline Post-Award Actions & & $\because$ \\
\hline $\begin{array}{l}\text { 1. Provide the PHMC access to key documentation, pre- } \\
\text { existing condition information, cornmitment data, } \\
\text { inventory data, etc. (as established in the pre-award } \\
\text { phase). Provide copies upon request. }\end{array}$ & $8 / 05 / 96$ & AL Young \\
\hline $\begin{array}{l}\text { 2. Provide any additional documentation or information } \\
\text { requested by the PHMC, if available, that were not } \\
\text { identified during the pre-award phase. }\end{array}$ & by $9 / 01 / 96$ & AL Young \\
\hline 3. Provide briefing(s) to the PHMC. & $8 / 12 / 96-9 / 02 / 96$ & $\begin{array}{l}\text { DJ McCain } \\
\text { SJ Eberlein }\end{array}$ \\
\hline 4. Provide a tour to the PHMC. & $8 / 12 / 96-9 / 02 / 96$ & $\begin{array}{l}\text { DJ McCain } \\
\text { SJ Eberlein }\end{array}$ \\
\hline $\begin{array}{l}\text { 5. Provide any recurring reports that the PHMC would } \\
\text { like to receive during transition. }\end{array}$ & by $9 / 15 / 96$ & DJ McCain \\
\hline 6. Provide necessary training to PHMC personnel. & $9 / 01 / 96-9 / 23 / 96$ & SJ Eberlein \\
\hline $\begin{array}{l}\text { 7. Perform a joint property and equipment inventory } \\
\text { with the PHMC. }\end{array}$ & by $9 / 16 / 96$ & YR Canns \\
\hline $\begin{array}{l}\text { 8. Provide an updated status of ongoing work prior to } \\
\text { contract turnover. }\end{array}$ & by $9 / 30 / 96$ & SJ Eberlein \\
\hline 9. Provide a categorized inventory of wastes. & by $9 / 30 / 96$ & $\begin{array}{l}\text { DJ McCain } \\
\text { I Husa }\end{array}$ \\
\hline
\end{tabular}

4.1.6.2 Waste Characterization - Operations (Field) Activities. Responsible for the following: Push-mode Core Sampling System (Truck \#1), Rotary-mode Core Sampling System \#1 (Truck \#2), Rotary-mode Core Sampling System \#2 (Truck \#3), and Rotary-mode Core Sampling System \#3 (Truck \#4).

\begin{tabular}{|c|c|c|}
\hline Pre-Award Actions & $\begin{array}{l}\text { Scheduled } \\
\text { Date }\end{array}$ & Actionee(s) \\
\hline $\begin{array}{l}\text { 1. Identify key documents. Provide listing to } \\
\text { program/project level for consolidation and RL review. }\end{array}$ & $\begin{array}{c}5 / 01 / 96 \\
\text { Completed }\end{array}$ & TJ Kelley \\
\hline $\begin{array}{l}\text { 2. Develop a comprehensive list of open-ended } \\
\text { compliance issues (HATS as an example). Provide to } \\
\text { RL POC for review. }\end{array}$ & $\begin{array}{l}5 / 01 / 96 \\
\text { Completed }\end{array}$ & $\begin{array}{l}\text { TJ Kelley } \\
\text { LD Pennington }\end{array}$ \\
\hline $\begin{array}{l}\text { 3. Identify POCs and input to consolidated } \\
\text { program/project listing. }\end{array}$ & $\begin{array}{c}5 / 15 / 96 \\
\text { Completed }\end{array}$ & JG Burton \\
\hline $\begin{array}{l}\text { 4. Develop a listing and description of subcontracts } \\
\text { supporting the program/project or facility (or } \\
\text { operation) and input to consolidated program/project } \\
\text { listing. Include any subcontracts planned to be } \\
\text { executed prior to contract takeover. }\end{array}$ & $\begin{array}{c}5 / 15 / 96 \\
\text { Completed }\end{array}$ & $\begin{array}{l}\text { TJ Kelley } \\
\text { GA Hanson }\end{array}$ \\
\hline
\end{tabular}




\begin{tabular}{|c|c|c|}
\hline Pre-Award Actions & $\begin{array}{c}\text { Scheduled } \\
\text { Date }\end{array}$ & Actionee(s) \\
\hline $\begin{array}{l}\text { 5. Develop a listing and description of databases. Provide } \\
\text { list to program/project level for consolidation. }\end{array}$ & $\begin{array}{c}5 / 15 / 96 \\
\text { Completed }\end{array}$ & RE Raymond \\
\hline $\begin{array}{l}\text { 6. Identify number of briefings, and prepare briefing(s) } \\
\text { for the PHMC. (Coordinate number and content with } \\
\text { RL POC.) }\end{array}$ & $7 / 15 / 96$ & LF Ermold \\
\hline $\begin{array}{l}\text { 7. Assemble key documentation, or identify its location, } \\
\text { for use by the PHMC. Includes preparation of a } \\
\text { "Smart Book," to include information develop in the } \\
\text { following actions. }\end{array}$ & $\begin{array}{c}6 / 01 / 96 \\
\text { Completed "Smart } \\
\text { Book" only } \\
\text { See item } \$ 20 \\
\end{array}$ & TJ Kelley \\
\hline $\begin{array}{l}\text { 8. Using the pre-existing checklist, compile pre-existing } \\
\text { condition information on the facility and/or major } \\
\text { equipment. Conduct, or schedule as a separate post- } \\
\text { award action(s), any assessments required to adequately } \\
\text { address all relevant items on the checklist. Provide } \\
\text { completed checklist to RL POC for review. }\end{array}$ & $6 / 15 / 96$ & JG Burton \\
\hline $\begin{array}{l}\text { 9. Develop a list of open action items and commitments, } \\
\text { including status (Environmental, Tri-Party Agreement, } \\
\text { DNFSB as examples). Provide to RL POC for review. }\end{array}$ & $\begin{array}{l}\text { 6/01/96 } \\
\text { Completed }\end{array}$ & $\begin{array}{l}\text { TJ Kelley } \\
\text { LD Pennington }\end{array}$ \\
\hline $\begin{array}{l}\text { 10. Identify current inventories of feed for the facility, and } \\
\text { materials to support continued operation (i.e., essential } \\
\text { materials, tools and equipment). }\end{array}$ & $7 / 15 / 96$ & JG Burton \\
\hline $\begin{array}{l}\text { 11. Develop a list of facility (or operation)-specific } \\
\text { mandatory training and qualification requirements for } \\
\text { employees. }\end{array}$ & $\begin{array}{l}\text { 6/01/96 } \\
\text { Completed }\end{array}$ & GA Hanson \\
\hline $\begin{array}{l}\text { 12. Develop a listing of information which constitutes the } \\
\text { "authorization basis" for the facility. }\end{array}$ & $\begin{array}{l}6 / 01 / 96 \\
\text { Completed } \\
\end{array}$ & $\begin{array}{l}\text { ML Cowen } \\
\text { JJ Klos }\end{array}$ \\
\hline $\begin{array}{l}\text { 13. Deveiop a description of ongoing and planned } \\
\text { construction projects. }\end{array}$ & $\begin{array}{c}\text { 6/01/96 } \\
\text { Completed } \\
\end{array}$ & TJ Kelley \\
\hline $\begin{array}{l}\text { 14. Develop a listing of special agreement services } \\
\text { provided to RL, other DOE sites, and other entities. }\end{array}$ & $\begin{array}{c}6 / 01 / 96 \\
\text { Completed } \\
\end{array}$ & $\begin{array}{l}\text { TJ Kelley } \\
\text { GA Hanson }\end{array}$ \\
\hline $\begin{array}{l}\text { 15. Develop a list of current work order services or other } \\
\text { form of agreement to provide work for other } \\
\text { organizations, on and offsite. }\end{array}$ & $\begin{array}{l}\text { 6/01/96 } \\
\text { Completed }\end{array}$ & $\begin{array}{l}\text { TJ Kelley } \\
\text { GA Hanson }\end{array}$ \\
\hline $\begin{array}{l}\text { 16. Develop a list of current work order services or other } \\
\text { form of agreement to obtain work from other } \\
\text { organizations, on and offsite. }\end{array}$ & $\begin{array}{l}\text { 6/01/96 } \\
\text { Completed }\end{array}$ & $\begin{array}{l}\text { TJ Kelley } \\
\text { GA Hanson }\end{array}$ \\
\hline $\begin{array}{l}\text { 17. Develop a list of permits governing the facility (or } \\
\text { operation), and provide a description of each as to } \\
\text { where and how it applies. }\end{array}$ & $\begin{array}{l}6 / 01 / 96 \\
\text { Completed }\end{array}$ & RD Gustavson \\
\hline $\begin{array}{l}\text { 18. Verify the accuracy of property assignments for the } \\
\text { facility (or operations) and correct any deficiencies. }\end{array}$ & $\begin{array}{l}6 / 01 / 96 \\
\text { Completed }\end{array}$ & $\begin{array}{l}\text { JG Burton } \\
\text { TJ Kelley } \\
\text { RE Raymond }\end{array}$ \\
\hline $\begin{array}{l}\text { 19. Identify space and equipment within program/project } \\
\text { area and facilities for use by the PHMC. }\end{array}$ & $\begin{array}{l}6 / 01 / 96 \\
\text { Completed }\end{array}$ & PD Cuiver \\
\hline 20. Update/submit "Smart Book"/assemble key documents. & $7 / 15 / 96$ & TJ Kelley \\
\hline 21. Ensure building assignments are correct in RLPS. & $7 / 22 / 96$ & JG Burton \\
\hline
\end{tabular}


WHC-SP-1181 Rev. 1

\begin{tabular}{|c|c|c|}
\hline Pre-Award Actions & $\begin{array}{l}\text { Scheduled } \\
\text { Date }\end{array}$ & Actionee(s) \\
\hline $\begin{array}{l}\text { 22. Verify pre-existing condition data is current and } \\
\text { supplement as necessary. }\end{array}$ & $7 / 22 / 96$ & JG Burton \\
\hline Post-Award Actions & & \\
\hline $\begin{array}{l}\text { 1. Provide the PHMC access to key documentation, pre- } \\
\text { existing condition information, commitment data, } \\
\text { inventory data, etc. (as established in the pre-award } \\
\text { phase). Provide copies upon request. }\end{array}$ & $8 / 05 / 96$ & TJ Kelley \\
\hline $\begin{array}{l}\text { 2. Provide any additional docurnentation or information } \\
\text { requested by the PHMC, if available, that were not } \\
\text { identified during the pre-award phase. }\end{array}$ & by $9 / 01 / 96$ & TJ Kelley \\
\hline 3. Provide briefing(s) to the PHMC. & $8 / 12 / 96-9 / 02 / 96$ & $\begin{array}{l}\text { LF Ermold } \\
\text { JG Burton }\end{array}$ \\
\hline 4. Provide a tour to the PHMC. & $8 / 12 / 96-9 / 02 / 96$ & JG Burton \\
\hline 5. Provide necessary training to PHMC personnel. & $9 / 01 / 96-9 / 23 / 96$ & JG Burton \\
\hline $\begin{array}{l}\text { 6. Assist the PHMC in the conduct of a Pre-Existing } \\
\text { Condition Assessment. }\end{array}$ & $8 / 12 / 96-9 / 16 / 96$ & JG Burton \\
\hline $\begin{array}{l}\text { 7. Provide an updated status of ongoing work prior to } \\
\text { contract turnover. }\end{array}$ & by $9 / 30 / 96$ & TI Kelley \\
\hline 8. Provide a categorized inventory of wastes. & by $9 / 30 / 96$ & $\begin{array}{l}\text { IG Burton } \\
\text { R. E. Raymond }\end{array}$ \\
\hline
\end{tabular}

\subsubsection{Waste Disposal Program}

The charter of the TWRS Disposal Program includes four projects: (1) the Waste Retrieval Project, (2) the Low-Level Waste (LLW) Project, (2) the HLW Project, and (4) the Storage and Disposal Project. The Waste Retrieval Project includes SST and DST retrieval and tank farm closure. The LLW Project covers pretreatment and immobilization of lowactivity waste for disposition onsite. The HLW Project is responsible for the pretreatment and immobilization of HLW for final disposition offsite. The Storage and Disposal Project includes LLW disposal onsite, the interim storage of HLW until final disposition offsite, and the treatment and interim storage of cesium and strontium capsules. The TWRS Disposal Program charter also includes all M\&O Contractor support to the Privatization effort for the LLW and the HLW Vitrification programs.

Facilities within the Disposal Program include Near-Surface Vaults 218-E-16-102, $-103,-104$, and -105 . These four vaults are all empty and clean.

All key equipment currently owned or expected to be owned by the end of FY 1996 by the Disposal Program falls within the Waste Retrieval Project. Current equipment includes the Tank C-106 Retrieval Sluicing System for Project W-320 and Tank AZ-101

\footnotetext{
organization.

${ }^{1}$ Waste generated during field sampling activities. Tank inventories covered by Technical Basis
} 
Retrieval System (decant pump, two mixer pumps, and instrumentation) for Project W-151. By the end of FY 1996, we expect to procure a Sonic Probe to supplement mixer pumps during DST retrieval.

\begin{tabular}{|c|c|c|}
\hline Pre-Award Actions & $\begin{array}{c}\text { Scheduled } \\
\text { Date }\end{array}$ & Actionee(s) \\
\hline $\begin{array}{l}\text { 1. Identify key documents. Provide listing to program/project level } \\
\text { for consolidation and RL review. }\end{array}$ & $\begin{array}{c}5 / 01 / 96 \\
\text { Completed }\end{array}$ & TW Crawford \\
\hline 2. Identify POCs and input to consolidated program/project listing. & $\begin{array}{c}5 / 15 / 96 \\
\text { Completed }\end{array}$ & TW Crawford \\
\hline $\begin{array}{l}\text { 3. Develop a listing and description of subcontracts supporting the } \\
\text { program/project or facility (or operation) and input to consolidated } \\
\text { program/project listing. Include any subcontracts planned to be } \\
\text { executed prior to contract takeover. }\end{array}$ & $\begin{array}{l}5 / 15 / 96 \\
\text { Completed }\end{array}$ & TW Crawford \\
\hline $\begin{array}{l}\text { 4. Develop a listing and description of databases. Provide list to } \\
\text { program/project level for consolidation. }\end{array}$ & $\begin{array}{c}5 / 15 / 96 \\
\text { Completed } \\
\end{array}$ & TW Crawford \\
\hline $\begin{array}{l}\text { 5. Identify number of briefings, and prepare briefing(s) for the } \\
\text { PHMC. (Coordinate number and content with RL POC.) }\end{array}$ & $7 / 15 / 96$ & TW Crawford \\
\hline $\begin{array}{l}\text { 6. Assemble key documentation, or identify its location, for use by } \\
\text { the PHMC. Includes preparation of a facility (or operations) } \\
\text { "Smart Book," to include information develop in the following } \\
\text { actions. }\end{array}$ & $\begin{array}{c}6 / 01 / 96 \\
\text { Completed "Smart } \\
\text { Book" only } \\
\text { See item } \$ 16 \\
\end{array}$ & TW Crawford \\
\hline $\begin{array}{l}\text { 7. Using the pre-existing checklist, compile pre-existing condition } \\
\text { information on the facility and/or major equipment. Conduct, or } \\
\text { schedule as a separate post-award action(s), any assessments } \\
\text { required to adequately address all relevant items on the checklist. } \\
\text { Provide completed checklist to RL POC for review. }\end{array}$ & $\begin{array}{l}6 / 15 / 96 \\
\text { Completed }\end{array}$ & TW Crawford \\
\hline $\begin{array}{l}\text { 8. Develop a list of open action items and commitments, including } \\
\text { status (Environmental, Tri-Party Agreement, DNFSB as examples). } \\
\text { Provide to RL POC for review. }\end{array}$ & $\begin{array}{l}\text { 6/01/96 } \\
\text { Completed }\end{array}$ & TW Crawford \\
\hline $\begin{array}{l}\text { 9. Develop a listing of information which constitutes the } \\
\text { "authorization basis" for the facility. }\end{array}$ & $\begin{array}{c}6 / 01 / 96 \\
\text { Completed } \\
\end{array}$ & TW Crawford \\
\hline $\begin{array}{l}\text { 10. Develop a description of ongoing and planned construction } \\
\text { projects. }\end{array}$ & $\begin{array}{c}6 / 01 / 96 \\
\text { Completed } \\
\end{array}$ & TW Crawford \\
\hline $\begin{array}{l}\text { 11. Develop a list of current work order services or other form of } \\
\text { agreement to provide work for other organizations, on and offsite. }\end{array}$ & $\begin{array}{c}6 / 01 / 96 \\
\text { Completed } \\
\end{array}$ & TW Crawford \\
\hline $\begin{array}{l}\text { 12. Develop a list of current work order services or other form of } \\
\text { agreement to obtain work from other organizations, on and offsite. }\end{array}$ & $\begin{array}{c}\text { 6/01/96 } \\
\text { Completed } \\
\end{array}$ & TW Crawford \\
\hline $\begin{array}{l}\text { 13. Identify space and equipment within program/project area and } \\
\text { facilities for use by the PHMC. }\end{array}$ & $\begin{array}{c}6 / 01 / 96 \\
\text { Completed } \\
\end{array}$ & TW Crawford \\
\hline $\begin{array}{l}\text { 14. Develop a list of permits governing the facility (or operation), and } \\
\text { provide a description of each as to where and how it applies. }\end{array}$ & $\begin{array}{c}6 / 01 / 96 \\
\text { Completed } \\
\end{array}$ & TW Crawford \\
\hline $\begin{array}{l}\text { 15. Verify the accuracy of property assignments for the facility (or } \\
\text { operations) and correct any deficiencies. }\end{array}$ & $\begin{array}{c}\text { 6/01/96 } \\
\text { Completed } \\
\end{array}$ & TW Crawford \\
\hline 16. Update/submit "Smart Book"/assemble key documents. & $7 / 15 / 96$ & TW Crawford \\
\hline
\end{tabular}




\begin{tabular}{|c|c|c|}
\hline Pre-Award Actions & $\begin{array}{c}\text { Scheduled } \\
\text { Date }\end{array}$ & Actionee(s) \\
\hline $\begin{array}{l}\text { 17. Verify pre-existing condition data is current and supplement as } \\
\text { necessary. }\end{array}$ & $7 / 22 / 96$ & TW Crawford \\
\hline 18. Ensure building assignments are current in RLPS. & $7 / 22 / 96$ & TW Crawford \\
\hline Post-Award Actions & & \\
\hline $\begin{array}{l}\text { 1. Provide the PHMC access to key documentation, pre-existing } \\
\text { condition information, commitment data, inventory data, etc. (as } \\
\text { established in the pre-award phase). Provide copies upon request. }\end{array}$ & $8 / 05 / 96$ & TW Crawford \\
\hline $\begin{array}{l}\text { 2. Provide any additional documentation or information requested by } \\
\text { the PHMC, if available, that were not identified during the pre- } \\
\text { award phase. }\end{array}$ & by $9 / 01 / 96$ & TW Crawford \\
\hline 3. Provide briefing(s) to the PHMC. & $8 / 12 / 96-9 / 02 / 96$ & TW Crawford \\
\hline 4. Provide a tour to the PHMC. & $8 / 12 / 96-9 / 02 / 96$ & TW Crawford \\
\hline $\begin{array}{l}\text { 5. Provide any recurring reports that the PHMC would like to } \\
\text { receive during transition. }\end{array}$ & by $9 / 15 / 96$ & TW Crawford \\
\hline 6. Provide necessary training to PHMC personnel. & $9 / 01 / 96-9 / 23 / 96$ & TW Crawford \\
\hline $\begin{array}{l}\text { 7. Assist the PHMC in the conduct of a Pre-Existing Condition } \\
\text { Assessment. }\end{array}$ & $8 / 12 / 96-9 / 16 / 96$ & TW Crawford \\
\hline $\begin{array}{l}\text { 8. Perform a joint property and equipment inventory with the } \\
\text { PHMC. }\end{array}$ & by $9 / 16 / 96$ & TW Crawford \\
\hline $\begin{array}{l}\text { 9. Provide an updated status of ongoing work prior to contract } \\
\text { turnover. }\end{array}$ & by $9 / 30 / 96$ & TW Crawford \\
\hline
\end{tabular}

\subsection{WASTE MANAGEMENT}

\subsubsection{Solid Waste} for RL.

The Solid Waste (SW) Program at the Hanford Site is currently directed by WHC

The SW Program is responsible for the development (and RL approval) of the Hanford Site Waste Acceptance Criteria (WAC), and the effective and efficient treatment, storage, and disposal of the hazardous, radioactive, and mixed solid wastes generated on the site, as well as selected offsite generators. The program's goal is to receive, store, treat, and dispose of solid radioactive and nonradioactive dangerous wastes in a safe, environmentally compliant, and cost effective manner.

The SW Program provides a comprehensive approach for the storage, treatment, and disposal of current and future solid waste received at the Hanford Site (from onsite and offsite generators) in a manner compliant with the current and evolving regulations, orders, permits, and safety analyses (Federal, state, DOE, and WHC). 
The SW Program addresses activities required for the exhumation and disposal of selected wastes currently in retrievable storage; provides a central focus for control of cost, scope, and schedule of Hanford Site non sanitary, non-HLW solid waste activities; and, provides a vehicle for ready communication of the scope of those activities to onsite and offsite organizations.

During remediation activities, large volumes of radioactive, nonradioactive hazardous, and mixed solid waste are expected to be produced; thus, creating the need for subsequent decontamination, treatment, storage, and/or waste disposal. The SW Program mission is to manage current and future contaminated solid waste streams in a safe, responsible, cost effective, and legally compliant manner.

Complex regulatory and technical requirements drive the scope of the SW Program services to receive, store, treat, and dispose of the radioactive and nonradioactive dangerous waste in support of the cleanup and remediation efforts onsite. In addition, the SW Program provides management and oversight to projects and programs which support the overall program mission.

The Solid Waste Disposal (SWD) facilities listed below have primary functions of treatment, storage, and disposal:

- Treatment

- 2336-W Waste Receiving and Processing Module (WRAP) 1

- Radioactive Mixed Waste (RMW) Treatment (via offsite contracts)

- 221-T Canyon (High Level Decon. Processing)

- 2706-T (LLW Decontamination Processing)

- Thermal Treatment (via offsite contract)

- Tri Butyl Phosphate (TBP) Treatment (via offsite contract)

- Storage

- 616 Facility

- Transuranic Assay and Storage Facility (TRUSAF)

- Central Waste Complex (CWC)

- W-112 Enhanced Radioactive Mixed Waste Storage

- Disposal

- Low Level Burial Grounds

- RMW Disposal (Trenches)

- Dangerous (Nonradioactive) Waste Disposal (offsite contract).

\section{Facility Descriptions}

2336-W Waste Receiving and Processing Module 1: WRAP 1 will receive retrieved and newly generated solid contact-handled radioactive wastes in 55-gal drums (some overpacked in salvage drums). Treatment activities will include repackaging and certification of design through-put waste streams. Drums will be inspected, assayed, and opened if necessary. Waste contents can be sorted and segregated, empty drums and wastes compacted as appropriate, repackaged, certified and shipped to final disposal (Waste Isolation Pilot Plant 
[WIPP]). Construction of the WRAP 1 facility scheduled to be complete in June 1996, with operation starting in March 1997. WRAP 1 will have a design operating life of 30 years on a one-shift per day basis. 6,825 drums of contact-handled waste can be received annually. This includes 2,100 drums of newly generated TRU/TRUMW, 2,625 drums of retrieved suspect transuranic (TRU) (assumed to be comprised of 50\% TRU and 50\% LLW), and 2,100 drums of newly generated LLW/low-level mixed waste (LLMW). The facility can also receive, certify, and ship 70 standard waste boxes of contact-handled TRU annually. WRAP 1 will be capable of accommodating the minimum required annual waste throughput in no more 175 operating days per year, with no more than five operating days per week. This is equivalent to $70 \%$, or 35 weeks, of the 50 weeks in a normal operating year. TRU/TRUMW processed will meet TRUPACT II shipping requirements and the waste acceptance criteria for disposal at WIPP, which will receive waste from WRAP 1 until 2023. WRAP 1 contains two gloveboxes in which limited small-scale treatment can be performed for more difficult waste in support of Federal Facilities Compliance Agreement (FFCA) or other small-scale treatment options. Currently funding guidance only provides for LLW processing and nondestructive examination (NDE)/nondestructive assay (NDA) activities, with start-up of TRU processing beyond FY 2002.

RMW Treatment Offsite Contracts: Since 1985, the Hanford Site has been storing LLMW for future treatment and disposal. It has been decided that the most efficient way to treat this waste is through utilization of offsite commercial contractors. Solid Waste has been investigating several different methods of treatment and has contracts either in place or in the process of being placed for these treatments. These include:

- TBP Treatment

- RMW Stabilization

- Macroencapsulation

- Volume Reduction with ATG (DOE contract SWD manages)

- Thermal Treatment.

221-T: The T Plant facility consists of a number of structures in the 200 West Area of the Hanford Site. The two primary treatment and storage facilities are the 221-T canyon and the 2706-T Building. T Plant was constructed in 1943 through 1944 to extract plutonium from production reactor fuel. The plant performed this function until it was deactivated in 1956. Most of the original process equipment was subsequently removed. In 1957 , $T$ Plant was placed in service as a beta-gamma decontamination facility and a support complex for experiments or other operations requiring containment or isolation. Today, 221-T is used for decontamination of high-level radioactive and hazardous contaminated items and process equipment. Support structures also have limited treatment of mixed and dangerous waste. The 221-T Facility has a waste container load/unload bay in the railcar tunnel that is used to place waste containers into the canyon for storage or treatment. This bay is also used for the cleanout and annual certification and maintenance of the liquid waste railcars.

2706-T: The 2706-T Building is a pre-engineered building erected during 1959 and 1960 to perform low level radioactive decontamination of railroad equipment. The facility has an open top tank to catch liquids during decontamination and piping to transfer the waste liquids to holding tanks. The building is fed by a railroad spur and the 
facility can accept a full size locomotive for treatment. 2706-T underwent extensive refurbishment in 1992 through 1994 and recently the building was expanded to include an additional 3000 square feet of floor area. This will allow an area to perform microencapsulation of waste under cover and in a separate containment if required. The additional space will also be utilized for staging of heavy equipment before decontamination and after decontamination for final release surveys. A second containment has been set up to allow limited alpha treatment in the facility making the facility a "dual survey" facility. The tank and piping systems are being upgraded with capital funds to bring the tank and liquid piping systems into full compliance by September 1999. The safety basis is being updated to change the facility from a fissile exempt facility to a fissile isolated facility. This will allow decontamination of alpha equipment and allow receipt of measurable amounts of fissile material into the facility. The 2706-T Facility is also used for LLW verification, sampling and repackaging for the Hanford Site and other waste generators. In the area surrounding the facility, there are numerous storage pads to stage waste, store empty containers and perform treatment. A $60-\mathrm{ft}$ by $90-\mathrm{ft}, 4,000$ psf pad to macroencapsulate tank farms long length equipment is being poured to allow that work to be performed in the area. A $70-\mathrm{ft}$ by $28-\mathrm{ft}$ change/office trailer was recently added to the complex and provides space for engineering and supervision offices and a step off pad for controlled movement in and out of the facility. A second, older change facility exists and could be used for a second step off pad when work load requires. The entire area is bounded by a fence which is used to control access and designate the permitted area.

Nonradioactive Dangerous Waste Storage Facility (616 Building): The Nonradioactive Dangerous Waste Storage Facility (NRDWSF) is classified as container storage and provides a centralized storage unit to receive, store, and prepare shipments of nonradioactive dangerous waste. This facility has the capacity to store 400 containers of dangerous waste. This facility is used on an exception basis only - preferred procedure is for generators to ship directly to offsite treatment, storage, or disposal facilities. The costs of offsite shipment are borne by the generators.

224-T Transuranic Assay and Storage Facility (TRUSAF): The 224-T Facility primarily stores TRU waste that meets the Hanford Site Solid WAC. This TRU waste will require processing through WRAP 1 to meet the WIPP WAC when finalized. In addition, other properly characterized and packaged LLMW could be accepted for storage. TRUSAF has the capacity to store $2,000,55$-gal (208 liter) drums. Waste is received primarily in DOT approved or equivalent $17 \mathrm{C}$ or $17 \mathrm{H}$ UN1A2 55 -gal containers or other DOT approved packages and overpacks.

Central Waste Complex (CWC): The CWC consists of a number of buildings, storage modules, and projects. Included among these facilities are twelve small mixed waste storage buildings (2402-W, 2402-WB, 2402-WC, 2402-WD, 2402-WE, 2402-WF, 2402-WG, 2402-WH, 2402-WI, 2402-WJ, 2402-WK, and 2402-WL), low-flash-point mixed waste storage modules, one plutonium/polychlorinated biphenyl mixed waste storage building (2401-W), large mixed waste storage (2403-WA, 2403-WB, 2403-WC, and 2403-WD) and a mixed waste storage pad. These facilities provide interim storage of mixed waste awaiting appropriate treatment and disposal methods being developed for waste disposition. The 2401-W, 2402-W series, 2403-W series, low-flashpoint mixed storage modules, alkali metal 
waste storage modules, and south alkali metal storage modules are currently operational. The 2401-W storage building has the capacity to store 1,072, 208-liter (55-gallon) drum equivalents of waste. Each of the 2402-W series buildings has the capacity to store 1,072 , 208-liter (55-gal) drum equivalents of waste. The 2403-W series buildings have a combined capacity of 52,300, 208-liter (55-gal) drum equivalents. Buildings 2403-WA, -WB, and -WC will accommodate 11,600, 208-liter (55-gal) drum equivalents each; and 2403-WD will accommodate $17,500,208$-liter (55-gal) drum equivalents. There are 19 low-flashpoint mixed storage modules providing a total capacity to store 400 , 208-liter (55-gal) drum equivalents of waste. There are 4 alkali metal waste storage modules providing a total capacity to store 84,208 -liter (55-gal) drum equivalents of waste. There are 8 south alkali metal waste storage modules providing a total capacity to store 168, 208-liter (55-gal) drum equivalents of waste. Waste in all CWC facilities is segregated according to hazardous characteristics. The characteristic types of LLMW/TRUMW stored will include acids, caustics, oxidizers, and Toxicity Characteristic Leaching Procedure toxic waste.

W-112 Enhanced Radioactive and Mixed Waste Storage Phase V Project: The Phase V storage facility will store wastes and distribute waste to various radioactive solid waste treatment facilities. This project is an addition to the existing CWC and includes the following storage buildings: 2404-WA, 2404-WB, 2404-WC. The Phase V project will provide interim and long term storage for waste. Project W-112 will also provide general office $(2740-\mathrm{W})$ and maintenance space $(2620-\mathrm{W})$ for the WRAP 1. Phase V will store contact-handled solid wastes received from offsite and onsite generators. Phase V will be used for interim storage of LLW and LLMW transferred from storage to treatment or disposal facilities. Storage will be provided for 13,300 drum equivalent (55-gallon drums) of contact-handled waste. Phase V will be able to accommodate low-level, mixed, and TRU wastes that do not meet the performance assessment. Classified and remote handled wastes will not be stored in the Phase V Facility.

Low-Level Burial Grounds: The Low-Level Burial Grounds (LLBG) provide disposal capability for Category 1 and Category 3 LLW. The SW Program currently disposes of LLW in burial grounds in the 200 Areas. The LLBG is capable of accommodating all forecasted Category 1 and Category $3 \mathrm{LLW}$.

Radioactive Mixed Waste Disposal: The RMW Disposal facility (RMW Trenches \#31 and \#34, and Burial Ground \#218-W-5) will provide permanent disposal capability for LLMW. Two disposal trenches have been constructed for RMW. Each disposal system will have a design life of 50 years minimum, 20 years are required for the operational phase, and 30 years for post closure monitoring. The capacity of each of the two RMW trench is a minimum of $7,600 \mathrm{~m}^{3}\left(270,000 \mathrm{ft}^{3}\right)$ of waste for a total of $15,200 \mathrm{~m}^{3}\left(540,000 \mathrm{ft}^{3}\right)$ of waste. Additional disposal capacity will be built in the future as demand requires. The site selected for disposal of mixed waste packages is the $218-\mathrm{W}-5$ Burial Ground. The RMW disposal system will meet the minimum functional standards for a dangerous waste landfill as required by Washington State regulations (Ecology 1991). 


\begin{tabular}{|c|c|c|}
\hline Pre-Award Actions & $\begin{array}{l}\text { Scheduled } \\
\text { Date }\end{array}$ & Actionee(s) \\
\hline $\begin{array}{l}\text { 1. Assemble key program documentation, or identify its } \\
\text { location, for use by the PHMC. Includes development } \\
\text { of a program "Smart Book." }\end{array}$ & $\begin{array}{l}\text { 6/01/96 } \\
\text { Completed }\end{array}$ & ME Kurts \\
\hline $\begin{array}{l}\text { 2. Develop a listing of key program individuals as POCs, } \\
\text { including facility and operations personnel. }\end{array}$ & $\begin{array}{l}\text { 6/01/96 } \\
\text { Completed }\end{array}$ & ME Kurts \\
\hline $\begin{array}{l}\text { 3. Develop listing and description of program databases, } \\
\text { including facility (or operations) databases. }\end{array}$ & $\begin{array}{c}6 / 01 / 96 \\
\text { Completed }\end{array}$ & ME Kurts \\
\hline $\begin{array}{l}\text { 4. Verify the accuracy of property assignments at the } \\
\text { program organizational level and correct any } \\
\text { deficiencies. }\end{array}$ & $\begin{array}{l}\text { 6/01/96 } \\
\text { Completed }\end{array}$ & NP Daniel \\
\hline $\begin{array}{l}\text { 5. Identify applicable milestones and commitments, } \\
\text { determine their status, and supply a copy of the } \\
\text { milestone description sheet. }\end{array}$ & $\begin{array}{l}\text { 6/01/96 } \\
\text { Completed }\end{array}$ & EF Mares \\
\hline $\begin{array}{l}\text { 6. Develop a list of open action items and commitments } \\
\text { and open-ended compliance issues. }\end{array}$ & $\begin{array}{c}6 / 01 / 96 \\
\text { Completed }\end{array}$ & ML Heinemeyer \\
\hline $\begin{array}{l}\text { 7. Develop a list of facility-specific mandatory trairing and } \\
\text { qualification requirements for employees. }\end{array}$ & $\begin{array}{c}6 / 01 / 96 \\
\text { Completed }\end{array}$ & ML Heinemeyer \\
\hline $\begin{array}{l}\text { 8. Develop a listing of information which constitutes the } \\
\text { "authorization basis" for all the SWM facilities. }\end{array}$ & $\begin{array}{c}6 / 01 / 96 \\
\text { Completed } \\
\end{array}$ & PL Hapke \\
\hline $\begin{array}{l}\text { 9. Develop a listing of information which constitutes the } \\
\text { "authorization basis" for the T Plant facilities. }\end{array}$ & $\begin{array}{c}6 / 01 / 96 \\
\text { Completed } \\
\end{array}$ & MS Wright \\
\hline $\begin{array}{l}\text { 10. Develop a listing and description of ongoing and } \\
\text { planned construction projects for SWD. }\end{array}$ & $\begin{array}{c}6 / 01 / 96 \\
\text { Completed }\end{array}$ & DE McKenney \\
\hline $\begin{array}{l}\text { 11. Develop a listing of "work for others" agreements, to } \\
\text { include onsite work orders and offsite special requests } \\
\text { and HQ ADSs. }\end{array}$ & $\begin{array}{l}6 / 01 / 96 \\
\text { Completed }\end{array}$ & ME Kurts \\
\hline $\begin{array}{l}\text { 12. Using the pre-existing condition checklist, compile pre- } \\
\text { existing condition information for all SWD facilities. } \\
\text { Conduct, or schedule as a separate post-closure } \\
\text { action(s), any assessments required to adequately } \\
\text { address all relevant items on the checklist. }\end{array}$ & $\begin{array}{l}\text { 6/15/96 } \\
\text { Completed }\end{array}$ & $\begin{array}{l}\text { RD Pierce } \\
\text { BM Barnes }\end{array}$ \\
\hline 13. Update/submit "Smart Book"/assemble key documents. & $7 / 15 / 96$ & ME Kurts \\
\hline 14. Complete briefings for PHMC. & $7 / 15 / 96$ & ME Kurts \\
\hline $\begin{array}{l}\text { 15. . Verify pre-existing condition evaluations are current. } \\
\text { Supplement as necessary. }\end{array}$ & $7 / 22 / 96$ & $\begin{array}{l}\text { RD Pierce } \\
\text { BM Barnes }\end{array}$ \\
\hline 16. Ensure building assignments are correct in RLPS. & $7 / 22 / 96$ & NP Daniel \\
\hline Post-Award Actions & $\therefore$ & \\
\hline $\begin{array}{l}\text { 1. Provide the PHMC access to the key program } \\
\text { documentation, any program level generated pre-existing } \\
\text { conditions information, listing of POCs, etc. Provide } \\
\text { copies upon request. }\end{array}$ & $8 / 05 / 96$ & DE McKenney \\
\hline
\end{tabular}




\begin{tabular}{|c|c|c|}
\hline Post-Award Actions & $\begin{array}{l}\text { Schedaled } \\
\text { Dace }\end{array}$ & Actionee(s) \\
\hline $\begin{array}{l}\text { 2. Program and facility space and services available for use } \\
\text { by the PHMC. }\end{array}$ & $8 / 05 / 96$ & ML Heinemeyer \\
\hline $\begin{array}{l}\text { 3. Provide Solid Waste Program business overview } \\
\text { briefing to the PHMC. }\end{array}$ & $8 / 08 / 96-8 / 19 / 96$ & $\begin{array}{l}\text { ML Heinemeyer } \\
\text { DE McKenney }\end{array}$ \\
\hline 4. Provide T Plant business review briefing to the PHMC. & $8 / 12 / 96-8 / 30 / 96$ & MS Wright \\
\hline $\begin{array}{l}\text { 5. Provide a WRAP } 1 \text { business review briefing to the } \\
\text { PHMC. }\end{array}$ & $8 / 12 / 96-8 / 30 / 96$ & PL Hapke \\
\hline 6. Provide SWM facilities tour to the PHMC. & $8 / 12 / 96 /-8 / 30 / 96$ & PL Hapke \\
\hline 7. Provide T Plant facilities tour to the PHMC. & $8 / 12 / 96-8 / 30 / 96$ & MS Wright \\
\hline 8. Provide WRAP 1 facilities tour to the PHMC. & $8 / 12 / 96-8 / 30 / 96$ & PL Hapke \\
\hline $\begin{array}{l}\text { 9. Discuss list of non-compliant issues and status with } \\
\text { PHMC and RL POC. }\end{array}$ & $8 / 12 / 96-9 / 16 / 96$ & $\begin{array}{l}\text { RD Pierce } \\
\text { CK Girres } \\
\text { KM McDonald }\end{array}$ \\
\hline $\begin{array}{l}\text { 10. Provide PHMC any recurring reports that they would } \\
\text { like to receive during transition. }\end{array}$ & $8 / 05 / 96-9 / 30 / 96$ & ML Heinemeyer \\
\hline 11. Provide necessary training to PHMC personnel. & $8 / 05 / 96-8 / 30 / 96$ & ML Heinemeyer \\
\hline $\begin{array}{l}\text { 12. Assist the PHMC in the conduct of Pre-existing } \\
\text { Condition Assessments. }\end{array}$ & $8 / 12 / 96-9 / 16 / 96$ & $\begin{array}{l}\text { RD Pierce } \\
\text { CK Girres } \\
\text { KM McDonald }\end{array}$ \\
\hline $\begin{array}{l}\text { 13. Provide an updated status on ongoing work prior to } \\
\text { contract turnover. }\end{array}$ & $9 / 30 / 96$ & WH Hamilton, Jr. \\
\hline $\begin{array}{l}\text { 14. Perform a joint property and equipment inventory with } \\
\text { the PHMC. }\end{array}$ & $9 / 16 / 96$ & NP Daniel \\
\hline
\end{tabular}

\subsubsection{Liquid Effluents Program}

The Liquid Effluents Program provides integrated liquid effluent management to support cleanup of the Hanford Site. The program mission is to manage current and future liquid effluent streams in a safe, responsible, cost-effective, and legally-compliant manner. The approach to implementing treatment and disposal of liquid effluents is based on the following strategy: stream characterization; identification of best available technology/all known and reasonable treatment (BAT/AKART); regulator acceptance of BAT/AKART; and obtaining appropriate state and/or Federal permits.

\begin{tabular}{|c|c|c|}
\hline Pre-Award Actions & $\begin{array}{l}\text { Scheduled } \\
\text { Date }\end{array}$ & Actionee(s) \\
\hline $\begin{array}{l}\text { 1. Develop a list of key program (or project) } \\
\text { documentation. Provide to RL POC for review. }\end{array}$ & $\begin{array}{l}5 / 15 / 96 \\
\text { Completed }\end{array}$ & FT Green \\
\hline
\end{tabular}




\begin{tabular}{|c|c|c|}
\hline Pre-Award Actions & $\begin{array}{c}\text { Scheduled } \\
\text { Date } \\
\end{array}$ & Actionee(s) \\
\hline $\begin{array}{l}\text { 2. Assemble key program (or project) documentation, or } \\
\text { identify its location, for use by the PHMC. Includes } \\
\text { development of a program/project "Smart Book," } \\
\text { including listings and descriptions developed below. }\end{array}$ & $\begin{array}{l}\text { 6/01/96 } \\
\text { Completed } \\
\text { "Smart Book" } \\
\text { only } \\
\text { See item } \# 9 \\
\end{array}$ & FT Green \\
\hline $\begin{array}{l}\text { 3. Identify number of briefings, and prepare program (or } \\
\text { project) briefing(s) for presentation to PHMC. } \\
\text { (Coordinate number and content with RL POC.) }\end{array}$ & $7 / 15 / 96$ & FT Green \\
\hline $\begin{array}{l}\text { 4. Develop a listing of Key program (or project) } \\
\text { individuals as POCs, including facility and operations } \\
\text { personnel. }\end{array}$ & $\begin{array}{l}\text { 6/01/96 } \\
\text { Completed }\end{array}$ & FT Green \\
\hline $\begin{array}{l}\text { 5. Develop listing and description of program (or project) } \\
\text { databases, including facility (or operations) databases. }\end{array}$ & $\begin{array}{c}6 / 01 / 96 \\
\text { Completed } \\
\end{array}$ & FT Green \\
\hline $\begin{array}{l}\text { 6. Verify the accuracy of property assigninents at the } \\
\text { program/project organizational level and correct any } \\
\text { deficiencies. }\end{array}$ & $\begin{array}{l}\text { 6/01/96 } \\
\text { Completed }\end{array}$ & FT Green \\
\hline $\begin{array}{l}\text { 7. Identify space and equipment within program (or } \\
\text { project) area and facilities for use by the PHMC. }\end{array}$ & $\begin{array}{c}\text { 6/01/96 } \\
\text { Completed }\end{array}$ & FT Green \\
\hline $\begin{array}{l}\text { 8. In outline form for all facilities/operations to follow, } \\
\text { define the appropriate contents and level of detail to be } \\
\text { included in facility/operations briefings to the PHMC. } \\
\text { (Review with RL POC.) }\end{array}$ & $\begin{array}{l}5 / 01 / 96 \\
\text { Completed }\end{array}$ & FT Green \\
\hline $\begin{array}{l}\text { 9. Update/submit program "Smart Book"/assemble key } \\
\text { documents. }\end{array}$ & $7 / 15 / 96$ & FT Green \\
\hline $\begin{array}{l}\text { 10. Ensure building assignments for all Liquid Effluent } \\
\text { Facilities are correct in RLPS. }\end{array}$ & $7 / 22 / 96$ & FT Green \\
\hline Post-Award Actions & . & \\
\hline $\begin{array}{l}\text { 1. Provide the PHMC access to the key program (or } \\
\text { project) documentation, any program level generated } \\
\text { pre-existing conditions information, listing of POCs, } \\
\text { and listing of subcontracts. Provide copies upon } \\
\text { request. }\end{array}$ & $8 / 05 / 96$ & FT Green \\
\hline $\begin{array}{l}\text { 2. Program/project and facility space and services } \\
\text { available for use by the PHMC. }\end{array}$ & $8 / 05 / 96$ & FT Green \\
\hline 3. Provide program/project briefing(s) to the PHMC. & $8 / 05 / 96-8 / 19 / 96$ & FT Green \\
\hline $\begin{array}{l}\text { 4. Perform a joint program/project level property and } \\
\text { equipment inventory with PHMC personnel. }\end{array}$ & $9 / 16 / 96$ & FT Green \\
\hline
\end{tabular}

4.2.2.1 200 Area Treated Effluent Disposal Facility. The 200 Area TEDF is a collection and disposal system for non-RCRA permitted waste streams which already meet discharge requirements. Implementation of BAT/AKART is the responsibility of the generating facilities. Facilities which discharge to the 200 Area TEDF include the PFP, 222-S Laboratory, T Plant, 284-W Power Plant, PUREX Plant, B Plant, and 242-A-81 
Water Services Building. Each facility must comply with discharge limits in the WAC 173-216 State Waste Discharge Permit without further treatment.

The 200 Area TEDF began operation in April 1995 and has a 30-year life. Design capacity of the 200 Area TEDF is $2300 \mathrm{gal} / \mathrm{min}$, although the Discharge Permit presently limits the average monthly flow to $640 \mathrm{gal} / \mathrm{min}$. About $130 \mathrm{Mgal}$ of treated effluent was discharged in 1995. The 200 Area TEDF includes more that 12 miles of polyvinyl chloride (PVC) pipe up to $14 \mathrm{in}$. in diameter. The effluent is discharged to two, 5-acre disposal ponds located east of the 200 East Area.

\begin{tabular}{|c|c|c|}
\hline Pre-Award Actions & $\begin{array}{l}\text { Scheduled } \\
\text { Date }\end{array}$ & Actionee(s) \\
\hline $\begin{array}{l}\text { 1. Identify number of briefings, and prepare facility (or } \\
\text { operations) briefing(s) for the PHMC. (Coordinate } \\
\text { number and content with RL POC.) }\end{array}$ & $\begin{array}{l}7 / 15 / 96 \\
\text { Completed }\end{array}$ & DW Lindsey \\
\hline $\begin{array}{l}\text { 2. Identify key documents for the facility (or operations). } \\
\text { Provide listing to program/project level for } \\
\text { consolidation and RL review. }\end{array}$ & $\begin{array}{l}5 / 01 / 96 \\
\text { Completed }\end{array}$ & DW Lindsey \\
\hline $\begin{array}{l}\text { 3. Assemble key facility (or operations) documentation, } \\
\text { or identify its location, for use by the PHMC. } \\
\text { Includes preparation of a facility (or operations) } \\
\text { "Smart Bock," to include information develop in the } \\
\text { following actions. }\end{array}$ & $\begin{array}{c}6 / 01 / 96 \\
\text { Completed } \\
\text { "Smart Book" Only } \\
\text { See Item } \sharp 17\end{array}$ & DW Lindsey \\
\hline $\begin{array}{l}\text { 4. Identify POCs for the facility (or operations) and input } \\
\text { to consolidated program/project listing. }\end{array}$ & $\begin{array}{c}5 / 15 / 96 \\
\text { Completed }\end{array}$ & DW Lindsey \\
\hline $\begin{array}{l}\text { 5. Develop a listing and description of subcontracts } \\
\text { supporting the facility (or operation) and input to } \\
\text { consolidated program/project listing. Include any } \\
\text { subcontracts planned to be executed prior to contract } \\
\text { takeover. }\end{array}$ & $\begin{array}{l}5 / 15 / 96 \\
\text { Completed }\end{array}$ & DW Lindsey \\
\hline $\begin{array}{l}\text { Using the pre-existing checklist, compile pre-existing } \\
\text { condition information on the facility and/or major } \\
\text { equipment. Conduct, or schedule as a separate post- } \\
\text { award action(s), any assessments required to } \\
\text { adequately address all relevant items on the checklist. } \\
\text { Provide completed checklist to RL POC for review. }\end{array}$ & $\begin{array}{l}\text { 6/15/96 } \\
\text { Completed }\end{array}$ & DW Lindsey \\
\hline $\begin{array}{l}\text { 7. Develop a list of open action items and commitments, } \\
\text { including status (Environmental, Tri-Party Agreement, } \\
\text { DNFSB as examples). Provide to RL POC for } \\
\text { review. }\end{array}$ & $\begin{array}{l}\text { 6/01/96 } \\
\text { Completed }\end{array}$ & DW Lindsey \\
\hline $\begin{array}{l}\text { 8. Identify current inventories of feed for the facility, and } \\
\text { materials to support continued operation (i.e., essential } \\
\text { materials, tools and equipment). }\end{array}$ & $\begin{array}{l}7 / 15 / 96 \\
\text { Completed }\end{array}$ & DW Lindsey \\
\hline $\begin{array}{l}\text { 9. Develop a list of facility (or operation)-specific } \\
\text { mandatory training and qualification requirements for } \\
\text { employees. }\end{array}$ & $\begin{array}{l}6 / 01 / 96 \\
\text { Completed }\end{array}$ & DW Lindsey \\
\hline
\end{tabular}




\begin{tabular}{|c|c|c|}
\hline Pre-Award Actions & $\begin{array}{l}\text { Scheduled } \\
\text { Date }\end{array}$ & Actionee(s) \\
\hline $\begin{array}{l}\text { 10. Develop a listing of information which constitutes the } \\
\text { "authorization basis" for the facility. }\end{array}$ & $\begin{array}{c}\text { 6/01/96 } \\
\text { Completed }\end{array}$ & DW Lindsey \\
\hline $\begin{array}{l}\text { 11. Develop a description of ongoing and planned } \\
\text { construction projects. }\end{array}$ & $\begin{array}{c}6 / 01 / 96 \\
\text { Completed }\end{array}$ & DW Lindsey \\
\hline $\begin{array}{l}\text { 12. Develop a listing and description of facility (or } \\
\text { operations) databases. Provide list to program/project } \\
\text { level for consolidation. }\end{array}$ & $\begin{array}{c}5 / 15 / 96 \\
\text { Completed }\end{array}$ & DW Lindsey \\
\hline $\begin{array}{l}\text { 13. Develop a list of current work order services or other } \\
\text { form of agreement to provide work for other } \\
\text { organizations, on and offsite. }\end{array}$ & $\begin{array}{c}6 / 01 / 96 \\
\text { Completed }\end{array}$ & DW Lindsey \\
\hline $\begin{array}{l}\text { 14. Develop a list of current work order services or other } \\
\text { form of agreement to obtain work from other } \\
\text { organizations, on and offsite. }\end{array}$ & $\begin{array}{l}6 / 01 / 96 \\
\text { Completed }\end{array}$ & DW Lindsey \\
\hline $\begin{array}{l}\text { 15. Develop a list of permits governing the facility (or } \\
\text { operation), and provide a description of each as to } \\
\text { where and how it applies. }\end{array}$ & $\begin{array}{c}\text { 6/01/96 } \\
\text { Completed }\end{array}$ & DW Lindsey \\
\hline $\begin{array}{l}\text { 16. Verify the accuracy of property assignments for the } \\
\text { facility (or operations) and correct any deficiencies. }\end{array}$ & $\begin{array}{l}6 / 01 / 96 \\
\text { Completed }\end{array}$ & DW Lindsey \\
\hline 17. Update/submit "Smart Book"/assemble key documents. & $7 / 15 / 96$ & DW Lindsey \\
\hline $\begin{array}{l}\text { 18. Verify pre-exising condition data is current and } \\
\text { supplement as necessary. }\end{array}$ & $7 / 22,36$ & DW Lindse $y$ \\
\hline 19. Update inventories of feed materials and waste. & $7 / 15 / 96$ & DW Lindsey \\
\hline Post-Award Actions & & \\
\hline $\begin{array}{l}\text { 1. Provide facility (or operations) briefing(s) to the } \\
\text { PHMC. }\end{array}$ & $8 / 12 / 96-8 / 30 / 96$ & DW Lindsey \\
\hline 2. Provide a facility (or operations) tour to the PHMC. & $8 / 12 / 96-8 / 30 / 96$ & DW Lindsey \\
\hline $\begin{array}{l}\text { 3. Provide the PHMC access to key documentation, pre- } \\
\text { existing condition information, commitment data, } \\
\text { inventory data, etc. (as established in the pre-award } \\
\text { phase) Provide copies upon request. }\end{array}$ & $8 / 05 / 96$ & DW Lindsey \\
\hline $\begin{array}{l}\text { 4. Provide any additional documentation or information } \\
\text { requested by the PHMC, if available, that was not } \\
\text { identified during the pre-award phase. }\end{array}$ & $8 / 30 / 96$ & DW Lindsey \\
\hline $\begin{array}{l}\text { 5. Provide PHMC any securring seports that they would } \\
\text { like to receive during transition. }\end{array}$ & $8 / 15 / 96-9 / 30 / 96$ & DW Lindsey \\
\hline 6. Provide necessary training to PHMC personnel. & $8 / 12 / 96-8 / 30 / 96$ & DW Lindsey \\
\hline $\begin{array}{l}\text { 7. Provide an updated status of ongoing work prior to } \\
\text { contract turnover. }\end{array}$ & by $9 / 30 / 96$ & DW Lindsey \\
\hline $\begin{array}{l}\text { 8. Perform a joint property and equipment inventory with } \\
\text { the PHMC. }\end{array}$ & by $9 / 16 / 96$ & DW Lindsey \\
\hline 9. Provide a categorized inventory of wastes. & by $9 / 30 / 96$ & DW Lindsey \\
\hline
\end{tabular}




\begin{tabular}{|l|r|l|}
\hline \multicolumn{1}{|c|}{ Post-Award Actions } & Scheduled & Actionee(s) \\
\hline 10. $\begin{array}{l}\text { Assist the PHMC in the conduct of Pre-existing } \\
\text { Condition Assessments. }\end{array}$ & $8 / 12 / 96-9 / 16 / 96$ & DW Lindsey \\
\hline
\end{tabular}

4.2.2.2 Liquid Effluent Retention Facility. The LERF consists of three RCRA-compliant surface impoundments for storing process condensate from the 242-A Evaporator and other feeds. The LERF provides equalization of the flow and $\mathrm{pH}$ of the feed to the ETF. Each LERF basin has a capacity of $6.5 \mathrm{Mgal}$. Two basins are used for normal operation and the third is used as contingency in the event a leak should develop in an operational basin. The basins are constructed of two high-density polyethylene (HDPE) flexible membrane liners. A system is provided to detect, collect, and remove leachate from between the primary and secondary liners. Beneath the secondary HDPE liner is a soil/bentonite barrier should the primary and secondary liners fail. Each basin has a mechanically-tensioned floating membrane cover constructed of very low-density polyethylene (VLDPE) to keep-out unwanted material and to minimize evaporation of the basin contents. The LERF began operation in April 1994 and is designed for a 20-year life. A total of $8.7 \mathrm{Mgal}$ of process condensate were stored in the LERF at the end of 1995.

\begin{tabular}{|c|c|c|}
\hline Pre-Award Actions & $\begin{array}{c}\text { Scheduled } \\
\text { Date }\end{array}$ & Actionee(s) \\
\hline $\begin{array}{l}\text { 1. Identify mumber af hrin finge, an atepare facility or } \\
\text { operations) briefing(s) for the PHMC. (Coordinate } \\
\text { number and content with RL POC.) }\end{array}$ & $\begin{array}{c}7 / 15 / 96 \\
\text { Completed }\end{array}$ & DW Linds=y \\
\hline $\begin{array}{l}\text { 2. Identify key docurnents for the facility (or operations). } \\
\text { Provide listing to program/project level for } \\
\text { consolidation and RL review. }\end{array}$ & $\begin{array}{l}5 / 01 / 96 \\
\text { Completed }\end{array}$ & DW Lindsey \\
\hline $\begin{array}{l}\text { 3. Assemble key facility (or operations) documentation, } \\
\text { or identify its location, for use by the PHMC. } \\
\text { Includes preparation of a facility (or operations) } \\
\text { "Smart Book," to include information develop in the } \\
\text { following actions. }\end{array}$ & $\begin{array}{l}6 / 01 / 96 \\
\text { Completed } \\
\text { "Smart Book" only } \\
\text { See item } \# 17\end{array}$ & DW Lindsey \\
\hline $\begin{array}{l}\text { 4. Identify POCs for the facility (or operations) and input } \\
\text { to consolidated program/project listing. }\end{array}$ & $\begin{array}{c}5 / 15 / 96 \\
\text { Completed }\end{array}$ & DW Lindsey \\
\hline $\begin{array}{l}\text { 5. Develop a listing and description of subcontracts } \\
\text { supporting the facility (or operation) and input to } \\
\text { consolidated program/project listing. Include any } \\
\text { subcontracts planned to be executed prior to contract } \\
\text { takeover. }\end{array}$ & $\begin{array}{c}5 / 15 / 96 \\
\text { Completed }\end{array}$ & DW Lindsey \\
\hline $\begin{array}{l}\text { Using the pre-existing checklist, compile pre-existing } \\
\text { condition information on the facitity and/or major } \\
\text { equipment. Conduct, or schedule as a separate post-. } \\
\text { award action(s), any assessments required to } \\
\text { adequately address all relevant items on the checklist. } \\
\text { Provide completed checklist to RL POC for review. }\end{array}$ & $\begin{array}{c}6 / 15 / 96 \\
\text { Completed }\end{array}$ & DW Lindsey \\
\hline
\end{tabular}




\begin{tabular}{|c|c|c|}
\hline Pre-Award Actions & $\begin{array}{c}\text { Scheduled } \\
\text { Date }\end{array}$ & Actionee(s) \\
\hline $\begin{array}{l}\text { 7. Develop a list of open action items and commitments, } \\
\text { inchuding staus (Environmental, Tri-Party Agreement, } \\
\text { DNFSB as examples). Provide to RL POC for } \\
\text { review. }\end{array}$ & $\begin{array}{l}6 / 01 / 96 \\
\text { Completed }\end{array}$ & DW Lindsey \\
\hline $\begin{array}{l}\text { 8. Identify current inventories of feed for the facility, and } \\
\text { materials to support continued operation (i.e., essential } \\
\text { materials, tools and equipment). }\end{array}$ & $\begin{array}{l}7 / 15 / 96 \\
\text { Completed }\end{array}$ & DW Lindsey \\
\hline $\begin{array}{l}\text { 9. Develop a list of facility (or operation)-specific } \\
\text { mandatory training and qualification requirements for } \\
\text { employees. }\end{array}$ & $\begin{array}{c}6 / 01 / 96 \\
\text { Completed }\end{array}$ & DW Lindsey \\
\hline $\begin{array}{l}\text { 10. Develop a listing of information which constitutes the } \\
\text { "authorization basis" for the facility. }\end{array}$ & $\begin{array}{c}\text { 6/01/96 } \\
\text { Completed }\end{array}$ & DW Lindsey \\
\hline $\begin{array}{l}\text { 11. Develop a description of ongoing and planned } \\
\text { construction projects. }\end{array}$ & $\begin{array}{c}\text { 6/01/96 } \\
\text { Completed }\end{array}$ & DW Lindsey \\
\hline $\begin{array}{l}\text { 12. Develop a listing and description of facility (or } \\
\text { operations) databases. Provide list to program/project } \\
\text { level for consolidation. }\end{array}$ & $\begin{array}{l}5 / 15 / 96 \\
\text { Completed }\end{array}$ & DW Lindsey \\
\hline $\begin{array}{l}\text { 13. Develop a list of current work order services or other } \\
\text { form of agreement to provide work for other } \\
\text { organizations, on and offsite. }\end{array}$ & $\begin{array}{l}\text { 6/01/96 } \\
\text { Completed }\end{array}$ & DW Lindsey \\
\hline $\begin{array}{l}\text { 14. Develop a list of current work order services or other } \\
\text { form of agreement to obtain work from other } \\
\text { organizations, on and offsite. }\end{array}$ & $\begin{array}{c}6 / 01 / 96 \\
\text { Completed }\end{array}$ & DW Lindsey \\
\hline $\begin{array}{l}\text { 15. Develop a list of permits governing the facility (or } \\
\text { operation), and provide a description of each as to } \\
\text { where and how it applies. }\end{array}$ & $\begin{array}{c}6 / 01 / 96 \\
\text { Completed }\end{array}$ & DW Lindsey \\
\hline $\begin{array}{l}\text { 16. Verify the accuracy of property assignments for the } \\
\text { facility (or operations) and correct any deficiencies. }\end{array}$ & $\begin{array}{l}6 / 01 / 96 \\
\text { Completed }\end{array}$ & DW Lindsey \\
\hline 17. Update/submit "Smart Book"/assemble key documents. & $7 / 15 / 96$ & DW Lindsey \\
\hline $\begin{array}{l}\text { 18. Verify pre-existing condition data is current and } \\
\text { supplement as necessary. }\end{array}$ & $7 / 22 / 96$ & DW Lindsey \\
\hline 19. Update inventories of feed materials and waste. & $7 / 15 / 96$ & DW Lindsey \\
\hline s. $\quad$ Post-Award Actions & & \\
\hline $\begin{array}{l}\text { 1. Provide facility (or operations) briefing(s) to the } \\
\text { PHMC. }\end{array}$ & $8 / 12 / 96-8 / 30 / 96$ & DW Lindsey \\
\hline 2. Provide a facility (or operations) tour to the PHMC. & $8 / 12 / 96-8 / 30 / 96$ & DW Lindsey \\
\hline $\begin{array}{l}\text { 3. Provide the PHMC access to key documentation, pre- } \\
\text { existing condition information, commitment data, } \\
\text { inventory data, etc. (as established in the pre-award } \\
\text { phase) Provide copies upon request. }\end{array}$ & $8 / 05 / 96$ & DW Lindsey \\
\hline $\begin{array}{l}\text { 4. Provide any additional documentation or information } \\
\text { requested by the PHMC, if available, that was not } \\
\text { identified during the pre-award phase. }\end{array}$ & $8 / 30 / 96$ & DW Lindsey \\
\hline
\end{tabular}




\begin{tabular}{|c|c|c|}
\hline Post-Award Actions & $\begin{array}{c}\text { Scheduled } \\
\text { Date }\end{array}$ & Actionee(s) \\
\hline $\begin{array}{l}\text { 5. Provide PHMC any recurring reports that they would } \\
\text { like to receive during transition. }\end{array}$ & $8 / 15 / 96-9 / 30 / 96$ & DW Lindsey \\
\hline 6. Provide necessary training to PHMC personnel. & $8 / 12 / 96-8 / 30 / 96$ & DW Lindsey \\
\hline $\begin{array}{l}\text { 7. Provide an updated status of ongoing work prior to } \\
\text { contract turnover. }\end{array}$ & by $9 / 30 / 96$ & DW Lindsey \\
\hline $\begin{array}{l}\text { 8. Perform a joint property and equipment inventory with } \\
\text { the PHMC. }\end{array}$ & by $9 / 16 / 96$ & DW Lindsey \\
\hline 9. Provide a categorized inventory of wastes. & by $9 / 30 / 96$ & DW Lindsey \\
\hline $\begin{array}{l}\text { 10. Assist the PHMC in the conduct of Pre-existing } \\
\text { Condition Assessments. }\end{array}$ & $8 / 12 / 96-9 / 16 / 96$ & DW Lindsey \\
\hline
\end{tabular}

4.2.2.3 200 Area Effluent Treatment Facility. The ETF provides for (1) collection of liquid effluents; (2) treatment to reduce the concentration of radioactive and hazardous waste constituents in the effluent streams to acceptable levels; (3) tanks to verify the treated effluent meets discharge requirements; and (4) disposal of the treated effluent. The treatment process constitutes BAT and includes ultraviolet light/peroxide (UV/Ox) destruction of organic compounds, reverse osmosis to remove dissolved solids, and ion exchange to remove the last traces of contaminants. Treatment capacity of the ETF is $150 \mathrm{gal} / \mathrm{min}$., with a design operating efficiency of 72 percent. The ETF began operation in December 1995 and has a 30 -year design life.

The treated effluent from the ETF is discharged via a dedicated pipeline to a stateapproved land disposal site (SALDS). The SALDS consists of an underground drain field. A delisting petition was approved by the EPA and exempts the treated process condensate from the requirements of hazardous waste regulations under RCRA, and imposes certain effluent quality restrictions. High concentrations of ammonia in the evaporator process condensate also make this stream a dangerous waste subject to WAC 173-303 Dangerous Waste Regulations. The SALDS was permitted under the WAC 173-216 State Waste Discharge Permit Program.

Secondary waste from treating the process condensate (or other feed streams) is a LLMW that will be concentrated, dried, and packaged in 55-gal drums. The ETF is a RCRA permitted storage facility and this secondary waste material is temporarily stored until it is transferred to the CWC for subsequent treatment (if needed to meet Land Disposal Restriction treatment standards) and disposal in the Mixed Waste Trench.

\begin{tabular}{|c|c|c|}
\hline \multicolumn{1}{|c|}{ Pre-Award Actions } & $\begin{array}{c}\text { Scheduled } \\
\text { Date }\end{array}$ & Actionee(s) \\
\hline $\begin{array}{l}\text { 1. Identify number of briefings, and prepare facility (or } \\
\text { operations) briefing(s) for the PHMC. (Coordinate } \\
\text { number and content with RL POC.) }\end{array}$ & $\begin{array}{c}7 / 15 / 96 \\
\text { Completed }\end{array}$ & DW Lindsey \\
\hline
\end{tabular}




\begin{tabular}{|c|c|c|}
\hline Pre-Award Actions & $\begin{array}{l}\text { Scheduled } \\
\text { Date }\end{array}$ & Actionee(s) \\
\hline $\begin{array}{l}\text { 2. Identify key documents for the facility (or operations). } \\
\text { Provide listing to program/project level for } \\
\text { consolidation and RL review. }\end{array}$ & $\begin{array}{l}5 / 01 / 96 \\
\text { Completed }\end{array}$ & DW Lindsey \\
\hline $\begin{array}{l}\text { 3. Assemble key facility (or operations) documentation, } \\
\text { or identify its location, for use by the PHMC. } \\
\text { Includes preparation of a facility (or operations) } \\
\text { "Smart Book," to include information develop in the } \\
\text { following actions. }\end{array}$ & $\begin{array}{c}6 / 01 / 96 \\
\text { Completed } \\
\text { "Smart Book" Only } \\
\text { See item \#17 }\end{array}$ & DW Lindsey \\
\hline $\begin{array}{l}\text { 4. Identify POCs for the facility (or operations) and input } \\
\text { to consolidated program/project listing. }\end{array}$ & $\begin{array}{c}5 / 15 / 96 \\
\text { Completed }\end{array}$ & DW Lindsey \\
\hline $\begin{array}{l}\text { 5. Develop a listing and description of subcontracts } \\
\text { supporting the facility (or operation) and input to } \\
\text { consolidated program/project listing. Include any } \\
\text { subcontracts planned to be executed prior to contract } \\
\text { takeover. }\end{array}$ & $\begin{array}{c}5 / 15 / 96 \\
\text { Completed }\end{array}$ & DW Lindsey \\
\hline $\begin{array}{l}\text { Using the pre-existing checklist, compile pre-existing } \\
\text { condition information on the facility and/or major } \\
\text { equipment. Conduct, or schedule as a separate post- } \\
\text { award action(s), any assessments required to } \\
\text { adequately address all relevant items on the checklist. } \\
\text { Provide completed checklist to RL POC for review. }\end{array}$ & $\begin{array}{l}6 / 15 / 96 \\
\text { Completed }\end{array}$ & DW Lindsey \\
\hline $\begin{array}{l}\text { 7. Develop a list of open action items and cornmitments, } \\
\text { including status (Environmental, Tri-Party Agreement, } \\
\text { DNFSB as examples). Provide to RL POC for } \\
\text { review. }\end{array}$ & $\begin{array}{l}6 / 01 / 96 \\
\text { Completed }\end{array}$ & DW Lindsey \\
\hline $\begin{array}{l}\text { 8. Identify current inventories of feed for the facility, and } \\
\text { materials to support continued operation (i.e., essential } \\
\text { materials, tools and equipment). }\end{array}$ & $\begin{array}{l}7 / 15 / 96 \\
\text { Completed }\end{array}$ & DW Lindsey \\
\hline $\begin{array}{l}\text { 9. Develop a list of facility (or operation)-specific } \\
\text { mandatory training and qualification requirements for } \\
\text { employees. }\end{array}$ & $\begin{array}{l}\text { 6/01/96 } \\
\text { Completed }\end{array}$ & DW Lindsey \\
\hline $\begin{array}{l}\text { 10. Develop a listing of information which constitutes the } \\
\text { "authorization basis" for the facility. }\end{array}$ & $\begin{array}{l}6 / 01 / 96 \\
\text { Completed }\end{array}$ & DW Lindsey \\
\hline $\begin{array}{l}\text { 11. Develop a description of ongoing and planned } \\
\text { construction projects. }\end{array}$ & $\begin{array}{l}\text { 6/01/96 } \\
\text { Completed }\end{array}$ & DW Lindsey \\
\hline $\begin{array}{l}\text { 12. Develop a listing and description of facility (or } \\
\text { operations) databases. Provide list to program/project } \\
\text { level for consolidation. }\end{array}$ & $\begin{array}{l}5 / 15 / 96 \\
\text { Completed }\end{array}$ & DW Lindsey \\
\hline $\begin{array}{l}\text { 13. Develop a list of current work order services or other } \\
\text { form of agreement to provide work for other } \\
\text { organizations, on and offsite. }\end{array}$ & $\begin{array}{l}6 / 01 / 96 \\
\text { Completed }\end{array}$ & DW Lindsey \\
\hline $\begin{array}{l}\text { 14. Develop a list of current work order services or other } \\
\text { form of agreement to obtain work from other } \\
\text { organizations, on and offsite. }\end{array}$ & $\begin{array}{c}6 / 01 / 96 \\
\text { Completed }\end{array}$ & DW Lindsey \\
\hline
\end{tabular}




\begin{tabular}{|c|c|c|}
\hline Post-A ward Actions & $\begin{array}{l}\text { Scheduled } \\
\text { Date }\end{array}$ & Actionee(s) \\
\hline $\begin{array}{l}\text { 15. Develop a list of permits governing the facility (or } \\
\text { operation), and provide a description of each as to } \\
\text { where and how it applies. }\end{array}$ & $\begin{array}{l}\text { 6/01/96 } \\
\text { Completed }\end{array}$ & DW Lindsey \\
\hline $\begin{array}{l}\text { 16. Verify the accuracy of propercy assignments for the } \\
\text { facility (or operations) and correct any deficiencies. }\end{array}$ & $\begin{array}{c}\text { 6/01/96 } \\
\text { Completed }\end{array}$ & DW Lindsey \\
\hline 17. Update/submit "Smart Book"/assemble key documents. & $7 / 15 / 96$ & DW Lindsey \\
\hline $\begin{array}{l}\text { 18. Verify pre-existing condition data is current and } \\
\text { supplement as necessary. }\end{array}$ & $7 / 22 / 96$ & DW Lindsey \\
\hline 19. Update inventories of feed materials and waste. & $7 / 15 / 96$ & DW Lindsey \\
\hline $\begin{array}{l}\text { 1. Provide facility (or operations) briefing(s) to the } \\
\text { PHMC. }\end{array}$ & $8 / 12 / 96-8 / 30 / 96$ & DW Lindsey \\
\hline 2. Provide a facility (or operations) tour to the PHMC. & $8 / 12 / 96-8 / 30 / 96$ & DW Lindsey \\
\hline $\begin{array}{l}\text { 3. Provide the PHMC access to key documentation, pre- } \\
\text { existing condition information, commitment data, } \\
\text { inventory data, etc. (as established in the pre-award } \\
\text { phase) Provide copies upon request. }\end{array}$ & $8 / 05 / 96$ & DW Lindsey \\
\hline $\begin{array}{l}\text { 4. Provide any additional documentation or information } \\
\text { requested by the PHMC, if available, that was not } \\
\text { identified during the pre-award phase. }\end{array}$ & $8 / 30 / 96$ & DW Lindsey . \\
\hline $\begin{array}{l}\text { 5. Provide PHMC any recurring reports that they would } \\
\text { like to receive during transition. }\end{array}$ & $8 / 15 / 96-9 / 30 / 96$ & DW Lindsey \\
\hline 6. Provide necessary training to PHMC personnel. & $8 / 15 / 96-9 / 30 / 96$ & DW Lindsey \\
\hline $\begin{array}{l}\text { 7. Provide an updated status of ongoing work prior to } \\
\text { contract turnover. }\end{array}$ & by $9 / 30 / 96$ & DW Lindsey \\
\hline $\begin{array}{l}\text { 8. Perform a joint property and equipment inventory with } \\
\text { the PHMC. }\end{array}$ & by $9 / 16 / 96$ & DW Lindsey \\
\hline 9. Provide a categorized inventory of wastes. & by $9 / 30 / 96$ & DW Lindsey \\
\hline $\begin{array}{l}\text { 10. Assist the PHMC in the conduct of Pre-existing } \\
\text { Condition Assessments. }\end{array}$ & $8 / 12 / 96-9 / 16 / 96$ & DW Lindsey \\
\hline
\end{tabular}

4.2.2.4 300 Area Treated Effluent Disposal Facility. Wastewater from laboratories, research facilities, office buildings, and reactor fuel fabrication facilities in the 300 Area is treated in the 300 Area TEDF. The wastewater consists of once-through cooling water, steam condensate, and other liquid wastes generated in non-contact radioactive processes. The 300 Area TEDF is designed for continuous receipt of waste waters with storage capacity of up to 5 days at the design flow rate of $300 \mathrm{gal} / \mathrm{min}$. The facility treats the wastewater using BAT. The treatment process includes iron co-precipitation to remove heavy metals, thiol functional resin ion exchange to remove mercury, and ultraviolet light/hydrogen peroxide oxidation to destroy organics and cyanide. The 300 Area TEDF began operation in December 1994 and has a 30-year design life. About $83 \mathrm{Mgal}$ of wastewater were treated in 1995. 
The treated liquid effluent is monitored and discharged through an outfall to the Columbia River under a NPDES permit. Sludge from the iron co-precipitation process is dewatered and used for backfill in the LLW trench.

\begin{tabular}{|c|c|c|}
\hline Pre-Award Actions & $\begin{array}{l}\text { Scheduled } \\
\text { Date }\end{array}$ & Actionee(s) \\
\hline $\begin{array}{l}\text { 1. Identify number of briefings, and prepare facility (or } \\
\text { operations) briefing(s) for the PHMC. (Coordinate } \\
\text { number and content with RL POC.) }\end{array}$ & $7 / 15 / 96$ & LW Roberts \\
\hline $\begin{array}{l}\text { 2. Identify key documents for the facility (or operations). } \\
\text { Provide listing to program/project level for } \\
\text { consolidation and RL review. }\end{array}$ & $\begin{array}{l}5 / 01 / 96 \\
\text { Completed }\end{array}$ & LW Roberts \\
\hline $\begin{array}{l}\text { 3. Assemble key facility (or operations) documentation, } \\
\text { or identify its location, for use by the PHMC. } \\
\text { Includes preparation of a facility (or operations) } \\
\text { "Smart Book," to include information develop in the } \\
\text { following actions. }\end{array}$ & $\begin{array}{c}6 / 01 / 96 \\
\text { Completed } \\
\text { "Smart Book" only } \\
\text { See item } \# 17\end{array}$ & LW Roberts \\
\hline $\begin{array}{l}\text { 4. Identify POCs for the facility (or operations) and input } \\
\text { to consolidated program/project listing. }\end{array}$ & $\begin{array}{c}5 / 15 / 96 \\
\text { Completed }\end{array}$ & LW Roberts \\
\hline $\begin{array}{l}\text { 5. Develop a listing and description of subcontracts } \\
\text { supporting the facility (or operation) and input to } \\
\text { consolidated program/project listing. Include any } \\
\text { subcontracts planned to be executed prior to contract } \\
\text { takeover. }\end{array}$ & $\begin{array}{l}5 / 15 / 96 \\
\text { Completed }\end{array}$ & LW Roberts \\
\hline $\begin{array}{l}\text { 6sing the pre-existing checklist, compile pre-existing } \\
\text { condition information on the facility and/or major } \\
\text { equipment. Conduct, or schedule as a separate post- } \\
\text { award action(s), any assessments required to } \\
\text { adequately address all relevant items on the checklist. } \\
\text { Provide completed checklist to RL POC for review. }\end{array}$ & $\begin{array}{c}6 / 15 / 96 \\
\text { Completed }\end{array}$ & LW Roberts \\
\hline $\begin{array}{l}\text { 7. Develop a list of open action items and commitments, } \\
\text { including status (Environmental, Tri-Party Agreement, } \\
\text { DNFSB as examples). Provide to RL POC for } \\
\text { review. }\end{array}$ & $\begin{array}{l}\text { 6/01/96 } \\
\text { Completed }\end{array}$ & LW Roberts \\
\hline $\begin{array}{l}\text { 8. Identify current inventories of feed for the facility, and } \\
\text { materials to support continued operation (i.e., essential } \\
\text { materials, tools and equipment). }\end{array}$ & $\begin{array}{l}\text { 6/01/96 } \\
\text { Completed }\end{array}$ & LW Roberts \\
\hline $\begin{array}{l}\text { 9. Develop a list of facility (or operation)-specific } \\
\text { mandatory training and qualification requirements for } \\
\text { employees. }\end{array}$ & $\begin{array}{l}\text { 6/01/96 } \\
\text { Completed }\end{array}$ & LW Roberts \\
\hline $\begin{array}{l}\text { 10. Develop a listing of infornation which constitutes the } \\
\text { "authorization basis" for the facility. }\end{array}$ & $\begin{array}{c}\text { 6/01/96 } \\
\text { Completed } \\
\end{array}$ & LW Roberts \\
\hline $\begin{array}{l}\text { 11. Develop a description of ongoing and planned } \\
\text { construction projects. }\end{array}$ & $\begin{array}{c}6 / 01 / 96 \\
\text { Completed }\end{array}$ & LW Roberts \\
\hline $\begin{array}{l}\text { 12. Develop a listing and description of facility (or } \\
\text { operations) databases. Provide list to program/project } \\
\text { level for consolidation. }\end{array}$ & $\begin{array}{l}5 / 15 / 96 \\
\text { Completed }\end{array}$ & LW Roberts \\
\hline $\begin{array}{l}\text { 13. Develop a list of current work order services or other } \\
\text { form of agreement to provide work for other } \\
\text { organizations, on and offsite. }\end{array}$ & $\begin{array}{l}\text { 6/01/96 } \\
\text { Completed }\end{array}$ & LW Roberts \\
\hline
\end{tabular}




\begin{tabular}{|c|c|c|}
\hline Pre-Award Actions & $\begin{array}{l}\text { Scheduled } \\
\text { Dâté }\end{array}$ & Actionet(s) \\
\hline $\begin{array}{l}\text { 14. Develop a list of current work order services or other } \\
\text { form of agreement to obtain work from other } \\
\text { organizations, on and offsite. }\end{array}$ & $\begin{array}{l}\text { 6/01/96 } \\
\text { Completed }\end{array}$ & LW Roberts \\
\hline $\begin{array}{l}\text { 15. Develop a list of permits governing the facility (or } \\
\text { operation), and provide a description of each as to } \\
\text { where and how it applies. }\end{array}$ & $\begin{array}{l}\text { 6/01/96 } \\
\text { Completed }\end{array}$ & LW Roberts \\
\hline $\begin{array}{l}\text { 16. Verify the accuracy of property assignments for the } \\
\text { facility (or operations) and correct any deficiencies. }\end{array}$ & $\begin{array}{c}6 / 01 / 96 \\
\text { Completed }\end{array}$ & LW Roberts \\
\hline 17. Update/submit "Smart Book"/assemble key documents. & $7 / 15 / 96$ & LW Roberts \\
\hline $\begin{array}{l}\text { 18. Verify pre-existing condition data is current and } \\
\text { supplement as necessary. }\end{array}$ & $7 / 22 / 96$ & LW Roberts \\
\hline 19. Update inventories of feed materials and waste. & $7 / 15 / 96$ & LW Roberts \\
\hline \multicolumn{3}{|l|}{ Post-Award Actions } \\
\hline $\begin{array}{l}\text { 1. Provide facility (or operations) briefing(s) to the } \\
\text { PHMC. }\end{array}$ & $8 / 12 / 96-8 / 30 / 96$ & LW Roberts \\
\hline 2. Provide a facility (or operations) tour to the PHMC. & $8 / 12 / 96-8 / 30 / 96$ & LW Roberts \\
\hline $\begin{array}{l}\text { 3. Provide the PHMC access to key documentition, pre- } \\
\text { existing condition information, commiment data, } \\
\text { inventory data, etc. (as established in the pre-award } \\
\text { phase) Provide copies upon request. }\end{array}$ & $8 / 05 / 96$ & LW Roberts \\
\hline $\begin{array}{l}\text { 4. Provide any additional documentation or information } \\
\text { requested by the PHMC, if available, that was not } \\
\text { identified during the pre-award phase. }\end{array}$ & $8 / 30 / 96$ & LW Roberts \\
\hline $\begin{array}{l}\text { 5. Develop a listing and description of subcontracts } \\
\text { supporting the facility (or operation) and input to } \\
\text { consolidated program/project listing. Include any } \\
\text { subcontracts planned to be executed prior to contract } \\
\text { takeover. }\end{array}$ & $8 / 15 / 96-9 / 30 / 96$ & LW Roberts \\
\hline 6. Provide necessary training to PHMC personnel. & $8 / 12 / / 96-8 / 30 / 96$ & LW Roberts \\
\hline $\begin{array}{l}\text { 7. Provide an updated status of ongoing work prior to } \\
\text { contract turnover. }\end{array}$ & by $9 / 30 / 96$ & LW Roberts \\
\hline $\begin{array}{l}\text { 8. Perform a joint property and equipment inventory with } \\
\text { the PHMC. }\end{array}$ & by $9 / 16 / 96$ & LW Roberts \\
\hline 9. Provide a categorized inventory of wastes. & by $9 / 30 / 96$ & LW Roberts \\
\hline $\begin{array}{l}\text { 10. Assist the PHMC in the conduct of Pre-existing } \\
\text { Condition Assessments. }\end{array}$ & $8 / 12 / 96-9 / 16 / 96$ & LW Roberts \\
\hline
\end{tabular}

4.2.2.5 340 Waste Handling Facility. The 340 Complex provides receipt, storage, and loadout capability for low-level liquid waste generated during laboratory operations in the 300 Area. The waste is accumulated and stored in two, 15,000-gal tanks located in a covered, below-grade vault in the 340 Building. Six additional 8,000 -gal tanks in the adjacent 340-A Building provide backup storage capability. The waste is pumped into rail cars and transported to the 204-AR Unloading Facility, for neutralization and transfer to 
DSTs in the 200 Area for storage. The 340 Facility is non-compliant with RCRA requirements and waste can only be stored for less than 90 days.

The 340 Facility will cease operation on or before the year 2000 following implementation of a replacement system in an existing facility such as the 325 Building. A new waste handling facility with storage and loadout capability will be provided at the 325 Building to handle radioactive and mixed waste from the 300 Area laboratory operations. This replacement facility will also serve any other generators which are still operating. The 340 Facility will be cleaned out and custody will be transferred to the Facility Stabilization Program for deactivation.

The 307 Basins are four retention basins located adjacent to the 340 Facility. the basins receive wastewater via the retention process sewer system, which is a collection system for non-regulated liquids which have the potential to become radioactively contaminated. Monitoring is provided at the 307 Basins with batch-release to either the 300 Area TEDF or the 340 Facility. A shutdown plan being developed for the 340 Facility will address possible future uses of the basins. The 307 Basins are included with the 340 Facility for transition planning purposes.

\begin{tabular}{|c|c|c|}
\hline Pre-Award Actions & $\begin{array}{l}\text { Scheduled } \\
\text { Date }\end{array}$ & Actionee(s) \\
\hline $\begin{array}{l}\text { 1. Identify number of briefings, and prepare facility (or } \\
\text { operations) briefing(s) for the PHMC. (Coordinate } \\
\text { number and content with RL POC.) }\end{array}$ & $7 / 15 / 96$ & LW Roberts \\
\hline $\begin{array}{l}\text { 2. Identify key documents for the facility (or operations). } \\
\text { Provide listing to program/project level for } \\
\text { consolidation and RL review. }\end{array}$ & $\begin{array}{l}5 / 01 / 96 \\
\text { Completed }\end{array}$ & LW Roberts \\
\hline $\begin{array}{l}\text { 3. Assemble key facility (or operations) documentation, } \\
\text { or identify its location, for use by the PHMC. } \\
\text { Includes preparation of a facility (or operations) } \\
\text { "Smart Book," to include information develop in the } \\
\text { following actions. }\end{array}$ & $\begin{array}{c}6 / 01 / 96 \\
\text { Completed } \\
\text { "Smart Book" Only } \\
\text { See item } \sharp 17\end{array}$ & LW Roberts \\
\hline $\begin{array}{l}\text { 4. Identify POCs for the facility (or operations) and input } \\
\text { to consolidated program/project listing. }\end{array}$ & $\begin{array}{c}5 / 15 / 96 \\
\text { Completed }\end{array}$ & LW Roberts \\
\hline $\begin{array}{l}\text { 5. Develop a listing and description of subcontracts } \\
\text { supporting the facility (or operation) and input to } \\
\text { consolidated program/project listing. Include any } \\
\text { subcontracts planned to be executed prior to contract } \\
\text { takeover. }\end{array}$ & $\begin{array}{c}5 / 15 / 96 \\
\text { Completed }\end{array}$ & LW Roberts \\
\hline $\begin{array}{l}\text { Using the pre-existing checklist, compile pre-existing } \\
\text { condition information on the facitity and/or major } \\
\text { equipment. Conduct, or schedule as a separate post- } \\
\text { award action(s), any assessments required to } \\
\text { adequately address all relevant items on the checklist. } \\
\text { Provide completed checklist to RL POC for review. }\end{array}$ & $\begin{array}{l}\text { 6/15/96 } \\
\text { Completed }\end{array}$ & LW Roberts \\
\hline $\begin{array}{l}\text { 7. Develop a list of open action items and cornmitments, } \\
\text { including status (Environmental, Tri-Party Agreement, } \\
\text { DNFSB as examples). Provide to RL POC for } \\
\text { review. }\end{array}$ & $\begin{array}{l}\text { 6/01/96 } \\
\text { Completed }\end{array}$ & LW Roberts \\
\hline
\end{tabular}




\begin{tabular}{|c|c|c|}
\hline Pre-Award Actions & $\begin{array}{c}\text { Scheduled } \\
\text { Date } \\
\end{array}$ & Actionee(s) \\
\hline $\begin{array}{l}\text { 8. Identify current inventories of feed for the facility, and } \\
\text { materials to support coninued operation (i.e., essential } \\
\text { materials, tools and equipment). }\end{array}$ & $\begin{array}{l}\text { 6/01/96 } \\
\text { Completed }\end{array}$ & LW Roberts \\
\hline $\begin{array}{l}\text { 9. Develop a list of facility (or operation)-specific } \\
\text { mandatory training and qualification requirements for } \\
\text { employees. }\end{array}$ & $\begin{array}{l}6 / 01 / 96 \\
\text { Completed }\end{array}$ & LW Roberts \\
\hline $\begin{array}{l}\text { 10. Develop a listing of information which constitutes the } \\
\text { "authorization basis" for the facility. }\end{array}$ & $\begin{array}{c}\text { 6/01/96 } \\
\text { Completed } \\
\end{array}$ & LW Roberts \\
\hline $\begin{array}{l}\text { 11. Develop a description of ongoing and planned } \\
\text { construction projects. }\end{array}$ & $\begin{array}{c}6 / 01 / 96 \\
\text { Completed }\end{array}$ & LW Roberts \\
\hline $\begin{array}{l}\text { 12. Develop a listing and description of facility (or } \\
\text { operations) databases. Provide list to program/project } \\
\text { level for consolidation. }\end{array}$ & $\begin{array}{c}5 / 15 / 96 \\
\text { Completed }\end{array}$ & LW Roberts \\
\hline $\begin{array}{l}\text { 13. Develop a list of current work order services or other } \\
\text { form of agreement to provide work for other } \\
\text { organizations, on and offsite. }\end{array}$ & $\begin{array}{l}\text { 6/01/96 } \\
\text { Completed }\end{array}$ & LW Roberts \\
\hline $\begin{array}{l}\text { 14. Develop a list of current work order services or other } \\
\text { form of agreement to obtain work from other } \\
\text { organizations, on and offsite. }\end{array}$ & $\begin{array}{l}\text { 6/01/96 } \\
\text { Completed }\end{array}$ & LW Roberts \\
\hline $\begin{array}{l}\text { 15. Develop a list of permits governing the facility (or } \\
\text { operation), and provide a description of each as to } \\
\text { where and how it applies. }\end{array}$ & $\begin{array}{l}\text { 6/01/96 } \\
\text { Comile:t }\end{array}$ & LW Roberts \\
\hline $\begin{array}{l}\text { 16. Verify the accuracy of property assignments for the } \\
\text { facility (or operations) and correct any deficiencies. }\end{array}$ & $\begin{array}{c}6 / 01 / 96 \\
\text { Completed }\end{array}$ & LW Roberts \\
\hline 17. Update/submit "Smart Book"/assemble key documents. & $7 / 15 / 96$ & LW Roberts \\
\hline $\begin{array}{l}\text { 18. Verify pre-existing condition data is current and } \\
\text { supplement as necessary. }\end{array}$ & $7 / 22 / 96$ & LW Roberts \\
\hline 19. Update inventories of feed materials and waste. & $7 / 15 / 96$ & LW Roberts \\
\hline \multicolumn{3}{|l|}{ Post-Award Actions } \\
\hline $\begin{array}{l}\text { 1. Provide facility (or operations) briefing(s) to the } \\
\text { PHMC. }\end{array}$ & $8 / 12 / 96-8 / 30 / 96$ & LW Roberts \\
\hline 2. Provide a facility (or operations) tour to the PHMC. & $8 / 12 / 96-8 / 30 / 96$ & LW Roberts \\
\hline $\begin{array}{l}\text { 3. Provide the PHMC access to key documentation, pre- } \\
\text { existing condition information, commitment data, } \\
\text {.inventory data, etc. (as established in the pre-award } \\
\text { phase). Provide copies upon request. }\end{array}$ & $8 / 05 / 96$ & LW Roberts \\
\hline $\begin{array}{l}\text { 4. Provide any additional documentation or information } \\
\text { requested by the PHMC, if available, that was not } \\
\text { identified during the pre-award phase. }\end{array}$ & $8 / 30 / 96$ & LW Roberts \\
\hline $\begin{array}{l}\text { 5. Provide PHMC any recurring reports that they would } \\
\text { like to receive during transition. }\end{array}$ & $8 / 15 / 96-9 / 30 / 96$ & LW Roberts \\
\hline 6. Provide necessary training to PHMC personnel. & $8 / 12 / 96-8 / 30 / 96$ & LW Roberts \\
\hline $\begin{array}{l}\text { 7. Provide an updated status of ongoing work prior to } \\
\text { contract turnover. }\end{array}$ & by $9 / 30 / 96$ & LW Roberts \\
\hline
\end{tabular}




\begin{tabular}{|l|c|l|}
\hline \multicolumn{1}{|c|}{ Post-Award Actions } & $\begin{array}{c}\text { Scheduled } \\
\text { Date }\end{array}$ & Actionee(s) \\
\hline $\begin{array}{l}\text { 8. } \\
\begin{array}{l}\text { Perform a joint property and equipment inventory with } \\
\text { the PHMC. }\end{array}\end{array}$ by 9/16/96 & LW Roberts \\
\hline 9. Provide a categorized inventory of wastes. & by $9 / 30 / 96$ & LW Roberts \\
\hline 10. $\begin{array}{l}\text { Assist the PHMC in the conduct of Pre-existing } \\
\text { Condition Assessments. }\end{array}$ & $8 / 12 / 96-9 / 16 / 96$ & LW Roberts \\
\hline
\end{tabular}

\subsection{SPENT NUCLEAR FUEL}

The scope of the SNF Project covers the maintenance and preparation of the $K$ Basins for safe storage and removal of all SNF, debris, and sludge; constructing, as necessary, new systems and facilities to condition and store the fuel prior to final disposition; relocating $\mathrm{K}$ Basins spent nuclear fuel to the interim storage facility; and accepting stewardship of spent nuclear fuel from other Hanford locations. The Project Management Plan sets forth the management basis for the SNF Project.

\begin{tabular}{|c|c|c|}
\hline Pre-A ward Actions & $\begin{array}{l}\text { Scheduled } \\
\text { Date }\end{array}$ & Actionee(s) \\
\hline $\begin{array}{l}\text { 1. Assemble documents that led to overall SNF Project } \\
\text { Strategy }\end{array}$ & $\begin{array}{c}5 / 31 / 96 \\
\text { Completed }\end{array}$ & EW Gerber \\
\hline $\begin{array}{l}\text { 2. Assemble key SNF Project Management documents } \\
\text { (Project Plan, Project Management Plan, SNF Interim } \\
\text { Storage Plan, MOUs, etc.). }\end{array}$ & $\begin{array}{l}5 / 31 / 96 \\
\text { Completed }\end{array}$ & $\begin{array}{l}\text { CC Hages } \\
\text { SL Magnani }\end{array}$ \\
\hline $\begin{array}{l}\text { 3. Develop a list of key project documentation. Provide } \\
\text { to RL POC for review. }\end{array}$ & $\begin{array}{c}5 / 15 / 96 \\
\text { Completed }\end{array}$ & CC Hages \\
\hline $\begin{array}{l}\text { 4. Identify key project documentation location for use by } \\
\text { the PHMC. }\end{array}$ & $\begin{array}{c}6 / 01 / 96 \\
\text { Completed }\end{array}$ & CC Hages \\
\hline 5. Prepare SNF Project briefing. & $7 / 24 / 96$ & CC Hages \\
\hline $\begin{array}{l}\text { 6. Develop a listing of key project individuals as POCs, } \\
\text { including facility and operations personnel. }\end{array}$ & $\begin{array}{c}6 / 01 / 96 \\
\text { Completed }\end{array}$ & CC Hages \\
\hline $\begin{array}{l}\text { 7. Develop listing and description of project subcontracts, } \\
\text { including operations subcontracts. Include any } \\
\text { subcontracts still planned to be executed prior to } \\
\text { contract takeover. }\end{array}$ & $\begin{array}{l}5 / 15 / 96 \\
\text { Completed }\end{array}$ & CC Hages \\
\hline $\begin{array}{l}\text { 8. Assemble copies of all SNF subcontracts the PHMC } \\
\text { will assume. }\end{array}$ & $\begin{array}{c}6 / 01 / 96 \\
\text { Completed } \\
\end{array}$ & CC Hages \\
\hline $\begin{array}{l}\text { 9. Develop listing and description of project databases, } \\
\text { including operations databases. }\end{array}$ & $\begin{array}{l}\text { 6/01/96 } \\
\text { Completed }\end{array}$ & CC Hages \\
\hline $\begin{array}{l}\text { 10. Verify the accuracy of property assignments at the } \\
\text { project organizational level and correct any } \\
\text { deficiencies. }\end{array}$ & $\begin{array}{l}\text { 6/01/96 } \\
\text { Completed }\end{array}$ & CC Hages \\
\hline $\begin{array}{l}\text { 11. Identify space and equipment within project area and } \\
\text { facilities for use by the PHMC. }\end{array}$ & $\begin{array}{l}\text { 6/01/96 } \\
\text { Completed }\end{array}$ & CC Hages \\
\hline
\end{tabular}


WHC-SP-1181 Rev. 1

\begin{tabular}{|c|c|c|}
\hline Pre-Award Actions & $\begin{array}{l}\text { Scheduled } \\
\text { Date }\end{array}$ & Actionee(s) \\
\hline $\begin{array}{l}\text { 12. In outline form for all operations to follow, define the } \\
\text { appropriate contents and level of detail to be included } \\
\text { in operations briefings to the PHMC. (Review with } \\
\text { RL POC.) }\end{array}$ & $\begin{array}{l}5 / 01 / 96 \\
\text { Completed }\end{array}$ & CC Hages \\
\hline 13. Verify and submit "Smart Book." & $7 / 15 / 96$ & CC Hages \\
\hline $\begin{array}{l}\text { 14. Furnish satellite office space (with supplies \& } \\
\text { equipment). }\end{array}$ & $7 / 24 / 96$ & $\begin{array}{l}\text { CC Hages } \\
\text { DW Siddoway }\end{array}$ \\
\hline $\begin{array}{l}\text { 15. Ensure organizational responsibility assignment with } \\
\text { the RLPS are correct for buildings and other } \\
\text { structures. }\end{array}$ & $7 / 22 / 96$ & $\begin{array}{l}\text { CC Hages } \\
\text { DW Siddoway }\end{array}$ \\
\hline \multicolumn{3}{|l|}{ Post-Award Actions } \\
\hline 1. Conduct overall briefing on SNF Project & $8 / 08 / 96-8 / 19 / 96$ & JC Fulton \\
\hline $\begin{array}{l}\text { 2. Perform a joint project level property inventory with } \\
\text { PHMC personnel. }\end{array}$ & by $9 / 16 / 96$ & $\begin{array}{l}\text { CC Hages } \\
\text { DW Siddoway }\end{array}$ \\
\hline
\end{tabular}

\subsubsection{Spent Nuclear Fuel Project Integration}

Project Integration encompasses the development of required project management planning documentation, establishment of the project technical, schedule, and cost baselines, and definition of work priorities and funding requirements. Project Evaluation defines project reporting, review, and commitment tracking requirements. Project Execution covers the implementation of the project control system through work authorization, baseline status accounting, and BCC. Workscope includes integration of environmental, safety, quality assurance, regulatory involvement, and external interfaces.

\begin{tabular}{|c|c|c|}
\hline Pre-Award Actions & $\begin{array}{c}\text { Scheduled } \\
\text { Date }\end{array}$ & Actionee(s) \\
\hline $\begin{array}{l}\text { 1. Provide key documents on cost, schedule, reporting, } \\
\text { administration, safety, and stakeholders involvement to } \\
\text { include Level } 0,1 \text {, and } 2 \text { resource loaded schedules, } \\
\text { MYPP, ADS, RDS, SMP, RRP, Construction. Project } \\
\text { Data Sheet, configuration control, etc. }\end{array}$ & $\begin{array}{c}5 / 31 / 96 \\
\text { Completed }\end{array}$ & CC Hages \\
\hline
\end{tabular}

\subsubsection{Spent Nuclear Fuel Systems Integration}

The Systems Integration element encompasses the overall technical direction for the SNF Project. The workscope includes identification and evaluation of technical approaches for safe dry interim storage of $K$ Basins SNF, implementation of the overall technical 
strategy for the Project, and resolution of technical issues as necessary to implement the strategy. System Integration consists of six major sub-elements:

- Systems Engineering

- Process Engineering

- Technology Acquisition

- Characterization

- Technical Program Integration

- EIS.

\begin{tabular}{|l|c|c|}
\hline \multicolumn{1}{|c|}{ Pre-Award Actions } & $\begin{array}{c}\text { Scheduled } \\
\text { Date }\end{array}$ & Actionee(s) \\
\hline 1. Provide key documents on SNF Systems Integration. & $\begin{array}{c}5 / 31 / 96 \\
\text { Completed }\end{array}$ & EW Gerber \\
\hline $\begin{array}{l}\text { 2. Provide background information on the EIS and Record } \\
\text { of Decision. }\end{array}$ & $\begin{array}{c}5 / 31 / 96 \\
\text { Completed }\end{array}$ & GC Mooers \\
\hline
\end{tabular}

\subsubsection{K Basins Maintenance and Operations}

$\mathrm{K}$ Basins $\mathrm{M} \& \mathrm{O}$ manages and directs all activities at the $\mathrm{K}$ Basins in a safe, economic, and environmentally sound manner, including facility operations and maintenance; handling and storage of spent nuclear fuel; and operational support of the SNF Project. All activities are conducted in compliance with DOE contractual orders and applicable Federal, state, and local laws.

\begin{tabular}{|c|c|c|}
\hline Pre-Award Actions & $\begin{array}{c}\text { Scheduled } \\
\text { Date }\end{array}$ & Actionee(s) \\
\hline 1. Provide the pre-existing conditions checklist. & $\begin{array}{c}6 / 15 / 96 \\
\text { Completed }\end{array}$ & DW Siddoway \\
\hline $\begin{array}{l}\text { 2. Provide } \mathbf{K} \text { Basin fuel inventory and arnover } \\
\text { documentation and certification. }\end{array}$ & $\begin{array}{l}\text { 6/01/96 } \\
\text { Completed }\end{array}$ & DW Siddoway \\
\hline $\begin{array}{l}\text { 3. Verify pre-existing condition evaluations are current, } \\
\text { and modify as necessary (including CSB). }\end{array}$ & $7 / 22 / 96$ & DW Siddoway \\
\hline \multicolumn{3}{|l|}{ Post-Award Actions } \\
\hline 1. Conduct facility tours. & $8 / 12 / 96-8 / 30 / 96$ & DW Siddoway \\
\hline $\begin{array}{l}\text { 2. Assist PHMC in pre-existing conditions assessment } \\
\text { (including CSB). }\end{array}$ & $8 / 12 / 96 \cdots 09 / 16 / 96$ & DW Siddoway \\
\hline $\begin{array}{l}\text { 3. Update pre-existing conditions packages (including } \\
\text { CSB). }\end{array}$ & $9 / 23 / 96$ & DW Siddoway \\
\hline
\end{tabular}




\subsubsection{K Basin Material Removal and Clean-Up Project}

The $\mathrm{K}$ Basin Material Removal and Clean-Up Project manages and executes projects and subprojects within $\mathrm{K}$ Area required to support the Path Forward, and projects required to support $\mathrm{K}$ Basins day-to-day maintenance and operations function consistent with SNF Project requirements. Projects/subprojects scope will be defined by specific acquisition strategies, but typically will be from design through turnover to operations. The Fuel Removal System Design and Acquisition Project is being managed separate from water, sludge, and debris.

\begin{tabular}{|l|c|l|}
\hline \multicolumn{1}{|c|}{ Presward Actions } & $\begin{array}{c}\text { Scheduled } \\
\text { Date }\end{array}$ & Actionee(s) \\
\hline 1. Provide facility projects documentation. & $\begin{array}{c}5 / 31 / 96 \\
\text { Completed }\end{array}$ & MJ Wiemers \\
\hline 2. Provide Fuel Removal Systems documentation. & $\begin{array}{c}5 / 31 / 96 \\
\text { Completed }\end{array}$ & BS Carlisle \\
\hline $\begin{array}{l}\text { 3. Provide Fuel Removal Water Purification } \\
\text { documentation. }\end{array}$ & $\begin{array}{c}5 / 31 / 96 \\
\text { Completed }\end{array}$ & DS Takasumi \\
\hline
\end{tabular}

\subsubsection{Multi-Canister Overpack (MCO) Acquisition}

The MCO represents activities that provide a container or containment boundary with known integrity for SNF. The MCO is common to systems associated with fuel retrieval, transport, drying, staging, conditioning, and storage. Therefore, the $\mathrm{MCO}$ is designed to the most restrictive constraints imposed by requirements for Fuel Retrieval, K Basins SNF Vacuum Drying, Cask and Transportation, Canister Storage Building (CSB), and Hot Vacuum Conditioning systems. The current $\mathrm{MCO}$ concept is a cylindrical vessel with removable fuel baskets that are capable of being stacked within the cylinder.

\begin{tabular}{|l|c|c|}
\hline \multicolumn{1}{|c|}{ Pre-Award Actions } & $\begin{array}{c}\text { Scheduled } \\
\text { Date }\end{array}$ & Actionee(s) \\
\hline $\begin{array}{l}\text { 1. Provide MCO design fabrication and testing } \\
\text { documentation and safety report. }\end{array}$ & $\begin{array}{c}5 / 31 / 96 \\
\text { Completed }\end{array}$ & $\begin{array}{c}\text { WD Gailo } \\
\text { Comples }\end{array}$ \\
\hline
\end{tabular}

\subsubsection{Cask/Transportation System}

The cask and transportation activities involve physically removing the $\mathrm{MCO} /$ Cask from the basin loadout pit, moving the cask to the K Basin SNF Vacuum Drying, and transporting the loaded cask from the $\mathrm{K}$ Basin to the CSB (or hot vacuum conditioning as an alternative) located in the 200 Area. Empty MCOs are also received at the CSB, transported, and placed in the $\mathrm{K}$ Basin loadout. 
WHC-SP-1181 Rev. 1

\begin{tabular}{|l|c|c|}
\hline \multicolumn{1}{|c|}{ Pre-Award Actions } & $\begin{array}{c}\text { Scheduled } \\
\text { Date }\end{array}$ & \multicolumn{1}{|c|}{ Actionet(s) } \\
\hline $\begin{array}{l}\text { 1. Provide Cask/Transportation Systems Acquisition, } \\
\text { Testing and Qualification documentation and Regulatory } \\
\text { Compliance information. }\end{array}$ & $\begin{array}{c}5 / 31 / 96 \\
\text { Completed }\end{array}$ & WD Gallo \\
\hline 2. Provide Basin facility upgrade plans for transportation. & $\begin{array}{c}5 / 31 / 96 \\
\text { Completed }\end{array}$ & WD Gallo \\
\hline 3. Provide SARP. & $\begin{array}{c}5 / 31 / 96 \\
\text { Completed }\end{array}$ & WD Gallo \\
\hline
\end{tabular}

\subsubsection{Canister Storage Building}

The CSB will be used for dry staging and storage of the $\mathrm{K}$ Basins fuel. The CSB will utilize the partially constructed building in the 200 East Area that was originally intended for a vitrification plant.

\begin{tabular}{|l|c|l|}
\hline \multicolumn{1}{|c|}{ Pre-Award Actions } & $\begin{array}{c}\text { Scheduled } \\
\text { Date }\end{array}$ & \multicolumn{1}{|c|}{ Actionee(s) } \\
\hline 1. Provide CSB Project Management Plan. & $\begin{array}{c}5 / 31 / 96 \\
\text { Completed }\end{array}$ & MK Mahaffey \\
\hline $\begin{array}{l}\text { 2. Provide Fluor Tille II and II statement of work and } \\
\text { performance specification S-0425. }\end{array}$ & $\begin{array}{c}5 / 31 / 96 \\
\text { Completed }\end{array}$ & J Bazinet \\
\hline 3. Provide CSB Startup Plan and Operations Stafting Plan. & $\begin{array}{c}5 / 31 / 96 \\
\text { Completed }\end{array}$ & C Thompson \\
\hline $\begin{array}{l}\text { 4. Provide Phased SARs and SRDS (depending on } \\
\text { funding). }\end{array}$ & $\begin{array}{c}6 / 01 / 96 \\
\text { Completed }\end{array}$ & J Garvin \\
\hline 5. Provide Construction Management Plan. & $\begin{array}{c}6 / 01 / 96 \\
\text { Completed }\end{array}$ & J Mortimer \\
\hline
\end{tabular}

\subsubsection{Fuel Conditioning Processes}

Cold Vacuum Drying. The Cold Vacuum Drying Facility will remove the water from the MCO before shipping it to the CSB by a combination of draining and cold vacuum drying. Vacuum drying will require only 24 to 48 hours to remove all of the free water. Removal of the free water greatly reduces the hydrogen generation rate and allows the MCO to be shipped to the CSB with the process valves closed, greatly enhancing safety during shipping.

The Hot Vacuum Conditioning is expected to be performed within the CSB. This process involves heating the fuel and any residual sludge to $300{ }^{\circ} \mathrm{C}$, where it is held under vacuum to remove water that is chemically bound to fuel and corrosion products, and to decompose uranium hydride that may be present. After the drying and uranium hydride decomposition step is completed, the fuel is cooled and exposed to a mixture of inert gas and 


\section{WHC-SP-1181 Rev. 1}

controlled concentrations of oxygen. This step exposes the fuel to increasing levels of oxygen in a controlled manner to oxidize high surface area uranium metal. Thus, high surface area unoxidized uranium metal formed by the decomposition of hydrides, or during fuel handling, will be eliminated. When this partial oxidation step is completed, the MCOs will be cooled, backfilled with inert gas and sealed for interim storage in the CSB.

\begin{tabular}{|l|c|l|}
\hline \multicolumn{1}{|c|}{ Pre-Award Actions } & $\begin{array}{c}\text { Scheduled } \\
\text { Date }\end{array}$ & \multicolumn{1}{|c|}{ Actionee(s) } \\
\hline $\begin{array}{l}\text { 1. Provide Cold Vacuum Drying Facility Design, } \\
\text { Acquisition, Safety, and Regulatory documentation. }\end{array}$ & $\begin{array}{c}5 / 31 / 96 \\
\text { Completed }\end{array}$ & FW Bradshaw \\
\hline $\begin{array}{l}\text { 2. Provide Hot Conditioning System Design, Acquisition, } \\
\text { Safety, and Regulatory docurnentation. }\end{array}$ & $\begin{array}{c}5 / 31 / 96 \\
\text { Completed }\end{array}$ & WD Gallo \\
\hline
\end{tabular}

\subsubsection{Other Hanford Fuel Retrieval Processing and Storage}

The Other Fuel Management sub-project encompasses SNF Project activities necessary to attain safe interim storage of the SNF at the Hanford Site that is not currently stored at the K Basins and transfer of these materials to INEL in accordance with the SNF and INEL EIS Record of Decision. Some of the SNF stored at the 200 Area ISA may be transferred to INEL for long-term interim storage.

\begin{tabular}{|l|c|c|}
\hline \multicolumn{1}{|c|}{ Pre-Award Actions } & Scheduled & Actionee(s) \\
\hline $\begin{array}{l}\text { 1. Provide Other Hanford SNF documentation and } \\
\text { certification. }\end{array}$ & Date & $5 / 15 / 96$ \\
Completed & EW Gerber \\
NoTE: Plans and schedules for Other Hanford SNF is \\
contained in DOE-owned SNF Interim Storage Plan and \\
Program Plan (identified under 4.3)
\end{tabular}

\subsection{FACILITY STABILIZATION}

\begin{tabular}{|l|c|c|}
\hline \multicolumn{1}{|c|}{ Pre-Award Actions } & $\begin{array}{c}\text { Scheduled } \\
\text { Date }\end{array}$ & Actionee(s) \\
\hline $\begin{array}{l}\text { 1. Develop a list of key Transition Projects documentation. } \\
\text { Provide to RL POC for review. }\end{array}$ & $\begin{array}{c}5 / 15 / 96 \\
\text { Completed }\end{array}$ & CL Nansen \\
\hline $\begin{array}{l}\text { 2. Assernble key Transition Projects documentation, or } \\
\text { identify its location, for use by the PHMC. Inciudes } \\
\text { development of a Transition Projects "Smart Book," } \\
\text { including listings and descriptions developed below. }\end{array}$ & DELETED & \\
\hline $\begin{array}{l}\text { 3. Identify number of briefings, and prepare Transition } \\
\text { Projects briefing(s) for presentation to PHMC. } \\
\text { (Coordinate number and content with RL POC.) }\end{array}$ & DELETED & \\
\hline
\end{tabular}


WHC-SP-1181 Rev. 1

\begin{tabular}{|l|c|l|}
\hline \multicolumn{1}{|c|}{ Pre-Award Actions } & $\begin{array}{c}\text { Scheduled } \\
\text { Date }\end{array}$ & Actionee(s) \\
\hline $\begin{array}{l}\text { 4. Develop a listing of key Transition Projects individuals } \\
\text { as POCs, including facility and operations personnel. }\end{array}$ & $\begin{array}{c}6 / 01 / 96 \\
\text { Completed }\end{array}$ & CL Nansen \\
\hline $\begin{array}{l}\text { 5. Develop listing and description of Transition Projects } \\
\text { subcontracts. Include any subcontracts still planned to } \\
\text { be executed prior to contract takeover. }\end{array}$ & $\begin{array}{c}6 / 01 / 96 \\
\text { Completed }\end{array}$ & CL Nansen \\
\hline $\begin{array}{l}\text { 6. Develop listing and description of Transition Projects } \\
\text { databases. }\end{array}$ & $\begin{array}{c}6 / 01 / 96 \\
\text { Completed }\end{array}$ & CL Nansen \\
\hline $\begin{array}{l}\text { 7. Identify space and equipment within 2753E and facilities } \\
\text { for use by the PHMC. }\end{array}$ & $\begin{array}{c}6 / 01 / 96 \\
\text { Completed }\end{array}$ & CL Nansen \\
\hline $\begin{array}{l}\text { 8. In outline form for all facilities/operations to follow, } \\
\text { define the appropriate contents and level of detail to be } \\
\text { included in facility/operations briefings to the PHMC. } \\
\text { (Review with RL POC.) }\end{array}$ & $\begin{array}{c}5 / 01 / 96 \\
\text { Completed }\end{array}$ & CL Nansen \\
\hline $\begin{array}{l}\text { Post-Award Actions } \\
\text { 1. Transition Projects and facility space and services } \\
\text { available for use by the PHMC. }\end{array}$ & $8 / 05 / 96$ & CL Nansen \\
\hline
\end{tabular}

\subsubsection{PUREX}

This complex was a processing facility to extract plutonium in spent fuels. In the process, uranium was separated, sent to the Uranium Trioxide $\left(\mathrm{UO}_{3}\right)$ Plant to become Uranium fluoride and then returned to Fernald to be enriched and reprocessed into fuel billets for new fuel elements for $\mathrm{N}$ Reactor, thus closing the fuel cycle.

In December, 1992, DOE authorized termination of the facilities and directed that actions be initiated to proceed with shutdown planning and subsequent transition to a passively safe and environmentally secure state pending final disposition. The $\mathrm{UO}_{3}$ transition was completed in FY 1995; the PUREX transition is targeted to be completed in FY 1997.

\begin{tabular}{|c|c|c|}
\hline Pre-Award Actions & $\begin{array}{l}\text { Scheduled } \\
\text { Date }\end{array}$ & Actionee(s) \\
\hline $\begin{array}{l}\text { 1. Identify number of briefings, and prepare PUREX } \\
\text { briefing(s) for the PHMC. (Coordinate number and } \\
\text { content with RL POC.) }\end{array}$ & $7 / 15 / 96$ & CF Schuster \\
\hline $\begin{array}{l}\text { 2. Identify key documents for PUREX. Provide listing to } \\
\text { CL Nansen for consolidation and RL review. }\end{array}$ & $\begin{array}{l}5 / 01 / 96 \\
\text { Completed }\end{array}$ & CF Schuster \\
\hline $\begin{array}{l}\text { 3. Assemble key PUREX documentation, or identify its } \\
\text { location, for use by the PHMC. Includes preparation } \\
\text { of a PUREX "Smart Book," to include information } \\
\text { developed in the foliowing actions. }\end{array}$ & $\begin{array}{c}6 / 01 / 96 \\
\text { Completed "Smart } \\
\text { Book" only } \\
\text { See item \#19 }\end{array}$ & CF Schuster \\
\hline 4. Identify POCs for PUREX and input to CL Nansen. & $\begin{array}{l}5 / 15 / 96 \\
\text { Completed }\end{array}$ & CF Schuster \\
\hline
\end{tabular}




\begin{tabular}{|c|c|c|}
\hline Pre-Award Actions & $\begin{array}{r}\text { Scheduled } \\
\text { Dafe }\end{array}$ & Actionee(s) \\
\hline $\begin{array}{l}\text { 5. Develop a listing and description of subcontracts } \\
\text { supporting PUREX and input to CL Nansen. Include } \\
\text { any subcontracts planned to be executed prior to } \\
\text { contract takeover. }\end{array}$ & $\begin{array}{l}5 / 15 / 96 \\
\text { Completed }\end{array}$ & CF Schuster \\
\hline $\begin{array}{l}\text { 6. Using the pre-existing checklist, compile pre-existing } \\
\text { condition information on PUREX facilities and/or } \\
\text { major equipment. Conduct, or schedule as a separate } \\
\text { post-award action(s), any assessments required to } \\
\text { adequately address all relevant items on the checklist. } \\
\text { Provide completed checklist to RL POC for review. }\end{array}$ & $\begin{array}{l}6 / 15 / 96 \\
\text { Completed }\end{array}$ & CF Schuster \\
\hline $\begin{array}{l}\text { 7. Identify applicable milestones and commitments (e.g., } \\
\text { Tri-Party Agreement, DNFSB and consent order } \\
\text { milestones), and determine their status. Describe the } \\
\text { form of the deliverable and what constitutes } \\
\text { completion. Provide to RL POC for review. }\end{array}$ & $\begin{array}{l}\text { 6/01/96 } \\
\text { Completed }\end{array}$ & CF Schuster \\
\hline $\begin{array}{l}\text { 8. Identify current inventories of feed for PUREX, and } \\
\text { materiais to support continued operation (i.e., essential } \\
\text { materials, tools and equipment). }\end{array}$ & $\begin{array}{l}\text { 6/01/96 } \\
\text { Completed }\end{array}$ & CF Schuster \\
\hline $\begin{array}{l}\text { 9. Develop a list of PUREX-specific mandatory training } \\
\text { and qualification requirements for employees. }\end{array}$ & $\begin{array}{l}6 / 01 / 96 \\
\text { Completed }\end{array}$ & CF Schuster \\
\hline $\begin{array}{l}\text { 10. Develop a listing of information which constitutes the } \\
\text { "authorization basis" for the PUREX facility. }\end{array}$ & $\begin{array}{c}6 / 01 / 96 \\
\text { Completed } \\
\end{array}$ & CF Schuster \\
\hline $\begin{array}{l}\text { 11. Develop a description of ongoing and planned } \\
\text { construction projects. }\end{array}$ & $\begin{array}{c}\text { 6/01/96 } \\
\text { Completed }\end{array}$ & CF Schuster \\
\hline $\begin{array}{l}\text { 12. Develop a listing of special agreement services } \\
\text { provided to RL, other DOE sites, and other entities. }\end{array}$ & $\begin{array}{c}6 / 01 / 96 \\
\text { Completed }\end{array}$ & CF Schuster \\
\hline $\begin{array}{l}\text { 13. Develop a listing and description of PUREX } \\
\text { databases. Provide list to CL Nansen for } \\
\text { consolidation. }\end{array}$ & $\begin{array}{l}5 / 15 / 96 \\
\text { Completed }\end{array}$ & CF Schuster \\
\hline $\begin{array}{l}\text { 14. Develop a list of current work order services or other } \\
\text { form of agreement to provide work for other } \\
\text { organizations, on and offsite. }\end{array}$ & $\begin{array}{l}\text { 6/01/96 } \\
\text { Completed }\end{array}$ & CF Schuster \\
\hline $\begin{array}{l}\text { 15. Develop a list of current work order services or other } \\
\text { form of agreement to obtain work from other } \\
\text { organizations, on and offsite. }\end{array}$ & $\begin{array}{l}\text { 6/01/96 } \\
\text { Completed }\end{array}$ & CF Schuster \\
\hline $\begin{array}{l}\text { 16. Develop a list of permits governing PUREX and } \\
\text { provide a description of each as to where and how it } \\
\text { applies. }\end{array}$ & $\begin{array}{l}6 / 01 / 96 \\
\text { Completed }\end{array}$ & CF Schuster \\
\hline $\begin{array}{l}\text { 17. Verify the accuracy of property assignments for } \\
\text { PUREX and correct any deficiencies. }\end{array}$ & $\begin{array}{c}\text { 6/01/96 } \\
\text { Completed } \\
\end{array}$ & CF Schuster \\
\hline $\begin{array}{l}\text { 18. Identify and make available key PUREX-specific } \\
\text { training and qualification records and documents. }\end{array}$ & $\begin{array}{c}6 / 01 / 96 \\
\text { Completed }\end{array}$ & CF Schuster \\
\hline 19. Update/submit "Smart Book"/assemble key documents. & $7 / 15 / 96$ & CF Schuster \\
\hline $\begin{array}{l}\text { 20. Update/finalize current inventories of feed, materials, } \\
\text { and/or wastes. }\end{array}$ & $7 / 15 / 96$ & CF Schuster \\
\hline
\end{tabular}


WHC-SP-1181 Rev. 1

\begin{tabular}{|c|c|c|}
\hline Pre-Award Actions & $\begin{array}{r}\text { Scheduled } \\
\text { Date }\end{array}$ & Actionee(s) \\
\hline $\begin{array}{l}\text { 21. Verify pre-existing condition data is current and } \\
\text { supplement as necessary. }\end{array}$ & $7 / 22 / 96$ & CF Schuster \\
\hline 22. Ensure building assignments are correct in RLPS. & $7 / 22 / 96$ & CF Schuster \\
\hline \multicolumn{3}{|l|}{ Post-Award Actions } \\
\hline 1. Provide PUREX briefing(s) to the PHMC. & 8/08/96-8/19/96 & CF Schuster \\
\hline 2. Provide a PUREX facilities tour to the PHMC. & $8 / 08 / 96-8 / 19 / 96$ & CF Schuster \\
\hline $\begin{array}{l}\text { 3. Provide PHMC access to key documentation, pre- } \\
\text { existing condition information, commitment data, } \\
\text { inventory data, etc. (as established in the pre-award } \\
\text { phase). Provide copies upon request. }\end{array}$ & $8 / 05 / 96$ & CF Schuster \\
\hline $\begin{array}{l}\text { 4. Provide any additional documentation or information } \\
\text { requested by the PHMC, if available, that was not } \\
\text { identified during the pre-award phase. }\end{array}$ & by $9 / 01 / 96$ & CF Schuster \\
\hline $\begin{array}{l}\text { 5. PHMC identify any recurring reports that they would } \\
\text { like to receive during transition. }\end{array}$ & by $8 / 15 / 96$ & PHMC \\
\hline $\begin{array}{l}\text { 6. Provide necessary PUREX-specific training to PHMC } \\
\text { personnel. }\end{array}$ & $8 / 12 / 96-9 / 01 / 96$ & CF Schuster \\
\hline $\begin{array}{l}\text { 7. Provide an updated status of ongoing work prior to } \\
\text { contract turnover. }\end{array}$ & by $9 / 30 / 96$ & CF Schuster \\
\hline 8. Perform a joint inventory of SNM with the PHMC. & by $9 / 16 / 96$ & CF Schuster \\
\hline $\begin{array}{l}\text { 9. Perform a joint property and equipment inventory with } \\
\text { the PHMC. }\end{array}$ & by $9 / 16 / 96$ & CF Schuster \\
\hline 10. Provide a categorized inventory of wastes. & by $9 / 30 / 96$ & CF Schuster \\
\hline $\begin{array}{l}\text { 11. Provide an updated status of end point criteria to } \\
\text { PHMC. }\end{array}$ & by $9 / 30 / 96$ & CF Schuster \\
\hline $\begin{array}{l}\text { 12. Assist the PHMC in the conduct of a pre-existing } \\
\text { condition assessment. }\end{array}$ & $8 / 12 / 96-9 / 16 / 96$ & CF Schuster \\
\hline
\end{tabular}

\subsubsection{Plutonium Finishing Plant}

This complex contains vaults for storage of special nuclear material and processing systems with associated support systems for stabilizing plutonium materials.

\begin{tabular}{|c|c|c|}
\hline Pre-Award Actions & $\begin{array}{l}\text { Scheduled } \\
\text { Date }\end{array}$ & Actionee(s) \\
\hline $\begin{array}{l}\text { 1. Identify number of briefings, and prepare PFP } \\
\text { briefing(s) for the PHMC. (Coordinate number and } \\
\text { content with RL POC.) }\end{array}$ & $7 / 15 / 96$ & CM Kronvall \\
\hline $\begin{array}{l}\text { 2. Identify key documents for PFP. Provide listing to } \\
\text { CL Nansen for consolidation and RL review. }\end{array}$ & $\begin{array}{l}5 / 01 / 96 \\
\text { Completed }\end{array}$ & CM Kronvall \\
\hline
\end{tabular}




\begin{tabular}{|c|c|c|}
\hline Pre-Award Actions & $\begin{array}{l}\text { Scheriuled } \\
\text { Date }\end{array}$ & Actionee(s) \\
\hline $\begin{array}{l}\text { 3. Assemble key PFP documentation, or identify its } \\
\text { location, for use by the PHMC. Includes preparation } \\
\text { of a PFP "Smart Book," to include information } \\
\text { developed in the following actions. }\end{array}$ & $\begin{array}{l}6 / 01 / 96 \\
\text { Completed "Smart } \\
\text { Book" only } \\
\text { See item } \# 19\end{array}$ & CM Kronvail \\
\hline 4. Identify POCs for PFP and input to CL Nansen. & $\begin{array}{c}5 / 15 / 96 \\
\text { Completed }\end{array}$ & CM Kronvall \\
\hline $\begin{array}{l}\text { 5. Develop a listing and description of subcontracts } \\
\text { supporting PFP and input to } C L \text { Nansen. Include any } \\
\text { subcontracts planned to be executed prior to contract } \\
\text { takeover. }\end{array}$ & $\begin{array}{l}5 / 15 / 96 \\
\text { Completed }\end{array}$ & CM Kronvall \\
\hline $\begin{array}{l}\text { 6. Using the pre-existing checklist, compile pre-existing } \\
\text { condition information on the PFP facilities and/or major } \\
\text { equipment. Conduct, or schedule as a separate post- } \\
\text { award action(s), any assessments required to adequately } \\
\text { address all relevant items on the checklist. Provide } \\
\text { completed checklist to RL POC for review. }\end{array}$ & $\begin{array}{l}\text { 6/15/96 } \\
\text { Completed }\end{array}$ & CM Kronvall \\
\hline $\begin{array}{l}\text { 7. Identify applicabie milestones and commitments (e.g., } \\
\text { Tri-Party Agreement, DNFSB and consent order } \\
\text { milestones), and determine their stans. Describe the } \\
\text { forn of the deliverable and what constitutes completion. } \\
\text { Provide to RL POC for review. }\end{array}$ & $\begin{array}{l}6 / 01 / 96 \\
\text { Completed }\end{array}$ & CM Kronvall \\
\hline $\begin{array}{l}\text { 8. Identify current inventories of feed for PFP, and } \\
\text { materials to support continued operation (i.e., essential } \\
\text { materials, tools and equipment). }\end{array}$ & $\begin{array}{l}7 / 15 / 96 \\
\text { Completed }\end{array}$ & CM Kronvall \\
\hline $\begin{array}{l}\text { 9. Develop a list of PFP-specific mandatory training and } \\
\text { qualification requirements for employees. }\end{array}$ & $\begin{array}{l}6 / 01 / 96 \\
\text { Completed }\end{array}$ & CM Kronvall \\
\hline $\begin{array}{l}\text { 10. Develop a listing of information which constitutes the } \\
\text { "authorization basis" for PFP. }\end{array}$ & $\begin{array}{c}6 / 01 / 96 \\
\text { Completed }\end{array}$ & CM Kronvall \\
\hline $\begin{array}{l}\text { 11. Develop a description of ongoing and planned } \\
\text { construction projects. }\end{array}$ & $\begin{array}{l}6 / 01 / 96 \\
\text { Completed }\end{array}$ & CM Kronvall \\
\hline $\begin{array}{l}\text { 12. Develop a listing of special agreement services provided } \\
\text { to } R L \text {, other DOE sites, and other entities. }\end{array}$ & $\begin{array}{c}6 / 01 / 96 \\
\text { Completed }\end{array}$ & CM Kronvall \\
\hline $\begin{array}{l}\text { 13. Develop a listing and description of PFP databases. } \\
\text { Provide list to CL Nansen for consolidation. }\end{array}$ & $\begin{array}{c}5 / 15 / 96 \\
\text { Completed }\end{array}$ & CM Kronvall \\
\hline $\begin{array}{l}\text { 14. Develop a list of current work order services or other } \\
\text { form of agreement to provide work for other } \\
\text { organizations, on and offsite. }\end{array}$ & $\begin{array}{l}6 / 01 / 96 \\
\text { Completed }\end{array}$ & CM Kronvall \\
\hline $\begin{array}{l}\text { 15. Develop a list of current work order services or other } \\
\text { form of agreement to obtain work from other } \\
\text { organizations, on and offsite. }\end{array}$ & $\begin{array}{l}\text { 6/01/96 } \\
\text { Completed }\end{array}$ & CM Kronvall \\
\hline $\begin{array}{l}\text { 16. Develop a list of permits governing PFP, and provide a } \\
\text { description of each as to where and how it applies. }\end{array}$ & $\begin{array}{c}6 / 01 / 96 \\
\text { Completed }\end{array}$ & CM Kronvall \\
\hline $\begin{array}{l}\text { 17. Verify the accuracy of property assignments for PFP } \\
\text { and correct any deficiencies. }\end{array}$ & $\begin{array}{l}6 / 01 / 96 \\
\text { Completed }\end{array}$ & CM Kronvall \\
\hline
\end{tabular}




\begin{tabular}{|c|c|c|}
\hline Pre-Award Actions & $\begin{array}{c}\text { Scheduled } \\
\text { Date } \\
\end{array}$ & Actionee(s) \\
\hline $\begin{array}{l}\text { 18. Identify and make available PFP-specific training and } \\
\text { qualification records and documents. }\end{array}$ & $\begin{array}{c}6 / 01 / 96 \\
\text { Completed }\end{array}$ & CM Kronvall \\
\hline 19. Update/submit "Smart Book"/assemble key documents. & $7 / 15 / 96$ & CM Kronvall \\
\hline $\begin{array}{l}\text { 20. Update/finalize inventories of feed, materials, and/or } \\
\text { wastes. }\end{array}$ & $7 / 15 / 96$ & CM Kronvall \\
\hline $\begin{array}{l}\text { 21. Verify pre-existing condition data is current and } \\
\text { supplement as necessary. }\end{array}$ & $7 / 22 / 96$ & CM Kronvall \\
\hline 22. Ensure building assignments are correct in RIPS. & $7 / 22 / 96$ & CM Kronval \\
\hline Post-Award Actions & & \\
\hline 1. Provide PFP briefing(s) to the PHMC. & $8 / 08 / 96-8 / 19 / 96$ & CM Kronvall \\
\hline 2. Provide a PFP facilities tour to the PHMC. & $8 / 08 / 96-8 / 19 / 96$ & $\begin{array}{l}\text { CM Kronvall } \\
\text { EC Vogt }\end{array}$ \\
\hline $\begin{array}{l}\text { 3. Provide PHMC access to key documentation, pre-existing } \\
\text { condition information, commitment data, inventory data, } \\
\text { etc. (as established in the pre-award phase). Provide } \\
\text { copies upon request. }\end{array}$ & $8 / 05 / 96$ & $\begin{array}{l}\text { CM Kronvall } \\
\text { AE Schilling } \\
\text { DT Mildon }\end{array}$ \\
\hline $\begin{array}{l}\text { 4. Provide any additional documentation or information } \\
\text { requested by the PHMC, if available, that was not } \\
\text { identified during the pre-award phase. }\end{array}$ & by $9 / 01 / 96$ & CM Kronvall \\
\hline $\begin{array}{l}\text { 5. PHMC identify any recurring reports that they would like } \\
\text { to receive during transition. }\end{array}$ & by $8 / 15 / 96$ & PHMC \\
\hline $\begin{array}{l}\text { 6. Provide necessary PFP-specific training to PHMC } \\
\text { personnel. }\end{array}$ & $8 / 12 / 96-9 / 01 / 96$ & CM Kronvall \\
\hline $\begin{array}{l}\text { 7. Provide an updated status of ongoing work prior to } \\
\text { contract turnover. }\end{array}$ & by $9 / 30 / 96$ & $\begin{array}{l}\text { CM Kronvall } \\
\text { EC Vogt }\end{array}$ \\
\hline 8. Perform a joint inventory of SNM with the PHMC. & by $9 / 16 / 96$ & $\begin{array}{l}\text { CM Kronvall } \\
\text { BR Fitzpatrick }\end{array}$ \\
\hline $\begin{array}{l}\text { 9. Perform a joint property and equipment inventory with } \\
\text { the PHMC. }\end{array}$ & by $9 / 16 / 96$ & CM Kronvall \\
\hline 10. Provide a categorized inventory of wastes. & by $9 / 30 / 96$ & CM Kronvall \\
\hline 11. Provide an updated status of end point criteria to PHMC. & by $9 / 30 / 96$ & CM Kronvall \\
\hline $\begin{array}{l}\text { 12. Assist the PHMC in the conduct of a Pre-existing } \\
\text { Condition Assessment. }\end{array}$ & $8 / 12 / 96-9 / 16 / 96$ & CM Kronvall \\
\hline
\end{tabular}

\subsection{3 - Fast Flux Test Facility}

The liquid metal reactor and support facilities served as the technology and materials development center for the liquid metal and breeder reactor programs. The FFTF is shut down and being prepared for turnover to D\&D. Options for alternative use are currently under consideration. 


\begin{tabular}{|c|c|c|}
\hline Pre-Award Actions & $\begin{array}{c}\text { Scheduled } \\
\text { Date }\end{array}$ & Actionee(s) \\
\hline $\begin{array}{l}\text { 1. Identify number of briefings, and prepare FFTF briefing(s) for the } \\
\text { PHMC. (Coordinate number and content with RL POC.) }\end{array}$ & $7 / 15 / 96$ & DA Gantt \\
\hline $\begin{array}{l}\text { 2. Identify key documents for FFTF. Provide listing to CL Nansen } \\
\text { for consolidation and RL review. }\end{array}$ & $\begin{array}{c}5 / 01 / 96 \\
\text { Completed }\end{array}$ & DA Gantt \\
\hline $\begin{array}{l}\text { 3. Assemble FFTF documentation, or identify its location, for use by } \\
\text { the PHMC. Includes preparation of an FFTF "Smart Book," to } \\
\text { include information developed in the following actions. }\end{array}$ & $\begin{array}{l}\text { 6/01/96 } \\
\text { Completed } \\
\text { "Smart Book" } \\
\text { only } \\
\text { See item \#19 }\end{array}$ & DA Gant \\
\hline 4. Identify POCs for FFTF and input to CL Nansen. & $\begin{array}{c}5 / 15 / 96 \\
\text { Completed }\end{array}$ & DA Gantt \\
\hline $\begin{array}{l}\text { 5. Develop a listing and description of subcontracts supporting FFTF } \\
\text { and input to CL Nansen. Include any subcontracts planned to be } \\
\text { executed prior to contract takeover. }\end{array}$ & $\begin{array}{l}5 / 15 / 96 \\
\text { Completed }\end{array}$ & DA Gantt \\
\hline $\begin{array}{l}\text { 6. Using the pre-existing checklist, compile pre-existing condition } \\
\text { information on FFTF facilities and/or major equipment. Conduct, } \\
\text { or schedule as a separate post-award action(s), any assessments } \\
\text { required to adequately address all relevant items on the checklist. } \\
\text { Provide completed checklist to RL POC for review. }\end{array}$ & $\begin{array}{l}6 / 15 / 96 \\
\text { Completed }\end{array}$ & DA Gantt \\
\hline $\begin{array}{l}\text { 7. Identify applicable milestones and commitments (e.g., Tri-Party } \\
\text { Agreement, DNFSB and consent order milestones), and determine } \\
\text { their status. Describe the form of the deliverable and what } \\
\text { constitutes completion. Provide to RL POC for review. }\end{array}$ & $\begin{array}{l}6 / 01 / 96 \\
\text { Completed }\end{array}$ & DA Gantt \\
\hline $\begin{array}{l}\text { 8. Identify current inventories of feed for FFTF, and materials to } \\
\text { support continued operation (i.e., essential materials, tools and } \\
\text { equipment). }\end{array}$ & $\begin{array}{l}6 / 01 / 96 \\
\text { Completed }\end{array}$ & DA Gantt \\
\hline $\begin{array}{l}\text { 9. Develop a list of FFTF-specific mandatory training and } \\
\text { qualification requirements for employees. }\end{array}$ & $\begin{array}{c}6 / 01 / 96 \\
\text { Completed }\end{array}$ & DA Gant: \\
\hline $\begin{array}{l}\text { 10. Develop a listing of information which constitutes the } \\
\text { "authorization basis" for FFTF. }\end{array}$ & $\begin{array}{c}6 / 01 / 96 \\
\text { Completed }\end{array}$ & DA Gantt \\
\hline $\begin{array}{l}\text { 11. Develop a description of ongoing and planned construction } \\
\text { projects. }\end{array}$ & $\begin{array}{c}6 / 01 / 96 \\
\text { Completed }\end{array}$ & DA Gantt \\
\hline $\begin{array}{l}\text { 12. Develop a listing of special agreement services provided to RL, } \\
\text { other DOE sites, and other entities. }\end{array}$ & $\begin{array}{c}6 / 01 / 96 \\
\text { Completed }\end{array}$ & DA Gantt \\
\hline $\begin{array}{l}\text { 13. Develop a listing and description of FFTF databases. Provide list } \\
\text { to CL Nansen for consolidation. }\end{array}$ & $\begin{array}{l}5 / 15 / 96 \\
\text { Completed }\end{array}$ & DA Gantt \\
\hline $\begin{array}{l}\text { 14. Develop a list of current work order services or other form of } \\
\text { agreement to provide work for other organizations, on and offsite. }\end{array}$ & $\begin{array}{c}6 / 01 / 96 \\
\text { Completed }\end{array}$ & DA Gantt \\
\hline $\begin{array}{l}\text { 15. Develop a list of current work order services or other form of } \\
\text { agreement to obtain work from other organizations, on and offsite. }\end{array}$ & $\begin{array}{l}6 / 01 / 96 \\
\text { Completed }\end{array}$ & DA Gantt \\
\hline $\begin{array}{l}\text { 16. Develop a list of permits governing FFTF, and provide a } \\
\text { description of each as to where and how it applies. }\end{array}$ & $\begin{array}{l}6 / 01 / 96 \\
\text { Completed }\end{array}$ & DA Gantt \\
\hline $\begin{array}{l}\text { 17. Verify the accuracy of property assignments for FFTF and correct } \\
\text { any deficiencies. }\end{array}$ & $\begin{array}{l}\text { 6/01/96 } \\
\text { Completed }\end{array}$ & DA Gantt \\
\hline
\end{tabular}




\begin{tabular}{|c|c|c|}
\hline Pre-Award Actions & $\begin{array}{l}\text { Scheduled } \\
\text { Date } \\
\end{array}$ & Actionee(s) \\
\hline $\begin{array}{l}\text { 18. Identify and make available FFTF-specific training and } \\
\text { qualification records and documents. }\end{array}$ & $\begin{array}{c}6 / 01 / 96 \\
\text { Completed }\end{array}$ & DA Gantt \\
\hline 19. Update/submit "Smart Book"/assemble key documents. & $7 / 15 / 96$ & DA Gantt \\
\hline $\begin{array}{l}\text { 20. Update/finalize current inventories of feed, materials, and/or } \\
\text { wastes. }\end{array}$ & $7 / 15 / 96$ & DA Gantt \\
\hline $\begin{array}{l}\text { 21. Verify pre-existing condition data is current and supplement as } \\
\text { necessary. }\end{array}$ & $7 / 22 / 96$ & DA Gantt \\
\hline 22. Ensure building assignments are correct in RLPS. & $7 / 22 / 96$ & DA Gantt \\
\hline Post-Award Actions & & \\
\hline I. Provide FFTF briefing(s) to the PHMC. & $8 / 08 / 96-8 / 19 / 96$ & DA Gantt \\
\hline 2. Provide FFTF facilities tour to the PHMC. & $8 / 08 / 96-8 / 19 / 96$ & DA Gantt \\
\hline $\begin{array}{l}\text { 3. Provide PHMC access to key documentation, pre-existing } \\
\text { condition information, commitment data, inventory data, etc. (as } \\
\text { established in the pre-award phase). Provide copies upon request. }\end{array}$ & $8 / 05 / 96$ & DA Gantt \\
\hline $\begin{array}{l}\text { 4. Provide any additional documentation or information requested by } \\
\text { the PHMC, if available, that was not identified during the pre- } \\
\text { award phase. }\end{array}$ & by $9 / 01 / 96$ & DA Gantt \\
\hline $\begin{array}{l}\text { 5. PHMC identify any recurring reports that they would like to } \\
\text { receive during transition. }\end{array}$ & by $8 / 15 / 96$ & PHDMC \\
\hline 6. Provide necessary FFTF-specific training to PHMC personnel. & $8 / 12 / 96-9 / 01 / 96$ & DA Gantt \\
\hline $\begin{array}{l}\text { 7. Provide an updated status of ongoing work prior to contract } \\
\text { turnover. }\end{array}$ & by $9 / 30 / 96$ & DA Gantt \\
\hline 8. Perform a joint inventory of SNM with the PHMC. & by $9 / 16 / 96$ & DA Gantt \\
\hline 9. Perform a joint property and equipment inventory with the PHMC. & by $9 / 16 / 96$ & DA Gantt \\
\hline 10. Provide a categorized inventory of wastes. & by $9 / 30 / 96$ & DA Gantt \\
\hline 11. Provide an updated status of end point criteria to PHMC. & by $9 / 30 / 96$ & DA Gantt \\
\hline $\begin{array}{l}\text { 12. Assist the PHMC in the conduct of a Pre-existing Condition } \\
\text { Assessment. }\end{array}$ & $8 / 12 / 96-9 / 16 / 96$ & DA Gantt \\
\hline
\end{tabular}

\subsubsection{B Plant/Waste Encapsulation and Storage Facility}

The B Plant facility was originally constructed as a chemical separations canyon facility to recover nuclear materials through a Bismuth Phosphate Process and was later operated as a "waste fractionization plant" to remove cesium and strontium from tank farm liquid waste. The B Plant deactivation order was received in September 1995. The Waste Encapsulation and Storage Facility (WESF) was constructed to encapsulate and store cesium and strontium obtained from the B Plant processing. The B Plant facility systems are required to maintain the safe environment for WESF, which currently stores the cesium and strontium capsules. An accelerated hazards reduction plan has been proposed that will complete plant deactivation by October 1998. 


\begin{tabular}{|c|c|c|}
\hline Pre-Award Actions & $\begin{array}{l}\text { Scheduled } \\
\text { Date }\end{array}$ & Actionee(s) \\
\hline $\begin{array}{l}\text { 1. Identify number of briefings, and prepare } \\
\text { B Plant/WESF briefing(s) for the PHMC. (Coordinate } \\
\text { number and content with RL POC.) }\end{array}$ & $7 / 15 / 96$ & JL Pappan \\
\hline $\begin{array}{l}\text { 2. Identify key documents for B Plant/WESF. Provide } \\
\text { listing to CL Nansen for consolidation and RL review. }\end{array}$ & $\begin{array}{c}5 / 01 / 96 \\
\text { Completed }\end{array}$ & $\begin{array}{l}\text { JL Pappan } \\
\text { TL Main }\end{array}$ \\
\hline $\begin{array}{l}\text { 3. Assemble key B Plant/WESF documentation, or } \\
\text { identify its location, for use by the PHMC. Includes } \\
\text { preparation of a B Plant/WESF "Smart Book," to } \\
\text { include information developed in the following actions. }\end{array}$ & $\begin{array}{l}6 / 01 / 96 \\
\text { Completed "Smart } \\
\text { Book" only } \\
\text { See item } \# 19 \\
\end{array}$ & IL Pappan \\
\hline $\begin{array}{l}\text { 4. Identify POCs for B Plant/WESF and inquit to } \\
\text { CL Nansen. }\end{array}$ & $\begin{array}{c}5 / 15 / 96 \\
\text { Completed }\end{array}$ & JL Pappan \\
\hline $\begin{array}{l}\text { 5. Develop a listing and description of subcontracts } \\
\text { supporting B Plant/WESF and input to CL Nansen. } \\
\text { Include any subcontracts planned to be executed prior } \\
\text { to contract takeover. }\end{array}$ & $\begin{array}{c}5 / 15 / 96 \\
\text { Completed }\end{array}$ & JL Pappan \\
\hline $\begin{array}{l}\text { 6. Using the pre-existing checklist, compile pre-existing } \\
\text { condition information on B Plant/WESF facilities } \\
\text { and/or major equipment. Conduct, or schedule as a } \\
\text { separate post-award action(s), any assessments } \\
\text { required to adequately address all relevant items on the } \\
\text { checklist. Provide completed checllist to RL POC for } \\
\text { review. }\end{array}$ & $\begin{array}{l}6 / 15 / 96 \\
\text { Completed }\end{array}$ & JL Pappan \\
\hline $\begin{array}{l}\text { 7. Identify applicable milestones and commitments (e.g., } \\
\text { Tri-Party Agreement, DNFSB and consent order } \\
\text { milestones), and determine their stans. Describe the } \\
\text { form of the deliverable and what constitutes } \\
\text { completion. Provide to RL POC for review. }\end{array}$ & $\begin{array}{l}6 / 01 / 96 \\
\text { Completed }\end{array}$ & JL Pappan \\
\hline $\begin{array}{l}\text { 8. Identify current inventories of feed for B Plant/WESF, } \\
\text { and materials to support continued operation (i.e., } \\
\text { essential materials, tools and equipment). }\end{array}$ & $\begin{array}{l}\text { 6/01/96 } \\
\text { Completed }\end{array}$ & IL Pappan \\
\hline $\begin{array}{l}\text { 9. Develop a list of B Plant/WESF-specific mandatory } \\
\text { training and qualification requirements for employees. }\end{array}$ & $\begin{array}{c}6 / 01 / 96 \\
\text { Completed }\end{array}$ & JL Pappan \\
\hline $\begin{array}{l}\text { 10. Develop a listing of information which constitutes the } \\
\text { "authorization basis" for B Plant/WESF. }\end{array}$ & $\begin{array}{c}6 / 01 / 96 \\
\text { Completed }\end{array}$ & JL Pappan \\
\hline $\begin{array}{l}\text { 11. Develop a description of ongoing and planned } \\
\text { construction projects. }\end{array}$ & $\begin{array}{c}6 / 01 / 96 \\
\text { Completed } \\
\end{array}$ & IL Pappan \\
\hline $\begin{array}{l}\text { 12. Develop a listing of special agreement services } \\
\text { provided to } R \mathbf{R} \text {, other DOE sites, and other entities. }\end{array}$ & $\begin{array}{c}\text { 6/01/96 } \\
\text { Completed } \\
\end{array}$ & JL Pappan \\
\hline $\begin{array}{l}\text { 13. Develop a listing and description of B Plant/WESF } \\
\text { databases. Provide list to CL Nansen for } \\
\text { consolidation. }\end{array}$ & $\begin{array}{l}5 / 15 / 96 \\
\text { Completed }\end{array}$ & JL Pappan \\
\hline $\begin{array}{l}\text { 14. Develop a list of current work order services or other } \\
\text { form of agreernent to provide work for other } \\
\text { organizations, on and offsite. }\end{array}$ & $\begin{array}{l}6 / 01 / 96 \\
\text { Completed }\end{array}$ & JL Pappan \\
\hline
\end{tabular}




\begin{tabular}{|c|c|c|}
\hline Pre-Award Actions & $\begin{array}{l}\text { Scheduled } \\
\text { Date }\end{array}$ & Actionee(s) \\
\hline $\begin{array}{l}\text { 15. Develop a list of current work order services or other } \\
\text { form of agreement to obtain work from other } \\
\text { organizations, on and offsite. }\end{array}$ & $\begin{array}{l}\text { 6/01/96 } \\
\text { Completed }\end{array}$ & JL Pappan \\
\hline $\begin{array}{l}\text { 16. Develop a list of permits governing B Plant/WESF, } \\
\text { and provide a description of each as to where and how } \\
\text { it applies. }\end{array}$ & $\begin{array}{l}6 / 01 / 96 \\
\text { Completed }\end{array}$ & JL Pappan \\
\hline $\begin{array}{l}\text { 17. Verify the accuracy of property assignments for } \\
\text { B Plant/WESF and correct any deficiencies. }\end{array}$ & $\begin{array}{l}6 / 01 / 96 \\
\text { Completed }\end{array}$ & JL Pappan \\
\hline $\begin{array}{l}\text { 18. Identify and make available B Plant/WESF -specific } \\
\text { training and qualification records and documents. }\end{array}$ & $\begin{array}{c}6 / 01 / 96 \\
\text { Completed }\end{array}$ & JL Pappan \\
\hline 19. Update/submit "Smart Book"/assemble key documents. & $7 / 15 / 96$ & JL Pappan \\
\hline $\begin{array}{l}\text { 20. Update/finalize current inventories of feed, materials, } \\
\text { and/or wastes. }\end{array}$ & $7 / 15 / 96$ & JL Pappan \\
\hline $\begin{array}{l}\text { 21. Verify pre-existing condition data is current and } \\
\text { supplement as necessary. }\end{array}$ & $7 / 22 / 96$ & JL Pappan \\
\hline 22. Ensure building assignments are correct in RLPS. & $7 / 22 / 96$ & JL Pappan \\
\hline Post-Award Actions & & \\
\hline 1. Provide B Plant/WESF briefing(s) to the PHMC. & $8 / 08 / 96-8 / 19 / 96$ & JL Pappan \\
\hline 2. Provide B Plant/WESF facilities tour to the PHMC. & $8 / 08 / 96-8 / 19 / 96$ & IL Pappan \\
\hline $\begin{array}{l}\text { 3. Provide PHMC access to key documentation, pre- } \\
\text { existing condition information, commitment data, } \\
\text { inventory data, etc. (as established in the pre-award } \\
\text { phase). Provide copies upon request. }\end{array}$ & $8 / 05 / 96$ & JL Pappan \\
\hline $\begin{array}{l}\text { 4. Provide any additional documentation or information } \\
\text { requested by the PHMC, if available, that was not } \\
\text { identified during the pre-award phase. }\end{array}$ & by $9 / 01 / 96$ & JL Pappan \\
\hline $\begin{array}{l}\text { 5. PHMC identify any recurring reports that they would } \\
\text { like to receive during transition. }\end{array}$ & by $8 / 15 / 96$ & PHMC \\
\hline $\begin{array}{l}\text { 6. Provide necessary B Plant/WESF-specific training to } \\
\text { PHMC personnel. }\end{array}$ & $8 / 12 / 96-9 / 01 / 96$ & JL Pappan \\
\hline $\begin{array}{l}\text { 7. Provide an updated status of ongoing work prior to } \\
\text { contract turnover. }\end{array}$ & by $9 / 30 / 96$ & JL Pappan \\
\hline $\begin{array}{l}\text { 8. Perform a joint property and equipment inventory with } \\
\text { the PHMC. }\end{array}$ & by $9 / 16 / 96$ & JL Pappan \\
\hline 9. Provide a categorized inventory of wastes. & by $9 / 30 / 96$ & JL Pappan \\
\hline $\begin{array}{l}\text { 10. Provide an updated status of end point criteria to } \\
\text { PHMC. }\end{array}$ & by $9 / 30 / 96$ & JL Pappan \\
\hline $\begin{array}{l}\text { 11. Assist the PHMC in the conduct of a Pre-existing } \\
\text { Condition Assessment. }\end{array}$ & $8 / 12 / 96-9 / 16 / 96$ & JL Pappan \\
\hline
\end{tabular}




\subsubsection{0/400 Area Fuel Fabrication Facilities and 400 Area Fuels and Materials Examination Facility}

Facilities include the 15 fuel fabrication facilities that were used for fabricating Hanford's production reactor fuel. The facilities are being deactivated but continue to store large quantities of uranium metal and have active RCRA TSDs. Also included are the fuels development Laboratory (308 Building) which was most recently used to fabricate FFTF plutonium fuels and the Plutonium Recycle Test Reactor (PRTR) (309 Building). Both are being deactivated, the 308 Building is scheduled for turnover to the ERC by October 1, 1996. Also included in this grouping is the Fuels and Materials Examination Facility (FMEF) in the 400 Area. It was never utilized for its original mission and is therefore not radiologically contaminated. It supports various Hanford missions by renting valuable space and capabilities. It continues to be studied for FFTF support activities and national weapons plutonium disposition alternatives.

\begin{tabular}{|c|c|c|}
\hline Pre-Award Actions & $\begin{array}{c}\text { Scheduled } \\
\text { Date }\end{array}$ & Actionee(s) \\
\hline $\begin{array}{l}\text { 1. Identify number of briefings, and prepare } 300 / 400 \\
\text { Area Fuel Fabrications briefing(s) for the PHMC } \\
\text { (Coordinate number and content with RL POC.) }\end{array}$ & $\begin{array}{c}6 / 01 / 96 \\
\text { Completed }\end{array}$ & M Steffen \\
\hline $\begin{array}{l}\text { 2. Identify key documents for the } 300 / 400 \text { Area Fuel } \\
\text { Fabrications. Provide listing to CL Nansen for } \\
\text { consolidation and RL review. }\end{array}$ & $\begin{array}{c}\text { 5/01/96 } \\
\text { Compicted }\end{array}$ & M Steffen \\
\hline $\begin{array}{l}\text { 3. Assemble } 300 / 400 \text { Area Fuel Fabrication Facilities } \\
\text { documentation, or identify its location, for use by the } \\
\text { PHMC. Includes preparation of a } 300 / 400 \text { Area Fuel } \\
\text { Fabrications "Smart Book," to include information } \\
\text { developed in the following actions. }\end{array}$ & $\begin{array}{l}6 / 01 / 96 \\
\text { Completed "Smart } \\
\text { Book" only } \\
\text { See item } \# 19\end{array}$ & M Steffen \\
\hline $\begin{array}{l}\text { 4. Identify POCs for the } 300 / 400 \text { Area Fuel Fabrication } \\
\text { Facilities and input to CL Nansen. }\end{array}$ & $\begin{array}{c}5 / 15 / 96 \\
\text { Completed } \\
\end{array}$ & JM Steffen \\
\hline $\begin{array}{l}\text { 5. Develop a listing and description of subcontracts } \\
\text { supporting the } 300 / 400 \text { Area Fuel Fabrication } \\
\text { Facilities and input to CL Nansen. Include any } \\
\text { subcontracts planned to be executed prior to contract } \\
\text { takeover. }\end{array}$ & $\begin{array}{c}5 / 15 / 96 \\
\text { Compjeted }\end{array}$ & JM Steffen \\
\hline $\begin{array}{l}\text { 6. Using the pre-existing checklist, compile pre-existing } \\
\text { condition information on the } 300 / 400 \text { Area Fuel } \\
\text { Fabrications Facilities and/or major equipment. } \\
\text { Conduct, or schedule as a separate post-award } \\
\text { action(s), any assessments required to adequately } \\
\text { address all relevant items on the checklist. Provide } \\
\text { completed checklist to RL POC for review. }\end{array}$ & $\begin{array}{c}\text { 6/15/96 } \\
\text { Completed }\end{array}$ & MM Steffen \\
\hline $\begin{array}{l}\text { 7. Identify applicable milestones and cornmitments (e.g., } \\
\text { Tri-Party Agreement, DNFSB and consent order } \\
\text { milestones), and determine their status. Describe the } \\
\text { form of the deliverable and what constitutes } \\
\text { completion. Provide to RL POC for review. }\end{array}$ & $\begin{array}{c}6 / 01 / 96 \\
\text { Completed }\end{array}$ & M Steffen \\
\hline
\end{tabular}




\begin{tabular}{|c|c|c|}
\hline Pre-Award Actions & $\begin{array}{l}\text { Scheduled } \\
\text { Date }\end{array}$ & Actionee(s) \\
\hline $\begin{array}{l}\text { 8. Identify current inventories of feed for the } 300 / 400 \\
\text { Area Puel Fabrications, and materials to support } \\
\text { continued operation (i.e., essential materials, tools and } \\
\text { equipment). }\end{array}$ & $\begin{array}{c}\text { 6/01/96 } \\
\text { Completed }\end{array}$ & JM Steffen \\
\hline $\begin{array}{l}\text { 9. Develop a list of } 300 / 400 \text { Area Fuel Fabrications - } \\
\text { specific mandatory training and qualification } \\
\text { requirements for employees. }\end{array}$ & $\begin{array}{l}\text { 6/01/96 } \\
\text { Completed }\end{array}$ & M Steffen \\
\hline $\begin{array}{l}\text { 10. Develop a listing of information which constitutes the } \\
\text { "authorization basis" for the } 300 / 400 \text { Area Fuel } \\
\text { Fabrications. }\end{array}$ & $\begin{array}{c}\text { 6/01/96 } \\
\text { Completed }\end{array}$ & JM Steffen \\
\hline $\begin{array}{l}\text { 11. Develop a description of ongoing and planned } \\
\text { construction projects. }\end{array}$ & $\begin{array}{c}6 / 01 / 96 \\
\text { Completed }\end{array}$ & JM Steffen \\
\hline $\begin{array}{l}\text { 12. Develop a listing of special agreement services } \\
\text { provided to } R L \text {, other DOE sites, and other entities. }\end{array}$ & $\begin{array}{c}6 / 01 / 96 \\
\text { Completed }\end{array}$ & M Steffen \\
\hline $\begin{array}{l}\text { 13. Develop a listing and description of } 300 / 400 \text { Area Fuel } \\
\text { Fabrications databases. Provide list to CL Nansen for } \\
\text { consolidation. }\end{array}$ & $\begin{array}{c}5 / 15 / 96 \\
\text { Completed }\end{array}$ & JM Steffen \\
\hline $\begin{array}{l}\text { 14. Develop a list of current work order services or other } \\
\text { form of agreement to provide work for other } \\
\text { organizations, on and offsite. }\end{array}$ & $\begin{array}{l}6 / 01 / 96 \\
\text { Completed }\end{array}$ & JM Steffen \\
\hline $\begin{array}{l}\text { 15. Develop a list of current work order services or other } \\
\text { form of agreement to obtain work from other } \\
\text { organizations, on and offsite. }\end{array}$ & $\begin{array}{l}\text { 6/01/96 } \\
\text { Completed }\end{array}$ & JM Steffen \\
\hline $\begin{array}{l}\text { 16. Develop a list of permits governing the } 300 / 400 \text { Area } \\
\text { Fuel Fabrications, and provide a description of each as } \\
\text { to where and how it applies. }\end{array}$ & $\begin{array}{l}6 / 01 / 96 \\
\text { Completed }\end{array}$ & JM Steffen \\
\hline $\begin{array}{l}\text { 17. Verify the accuracy of property assignments for the } \\
300 / 400 \text { Area Fuel Fabrications and correct any } \\
\text { deficiencies. }\end{array}$ & $\begin{array}{l}6 / 01 / 96 \\
\text { Completed }\end{array}$ & M Steffen \\
\hline $\begin{array}{l}\text { 18. Identify and make available } 300 / 400 \text { Area Fuel } \\
\text { Fabrications -specific training and qualification records } \\
\text { and documents. }\end{array}$ & $\begin{array}{c}\text { 6/01/96 } \\
\text { Completed }\end{array}$ & JM Steffen \\
\hline 19. Update/submit "Smart Book"/assemble key documents. & $7 / 15 / 96$ & JM Steffen \\
\hline 20. Update/finalize briefing to PHMC. & $7 / 15 / 96$ & M Steffen \\
\hline $\begin{array}{l}\text { 21. Update/finalize current inventories of feed, materials, } \\
\text { and/or wastes. }\end{array}$ & $7 / 15 / 96$ & JM Steffen \\
\hline $\begin{array}{l}\text { 22. Verify pre-existing condition data is current and } \\
\text { supplement as necessary. }\end{array}$ & $7 / 22 / 96$ & M Steffen \\
\hline 23. Ensure building assignments are correct in RLPS. & $\begin{array}{c}7 / 22 / 96 \\
\text { Completed }\end{array}$ & JM Steffen \\
\hline Post-Award Actions & 3 & \\
\hline $\begin{array}{l}\text { 1. Provide } 300 / 400 \text { Area Fuel Facility briefing(s) to the } \\
\text { PHMC. }\end{array}$ & $8 / 08 / 96-8 / 19 / 96$ & JM Steffen \\
\hline
\end{tabular}




\begin{tabular}{|c|c|c|}
\hline Post-Award Actions & $\begin{array}{c}\text { Scheduled } \\
\text { Date }\end{array}$ & Actionee(s) \\
\hline $\begin{array}{l}\text { 2. Provide } 300 / 400 \text { Area Puel Facility tour to the } \\
\text { PHMC. }\end{array}$ & $8 / 08 / 96-8 / 19 / 96$ & JM Steffen \\
\hline $\begin{array}{l}\text { 3. Provide PHMC access to key documentation, pre- } \\
\text { existing condition information, commitment data, } \\
\text { inventory data, etc. (as established in the pre-award } \\
\text { phase). Provide copies upon request. }\end{array}$ & $8 / 05 / 96$ & JM Steffen \\
\hline $\begin{array}{l}\text { 4. Provide any additional documentation or information } \\
\text { requested by the PHMC, if available, that was not } \\
\text { identified during the pre-award phase. }\end{array}$ & by $9 / 01 / 96$ & JM Steffen \\
\hline $\begin{array}{l}\text { 5. PHMC identify any recurring reports that they would } \\
\text { like to receive during transition. }\end{array}$ & by $8 / 15 / 96$ & PHMC \\
\hline $\begin{array}{l}\text { 6. Provide necessary } 300 / 400 \text { Area Fuel Facility-specific } \\
\text { training to PHMC personnel. }\end{array}$ & $8 / 12 / 96-9 / 01 / 96$ & JM Steffen \\
\hline $\begin{array}{l}\text { 7. Provide an updated status of ongoing work prior to } \\
\text { contract turnover. }\end{array}$ & by $9 / 30 / 96$ & M Steffen \\
\hline 8. Perform a joint inventory of SNM with the PHMC. & by $9 / 16 / 96$ & IM Steffen \\
\hline $\begin{array}{l}\text { 9. Perform a joint property and equipment inventory with } \\
\text { the PHMC. }\end{array}$ & by $9 / 16 / 96$ & M Steffen \\
\hline 10. Provide a categorized inventory of wastes. & by $9 / 30 / 96$ & $\mathrm{M}$ Steffen \\
\hline $\begin{array}{l}\text { 11. Provide an updated status of end point criteria to } \\
\text { PHMC. }\end{array}$ & by $9 / 30 / 96$ & JM Steffen \\
\hline $\begin{array}{l}\text { 12. Assist the PHMC in the conduct of a Pre-existing } \\
\text { Condition Assessment. }\end{array}$ & $8 / 12 / 96-9 / 16 / 96$ & IM Steffen \\
\hline
\end{tabular}

\subsubsection{Transfer of 324 and 327 Building PNNL Facilities}

The 324 Building provided for the development of liquid metal systems and the study of chemical and metallurgical processes in a single integrated facility for the orderly progress of nonradioactive or radioactive developmental studies from laboratory or bench-scale to full engineering scale pilot plant demonstrations. It houses a nuclear materials storage vault, plutonium glovebox laboratory, enriched uranium hood, and assay equipment for fuel and waste material.

The 327 Building provided specially shielded, ventilated and equipped laboratories for physical and metallurgical examination of irradiated nuclear materials including measurements, metallography, transient testing at room and elevated temperatures, density and in-cell machining. Radiation effects can be determined as and aid in developing stable materials. 


\begin{tabular}{|c|c|c|}
\hline Pre-Award Actions & $\begin{array}{c}\text { Scheduled } \\
\text { Date }\end{array}$ & Actionee(s) \\
\hline $\begin{array}{l}\text { 1. Identify number of briefings, and prepare Buildings } \\
324 \text { and } 327 \text { briefing(s) for the PHMC. (Coordinate } \\
\text { number and content with RL POC.) }\end{array}$ & $\begin{array}{l}\text { 6/01/96 } \\
\text { Completed }\end{array}$ & $\begin{array}{l}\text { TW Halverson } \\
\text { D Knowlton (PNNL) }\end{array}$ \\
\hline $\begin{array}{l}\text { 2. Identify key documents for Buildings } 324 \text { and } 327 . \\
\text { Provide listing to CL Nansen for consolidation and RL } \\
\text { review. }\end{array}$ & $\begin{array}{l}5 / 15 / 96 \\
\text { Completed }\end{array}$ & $\begin{array}{l}\text { TW Halverson } \\
\text { D Rnowlton (PNNL) }\end{array}$ \\
\hline $\begin{array}{l}\text { 3. Assemble key Buildings } 324 \text { and } 327 \text { documentation, } \\
\text { or identify its location, for use by the PHMC. } \\
\text { Includes preparation of a Buildings } 324 \text { and } 327 \\
\text { "Smart Book," to include information developed in the } \\
\text { following actions. }\end{array}$ & $\begin{array}{l}6 / 01 / 96 \\
\text { Completed "Smart } \\
\text { Book" only } \\
\text { See item } \# 19\end{array}$ & $\begin{array}{l}\text { TW Halverson } \\
\text { D Knowlton (PNNL) }\end{array}$ \\
\hline $\begin{array}{l}\text { 4. Identify POCs for Buildings } 324 \text { and } 327 \text { and input to } \\
\text { CL Nansen. }\end{array}$ & $\begin{array}{c}5 / 15 / 96 \\
\text { Completed }\end{array}$ & $\begin{array}{l}\text { TW Halverson } \\
\text { D Knowlton (PNNL) }\end{array}$ \\
\hline $\begin{array}{l}\text { 5. Develop a listing and description of subcontracts } \\
\text { supporting Buildings } 324 \text { and } 327 \text { and input to } \\
\text { CL Nansen. Include any subcontracts planned to be } \\
\text { executed prior to contract takeover. }\end{array}$ & $\begin{array}{c}5 / 15 / 96 \\
\text { Completed }\end{array}$ & $\begin{array}{l}\text { TW Halverson } \\
\text { D Knowlton (PNNL) }\end{array}$ \\
\hline $\begin{array}{l}\text { 6sing the pre-existing checklist, compile pre-existing } \\
\text { condition information on Buildings } 324 \text { and } 327 \text { and/or } \\
\text { major equipment. Conduct, or schedule as a separate } \\
\text { post-award action(s), any assessments required to } \\
\text { adequately address all relevant items on the checklist. } \\
\text { Provide completed checklist to RL POC for review. }\end{array}$ & $\begin{array}{c}6 / 15 / 96 \\
\text { Completed }\end{array}$ & $\begin{array}{l}\text { TW Halverson } \\
\text { D Knowlton (PNNL) }\end{array}$ \\
\hline $\begin{array}{l}\text { 7. Identify applicable milestones and commitments (e.g., } \\
\text { Tri-Party Agreement, DNFSB and consent order } \\
\text { milestones), and determine their status. Describe the } \\
\text { form of the deliverable and what constitutes } \\
\text { completion. Provide to RL POC for review. }\end{array}$ & $\begin{array}{l}\text { 6/01/96 } \\
\text { Completed }\end{array}$ & $\begin{array}{l}\text { TW Halverson } \\
\text { D Knowlton (PNNL) }\end{array}$ \\
\hline $\begin{array}{l}\text { 8. Identify current inventories of feed for Buildings } 324 \\
\text { and } 327 \text {, and materials to support continued operation } \\
\text { (i.e., essential materials, tools and equipment). }\end{array}$ & $\begin{array}{l}\text { 6/01/96 } \\
\text { Completed }\end{array}$ & $\begin{array}{l}\text { TW Halverson } \\
\text { D Knowlton (PNNI) }\end{array}$ \\
\hline $\begin{array}{l}\text { 9. Develop a list of Buildings } 324 \text { and } 327 \text {-specific } \\
\text { mandatory training and qualification requirements for } \\
\text { employees. }\end{array}$ & $\begin{array}{l}6 / 01 / 96 \\
\text { Completed }\end{array}$ & $\begin{array}{l}\text { TW Halverson } \\
\text { D Knowiton (PNNL) }\end{array}$ \\
\hline $\begin{array}{l}\text { 10. Develop a listing of information which constitutes the } \\
\text { "authorization basis" for Buildings } 324 \text { and } 327 \text {. }\end{array}$ & $\begin{array}{c}\text { 6/01/96 } \\
\text { Completed }\end{array}$ & $\begin{array}{l}\text { TW Halverson } \\
\text { D Knowlton (PNNL) }\end{array}$ \\
\hline $\begin{array}{l}\text { 11. Develop a description of ongoing and planned } \\
\text { construction projects. }\end{array}$ & $\begin{array}{c}6 / 01 / 96 \\
\text { Completed }\end{array}$ & $\begin{array}{l}\text { TW Halverson } \\
\text { D Knowlton (PNNL) }\end{array}$ \\
\hline $\begin{array}{l}\text { 12. Develop a listing of special agreement services } \\
\text { provided to } R L \text {, other DOE sites, and other entities. }\end{array}$ & $\begin{array}{c}6 / 01 / 96 \\
\text { Completed } \\
\end{array}$ & $\begin{array}{l}\text { TW Halverson } \\
\text { D Knowlton (PNNL) }\end{array}$ \\
\hline $\begin{array}{l}\text { 13. Develop a listing and description of Buildings } 324 \text { and } \\
327 \text { databases. Provide list to for consolidation. }\end{array}$ & $\begin{array}{c}5 / 15 / 96 \\
\text { Completed } \\
\end{array}$ & $\begin{array}{l}\text { TW Halverson } \\
\text { D Knowlton (PNNL) }\end{array}$ \\
\hline $\begin{array}{l}\text { 14. Develop a list of current work order services or other } \\
\text { form of agreement to provide work for other } \\
\text { organizations, on and offsite. }\end{array}$ & $\begin{array}{c}6 / 01 / 96 \\
\text { Completed }\end{array}$ & $\begin{array}{l}\text { TW Halverson } \\
\text { D Knowlton (PNNL) }\end{array}$ \\
\hline
\end{tabular}




\begin{tabular}{|c|c|c|}
\hline Pre-Award Actions & $\begin{array}{l}\text { Scheduled } \\
\text { Date }\end{array}$ & Actionee(s) \\
\hline $\begin{array}{l}\text { 15. Develop a list of current work order services or other } \\
\text { form of agreement to obtain work from other } \\
\text { organizations, on and offsite. }\end{array}$ & $\begin{array}{l}\text { 6/01/96 } \\
\text { Completed }\end{array}$ & $\begin{array}{l}\text { TW Halverson } \\
\text { D Knowlton (PNNL) }\end{array}$ \\
\hline $\begin{array}{l}\text { 16. Develop a list of permits governing Buildings } 324 \text { and } \\
327 \text {, and provide a description of each as to where and } \\
\text { how it applies. }\end{array}$ & $\begin{array}{l}\text { 6/01/96 } \\
\text { Completed }\end{array}$ & $\begin{array}{l}\text { TW Halverson } \\
\text { D Knowlton (PNNL) }\end{array}$ \\
\hline $\begin{array}{l}\text { 17. Verify the accuracy of property assignments for } \\
\text { Buildings } 324 \text { and } 327 \text { and correct any deficiencies. }\end{array}$ & $\begin{array}{c}6 / 01 / 96 \\
\text { Completed } \\
\end{array}$ & $\begin{array}{l}\text { TW Halverson } \\
\text { D Knowlion (PNNL) }\end{array}$ \\
\hline $\begin{array}{l}\text { 18. Identify and make available Buildings } 324 \text { and } 327 \text { - } \\
\text { specific training and qualification records and } \\
\text { documents. }\end{array}$ & $\begin{array}{l}\text { 6/01/96 } \\
\text { Completed }\end{array}$ & $\begin{array}{l}\text { TW Halverson } \\
\text { D Knowlton (PNNL) }\end{array}$ \\
\hline 19. Update/subrnit "Smart Book"/assemble key documents. & $7 / 15 / 96$ & $\begin{array}{l}\text { TW Halverson } \\
\text { D Knowlton (PNNL) }\end{array}$ \\
\hline 20. Update/finalize briefing to PHMC. & $7 / 15 / 96$ & $\begin{array}{l}\text { TW Halverson } \\
\text { D Knowiton (PNNL) }\end{array}$ \\
\hline $\begin{array}{l}\text { 21. Update/finalize current inventories of feed, materials, } \\
\text { and/or wastes. }\end{array}$ & $7 / 15 / 96$ & $\begin{array}{l}\text { TW Halverson } \\
\text { D Knowlton (PNNL) }\end{array}$ \\
\hline $\begin{array}{l}\text { 22. Verify pre-existing condition data is current and } \\
\text { supplement as necessary. }\end{array}$ & $7 / 22 / 96$ & $\begin{array}{l}\text { TW Halverson } \\
\text { D Knowlton (PNNL) }\end{array}$ \\
\hline 23. Ensure building assignments are correct in RLPS. & $7 / 22 / 96$ & $\begin{array}{l}\text { TW Halverson } \\
\text { D Knowiton (PNNL) }\end{array}$ \\
\hline \multicolumn{3}{|l|}{ Post-Award Actions } \\
\hline $\begin{array}{l}\text { 1. Provide } 324 \text { and } 327 \text { Facilities briefing(s) to the } \\
\text { PHMC. }\end{array}$ & $8 / 08 / 96-8 / 19 / 96$ & $\begin{array}{l}\text { TW Halverson } \\
\text { D Knowlton (PNNL) }\end{array}$ \\
\hline 2. Provide 324 and 327 Facilities tour to the PHMC. & $8 / 08 / 96-8 / 19 / 96$ & $\begin{array}{l}\text { TW Halverson } \\
\text { D Knowlton (PNNL) } \\
\end{array}$ \\
\hline $\begin{array}{l}\text { 3. Provide PHMC access to key documentation, pre- } \\
\text { existing condition information, commitment data, } \\
\text { inventory data, etc. (as established in the pre-award } \\
\text { phase). Provide copies upon request. }\end{array}$ & $8 / 05 / 96$ & $\begin{array}{l}\text { TW Halverson } \\
\text { D Knowlton (PNNL) }\end{array}$ \\
\hline $\begin{array}{l}\text { 4. Provide any additional documentation or information } \\
\text { requested by the PHMC, if available, that was not } \\
\text { identified during the pre-award phase. }\end{array}$ & by $9 / 01 / 96$ & $\begin{array}{l}\text { TW Halverson } \\
\text { D Knowiton (PNNL) }\end{array}$ \\
\hline $\begin{array}{l}\text { 5. PHMC identify any recurring reports that they would } \\
\text { like to receive during transition. }\end{array}$ & by $8 / 15 / 96$ & PHMC \\
\hline $\begin{array}{l}\text { 6. Provide necessary } 324 \text { and } 327 \text { Facilities -specific } \\
\text { training to PHMC personnel. }\end{array}$ & $8 / 12 / 96-9 / 01 / 96$ & $\begin{array}{l}\text { Tw Hajverson } \\
\text { D Knowlton (PNNL) } \\
\end{array}$ \\
\hline $\begin{array}{l}\text { 7. Provide an updated starus of ongoing work prior to } \\
\text { contract turnover. }\end{array}$ & by $9 / 30 / 96$ & \begin{tabular}{|l} 
TW Halverson \\
D Knowlton (PNNL) \\
\end{tabular} \\
\hline 8. Perform a joint inventory of SNM with the PHMC. & by $9 / 16 / 96$ & $\begin{array}{l}\text { TW Haiverson } \\
\text { D Knowlton (PNNL) } \\
\end{array}$ \\
\hline $\begin{array}{l}\text { 9. Perform a joint property and equipment inventory with } \\
\text { the PHMC. }\end{array}$ & by $9 / 16 / 96$ & $\begin{array}{l}\text { TW Halverson } \\
\text { D Knowlton (PNNL) }\end{array}$ \\
\hline
\end{tabular}




\begin{tabular}{|c|c|c|}
\hline Post-Award Actions & $\begin{array}{l}\text { Scheduled } \\
\text { Date }\end{array}$ & Actionee(s) \\
\hline 10. Provide a categorized inventory of wastes. & by $9 / 30 / 96$ & $\begin{array}{l}\text { TW Halverson } \\
\text { D Knowlton (PNNL) }\end{array}$ \\
\hline $\begin{array}{l}\text { 11. Provide an updated status of end point criteria to } \\
\text { PHMC. }\end{array}$ & by $9 / 30 / 96$ & $\begin{array}{l}\text { TW Halverson } \\
\text { D Knowiton (PNNL) }\end{array}$ \\
\hline $\begin{array}{l}\text { 12. Assist the PHMC in the conduct of a Pre-existing } \\
\text { Condition Assessment }\end{array}$ & $8 / 12 / 96-9 / 16 / 96$ & $\begin{array}{l}\text { TW Halverson } \\
\text { D Knowlton (PNNL) }\end{array}$ \\
\hline
\end{tabular}

\subsubsection{Materials and Future Transition Programs}

Long-term disposition of nuclear material and special nuclear materials. Identification and development of facilities and facility group transitions above and beyond the current scopes of work.

Note: Materials and Future Transition Programs section covers facility stabilization areas that may not be covered by individual plants. It may therefore be possible that response to some Section 4.4.7 actions may become "Not applicable" when a specific item is completely covered by individual plants.

\begin{tabular}{|c|c|c|}
\hline Pre-Award Actions & $\begin{array}{c}\text { Scheduled } \\
\text { Date }\end{array}$ & Actionee(s) \\
\hline $\begin{array}{l}\text { 1. Prepare Materials and Future Transition Programs } \\
\text { briefing for the PHMC. (Coordinate with RL POC.) }\end{array}$ & $7 / 15 / 96$ & G Cox \\
\hline $\begin{array}{l}\text { 2. Identify key documents for the Materials and Future } \\
\text { Transition Programs. Provide listing to CL Nansen } \\
\text { for consolidation and RL review. }\end{array}$ & $\begin{array}{l}5 / 01 / 96 \\
\text { Completed }\end{array}$ & G Cox \\
\hline $\begin{array}{l}\text { 3. Assemble Materials and Future Transition Programs } \\
\text { documentation, or identify its location, for use by the } \\
\text { PHMC. Includes preparation of a Materials and } \\
\text { Future Transition Programs "Smart Book," to include } \\
\text { information developed in the following actions. }\end{array}$ & $\begin{array}{c}6 / 01 / 96 \\
\text { Completed "Smart } \\
\text { Book" only } \\
\text { See item } \# 14\end{array}$ & G Cox \\
\hline $\begin{array}{l}\text { 4. Identify POCs for the Materials and Future Transition } \\
\text { Programs and input to CL Nansen. }\end{array}$ & $\begin{array}{l}5 / 15 / 96 \\
\text { Completed }\end{array}$ & G Cox \\
\hline $\begin{array}{l}\text { 5. Develop a listing and description of subcontracts } \\
\text { supporting the Materials and Future Transition } \\
\text { Programs and input to consolidated program/project } \\
\text { listing. Include any subcontracts planned to be } \\
\text { executed prior to contract takeover. }\end{array}$ & $\begin{array}{c}5 / 15 / 96 \\
\text { Completed }\end{array}$ & G Cox \\
\hline $\begin{array}{l}\text { 6. Identify applicable milestones and commitments (e.g., } \\
\text { Tri-Party Agreement, DNFSB and consent order } \\
\text { milestones), and determine their status. Describe the } \\
\text { form of the deliverable and what constiutes } \\
\text { completion. Provide to RL POC for review. }\end{array}$ & $\begin{array}{l}6 / 15 / 96 \\
\text { Completed }\end{array}$ & G Cox \\
\hline $\begin{array}{l}\text { 7. Develop a listing of special agreement services } \\
\text { provided to RL, other DOE sites, and other entities. }\end{array}$ & $\begin{array}{l}\text { 6/01/96 } \\
\text { Completed }\end{array}$ & G Cox \\
\hline
\end{tabular}




\begin{tabular}{|c|c|c|}
\hline Pre-Award Actions & $\begin{array}{l}\text { Scheduled } \\
\text { Date } \\
\end{array}$ & Actionee(s) \\
\hline $\begin{array}{l}\text { 8. Develop a listing of Materials and Future Transition } \\
\text { Programs facility POCs to be available for the } \\
\text { transition phase. Provide list to CL Nansen for } \\
\text { consolidation. }\end{array}$ & $\begin{array}{l}5 / 15 / 96 \\
\text { Completed }\end{array}$ & G Cox \\
\hline $\begin{array}{l}\text { 9. Develop a listing and description of Materials and } \\
\text { Future Transition Programs databases. Provide list to } \\
\text { CL Nansen for consolidation. }\end{array}$ & $\begin{array}{l}5 / 15 / 96 \\
\text { Completed }\end{array}$ & G Cox \\
\hline $\begin{array}{l}\text { 10. Develop a list of current work order services or other } \\
\text { form of agreement to provide work for other } \\
\text { organizations, on and offsite. }\end{array}$ & $\begin{array}{l}\text { 6/01/96 } \\
\text { Completed }\end{array}$ & G Cox \\
\hline $\begin{array}{l}\text { 11. Develop a list of current work order services or other } \\
\text { form of agreement to obtain work from other } \\
\text { organizations, on and offsite. }\end{array}$ & $\begin{array}{c}6 / 01 / 96 \\
\text { Completed }\end{array}$ & G Cox \\
\hline $\begin{array}{l}\text { 12. Verify the accuracy of property assignments for } \\
\text { Materials and Future Transition Programs and correct } \\
\text { any deficiencies. }\end{array}$ & $\begin{array}{c}\text { 6/01/96 } \\
\text { Completed }\end{array}$ & G Cox \\
\hline $\begin{array}{l}\text { 13. Identify and make available Materials and Future } \\
\text { Transition Programs -specific training and qualification } \\
\text { records and documents. }\end{array}$ & $\begin{array}{c}6 / 01 / 96 \\
\text { Completed }\end{array}$ & G Cox \\
\hline 14. Update/submit "Smart Book"/assemble key documents. & $7 / 15 / 96$ & $G \operatorname{Cox}$ \\
\hline \multicolumn{3}{|l|}{ Post-Award Actions } \\
\hline $\begin{array}{l}\text { 1. Provide Materials and Future Transition briefing(s) to } \\
\text { the PHMC. }\end{array}$ & $8 / 08 / 96-8 / 19 / 96$ & G. $\operatorname{Cox}$ \\
\hline $\begin{array}{l}\text { 2. Provide PHMC access to key documentation, } \\
\text { commitment data, inventory data, etc. (as established } \\
\text { in the pre-award phase). Provide copies upon request. }\end{array}$ & $8 / 05 / 96$ & G. Cox \\
\hline $\begin{array}{l}\text { 3. Provide any additional documentation or information } \\
\text { requested by the PHMC, if available, that was not } \\
\text { identified during the pre-award phase. }\end{array}$ & by $9 / 01 / 96$ & G. Cox \\
\hline $\begin{array}{l}\text { 4. PHMC identify any recurring reports that they would } \\
\text { like to receive during transition. }\end{array}$ & by $8 / 15 / 96$ & PHMC \\
\hline $\begin{array}{l}\text { 5. Provide an updated status of ongoing work prior to } \\
\text { contract turnover. }\end{array}$ & by $9 / 30 / 96$ & G. $\operatorname{cox}$ \\
\hline $\begin{array}{l}\text { 6. Perform a joint property and equipment inventory with } \\
\text { the PHMC. }\end{array}$ & by $9 / 16 / 96$ & G. Cox \\
\hline
\end{tabular}




\subsection{ENVIRONMENTAL RESTORATION (ER) (INTEGRATION)}

BHI is under separate prime contract to RL to perform ER work for the Hanford Site. The contract runs through FY 1999, with an option for three additional years.

ER work includes removal of contamination in soils and groundwater, D\&D of Site facilities, exhuming of buried waste, installation of environmental barriers (where appropriate), and the management and support activities required to accomplish this work. Operation of the Environmental Restoration Disposal Facility (ERDF) for disposal of ER exhumed and generated wastes is included within the BHI workscope. BHI has responsibility for management and coordination of the sitewide groundwater monitoring activities (refer to Section 6.6.2), sitewide asbestos removal/abatement program, and (non-radioactive) underground storage tank removal. BHI also is responsible for management of three sitewide environmental databases: the Hanford Geological Information System (GIS), Hanford Environmental Information System (HEIS), and Waste Information Data System (WIDS).

The PHMC integrates data generated and provided by BHI for the Richland ER Project into the overall Hanford Site data systems, including the Sitewide Baseline. Examples of such data include cost, schedule, milestone, and any other data requested by DOE. The PHMC is responsible for integrating data and planning information from $\mathrm{BHI}$ into the sitewide planning and reporting systems, and is not responsible for management of or accomplishing any of the workscope within the BHI contract.

Refer to Section 6.2.2 of this plan for a description of the BHI ER Project workscope for CERCLA monitoring and related pre- and post-award transition actions.

\begin{tabular}{|l|c|l|}
\hline \multicolumn{1}{|c|}{ Pre-Award Actions } & Scheduled & Date \\
\hline $\begin{array}{l}\text { 1. Identify all applicable documents serving to define the } \\
\text { business relationships and workscope interactions } \\
\text { between the PHMC and BHI organizations (contractual } \\
\text { requirements, Sitewide agreements, procedures, MOUs, } \\
\text { end point criteria, etc.). }\end{array}$ & $7 / 12 / 96$ & $\begin{array}{l}\text { DD von der Linden } \\
\text { JH Dunkirk }\end{array}$ \\
\hline $\begin{array}{l}\text { 2. Prepare a briefing for PHMC management } \\
\text { Iepresentatives, describing relations and interactions } \\
\text { between BHI and the PHMC in ail applicable } \\
\text { workscope areas and functions. }\end{array}$ & $7 / 26 / 96$ & GF Jones \\
\hline $\begin{array}{l}\text { 3. Identify appropriate BHI points-of -contact for the } \\
\text { PHMC. }\end{array}$ & $7 / 01 / 96$ & GF Jones \\
\hline $\begin{array}{l}\text { 4. Document the status of ongoing and near-term planned } \\
\text { sub-projects and activities requiring involvement by or } \\
\text { support from the PHMC. }\end{array}$ & $\begin{array}{c}5 / 01 / 96 \\
\text { Completed }\end{array}$ & MC Hughes \\
\hline SC Foelber \\
\hline 5. List all active work orders and their expiration dates. & $4 / 22 / 96$ & DD von der Linden \\
\hline $\begin{array}{l}\text { 6. Determine which support services work orders will be } \\
\text { continued and/or renewed, and which are candidates for } \\
\text { discontinuation. Document rationale. }\end{array}$ & $7 / 12 / 96$ & (Numerous) \\
\hline
\end{tabular}


WHC-SP-1181 Rev. 1

\begin{tabular}{|c|c|c|}
\hline Pre-Award Actions & $\begin{array}{r}\text { Scheduled } \\
\text { Date } \\
\end{array}$ & Actionee(6) \\
\hline $\begin{array}{l}\text { 7. Determine and document status of Site facilities being } \\
\text { transitioned from WHC to ER; incluđe status of pre- } \\
\text { existing conditions records, as appropriate. }\end{array}$ & $7 / 19 / 96$ & $\begin{array}{l}\text { MC Hughes } \\
\text { JH Dunkirk }\end{array}$ \\
\hline $\begin{array}{l}\text { 8. Complete resolution of open contract liability issues } \\
\text { involving WHC, BHI, and DOE. }\end{array}$ & $8 / 01 / 96$ & $\begin{array}{l}\text { DD von der Linden } \\
\text { JH Dunkirk }\end{array}$ \\
\hline \multicolumn{3}{|l|}{ Post-Award Actions } \\
\hline $\begin{array}{l}\text { 1. Provide briefing for the PHMC relevant to interactions } \\
\text { between BHI and PHMC in all applicable workscope } \\
\text { areas and functions. }\end{array}$ & $8 / 08 / 96-8 / 19 / 96$ & $\begin{array}{l}\text { MC Hughes } \\
\text { SC Foelber } \\
\text { (and others) }\end{array}$ \\
\hline $\begin{array}{l}\text { 2. Identify to PHMC those work orders for PHMC } \\
\text { services that BHI will continue and/or renew, and those } \\
\text { work orders BHI is discontinuing (including effective } \\
\text { date of discontinuation). }\end{array}$ & $8 / 08 / 96$ & (Numerous) \\
\hline $\begin{array}{l}\text { 3. Establish routine points of contact and agreed method } \\
\text { and frequency of interface between PHMC and BHI } \\
\text { during Transition Period. }\end{array}$ & $8 / 08 / 96$ & $\begin{array}{l}\text { MC Hughes } \\
\text { SC Foelber }\end{array}$ \\
\hline $\begin{array}{l}\text { 4. Reach concurrence and document agreement on } \\
\text { continuation of all work in-progress involving support } \\
\text { from PHMC. }\end{array}$ & $9 / 06 / 96$ & TBD \\
\hline $\begin{array}{l}\text { 5. If requested, provide the PHMC representatives a tour } \\
\text { of ER Project sites to clarify areas of BHI responsibility } \\
\text { (vs. PHMC or other contractor), including discussion of } \\
\text { relevant pre-existing site conditions. }\end{array}$ & TBD & TBD \\
\hline 6. Update/senew active work orders, as appropriate. & 9/13/96 & (Numerous) \\
\hline
\end{tabular}


WHC-SP-1181 Rev. 1

This page intentionally left blank. 

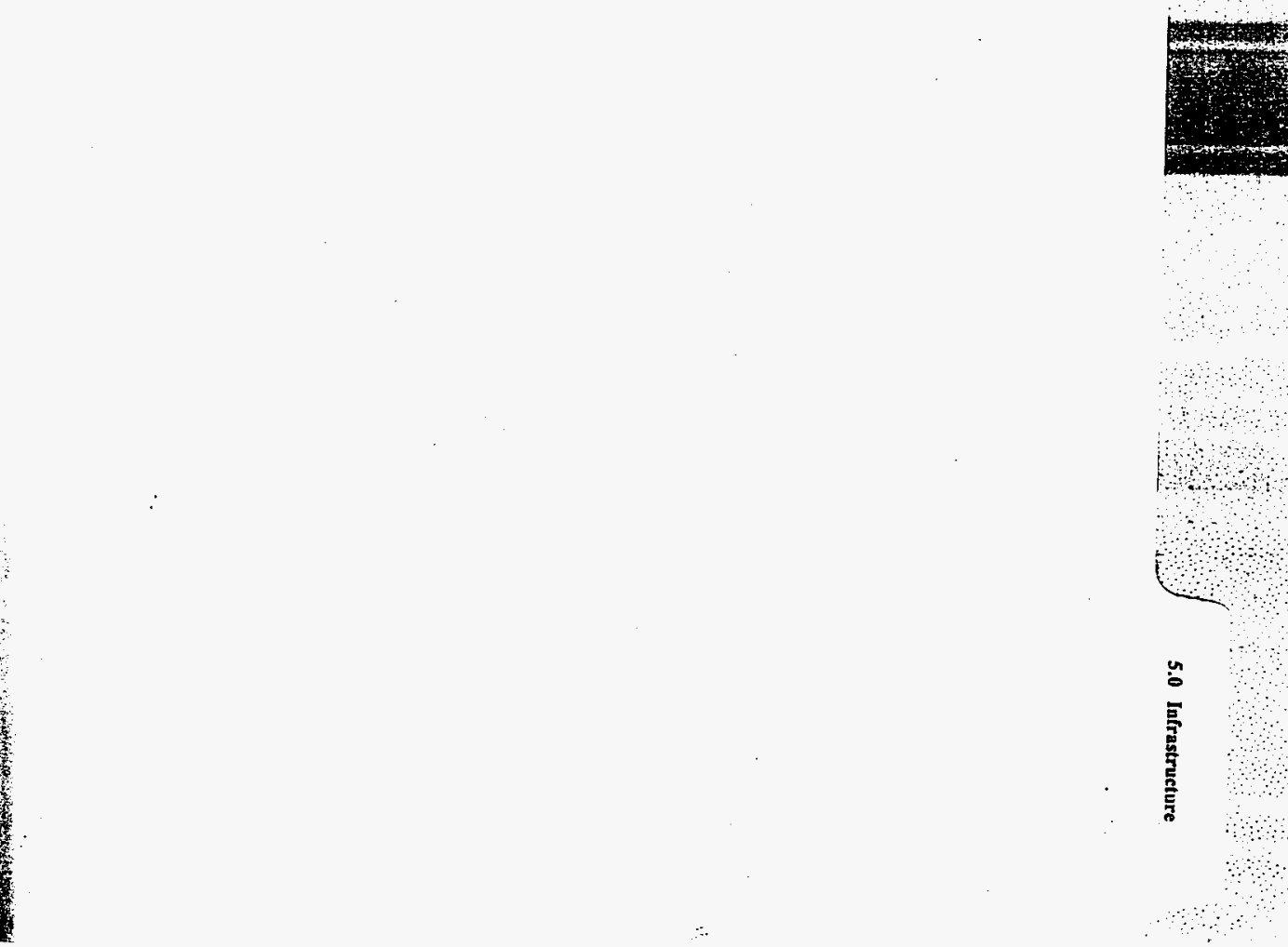
grt 


\subsection{INFRASTRUCTURE}

\subsection{INFRASTRUCTURE OPERATIONS AND MAINTENANCE}

\subsubsection{Facility Acquisition, Operations, and Maintenance}

The Maintenance Services organization provides expertise and funding for acquisition, management, disposal, and reporting of 25 commercial and intercontractor lease space. Provide oversite of major modifications to leased facilities. Serve as RL contact point for General Services Administration (GSA) leased facilities and site outleasing activities. Property/space and maintenance management to 378 government facilities and 25 commercial lease facilities are also provided. Facilities and Maintenance management manages the Occupancy Pool budget and maintenance activities in general purpose facilities in addition to programmatic support. Maintenance Services organization also provides landlord related maintenance (corrective, preventive, and predictive) for approximately 378 general purpose facilities (GPF), such as office buildings, mobile offices, fabrication shops, and warehouses. As of January 1996, this included 1.4 million square feet of useable floor space. Additionally, maintenance provides support to programmatic facilities, including PFP, PUREX, T and B Plants, Tank Farms, Solid Waste Disposal, Spent Nuclear Fuels, Transportation, Analytical Labs, FMEF, PNNL, Central Stores, Hanford Fire Department (HFD), Steam \& Water Utilities (Powerhouse), HAMMER Training Facility, and TEDF. Maintenance Services provides custodial services and management direction for approximately 222 general purpose facilities in the 100,200,300, 400, and 600 Areas. Included is 145 Programmatic facilities.

Assumptions

- Space utilization during transition will follow the hierarchy of occupying government-owned space first, followed by government-leased space and finally commercially-leased space.

- Current site moves workload is at capacity (approximately 500 moves per month) and requires a two-week advance notice. Significant additional moves, if required by transition, will exceed current workforce capabilities.

- Funding for upgrades and maintenance to the infrastructure system will be maintained to ensure services, economic transition, and diversification will succeed.

- Continued efforts using a graded approach in the development and implementation of commercial standards will continue. 
- The current Computerized Maintenance Management System will continue to be the methodology to identify, plan/schedule, and document performance measures for maintenance work.

- There will be potential HAMTC issues related to outsourcing which will have to be managed by the current $M \& O$ staff.

\begin{tabular}{|c|c|c|}
\hline Pre-Award Actions & $\begin{array}{l}\text { Scheduled } \\
\text { Date }\end{array}$ & Actionee(s) \\
\hline $\begin{array}{l}\text { 1. Identify number of briefings, and prepare briefing(s) } \\
\text { for the PHMC on facility acquisition, occupancy, } \\
\text { maintenance, and custodial. (Provide for RL POC } \\
\text { review) }\end{array}$ & $\begin{array}{l}5 / 15 / 96 \\
\text { Completed }\end{array}$ & JG Holyoak \\
\hline $\begin{array}{l}\text { 2. Identify key documents for facility acquisition, } \\
\text { occupancy, maintenance, and custodial. (Provide for } \\
\text { RL POC review) }\end{array}$ & $\begin{array}{c}5 / 01 / 96 \\
\text { Completed }\end{array}$ & JG Holyoak \\
\hline $\begin{array}{l}\text { 3. Assemble key documentation, or identify its location, } \\
\text { for use by the PHMC. Includes preparation of a } \\
\text { "Smart Book," to include information develop in the } \\
\text { following actions. }\end{array}$ & $\begin{array}{l}\text { 6/01/96 } \\
\text { Completed }\end{array}$ & JG Holyoak \\
\hline $\begin{array}{l}\text { 4. Develop a listing and description of subcontracts. } \\
\text { Include any subcontracts planned to be executed prior } \\
\text { to contract takeover. }\end{array}$ & $\begin{array}{l}5 / 15 / 96 \\
\text { Completed }\end{array}$ & JG Holyoak \\
\hline $\begin{array}{l}\text { 5. Using the pre-existing checklist, compile pre-existing } \\
\text { condition information on facilities and/or major } \\
\text { equipment. Conduct, or schedule as a separate post- } \\
\text { award action(s), any assessments required to } \\
\text { adequately address all relevant items on the checklist. } \\
\text { Provide completed checklist for RL POC review. }\end{array}$ & $\begin{array}{l}\text { 6/15/96 } \\
\text { Completed }\end{array}$ & JG Holyoak \\
\hline $\begin{array}{l}\text { 6. Develop a list of open action items and commitments, } \\
\text { including status. (Environmental, Tri-Party } \\
\text { Agreement, DNFSB as examples) }\end{array}$ & $\begin{array}{c}6 / 01 / 96 \\
\text { Completed }\end{array}$ & JG Holyoak \\
\hline $\begin{array}{l}\text { 7. Develop a list of facility (or operation)-specific } \\
\text { mandatory training and qualification requirements for } \\
\text { employees. }\end{array}$ & $\begin{array}{l}6 / 01 / 96 \\
\text { Completed }\end{array}$ & JG Holyoak \\
\hline $\begin{array}{l}\text { 8. Develop a listing of special agreement services } \\
\text { provided to RL, other DOE sites, and other entities. }\end{array}$ & $\begin{array}{c}6 / 01 / 96 \\
\text { Completed } \\
\end{array}$ & JG Holyoak \\
\hline $\begin{array}{l}\text { 9. Develop a listing of POCs to be available for the } \\
\text { transition phase. }\end{array}$ & $\begin{array}{c}5 / 15 / 96 \\
\text { Compieted }\end{array}$ & JG Holyoak \\
\hline 10. Develop a listing and description of databases. & $\begin{array}{l}5 / 15 / 96 \\
\text { Completed }\end{array}$ & JG Holyoak \\
\hline $\begin{array}{l}\text { 11. Develop a list of current work order services or other } \\
\text { form of agreement to provide work for other } \\
\text { organizations, on and offsite. }\end{array}$ & $\begin{array}{l}\text { 6/01/96 } \\
\text { Completed }\end{array}$ & JG Holyoak \\
\hline $\begin{array}{l}\text { 12. Develop a list of current work order services or other } \\
\text { form of agreement to obtain work from other } \\
\text { organizations, on and offsite. }\end{array}$ & $\begin{array}{l}6 / 01 / 96 \\
\text { Completed }\end{array}$ & JG Holyoak \\
\hline
\end{tabular}




\begin{tabular}{|c|c|c|}
\hline Pre-Award Actions & $\begin{array}{l}\text { Scheduled } \\
\text { Date }\end{array}$ & Actionee(s) \\
\hline $\begin{array}{l}\text { 13. Develop a list of permits governing facilities or } \\
\text { operation, and provide a description of each as to } \\
\text { where and how it applies. }\end{array}$ & $\begin{array}{l}6 / 01 / 96 \\
\text { Completed }\end{array}$ & JG Holyoak \\
\hline $\begin{array}{l}\text { 14. Verify the accuracy of property assignments and } \\
\text { correct any deficiencies. }\end{array}$ & $\begin{array}{l}6 / 01 / 96 \\
\text { Completed }\end{array}$ & JG Holyoak \\
\hline 15. Update/submit "Smart Book"/assemble key documents. & $7 / 15 / 96$ & JG Holyoak \\
\hline $\begin{array}{l}\text { 16. Verify pre-existing condition data is current and } \\
\text { supplement as necessary. }\end{array}$ & $7 / 22 / 96$ & JG Holyoak \\
\hline $\begin{array}{l}\text { 17. Ensure building responsibility assignments are correct } \\
\text { in RLPS. }\end{array}$ & $7 / 22 / 96$ & JG Holyoak \\
\hline Post-Award Actions & & \\
\hline 1. Provide briefing(s) to the PHMC. & $8 / 12 / 96-8 / 30 / 96$ & JG Holyoak \\
\hline 2. Provide tour(s) to the PHMC. & $8 / 12 / 96-8 / 30 / 96$ & JG Holyoak \\
\hline $\begin{array}{l}\text { 3. Provide the PHMC access to key documentation, pre- } \\
\text { existing condition information, commitment data, } \\
\text { inventory data, etc. (as established in the pre-award } \\
\text { phase). Provide copies upon request. }\end{array}$ & $8 / 05 / 96$ & JG Holyoak \\
\hline $\begin{array}{l}\text { 4. Provide any additional documentation or information } \\
\text { requested by the } P H M C \text {, if available, that was not } \\
\text { identified during the pre-award phase. }\end{array}$ & by $8 / 30 / 96$ & JG Holyoak \\
\hline $\begin{array}{l}\text { 5. PHMC identify any recurring reports that they would } \\
\text { like to receive during transition. }\end{array}$ & $8 / 19 / 96$ & JG Holyoak \\
\hline 6. Provide necessary training to PHMC personnel. & $8 / 12 / 96-8 / 30 / 96$ & JG Holyoak \\
\hline $\begin{array}{l}\text { 7. Provide an updated stanus of ongoing work prior to } \\
\text { contract turnover. }\end{array}$ & by $9 / 30 / 96$ & JG Holyoak \\
\hline $\begin{array}{l}\text { 8. Perform a joint property and equipment inventory with } \\
\text { the PHMC. }\end{array}$ & by $9 / 16 / 96$ & JG Holyoak \\
\hline $\begin{array}{l}\text { 9. Provide a categorized inventory of wastes or hazardous } \\
\text { materials. }\end{array}$ & by $9 / 30 / 96$ & JG Holyoak \\
\hline $\begin{array}{l}\text { 10. Assist the PHMC in the conduct of pre-existing } \\
\text { condition assessments. }\end{array}$ & $8 / 12 / 96-9 / 16 / 96$ & JG Holyoak \\
\hline
\end{tabular}

\subsubsection{Utilities}

5.1.2.1 Steam Plant Operations. Utilities operates three steam generating plants; 300 Powerhouse, 200 East Powerhouse and the 200 West Package Boiler, and the steam distribution systems. Presently, there is an ESPC proposal to find and institute alternate energy sources, which allows for the decommissioning of the steam plants. The central steam generating plans are scheduled to be shut down in the second and third quarters of FY 1997, dependent upon ESPC schedule and implementation. 


\begin{tabular}{|c|c|c|}
\hline Pre-Award Actions & $\begin{array}{l}\text { Scheduled } \\
\text { Date }\end{array}$ & Actionee(s) \\
\hline 1. Identify steam plant transition team. & $\begin{array}{c}5 / 01 / 96 \\
\text { Completed }\end{array}$ & $\begin{array}{l}\text { HL Debban } \\
\text { WA Retterer }\end{array}$ \\
\hline $\begin{array}{l}\text { 2. Identify number of briefings, and prepare briefing(s) } \\
\text { for the PHMC. }\end{array}$ & $7 / 15 / 96$ & $\begin{array}{l}\text { HL. Debban } \\
\text { WA Retterer }\end{array}$ \\
\hline $\begin{array}{l}\text { 3. Identify key documents for the facility/operations. } \\
\text { Assemble or identify their location for the PHMC. }\end{array}$ & $\begin{array}{c}\text { 6/01/96 } \\
\text { Completed } \\
\end{array}$ & $\begin{array}{l}\text { HL Debban } \\
\text { DS Gleason }\end{array}$ \\
\hline $\begin{array}{l}\text { 4. Develop a listing and description of subcontracts and } \\
\text { other supporting activity organizations. Include any } \\
\text { subcontracts planned to be executed prior to contract } \\
\text { takeover. }\end{array}$ & $\begin{array}{l}\text { 6/01/96 } \\
\text { Completed }\end{array}$ & $\begin{array}{l}\text { HL Debban } \\
\text { DS Gleason }\end{array}$ \\
\hline $\begin{array}{l}\text { Using the pre-existing checklist, compile pre-existing } \\
\text { condition information on facilities and/or major } \\
\text { equipment. Conduct, or schedule as a separate post- } \\
\text { award action(s), any assessments required to } \\
\text { adequately address all relevant items on the checklist. } \\
\text { Provide completed checklist for RL POC for review. } \\
\text { Include a list of open-ended compliance issues; } \\
\text { HATS, SATS, shutdown plans, ESPC, etc. }\end{array}$ & $\begin{array}{c}\text { 6/15/96 } \\
\text { Completed }\end{array}$ & $\begin{array}{l}\text { HL Debban } \\
\text { DS Gleason }\end{array}$ \\
\hline 6. Develop a listing of POCs for operations/ facilities. & $\begin{array}{c}5 / 01 / 96 \\
\text { Compieted }\end{array}$ & $\begin{array}{l}\text { HL Debban } \\
\text { WA Retterer }\end{array}$ \\
\hline $\begin{array}{l}\text { 7. Verify "Smart Book" to include any upcates and } \\
\text { submit. }\end{array}$ & $7 / 15 / 96$ & DS Gleason \\
\hline $\begin{array}{l}\text { 8. Verify PEC package, modify/supplement as } \\
\text { necessary. }\end{array}$ & $7 / 22 / 96$ & DS Gleason \\
\hline $\begin{array}{l}\text { 9. Ensure building assignnents responsibility are correct } \\
\text { in RLPS. }\end{array}$ & $7 / 22 / 96$ & DS Gleason \\
\hline Post-Award Actions & & $\because$ \\
\hline 1. Provide facility briefing(s) to the PHMC. & $8 / 12 / 96-8 / 30 / 96$ & $\begin{array}{l}\text { SL Camp, Jr. } \\
\text { JL Day }\end{array}$ \\
\hline 2. Provide facility tour(s) to the PHMC. & $8 / 12 / 96-8 / 30 / 96$ & $\begin{array}{l}\text { SL Camp, Jr. } \\
\text { JL Day }\end{array}$ \\
\hline $\begin{array}{l}\text { 3. Provide the PHMC access to key documentation, pre- } \\
\text { existing condition information, commitment data, } \\
\text { inventory data, etc. (as established in the pre-award } \\
\text { phase). Provide copies upon request. }\end{array}$ & $8 / 05 / 96$ & $\begin{array}{l}\text { SL Camp, Jr. } \\
\text { JL Day }\end{array}$ \\
\hline $\begin{array}{l}\text { 4. Provide additional documentation as requested to } \\
\text { PHMC. }\end{array}$ & by $8 / 30 / 96$ & DS Gleason \\
\hline $\begin{array}{l}\text { 5. Provide PHMC with events as they occur, in a timely } \\
\text { manner. }\end{array}$ & by $9 / 30 / 96$ & HL Debban \\
\hline 6. Provide necessary training to PHMC personnel. & $8 / 12 / 96-8 / 30 / 96$ & RR Grantham \\
\hline $\begin{array}{l}\text { 7. Provide an updated status of ongoing work prior to } \\
\text { contract nurnover. }\end{array}$ & by $9 / 30 / 96$ & $\begin{array}{l}\text { SL Camp, Jr. } \\
\text { JL Day }\end{array}$ \\
\hline
\end{tabular}




\begin{tabular}{|c|c|c|}
\hline \multicolumn{1}{|c|}{ Post-Award Actions } & $\begin{array}{c}\text { Scheduled } \\
\text { Date }\end{array}$ & Actionee(s) \\
\hline 8. $\begin{array}{l}\text { Perform a joint property and equipment inventory } \\
\text { with the PHMC. }\end{array}$ & by $9 / 16 / 96$ & DR Herman \\
\hline $\begin{array}{l}\text { 9. } \\
\begin{array}{l}\text { Provide a categorized inventory of wastes or } \\
\text { hazardous materials. }\end{array}\end{array}$ & by $9 / 30 / 96$ & DR Herman \\
\hline 10. $\begin{array}{l}\text { Assist the PHMC in the conduct of pre-existing } \\
\text { condition assessments. }\end{array}$ & $8 / 12 / 96-9 / 16 / 96$ & DR Herman \\
\hline
\end{tabular}

\subsubsection{300 Area Water/Sewer Operations. The 300 Area Utilities organization produces} potable water for distribution to facilities in the 300 Area. The organization also maintains and operates the sanitary sewer collection and treatment system. In the fall of 1996 the sanitary sewer will be re-routed to the City of Richland for treatment; 300 Area Operations will retain responsibility for the collection and pumping of the sanitary sewer.

\begin{tabular}{|c|c|c|}
\hline Pre-Award Actions & $\begin{array}{c}\text { Scheduled } \\
\text { Date }\end{array}$ & Actionee(s) \\
\hline 1. Identify 300 area water/sewer transition team. & $\begin{array}{l}5 / 01 / 96 \\
\text { Completed }\end{array}$ & $\begin{array}{l}\text { HL Debban } \\
\text { WA Retterer }\end{array}$ \\
\hline $\begin{array}{l}\text { 2.. Identify number of briefines, and prepare briefirg(s) } \\
\text { for the PHMC. }\end{array}$ & $7 / 15 / 96$ & $\begin{array}{l}\text { ELL Debban } \\
\text { WA Retterer }\end{array}$ \\
\hline $\begin{array}{l}\text { 3. Identify key documents for the facility/operations. } \\
\text { Assemble or identify their location for the PHMC. }\end{array}$ & $\begin{array}{c}\text { 6/01/96 } \\
\text { Completed }\end{array}$ & $\begin{array}{l}\text { HL Debban } \\
\text { DS Gleason }\end{array}$ \\
\hline $\begin{array}{l}\text { 4. Develop a listing and description of subcontracts and } \\
\text { other supporting activity organizations. Include any } \\
\text { subcontracts planned to be executed prior to contract } \\
\text { takeover. }\end{array}$ & $\begin{array}{l}\text { 6/01/96 } \\
\text { Completed }\end{array}$ & $\begin{array}{l}\text { HL. Debban } \\
\text { DS Gleason }\end{array}$ \\
\hline $\begin{array}{l}\text { 5. Using the pre-existing checklist, compile pre-existing } \\
\text { condition information on facilities and/or major } \\
\text { equipment. Conduct, or schedule as a separate post- } \\
\text { award action(s), any assessments required to } \\
\text { adequately address all relevant items on the checktist. } \\
\text { Provide completed checklist for RL, POC for review. } \\
\text { Include a list of open-ended compliance issues: HATS, } \\
\text { SATS, shutdown plans, ESPC, etc. }\end{array}$ & $\begin{array}{l}6 / 15 / 96 \\
\text { Completed }\end{array}$ & $\begin{array}{l}\text { HL Debban } \\
\text { DS Gleason }\end{array}$ \\
\hline 6. - Develop a listing of POCs for operations/ facilities. & $\begin{array}{c}5 / 01 / 96 \\
\text { Completed } \\
\end{array}$ & $\begin{array}{l}\text { HL Debban } \\
\text { WA Retterer }\end{array}$ \\
\hline $\begin{array}{l}\text { 7. Verify "Smart Book" to include any updates and } \\
\text { submit. }\end{array}$ & $7 / 15 / 96$ & DS Gleason \\
\hline 8. Verify PEC package, modify/supplement as necessary. & $7 / 22 / 96$ & DS Gleason \\
\hline $\begin{array}{l}\text { 9. Ensure building assignments responsibility are correct } \\
\text { in RLPS. }\end{array}$ & $\begin{array}{c}7 / 22 / 96 \\
. \\
\end{array}$ & DS Gleason \\
\hline Post-Award Actions & & \\
\hline 1. Provide facility briefing(s) to the PHMC. & $8 / 12 / 96-8 / 30 / 96$ & SL Camp, Jr. \\
\hline
\end{tabular}




\begin{tabular}{|c|c|c|}
\hline Post-Award Actions & $\begin{array}{l}\text { Scheduled } \\
\text { Date }\end{array}$ & Actionee(s) \\
\hline 2. Provide facility tour(s) to the PHMC. & $8 / 12 / 96-8 / 30 / 96$ & SL Camp, Jr. \\
\hline $\begin{array}{l}\text { 3. Provide the PHMC access to key documentation, pre- } \\
\text { existing condition information, commitment data, } \\
\text { inventory data, etc. (as established in the pre-award } \\
\text { phase). Provide copies upon request. }\end{array}$ & $8 / 05 / 96$ & SL Camp, Ir. \\
\hline $\begin{array}{l}\text { 4. Provide additional documentation as requested to } \\
\text { PHMC. }\end{array}$ & by $9 / 30 / 96$ & DS Gleason \\
\hline $\begin{array}{l}\text { 5. Provide PHMC with events as they occur, in a timely } \\
\text { manner. }\end{array}$ & by $9 / 30 / 96$ & HL Debban \\
\hline 6. Provide necessary training to PHMC personnel. & $8 / 12 / 96-8 / 30 / 96$ & RR Grantham \\
\hline $\begin{array}{l}\text { 7. Provide an updated stanus of ongoing work prior to } \\
\text { contract turnover. }\end{array}$ & by $9 / 30 / 96$ & SL Camp, Jr. \\
\hline $\begin{array}{l}\text { 8. Perform a joint property and equipment inventory with } \\
\text { the PHMC. }\end{array}$ & by $9 / 16 / 96$ & DR Herman \\
\hline $\begin{array}{l}\text { 9. Provide a categorized inventory of wastes or hazardous } \\
\text { materials. }\end{array}$ & by $9 / 30 / 96$ & DR Herman \\
\hline $\begin{array}{l}\text { 10. Assist the PHMC in the conduct of pre-existing } \\
\text { condition assessments. }\end{array}$ & $8 / 12 / 96-9 / 16 / 96$ & DR Herman \\
\hline
\end{tabular}

\subsubsection{200 Area Water/Sewer Operations. The 200 Area Utilities organization maintains} and operates the export water system, the 200 East water treatment plant, the 200 West water treatment plant and associated distribution systems. This organization also performs septic system pumping, maintenance and reporting.

\begin{tabular}{|c|c|c|}
\hline Pre-Award Actions & $\begin{array}{l}\text { Scheduled } \\
\text { Date }\end{array}$ & Actionee(s) \\
\hline 1. Identify 200 area water/sewer transition team. & $\begin{array}{c}5 / 01 / 96 \\
\text { Completed }\end{array}$ & $\begin{array}{l}\text { HL Debban } \\
\text { WA Retterer }\end{array}$ \\
\hline $\begin{array}{l}\text { 2. Identify number of briefings, and prepare briefing(s) } \\
\text { for the PHMC. }\end{array}$ & $7 / 15 / 96$ & $\begin{array}{l}\text { HL Debban } \\
\text { WA Retterer }\end{array}$ \\
\hline $\begin{array}{l}\text { 3. Identify key documents for the facility/operations. } \\
\text { Assemble or identify their location for the PHMC. }\end{array}$ & $\begin{array}{l}6 / 01 / 96 \\
\text { Completed }\end{array}$ & $\begin{array}{l}\text { HL Debban } \\
\text { DS Gleason }\end{array}$ \\
\hline $\begin{array}{l}\text { 4. Develop a listing and description of subcontracts and } \\
\text { other supporting activity organizations. Include any } \\
\text { subcontracts planned to be executed prior to contract } \\
\text { takeover. }\end{array}$ & $\begin{array}{l}\text { 6/01/96 } \\
\text { Completed }\end{array}$ & $\begin{array}{l}\text { HL Debban } \\
\text { DS Gleason }\end{array}$ \\
\hline $\begin{array}{l}\text { 5. Using the pre-existing checklist, compile pre-existing } \\
\text { condition information on facilities and/or major } \\
\text { equipment. Conduct, or schedule as a separate post- } \\
\text { award action(s), any assessments required to } \\
\text { adequately address all relevant items on the checklist. } \\
\text { Provide completed checklist for RL POC for review. } \\
\text { Include a list of open-ended compliance issues: HATS, } \\
\text { SATS, shutdown plans, ESPC, etc. }\end{array}$ & $\begin{array}{l}6 / 15 / 96 \\
\text { Completed }\end{array}$ & $\begin{array}{l}\text { HL Debban } \\
\text { DS Gleason }\end{array}$ \\
\hline
\end{tabular}




\begin{tabular}{|c|c|c|}
\hline sis & $\begin{array}{l}\text { Scheduled } \\
\text { Date }\end{array}$ & Actionee(s) \\
\hline 6. Develop a listing of POCs for operations/ facilities. & $\begin{array}{c}5 / 01 / 96 \\
\text { Completed }\end{array}$ & $\begin{array}{l}\text { HL Debban } \\
\text { WA Retterer }\end{array}$ \\
\hline $\begin{array}{l}\text { 7. Verify "Smart Book" to include any updates and } \\
\text { submit. }\end{array}$ & $7 / 15 / 96$ & DS Gleason \\
\hline 8. Verify PEC package, modify/supplement as necessary. & $7 / 22 / 96$ & DS Gleason \\
\hline $\begin{array}{l}\text { 9. Ensure building assignments responsibility are correct } \\
\text { in RLPS. }\end{array}$ & $7 / 22 / 96$ & DS Gleason \\
\hline Post-Award Actions & & \\
\hline 1. Provide facility briefing(s) to the PHMC. & $8 / 12 / 96-8 / 30 / 96$ & IL Day \\
\hline 2. Provide facility tour(s) to the PHMC. & $8 / 12 / 96-8 / 30 / 96$ & JL Day \\
\hline $\begin{array}{l}\text { 3. Provide the PHMC access to key documentation, pre- } \\
\text { existing condition information, commitment data, } \\
\text { inventory data, etc. (as established in the pre-award } \\
\text { phase) Provide copies upon request. }\end{array}$ & $8 / 05 / 96$ & JL Day \\
\hline $\begin{array}{l}\text { 4. Provide additional documentation as requested to } \\
\text { PHMC. }\end{array}$ & by $8 / 30 / 96$ & DS Gleason \\
\hline $\begin{array}{l}\text { 5. Provide PHMC with events as they occur, in a timely } \\
\text { manner. }\end{array}$ & by $9 / 30 / 96$ & HL Debban \\
\hline 6. Provide necessary training to PHMC personnel. & $8 / 12 / 96-8 / 30 / 96$ & RR Grantham \\
\hline $\begin{array}{l}\text { 7. Provide an updated status of ongoing work prior to } \\
\text { contract turnover. }\end{array}$ & by $9 / 30 / 96$ & JL Day \\
\hline $\begin{array}{l}\text { 8. Perform a joint property and equipment inventory with } \\
\text { the PHMC. }\end{array}$ & by $9 / 16 / 96$ & DR Herman \\
\hline $\begin{array}{l}\text { 9. Provide a categorized inventory of wastes or hazardous } \\
\text { materials. }\end{array}$ & by $9 / 30 / 96$ & DR Herman \\
\hline $\begin{array}{l}\text { 10. Assist the PHMC in the conduct of pre existing } \\
\text { condition assessments. }\end{array}$ & $8 / 12 / 96-9 / 16 / 96$ & DR Herman \\
\hline
\end{tabular}

5.1.2.4 Site Electrical Systems. Electrical Utilities (EU) is responsible for the operation, maintenance, engineering, and configuration management of the Hanford Site Electrical Transmission and Distribution systems to the connection point at the site facilities, in accordance with applicable RL/ICF KH policies and requirements, and federal/state regulations.

\begin{tabular}{|c|c|c|}
\hline Pre-Award Actions & $\begin{array}{l}\text { Scheduled } \\
\text { Date }\end{array}$ & Actionee(s) \\
\hline 1. Identify electrical transition team. & $\begin{array}{l}5 / 01 / 96 \\
\text { Completed }\end{array}$ & $\begin{array}{l}\text { HL Debban } \\
\text { WA Retterer }\end{array}$ \\
\hline $\begin{array}{l}\text { 2. Identify number of briefings, and prepare briefing(s) } \\
\text { for the PHMC. }\end{array}$ & $7 / 15 / 96$ & $\begin{array}{l}\text { HL Debban } \\
\text { WA Retterer }\end{array}$ \\
\hline
\end{tabular}




\begin{tabular}{|c|c|c|}
\hline $\begin{array}{l}\text { Pre-Award Actions } \\
\end{array}$ & $\begin{array}{l}\text { Scheduled } \\
\text { Date } \\
\end{array}$ & Actionee(s) \\
\hline $\begin{array}{l}\text { 3. Identify key documents for the facility/operations. } \\
\text { Assemble or identify their location for the PHMC. }\end{array}$ & $\begin{array}{c}6 / 01 / 96 \\
\text { Completed } \\
\end{array}$ & $\begin{array}{l}\text { HL Debban } \\
\text { DS Gleason } \\
\end{array}$ \\
\hline $\begin{array}{l}\text { 4. Develop a listing and description of subcontracts and } \\
\text { other supporting activity organizations. Include any } \\
\text { subcontracts planned to be executed prior to contract } \\
\text { takeover. }\end{array}$ & $\begin{array}{c}\text { 6/01/96 } \\
\text { Completed }\end{array}$ & $\begin{array}{l}\text { HL Debban } \\
\text { DS Gleason }\end{array}$ \\
\hline $\begin{array}{l}\text { 5. Using the pre-existing checklist, compile pre-existing } \\
\text { condition information on facilities and/or major } \\
\text { equipment. Conduct, or schedule as a separate post- } \\
\text { award action(s), any assessments required to adequately } \\
\text { address all relevant items on the checklist. Provide } \\
\text { completed checklist for RL POC for review. Include a } \\
\text { list of open-ended compliance issues: HATS, SATS, } \\
\text { shutdown plans, ESPC, etc. }\end{array}$ & $\begin{array}{c}6 / 15 / 96 \\
\text { Completed }\end{array}$ & $\begin{array}{l}\text { HL Debban } \\
\text { DS Gleason }\end{array}$ \\
\hline 6. Develop a listing of POCs for operations/facilities. & $\begin{array}{c}5 / 01 / 96 \\
\text { Completed }\end{array}$ & $\begin{array}{l}\text { HL Debban } \\
\text { WA Retterer }\end{array}$ \\
\hline $\begin{array}{l}\text { 7. Verify "Smart Book" to include any updates and } \\
\text { submit. }\end{array}$ & $7 / 15 / 96$ & DS Gleason \\
\hline 8. Verify PEC package, modify/supplement as necessary. & $7 / 22 / 96$ & DS Gleason \\
\hline $\begin{array}{l}\text { 9. Ensure building assignments responsibility are correct } \\
\text { in RLPS. }\end{array}$ & $7 / 22 / 96$ & DS Gleason \\
\hline \multicolumn{3}{|l|}{ Post-Award Actions } \\
\hline 1. Provide facility briefing(s) to the PHMC. & $8 / 12 / 96-8 / 30 / 96$ & RL Fritz \\
\hline 2. Provide facility tour(s) to the PHMC. & $8 / 12 / 96-8 / 30 / 96$ & RL Fritz \\
\hline $\begin{array}{l}\text { 3. Provide the PHMC access to key documentation, pre- } \\
\text { existing condition information, conmitment data, } \\
\text { inventory cata, etc. (as established in the pre-award } \\
\text { phase). Provide copies upon request. }\end{array}$ & $8 / 05 / 96$ & RL Fritz \\
\hline $\begin{array}{l}\text { 4. Provide additional documentation as requested to } \\
\text { PHMC. }\end{array}$ & by $8 / 30 / 96$ & DS Gleason \\
\hline $\begin{array}{l}\text { 5. Provide PHMC with events as they occur, in a timely } \\
\text { manner. }\end{array}$ & by $9 / 30 / 96$ & HL Debban \\
\hline 6. Provide necessary training to PHMC personnel. & $8 / 12 / 96-8 / 30 / 96$ & RR Grantham \\
\hline $\begin{array}{l}\text { 7. Provide an updated status of ongoing work prior to } \\
\text { contract turnover. }\end{array}$ & by $9 / 30 / 96$ & RL Fritz \\
\hline $\begin{array}{l}\text { 8. Perform a joint property and equipment inventory with } \\
\text { the PHMC. }\end{array}$ & by $9 / 16 / 96$ & DR Herman \\
\hline $\begin{array}{l}\text { 9. Provide a categorized inventory of wastes or hazardous } \\
\text { materials. }\end{array}$ & by $9 / 30 / 96$ & DR Herman \\
\hline $\begin{array}{l}\text { 10. Assist the PHMC in the conduct of pre-existing } \\
\text { condition assessments. }\end{array}$ & $8 / 12 / 96-9 / 16 / 96$ & DR Herman \\
\hline
\end{tabular}




\subsubsection{Transportation Infrastructure and Services}

Manage Hanford Sitewide fleet, including inventory, procurement, and costing for Hanford Site contractors. Interface with GSA, RL, and contractors on GSA-provided fleet issues. Provide preventive maintenance and repair services for Hanford Site vehicle and construction equipment. Services include fueling, lubrication, and field and shop maintenance. Provide personnel and material transportation services for the Hanford Site. Modes used include rail, truck, bus, and light vehicle. Transportation support functions include road and rail system maintenance, snow removal, and fire support.

Assumptions:

- MOU between RL and GSA will remain in effect.

- GSA will use Fleet Maintenance for preventive maintenance (PM) and repair.

- BHI will continue to use Fleet Maintenance for PM and repair work via work order.

- Fleet Maintenance personnel will not be consolidated into one facility prior to October 1996.

- Currently planned consolidation of transportation functions will occur.

\begin{tabular}{|l|c|l|}
\hline \multicolumn{1}{|c|}{ Pre-Award Actions } & $\begin{array}{c}\text { Scheduled } \\
\text { Date }\end{array}$ & Actionee(s) \\
\hline $\begin{array}{l}\text { 1. Identify number of briefings, and prepare briefing(s) } \\
\text { on transportation management, maintenance, and } \\
\text { operations for the PHMC. (Provide for RL POC } \\
\text { review.) }\end{array}$ & $\begin{array}{c}5 / 15 / 96 \\
\text { Completed }\end{array}$ & $\begin{array}{l}\text { AC Metz (Management) } \\
\text { JF Woods (Maintenance) } \\
\text { RG Ciccone (Operations) }\end{array}$ \\
\hline $\begin{array}{l}\text { 2. Identify key documents for the transportation } \\
\text { management, maintenance, and operations. (Provide } \\
\text { for RL POC review.) }\end{array}$ & $\begin{array}{c}5 / 01 / 96 \\
\text { Completed }\end{array}$ & $\begin{array}{l}\text { AC Metz } \\
\text { JF Woods } \\
\text { RG Ciccone }\end{array}$ \\
\hline $\begin{array}{l}\text { 3. Assemble key documentation, or identify its location, } \\
\text { for use by the PHMC. Includes preparation of a } \\
\text { "Smart Book," to include information develop in the } \\
\text { following actions. }\end{array}$ & $\begin{array}{c}\text { 6/01/96 } \\
\text { Completed } \\
\text { Smart Book" only } \\
\text { See item \#15 }\end{array}$ & $\begin{array}{l}\text { AC Metz } \\
\text { JF Woods } \\
\text { RG Ciccone }\end{array}$ \\
\hline $\begin{array}{l}\text { 4. Develop a listing and description of subcontracts. } \\
\text { Include any subcontracts planned to be executed prior } \\
\text { to contract takeover. }\end{array}$ & $\begin{array}{c}5 / 15 / 96 \\
\text { Completed }\end{array}$ & BA Holrnberg \\
\hline $\begin{array}{l}\text { 5. Using the pre-existing checklist, compile pre-existing } \\
\text { condition information on facilities and/or major } \\
\text { equipment. Conduct, or schedule as a separate post- } \\
\text { award action(s), any assessments required to adequately } \\
\text { address all relevant items on the checklist. (Provide } \\
\text { completed checklist for RL POC review.) }\end{array}$ & $\begin{array}{c}6 / 15 / 96 \\
\text { Completed }\end{array}$ & $\begin{array}{l}\text { BA Holmberg } \\
\text { AC Metz } \\
\text { JF Woods } \\
\text { RG Ciccone }\end{array}$ \\
\hline
\end{tabular}




\begin{tabular}{|c|c|c|}
\hline Pre-Award Actions & $\begin{array}{l}\text { Scheduled } \\
\text { Date }\end{array}$ & Actionee(s) \\
\hline $\begin{array}{l}\text { 6. Develop a list of open action items and commitments, } \\
\text { including status (Environmental, Tri-Party Agreement, } \\
\text { DNFSB as examples). }\end{array}$ & $\begin{array}{l}6 / 01 / 96 \\
\text { Completed }\end{array}$ & BA Holmberg \\
\hline $\begin{array}{l}\text { 7. Develop a list of facility (or operation)-specific } \\
\text { mandatory training and qualification requirements for } \\
\text { employees. }\end{array}$ & $\begin{array}{l}6 / 01 / 96 \\
\text { Completed }\end{array}$ & BA Holmberg \\
\hline $\begin{array}{l}\text { 8. Develop a listing of special agreement services } \\
\text { provided to RL, other DOE sites, and other entities. }\end{array}$ & $\begin{array}{c}6 / 01 / 96 \\
\text { Completed }\end{array}$ & BA Holmberg \\
\hline $\begin{array}{l}\text { 9. Develop a listing of POCs to be available for the } \\
\text { transition phase. }\end{array}$ & $\begin{array}{c}5 / 15 / 96 \\
\text { Completed }\end{array}$ & BA Holmberg \\
\hline 10. Develop a listing and description of databases. & $\begin{array}{c}5 / 15 / 96 \\
\text { Completed }\end{array}$ & BA Holmberg \\
\hline $\begin{array}{l}\text { 11. Develop a list of current work order services or other } \\
\text { form of agreement to provide work for other } \\
\text { organizations, on and offsite. }\end{array}$ & $\begin{array}{c}\text { 6/01/96 } \\
\text { Completed }\end{array}$ & BA Holmberg \\
\hline $\begin{array}{l}\text { 12. Develop a list of current work order services or other } \\
\text { form of agreement to obtain work from other } \\
\text { organizations, on and offsite. }\end{array}$ & $\begin{array}{c}6 / 01 / 96 \\
\text { Completed }\end{array}$ & BA Holmberg \\
\hline $\begin{array}{l}\text { 13. Develop a list of permits governing facilities or } \\
\text { operation, and provide a description of each as to } \\
\text { where and how it applies. }\end{array}$ & $\begin{array}{l}\text { 6/01/96 } \\
\text { Completed }\end{array}$ & BA Holmberg \\
\hline $\begin{array}{l}\text { 14. Verify the accuracy of property assignments and } \\
\text { correct any deficiencies. }\end{array}$ & $\begin{array}{c}6 / 01 / 96 \\
\text { Completed }\end{array}$ & BA Holmberg \\
\hline $\begin{array}{l}\text { 15. Verify "Smart Book" to include any updates and } \\
\text { submit. }\end{array}$ & $7 / 15 / 96$ & BA Holmberg \\
\hline 16. Verify PEC package, modify/supplement as necessary. & $7 / 22 / 96$ & BA Holmberg \\
\hline $\begin{array}{l}\text { 17. Ensure building responsibility assigmments are correct } \\
\text { in RLPS. }\end{array}$ & $7 / 22 / 96$ & BA Holmberg \\
\hline Post-Award Actions & & \\
\hline 1. Provide briefing(s) to the PHMC. & $8 / 12 / 96-8 / 30 / 96$ & $\begin{array}{l}\text { AC Metz (Management) } \\
\text { JF Woods (Maintenance) } \\
\text { RG Ciccone (Operations) }\end{array}$ \\
\hline 2. Provide tour(s) to the PHMC. & $8 / 12 / 96-8 / 30 / 96$ & $\begin{array}{l}\text { AC Metz } \\
\text { JF Woods } \\
\text { RG Ciccone }\end{array}$ \\
\hline $\begin{array}{l}\text { 3. Provide the PHMC access to key documentation, pre- } \\
\text { existing condition information, commitment data, } \\
\text { inventory data, etc. (as established in the pre-award } \\
\text { phase). Provide copies upon request. }\end{array}$ & $8 / 05 / 96$ & $\begin{array}{l}\text { AC Metz } \\
\text { JF Woods } \\
\text { RG Ciccone }\end{array}$ \\
\hline $\begin{array}{l}\text { 4. Provide any additional documentation or information } \\
\text { requested by the PHMC, if available, that was not } \\
\text { identified during the pre-award phase. }\end{array}$ & by $8 / 30 / 96$ & BA Holmberg \\
\hline
\end{tabular}




\begin{tabular}{|l|c|l|}
\hline \multicolumn{1}{|c|}{ Post-Award Actions } & $\begin{array}{c}\text { Scheduled } \\
\text { Date }\end{array}$ & \multicolumn{1}{|c|}{ Actionee(s) } \\
\hline $\begin{array}{l}\text { 5. PHMC identify any recurring reports that they would } \\
\text { like to receive during transition. }\end{array}$ & by $8 / 19 / 96$ & BA Holmberg \\
\hline 6. Provide necessary training to PHMC personnel. & $8 / 12 / 96-8 / 30 / 96$ & BA Holmberg \\
\hline $\begin{array}{l}\text { 7. Provide an updated status of ongoing work prior to } \\
\text { contract turnover. }\end{array}$ & by $9 / 30 / 96$ & BA Holmberg \\
\hline $\begin{array}{l}\text { 8. Perform a joint property and equipment inventory with } \\
\text { the PHMC. }\end{array}$ & by $9 / 16 / 96$ & BA Holmberg \\
\hline $\begin{array}{l}\text { 9. Provide a categorized inventory of wastes or hazardous } \\
\text { materials. }\end{array}$ & by $9 / 30 / 96$ & BA Holmberg \\
\hline $\begin{array}{l}\text { 10. Assist the PHMC in the conduct of pre-existing } \\
\text { condition assessments. }\end{array}$ & $8 / 12 / 96-9 / 16 / 96$ & BA Holmberg \\
\hline
\end{tabular}

\subsubsection{Fire and Emergency Response}

The HFD is a multi-disciplined, multi-functional organization which provides fire suppression, rescue, emergency medical and ambulance services, and hazardous material responses that are capable of containing, controlling, and terminating emergency situations

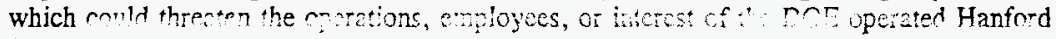
Site or the various companies and agencies operating on the Hanford Site. This includes emergency support to Bonneville Power Administration facilities, mutual aid response to surrounding fire districts under a Mutual Aid Agreement, and contractual firefighting and ambulance response to Washington Public Power Supply System (Supply System). The fire department's sitewide safety and fire prevention program includes fire alarm system functional testing and inspection, preventive and corrective maintenance, respiratory protection, building tours and inspections, prefire planning, and employee fire safety training.

5.1.4.1 Fire Department Emergency Services. The emergency services group operates out of four fire stations, strategically located on the Hanford Site to provide emergency support services and staffed with trained fire fighters and fire service officers. Personnel are typically cross-trained in several areas; a fire fighter may hold certifications as an Emergency Medical Technician (EMT), a paramedic, a hazardous material technician, a driver/pumper operator, an emergency dispatcher, a high angle/low angle and confined space rescue responder, etc. Similarly, fire service officers are trained in incident command and in various emergency response specialties. This cross training optimizes personnel utilization by assuring certified responders are available to cover a wide range of emergency situations with the lowest possible number of response staff. All emergency services are provided on a 24-hour-a-day, 7-day-a-week basis.

5.1.4.2 Fire Systems Testing and Emergency Response. The fire systems testing and emergency response group provide operational assurance testing of fire alarm, fire suppression, and fire water distribution systems in and around site building and facilities. 
Testing is performed to the frequencies required by NFPA codes and standards as implemented by DOE Orders and as modified by approved waivers and exemption requests.

5.1.4.3 Services Provided to Others. HFD services are being utilized to varying degrees by private companies doing business on the site and by other government agencies. Current recipients of these services include the Supply System complex, Kaiser Aluminum, Bonneville Power Administration 1Ashe Sub-Station, the Port of Benton 400 Area Incubator Project Facilities, and the Laser Interferometer Gravitational-Wave Observatory.

5.1.4.4 Other - New RL Work Scope Planned for the HFD. WHC has the lead (as designated by RL) for a Hanford Site Emergency Response Organization re-engineering project, which is a performanced-based RL milestone. This effort will provide a streamlined emergency response organization, while maintaining a strong response capability for the site. The HFD is providing key input and support to the re-engineering project, which when implemented will expand HFD responsibilities sitewide for incident management during emergencies.

5.1.4.5 Specialized Training for Emergency Response Fire Department Personnel. Except for the special classification of paramedic, all HFD personnel are qualified as Firefighter I before being allowed to respond to an emergency. Job specific training includes, but is not limited to, pumper operators, EMT; officer, hazardous materials, special operations, and fire cause \& origin. Both DOE and the Washington State mandates certain levels of competencies based on National Codes and Standards.

5.1.4.6 Fire Prevention. The Fire Marshal has the responsibility for fire prevention programs, public education, fire investigation, pre-design and pre-construction briefings, plans review, and special considerations for decommissioning and decontamination projects.

Annual programmatic activities include, but are not limited to, fire prevention, handson fire extinguisher training for site employees, Fire Marshal Permits (as listed in the NFPA Fire Prevention Code), final authority for pre-fire plans, developing and directing the inspection programs for flammable \& reactive waste sites, HFD building tours \& inspections, hydrant flow testing, and storage, use, and disposal of combustible, flammable, explosive or reactive material.

5.1.4.7 Fire Systems Maintenance. Fire Systems Maintenance (FSM) provides preventive, and repair maintenance of fire alarm, fire suppression, and fire water distribution systems in and around site buildings and facilities. Maintenance is performed to the frequencies required by National Fire Protection Association codes and standards as implemented by DOE orders, and as modified by approved waivers and current exemption requests.

5.1.4.8 Respiratory Protection Equipment Services. Self-contained breathing apparatus (SCBA), escape packs, Powered Air Purifying Respirator (PAPR) systems, regulator and cylinder maintenance, inspection, certified testing and servicing are provided for Hanford contractors who operate/use respiratory protection equipment. Services include the performance of inspections and tests on SCBA valves and low and high pressure breathing 
air cylinders, PAPR, and escape packs, and the refilling of approximately 4,400 SCBA cylinders per year.

\subsubsection{Contracts for Service and Purchase Requisitions that Extend Past}

October 1, 1996. The HFD has numerous contracts in place that either extend over more than one year or have renewal clauses. This is necessary to in order to avoid any delay in the receipt of equipment or services required to support emergency operations.

\subsubsection{Emergency Services Facilities, Buildings, and Capital Projects for}

Replacement. Many of the HFD facilities and buildings were built decades ago and are nearing the end of their useful life without major restoration and/or replacement. These facilities exist throughout the Hanford Site.

The following actions will be completed to ensure a complete and orderly turnover of HFD operations to the PHMC.

\begin{tabular}{|c|c|c|}
\hline Pre-Award Actions & $\begin{array}{l}\text { Scheduled } \\
\text { Date }\end{array}$ & Actionec(s) \\
\hline $\begin{array}{l}\text { 1. Develop a list of key HFD documentation. Provide to } \\
\text { RL POC for review. }\end{array}$ & $\begin{array}{c}5 / 15 / 96 \\
\text { Completed } \\
\end{array}$ & DE Good \\
\hline $\begin{array}{l}\text { 2. Assernble key HFD documentation, or identify its } \\
\text { location, for use by the PHMC. Incjudes devtlopment } \\
\text { of a HFD "Srnart Book," including listings and } \\
\text { descriptions developed below. }\end{array}$ & $\begin{array}{l}6 / 01 / 96 \\
\text { Completed } \\
\text { "Smart Book" only } \\
\text { See item } \# 22\end{array}$ & WR Hayes \\
\hline $\begin{array}{l}\text { 3. Identify number of briefings, and prepare HFD briefing } \\
\text { for presentation to PFMC. (Coordinate number and } \\
\text { content with RL POC.) }\end{array}$ & $7 / 15 / 96$ & DE Good \\
\hline $\begin{array}{l}\text { 4. Develop a listing of key HFD individuals as POCs, } \\
\text { including facility and operations personnel. }\end{array}$ & $\begin{array}{l}6 / 01 / 96 \\
\text { Completed }\end{array}$ & DE Good \\
\hline $\begin{array}{l}\text { 5. Deveiop listing and description of HFD subcontracts, } \\
\text { including facility (or operations) subcontracts. Include } \\
\text { any subcontracts still planned to be executed prior to } \\
\text { contract takeover. }\end{array}$ & $\begin{array}{l}\text { 6/01/96 } \\
\text { Completed }\end{array}$ & WR Hayes \\
\hline $\begin{array}{l}\text { 6. Develop listing and description of HFD databases, } \\
\text { including facility (or operations) databases. }\end{array}$ & $\begin{array}{l}6 / 01 / 96 \\
\text { Cornpleted }\end{array}$ & WR Hayes \\
\hline $\begin{array}{l}\text { 7. Verify the accuracy of property assignments at the HFD } \\
\text { organizational level and correct any deficiencies. }\end{array}$ & $\begin{array}{l}\text { 6/01/96 } \\
\text { Completed }\end{array}$ & WR Hayes \\
\hline $\begin{array}{l}\text { 8. Identify space and equipment within HFD facilities for } \\
\text { use by the PHMC. }\end{array}$ & $\begin{array}{l}\text { 6/01/96 } \\
\text { Completed }\end{array}$ & WR Hayes \\
\hline $\begin{array}{l}\text { 9. Using the pre-existing condition checklist, compite pre- } \\
\text { existing condition information on any facilities or major } \\
\text { equipment not addressed separately. Conduct, or } \\
\text { schedule as a separate post-closure action(s), any } \\
\text { assessments required to adequately address all relevant } \\
\text { items on the checklist. }\end{array}$ & $\begin{array}{l}\text { 6/15/96 } \\
\text { Completed }\end{array}$ & DE Good \\
\hline
\end{tabular}




\begin{tabular}{|c|c|c|}
\hline Pre-Award Actions & $\begin{array}{l}\text { Scheduled } \\
\text { Date }\end{array}$ & Actionee(s) \\
\hline $\begin{array}{l}\text { 10. Conduct an assessment (e.g., OSHA assessment) of } \\
\text { HFD facilities. (If there are any significant assessments } \\
\text { required for facilities or major equipment then include } \\
\text { them as separate actions). }\end{array}$ & $\begin{array}{l}6 / 15 / 96 \\
\text { Completed }\end{array}$ & DE Good \\
\hline $\begin{array}{l}\text { 11. In outline form for all facilities/operations to follow, } \\
\text { define the appropriate contents and level of detail to be } \\
\text { included in facility/operations briefings to the PHMC. } \\
\text { (Review with RL POC.) }\end{array}$ & $\begin{array}{l}5 / 01 / 96 \\
\text { Completed }\end{array}$ & DE Good \\
\hline $\begin{array}{l}\text { 12. Develop a list of open action items and commitments, } \\
\text { including stanus (Environmental, Tri-Party Agreernent, } \\
\text { DNFSB as examples). Provide to RL POC for review. }\end{array}$ & $\begin{array}{l}6 / 01 / 96 \\
\text { Completed }\end{array}$ & WR Hayes \\
\hline $\begin{array}{l}\text { 13. Identify current inventories of materials to support } \\
\text { continued operation (i.e., essential materials, tools and } \\
\text { equipment). }\end{array}$ & $\begin{array}{l}7 / 15 / 96 \\
\text { Completed }\end{array}$ & WR Hayes \\
\hline $\begin{array}{l}\text { 14. Develop a list of facility (or operation)-specific } \\
\text { mandatory training and qualification requirements for } \\
\text { employees. }\end{array}$ & $\begin{array}{l}6 / 01 / 96 \\
\text { Completed }\end{array}$ & WR Hayes \\
\hline $\begin{array}{l}\text { 15. Develop a listing of information which constitutes the } \\
\text { "authorization basis" for each facility. }\end{array}$ & $\begin{array}{c}6 / 01 / 96 \\
\text { Completed }\end{array}$ & WR Hayes \\
\hline $\begin{array}{l}\text { 16. Develop a description of ongoing and planned } \\
\text { construction projects. }\end{array}$ & $\begin{array}{c}6 / 01 / 96 \\
\text { Completed }\end{array}$ & WR Hayes \\
\hline $\begin{array}{l}\text { 17. Develop a listing of special agreement services provided } \\
\text { to RL, other DOE sites, and other entities. }\end{array}$ & $\begin{array}{c}6 / 01 / 96 \\
\text { Completed }\end{array}$ & WR Hayes \\
\hline $\begin{array}{l}\text { 18. Develop a listing of facility POCs to be available for the } \\
\text { transition phase. Provide list to program/project level } \\
\text { for consolidation. }\end{array}$ & $\begin{array}{c}5 / 15 / 96 \\
\text { Completed }\end{array}$ & DE Good \\
\hline $\begin{array}{l}\text { 19. Develop a list of current work order services or other } \\
\text { form of agreement to provide work for other } \\
\text { organizations, on and offsite. }\end{array}$ & $\begin{array}{l}6 / 01 / 96 \\
\text { Completed }\end{array}$ & WR Hayes \\
\hline $\begin{array}{l}\text { 20. Develop a list of current work order services or other } \\
\text { form of agreement to obtain work from other } \\
\text { organizations, on and offsite. }\end{array}$ & $\begin{array}{c}6 / 01 / 96 \\
\text { Completed }\end{array}$ & WR Hayes \\
\hline $\begin{array}{l}\text { 21. Develop a list of permits governing the facility (or } \\
\text { operation), and provide a description of each as to where } \\
\text { and how it applies. }\end{array}$ & $\begin{array}{c}6 / 01 / 96 \\
\text { Completed }\end{array}$ & WR Hayes \\
\hline 22. Update/submit "Smart Book"/assemble key documents. & $7 / 15 / 96$ & WR Hayes \\
\hline $\begin{array}{l}\text { 23. Verify pre-existing condition data is current and } \\
\text { supplement as necessary. }\end{array}$ & $7 / 22 / 96$ & DE Good \\
\hline 24. Ensure building assignments are correct in RLPS. & $7 / 22 / 96$ & WR Hayes \\
\hline Post-Award Actions & & \\
\hline $\begin{array}{l}\text { 1. Provide the PHMC access to the key HFD } \\
\text { documentation, any program level generated pre-existing } \\
\text { conditions information, listing of POCs, and listing of } \\
\text { subcontracts. Provide copies upon request. }\end{array}$ & $8 / 05 / 96$ & WR Hayes \\
\hline
\end{tabular}




\begin{tabular}{|c|c|c|}
\hline Post-A rrard Actions & $\begin{array}{l}\text { Scheduled } \\
\text { Date }\end{array}$ & Actionee(s) \\
\hline $\begin{array}{l}\text { 3. HFD facility space and services available for use by the } \\
\text { PHMC. }\end{array}$ & $8 / 05 / 96$ & WR Hayes \\
\hline 4. Provide HFD briefing to the PHMC. & $8 / 12 / 96-8 / 30 / 96$ & DE Good \\
\hline 5. Provide HFD cours to the PHMC. & $8 / 12 / 96-8 / 30 / 96$ & DE Good \\
\hline $\begin{array}{l}\text { 6. Perform a joint HFD property and equipment inventory } \\
\text { with PHMC personnel. }\end{array}$ & by $9 / 16 / 96$ & DE Good \\
\hline $\begin{array}{l}\text { 7. Provide any additional documentation or information } \\
\text { requested by the PHMC, if available, that was not } \\
\text { identified during the pre-award phase. }\end{array}$ & $8 / 30 / 96$ & WR Hayes \\
\hline $\begin{array}{l}\text { 8. PHMC identify any recurring reports that they would } \\
\text { like to receive during transition. }\end{array}$ & by $8 / 19 / 96$ & PHMC \\
\hline 9. Provide necessary training to PHMC personnel. & $8 / 12 / 96-8 / 30 / 96$ & WR Hayes \\
\hline $\begin{array}{l}\text { 10. Provide an updated status of ongoing work prior to } \\
\text { contract turnover. }\end{array}$ & by $9 / 30 / 96$ & DE Good \\
\hline 11. Provide a categorized inventory of wastes. & by $9 / 16 / 96$ & WR Hayes \\
\hline
\end{tabular}

\subsubsection{Fabrication Shops}

The Site Fabrication Services (SFS) mission is to maintain a center of excellence with capability for the fabrication of prototype assemblies, repair of parts, components, and specified equipment repairs. Specific emphasis will be placed on prototypical and emergency work, which requires extensive engineering and manufacturing coordination. SFS will encourage a one-stop shopping center by maintaining a work brokering activity that will route and manage fabrication and manufacturing work to others onsite or offsite as required to support clean-up activities and schedules.

Assumptions:

- Welding Procedures and Welder Qualifications are currently ICF KH and cannot be transferred to the new contractor. The new contractor must use their own procedures and re-qualify the welders to their program for code work. If the contractor does not have the necessary procedures to perform the work currently in progress, they will need to qualify those procedures prior to the continuation of that work.

- The ICF KH QA Program cannot be transferred to the new contractor. The new contractor must submit for approval their own program. This will require the recertification of all ICF KH Inspection Personnel. 
- The ICF KH American Society of Mechanical Engineers (ASME) Code Stamps cannot be transferred to the new contractor. If code work is required, the new contractor will be required to be surveyed and qualified by the code community.

- Magnetic Particle Testing, Liquid Penetrant Testing, Radiographic Testing, and Ultrasonic Testing is currently performed by WHC as a service to SFS. When this function is terminated, the new contractor will have to supply their own NDE Service, or hire these services in accordance with their QA Program if they are to comply with American Society for Nondestructive Testing requirements.

\begin{tabular}{|c|c|c|}
\hline Pre-Award Actions & $\begin{array}{l}\text { Scheduled } \\
\text { Date }\end{array}$ & Actionet(s) \\
\hline $\begin{array}{l}\text { 1. Identify number of briefings, and prepare briefing(s) } \\
\text { for the PHMC. (Provide for RL POC review.) }\end{array}$ & $\begin{array}{c}5 / 15 / 96 \\
\text { Completed }\end{array}$ & MA Butterworth \\
\hline $\begin{array}{l}\text { 2. Identify key documents for the facility (or operations). } \\
\text { (Provide for RL POC review.) }\end{array}$ & $\begin{array}{c}5 / 01 / 96 \\
\text { Completed }\end{array}$ & MA Butterworth \\
\hline $\begin{array}{l}\text { 3. Assemble key documentation, or identify its location, } \\
\text { for use by the PHMC. Includes preparation of a } \\
\text { "Smart Book," to include infornation develop in the } \\
\text { following actions. }\end{array}$ & $\begin{array}{c}6 / 01 / 96 \\
\text { Completed "Smart } \\
\text { Book" only } \\
\text { See item \#15 }\end{array}$ & MA Butterworth \\
\hline $\begin{array}{l}\text { 4. Develop a listing and description of subcontracts. } \\
\text { Include any subcontracts planned to be executed prior } \\
\text { to contract takeover. }\end{array}$ & $\begin{array}{l}5 / 15 / 96 \\
\text { Completed }\end{array}$ & MA Butterworth \\
\hline $\begin{array}{l}\text { 5. Using the pre-existing checklist, compile pre-existing } \\
\text { condition information on facilities and/or major } \\
\text { equipment. Conduct, or schedule as a separate post- } \\
\text { award action(s), any assessments required to adequately } \\
\text { address all relevant items on the checklist. Provide } \\
\text { completed checklist for RL POC review. }\end{array}$ & $\begin{array}{l}6 / 15 / 96 \\
\text { Completed }\end{array}$ & MA Butterworth \\
\hline $\begin{array}{l}\text { 6. Develop a list of open action items and commitments, } \\
\text { including status (Environmental, Tri-Party Agreement, } \\
\text { DNFSB as examples). }\end{array}$ & $\begin{array}{l}\text { 6/01/96 } \\
\text { Completed }\end{array}$ & MA Butterworth \\
\hline $\begin{array}{l}\text { 7. Develop a list of facility (or operation)-specific } \\
\text { mandatory training and qualification requirements for } \\
\text { employees. }\end{array}$ & $\begin{array}{l}\text { 6/01/96 } \\
\text { Completed }\end{array}$ & MA Butterworth \\
\hline $\begin{array}{l}\text { 8. Develop a listing of special agreement services } \\
\text { provided to } \mathrm{RL} \text {, other DOE sites, and other entities. }\end{array}$ & $\begin{array}{c}\text { 6/01/96 } \\
\text { Completed }\end{array}$ & MA Butterworth \\
\hline $\begin{array}{l}\text { 9. Develop a listing of POCs to be available for the } \\
\text { transition phase. }\end{array}$ & $\begin{array}{l}5 / 15 / 96 \\
\text { Completed }\end{array}$ & MA Butterworth \\
\hline 10. Develop a listing and description of databases. & $\begin{array}{l}5 / 15 / 96 \\
\text { Completed }\end{array}$ & MA Butterworth \\
\hline $\begin{array}{l}\text { 11. Develop a list of current work order services or other } \\
\text { forn of agreement to provide work for other } \\
\text { organizations, on and offsite. }\end{array}$ & $\begin{array}{l}\text { 6/01/96 } \\
\text { Completed }\end{array}$ & MA Butterworth \\
\hline $\begin{array}{l}\text { 12. Develop a list of current work order services or other } \\
\text { form of agreement to obtain work from other } \\
\text { organizations, on and offsite. }\end{array}$ & $\begin{array}{l}6 / 01 / 96 \\
\text { Completed }\end{array}$ & MA Butterworth \\
\hline
\end{tabular}




\begin{tabular}{|c|c|c|}
\hline Pre-Award Actions & $\begin{array}{c}\text { Scheduled } \\
\text { Date }\end{array}$ & Actionee(s) \\
\hline $\begin{array}{l}\text { 13. Develop a list of permits governing facilities or } \\
\text { operation, and provide a description of each as to } \\
\text { where and how it applies. }\end{array}$ & $\begin{array}{c}6 / 01 / 96 \\
\text { Completed }\end{array}$ & MA Butterworth \\
\hline $\begin{array}{l}\text { 14. Verify the accuracy of property assignments and } \\
\text { correct any deficiencies. }\end{array}$ & $\begin{array}{c}6 / 01 / 96 \\
\text { Compieted }\end{array}$ & MA Butterworth \\
\hline $\begin{array}{l}\text { 15. Verify "Smart Book" to include any updates and } \\
\text { submit. }\end{array}$ & $7 / 15 / 96$ & MA Butterworth \\
\hline 16. Verify PEC package, modify/supplement as necessary. & $7 / 22 / 96$ & MA Butterworth \\
\hline $\begin{array}{l}\text { 17. Ensure building responsibility assignments are correct } \\
\text { in RLPS. }\end{array}$ & $7 / 22 / 96$ & MA Butterworth \\
\hline Post-Award Actions & & \\
\hline 1. Provide briefing(s) to the PHMC. & $8 / 12 / 96-8 / 30 / 96$ & MA Butterworth \\
\hline 2. Provide tour(s) to the PHMC. & $8 / 12 / 96-8 / 30 / 96$ & MA Butterworth \\
\hline $\begin{array}{l}\text { 3. Provide the PHMC access to key documentation, pre- } \\
\text { existing condition information, commitment data, } \\
\text { inventory data, etc. (as established in the pre-award } \\
\text { phase). Provide copies upon request. }\end{array}$ & $8 / 05 / 96$ & MA Butterworth \\
\hline $\begin{array}{l}\text { 4. Provide any additional documentation or information } \\
\text { requested by the PHMC, if avaitahle, that was not } \\
\text { identitied during the pre-award phase. }\end{array}$ & by $8 / 30 / 96$ & MA Butterworth \\
\hline $\begin{array}{l}\text { 5. PHMC identify any recurring reports that they would } \\
\text { like to receive during transition. }\end{array}$ & by $8 / 19 / 96$ & MA Butterworth \\
\hline 6. Provide necessary training to PHMC personnel. & $8 / 12 / 96-8 / 30 / 96$ & MA Butterworth \\
\hline $\begin{array}{l}\text { 7. Provide an updated status of ongoing work prior to } \\
\text { contract turnover. }\end{array}$ & by $9 / 30 / 96$ & MA Butterworth \\
\hline $\begin{array}{l}\text { 8. Perform a joint property and equipment inventory with } \\
\text { the PHMC. }\end{array}$ & by $9 / 16 / 96$ & MA Butterworth \\
\hline $\begin{array}{l}\text { 9. Provide a categorized inventory of wastes or hazardous } \\
\text { materials. }\end{array}$ & by $9 / 30 / 96$ & MA Butterworth \\
\hline $\begin{array}{l}\text { 10. Assist the PHMC in the conduct of pre-existing } \\
\text { condition assessments. }\end{array}$ & $8 / 12 / 96-9 / 16 / 96$ & MA Butterworth \\
\hline
\end{tabular}

\subsubsection{Integrated Pest Management Services}

Integrated Pest Management manages and controls plant and animal pest species at Hanford using a combination of safe and cost effective control alternatives such as administrative controls, engineering controls, and chemical applications. 


\begin{tabular}{|c|c|c|}
\hline Pre-Award Actions & $\begin{array}{l}\text { Scheduled } \\
\text { Date }\end{array}$ & Actionee(s) \\
\hline $\begin{array}{l}\text { 1. Prepare for a comprehensive briefing for the PHMC describing } \\
\text { all operational aspects of the organization. }\end{array}$ & $\begin{array}{c}5 / 01 / 96 \\
\text { Completed } \\
\end{array}$ & CL Looney \\
\hline $\begin{array}{l}\text { 2. Identify all key documents relevant to the organizations } \\
\text { operations, including a listing of key facility and field } \\
\text { documents. (Provide a listing to RL for review.) }\end{array}$ & $\begin{array}{l}\text { 5/01/96 } \\
\text { Completed }\end{array}$ & CL Looney \\
\hline $\begin{array}{l}\text { 3. Obtain copies of key facility documents and assemble, or } \\
\text { identify their location for access. Develop an operations } \\
\text { "Smart Book." }\end{array}$ & $\begin{array}{c}\text { 5/15/96 } \\
\text { Completed } \\
\text { "Smart Book" } \\
\text { only } \\
\text { See itern \#9 } \\
\end{array}$ & CL Looney \\
\hline 4. Identify points-of-contact for the organization. & $\begin{array}{c}5 / 15 / 96 \\
\text { Completed } \\
\end{array}$ & CL Looney \\
\hline $\begin{array}{l}\text { 5. Using the pre-existing conditions checklist, evaluate pre- } \\
\text { existing conditions. Conduct assessments as necessary to } \\
\text { adequately address checklist. }\end{array}$ & $\begin{array}{l}6 / 15 / 96 \\
\text { Completed }\end{array}$ & CL Looney \\
\hline $\begin{array}{l}\text { 6. Identify applicable milestones and commitments (e.g., Tri- } \\
\text { Party Agreement and Consent Order milestones), and } \\
\text { determine their stans. Describe the form of the deliverable } \\
\text { and what constitutes completion. }\end{array}$ & $\begin{array}{l}5 / 15 / 96 \\
\text { Completed }\end{array}$ & CL Looney \\
\hline $\begin{array}{l}\text { 7. Verify the accuracy of property assignments for the facility and } \\
\text { correct any deficiencies. }\end{array}$ & $\begin{array}{c}5 / 15 / 96 \\
\text { Completed }\end{array}$ & CL Looney \\
\hline $\begin{array}{l}\text { 8. Verify the completeness of all RIDS and correct any } \\
\text { deficiencies. }\end{array}$ & $\begin{array}{c}5 / 15 / 96 \\
\text { Completed }\end{array}$ & CL Loney \\
\hline 9. Update/submit "Smart Book"/assemble key documents. & $7 / 15 / 96$ & CL Looney \\
\hline $\begin{array}{l}\text { 10. Verify pre-existing condition data is current and supplement as } \\
\text { necessary. }\end{array}$ & $7 / 22 / 96$ & CL Looney \\
\hline 11. Ensure building assignments are correct in RLPS. & $7 / 22 / 96$ & CL Looney \\
\hline \multicolumn{3}{|l|}{ Post-Award Actions } \\
\hline $\begin{array}{l}\text { 1. Provide a comprehensive briefing for the PHMC complete with } \\
\text { all facility, personnel, and organizational documentation. } \\
\text { (Provide copies upon request.) }\end{array}$ & $8 / 12 / 96-8 / 30 / 96$ & CL Looney \\
\hline $\begin{array}{l}\text { 2. Conduct a comprehensive tour of all facilities and field } \\
\text { operations relevant to the organization. }\end{array}$ & $8 / 12 / 96-8 / 30 / 96$ & CL Looney \\
\hline $\begin{array}{l}\text { 3. Assist the PHMC in a survey of pre-existing conditions at the } \\
\text { facility. }\end{array}$ & $8 / 12 / 96-9 / 16 / 96$ & CL Looney \\
\hline $\begin{array}{l}\text { 4. Provide an update on work completed and work in progress } \\
\text { during the transition to the PHMC. }\end{array}$ & 9/15/96 & CL Looney \\
\hline $\begin{array}{l}\text { 5. Complete a joint property and equipment inventory with the } \\
\text { PHMC. }\end{array}$ & $9 / 16 / 96$ & CL Looney \\
\hline 6. Provide any additional documentation requested by the PHMC. & $8 / 30 / 96$ & CL Looney \\
\hline 7. Provide necessary training to PHMC personnel. & $8 / 12 / 96-8 / 30 / 96$ & CL Looney \\
\hline 8. Provide a categorized inventory of wastes. & $9 / 30 / 96$ & CL Looney \\
\hline
\end{tabular}




\subsubsection{Non-Radioactive/Non-Dangerous Waste Handling}

Provide disposal services for municipal, asbestos, drummed, and medical wastes. Manage contracts and interface with the City of Richland, Waste Management of Kennewick, and Basin Disposal, Inc. Pick up waste containers throughout the Hanford Site and deliver to the City of Richland landfill. Transport hazardous materials.

\section{Assumptions:}

- Contracts with the City of Richland, Waste Management of Kennewick, and Basin Disposal, Inc., will remain in effect.

- Municipal waste will be delivered to the City of Richland landfill by HAMTC workers.

- Hazardous material transportation groups will be consolidated.

\begin{tabular}{|c|c|c|}
\hline Pre-Award Actions & $\begin{array}{l}\text { Scheduled } \\
\text { Date }\end{array}$ & Actionee(s) \\
\hline $\begin{array}{l}\text { 1. Identify number of briefings, and prepare briefing(s) } \\
\text { for the PHMC. (Provide for RL POC review.) }\end{array}$ & $\begin{array}{c}5 / 15 / 96 \\
\text { Completed } \\
\end{array}$ & KD Johnson \\
\hline $\begin{array}{l}\text { 2. Identify key documents for the facility (or operations). } \\
\text { (Provide for RL POC review.) }\end{array}$ & $\begin{array}{c}5 / 01 / 96 \\
\text { Completed }\end{array}$ & KD Johnson \\
\hline $\begin{array}{l}\text { 3. Assemble key documentation, or identify its location, } \\
\text { for use by the PHMC. Includes preparation of a } \\
\text { "Smart Book," to include information develop in the } \\
\text { following actions. }\end{array}$ & $\begin{array}{c}6 / 01 / 96 \\
\text { Completed "Smart } \\
\text { Book" only } \\
\text { See item } \# 15 \\
\end{array}$ & KD Johrison \\
\hline $\begin{array}{l}\text { 4. Develop a listing and description of subcontracts. } \\
\text { Include any subcontracts planned to be executed prior } \\
\text { to contract takeover. }\end{array}$ & $\begin{array}{l}5 / 15 / 96 \\
\text { Completed }\end{array}$ & KD Johnson \\
\hline $\begin{array}{l}\text { 5. Using the pre-existing checklist, compile pre-existing } \\
\text { condition information on facilities and/or major } \\
\text { equipment. Conduct, or schedule as a separate post- } \\
\text { award action(s), any assessments required to adequately } \\
\text { address all relevant iterns on the checklist. Provide } \\
\text { completed checklist for RL POC review. }\end{array}$ & $\begin{array}{l}\text { 6/15/96 } \\
\text { Completed }\end{array}$ & KD Johnson \\
\hline $\begin{array}{l}\text { 6. Develop a list of open action items and commitments, } \\
\text { including starus (Environmental, Tri-Party Agreement, } \\
\text { DNFSB as examples). }\end{array}$ & $\begin{array}{l}\text { 6/01/96 } \\
\text { Completed }\end{array}$ & KD Johnson \\
\hline $\begin{array}{l}\text { 7. Develop a list of facility (or operation)-specific } \\
\text { mandatory training and qualification requirements for } \\
\text { employees. }\end{array}$ & $\begin{array}{l}6 / 01 / 96 \\
\text { Completed }\end{array}$ & KD Johnson \\
\hline $\begin{array}{l}\text { 8. Develop a listing of special agreement services } \\
\text { provided to RL, other DOE sites, and other entities. }\end{array}$ & $\begin{array}{c}6 / 01 / 96 \\
\text { Completed }\end{array}$ & $\mathrm{KD}$ Johnson \\
\hline $\begin{array}{l}\text { 9. Develop a listing of POCs to be available for the } \\
\text { transition phase. }\end{array}$ & $\begin{array}{l}5 / 15 / 96 \\
\text { Completed }\end{array}$ & KD Johnson \\
\hline
\end{tabular}




\begin{tabular}{|c|c|c|}
\hline Pre-Award Actions & $\begin{array}{l}\text { Scheduled } \\
\text { Date } \\
\end{array}$ & Actionee(s) \\
\hline 10. Develop a iisting and description of databases. & $\begin{array}{c}5 / 15 / 96 \\
\text { Completed }\end{array}$ & KD Johnson \\
\hline $\begin{array}{l}\text { 11. Develop a list of current work order services or other } \\
\text { form of agreement to provide work for other } \\
\text { organizations, on and offsite. }\end{array}$ & $\begin{array}{c}\text { 6/01/96 } \\
\text { Completed }\end{array}$ & KD Johnson \\
\hline $\begin{array}{l}\text { 12. Develop a list of current work order services or other } \\
\text { form of agreement to obtain work from other } \\
\text { organizations, on and offsite. }\end{array}$ & $\begin{array}{l}6 / 01 / 96 \\
\text { Completed }\end{array}$ & KD Johnson \\
\hline $\begin{array}{l}\text { 13. Develop a list of permits governing facilities or } \\
\text { operation, and provide a description of each as to } \\
\text { where and how it applies. }\end{array}$ & $\begin{array}{l}\text { 6/01/96 } \\
\text { Completed }\end{array}$ & KD Johnson \\
\hline $\begin{array}{l}\text { 14. Verify the accuracy of property assignments and } \\
\text { correct any deficiencies. }\end{array}$ & $\begin{array}{c}6 / 01 / 96 \\
\text { Completed }\end{array}$ & KD Johnson \\
\hline $\begin{array}{l}\text { 15. Verify "Smart Book" to include any updates and } \\
\text { submit. }\end{array}$ & $7 / 15 / 96$ & KD Johnson \\
\hline $\begin{array}{l}\text { 16. Verify PEC package, modify/supplement as } \\
\text { necessary. }\end{array}$ & $7 / 22 / 96$ & KD Johnson \\
\hline $\begin{array}{l}\text { 17. Ensure building responsibility assignments are correct } \\
\text { in RLPS. }\end{array}$ & $7 / 22 / 96$ & KD Johnson \\
\hline 18. Prepare satellite areas for use by PHMC. & $7 / 22 / 96$ & KD Johnson \\
\hline Post-Award Actions & 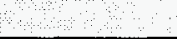 & 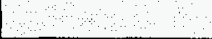 \\
\hline 1. Provide briefing(s) to the PHMC. & $8 / 12 / 96-8 / 30 / 96$ & KD Johnson \\
\hline 2. Provide tour(s) to the PHMC. & $8 / 12 / 96-8 / 30 / 96$ & KD Johnson \\
\hline $\begin{array}{l}\text { 3. Provide the PHMC access to key documentation, pre- } \\
\text { existing condition information, commitment data, } \\
\text { inventory data, eic. (as established in the pre-award } \\
\text { phase). Provide copies upon request. }\end{array}$ & $8 / 05 / 96$ & KD Johrison \\
\hline $\begin{array}{l}\text { 4. Provide any additional documentation or information } \\
\text { requested by the PHMC, if available, that was not } \\
\text { identified during the pre-award phase. }\end{array}$ & by $8 / 30 / 96$ & KD Johnson \\
\hline $\begin{array}{l}\text { 5. PHMC identify any recurring reports that they would } \\
\text { like to receive during transition. }\end{array}$ & by $8 / 19 / 96$ & KD Johnson \\
\hline 6. Provide necessary training to PHMC personnel. & $8 / 12 / 96-8 / 30 / 96$ & KD Johnson \\
\hline $\begin{array}{l}\text { 7. Provide an updated status of ongoing work prior to } \\
\text { contract turnover. }\end{array}$ & by $9 / 30 / 96$ & KD Johnson \\
\hline $\begin{array}{l}\text { 8. Perform a joint property and equipment inventory } \\
\text { with the PHMC. }\end{array}$ & by $9 / 16 / 96$ & KD Johnson \\
\hline $\begin{array}{l}\text { 9. Provide a categorized inventory of wastes or } \\
\text { hazardous materials. }\end{array}$ & by $9 / 30 / 96$ & KD Johnson \\
\hline $\begin{array}{l}\text { 10. Assist the PHMC in the conduct of pre-existing } \\
\text { condition assessments. }\end{array}$ & $8 / 12 / 96-9 / 16 / 96$ & KD Johnson \\
\hline
\end{tabular}




\subsubsection{Site Crane \& Rigging Services}

The Mission of ICF KH Site Crane and Rigging Services is to support various programs dedicated to the maintenance, cleanup, and restoration of the Hanford Site. This support is provided through the lifting, handling, movement, and placement of material and equipment; inspection and maintenance of lifting equipment; erection, inspection, and removal of scaffold; fabrication, testing, and control of below-the-hook rigging equipment; and the management of mobile lifting equipment. All of this support is provided within the boundaries and scope of WHC, ICF KH, or Hanford procedures, directives and orders.

\begin{tabular}{|c|c|c|}
\hline Pre-Award Actions & $\begin{array}{c}\text { Scheduled } \\
\text { Date }\end{array}$ & Actionee(s) \\
\hline $\begin{array}{l}\text { 1. Identify number of briefings, and prepare briefing(s) } \\
\text { for the PHMC. (Provide for RL POC review.) }\end{array}$ & $\begin{array}{c}5 / 15 / 96 \\
\text { Completed }\end{array}$ & WA Ferree \\
\hline $\begin{array}{l}\text { 2. Identify key documents for the facility (or operations). } \\
\text { (Provide for RL POC review.) }\end{array}$ & $\begin{array}{c}5 / 01 / 96 \\
\text { Completed }\end{array}$ & CE Brewer \\
\hline $\begin{array}{l}\text { 3. Assemble key documentation and org. data, or identify } \\
\text { its location, for use by the PHMC. Includes } \\
\text { preparation of a "Smart Book," to include infornation } \\
\text { develop in the following accions. }\end{array}$ & $\begin{array}{c}6 / 01 / 96 \\
\text { Completed "Smart } \\
\text { Book" only } \\
\text { See item } \# 15 \\
\end{array}$ & CE Brewer \\
\hline $\begin{array}{l}\text { 4. Develop a listing and description of subcontracts. } \\
\text { Include any subcontracts planned to be executed prior } \\
\text { to contract takeover. }\end{array}$ & $\begin{array}{c}5 / 15 / 96 \\
\text { Completed }\end{array}$ & WA Ferree \\
\hline $\begin{array}{l}\text { 5. Using the pre-existing checklist, compile pre-existing } \\
\text { condition information on facilities and/or major } \\
\text { equipment. Conduct, or schedule as a separate post- } \\
\text { award action(s), any assessments required to adequately } \\
\text { address all relevant iterns on the checklist. Provide } \\
\text { completed checklist for RL POC review. }\end{array}$ & $\begin{array}{l}\text { 6/15/96 } \\
\text { Completed }\end{array}$ & WA Ferree \\
\hline $\begin{array}{l}\text { 6. Develop a list of open action iterns and commitments, } \\
\text { including status (Environmental, Tri-Party Agreement, } \\
\text { DNFSB as examples). }\end{array}$ & $\begin{array}{l}\text { 6/01/96 } \\
\text { Completed }\end{array}$ & WA Ferree \\
\hline $\begin{array}{l}\text { 7. Develop a list of facility (or operation)-specific } \\
\text { mandatory training and qualification requirements for } \\
\text { employees. }\end{array}$ & $\begin{array}{l}\text { 6/01/96 } \\
\text { Completed }\end{array}$ & SM Holloman \\
\hline $\begin{array}{l}\text { 8. Develop a listing of special agreement services } \\
\text { provided to RL, other DOE sites, and other entities. }\end{array}$ & $\begin{array}{c}6 / 01 / 96 \\
\text { Completed }\end{array}$ & WA Ferree \\
\hline $\begin{array}{l}\text { 9. Develop a listing of POCs to be available for the } \\
\text { transition phase. }\end{array}$ & $\begin{array}{c}5 / 15 / 96 \\
\text { Completed }\end{array}$ & WA Ferree \\
\hline 10. Develop a listing and description of databases. & $\begin{array}{c}5 / 15 / 96 \\
\text { Completed }\end{array}$ & WA Ferree \\
\hline $\begin{array}{l}\text { 11. Develop a list of current work order services or other } \\
\text { form of agreement to provide work for other } \\
\text { organizations, on and offsite. }\end{array}$ & $\begin{array}{l}\text { 6/01/96 } \\
\text { Completed }\end{array}$ & WA Ferree \\
\hline $\begin{array}{l}\text { 12. Develop a list of current work order services or other } \\
\text { form of agreement to obtain work from other } \\
\text { organizations, on and offsite. }\end{array}$ & $\begin{array}{l}\text { 6/01/96 } \\
\text { Completed }\end{array}$ & WA Ferree \\
\hline
\end{tabular}




\begin{tabular}{|c|c|c|}
\hline Pre-Award Actions & $\begin{array}{l}\text { Scheduled } \\
\text { Date }\end{array}$ & Actionee(s) \\
\hline $\begin{array}{l}\text { 13. Develop a list of permits governing facilities or } \\
\text { operation, and provide a description of each as to } \\
\text { where and how it applies. }\end{array}$ & $\begin{array}{l}6 / 01 / 96 \\
\text { Completed }\end{array}$ & WA Ferree \\
\hline $\begin{array}{l}\text { 14. Verify the accuracy of property assignments and } \\
\text { correct any deficiencies. }\end{array}$ & $\begin{array}{c}\text { 6/01/96 } \\
\text { Completed }\end{array}$ & WA Ferree \\
\hline $\begin{array}{l}\text { 15. Verify "Smart Book" to include any updates and } \\
\text { submit. }\end{array}$ & $7 / 15 / 96$ & WA Ferree \\
\hline 16. Verify PEC package, modify/supplement as necessary. & $7 / 22 / 96$ & WA Ferree \\
\hline $\begin{array}{l}\text { 17. Ensure building responsibility assignments are correct } \\
\text { in RLPS. }\end{array}$ & $7 / 22 / 96$ & WA Ferree \\
\hline Post-Award Actions & & \\
\hline 1. Provide briefing(s) to the PHMC. & $8 / 12 / 96-8 / 30 / 96$ & WA Ferree \\
\hline 2. Provide tour(s) to the PHMC. & $8 / 12 / 96-8 / 30 / 96$ & WA Ferree \\
\hline $\begin{array}{l}\text { 3. Provide the PHMC access to key documentation, pre- } \\
\text { existing condition information, commimnent data, } \\
\text { inventory data, etc. (as established in the pre-award } \\
\text { phase). Provide copies upon request. }\end{array}$ & $8 / 05 / 96$ & WA Ferree \\
\hline $\begin{array}{l}\text { 4. Provide any additional documentation or information } \\
\text { requested by the PHMC, if available, that was not } \\
\text { identified during the pre-award phase. }\end{array}$ & by $8 / 30 / 96$ & WA Ferree \\
\hline $\begin{array}{l}\text { 5. PHMC identify any recuring reports that they would } \\
\text { like to receive during transition. }\end{array}$ & by $8 / 19 / 96$ & WA Ferree \\
\hline 6. Provide necessary training to PHMC personnel. & $8 / 12 / 96-8 / 30 / 96$ & WA Ferree \\
\hline $\begin{array}{l}\text { 7. Provide an updated status of ongoing work prior to } \\
\text { contract turnover. }\end{array}$ & by $9 / 30 / 96$ & WA Ferree \\
\hline $\begin{array}{l}\text { 8. Perform a joint property and equipment inventory with } \\
\text { the PHMC. }\end{array}$ & by $9 / 16 / 96$ & WA Ferree \\
\hline $\begin{array}{l}\text { 9. Provide a categorized inventory of wastes or hazardous } \\
\text { materials. }\end{array}$ & by $9 / 30 / 96$ & WA Ferree \\
\hline 10. Provide overview of financial sinuation and work plans. & by $8 / 30 / 96$ & WA Ferree \\
\hline 11. Status audit/surveillance items & by $8 / 30 / 96$ & WA Ferree \\
\hline $\begin{array}{l}\text { 12. Assist the PHMC in the conduct of pre-existing } \\
\text { condition assessments. }\end{array}$ & $8 / 12 / 96-9 / 16 / 96$ & WA Ferree \\
\hline
\end{tabular}

\subsubsection{Landlord Program}

The Landlord Program (LP) replaces, repairs, or upgrades the existing Hanford Site (WHC, PNNL, HEHF, and ICF KH) general purpose infrastructure in support of the Hanford Cleanup and other missions. The general purpose infrastructure consists of buildings, systems, and equipment that, by design or use are not essentially dedicated to a 
single program mission. The LP provides capital funding for ongoing replacements or repairs to general purpose equipment and facilities to sustain support to the Hanford cleanup and other missions.

\begin{tabular}{|c|c|c|c|}
\hline & PreAward Actions & $\begin{array}{c}\text { Scheduled } \\
\text { Date }\end{array}$ & Actionee(s) \\
\hline 1. & $\begin{array}{l}\text { Develop a list of key program documentation. } \\
\text { Provide for RL POC review. }\end{array}$ & $\begin{array}{c}5 / 15 / 96 \\
\text { Completed } \\
\end{array}$ & AD Gadeken \\
\hline 2. & $\begin{array}{l}\text { Assemble key program documentation, or identify its } \\
\text { location, for use by the PHMC. Includes } \\
\text { development of a program/project "Smart Book," } \\
\text { including listings and descriptions developed below. }\end{array}$ & $\begin{array}{l}6 / 01 / 96 \\
\text { Completed "Smart } \\
\text { Book" only } \\
\text { See item } \# 10\end{array}$ & AD Gadeken \\
\hline 3. & $\begin{array}{l}\text { Identify number of briefings, and prepare program } \\
\text { briefing(s) for presentation to PHMC. (Provide for } \\
\text { coordination of number and content with RL POC.) }\end{array}$ & $\begin{array}{l}\text { 6/01/96 } \\
\text { Completed }\end{array}$ & AD Gadeken \\
\hline 4. & $\begin{array}{l}\text { Develop a listing of Key program individuals as } \\
\text { POCs, including facility and operations personnel. }\end{array}$ & $\begin{array}{l}\text { 6/01/96 } \\
\text { Completed }\end{array}$ & AD Gadeken \\
\hline 5. & $\begin{array}{l}\text { Develop listing and description of program } \\
\text { subcontracts. Include any subcontracts still planned to } \\
\text { be executed prior to contract takeover. }\end{array}$ & $\begin{array}{l}\text { 6/01/96 } \\
\text { Completed }\end{array}$ & AD Gadeken \\
\hline & Develop listing and description of program databases. & $\begin{array}{c}6 / 01 / 96 \\
\text { Completed }\end{array}$ & AD Gadeken \\
\hline & $\begin{array}{l}\text { Verify the accuracy of property assignments and } \\
\text { correct any deficiencies. }\end{array}$ & $\begin{array}{c}6 / 01 / 96 \\
\text { Completed }\end{array}$ & AD Gadeken \\
\hline & $\begin{array}{l}\text { Identify space and equipment within program area and } \\
\text { facilities for use by the PHMC. }\end{array}$ & $\begin{array}{c}6 / 01 / 96 \\
\text { Completed }\end{array}$ & AD Gadeken \\
\hline & $\begin{array}{l}\text { Develop a listing and description of ongoing and } \\
\text { planned construction projects. }\end{array}$ & $\begin{array}{c}6 / 01 / 96 \\
\text { Completed } \\
\end{array}$ & AD Gadeken \\
\hline & Verify "Smart Book," include updates and submit. & $7 / 15 / 96$ & DG Hay \\
\hline & Post-Award Actions & & \\
\hline & $\begin{array}{l}\text { Provide the PHMC access to the key program } \\
\text { documentation, listing of POCs, and listing of } \\
\text { subcontracts. Provide copies upon request. }\end{array}$ & $8 / 05 / 96$ & AD Gadeken \\
\hline & Provide program briefing(s) to the PHMC. & $8 / 12 / 96-8 / 30 / 96$ & AD Gadeken \\
\hline & Provide program level tour to the PHMC. & $8 / 12 / 96-8 / 30 / 96$ & AD Gadeken \\
\hline & $\begin{array}{l}\text { Perform a joint program level property and equipment } \\
\text { inventory with PHMC personnel. }\end{array}$ & by $9 / 16 / 96$ & AD Gadeken \\
\hline
\end{tabular}

\subsubsection{Resource and Energy Management}

Resource \& Energy Management (R\&EM) has the responsibility to reduce mortgage costs by sponsoring and validating life-cycle, cost effective, energy efficient projects. 
R\&EM promotes employee culture change for efficient resource use onsite. Documented resource and energy savings show progress towards reducing mortgage costs, allows more taxpayer dollars to be focused into the actual cleanup of the Hanford Site, and validates payback for the program.

\section{Assumptions:}

- Program will support Energy Savings Performance Contract in FY 1997 and outyears.

- Program will receive no Landlord funding in FY 1997 for life-cycle cost effective project development.

- Program will receive In-house Energy Management funding to support some energy related duties.

- Bonneville Power Administration will provide some level of project funding for the FY 1997 program.

- Related areas, such as electrical and other resource metering, will continue to be supported by RL and Site programs.

\begin{tabular}{|c|c|c|}
\hline Pre-Award Actions & $\begin{array}{l}\text { Scheduled } \\
\text { Date }\end{array}$ & Actionee(s) \\
\hline $\begin{array}{l}\text { 1. Develop a list of key program documentation. } \\
\text { Provide for RL POC review. }\end{array}$ & $\begin{array}{c}5 / 15 / 96 \\
\text { Completed }\end{array}$ & WS Dunnivant \\
\hline $\begin{array}{l}\text { 2. Assemble key program documentation, or identify its } \\
\text { location, for use by the PHMC. Includes } \\
\text { development of a program/project "Smart Book," } \\
\text { including listings and descriptions developed below. }\end{array}$ & $\begin{array}{l}6 / 01 / 96 \\
\text { Completed "Smart } \\
\text { Book" only } \\
\text { See item } \# 10\end{array}$ & WS Dunnivant \\
\hline $\begin{array}{l}\text { 3. Identify number of briefings, and prepare program } \\
\text { briefing(s) for presentation to PHMC. (Provide for } \\
\text { coordination of number and content with RL POC.) }\end{array}$ & $7 / 15 / 96$ & WS Dunnivant \\
\hline $\begin{array}{l}\text { 4. Develop a listing of Key program individuals as } \\
\text { POCs, including facility and operations personnel. }\end{array}$ & $\begin{array}{c}6 / 01 / 96 \\
\text { Completed }\end{array}$ & Ws Dunnivant \\
\hline $\begin{array}{l}\text { 5. Develop listing and description of program } \\
\text { subcontracts. Include any subcontracts still planned to } \\
\text { be executed prior to contract takeover. }\end{array}$ & $\begin{array}{c}6 / 01 / 96 \\
\text { Completed }\end{array}$ & WS Dunnivant \\
\hline 6. Develop listing and description of program databases. & $\begin{array}{c}6 / 01 / 96 \\
\text { Completed }\end{array}$ & WS Dunnivant \\
\hline $\begin{array}{l}\text { 7. Verify the accuracy of property assignments and } \\
\text { correct any deficiencies. }\end{array}$ & $\begin{array}{c}\text { 6/01/96 } \\
\text { Completed }\end{array}$ & WS Dunnivant \\
\hline $\begin{array}{l}\text { 8. Identify space and equipment within program area and } \\
\text { facilities for use by the PHMC. }\end{array}$ & $\begin{array}{c}6 / 01 / 96 \\
\text { Completed }\end{array}$ & WS Dunnivant \\
\hline 9. Conduct an energy assessment of site facilities. & $\begin{array}{l}5 / 15 / 96 \\
\text { Completed }\end{array}$ & WS Dunnivant \\
\hline
\end{tabular}


WHC-SP-1181 Rev. 1

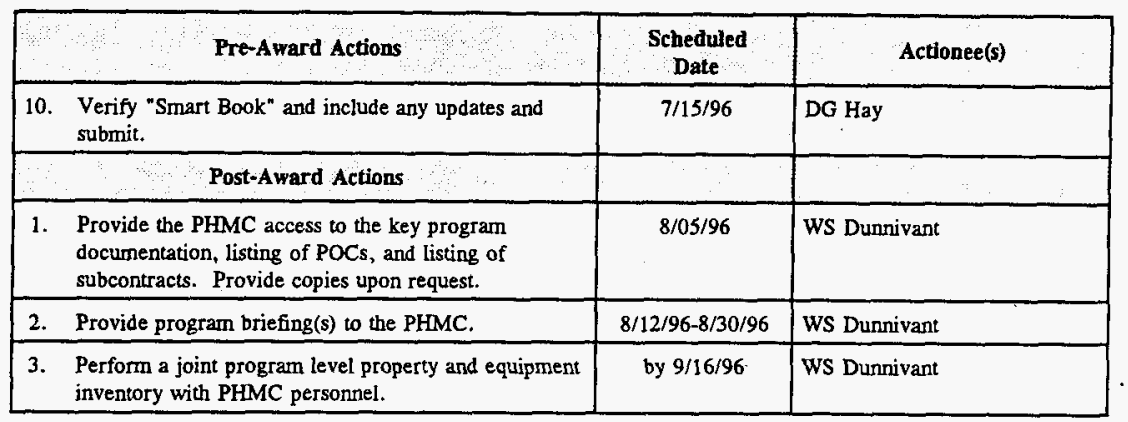

\subsection{REAL ESTATE AND MATERIAL MANAGEMENT}

(Also see Section 3.6, Procurement and Materials Management.)

\subsubsection{Land-Use Planning and Management}

This activity consists of the integration of land use activities at the 1,450 square kilometers (560 square miles) Hanford Site. This includes (1) site selection for individual construction projects; (2) site plans for major complexes and master plans within each operating area at Hanford; (3) comprehensive land use planning for the overall Site including integration with other sitewide plans such as environmental, strategic, systems engineering, ecologic/cultural, infrastructure, demolition, economic development/transition/asset conversion, site restoration, facility use/upgrades, safety, security, coordination of construction schedules, excavation permit approval, etc.; (4) development and operation of automated planning and mapping systems; and (5) information management, including building numbering system.

Regulatory Drivers:

- 48 CFR 970.5204-60, "Facilities Management"

- Secretary of Energy "Land and Facility Use Policy," dated December 21, 1994

- DOE 4320.2, "Capital Asset Management Process," dated March 13, 1992

- DOE 4300.1C, "Real Property Management," dated June 28, 1992

- DOE 4320.1B, "Site Development Planning," dated March 26, 1992

- DOE 6430.1A, "General Design Criteria," dated April 6, 1989 
- Draft DOE 4310.00, "Land and Facility Use Management," dated May 1, 1995

- DOE 0 430.1, "Life Cycle Asset Management," dated August 24, 1995

- RL 4310.1B, "Policy for Use of Land on the Crest of Rattlesnake Mountain," dated November 17, 1988

- RL 4320.2C, "Site Selection," dated March 16, 1990

- RL Letter to President of Westinghouse Hanford Company, "Interim Process for RL Review/Approval of Site Selection Requests, " dated October 16, 1995

- WHC-CM-8-7, Section 905, Rev. 2, "Site Selection"

Note: All of the regulatory drivers remain in effect until incorporated into the new operating contract, via DOE Order 430.1 . Even though some of the orders may be canceled, the specific work related to these orders will continue.

\begin{tabular}{|c|c|c|}
\hline Pre-Award Actions & $\begin{array}{l}\text { Scheduled } \\
\text { Date }\end{array}$ & Actionee(s) \\
\hline $\begin{array}{l}\text { 1. Develop a list of key program docurnentation. } \\
\text { Provide for RL POC review. }\end{array}$ & $\begin{array}{c}5 / 15 / 96 \\
\text { Cormpleted }\end{array}$ & EF Yancey \\
\hline $\begin{array}{l}\text { 2. Assemble key program documentation, or identify its } \\
\text { location, for use by the PHMC. Includes } \\
\text { development of a program/project "Smart Book," } \\
\text { including listings and descriptions developed below. }\end{array}$ & $\begin{array}{l}6 / 01 / 96 \\
\text { Completed "Smart } \\
\text { Book" only } \\
\text { See item } \# 9\end{array}$ & EF Yancey \\
\hline $\begin{array}{l}\text { 3. Identify number of briefings, and prepare program } \\
\text { briefing(s) for presentation to PHMC. (Provide for } \\
\text { coordination of number and content with RL POC.) }\end{array}$ & $7 / 15 / 96$ & EF Yancey \\
\hline $\begin{array}{l}\text { 4. Develop a listing of Key program individuals as } \\
\text { POCs, including facility and operations personnel. }\end{array}$ & $\begin{array}{c}\text { 6/01/96 } \\
\text { Completed }\end{array}$ & EF Yancey \\
\hline $\begin{array}{l}\text { 5. Develop listing and description of program } \\
\text { subcontracts. Include any subcontracts still planned to } \\
\text { be executed prior to contract takeover. }\end{array}$ & $\begin{array}{c}6 / 01 / 96 \\
\text { Completed }\end{array}$ & EF Yancey \\
\hline 6. Develop listing and description of program databases. & $\begin{array}{c}\text { 6/01/96 } \\
\text { Completed }\end{array}$ & EF Yancey \\
\hline $\begin{array}{l}\text { 7. Verify the accuracy of property assignments and } \\
\text { correct any deficiencies. }\end{array}$ & $\begin{array}{c}\text { 6/01/96 } \\
\text { Completed }\end{array}$ & EF Yancey \\
\hline $\begin{array}{l}\text { 8. Identify space and equipment within program area and } \\
\text { facilities for use by the PHMC. }\end{array}$ & $\begin{array}{c}\text { 6/01/96 } \\
\text { Completed } \\
\end{array}$ & EF Yancey \\
\hline $\begin{array}{l}\text { 9. Verify "Smart Book" and include any updates and } \\
\text { submit. }\end{array}$ & $7 / 15 / 96$ & DG Hay \\
\hline
\end{tabular}


WHC-SP-1181 Rev. 1

\begin{tabular}{|l|c|l|}
\hline \multicolumn{1}{|c|}{ Post-Award Actions } & $\begin{array}{c}\text { Scheduled } \\
\text { Date }\end{array}$ & Actionee(s) \\
\hline $\begin{array}{l}\text { 1. } \\
\begin{array}{l}\text { Provide the PHMC access to the key program } \\
\text { documentation, listing of POCs, and listing of } \\
\text { subcontracts. Provide copies upon request. }\end{array}\end{array}$ & $8 / 05 / 96$ & EF Yancey \\
\hline 2. $\quad$ Provide program briefing(s) to the PHMC. & $8 / 12 / 96-8 / 30 / 96$ & EF Yancey \\
\hline 3. Provide program level tour to the PHMC. & $8 / 12 / 96-8 / 30 / 96$ & EF Yancey \\
\hline 4. $\quad \begin{array}{l}\text { Perform a joint program level property and equipment } \\
\text { inventory with PHMC personnel. }\end{array}$ & by 9/16/96 & EF Yancey \\
\hline
\end{tabular}

\subsubsection{Calibration and Engineering Laboratories}

5.2.2.1 Engineering Laboratories. The objective of the Engineering Laboratories (EL) is to be the supplier of choice for EL services for development, assembly, testing, and evaluation to support the resolution of WHC, Hanford, and DOE complex-wide engineering issues for which we have a unique experience or capability and are competitively priced. Presently (June 1996), the ELs are staffed with 34 exempt technical staff and 30 non-exempt staff. The EL organizations operate under a laboratory oriented COO, operate two major testing facilities and offer use of their services and facilities at competitive rates, approximately $\$ 85 / \mathrm{hr}$ exempt, and $\$ 47 / \mathrm{hr}$ non-exempt; no additional charge is made for use of the facilities and its resources if EL labor is used for a job.

The ELs provide "one-of-a-kind" in-house prototype process and system development or multiple unit fabrication outsourcing experience and skills, model/test unit and pilot plant design/fabrication capability to determine limitations/operability and ALARA impact of new designs. Acceptance test procedure (ATP) testing capability of first unit production, both inhouse or outsourced, to assure operability, before introduction into a contaminated environment where the item may be lost if it does not work as designed.

Operator/Maintenance start-up training on mock-up unit prior to permanent installation is also provided for customers. EL staff have the training and practical experience to prepare fabrication specifications and offsite work job surveillance.

The ELs have an efficient record of responding to emergency field problems, e.g., recent 101-SY support and providing rapid turn-around design, machining, assembly, testing, debugging, redesign/alteration, process modification and analyses, mock-up testing, and operator training capability for either the PHMC.

\begin{tabular}{|l|c|l|}
\hline Post-Award Actions & $\begin{array}{c}\text { Scheduled } \\
\text { Date }\end{array}$ & Actionee(s) \\
\hline $\begin{array}{l}\text { 1. Provide an EL business review briefing to the PHMC major } \\
\text { subcontractors responsible for the major programmatic efforts } \\
\text { onsite. This would include TWRS, SNF and transition projects. }\end{array}$ & $8 / 12 / 96-8 / 30 / 96$ & LC Brown \\
\hline $\begin{array}{l}\text { 2. Tour PHMC through ELs for their understanding of capabilities } \\
\text { as part of their FY 1997 "make-buy" decision evaluation. }\end{array}$ & $8 / 12 / 96-8 / 30 / 96$ & DG Panther \\
\hline
\end{tabular}


5.2.2.2 Calibration Laboratory (AKA Standards Laboratory). The objective of the Standards Laboratory (SL) is to manage the Site's centralized Calibration and Repair Program to ensure safe, cost efficient and responsive services in support of all Hanford mission areas that will assure the necessary data quality and tractability for the Site's highly regulated environment.

The Standards Laboratory operates using a Self-Liquidating Pool concept. Funding sources are provided through users, approximately 500 plus customers annually, of the calibration program. Calibration services also extends to all other RL contractors and a few DOE approved outside groups. The Standards Laboratory provides physical and electrical calibration services directly traceable to the National Institute of Standards and Technology (NIST). The laboratory also provides pick-up and delivery, recall and records services to assist customers ensure their equipment is ready and able to provide accurate measurements and the necessary data quality traceability.

\begin{tabular}{|l|c|c|}
\hline \multicolumn{1}{|c|}{ Post-Award Actions } & $\begin{array}{c}\text { Scheduled } \\
\text { Date }\end{array}$ & Actionee(s) \\
\hline $\begin{array}{l}\text { 1. Provide a SL business review briefing to the PHMC major } \\
\text { subcontractors responsible for the major programmatic efforts } \\
\text { onsite for their understanding of capabilities as part of their FY } \\
\text { 1997 "make-buy" decision evaluation. }\end{array}$ & $8 / 12 / 96-8 / 30 / 96$ & JC Krogness \\
\hline
\end{tabular}

\subsection{INFORMATION AND COMMUNICATION}

Information and Communication includes the workscope currently performed by BCSR, as well as two areas performed in WHC.

BCSR provides RL and the Hanford Site contractors the ability to generate, store, access, and communicate information quickly, reliably, and cost effectively. BCSR plans, implements, and operates electronic communication, computing information management systems that enable effective operation of the Hanford Site.

The Transition Plan is compiled by major processes within BCSR (Sections 5.3.1 through 5.3.7), and the WHC areas (Sections 5.3.8 and 5.3.9). To simplify the plan, several of the smaller processes or organizations were consolidated into Section 5.3.3, "President's Office/Business Management/Technical Staff." A brief description of each area precedes the pre- and post-award actions. The primary pre-award deliverable is a baseline document for each area that fully documents the condition of the area as of July 1996.

\section{Assumptions}

- All active DOE clearances will remain in effect with the PHMC.

- Software and hardware nondisclosure agreements do not transfer, and will have to be reinitiated. 


\subsubsection{Operations and Support}

Boeing Operations and Support operates, maintains, and supports centralized and distributed computing and network infrastructure at Hanford.

\begin{tabular}{|c|c|c|}
\hline Pre-Award Actions & $\begin{array}{l}\text { Scheduled } \\
\text { Date }\end{array}$ & Actionee(s) \\
\hline $\begin{array}{l}\text { 1. Complete a baseline summary as defined by the outline in 5.3.7. } \\
\text { Prepare copies and briefing material. }\end{array}$ & $\begin{array}{c}5 / 31 / 96 \\
\text { Completed }\end{array}$ & SA Estey \\
\hline 2. Conduct 55 . & $\begin{array}{c}7 / 15 / 96 \\
\text { Completed }\end{array}$ & SA Estey \\
\hline $\begin{array}{l}\text { 3. Conduct and reconcile an inventory of all assigned property } \\
\text { including software. }\end{array}$ & $\begin{array}{c}5 / 31 / 96 \\
\text { Completed }\end{array}$ & SA Estey \\
\hline 4. Consolidate enterprise server implementation. & $\begin{array}{c}5 / 31 / 96 \\
\text { Completed }\end{array}$ & SA Estey \\
\hline $\begin{array}{l}\text { 5. Develop a plan for PHMC transition support including } \\
\text { computers and telephones. }\end{array}$ & $7 / 15 / 96$ & SA Estey \\
\hline Post-Award Actions & & \\
\hline 1. Applicable policies/procedures selected and in place. & $9 / 30 / 96$ & SA Estey \\
\hline 2. Identify performance measures for FY 1997. & $9 / 30 / 96$ & SA Estey \\
\hline 3. Finalize closeout agreements. & $9 / 30 / 96$ & SA Estey \\
\hline 4. Monitor contract performance during transition. & ongoing & SA Estey \\
\hline $\begin{array}{l}\text { 5. Finalize Memorandum of Understanding for services with other } \\
\text { contractors. }\end{array}$ & $9 / 30 / 96$ & SA Estey \\
\hline 6. Assign software licenses. & $9 / 30 / 96$ & SA Estey \\
\hline 7. Assign hardware/software maintenance agreements. & $9 / 30 / 96$ & SA Estey \\
\hline
\end{tabular}

\subsubsection{Information and Scientific Systems}

Information and Scientific Systems (ISS) provides planning, development, maintenance, and enhancement of automated information systems. It provides dedicated mainframe and microcomputer software applications support to programmatic, functional overhead, and general/administrative activities across the Hanford Site, as well as support to several identified national programs, using a wide range of hardware and software technologies.

\section{Assumption}

- The BCSR certification at Level 3 in the Software Engineering Institute Capability Maturity Model is not transferrable to the PHMC. 


\begin{tabular}{|c|c|c|}
\hline Pre-Award Actions & $\begin{array}{l}\text { Scheduled } \\
\text { Date }\end{array}$ & Actionee(s) \\
\hline $\begin{array}{l}\text { 1. Complete a baseline summary as defined by the outline in 5.3.7. } \\
\text { Prepare copies and briefing material. }\end{array}$ & $\begin{array}{c}5 / 31 / 96 \\
\text { Completed }\end{array}$ & RL Larson \\
\hline 2. Conduct 5S. & $7 / 15 / 96$ & RL Larson \\
\hline $\begin{array}{l}\text { 3. Conduct and reconcile an inventory of all assigned property } \\
\text { including software. }\end{array}$ & $\begin{array}{l}5 / 31 / 96 \\
\text { Completed }\end{array}$ & RL Larson \\
\hline \multicolumn{3}{|l|}{ Post-Award Actions } \\
\hline 1. Applicable policies/procedures selected and in place. & 9/30/96 & RL Larson \\
\hline 2. Identify performance measures for FY 1997. & 9/30/96 & RL Larson \\
\hline 3. Finalize closeout agreements. & 9/30/96 & RL Larson \\
\hline 4. Monitor contract performance during transition. & ongoing & RL Larson \\
\hline 5. Finalize MOU for services with other contractors. & $9 / 30 / 96$ & RL Larson \\
\hline
\end{tabular}

\subsubsection{President's Office/Technical Staff/Business Management}

The President's Office includes the BCSR President and Chief Information Officer (CIO), a small support staff, and Business Area Managers, acting as CIO's for the major WHC programs.

The Technical Staff supports the President's Office by conducting integrated planning and special studies; coordinating technology management activities including architecture, standards, and technology infusion; and managing complex cross-functional projects. The Technical Staff is managed by BCSR's Chief Engineer.

The Business Area Managers are members of the senior staff responsible for providing information management, planning, and integration services within assigned business areas. Business Area Managers focus on meeting the mission needs for effective electronic communication, computing, and information system capabilities.

Business Management's processes span the entire BCSR organization, providing centralized contract management, budget and pricing support, administrative liaison among contracting organizations, continuous quality improvement, and program planning and control functions.

\section{Assumption}

- The Boeing Learning Center is not transferrable to the PHMC.

\begin{tabular}{|c|c|c|}
\hline औ Pre-Award Actions & $\begin{array}{l}\text { Scheduled } \\
\text { Date }\end{array}$ & Actionee(s) \\
\hline $\begin{array}{l}\text { 1. Complete a baseline summary as defined by the outline in } 5.3 .7 \text {. } \\
\text { Prepare copies and briefing material. }\end{array}$ & $\begin{array}{l}5 / 31 / 96 \\
\text { Completed }\end{array}$ & JO Perkins \\
\hline
\end{tabular}




\begin{tabular}{|l|c|l|}
\hline \multicolumn{1}{|c|}{ Pre-Award Actions } & Scheduled & \multicolumn{1}{|c|}{ Actionee(s) } \\
\hline 2. Conduct 5S. & $7 / 15 / 96$ & JO Perkins \\
\hline $\begin{array}{l}\text { 3. Conduct and reconcile an inventory of all assigned property } \\
\text { including software. }\end{array}$ & $\begin{array}{c}5 / 31 / 96 \\
\text { Completed }\end{array}$ & JO Perkins \\
\hline $\begin{array}{l}\text { 4. Update as necessary the Baseline Summary for all processes and } \\
\text { forward a copy to the Transition Manager. }\end{array}$ & $7 / 15 / 96$ & JO Perkins \\
\hline 5. Issue preliminary rates for FY 1997. & $7 / 10 / 96$ & Jo Perkins \\
\hline \multicolumn{1}{|c|}{ Post-Award Actions } & $8 / 15 / 96$ & jO Perkins \\
\hline 1. Submit SSPP. & $9 / 01 / 96$ & JO Perkins \\
\hline 2. Issue final rates for FY 1997. & $9 / 30 / 96$ & JO Perkins \\
\hline 3. Applicable policies/procedures selected and in place. & $9 / 30 / 96$ & JO Perkins \\
\hline 4. Identify performance measures for FY 1997. & $9 / 30 / 96$ & JO Perkins \\
\hline 5. Finalize closeout agreements. & $8 / 01 / 96$ & CM Riddell \\
\hline 6. Identify a closeout manager. & ongoing & JO Perkins \\
\hline 7. Monitor contract performance during transition. & $9 / 30 / 96$ & JO Perkins \\
\hline 8. Finalize MOU for services with other contractors. & & \\
\hline
\end{tabular}

\subsubsection{Documentation and Records Management}

Establish a Records Management and Document Control Program that provides document receipt, release, clearance, and distribution; records identification, inventory, scheduling, transfer, collection, indexing, and disposition; vault storage; classified and unclassified document control; site forms administration and design; special project files management; database development and management; oversight of technology development and integration for electronic records and documents; and company policy, plans, and procedure oversight to ensure company and agency level compliance with all requirements (National Archives and Records Administration, DOE, Ecology, State of Washington, TriParty Agreement) governing the records management and document control program at Hanford.

\section{Assumption}

- The assignment by DOE-RL of responsibility for the Records Holding Area to the PHMC or another contractor will be made during transition. 


\begin{tabular}{|c|c|c|}
\hline as & $\begin{array}{l}\text { Schedoled } \\
\text { Date }\end{array}$ & Actionee(s) \\
\hline $\begin{array}{l}\text { 1. Complete a baseline summary as defined by the outline in 5.3.7. } \\
\text { Prepare copies and briefing material. }\end{array}$ & $\begin{array}{c}5 / 31 / 96 \\
\text { Completed }\end{array}$ & CN Potter \\
\hline 2. Conduct $5 S$. & $\begin{array}{l}7 / 15 / 96 \\
\text { Completed }\end{array}$ & CN Potter \\
\hline 3. Conduct and reconcile a classified document inventory. & Deleted & \\
\hline $\begin{array}{l}\text { 4. Conduct and reconcile an inventory of all assigned property } \\
\text { including software. }\end{array}$ & $\begin{array}{l}5 / 31 / 96 \\
\text { Completed }\end{array}$ & CN Potter \\
\hline Post-Award Actions & & 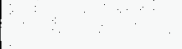 \\
\hline 1. Applicable policies/procedures selected and in place. & $9 / 30 / 96$ & CN Potter \\
\hline 2. Identify performance measures for FY 1997. & $9 / 30 / 96$ & CN Potter \\
\hline 3. Finalize closeout agreements. & $9 / 30 / 96$ & CN Potter \\
\hline 4. Monitor contract performance during transition. & ongoing & CN Potter \\
\hline 5. Finalize MOU for services with other contractors. & $9 / 30 / 96$ & CN Potter \\
\hline
\end{tabular}

\subsubsection{Media Management Systems}

Media Management Systems (MMS) offers multimedia solutions through integrated product development of computer-generated media products to meet the communication needs of the cleanup mission.

MMS develops, manages, and operates delivery systems using various media, including photographs, text, art, video, and sound. MMS also provides computer-generated, fully integrated information support and communication products and services, including technical illustration and graphic design, three-dimensional design, exhibit and display design, still photography, video production, technical writing and editing, custom photographic processing, digital imaging, macro writing, desktop publishing, internet publishing, and document analysis and data set tagging using Standard Generalized Markup Language (SGML).

MMS process analysis and automated work flow save time, money, and resources, and provide easy access to, and use of, information. MMS is helping today's users manage information for tomorrow. 


\begin{tabular}{|l|c|l|}
\hline \multicolumn{1}{|c|}{ Pre-Award Actions } & $\begin{array}{c}\text { Scheduled } \\
\text { Date }\end{array}$ & Actionee(s) \\
\hline $\begin{array}{l}\text { 1. Complete a baseline summary as defined by the outline in 5.3.7. } \\
\text { Prepare copies and briefing material. }\end{array}$ & $\begin{array}{c}5 / 31 / 96 \\
\text { Completed }\end{array}$ & NE Kenny \\
\hline $\begin{array}{l}\text { 2. Conduct 5S. } \\
\text { 3. Conduct and reconcile an inventory of all assigned property } \\
\text { including software. }\end{array}$ & $\begin{array}{c}5 / 31 / 96 \\
\text { Completed }\end{array}$ & NE Kenny \\
\hline $\begin{array}{l}\text { 4. Identify and inventory all physical and electronic information } \\
\text { repositories. }\end{array}$ & $\begin{array}{c}5 / 31 / 96 \\
\text { Completed }\end{array}$ & NE Kenny \\
\hline $\begin{array}{l}\text { 2. Award Actions } \\
\text { 1. Applicable policies/procedures selected and in place. }\end{array}$ & $9 / 30 / 96$ & NE Kenny \\
\hline $\begin{array}{l}\text { 2. Identify performance measures for FY 1997. } \\
\text { pages, etc. }\end{array}$ & $9 / 30 / 96$ & NE Kenny \\
\hline 3. Finalize closeout agreements. & $9 / 30 / 96$ & NE Kenny \\
\hline 4. Monitor contract performance during transition. & $9 / 30 / 96$ & NE Kenny \\
\hline 5. Finalize MOU for services with other contractors. & $9 / 30 / 96$ & NE Kenny \\
\hline $\begin{array}{l}\text { 6. Idencify redundant manuals, guides, and procedures that need to } \\
\text { be consolidated/integrated. }\end{array}$ & $9 / 30 / 96$ & NE Kenny \\
\hline 7. Establish plan for printing/distribution of information with & NE Kenny \\
\hline
\end{tabular}

\subsubsection{Telecommunications and Network Engineering}

The Telecommunications and Network Engineering Process plans, evaluates, designs, integrates, implements, and upgrades voice, video, data network, radio and computer systems. This process is responsible for technology infusion and upgrades to (1) Physical Infrastructure (i.e., inside and outside cable plant, facilities (building, electrical, UPS), site fiber, infrastructure grid, and GIS formatted database), (2) Operation and Control Systems (i.e., logical and physical drawings, cable assignment systems, software libraries, billing/usage tracking systems, monitoring systems, and asset management systems), and (3) Logical Systems (i.e., electronics - bridges, routers, concentrators, microwave, PAX, paging radio base stations; operating software - MS NTAS, WFW, OS2/Lanman, protocols, name services, domain controllers; client/server software services - MS SQL SVR, Oracle, Sybase SQL SVR; applications [i.e., voice mail, electronic mail, alpha numeric paging, software distribution, office automation applications]). This process additionally provides technical oversight of third party service-provider contracts, ADP and Telecommunications Information and Work Flow Management. 
WHC-SP-1181 Rev. 1

\begin{tabular}{|c|c|c|}
\hline Pre-Award Actions & Scheduled & Actionee(s) \\
\hline $\begin{array}{l}\text { 1. Complete a baseline summary as defined by the outine in 5.3.7. } \\
\text { Prepare copies and briefing material. }\end{array}$ & $\begin{array}{l}5 / 31 / 96 \\
\text { Completed }\end{array}$ & RE Harding \\
\hline 2. Conduct $5 \mathrm{~S}$. & $\begin{array}{l}7 / 15 / 96 \\
\text { Completed }\end{array}$ & RE Harding \\
\hline $\begin{array}{l}\text { 3. Conduct and reconcile an inventory of all assigned property } \\
\text { including software. }\end{array}$ & $\begin{array}{c}5 / 31 / 96 \\
\text { Completed }\end{array}$ & RE Harding \\
\hline Post-Apard Actions & & \\
\hline 1. Applicable policies/procedures selected and in place. & $9 / 30 / 96$ & RE Harding \\
\hline 2. Identify performance measures for FY 1997. & $9 / 30 / 96$ & RE Harding \\
\hline 3. Finalize closeout agreements. & 9/30/96 & RE Harding \\
\hline 4. Monitor contract performance during transition. & ongoing & RE Harding \\
\hline 5. Finalize MOU for services with other contractors. & 9/30/96 & RE Harding \\
\hline
\end{tabular}

\subsubsection{Baseline Summary Outline}

The following outline will be used for preparation of baseline summaries called for under each pre-award item \#1:

1.0 Program Guidance and Direction

1.1 Workscope Description

1.2 Performance Standards (Service/Commitment Levels)

1.3 Applicable DOE Orders, Directives, Letters, other

1.4 Identification of open Audit or other non-compliance items (HATS)

2.0 Program/Operations Management Systems

2.1 Automated Systems (hardware/software/applications)

2.2 Current Projects

2.3 Status of Acquisitions

2.4 Liquidation Methods

2.5 Budgets/Cost

3.0 Organization and Personnel

3.1 Workforce Characterization

3.2 EEO Statistics

4.0 Resource Management

4.1 Equipment

4.2 Facilities 
5.0 Interfaces

\subsection{RL Contacts}

5.2 WHC Contacts - Monitors, customers

5.3 Other Contractors, others

5.4 Memorandums of Understanding

6.0 Reporting Requirements
6.1 WHC
$6.2 \mathrm{RL}$
6.3 Other

7.0 Environmental, Safety and Health

7.1 Safety Program and Performance

7.2 Hazardous Waste Streams

7.3 Work Restrictions

8.0 Quality Assurance (if applicable)

8.1 Description and Implications

9.0 Safeguards and Security (if applicable)

9.1 Security Program and Performance

9.2 Classified Information

\subsubsection{Hanford Information System Inventory}

The Hanford Information System Inventory (HISI) is a valuable tool that provides a catalog of data about the information systems used at the Hanford Site. Registration of all Hanford systems in HISI is required by RL. HISI helps avoid redundancy of applications, and is a tool that can be used to belp define the site information architecture.

\begin{tabular}{|l|c|l|}
\hline \multicolumn{1}{|c|}{ Pre-Award Actions } & $\begin{array}{c}\text { Scheduled } \\
\text { Date }\end{array}$ & \multicolumn{1}{|c|}{ Actionee(s) } \\
\hline $\begin{array}{l}\text { 1. Update HISI data on key systems. Verify key systems with RL } \\
\text { POC. }\end{array}$ & $\begin{array}{c}5 / 20 / 96 \\
\text { Completed }\end{array}$ & $\begin{array}{l}\text { MK Britton } \\
\text { JM Knoll, Jr. }\end{array}$ \\
\hline $\begin{array}{l}\text { 2. Publish HISI System Document, documenting system design and } \\
\text { processes. }\end{array}$ & $\begin{array}{c}4 / 22 / 96 \\
\text { Completed }\end{array}$ & $\begin{array}{l}\text { MK Britton } \\
\text { M Knoll, Jr. }\end{array}$ \\
\hline $\begin{array}{l}\text { 3. Develop status of action items from the Hanford Information } \\
\text { System Inventory Anatysis of Potentialy Duplicate Functionality. }\end{array}$ & $\begin{array}{c}5 / 20 / 96 \\
\text { Completed }\end{array}$ & $\begin{array}{l}\text { MK Britton } \\
\text { JM Knoll, Jr. }\end{array}$ \\
\hline $\begin{array}{l}\text { Post-Award Actions } \\
\text { 1. Conduct a presentation covering the HISI database and process, } \\
\text { completed and open actions from the duplicate system study. }\end{array}$ & $9 / 15 / 96$ & MK Britton \\
\hline $\begin{array}{l}\text { 2. Provide listing of all registered systems in HISI, including list of } \\
\text { key systems. }\end{array}$ & $8 / 30 / 96$ & MK Britton \\
\hline
\end{tabular}




\subsubsection{Data Management and Standards}

Data Management guidance and Data Standards facilitate integration and help provide consistency in data usage among systems, functions and contractors. Data Management guidance is provided through strategic and operational tools and resources. RL requires compliance by all contractors to the Data Standards listed in the Data Standards Library.

\begin{tabular}{|c|c|c|}
\hline Pre-Award Actions & $\begin{array}{l}\text { Scheduled } \\
\text { Date }\end{array}$ & Actionee(s) \\
\hline 1. Update the Data Standards Library. & $\begin{array}{l}5 / 20 / 96 \\
\text { Completed }\end{array}$ & $\begin{array}{l}\text { MK Britton } \\
\text { JM Knoll, Jr. }\end{array}$ \\
\hline $\begin{array}{l}\text { 2. Develop a presentation describing Data Management concepts } \\
\text { and resources. }\end{array}$ & $\begin{array}{c}5 / 20 / 96 \\
\text { Completed }\end{array}$ & $\begin{array}{l}\text { MK Britton } \\
\text { JM Knoll, Jr. }\end{array}$ \\
\hline Post-Award Actions & $\because$ & 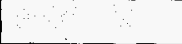 \\
\hline $\begin{array}{l}\text { 1. Conduct a presentation covering the Data Standards process and } \\
\text { Library. }\end{array}$ & $8 / 12 / 96-8 / 30 / 96$ & $\begin{array}{l}\text { MR Britton } \\
\text { MM Knoll, Jr. }\end{array}$ \\
\hline $\begin{array}{l}\text { 2. Conduct a presentation covering Data Management concepts and } \\
\text { resources. }\end{array}$ & $8 / 12 / 96-8 / 30 / 96$ & $\begin{array}{l}\text { MK Britton } \\
\text { MM Knoll, Jr. }\end{array}$ \\
\hline
\end{tabular}




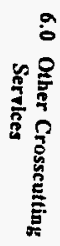

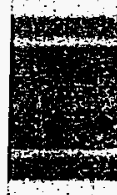


THT 


\subsection{OTHER CROSSCUTTING SERVICES}

\subsection{SAFEGUARDS AND SECURITY}

Safeguards and Security (SAS) maintains a standardized SAS program consistent with current DOE Orders, RL's Directives and company security policies and procedures. SAS program elements are designed to:

- Physically protect special nuclear material (SNM), classified matter, U.S. Government property, and personnel located within the confines of the Hanford Site and leased facilities.

- Maintain an armed protective force capable of responding to security alarms and incidents throughout the Site.

- Develop, maintain, and implement policies and procedures governing the use, control, and accountability of nuclear materials.

- Provide guidance, training, and procedures to ensure proper protection is afforded to classified matter and sensitive unclassified information which is processed, stored, transmitted, or discussed throughout the Site.

- Manage the Classification Program to assure the identification and proper classification of information, documents, or material.

- Manage the Declassification Project to meet the requirements of the RL LargeScale Declassification Review Plan.

- Provide technical and analytical services which assist DOE in establishing and maintaining uniform and cost-effective safeguards and security programs.

- Coordinate and implement new safeguards and security programs, systems, and upgrades for newly-constructed and existing facilities in order to protect Hanford interests.

- Provide protection program planning, evaluation, and validation testing to ensure that levels of protection and risk are appropriate to Site security interests that affect the protection of SNM and the health and safety of the public, onsite employees, and the environment.

- Develop, implement, and maintain policies and procedures governing the use and operation of government vehicles, roadways, and pedestrian walkways onsite in compliance with DOE Orders and Federal regulations. 
The following assumptions as noted below are assumed to be agreed upon by RL and the PHMC in order to effect an orderly and complete transition of SAS activities from WHC to the PHMC.

- Safeguards and Security

- For the near term, the PHMC will continue the current SAS manpower, organization, and budget.

- The SAS Training Matrix will be used to identify and monitor training requirements.

- Security Operations

- The schedule for asset protection surveys will continue using the current Asset Protection Agreement for high, medium, and low facilities.

- The planned security upgrade projects will continue.

- Key SAS documents such as controlled manuals, internal procedures, and MOUs will be adopted by the PHMC until normal revisions are required.

- $\quad$ Protective Force

- Protective force training plans will continue as scheduled to meet DOE requirements.

- $\quad$ Protective force organization, contingency plans, post orders, armament, and equipment will be continued.

- The current shift schedule and HGU collective bargaining agreement will continue (see Section 3.3).

- Information Security

- The PHMC will receive a favorable Foreign Ownership Control and Influence (FOCI) determination and a facility clearance, recorded on DOE D 5634.3, "Facility Data and Approval Request" by RL based on the criteria listed in Chapter V, DOE O 470.1.

- Appropriate PHMC personnel will receive necessary security clearances required to perform transition activities..

- A COMSEC account will be established for the PHMC to maintain/process COMSEC material. 


\section{WHC-SP-1181 Rev. 1}

- The PHMC will accept the Large Scale Declassification Review Project schedule and procedures.

- Personnel Security

A period of three months will be required for processing and obtaining a "L" clearance.

- A period of five months will be required for processing and obtaining a "Q" clearance.

- Employees with active DOE clearances at other DOE sites will have their clearance transferred upon approval of clearance justification at Hanford (assuming the clearance was not terminated by the host site).

- Employees with previously terminated clearances will be processed for reinstatement of the DOE clearance with processing and grant time determined by a number of variables, i.e., date of last investigation, date of termination, updated QSP, etc.

- Employees will be badged with a standard DOE security badge with the " 1 " access symbol (no clearance) pending receipt of the clearance.

- Protection Program Management

- The current Site Safeguards and Security Plan will be adopted by the PHMC until normal revision.

- The Vulnerability Assessment results and schedule will be approved until required revision.

- Corrective action plans for external and internal surveys will be accepted by the PHMC.

- Performance tests will be continued using the current test plans and schedules.

- Existing deviations will be continued until normal revision.

- Material Control and Accountability

- By August 15, 1996, the PHMC will have approved the Nuclear Material Transfer Plan to include: the availability of PHMC representatives to conduct the material inventories, acceptance of WHC NDA measurements, and the PHMC selectively verifying areas in question. 
- The Material Control and Accountability Plan will be adopted by the PHMC until normal revision.

- The current Material Balance Area configurations, categorization, inventory schedules and procedures will be continued.

- Technical Security

- Ongoing PFP system replacements will be continued as scheduled.

\begin{tabular}{|c|c|c|}
\hline Pre-Award Actions & $\begin{array}{l}\text { Scheduled } \\
\text { Date }\end{array}$ & Actionee(s) \\
\hline $\begin{array}{l}\text { 1. Using the pre-existing checklist, compile pre-existing } \\
\text { condition information on the facility and/or major equipment. } \\
\text { Conduct, or schedule as a separate post-award action(s), any } \\
\text { assessments required to adequately address all relevant items } \\
\text { on the checklist. Provide completed checklist to RL POC for } \\
\text { review. }\end{array}$ & $\begin{array}{c}\text { 6/15/96 } \\
\text { Completed }\end{array}$ & K Dolan \\
\hline $\begin{array}{l}\text { 2. Prepare SAS mission briefing to include manpower, budget, } \\
\text { and organizational roles. }\end{array}$ & $\begin{array}{c}5 / 15 / 96 \\
\text { Completed }\end{array}$ & FD Lee \\
\hline $\begin{array}{l}\text { 3. Prepare overview/copies of SAS Training Plan to include } \\
\text { Training Matrix. }\end{array}$ & $\begin{array}{c}5 / 15 / 96 \\
\text { Completed } \\
\end{array}$ & M Spracklen \\
\hline $\begin{array}{l}\text { 4. Prepare list of key SAS documents such as DOE Orders, } \\
\text { Manuals, and RLDDs. }\end{array}$ & $\begin{array}{c}5 / 15 / 96 \\
\text { Completed }\end{array}$ & D Haskins \\
\hline $\begin{array}{l}\text { 5. Identify space and make ready a functional office within } \\
\text { existing facilities for the PHMC. }\end{array}$ & $\begin{array}{c}7 / 15 / 96 \\
\text { Completed }\end{array}$ & K Dolan \\
\hline 6. Verify accuracy of various facility and vehicle inventories. & $7 / 15 / 96$ & K Dolan \\
\hline $\begin{array}{l}\text { 7. Prepare the Site Safeguards and Security Plan for transition } \\
\text { to new PHMC. }\end{array}$ & $7 / 31 / 96$ & $s$ Udell \\
\hline 8. Update/submit "Smart Book"/assemble key documents. & $7 / 15 / 96$ & VG Heiman \\
\hline Post-Award Actions & & \\
\hline 1. Conduct comprehensive SAS overview and mission briefing. & $\begin{array}{l}8 / 08 / 96- \\
8 / 19 / 96\end{array}$ & C Walton \\
\hline 2. Provide access to copies of assembled documents. & $8 / 05 / 96$ & VG Heiman \\
\hline $\begin{array}{l}\text { 3. Provide access to a copy of the new Site Safeguards and } \\
\text { Security Plan to new PHMC. }\end{array}$ & $8 / 05 / 96$ & S Udell \\
\hline
\end{tabular}

\subsubsection{Security Operations}

\begin{tabular}{|c|c|c|}
\hline Pre-Award Actions & $\begin{array}{l}\text { Scheduled } \\
\text { Date }\end{array}$ & Actionee(s) \\
\hline $\begin{array}{l}\text { 1. Collect listing and locations of all security areas (SCIF, PPA, } \\
\text { LA, PA). }\end{array}$ & $\begin{array}{l}5 / 15 / 96 \\
\text { Completed }\end{array}$ & D Haskins \\
\hline
\end{tabular}




\begin{tabular}{|c|c|c|}
\hline Pre-Award Actions & $\begin{array}{l}\text { Scheduled } \\
\text { Date }\end{array}$ & Actionee(s) \\
\hline $\begin{array}{l}\text { 2. Collect a sample copy of the Asset Protection Agreement and } \\
\text { a list of all high, medium, and low asset protection facilities } \\
\text { with a schedule of asset protection facilities. }\end{array}$ & $\begin{array}{c}5 / 15 / 96 \\
\text { Completed }\end{array}$ & D Haskins \\
\hline $\begin{array}{l}\text { 3. Collect list of ongoing or planned security projects or } \\
\text { upgrades. }\end{array}$ & $\begin{array}{c}5 / 15 / 96 \\
\text { Completed } \\
\end{array}$ & $\begin{array}{l}\text { D Haskins } \\
\text { VG Heiman } \\
\end{array}$ \\
\hline $\begin{array}{l}\text { 4. Prepare a list of key WHC SAS documents such as } \\
\text { Controlled Manuals, internal procedures, and MOUs. }\end{array}$ & $\begin{array}{c}5 / 15 / 96 \\
\text { Completed }\end{array}$ & D Haskins \\
\hline 5. Prepare a comprehensive briefing of SAS operations. & $7 / 15 / 96$ & $\begin{array}{l}\text { D Haskins } \\
\text { VG Heiman }\end{array}$ \\
\hline \multicolumn{3}{|l|}{ Post-Award Actions } \\
\hline 1. Conduct comprehensive briefing of SAS operations. & $\begin{array}{l}8 / 12 / 96- \\
8 / 30 / 96\end{array}$ & $\begin{array}{l}\text { D Haskins } \\
\text { VG Heiman }\end{array}$ \\
\hline 2. Conduct tours of SAS areas. & $\begin{array}{l}8 / 12 / 96- \\
8 / 30 / 96\end{array}$ & $\begin{array}{l}\text { D Haskins } \\
\text { VG Heiman }\end{array}$ \\
\hline $\begin{array}{l}\text { 3. Have available copies of key SAS documents, and offer to } \\
\text { PHMC for acceptance to continue operations. }\end{array}$ & $8 / 05 / 96$ & $\begin{array}{l}\text { D Haskins } \\
\text { VG Heiman }\end{array}$ \\
\hline $\begin{array}{l}\text { 4. Provide special orientation tour of security investigations to } \\
\text { include SEMS database and FY } 1996 \text { quarterly trends. }\end{array}$ & $9 / 16 / 96$ & G Curtis \\
\hline
\end{tabular}

\subsubsection{Protective Forces}

\begin{tabular}{|c|c|c|}
\hline Pre-Award Actions & $\begin{array}{l}\text { Scheduled } \\
\text { Date }\end{array}$ & Actionee(s) \\
\hline $\begin{array}{l}\text { 1. Provide overview/copies of Patrol Training Program to } \\
\text { include initial, recurring, firearms, Special Response Team } \\
\text { and Field Training Officer operations. }\end{array}$ & $\begin{array}{c}5 / 15 / 96 \\
\text { Completed }\end{array}$ & G McDowell \\
\hline $\begin{array}{l}\text { 2. Prepare briefing on Patrol Operations and contingency plans, } \\
\text { weapons and equipment, and organization. }\end{array}$ & $\begin{array}{c}5 / 15 / 96 \\
\text { Completed }\end{array}$ & G McDowell \\
\hline $\begin{array}{l}\text { 3. Prepare a list of post orders and prepare tours of all posts } \\
\text { and patrols. }\end{array}$ & $\begin{array}{c}5 / 15 / 96 \\
\text { Completed }\end{array}$ & G McDowell \\
\hline $\begin{array}{l}\text { 4. Prepare briefing on explosive detector dog program. (This } \\
\text { briefing included in item } \# 2 \text { above). }\end{array}$ & $\begin{array}{l}5 / 15 / 96 \\
\text { Completed }\end{array}$ & G McDowell \\
\hline $\begin{array}{l}\text { 5. Prepare inventory of unique protective force equipment such } \\
\text { as weapons, ammunition, night vision devices, } \\
\text { training/exercise aids, etc. }\end{array}$ & $\begin{array}{l}5 / 15 / 96 \\
\text { Completed }\end{array}$ & G McDowell \\
\hline $\begin{array}{l}\text { 6. Prepare listing of certified personnel such as instructors, } \\
\text { armorers, SPOII, etc. }\end{array}$ & $\begin{array}{l}5 / 15 / 96 \\
\text { Completed }\end{array}$ & G McDowell \\
\hline
\end{tabular}


WHC-SP-1181 Rev. 1

\begin{tabular}{|l|l|l|}
\hline \multicolumn{1}{|c|}{ Post-Award Actions } & $\begin{array}{c}\text { Schednled } \\
\text { Date }\end{array}$ & \\
\hline $\begin{array}{l}\text { 1. Conduct comprehensive briefing on protective force } \\
\text { operations and capabilities. }\end{array}$ & $\begin{array}{r}8 / 12 / 96- \\
8 / 30 / 96\end{array}$ & G McDowell \\
\hline 2. Conduct tours of all Patrol operations. & $\begin{array}{c}8 / 12 / 96- \\
8 / 30 / 96\end{array}$ & G McDowell \\
\hline 3. Provide access to list of all key protective force documents. & $8 / 05 / 96$ & G McDowell \\
\hline 4. Be prepared to facilitate inventory and turnover of unique \\
$\begin{array}{l}\text { protective force equipment such as weapons, ammunition, } \\
\text { night vision devices, training/exercise aids, etc. }\end{array}$ & $9 / 16 / 96$ & G MCDowell \\
\hline 5. Be prepared to facilitate introductions and tour of Benton & $9 / 16 / 96$ & G McDowell \\
\hline
\end{tabular}

\subsubsection{Information Security}

\begin{tabular}{|c|c|c|}
\hline Pre-Award Actions & $\begin{array}{l}\text { Scheduled } \\
\text { Date }\end{array}$ & Actionee(s) \\
\hline $\begin{array}{l}\text { 1. Prepare listing and location of classified repositories and } \\
\text { ADP systems to include names of custodians. }\end{array}$ & $\begin{array}{c}5 / 15 / 96 \\
\text { Completed }\end{array}$ & J Stowe \\
\hline 2. Prepare listing of unclassified sensitive AIS systems. & $\begin{array}{l}5 / 15 / 96 \\
\text { Completed }\end{array}$ & J Stowe \\
\hline $\begin{array}{l}\text { 3. Prepare listing of special position appointments such as } \\
\text { Classification Officer, Authorized Derivative Classifiers and } \\
\text { Declassifiers, Computer Security Site Manager, Computer } \\
\text { System Security Officer, Computer Protection Program } \\
\text { Manager, and Classified Matter Protection and Control } \\
\text { Coordinator, COMSEC, TSCM, TEMPEST. }\end{array}$ & $\begin{array}{c}5 / 15 / 96 \\
\text { Completed }\end{array}$ & $J$ Stowe \\
\hline $\begin{array}{l}\text { 4. Prepare for transfer of classified holdings to the new } \\
\text { contractor. }\end{array}$ & $\begin{array}{c}5 / 15 / 96 \\
\text { Completed }\end{array}$ & J Stowe \\
\hline $\begin{array}{l}\text { 5. Prepare for certification of possession or non-possession of } \\
\text { classified material. }\end{array}$ & $\begin{array}{c}5 / 15 / 96 \\
\text { Completed }\end{array}$ & I Stowe \\
\hline $\begin{array}{l}\text { 6. Establish an agreement with } R L \text { to transfer the Large Scale } \\
\text { Declassification Review project to the PHMC. }\end{array}$ & $\begin{array}{c}5 / 31 / 96 \\
\text { Completed }\end{array}$ & W Bernard \\
\hline $\begin{array}{l}\text { 7. Prepare comprehensive briefing of Information Security } \\
\text { activities. }\end{array}$ & $7 / 15 / 96$ & J Stowe \\
\hline $\begin{array}{l}\text { 8. Prepare status briefing for RL and the PHMC on the Large } \\
\text { Scale Declassification Review Project and arrange for project } \\
\text { transfer. }\end{array}$ & $7 / 15 / 96$ & W Bernard \\
\hline - Post-Award Actions & & \\
\hline $\begin{array}{l}\text { 1. Conduct comprehensive briefing of Information Security } \\
\text { activities. }\end{array}$ & $\begin{array}{l}8 / 12 / 96- \\
8 / 30 / 96\end{array}$ & J Stowe \\
\hline 2. Conduct tour of Information Security Areas. & $\begin{array}{l}8 / 12 / 96- \\
8 / 30 / 96\end{array}$ & J Stowe \\
\hline 3. Prepare certificates of possession and/or non-possession. & $9 / 23 / 96$ & J Stowe \\
\hline
\end{tabular}




\begin{tabular}{|c|c|c|}
\hline Post-Award Actions & $\begin{array}{l}\text { Schedaled } \\
\text { Date }\end{array}$ & Actionee(s) \\
\hline $\begin{array}{l}\text { 4. Provide guidance for facility inventories of classified } \\
\text { repositories/ADP systems, and transfer of classified/sensitive } \\
\text { unclassified systems. }\end{array}$ & $\begin{array}{l}8 / 19 / 96- \\
9 / 23 / 96\end{array}$ & J Stowe \\
\hline $\begin{array}{l}\text { 5. Conduct status briefing for } \mathrm{RL} \text { and the PHMC on the Large } \\
\text { Scale Declassification Review project and arrange for project } \\
\text { transfer. }\end{array}$ & $\begin{array}{l}8 / 12 / 96- \\
9 / 16 / 96\end{array}$ & W Bernard \\
\hline $\begin{array}{l}\text { 6. Provide access to assembled listings of position appointments, } \\
\text { classified repositories, classified/sensitive AIS systems. }\end{array}$ & $8 / 19 / 96$ & J Stowe \\
\hline
\end{tabular}

\subsubsection{Personnel Security and Central Badging}

\begin{tabular}{|c|c|c|}
\hline Pre-Award Actions & $\begin{array}{l}\text { Scheduled } \\
\text { Date }\end{array}$ & Actionee(s) \\
\hline $\begin{array}{l}\text { 1. Prepare for issuance of security badges for PHMC } \\
\text { employees. }\end{array}$ & $\begin{array}{c}5 / 15 / 96 \\
\text { Completed }\end{array}$ & W Hawkins \\
\hline 2. Prepare security awareness briefings for PHMC employees. & $7 / 15 / 96$ & W Hawkins \\
\hline \multicolumn{3}{|l|}{$\begin{array}{l}\text { 3. Coordinate with RL Personnel Security and monitor } \\
\text { activities, as requested, associated with Foreign Ownership, } \\
\text { Control, and Influence processing for clearance approval. }\end{array}$} \\
\hline \multirow{2}{*}{$\begin{array}{l}\text { 4. Prepare a comprehensive briefing on Personnel Security } \\
\text { activities to include pre-employment investigations, human } \\
\text { reliability, employee badging, and foreign national visits. } \\
\text { Post-Award Actions }\end{array}$} & $7 / 15 / 96$ & W Hawkins \\
\hline & & \\
\hline $\begin{array}{l}\text { 1. Initiate actions to facilitate security clearance processing for } \\
\text { PHMC employees. }\end{array}$ & $8 / 05 / 96$ & W Hawkins \\
\hline $\begin{array}{l}\text { 2. Be prepared to start issuance of site security badges to } \\
\text { PHMC employees. }\end{array}$ & $8 / 05 / 96$ & W Hawkins \\
\hline $\begin{array}{l}\text { 3. Be prepared to start providing security awareness briefings } \\
\text { for PHMC employees. }\end{array}$ & $8 / 05 / 96$ & W Hawkins \\
\hline $\begin{array}{l}\text { 4. Conduct comprehensive briefing and tour for personnel } \\
\text { security and badging operations. }\end{array}$ & $\begin{array}{l}8 / 12 / 96- \\
8 / 30 / 96\end{array}$ & W Hawkins \\
\hline $\begin{array}{l}\text { 5. Initiate transfer of required security clearances following } \\
\text { FOCl approval. }\end{array}$ & $9 / 30 / 96$ & W Hawkins \\
\hline
\end{tabular}

\subsubsection{Protection Program Management}

\begin{tabular}{|l|c|c|}
\hline Pre-Award Actions & Scheduled & Actionee(s) \\
\hline $\begin{array}{l}\text { 1. Prepare briefing on DoE Threat Guidance, Site Safeguards } \\
\text { and Security Plan, Vulnerability Assessment (VA) process } \\
\text { and schedule to include risk results for each area. }\end{array}$ & $\begin{array}{c}5 / 15 / 96 \\
\text { Completed }\end{array}$ & S Udell \\
\hline
\end{tabular}




\begin{tabular}{|c|c|c|}
\hline Pre-Award Actions & $\begin{array}{c}\text { Schedaled } \\
\text { Date }\end{array}$ & Actionee(s) \\
\hline $\begin{array}{l}\text { 2. Collect copy of last HQ DOE and RL/SAS audit and survey } \\
\text { to include corrective action plans. }\end{array}$ & $\begin{array}{c}5 / 15 / 96 \\
\text { Completed }\end{array}$ & S Udell \\
\hline $\begin{array}{l}\text { 3. Collect copies of most recent internal SAS survey reports to } \\
\text { include corrective action plans. }\end{array}$ & $\begin{array}{c}5 / 15 / 96 \\
\text { Completed }\end{array}$ & S Udell \\
\hline 4. Collect copies of most recent MBA audit reports. & $\begin{array}{c}5 / 15 / 96 \\
\text { Completed }\end{array}$ & S Udell \\
\hline $\begin{array}{l}\text { 5. Collect listing and schedule of performance tests and copies } \\
\text { of performance test reports. }\end{array}$ & $\begin{array}{c}5 / 15 / 96 \\
\text { Completed }\end{array}$ & S Udell \\
\hline 6. Collect listing of all current SAS deviations. & $\begin{array}{l}5 / 15 / 96 \\
\text { Completed }\end{array}$ & S Udell \\
\hline $\begin{array}{l}\text { 7. Collect copies of Site Safeguards and Security Plan and VA } \\
\text { reports. }\end{array}$ & $\begin{array}{c}5 / 15 / 96 \\
\text { Completed } \\
\end{array}$ & S Udell \\
\hline Post-Award Actions & & \\
\hline 1. Conduct comprehensive briefing of PPM activities. & $\begin{array}{l}8 / 12 / 96- \\
8 / 30 / 96\end{array}$ & s Udell \\
\hline 2. Conduct tour of PPM areas. & $\begin{array}{l}8 / 12 / 96 \\
8 / 30 / 96 \\
\end{array}$ & s Udell \\
\hline $\begin{array}{l}\text { 3. Provide access to key documents, to include classified } \\
\text { documents as appropriate (classified documents will require } \\
\text { approprate protection, clearances, and dissemination). }\end{array}$ & $8 / 05 / 96$ & $S$ Udell \\
\hline $\begin{array}{l}\text { 4. Conduct a risk assessment briefing for each area on the } \\
\text { existing risk levels and planned corrective/upgrade actions. }\end{array}$ & $\begin{array}{l}8 / 19 / 96 \\
9 / 16 / 96 \\
\end{array}$ & S Udell \\
\hline $\begin{array}{l}\text { 5. Allow PHMC employees to observe surveys and performance } \\
\text { tests conducted during transition period. }\end{array}$ & $9 / 30 / 96$ & S Udell \\
\hline
\end{tabular}

\subsubsection{Material Control and Accountability}

\begin{tabular}{|l|l|l|}
\hline \multicolumn{1}{|c|}{ Pre-Award Actions } & Scheduled \\
Date & Actionee(s) \\
\hline $\begin{array}{l}\text { 1. Prepare draft of Nuclear Material Transfer Plan for RL } \\
\text { review. }\end{array}$ & $\begin{array}{c}5 / 15 / 96 \\
\text { Completed }\end{array}$ & B Fitzpatrick \\
\hline $\begin{array}{l}\text { 2. Prepare copy of Material Control and Accountability Plan. } \\
\text { Completed }\end{array}$ & $\begin{array}{c}5 / 15 / 96 \\
\text { B Fitzpatrick }\end{array}$ \\
\hline $\begin{array}{l}\text { 3. Prepare listing of all Material Balance Areas to include } \\
\text { location, program, category of material, name of custodian } \\
\text { and MBA representative. }\end{array}$ & Bompleted \\
\hline
\end{tabular}




\begin{tabular}{|c|c|c|}
\hline Pre-Award Actions & $\begin{array}{l}\text { Scheduled } \\
\text { Date }\end{array}$ & Actionee(s) \\
\hline 4. Prepare schedule of MBA inventories for FY 1997. & $\begin{array}{l}5 / 15 / 96 \\
\text { Completed }\end{array}$ & B Fitzpatrick \\
\hline $\begin{array}{l}\text { 5. Prepare a comprehensive briefing on MC\&A activities to } \\
\text { include HANMAS and IAEA procedures. }\end{array}$ & $7 / 15 / 96$ & B Fitzpatrick \\
\hline Post-Award Actions & & \\
\hline $\begin{array}{l}\text { 1. Conduct comprehensive briefing on MC\&A activities to } \\
\text { include HANMAS and LAEA procedures. }\end{array}$ & $\begin{array}{l}8 / 12 / 96 \\
8 / 30 / 96\end{array}$ & B Fitzpatrick \\
\hline $\begin{array}{l}\text { 2. Conduct tours of MC\&A facilities to include tours of selected } \\
\text { MBAs. }\end{array}$ & $\begin{array}{l}8 / 12 / 96 \\
8 / 30 / 96\end{array}$ & B Fitzpatrick \\
\hline $\begin{array}{l}\text { 3. Provide access to copies of assembled documents, and offer } \\
\text { MCAP to PHMC for acceptance to continue operations. }\end{array}$ & $8 / 05 / 96$ & B Fitzpatrick \\
\hline $\begin{array}{l}\text { 4. Allow PHMC personnel to observe LAEA visits during the } \\
\text { transition period. }\end{array}$ & $\begin{array}{l}8 / 12 / 96 \\
9 / 23 / 96\end{array}$ & B Fitzpatrick \\
\hline $\begin{array}{l}\text { 5. Conduct joint inventory and complete transfer of special } \\
\text { nuclear material. }\end{array}$ & $\begin{array}{l}8 / 12 / 96 \\
9 / 30 / 96\end{array}$ & B Fitzpatrick \\
\hline
\end{tabular}

\subsubsection{Technical Security}

\begin{tabular}{|l|c|c|}
\hline \multicolumn{1}{|c|}{ Pre-Award Actions } & $\begin{array}{c}\text { Scheduled } \\
\text { Date }\end{array}$ & Actionee(s) \\
\hline 1. Prepare overview briefing of ongoing projects. & $\begin{array}{c}5 / 15 / 96 \\
\text { Completed }\end{array}$ & D Palmer \\
\hline 2. Organize tour and appropriate demonstrations of projects. & $\begin{array}{c}5 / 15 / 96 \\
\text { Completed }\end{array}$ & D Palmer \\
\hline Post-Award Actions & & \\
\hline 1. Conduct overview briefing of ongoing projects and how they & $\begin{array}{c}8 / 12 / 96- \\
8 / 30 / 96\end{array}$ & D Palmer \\
are integrated in the Site protection programs. & $8 / 12 / 96-$ & D Palmer \\
\hline 2. Conduct tour and demonstrations of Technical Security & $8 / 30 / 96$ & \\
areas/projects. & & \\
\hline
\end{tabular}

\subsection{HANFORD ANALYTICAL SERVICES PROGRAM}

The Hanford Analytical Services Program (HASP) will provide analytical, field support and process development services for the Hanford mission. HASP will manage and integrate onsite capabilities as well as commercially contracted services. Operations of onsite facilities will be organizationally independent from the management and evaluation function. HASP will oversee analytical facility operations to assure safe and effective use of resources and to assure cost effective services are provided. HASP will establish and maintain a Quality Assurance Program for all analytical services and provide sitewide integration of the Data Quality Objectives (DQO) process. 
HASP consists of the following elements:

- Site Sample Management Office

- Including integration of all site contractors

- High-level analytical services

- Low-level analytical services.

\subsubsection{Sample Management Office}

A centralized Hanford Sample Management Office (SMO) is required by DOE. The SMO will be responsible for developing site strategies for the Analytical Services Program, including managing site sampling and analytical needs and capabilities, consolidating commercial contracts, overseeing data quality and providing data management services. All Hanford contractors will be integrated under the SMO.

\begin{tabular}{|c|c|c|}
\hline Pre-Award Actions & $\begin{array}{l}\text { Scheduled } \\
\text { Date }\end{array}$ & Actionee(s) \\
\hline $\begin{array}{l}\text { 1. Issue letter to contractors formalizing the Sample } \\
\text { Management Working Group and directing SMO } \\
\text { Consolidation. }\end{array}$ & $\begin{array}{c}4 / 15 / 96 \\
\text { Completed }\end{array}$ & RP Carter \\
\hline $\begin{array}{l}\text { 2. Sample Management Working Group to recommend } \\
\text { Consolidation Strategy. }\end{array}$ & $\begin{array}{c}5 / 10 / 96 \\
\text { Completed } \\
\end{array}$ & CR Stroup \\
\hline $\begin{array}{l}\text { 3. Consolidation Strategy and pre-award actions approved } \\
\text { by RL. }\end{array}$ & $\begin{array}{c}5 / 15 / 96 \\
\text { Completed } \\
\end{array}$ & RP Carter \\
\hline $\begin{array}{l}\text { 4. Submit the SMO Consolidation Strategy to DOE-HQ } \\
\text { complying with } 2 / 29 / 96 \text { DOE-HQ directive. }\end{array}$ & $\begin{array}{c}5 / 21 / 96 \\
\text { Completed }\end{array}$ & RP Carter \\
\hline $\begin{array}{l}\text { 5. Memoranda of Understanding or equivalent approved } \\
\text { by ERC and PNNL for SMO consolidation. }\end{array}$ & DELETED & \\
\hline $\begin{array}{l}\text { 6. Develop a list of key Analytical Services Program } \\
\text { documentation. Provide to RL POC for review. }\end{array}$ & $\begin{array}{c}5 / 15 / 96 \\
\text { Completed } \\
\end{array}$ & AG King \\
\hline $\begin{array}{l}\text { 7. Assemble key Aralytical Services Program } \\
\text { documentation and identify its location, for use by the } \\
\text { PHMC. Includes development of a Analytical Services } \\
\text { Program "Smart Book." }\end{array}$ & $\begin{array}{l}6 / 01 / 96 \\
\text { Completed "Smart } \\
\text { Book" only } \\
\text { See itemn } \# 15\end{array}$ & AG King \\
\hline $\begin{array}{l}\text { 8. Prepare Analytical Services Program briefing for } \\
\text { presentation to PHMC (coordinate with RL POC.) }\end{array}$ & $\begin{array}{c}6 / 01 / 96 \\
\text { Completed }\end{array}$ & CR Stroup \\
\hline $\begin{array}{l}\text { 9. Develop a listing of key Analytical Services Program } \\
\text { individuals as POCs. Include facility and operations } \\
\text { POC list. }\end{array}$ & $\begin{array}{l}\text { 6/01/96 } \\
\text { Completed }\end{array}$ & AG King \\
\hline $\begin{array}{l}\text { 10. Develop listing and description of Analytical Services } \\
\text { Program subcontracts, including facility subcontracts. } \\
\text { Include any subcontracts still planned to be executed } \\
\text { prior to contract takeover. }\end{array}$ & $\begin{array}{l}\text { 6/01/96 } \\
\text { Completed }\end{array}$ & JL Deichman \\
\hline
\end{tabular}




\begin{tabular}{|c|c|c|}
\hline 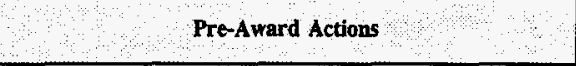 & $\begin{array}{l}\text { Scheduled } \\
\text { Date }\end{array}$ & Actionee(s) \\
\hline $\begin{array}{l}\text { 11. Develop listing and description of Analytical Services } \\
\text { Program databases, including facility databases. }\end{array}$ & $\begin{array}{c}6 / 01 / 96 \\
\text { Completed } \\
\end{array}$ & SL Brey \\
\hline $\begin{array}{l}\text { 12. Verify the accuracy of property assignments at the } \\
\text { Analytical Services Program organizational level and } \\
\text { correct any deficiencies. }\end{array}$ & $\begin{array}{l}\text { 6/01/96 } \\
\text { Completed }\end{array}$ & AG King \\
\hline $\begin{array}{l}\text { 13. Identify space and equipment within the Analytical } \\
\text { Services Program area and facilities for use by the } \\
\text { PHMC. }\end{array}$ & $\begin{array}{l}\text { 6/01/96 } \\
\text { Completed }\end{array}$ & AG King \\
\hline $\begin{array}{l}\text { 14. Using the pre-existing condition checklist, compile pre- } \\
\text { existing condition information on } 1706 \mathrm{KE} \text {. Conduct, or } \\
\text { schedule as a separate post-closure action, any } \\
\text { assessments required to adequately address all relevant } \\
\text { items on the checklist. Provide completed checklist to } \\
\text { RL POC for review. }\end{array}$ & $\begin{array}{c}6 / 15 / 96 \\
\text { Completed }\end{array}$ & AG King \\
\hline 15. Update/submit "Smart Book"/assemble key documents. & $7 / 15 / 96$ & AG King \\
\hline $\begin{array}{l}\text { 16. Verify } 1706 \mathrm{KE} \text { pre-existing condition data is current } \\
\text { and supplement as necessary. }\end{array}$ & $7 / 22 / 96$ & AG King \\
\hline 17. Ensure building assignments are correct in RLPS. & $7 / 22 / 96$ & AG King \\
\hline Post-Award Actions & & \\
\hline $\begin{array}{l}\text { 1. Make satellite space available and provide PHMC } \\
\text { access to the Analytical Services Program "Smart } \\
\text { Book." }\end{array}$ & $8 / 05 / 96$ & AG King \\
\hline $\begin{array}{l}\text { 2. Provide Analytical Services Prograrn briefing to the } \\
\text { PHMC. }\end{array}$ & $8 / 08 / 96-8 / 19 / 96$ & AG King \\
\hline $\begin{array}{l}\text { 3. PHMC review Pre-Existing Condition Assessment for } \\
\text { the 1706KE Facility, assess areas of uncertainty and } \\
\text { selectively verify other areas. }\end{array}$ & $8 / 12 / 96-9 / 16 / 96$ & PHMC \\
\hline $\begin{array}{l}\text { 4. Brief appropriate regulatory agencies and establish } \\
\text { method for transition of laboratory } \\
\text { accreditations/certifications to PHMC. }\end{array}$ & $9 / 01 / 96$ & RP Carter \\
\hline
\end{tabular}

\subsubsection{High Level Analytical Services}

The 222-S Laboratory and associated facilities provide high-activity analytical and development services. The 222-S Laboratory includes hot cells, gloveboxes shielded hoods for analysis of highly radioactive materials. 


\begin{tabular}{|c|c|c|}
\hline Pre-Award Actions & $\begin{array}{c}\text { Scheduled } \\
\text { Date } \\
\end{array}$ & Actionee(s) \\
\hline $\begin{array}{l}\text { 1. Prepare 222-S Laboratory complex briefing for the } \\
\text { PHMC (coordinate with RL POC). }\end{array}$ & $\begin{array}{c}6 / 01 / 96 \\
\text { Completed }\end{array}$ & AG King \\
\hline $\begin{array}{l}\text { 2. Identify key documents for the } 222-S \text { Laboratory } \\
\text { complex and review with RL. Provide list for } \\
\text { consolidation with the Analytical Services Program list. }\end{array}$ & $\begin{array}{c}5 / 01 / 96 \\
\text { Completed }\end{array}$ & AG King \\
\hline $\begin{array}{l}\text { 3. Assemble key documentation, or identify its location, } \\
\text { for use by the PHMC. Includes preparation of a } \\
222-S \text { Laboratory complex "Smart Book." }\end{array}$ & $\begin{array}{c}6 / 01 / 96 \\
\text { Completed "Smart } \\
\text { Book" only } \\
\text { See item } \# 18 \\
\end{array}$ & AG King \\
\hline $\begin{array}{l}\text { 4. Identify POCs for the } 222-S \text { Laboratory complex and } \\
\text { provide for consolidation with the Analytical Services } \\
\text { Program list. }\end{array}$ & $\begin{array}{c}5 / 17 / 96 \\
\text { Completed }\end{array}$ & AG King \\
\hline $\begin{array}{l}\text { 5. Develop a listing and description of subcontracts } \\
\text { supporting the 222-S Laboratory complex and } \\
\text { consolidate with the Analytical Services Program list. } \\
\text { lnclude any subcontracts planned to be executed prior } \\
\text { to contract takeover. }\end{array}$ & $\begin{array}{c}5 / 15 / 96 \\
\text { Completed }\end{array}$ & JL Deichman \\
\hline $\begin{array}{l}\text { 6. Using the pre-existing condition checklist, compile pre- } \\
\text { existing condition information on the 222-S Laboratory } \\
\text { complex. Conduct, or schedule as a separate post- } \\
\text { award action(s), any assessments required to adequately } \\
\text { address all relevant iterns on the checklist. Provide } \\
\text { completed checklist to RL POC for review. }\end{array}$ & $\begin{array}{c}6 / 15 / 96 \\
\text { Completed }\end{array}$ & GB Griffin \\
\hline $\begin{array}{l}\text { 7. Develop a list of open action iterns and commitments, } \\
\text { including status (Environmental, Tri-Party Agreement, } \\
\text { DNFSB as examples). Provide to RL POC for review. }\end{array}$ & $\begin{array}{c}6 / 01 / 96 \\
\text { Completed }\end{array}$ & JL Deichman \\
\hline $\begin{array}{l}\text { 8. Develop a list of } 222-S \text { specific mandatory training and } \\
\text { qualification requirements for employees. }\end{array}$ & $\begin{array}{c}6 / 01 / 96 \\
\text { Completed }\end{array}$ & AG King \\
\hline $\begin{array}{l}\text { 9. Develop a listing of information which constitutes the } \\
\text { "authorization basis" for the 222-S Laboratory. }\end{array}$ & $\begin{array}{c}6 / 01 / 96 \\
\text { Completed } \\
\end{array}$ & AG King \\
\hline $\begin{array}{l}\text { 10. Develop a description of ongoing and planned } \\
\text { construction projects and upgrades at the } \\
222-S \text { Laboratory complex. }\end{array}$ & $\begin{array}{l}\text { 6/01/96 } \\
\text { Completed }\end{array}$ & SL Brey \\
\hline $\begin{array}{l}\text { 11. Develop a listing of special agreernent services } \\
\text { provided to RL, other DOE sites, and other entities. }\end{array}$ & $\begin{array}{c}6 / 01 / 96 \\
\text { Completed }\end{array}$ & AG King \\
\hline $\begin{array}{l}\text { 12. Develop a listing and description of } 222-5 \text { Laboratory } \\
\text { complex databases. Include information on } \\
\text { LABCORE. Provide list to the Analytical Services } \\
\text { Program level for consolidation. }\end{array}$ & $\begin{array}{c}5 / 15 / 96 \\
\text { Completed }\end{array}$ & SL Brey \\
\hline $\begin{array}{l}\text { 13. Develop a list of current work order services or other } \\
\text { form of agreement to provide work for other } \\
\text { organizations, on and offsite. }\end{array}$ & $\begin{array}{c}6 / 01 / 96 \\
\text { Completed }\end{array}$ & JL Deichman \\
\hline $\begin{array}{l}\text { 14. Develop a list of current work order services or other } \\
\text { form of agreement to obtain work from other } \\
\text { organizations, on and offsite. }\end{array}$ & $\begin{array}{c}6 / 01 / 96 \\
\text { Completed }\end{array}$ & JL Deichman \\
\hline
\end{tabular}


WHC-SP-1181 Rev. 1

\begin{tabular}{|c|c|c|}
\hline Pre-Award Actions & $\begin{array}{l}\text { Scheduled } \\
\text { Date }\end{array}$ & Actionee(s) \\
\hline $\begin{array}{l}\text { 15. Develop a list of permits governing the } \\
222-S \text { Laboratory complex, and provide a description } \\
\text { of each as to where and how it applies. }\end{array}$ & $\begin{array}{l}\text { 6/01/96 } \\
\text { Completed }\end{array}$ & MJ Hall \\
\hline $\begin{array}{l}\text { 16. Verify the accuracy of property assignments for the } \\
222-S \text { Laboratory complex and correct any } \\
\text { deficiencies. }\end{array}$ & $\begin{array}{l}\text { 6/01/96 } \\
\text { Completed }\end{array}$ & AG King \\
\hline $\begin{array}{l}\text { 17. Develop a comprehensive list of open-ended } \\
\text { compliance issues at the 222-S Laboratory complex } \\
\text { (HATS as an example). Provide to RL POC for } \\
\text { review. }\end{array}$ & $\begin{array}{l}\text { 5/01/96 } \\
\text { Completed }\end{array}$ & AG King \\
\hline 18. Update/submit "Smart Book"/assemble key documents. & $7 / 15 / 96$ & AG King \\
\hline $\begin{array}{l}\text { 19. Verify } 222-S \text { Complex pre-existing condition data is } \\
\text { current and supplement as necessary. }\end{array}$ & $7 / 22 / 96$ & AG King \\
\hline Post-Award Actions & & $\therefore$ \\
\hline $\begin{array}{l}\text { 1. Provide a 222-S Laboratory complex tour to the } \\
\text { PHMC. }\end{array}$ & after $8 / 12 / 96$ & GB Griffin \\
\hline $\begin{array}{l}\text { 2. Provide any additional documentation or information } \\
\text { requested by the PHMC, if available, that was not } \\
\text { identified during the pre-award phase. }\end{array}$ & $8 / 30 / 96$ & AG King \\
\hline $\begin{array}{l}\text { 3. PHMC identify any recurring reports that they would } \\
\text { like to receive during transition. }\end{array}$ & $8 / 16 / 96$ & PHMC \\
\hline 4. Provide necessary training to PHMC personnel. & $8 / 05 / 96-8 / 20 / 96$ & AG King \\
\hline $\begin{array}{l}\text { 5. Provide an updated status of ongoing work at the } \\
222-S \text { Laboratory complex prior to contract turnover. }\end{array}$ & $9 / 16 / 96$ & JL Deichman \\
\hline $\begin{array}{l}\text { 6. PHMC selectively verify property and equipment } \\
\text { inventory of the } 222 \text {-S Laboratory complex. }\end{array}$ & $8 / 05 / 96-8 / 31 / 96$ & phmc \\
\hline $\begin{array}{l}\text { 7. Provide a categorized inventory of wastes at the } \\
\text { 222-S Laboratory complex. }\end{array}$ & $8 / 16 / 96$ & MJ Hall \\
\hline $\begin{array}{l}\text { 8. PHMC review Pre-Existing Condition Assessment for } \\
\text { the } 222-S \text { Laboratory, and assess areas of uncertainty } \\
\text { and selectively verify other areas. }\end{array}$ & $8 / 12 / 96-9 / 16 / 96$ & PHMC \\
\hline
\end{tabular}

\subsubsection{Low-Level Analytical Services}

The WSCF Complex provides low-level analytical, development and field services to Hanford. Emphasis is placed on faster turn around support to programs and includes process control and environmental analysis, field screening and analysis, stack counting and industrial hygiene services. 


\begin{tabular}{|c|c|c|}
\hline Pre-Award Actions & $\begin{array}{l}\text { Scheduled } \\
\text { Date }\end{array}$ & Actionee(s) \\
\hline $\begin{array}{l}\text { 1. Prepare WSCF complex briefing for the PHMC } \\
\text { (coordinate with RL POC). }\end{array}$ & $\begin{array}{l}6 / 01 / 96 \\
\text { Completed }\end{array}$ & AG King \\
\hline $\begin{array}{l}\text { 2. Identify key documents for the WSCF complex and } \\
\text { review with DOE-RL. Provide list for consolidation } \\
\text { with the Analytical Services Program list. }\end{array}$ & $\begin{array}{c}5 / 01 / 96 \\
\text { Completed }\end{array}$ & AG King \\
\hline $\begin{array}{l}\text { 3. Assembie key documentation, or identify its location, } \\
\text { for use by the PHMC. Includes preparation of a } \\
\text { WSCF complex "Smart Book." }\end{array}$ & $\begin{array}{c}6 / 01 / 96 \\
\text { Completed "Smart } \\
\text { Book" only } \\
\text { See item \#18 }\end{array}$ & AG King \\
\hline $\begin{array}{l}\text { 4. Identify POCs for the WSCF complex and provide for } \\
\text { consolidation with the Aralytical Services Program list. }\end{array}$ & $\begin{array}{l}5 / 17 / 96 \\
\text { Completed }\end{array}$ & AG King \\
\hline $\begin{array}{l}\text { 5. Develop a listing and description of subcontracts } \\
\text { supporting the WSCF complex and consolidate with the } \\
\text { Analytical Services Program list. Include any } \\
\text { subcontracts planned to be executed prior to contract } \\
\text { takeover. }\end{array}$ & $\begin{array}{c}5 / 15 / 96 \\
\text { Completed }\end{array}$ & JL Deichman \\
\hline $\begin{array}{l}\text { 6sing the pre-existing checklist, compile pre-existing } \\
\text { condition information on the WSCF complex. } \\
\text { Conduct, or schedule as a separate post-award. } \\
\text { action(s), any assessments required to adequately } \\
\text { address all relevant items on the checklist. Provide } \\
\text { completed checklist to RL POC for review. }\end{array}$ & $\begin{array}{c}6 / 15 / 96 \\
\text { Completed }\end{array}$ & CR Stroup \\
\hline $\begin{array}{l}\text { 7. Develop a list of open action items and commitments, } \\
\text { including status (Environmental, Tri-Party Agreement, } \\
\text { DNFSB as examples). Provide to RI POC for review. }\end{array}$ & $\begin{array}{c}\text { 6/01/96 } \\
\text { Completed }\end{array}$ & JL Deichman \\
\hline $\begin{array}{l}\text { 8. Develop a list of WSCF specific mandatory training } \\
\text { and qualification requirements for employees. }\end{array}$ & $\begin{array}{l}\text { 6/01/96 } \\
\text { Completed }\end{array}$ & CR Stroup \\
\hline $\begin{array}{l}\text { 9. Develop a listing of information which constitutes the } \\
\text { "authorization basis" for the WSCF. }\end{array}$ & $\begin{array}{l}6 / 01 / 96 \\
\text { Completed }\end{array}$ & CR Stroup \\
\hline $\begin{array}{l}\text { 10. Develop a description of ongoing and planned facility } \\
\text { upgrades at the WSCF complex. }\end{array}$ & $\begin{array}{l}6 / 01 / 96 \\
\text { Completed }\end{array}$ & SL Brey \\
\hline $\begin{array}{l}\text { 11. Develop a listing of special agreement services } \\
\text { provided to RI, other DOE sites, and other entities. }\end{array}$ & $\begin{array}{c}6 / 01 / 96 \\
\text { Completed }\end{array}$ & AG King \\
\hline $\begin{array}{l}\text { 12. Develop a listing and description of WSCF complex } \\
\text { databases. Include information on LABCORE. } \\
\text { Provide list for consolidation with the Analytical } \\
\text { Services Program list. }\end{array}$ & $\begin{array}{c}5 / 15 / 96 \\
\text { Completed }\end{array}$ & SL Brey \\
\hline $\begin{array}{l}\text { 13. Develop a list of current work order services or other } \\
\text { form of agreement to provide work for other } \\
\text { organizations, on and offsite. }\end{array}$ & $\begin{array}{c}\text { 6/01/96 } \\
\text { Completed }\end{array}$ & JL Deichman \\
\hline $\begin{array}{l}\text { 14. Develop a list of current work order services or other } \\
\text { form of agreement to obtain work from other } \\
\text { organizations, on and offsite. }\end{array}$ & $\begin{array}{c}\text { 6/01/96 } \\
\text { Completed }\end{array}$ & JL Deichman \\
\hline
\end{tabular}




\begin{tabular}{|c|c|c|}
\hline Pre-Award Actions: & $\begin{array}{l}\text { Scheduled } \\
\text { Date }\end{array}$ & Actionee(s) \\
\hline $\begin{array}{l}\text { 15. Develop a list of permits governing the WSCF } \\
\text { complex and provide a description of each as to where } \\
\text { and how it applies. }\end{array}$ & $\begin{array}{l}\text { 6/01/96 } \\
\text { Completed }\end{array}$ & MJ Hall \\
\hline $\begin{array}{l}\text { 16. Verify the accuracy of property assignments for the } \\
\text { WSCF complex and correct any deficiencies. }\end{array}$ & $\begin{array}{l}6 / 01 / 96 \\
\text { Completed }\end{array}$ & AG King \\
\hline $\begin{array}{l}\text { 17. Develop a comprehensive list of open-ended } \\
\text { compliance issues at the WSCF complex. (HATS as an } \\
\text { example). Provide to RL POC for review. }\end{array}$ & $\begin{array}{l}5 / 01 / 96 \\
\text { Completed }\end{array}$ & AG King \\
\hline 18. Update/submit "Smart Book"/assemble key documents. & $7 / 15 / 96$ & AG King \\
\hline $\begin{array}{l}\text { 19. Verify WSCF Complex pre-existing condition data is } \\
\text { current and supplement as necessary. }\end{array}$ & $7 / 22 / 96$ & AG King \\
\hline Post-Award Actions & $\therefore$ & $\because$ \\
\hline 1. Provide a WSCF complex tour to the PHMC. & after $8 / 12 / 96$ & CR Stroup \\
\hline $\begin{array}{l}\text { 2. Provide any additional documentation or information } \\
\text { requested by the PHMC, if available, that was not } \\
\text { identified during the pre-award phase. }\end{array}$ & $8 / 30 / 96$ & AG King \\
\hline $\begin{array}{l}\text { 3. PHMC identify any recurring reports that they would } \\
\text { like to receive during transition. }\end{array}$ & $8 / 16 / 96$ & PHMC \\
\hline 4. Provide necessary training to PHMC personnel. & $8 / 05 / 96-8 / 30 / 96$ & AG King \\
\hline $\begin{array}{l}\text { 5. Provide an updated status of ongoing work at the } \\
\text { WSCF complex prior to contract turnover. }\end{array}$ & $9 / 16 / 96$ & IL Deichman \\
\hline $\begin{array}{l}\text { 6. PHMC selectively verify property and equipment } \\
\text { inventory of the WSCF complex. }\end{array}$ & $8 / 05 / 96-8 / 31 / 96$ & PHMC \\
\hline $\begin{array}{l}\text { 7. Provide a categorized inventory of wastes at the WSCF } \\
\text { complex. }\end{array}$ & $8 / 16 / 96$ & MJ Hall \\
\hline $\begin{array}{l}\text { 8. PHMC review Pre-existing Condition Assessment for } \\
\text { WSCF Complex, and assess areas of uncertainty and } \\
\text { selectively verify other areas. }\end{array}$ & $8 / 12 / 96-9 / 10 / 96$ & PHMC \\
\hline
\end{tabular}

\subsection{HANFORD SITE TRAFFIC MANAGER}

The PHMC will need to establish and maintain sufficient organization, staffing and expertise to provide the following cross-cutting services that have significant safety and regulatory impact. This organization not only supports site missions but also provides unique technical support to DOE Headquarters and DOE contractors throughout the nationwide complex, and to other non-DOE entities through the Work For Others Program. 


\subsubsection{Hanford Site Transportation and Packaging Support}

The mission of this organization (Transportation and Packaging) is to manage a comprehensive transportation and packaging program that ensures safe, efficient and cost effective movement of materials, especially hazardous materials and hazardous waste, to, across, or from the Hanford Site in support of all site mission areas. In this capacity the organization acts as the sole designated shipping agent for DOE at the Hanford Site. This effort includes site operational support, packaging and transportation system development, and a full range of transportation and traffic management services, it does not include actual fleet operations or maintenance. All Transportation and Packaging activities are conducted in accordance with DOE Orders, State Regulations, applicable company directives and procedures, and Federal laws and regulations codified in Title 10 and Title 49 of the CFR.

The WHC-CM-2-14, "Hazardous Material Packaging and Shipping" manual, implements the site transportation safety program through overall policies and procedures and through Safety Analysis Reports for Packaging (SARPs) and Safety Evaluations for Packagings (SEPs) for individual packaging systems. The technical content of these documents evaluates site hazardous materials packaging in accordance with applicable Federal regulations and DOE directives. Specifically, these evaluations include package and transport system design, assessment of authorized payload, structural, thermal, shielding, criticality, risk assessment and containment analyses, and performance test data.

Package design and engineering support is provided for packaging and transportation systems development, procurement and maintenance, and package testing.

Facilities are provided specific operational support on package selection and use, classification of hazards, preparation of containers and shipments, and coordination of support services required for the movement.

Transportation and traffic management services are available to all Hanford Site contractors. Activities include support for the movement of general freight, hazardous materials, import and export, freight rate negotiations, carrier selection, freight bill prepayment auditing and processing, and the movement of household goods of new and transferring employees. As the designated shipping agent for DOE, Transportation Packaging manages all inbound and outbound hazardous material shipments and cross site transfers.

The following pre-award and post-award actions will be completed to ensure a complete and orderly turnover of the Transportation and Packaging organization to the PHMC.

\begin{tabular}{|l|c|c|}
\hline \multicolumn{1}{|c|}{ Pre-Award Actions } & Scheduled & Date \\
\hline $\begin{array}{l}\text { 1. Develop a list of key program documentation, } \\
\text { including regulatory drivers, workscope and charter, } \\
\text { customer and site contacts, staffing requirements and } \\
\text { funding sources/requirements. Provide to RL POC for } \\
\text { review. }\end{array}$ & $\begin{array}{c}5 / 15 / 96 \\
\text { Completed }\end{array}$ & WF Irvine \\
\hline
\end{tabular}




\begin{tabular}{|c|c|c|}
\hline Pre-Award Actions & $\begin{array}{l}\text { Scheduled } \\
\text { Date }\end{array}$ & Actionee(s) \\
\hline $\begin{array}{l}\text { 2. Assemble key program documentation, or identify its } \\
\text { location, for use by the PHMC. Includes development } \\
\text { of a program "Smart Book." }\end{array}$ & $\begin{array}{l}\text { 6/01/96 } \\
\text { Completed } \\
\text { "Smart Book" only } \\
\text { See item \#9 }\end{array}$ & WF Irvine \\
\hline $\begin{array}{l}\text { 3. Identify number of briefings, and prepare program } \\
\text { briefing(s) for presentation to PHMC. (Coordinate } \\
\text { number and content with RL POC.) }\end{array}$ & $7 / 15 / 96$ & WF Irvine \\
\hline 4. Develop a listing of Key program individuals as POCs. & $\begin{array}{c}6 / 01 / 96 \\
\text { Completed }\end{array}$ & WF Irvine \\
\hline $\begin{array}{l}\text { 5. Develop listing and description of program } \\
\text { subcontracts. Include any subcontracts still planned to } \\
\text { be executed prior to contract takeover. }\end{array}$ & $\begin{array}{l}6 / 01 / 96 \\
\text { Completed }\end{array}$ & WF Irvine \\
\hline 6. Develop listing and description of program databases. & $\begin{array}{c}6 / 01 / 96 \\
\text { Completed }\end{array}$ & WF Irvine \\
\hline $\begin{array}{l}\text { 7. Verify the accuracy of property assignments at the } \\
\text { program organizational level and correct any } \\
\text { deficiencies. }\end{array}$ & $\begin{array}{l}\text { 6/01/96 } \\
\text { Completed }\end{array}$ & WF Irvine \\
\hline $\begin{array}{l}\text { 8. Identify space and equipment within facility for use by } \\
\text { the PHMC. }\end{array}$ & $\begin{array}{c}6 / 01 / 96 \\
\text { Completed }\end{array}$ & WF Irvine \\
\hline 9. Update/submit "Smart Book"/assemble key documents. & $7 / 15 / 96$ & WF Irvine \\
\hline Post-Award Actions & 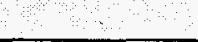 & \\
\hline $\begin{array}{l}\text { 1. Provide the PFMC access to the key program } \\
\text { documentation to include listing of POCs, listing of } \\
\text { subcontracts, etc. Provide copies upon request. }\end{array}$ & $8 / 05 / 96$ & WF Irvine \\
\hline 2. Provide space and services for use by the PHMC. & $8 / 05 / 96$ & WF Irvine \\
\hline 3. Provide program briefing(s) to the PHMC. & $8 / 12 / 96-8 / 30 / 96$ & WF Irvine \\
\hline $\begin{array}{l}\text { 4. Prepare and deliver briefing on how services are } \\
\text { provided across the site between multiple site facilities } \\
\text { and contractors, and identify strategies for } \\
\text { improvement. }\end{array}$ & $8 / 12 / 96-8 / 30 / 96$ & WF Irvine \\
\hline 5. Provide program level tour to the PHMC. & $8 / 12 / 96-8 / 30 / 96$ & WF Irvine \\
\hline $\begin{array}{l}\text { 6. Prepare and deliver management briefing on strategies } \\
\text { to leverage Transportation and Packaging expertise } \\
\text { through the WFO Program or outsourcing } \\
\text { (privatization). }\end{array}$ & $8 / 12 / 96-8 / 30 / 96$ & WF Irvine \\
\hline
\end{tabular}


WHC-SP-1181 Rev. 1

\begin{tabular}{|l|c|l|}
\hline \multicolumn{1}{|c|}{ Post-Award Actions } & $\begin{array}{c}\text { Scheduled } \\
\text { Date }\end{array}$ & Actionee(s) \\
\hline $\begin{array}{l}\text { 7. Perform a joint program level property and equipment } \\
\text { inventory with PHMC personnel. }\end{array}$ & by $9 / 16 / 96$ & WF Irvine \\
\hline $\begin{array}{l}\text { 8. Prepare a white paper describing how services are } \\
\text { provided across the site between multiple site facilities } \\
\text { and contractors, and identify strategies for } \\
\text { improvement. }\end{array}$ & $9 / 15 / 96$ & WF Irvine \\
\hline $\begin{array}{l}\text { 9. Prepare a white paper discussing strategies to leverage } \\
\text { transportation and packaging expertise prior to and } \\
\text { after contract award. }\end{array}$ & $9 / 15 / 96$ & WF Irvine \\
\hline
\end{tabular}

\subsubsection{DOE Headquarters EM Transportation Program Support}

The objective of this activity is to leverage the expertise and experience of the Transportation and Packaging staff to provide technical support to the DOE-wide transportation program managed by DOE-HQ EM-76. Related products and services provided are as follows.

Transportation Logistics - motor carrier evaluations, transportation plans for unique shipment campaigns, logistics services to smaller DOE sites, and site operational assessments upon request.

Transportation Training - safety and regulatory compliance training to DOE and contractor personnel, other state and Federal agency personnel, tribal and local officials, commercial carrier employees, and others.

Transportation Automation - development and implementation of automated systems to manage overall DOE transportation operations.

Regulatory Compliance and Technical Assessment - review and analysis of regulatory changes and high-visibility issues that affect DOE transportation management. Assessment teams are also provided to evaluate field element and contractor transportation operations.

Explosives Classification and DOE Explosives Program Management - explosive classification reviews, registration with DOT, site safety assessments, information management support, and other related activities.

Packaging Engineering and Analysis - design, analysis, and testing of packaging systems needed across the DOE. This includes assessment of packaging system needs throughout DOE.

Packaging Safety Assessment - risk assessment and NEPA documentation. Includes package proof-testing, confirmatory analysis, and technical design reviews. 


\section{WHC-SP-1181 Rev. 1}

Package Testing - DOT-7A Type A package testing program for the DOE complex. Both Type A and Type B package testing capability exists.

\subsubsection{Transportation and Packaging Work For Others Services}

The objective of this activity is to leverage the expertise and experience of the Transportation and Packaging staff to provide a number of products and services to other DOE contractors across the country within the framework of DOE's Work For Others. program, and to other government agencies and the commercial sector as authorized by DOE.

This activity benefits the site, the community and the entire DOE complex. The site benefits from the national experience of staff which translates to effective Hanford Site solutions. It preserves key personnel to meet site needs and benefits the community by retaining the core competency for potential business ventures. It benefits the DOE complex through effective utilization of resources and unique expertise.

Products and services are provided in four specific areas.

- Packaging and transportation system development (package design, analysis and testing).

- Operations support, shipment coordination and planning.

- Traffic and transportation management and automated management systems.

- Transportation safety and compliance training.

\subsection{ARCHITECT ENGINEER/CONSTRUCTION MANAGEMENT}

Scope of Support and Administrative Services - Furnish the administrative services described below, subject to such further detailed requirements as may be issued from time to time. These services are provided by ICF KH to support the ICF KH scope of work.

- Financial management - Including general and cost accounting, payroll, internal audit, and budget functions.

- Property management - Maintain a property management program for personal property and equipment assigned.

- Shop and warehouse operations - Operate centralized shops and warehouses in support of construction. Warehouse activities will generally be limited to receiving and distribution in support of specific project activities. 
- Facility custodianship - Perform maintenance and upgrading of facilities and equipment as required for assigned Government furnished buildings and grounds.

- Project management system - Provide a project management system for controlling the engineering and construction work including a project cost and schedule control system, an estimating system, and a scheduling system.

- Quality assurance - Perform those planned and systematic actions necessary to provide adequate confidence that a structure, facility, system, or component will perform satisfactorily in service. Quality assurance is to assure that components, systems, and processes are designed, developed, constructed, and tested according to sound engineering standards, quality practices, and technical specifications.

- Safety - Maintain effective environmental, safety, health, and emergency preparedness programs.

- Personnel management and labor relations - Provide work force mobilization and demobilization, labor-management relations, collective bargaining, etc. Maintenance of a qualified central and field staff commensurate with the workload.

- Procurement and Subcontracting - Solicit, award, and administer AE and construction subcontracts and agreements, including coordinating and expediting the work of suppliers/subcontractors. Develop and utilize a small/small disadvantaged business subcontracting program.

- Value Engineering (VE) - Maintain an effective VE program and perform VE analyses and studies.

\begin{tabular}{|l|c|c|}
\hline Pre-Award Actions & $\begin{array}{c}\text { Scheduled } \\
\text { Date }\end{array}$ & \multicolumn{1}{|c|}{ Actionee(s) } \\
\hline 1. Provide design authority and design agent staff listing. & $\begin{array}{c}6 / 01 / 96 \\
\text { Completed }\end{array}$ & $\begin{array}{l}\text { RL Benedetti } \\
\text { SE Gregg } \\
\text { CB Organ }\end{array}$ \\
\hline
\end{tabular}

\subsubsection{Architect Engineer Services}

Maintain and manage an engineering staff for the purpose of providing engineering support and such technical services as may be specifically required to support the Hanford Site.

Perform various onsite engineering services and such other related technical services as may be required and assigned. These engineering services will include Title I Preliminary Design Services, Title II - Definitive Design Services, and Title III Engineering and Inspection during Construction. In addition, perform engineering studies 
and conceptual design in support of anticipated construction projects; maintain an up to date and accurate engineering catalog file; maintain accurate and up to date Hanford Staridards and Specifications; and provide field survey services. Provide necessary administrative support services to accomplish this scope.

The preponderance of the work assigned may be generated via work orders from other onsite contractors. Accordingly, for each assigned task it will be necessary to work with, receive direction and guidance from, and maintain liaison with the other onsite contractors.

\begin{tabular}{|c|c|c|}
\hline Pre-Award Actions & $\begin{array}{l}\text { Scheduled } \\
\text { Date }\end{array}$ & Actionee(s) \\
\hline $\begin{array}{l}\text { 1. Identify number of briefings, and prepare briefing(s) } \\
\text { for the PHMC. Provide for RL POC review. }\end{array}$ & $7 / 15 / 96$ & $\begin{array}{l}\text { CD Maxson } \\
\text { CB Organ }\end{array}$ \\
\hline 2. Identify key documents. Provide for RL POC review. & $\begin{array}{l}5 / 01 / 96 \\
\text { Completed }\end{array}$ & $\begin{array}{l}\text { CD Maxson } \\
\text { CB Organ } \\
\text { MD Olsen }\end{array}$ \\
\hline $\begin{array}{l}\text { 3. Assemble key documentation, or identify its location, } \\
\text { for use by the PHMC. Includes preparation of a } \\
\text { "Smart Book," to include information develop in the } \\
\text { following actions. }\end{array}$ & $\begin{array}{l}6 / 01 / 96 \\
\text { Cormpleted "Smart } \\
\text { Book" Only } \\
\text { See new item \#18 }\end{array}$ & $\begin{array}{l}\text { CD Maxson } \\
\text { CB Organ }\end{array}$ \\
\hline $\begin{array}{l}\text { 4. Identify POCs and input to consolidated } \\
\text { program/project listing. }\end{array}$ & $\begin{array}{c}5 / 15 / 96 \\
\text { Completed }\end{array}$ & CD Maxson \\
\hline $\begin{array}{l}\text { 5. Develop a listing and description of supportive } \\
\text { subcontracts and input to the consolidated listing. } \\
\text { Include any subcontracts planned to be executed prior } \\
\text { to contract takeover. }\end{array}$ & $\begin{array}{c}5 / 15 / 96 \\
\text { Completed }\end{array}$ & $\begin{array}{l}\text { CD Maxson } \\
\text { CB Organ }\end{array}$ \\
\hline $\begin{array}{l}\text { 6. Using the pre-existing checktist, compile pre-existing } \\
\text { condition information. Conduct, or schedule as a } \\
\text { separate post-award action(s), any assessments required } \\
\text { to adequately address all relevant items on the } \\
\text { checktist. Provide completed checklist for RL POC } \\
\text { review. }\end{array}$ & $\begin{array}{l}\text { 6/15/96 } \\
\text { Completed }\end{array}$ & $\begin{array}{l}\text { CD Maxson } \\
\text { CB Organ } \\
\text { BJ Dixon }\end{array}$ \\
\hline $\begin{array}{l}\text { 7. Develop a tist of open action items and commitments, } \\
\text { including status. Provide to WHC for RL review. }\end{array}$ & $\begin{array}{l}\text { 6/01/96 } \\
\text { Completed }\end{array}$ & $\begin{array}{l}\text { CD Maxson } \\
\text { CB Organ } \\
\text { JH Arslanian } \\
\text { MD Olsen }\end{array}$ \\
\hline $\begin{array}{l}\text { 8. Develop a list of specific mandatory training and } \\
\text { qualification requirements for employees. }\end{array}$ & $\begin{array}{c}6 / 01 / 96 \\
\text { Completed }\end{array}$ & $\begin{array}{l}\text { CD Maxson } \\
\text { CB Organ }\end{array}$ \\
\hline 9. Develop a description of ongoing and planned projects. & $\begin{array}{c}\text { 6/01/96 } \\
\text { Completed }\end{array}$ & $\begin{array}{l}\text { CB Organ } \\
\text { CD Maxson }\end{array}$ \\
\hline $\begin{array}{l}\text { 10. Develop a listing of special agreement services } \\
\text { provided to RL, other DOE sites, and other entities. }\end{array}$ & $\begin{array}{l}\text { 6/01/96 } \\
\text { Completed }\end{array}$ & $\begin{array}{l}\text { CD Maxson } \\
\text { CB Organ }\end{array}$ \\
\hline $\begin{array}{l}\text { 11. Develop a listing and description of databases. Provide } \\
\text { for consolidation include developed software. }\end{array}$ & $\begin{array}{l}5 / 15 / 96 \\
\text { Completed }\end{array}$ & $\begin{array}{l}\text { CB Organ } \\
\text { MD Olsen }\end{array}$ \\
\hline
\end{tabular}




\begin{tabular}{|c|c|c|}
\hline Pre-Award Actions & $\begin{array}{l}\text { Scheduled } \\
\text { Date }\end{array}$ & Actionee(s) \\
\hline $\begin{array}{l}\text { 12. Develop a list of current work order services or ather } \\
\text { form of agreement to provide work for other } \\
\text { organizations, on and offsite. }\end{array}$ & $\begin{array}{l}\text { 6/01/96 } \\
\text { Completed }\end{array}$ & $\begin{array}{l}\text { CB Organ } \\
\text { CL Stice }\end{array}$ \\
\hline $\begin{array}{l}\text { 13. Develop list of accountability and litigation issues and } \\
\text { provide to WHC. }\end{array}$ & $\begin{array}{c}6 / 01 / 96 \\
\text { Completed }\end{array}$ & MD Olsen \\
\hline 14. Develop a list of patents and inteliectual property. & $\begin{array}{l}6 / 01 / 96 \\
\text { Completed }\end{array}$ & $\begin{array}{l}\text { CD Maxson } \\
\text { MD Olsen } \\
\text { CB Organ }\end{array}$ \\
\hline $\begin{array}{l}\text { 15. Develop a list of current work order services or other } \\
\text { form of agreement to obtain work from other } \\
\text { organizations, on and offsite. }\end{array}$ & $\begin{array}{l}6 / 01 / 96 \\
\text { Completed }\end{array}$ & $\begin{array}{l}\text { CB Organ } \\
\text { CL Stice }\end{array}$ \\
\hline $\begin{array}{l}\text { 16. Develop a list of licenses, permits, and code } \\
\text { certifications and provide a description of each as to } \\
\text { where and how it applies. }\end{array}$ & $\begin{array}{l}6 / 01 / 96 \\
\text { Completed }\end{array}$ & $\begin{array}{l}\text { CD Maxson } \\
\text { CB Organ }\end{array}$ \\
\hline $\begin{array}{l}\text { 17. Verify the accuracy of property assignments and } \\
\text { correct any deficiencies. }\end{array}$ & $\begin{array}{c}\text { 6/01/96 } \\
\text { Completed }\end{array}$ & CD Maxson \\
\hline $\begin{array}{l}\text { 18. Verify "Smart Book" to include any updates and } \\
\text { submit. }\end{array}$ & $7 / 15 / 96$ & CB Organ \\
\hline 19. Verify PEC package, modify/supplement as necessary. & $7 / 22 / 96$ & CB Organ \\
\hline Post-Award Actions & $\ldots$ & . \\
\hline 1. Provide briefing(s) to the PHMC. & $8 / 12 / 96-8 / 30 / 96$ & $\begin{array}{l}\text { CD Maxson } \\
\text { CB Organ }\end{array}$ \\
\hline 2. Provide tour(s) to the PHMC. & $8 / 12 / 96-8 / 30 / 96$ & $\begin{array}{l}\text { CD Maxson } \\
\text { CB Orgar }\end{array}$ \\
\hline $\begin{array}{l}\text { 3. Provide the PHMC access to key documentation, pre- } \\
\text { existing condition information, commitment data, } \\
\text { inventory data, etc. (as established in the pre-award } \\
\text { phase). Provide copies upon request. }\end{array}$ & $8 / 05 / 96$ & $\begin{array}{l}\text { CD Maxson } \\
\text { CB Organ }\end{array}$ \\
\hline $\begin{array}{l}\text { 4. Provide any additional documentation or information } \\
\text { requested by the PHMC, if available, that was not } \\
\text { identifed during the pre-award phase. }\end{array}$ & by $8 / 30 / 96$ & $\begin{array}{l}\text { CD Maxson } \\
\text { CB Organ }\end{array}$ \\
\hline $\begin{array}{l}\text { 5. PHMC identify any recurring reports that they would } \\
\text { like to receive during transition. }\end{array}$ & by $8 / 19 / 96$ & $\begin{array}{l}\text { CD Maxson } \\
\text { CB Organ }\end{array}$ \\
\hline 6. Provide necessary training to PHMC personnel. & $8 / 12 / 96-8 / 30 / 96$ & $\begin{array}{l}\text { CD Maxson } \\
\text { CB Organ }\end{array}$ \\
\hline $\begin{array}{l}\text { 7. Provide an updated status of ongoing work prior to } \\
\text { contract turnover. }\end{array}$ & by $9 / 30 / 96$ & $\begin{array}{l}\text { CD Maxson } \\
\text { CB Organ }\end{array}$ \\
\hline $\begin{array}{l}\text { 8. Perform a joint property and equipment inventory with } \\
\text { the PHMC. }\end{array}$ & by $9 / 16 / 96$ & $\begin{array}{l}\text { CD Maxson } \\
\text { CB Organ }\end{array}$ \\
\hline $\begin{array}{l}\text { 9. Provide a categorized inventory of wastes or hazardous } \\
\text { materials. }\end{array}$ & by $9 / 30 / 96$ & $\begin{array}{l}\text { CD Maxson } \\
\text { CB Organ }\end{array}$ \\
\hline
\end{tabular}


WHC-SP-1181 Rev. 1

\begin{tabular}{|l|c|l|}
\hline \multicolumn{1}{|c|}{ Post-Award Actions } & \multicolumn{1}{|c|}{$\begin{array}{c}\text { Scheduled } \\
\text { Date }\end{array}$} & Actioneefs) \\
\hline $\begin{array}{l}\text { 10. Update and disposition accountability and litigation } \\
\text { issues. }\end{array}$ & by 9/30/96 & $\begin{array}{l}\text { CD Maxson } \\
\text { CB Organ } \\
\text { MD Olsen }\end{array}$ \\
\hline $\begin{array}{l}\text { 11. Assist the PHMC in the conduct of pre-existing } \\
\text { condition assessments. }\end{array}$ & $8 / 12 / 96-9 / 16 / 96$ & $\begin{array}{l}\text { CD Maxson } \\
\text { CB Organ }\end{array}$ \\
\hline
\end{tabular}

\subsubsection{Construction Management Services}

- Furnish all labor, material, management, and supervision necessary for the performance of construction, construction management, maintenance, repair, and other construction related services. Provide necessary administrative support services to accomplish this scope.

- The construction work shall consist of two distinct types: construction and construction management.

- Perform work involving force account construction under radiological and nonradiological conditions and/or during scheduled and unscheduled plant shutdowns. These conditions often make scheduling difficult and preparation of definitive design and competitive subcontracting sometimes impractical. However, cost-type work assigned shall, when appropriate and to the extent possible, be subcontracted as competitive fixed-price subcontracts.

- In addition to industrial grade construction, the work will include fabrication of nuclear vessels, piping, and appurtenances which are subject to very stringent requirements. The work entails providing highly skilled craftsmen (electricians, pipefitters, boilermakers, millwright, sheetmetal workers, ironworkers, etc.) as necessary to support force account work.

- Perform construction management (CM) services on projects and provide dedicated CM staff and services on major projects which will include providing a wide range of professional services relating to management of a project during the predesign, design, and/or construction phases.

- The preponderance of the work assigned may be generated via work orders from other onsite contractors. Accordingly, for each assigned task it will be necessary to work with, receive direction and guidance from, and maintain liaison with the other onsite contractors.

\begin{tabular}{|l|c|c|c|}
\hline & Scheduled & Pre-Award Actions & Actionee(s) \\
\hline $\begin{array}{l}\text { 1. Identify number of briefings, and prepare briefing(s) } \\
\text { for the PHMC. Provide for RL POC review. }\end{array}$ & $7 / 15 / 96$ & LE Peterson \\
\hline
\end{tabular}




\begin{tabular}{|c|c|c|}
\hline Pre-Award Actions & $\begin{array}{r}\text { Scheduled } \\
\text { Date } \\
\end{array}$ & Actionee(s) \\
\hline 2. Identify key documents. Provide for RL POC review. & $\begin{array}{c}5 / 01 / 96 \\
\text { Completed }\end{array}$ & LE Peterson \\
\hline $\begin{array}{l}\text { 3. Assemble key docurnentation, or identify its location, } \\
\text { for use by the PHMC. Includes preparation of a } \\
\text { "Smart Book," to include information develop in the } \\
\text { following actions. }\end{array}$ & $\begin{array}{l}6 / 01 / 96 \\
\text { Completed "Smart } \\
\text { Book" only } \\
\text { See item \#16 }\end{array}$ & LE Peterson \\
\hline 4. Identify POCs and input to consolidated listing. & $\begin{array}{c}5 / 15 / 96 \\
\text { Completed }\end{array}$ & LE Peterson \\
\hline $\begin{array}{l}\text { 5. Develop a listing and description of supporting } \\
\text { subcontracts and input to consolidated listing. Include } \\
\text { any subcontracts planned to be executed prior to } \\
\text { contract takeover. }\end{array}$ & $\begin{array}{l}5 / 15 / 96 \\
\text { Completed }\end{array}$ & LE Peterson \\
\hline $\begin{array}{l}\text { 6. Using the pre-existing checklist, compile pre-existing } \\
\text { condition information. Conduct, or schedule as a } \\
\text { separate post-award action(s), any assessments required } \\
\text { to adequately address all relevant items on the } \\
\text { checklist. Provide completed checklist to WHC for } \\
\text { review and consolidation. }\end{array}$ & $\begin{array}{l}6 / 15 / 96 \\
\text { Completed }\end{array}$ & $\begin{array}{l}\text { LE Peterson } \\
\text { BJ Dixon }\end{array}$ \\
\hline $\begin{array}{l}\text { 7. Develop a list of open action items and commitments, } \\
\text { including status (Environmental, Tri-Party Agreement, } \\
\text { DNFSB as examples). }\end{array}$ & $\begin{array}{l}6 / 01 / 96 \\
\text { Completed }\end{array}$ & $\begin{array}{l}\text { LE Peterson } \\
\text { BJ Dixon }\end{array}$ \\
\hline $\begin{array}{l}\text { 8. Develop a list of specific mandatory training and } \\
\text { qualification requirements for employees. }\end{array}$ & $\begin{array}{c}6 / 01 / 96 \\
\text { Completed }\end{array}$ & $\begin{array}{l}\text { LE Peterson } \\
\text { BJ Dixon }\end{array}$ \\
\hline $\begin{array}{l}\text { 9. Develop a description of ongoing and planned } \\
\text { construction projects. }\end{array}$ & $\begin{array}{c}6 / 01 / 96 \\
\text { Completed }\end{array}$ & $\begin{array}{l}\text { LE Peterson } \\
\text { BJ Dixon }\end{array}$ \\
\hline $\begin{array}{l}\text { 10. Develop a listing of special agreement services } \\
\text { provided to RL, other DOE sites, and other entities. }\end{array}$ & $\begin{array}{c}\text { 6/01/96 } \\
\text { Completed }\end{array}$ & $\begin{array}{l}\text { LE Peterson } \\
\text { BJ Dixon } \\
\end{array}$ \\
\hline $\begin{array}{l}\text { 11. Develop a listing and description of databases. Provide } \\
\text { for consolidation. }\end{array}$ & $\begin{array}{l}5 / 15 / 96 \\
\text { Completed }\end{array}$ & LE Peterson \\
\hline $\begin{array}{l}\text { 12. Develop a list of current work order services or other } \\
\text { form of agreement to provide work for other } \\
\text { organizations, on and offsite. }\end{array}$ & $\begin{array}{l}\text { 6/01/96 } \\
\text { Completed }\end{array}$ & LE Peterson \\
\hline $\begin{array}{l}\text { 13. Develop a list of current work order services or other } \\
\text { form of agreement to obtain work from other } \\
\text { organizations, on and offsite. }\end{array}$ & $\begin{array}{l}\text { 6/01/96 } \\
\text { Completed }\end{array}$ & LE Peterson \\
\hline $\begin{array}{l}\text { 14. Develop a list of permits and provide a description of } \\
\text { each as to where and how it applies. }\end{array}$ & $\begin{array}{l}6 / 01 / 96 \\
\text { Completed }\end{array}$ & LE Peterson \\
\hline $\begin{array}{l}\text { 15. Verify the accuracy of property assignments and } \\
\text { correct any deficiencies. }\end{array}$ & $\begin{array}{c}6 / 01 / 96 \\
\text { Completed }\end{array}$ & LE Peterson \\
\hline $\begin{array}{l}\text { 16. Verify "Smart Book" to include any updates and } \\
\text { submit. }\end{array}$ & $7 / 15 / 96$ & LE Peterson \\
\hline 17. Verify PEC package, modify/supplement as neces & $7 / 22 / 96$ & LE Peterson \\
\hline
\end{tabular}




\begin{tabular}{|c|c|c|}
\hline Post-Award Actions & $\begin{array}{l}\text { Scheduled } \\
\text { Date }\end{array}$ & Actionee(s) \\
\hline 1. Provide briefing(s) to the PHMC. & $8 / 12 / 96-8 / 30 / 96$ & LE Peterson \\
\hline 2. Provide tour(s) to the PHMC. & $8 / 12 / 96-8 / 30 / 96$ & LE Peterson \\
\hline $\begin{array}{l}\text { 3. Provide the PHMC access to key documentation, pre- } \\
\text { existing condition information, commitment data, } \\
\text { inventory data, etc. (as established in the pre-award } \\
\text { phase). Provide copies upon request. }\end{array}$ & $8 / 05 / 96$ & LE Peterson \\
\hline $\begin{array}{l}\text { 4. Provide any additional documentation or information } \\
\text { requested by the PHMC, if available, that was not } \\
\text { identified during the pre-award phase. }\end{array}$ & by $8 / 30 / 96$ & LE Peterson \\
\hline $\begin{array}{l}\text { 5. PHMC identify any recurring reporss that they would } \\
\text { like to receive during transition. }\end{array}$ & by $8 / 19 / 96$ & LE Peterson \\
\hline 6. Provide necessary training to PHMC personnel. & $8 / 12 / 96-8 / 30 / 96$ & LE Peterson \\
\hline $\begin{array}{l}\text { 7. Provide an updated status of ongoing work prior to } \\
\text { contract turnover. }\end{array}$ & by $9 / 30 / 96$ & LE Peterson \\
\hline $\begin{array}{l}\text { 8. Perform a joint property and equipment inventory with } \\
\text { the PHMC. }\end{array}$ & by $9 / 16 / 96$ & LE Peterson \\
\hline $\begin{array}{l}\text { 9. Provide a categorized inventory of wastes or hazardous } \\
\text { materials. }\end{array}$ & by $9 / 30 / 96$ & LE Peterson \\
\hline $\begin{array}{l}\text { 10. Assist the PHMC in the conduct of pre-existing } \\
\text { condition assessments. }\end{array}$ & $8 / 12 / 96-9 / 16 / 96$ & LE Peterson \\
\hline
\end{tabular}

\subsection{CONSTRUCTION PROJECT MANAGEMENT}

Construction Projects typically involved capital funded activities involving design, procurement, and construction efforts governed by DOE 4700.1A "Project Management System" and covered by WHC-CM-6-2, "Project Management."

This scope includes the expense funded conceptual design phase where the project design criteria/requirements are established (scope baseline) and the associated cost and schedule baselines are developed. These three baselines are typically documented in the Conceptual Design Report (CDR) and are "validated" by RL (and HQ for Line Item projects, $>$ \$2.0M Total Estimated Cost [TEC]). Construction Projects are also responsible for the expense funded "Other Project Costs" (OPC) as reported on the Project Data Sheet approved by Congress. The TEC plus the OPC constitute the Total Project Cost (TPC).

Project Management involves either the onsite architect Engineer (ICF KH) or procurement of an offsite Architect-Engineer (A-E) through WHC Procurement. Typically, construction management services are provided by ICF $\mathrm{KH}$ for all projects except a very large project (MSA) which might involve a separate construction management contract strategy. 
An exception is a project funded with expense dollars, (e.g., an in-tank demonstration scope). A second significant scope of work are "project-like" activities that are not formal authorized projects but involve design and construction activities. Support activities such as maintenance of the project management process/procedures, financial management, forecasts, etc., are also required.

\begin{tabular}{|l|c|l|}
\hline \multicolumn{1}{|c|}{ Pre-Award Actions } & \multicolumn{1}{|c|}{$\begin{array}{c}\text { Scheduled } \\
\text { Date }\end{array}$} & Actionee(s) \\
\hline $\begin{array}{l}\text { 1. Prepare Overview Package containing all active formal } \\
\text { construction projects and supporting activities. }\end{array}$ & $7 / 15 / 96$ & RR Gadd \\
\hline 2. Prepare a Significant Issues List. & $7 / 15 / 96$ & RR Gadd \\
\hline $\begin{array}{l}\text { 3. Provide Project Management Process Road Map and significant } \\
\text { issues, if any. }\end{array}$ & $7 / 15 / 96$ & RR Gadd \\
\hline $\begin{array}{l}\text { 4. Provide list of all existing external contracts (e.g., A-E's, WHC } \\
\text { procurement or construction contracts, consultants). }\end{array}$ & $7 / 15 / 96$ & RR Gadd \\
\hline $\begin{array}{l}\text { 5. Provide accurate property assignments for each organization. } \\
\text { Post-Award Actions }\end{array}$ & $7 / 15 / 96$ \\
\hline $\begin{array}{l}\text { Completed } \\
\text { 1. Provide Project Reviews for key projects. }\end{array}$ & RR Gadd \\
\hline 2. Provide Site walk-through for projects in construction. & $8 / 12 / 96-8 / 30 / 96$ & RR Gadd \\
\hline
\end{tabular}

\subsection{ENVIRONMENTAL MONITORING AND SERVICES}

The following section address the scope of work as currently assigned to each contractor (M\&O, BHI, and PNNL), and do not reflect any potential transfer of scope between contractors.

\subsubsection{Hanford Technical Services}

Hanford Technical Services (HTS) conducts the RCRA Operational Monitoring (ROM) Program and provides technical expertise to support DOE Waste Management, Environmental, and Technology Development Programs. Services include: Well installation, monitoring, and maintenance, environmental sampling, geoscience expertise, environmental monitoring, geotechnical design and installation and geophysics monitoring/characterization. Pest Management (i.e., herbicide and pesticide program) is also provided and is addressed in Section 5.1.6.

\begin{tabular}{|l|l|l|l|}
\hline Pre-Award Actions & $\begin{array}{c}\text { Scheduled } \\
\text { Date }\end{array}$ & Actionee(s) \\
\hline 1. Update/submit HTS "Smart Book"/assemble key documents. & $7 / 15 / 96$ & RA Meznarich \\
\hline
\end{tabular}


WHC-SP-1181 Rev. 1

\begin{tabular}{|l|l|l|}
\hline \multicolumn{1}{|c|}{ Pre-Award Actions } & $\begin{array}{c}\text { Scheduled } \\
\text { Date }\end{array}$ & \multicolumn{1}{|c|}{ Actionee(s) } \\
\hline $\begin{array}{l}\text { 2. Ensure building assignments are correct in RLPS for all HTS } \\
\text { areas. }\end{array}$ & $7 / 22 / 96$ & RA Meznarich \\
\hline $\begin{array}{l}\text { 3. Verify pre-existing condition data is current and supplement } \\
\text { as necessary for all HTS areas. }\end{array}$ & $7 / 22 / 96$ & RA Meznarich \\
\hline
\end{tabular}

\subsubsection{RCRA Operational Monitoring Office. The RCRA Operational Monitoring} program office manages the ROM program activities, expense and capital equipment budgets, develops and status schedules and cost accounts, and administers subcontracts through task ordering agreements. The program office is the prime interface for the ROM program with the RL.

\begin{tabular}{|c|c|c|}
\hline Pre-Award Actions & $\begin{array}{l}\text { Scheduled } \\
\text { Date }\end{array}$ & Actionee(s) \\
\hline $\begin{array}{l}\text { 1. Identify key documents for the ROM program. (Provide a } \\
\text { listing of key program documents to RL for review). }\end{array}$ & $\begin{array}{c}5 / 01 / 96 \\
\text { Completed }\end{array}$ & FT Green \\
\hline 2. Obtain copies of key program documents and assemble. & $\begin{array}{c}5 / 15 / 96 \\
\text { Completed } \\
\end{array}$ & FT Green \\
\hline $\begin{array}{l}\text { 3. Identify number of briefings, and prepare program briefing(s) } \\
\text { for presentation to PHMC. (Coordinate number and content } \\
\text { with RL POC.) }\end{array}$ & $\begin{array}{l}6 / 01 / 96 \\
\text { Completed }\end{array}$ & FT Green \\
\hline $\begin{array}{l}\text { 4. Develop a listing of key program individuals as POCs, } \\
\text { including facility and operations personnel. }\end{array}$ & $\begin{array}{c}\text { 6/01/96 } \\
\text { Completed } \\
\end{array}$ & FT Green \\
\hline $\begin{array}{l}\text { 5. Develop listing and description of program databases, } \\
\text { including facility (or operations) databases. }\end{array}$ & $\begin{array}{c}\text { 6/01/96 } \\
\text { Completed } \\
\end{array}$ & FT Green \\
\hline Post-Award Actions & & \\
\hline $\begin{array}{l}\text { 1. Make the key program documents available to the PHMC. } \\
\text { Provide copies upon request. }\end{array}$ & $8 / 05 / 96$ & FT Green \\
\hline 2. Provide program briefing(s) to the PHMC. & $\begin{array}{l}8 / 12 / 96- \\
8 / 30 / 96\end{array}$ & FT Green \\
\hline
\end{tabular}

6.6.1.2 Geosciences. Geosciences is responsible for geochemistry and hydrochemistry activities conducted under RCRA and other projects. The organization ensures quality control and environmental protection requirements are met for sampling and analysis associated with groundwater monitoring and other programs. Other environmental and earth sciences related projects are conducted on a work order or contract basis. Services include field operations personnel and equipment, preparing reports, mapping and graphics services, seismic monitoring, and natural hazards analysis.

Groundwater management (GWM) supports design, installation and testing of groundwater monitoring wells and networks and provides technical services to support geologic, hydrologic, and geotechnical investigations. The group maintains a large data base and provides expertise in investigating, mapping, and documenting Hanford geology and 
hydrogeology. The group also assembles and reports groundwater data to regulatory agencies as required.

\begin{tabular}{|c|c|c|}
\hline Pre-Award Actions & $\begin{array}{c}\text { Scheduled } \\
\text { Date }\end{array}$ & Actionee(s) \\
\hline $\begin{array}{l}\text { 1. Prepare for a comprehensive briefing for the PHMC } \\
\text { describing all operational aspects of the organization. }\end{array}$ & $\begin{array}{c}5 / 01 / 96 \\
\text { Completed } \\
\end{array}$ & D Horton \\
\hline $\begin{array}{l}\text { 2. Identify all key documents relevant to the organization's } \\
\text { operations, including a listing of key facility and field } \\
\text { documents. }\end{array}$ & $\begin{array}{c}5 / 01 / 96 \\
\text { Completed }\end{array}$ & D Horton \\
\hline $\begin{array}{l}\text { 3. Obrain copies of key facility documents and assemble, or } \\
\text { identify their location for access. Develop operations "Smart } \\
\text { Book." }\end{array}$ & $\begin{array}{c}6 / 01 / 96 \\
\text { Completed } \\
\text { "Smart } \\
\text { Book" only } \\
\text { See item } \# 9 \\
\end{array}$ & D Horton \\
\hline 4. Identify points-of-contact for the organization. & $\begin{array}{c}5 / 15 / 96 \\
\text { Completed } \\
\end{array}$ & D Horton \\
\hline $\begin{array}{l}\text { 5. Using the pre-existing condition checklist, evaluate pre- } \\
\text { existing conditions. Conduct assessments as necessary to } \\
\text { adequately address the checklist. }\end{array}$ & $\begin{array}{c}6 / 15 / 96 \\
\text { Completed }\end{array}$ & D Horton \\
\hline $\begin{array}{l}\text { 6. Identify applicable milestones and commitments (e.g., Tri- } \\
\text { Party Agreement and Consent Order milestones), and } \\
\text { determine their status. Describe the form of the deliverable } \\
\text { and what constitutes completion. }\end{array}$ & $\begin{array}{c}5 / 15 / 96 \\
\text { Completed }\end{array}$ & D Horton \\
\hline $\begin{array}{l}\text { 7. Verify the accuracy of property assignments for the facility } \\
\text { and correct any deficiencies. }\end{array}$ & $\begin{array}{c}5 / 15 / 96 \\
\text { Completed }\end{array}$ & D Horton \\
\hline $\begin{array}{l}\text { 8. Verify the completeness of all RIDS and correct any } \\
\text { deficiencies. }\end{array}$ & $\begin{array}{c}5 / 15 / 96 \\
\text { Completed }\end{array}$ & D Horton \\
\hline 9. Update/submit "Smart Book"/assemble key documents. & $7 / 15 / 96$ & D Horton \\
\hline Post-Award Actions & & $\cdots$ \\
\hline $\begin{array}{l}\text { 1. Provide a cornprehensive briefing for the PHMC complete } \\
\text { with all facility, personnel, and organizational documentation. } \\
\text { Provide copies upon request. }\end{array}$ & $\begin{array}{l}8 / 12 / 96 \\
8 / 30 / 96\end{array}$ & D Horton \\
\hline $\begin{array}{l}\text { 2. Conduct a comprehensive tour of all facilities and field } \\
\text { operations relevant to the organization. }\end{array}$ & $\begin{array}{l}8 / 12 / 96 \\
8 / 30 / 96\end{array}$ & D Horton. \\
\hline $\begin{array}{l}\text { 3. Assist the PHMC in a survey of pre-existing conditions at the } \\
\text { facility. }\end{array}$ & $\begin{array}{l}8 / 12 / 96- \\
9 / 16 / 96 \\
\end{array}$ & D Horton \\
\hline $\begin{array}{l}\text { 4. Provide an update on work completed and work in progress } \\
\text { during the transition to the PHMC. }\end{array}$ & 9/15/96 & D Horton \\
\hline $\begin{array}{l}\text { 5. Complete a joint property and equipment inventory with the } \\
\text { PHMC. }\end{array}$ & by $9 / 16 / 96$ & D Horton \\
\hline $\begin{array}{l}\text { 6. Provide any additional documentation requested by the } \\
\text { PHMC. }\end{array}$ & $8 / 30 / 96$ & D Horton \\
\hline
\end{tabular}




\begin{tabular}{|l|c|c|}
\hline \multicolumn{1}{|c|}{ Post-Award Actions } & Scheduled & Actionee(s) \\
\hline 7. Provide necessary training to PHMC personnel. & Date & $8 / 12 / 96-$ \\
& $8 / 30 / 96$ & D Horton \\
\hline 8. Provide a categorized inventory of wastes. & $9 / 30 / 96$ & D Horton \\
\hline
\end{tabular}

6.6.1.3 Well Services. Well services plans, designs, and provides field support to install, refurbish, and abandon groundwater and vadose zone monitoring wells. The organization maintains a database of wells installed at Hanford and coordinates use of these wells. The database includes information on well design and construction, lithographic and stratigraphic logs, and responsible parties.

\begin{tabular}{|c|c|c|}
\hline 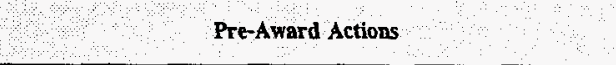 & $\begin{array}{l}\text { Scheduled } \\
\text { Date }\end{array}$ & Actionee(s) \\
\hline $\begin{array}{l}\text { 1. Prepare for a comprehensive briefing for the PHMC } \\
\text { contractor describing all operational aspects of the } \\
\text { organization. }\end{array}$ & $\begin{array}{c}5 / 01 / 96 \\
\text { Completed }\end{array}$ & MG Gardner \\
\hline $\begin{array}{l}\text { 2. Identify all key documents relevant to the organization's } \\
\text { operations, including a listing of key facility and field } \\
\text { documents. }\end{array}$ & $\begin{array}{c}5 / 01 / 96 \\
\text { Completed }\end{array}$ & MG Gardner \\
\hline $\begin{array}{l}\text { 3. Obtain copies of key facility documents and assemble, or } \\
\text { identify their location for access. Develop operations "Smart } \\
\text { Book." }\end{array}$ & $\begin{array}{l}6 / 01 / 96 \\
\text { Completed } \\
\text { "Smart } \\
\text { Book" only } \\
\text { See jtem \#9 }\end{array}$ & MG Gardner \\
\hline 4. Identify points-of-contact for the organization. & $\begin{array}{c}5 / 15 / 96 \\
\text { Completed }\end{array}$ & MG Gardner \\
\hline $\begin{array}{l}\text { 5. Using the pre-existing conditions checklist, evaluate pre- } \\
\text { existing conditions. Conduct assessments as necessary to } \\
\text { adequately address the checklist. }\end{array}$ & $\begin{array}{c}6 / 15 / 96 \\
\text { Completed }\end{array}$ & MG Gardner \\
\hline $\begin{array}{l}\text { 6. Identify applicable milestones and commitments (e.g., Tri- } \\
\text { Party Agreement and Consent Order milestones), and } \\
\text { determine their status. Describe the form of the deliverable } \\
\text { and what constitutes completion. }\end{array}$ & $\begin{array}{l}5 / 15 / 96 \\
\text { Completed }\end{array}$ & MG Gardner \\
\hline $\begin{array}{l}\text { 7. Verify the accuracy of property assignments for the facility } \\
\text { and correct any deficiencies. }\end{array}$ & $\begin{array}{c}5 / 15 / 96 \\
\text { Completed }\end{array}$ & MG Gardner \\
\hline $\begin{array}{l}\text { 8. Verify the completeness of all RIDS and correct any } \\
\text { deficiencies. }\end{array}$ & $\begin{array}{c}5 / 15 / 96 \\
\text { Completed }\end{array}$ & MG Gardner \\
\hline 9. Update/submit "Smart Book"/assemble key documents. & $7 / 15 / 96$ & MG Gardner \\
\hline । & & \\
\hline $\begin{array}{l}\text { 1. Provide a comprehensive briefing for the PHMC complete } \\
\text { with all facility, personnel, and organizational documentation. } \\
\text { Provide copies upon request. }\end{array}$ & $\begin{array}{l}8 / 12 / 96 \\
8 / 30 / 96\end{array}$ & MG Gardner \\
\hline
\end{tabular}




\begin{tabular}{|l|c|l|}
\hline \multicolumn{1}{|c|}{ Post-Award Actions } & $\begin{array}{r}\text { Scheduled } \\
\text { Date }\end{array}$ & Actionee(s) \\
\hline $\begin{array}{l}\text { 2. Conduct a comprehensive tour of all facilities and field } \\
\text { operations relevant to the organization. }\end{array}$ & $\begin{array}{r}8 / 12 / 96- \\
8 / 30 / 96\end{array}$ & MG Gardner \\
\hline $\begin{array}{l}\text { 3. Provide an update on work completed and work in progress } \\
\text { during the transition to the PHMC. }\end{array}$ & $9 / 15 / 96$ & MG Gardner \\
\hline $\begin{array}{l}\text { 4. Complete a joint property and equipment inventory with the } \\
\text { PHMC. }\end{array}$ & by 9/16/96 & MG Gardner \\
\hline $\begin{array}{l}\text { 5. Provide any additional documentation requested by the } \\
\text { PHMC. }\end{array}$ & $8 / 30 / 96$ & MG Gardner \\
\hline $\begin{array}{l}\text { 6. Assist the PHMC in a survey of pre-existing conditions at the } \\
\text { facility. }\end{array}$ & $\begin{array}{c}8 / 12 / 96- \\
9 / 16 / 96\end{array}$ & MG Gardner \\
\hline 7. Provide necessary training to PHMC personnel. & $\begin{array}{r}8 / 12 / 96- \\
8 / 30 / 96\end{array}$ & MG Gardner \\
\hline 8. Provide a categorized inventory of wastes. & $9 / 30 / 96$ & MG Gardner \\
\hline
\end{tabular}

6.6.1.4 Near Field Monitoring. Near Field Monitoring directs facility specific monitoring of environmental media and performs engineering evaluations/studies pertaining to the monitoring and control of environmental pollutants.

\begin{tabular}{|c|c|c|}
\hline Pre-Award Actions & $\begin{array}{l}\text { Scheduled } \\
\text { Date }\end{array}$ & Actionee(s) \\
\hline $\begin{array}{l}\text { 1. Prepare for a comprehensive briefing for the PHMC } \\
\text { contractor describing all operational aspects of the } \\
\text { organization. }\end{array}$ & $\begin{array}{c}5 / 01 / 96 \\
\text { Completed }\end{array}$ & JJ Dorian \\
\hline $\begin{array}{l}\text { 2. Identify all key documents relevant to the organization's } \\
\text { operations, including a listing of key facility and field } \\
\text { documents. }\end{array}$ & $\begin{array}{c}5 / 01 / 96 \\
\text { Completed }\end{array}$ & JJ Dorian \\
\hline $\begin{array}{l}\text { 3. Obtain copies of key facility documents and assemble, or } \\
\text { identify their location for access. Develop operations "Smart } \\
\text { Book." }\end{array}$ & $\begin{array}{c}6 / 01 / 96 \\
\text { Completed } \\
\text { "Smart } \\
\text { Book" only } \\
\text { See item } \$ 9\end{array}$ & JJ Dorian \\
\hline 4. Identify points-of-contact for the organization. & $\begin{array}{c}5 / 15 / 96 \\
\text { Completed }\end{array}$ & IJ Dorian \\
\hline $\begin{array}{l}\text { 5. Using the pre-existing conditions checklist, evaluate pre- } \\
\text { existing conditions. Conduct assessments as necessary to } \\
\text { adequately address the checklist. }\end{array}$ & $\begin{array}{c}6 / 15 / 96 \\
\text { Completed }\end{array}$ & Jj Dorian \\
\hline $\begin{array}{l}\text { 6. Identify applicable milestones and commioments (e.g., Tri- } \\
\text { Party Agreement and Consent Order milestones), and } \\
\text { determine their status. Describe the form of the deliverable } \\
\text { and what constitutes completion. }\end{array}$ & $\begin{array}{c}5 / 15 / 96 \\
\text { Completed }\end{array}$ & JJ Dorian \\
\hline $\begin{array}{l}\text { 7. Verify the accuracy of property assignments for the facility } \\
\text { and correct any deficiencies. }\end{array}$ & $\begin{array}{r}5 / 15 / 96 \\
\text { Completed } \\
\end{array}$ & J Dorian \\
\hline $\begin{array}{l}\text { 8. Verify the completeness of all RDS and correct any } \\
\text { deficiencies. }\end{array}$ & $\begin{array}{c}5 / 15 / 96 \\
\text { Completed }\end{array}$ & JJ Dorian \\
\hline
\end{tabular}




\begin{tabular}{|c|c|c|}
\hline Pre-Award Actions & $\begin{array}{l}\text { Scheduled } \\
\text { Date }\end{array}$ & Actionee(s) \\
\hline 9. Update/submit "Smart Book"/assemble key documents. & $7 / 15 / 96$ & JJ Dorian \\
\hline Post-Award Actions & & \\
\hline $\begin{array}{l}\text { 1. Provide a comprehensive briefing for the PHMC complete } \\
\text { with all facility, personnel, and organizational documentation. } \\
\text { Provide copies upon request. }\end{array}$ & $\begin{array}{l}8 / 12 / 96- \\
8 / 30 / 96\end{array}$ & JJ Dorian \\
\hline $\begin{array}{l}\text { 2. Conduct a comprehensive tour of all facilities and field } \\
\text { operations relevant to the organization. }\end{array}$ & $\begin{array}{l}8 / 12 / 96 \\
8 / 30 / 96\end{array}$ & JJ Dorian \\
\hline $\begin{array}{l}\text { 3. Assist the PHMC in a survey of pre-existing conditions at the } \\
\text { facility. }\end{array}$ & $\begin{array}{l}8 / 12 / 96- \\
9 / 16 / 96\end{array}$ & JJ Dorian \\
\hline $\begin{array}{l}\text { 4. Provide an update on work completed and work in progress } \\
\text { during the transition to the PHMC. }\end{array}$ & $9 / 15 / 96$ & JJ Dorian \\
\hline $\begin{array}{l}\text { 5. Complete a joint property and equipment inventory with the } \\
\text { PHMC. }\end{array}$ & by $9 / 16 / 96$ & JJ Dorian \\
\hline $\begin{array}{l}\text { 6. Provide any additional documentation requested by the } \\
\text { PHMC. }\end{array}$ & $8 / 30 / 96$ & JJ Dorian \\
\hline 7. Provide necessary training to PHMC personnel. & $\begin{array}{l}8 / 12 / 96- \\
8 / 30 / 96\end{array}$ & JJ Dorian \\
\hline 8. Provide a categorized inventory of wastes. & $9 / 30 / 96$ & JJ Dorian \\
\hline
\end{tabular}

6.6.1.5 Geophysics Investigations. The geophysics investigations team conducts surface and vadose zone geophysical testing. Primary support is to the RCRA Operational Monitoring program and Environmental Remediation Program.

\begin{tabular}{|l|c|l|}
\hline \multicolumn{1}{|c|}{ Pre-Award Actions } & $\begin{array}{c}\text { Scheduled } \\
\text { Date }\end{array}$ & Actionee(s) \\
\hline $\begin{array}{l}\text { 1. Prepare for a comprehensive briefing for the PHMC } \\
\text { contractor describing all operational aspects of the } \\
\text { organization. }\end{array}$ & $\begin{array}{c}5 / 01 / 96 \\
\text { Completed }\end{array}$ & JW Fassett \\
\hline $\begin{array}{l}\text { 2. Identify all key documents relevant to the organization's } \\
\text { operations, including a listing of key facility and field } \\
\text { documents. }\end{array}$ & $\begin{array}{c}5 / 01 / 96 \\
\text { Completed }\end{array}$ & JW Fassett \\
\hline $\begin{array}{l}\text { 3. Obtain copies of key facility documents and assemble, or } \\
\text { identify their location for access. Develop operations "Smart } \\
\text { Book." }\end{array}$ & $\begin{array}{c}\text { Completed } \\
\text { "Smart } \\
\text { Book" only } \\
\text { See item } \# 9\end{array}$ \\
\hline $\begin{array}{l}\text { 4. Identify POCs for the organization. } \\
\text { S. Using the pre-existing conditions checklist, evaluate pre- } \\
\text { existing conditions. Conduct assessments as necessary to } \\
\text { adequately address the checklist. }\end{array}$ & $\begin{array}{c}5 / 15 / 96 \\
\text { Completed }\end{array}$ & JW Fassett \\
\hline
\end{tabular}




\begin{tabular}{|c|c|c|}
\hline Pre-Award Actions & $\begin{array}{l}\text { Scheduled } \\
\text { Date }\end{array}$ & Actionee(s) \\
\hline $\begin{array}{l}\text { 6. Identify applicable milestones and commitments (e.g., Tri- } \\
\text { Party Agreement and Consent Order milestones), and } \\
\text { determine their status. Describe the form of the deliverable } \\
\text { and what constifutes completion. }\end{array}$ & $\begin{array}{c}5 / 15 / 96 \\
\text { Completed }\end{array}$ & JW Fassett \\
\hline $\begin{array}{l}\text { 7. Verify the accuracy of property assignments for the facility } \\
\text { and correct any deficiencies. }\end{array}$ & $\begin{array}{c}5 / 15 / 96 \\
\text { Completed }\end{array}$ & JW Fassett \\
\hline $\begin{array}{l}\text { 8. Verify the completeness of all RIDS and correct any } \\
\text { deficiencies. }\end{array}$ & $\begin{array}{c}5 / 15 / 96 \\
\text { Completed }\end{array}$ & JW Fassett \\
\hline 9. Update/subrnit "Smart Book"/assemble key docurnents. & $7 / 15 / 96$ & JW Fassett \\
\hline \multicolumn{3}{|l|}{ Post-Award Actions } \\
\hline $\begin{array}{l}\text { 1. Provide a comprehensive briefing for the PHMC complete } \\
\text { with all facility, personnel, and organizational documentation. } \\
\text { Provide copies upon request. }\end{array}$ & $\begin{array}{l}8 / 12 / 96 \\
8 / 30 / 96\end{array}$ & JW Fassett \\
\hline $\begin{array}{l}\text { 2. Conduct a comprehensive tour of all facifities and field } \\
\text { operations relevant to the organization. }\end{array}$ & $\begin{array}{l}8 / 12 / 96- \\
8 / 30 / 96 \\
\end{array}$ & JW Fassett \\
\hline $\begin{array}{l}\text { 3. Assist the PHMC in a survey of pre-existing conditions at the } \\
\text { facility. }\end{array}$ & $\begin{array}{l}8 / 12 / 96- \\
9 / 16 / 96\end{array}$ & JW Fassett \\
\hline $\begin{array}{l}\text { 4. Provide an update on work completed and work in progress } \\
\text { during the transition to the PHMC. }\end{array}$ & $9 / 15 / 96$ & IW Fassett \\
\hline $\begin{array}{l}\text { 5. Complete a joint property and equipment inventory with the } \\
\text { PHMC. }\end{array}$ & by $9 / 16 / 96$ & JW Fassett \\
\hline $\begin{array}{l}\text { 6. Provide any additional documentation requested by the } \\
\text { PHMC. }\end{array}$ & $8 / 30 / 96$ & JW Fassett \\
\hline 7. Provide necessary training to PHMC personnel. & $\begin{array}{l}8 / 12 / 96- \\
8 / 30 / 96\end{array}$ & JW Fassett \\
\hline 8. Provide a categorized inventory of wastes. & 9/30/96 & JW Fassett \\
\hline
\end{tabular}

6.6.1.6 Environmental Operations Sampling. The environmental operations sampling team conducts regulatory driven protocol sampling. Sampling applications include characterization, waste designation, recycling, and safety screening. Media sampled include but is not limited to soil, water, gas, vapor, oil, sludge, tar, concrete, facility effluents, filters, and asbestos. The team collects samples from wells, boreholes, cribs, ponds, ditches, drums, tanks, inside water lines, sewer lines, ducts, ion exchange columns, burial boxes, orphan containers, etc.

\begin{tabular}{|l|c|c|}
\hline \multicolumn{1}{|c|}{ Pre-Award Actions } & $\begin{array}{c}\text { Scheduled } \\
\text { Date }\end{array}$ & Actionee(s) \\
\hline $\begin{array}{l}\text { 1. Prepare for a comprehensive briefing for the PHMC } \\
\text { contractor describing all operational aspects of the } \\
\text { organization. }\end{array}$ & $\begin{array}{c}5 / 01 / 96 \\
\text { Completed }\end{array}$ & DL Edwards \\
\hline
\end{tabular}




\begin{tabular}{|c|c|c|}
\hline Pre-Award Actions & $\begin{array}{l}\text { Scheduled } \\
\text { Date }\end{array}$ & Actionee(s) \\
\hline $\begin{array}{l}\text { 2. Identify all key documents relevant to the organization's } \\
\text { operations, including a listing of key facility and field } \\
\text { documents. }\end{array}$ & $\begin{array}{l}5 / 01 / 96 \\
\text { Completed }\end{array}$ & DL Edwards \\
\hline $\begin{array}{l}\text { 3. Obtain copies of key facility documents and assemble, or } \\
\text { identify their location for access. Develop operations "Smart } \\
\text { Book." }\end{array}$ & $\begin{array}{l}\text { 6/01/96 } \\
\text { Completed } \\
\text { "Smart } \\
\text { Book" only } \\
\text { See item \#9 }\end{array}$ & DL Edwards \\
\hline 4. Identify POCs for the organization. & $\begin{array}{l}5 / 15 / 96 \\
\text { Completed }\end{array}$ & DL Edwards \\
\hline $\begin{array}{l}\text { 5. Using the pre-existing conditions checkist, evaluate pre- } \\
\text { existing conditions. Conduct assessments as necessary to } \\
\text { adequately address the checklist. }\end{array}$ & $\begin{array}{l}6 / 15 / 96 \\
\text { Completed }\end{array}$ & DL Edwards \\
\hline $\begin{array}{l}\text { 6. Identify applicable milestones and commitments (e.g., Tri- } \\
\text { Party Agreement and Consent Order milestones), and } \\
\text { determine their status. Describe the form of the deliverable } \\
\text { and what constiutes completion. }\end{array}$ & $\begin{array}{c}5 / 15 / 96 \\
\text { Completed }\end{array}$ & DL Edwards \\
\hline $\begin{array}{l}\text { 7. Verify the accuracy of property assigmments for the facility } \\
\text { and correct any deficiencies. }\end{array}$ & $\begin{array}{c}5 / 15 / 96 \\
\text { Completed }\end{array}$ & DL Edwards \\
\hline $\begin{array}{l}\text { 8. Verify the completeness of all RIDS and correct any } \\
\text { deficiencies. }\end{array}$ & $\begin{array}{c}5 / 15 / 96 \\
\text { Completed }\end{array}$ & DL Edwards \\
\hline 9. Update/submit "Smart Book"/assemble key documents. & $7 / 15 / 96$ & DL Edwards \\
\hline Post-Award Actions & & $\therefore$ \\
\hline $\begin{array}{l}\text { 1. Provide a comprehensive briefing for the PHMC complete } \\
\text { with all facility, personnel, and organizational documentation. } \\
\text { Provide copies upon request. }\end{array}$ & $\begin{array}{l}8 / 12 / 96- \\
8 / 30 / 96\end{array}$ & DL Edwards \\
\hline $\begin{array}{l}\text { 2. Conduct a comprehensive tour of all facilities and field } \\
\text { operations relevant to the organization. }\end{array}$ & $\begin{array}{l}8 / 12 / 96- \\
8 / 30 / 96\end{array}$ & DL Edwards \\
\hline $\begin{array}{l}\text { 3. Assist the PHMC in a survey of pre-existing conditions at the } \\
\text { facility. }\end{array}$ & $\begin{array}{l}8 / 12 / 96 \\
9 / 16 / 96\end{array}$ & DL Edwards \\
\hline $\begin{array}{l}\text { 4. Provide an update on work completed and work in progress } \\
\text { during the transition to the PHMC. }\end{array}$ & $9 / 15 / 96$ & DL Edwards \\
\hline $\begin{array}{l}\text { 5. Complete a joint property and equipment inventory with the } \\
\text { PHMC. }\end{array}$ & by $9 / 16 / 96$ & DL Edwards \\
\hline $\begin{array}{l}\text { 6. Provide any additional documentation requested by the } \\
\text { PHMC. }\end{array}$ & $8 / 30 / 96$ & DL Edwards \\
\hline 7. Provide necessary training to PHMC personnel. & $\begin{array}{l}8 / 12 / 96 \\
8 / 30 / 96\end{array}$ & DL Edwards \\
\hline 8. Provide a categorized listing of wastes. & $9 / 30 / 96$ & DL Edwards \\
\hline
\end{tabular}


6.6.1.7 Special Projects. Post-Award Actions Deleted. Ongoing special projects to be terminated by end of FY 1996.

\begin{tabular}{|l|c|c|}
\hline \multicolumn{1}{|c|}{ Pre-Award Actions } & $\begin{array}{c}\text { Scheduled } \\
\text { Date }\end{array}$ & Actionee(s) \\
\hline $\begin{array}{l}\text { 1. Prepare for a comprehensive briefing for the PHMC } \\
\text { contractor describing all planning, implementation, and } \\
\text { operational aspects of the project. }\end{array}$ & $\begin{array}{c}5 / 01 / 96 \\
\text { Completed }\end{array}$ & DI Herborn \\
\hline $\begin{array}{l}\text { 2. Identify all key documents relevant to the project, including a } \\
\text { listing of key agreements and plans. }\end{array}$ & $\begin{array}{c}5 / 01 / 96 \\
\text { Completed }\end{array}$ & DI Herborn \\
\hline $\begin{array}{l}\text { 3. Identify points-of-contact for all internal and external } \\
\text { organizations involved in the projects. }\end{array}$ & $\begin{array}{c}5 / 15 / 96 \\
\text { Completed }\end{array}$ & DI Herborn \\
\hline $\begin{array}{l}\text { 4. Identify applicable commitments and projects and determine } \\
\text { their status. Describe the form of the deliverable and what } \\
\text { constitutes completion. }\end{array}$ & $\begin{array}{c}5 / 15 / 96 \\
\text { Completed }\end{array}$ & DI Herborn \\
\hline $\begin{array}{l}\text { 5. Verify the accuracy of property assignments for the project } \\
\text { and correct deficiencies. }\end{array}$ & $\begin{array}{c}5 / 15 / 96 \\
\text { Cornpleted }\end{array}$ & DI Herborn \\
\hline
\end{tabular}

\subsubsection{CERCLA Monitoring}

Under its separate prime contract with RL BHI has responsibility for the management and coordination of Sitewide Comprehensive Environmental Response, Compensation, and Liability Act (CERCLA) monitoring activities. The scope includes groundwater characterization and monitoring services conducted by $\mathrm{BHI}$ to support investigations under the CERCLA program. CERCLA investigations are underway for a number of areas where known or suspected environmental contamination may have resulted from past Site practices.

PHMC Transition activities relevant to the CERCLA Monitoring program should focus on understanding the working relationships between PHMC, PNNL, and BHI for environmental monitoring; continuation of support services to BHI through select work orders; and PHMC assuming responsibility for applying the data collected by these activities for sitewide integration.

\begin{tabular}{|l|c|c|}
\hline \multicolumn{1}{|c|}{ Pre-Award Actions } & $\begin{array}{c}\text { Scheduled } \\
\text { Date }\end{array}$ & Actionee(s) \\
\hline $\begin{array}{l}\text { 1. Prepare a briefing for the PHMC's environmental monitoring } \\
\text { programs representatives, describing interactions between } \\
\text { BHI, PNNL, and the PHMC, and relevant operational } \\
\text { aspects of BHI's organization. }\end{array}$ & $7 / 26 / 96$ & $\begin{array}{l}\text { SC Foelber } \\
\text { WL Pamplin }\end{array}$ \\
\hline $\begin{array}{l}\text { 2. Identify all key documents relevant to the workscopes and } \\
\text { interactions between the PHMC and BHI organizations for } \\
\text { environmental monitoring activities (including Sitewide } \\
\text { agreements and MOUs). }\end{array}$ & $\begin{array}{c}5 / 01 / 96 \\
\text { Completed }\end{array}$ & WL Pamplin \\
\hline 3. Identify appropriate BHI points-of-contact for the PHMC. & $\begin{array}{c}5 / 01 / 96 \\
\text { Completed }\end{array}$ & SC Foelber \\
\hline
\end{tabular}


WHC-SP-1181 Rev. 1

\begin{tabular}{|c|c|c|}
\hline Pre-Award Actions & $\begin{array}{l}\text { Scheduled } \\
\text { Date }\end{array}$ & Actionee(s) \\
\hline 4. List active work orders and their expiration dates. & $\begin{array}{c}4 / 22 / 96 \\
\text { Completed }\end{array}$ & WL Pamplin \\
\hline $\begin{array}{l}\text { 5. Determine which support services work orders will be } \\
\text { continued and/or renewed, and which are candidates for } \\
\text { discontinuation. Document rationaie. }\end{array}$ & $7 / 12 / 96$ & $\begin{array}{l}\text { SC Foelber } \\
\text { WL Pamplin }\end{array}$ \\
\hline $\begin{array}{l}\text { 6. Identify applicable in-process milestones and commitments } \\
\text { for which PHMC must pick up supporting or contributing } \\
\text { responsibility (e.g., Tri-Party Agreement and Consent Order } \\
\text { milestones), and determine their status. Describe the form of } \\
\text { the support/involvement and what constintes completion. }\end{array}$ & $7 / 19 / 96$ & WL Pamplin \\
\hline 7. Identify pre-existing conditions, as applicable. & $7 / 19 / 96$ & WL Pamplin \\
\hline Post-Award Actions & & \\
\hline $\begin{array}{l}\text { 1. Provide a briefing for the PHMC relevant to interactions } \\
\text { between BHI and PHMC, and PHMC support services for } \\
\text { ongoing and planned CERCLA monitoring activities. }\end{array}$ & $8 / 08 / 96$ & $\begin{array}{l}\text { SC Foelber } \\
\text { WL Pamplin }\end{array}$ \\
\hline $\begin{array}{l}\text { 2. Identify to PHMC those work orders for PHMC services that } \\
\text { BHI will continue and/or renew, and any work orders BHI is } \\
\text { considering discontinuing (offer PHMC opportunity to } \\
\text { improve by specified date). }\end{array}$ & $8 / 08 / 96$ & $\begin{array}{l}\text { SC Foelber } \\
\text { WL Pamplin }\end{array}$ \\
\hline $\begin{array}{l}\text { 3. Establish routine points of contact and agreed method and } \\
\text { frequency of interface between PHMC and BHI. }\end{array}$ & $8 / 08 / 96$ & WL Pamplin \\
\hline $\begin{array}{l}\text { 4. Reach concurrence and document agreement on continuation } \\
\text { of all work in-progress involving support from PHMC. }\end{array}$ & $9 / 06 / 96$ & (Numerous) \\
\hline $\begin{array}{l}\text { 5. If requested, provide the PHMC a tour of field sites to clarify } \\
\text { areas of BHI program responsibility (vs. PHMC and PNNL), } \\
\text { including discussion of relevant pre-existing site conditions. }\end{array}$ & TBD & WL Pamplin \\
\hline 6. Update/renew active work orders, as appropriate. & $9 / 13 / 96$ & WL Pamplin \\
\hline
\end{tabular}

\subsubsection{Groundwater Surveillance Monitoring Project}

\begin{tabular}{|l|c|c|}
\hline Pre-Award Actions & Scheduled & Actionee(s) \\
\hline $\begin{array}{l}\text { 1. Prepare briefing for PHMC, describing relevant scope and } \\
\text { operational aspects of PNNL program and interactions } \\
\text { between PNNL, BHI, and PHMC. }\end{array}$ & $7 / 21 / 96$ & TL Page \\
\hline $\begin{array}{l}\text { 1. Provide a briefing for PHMC, describing relevant scope and } \\
\text { operational aspects of PNNL program and interactions } \\
\text { between PNNL, BHI, and PHMC. }\end{array}$ & by 8/30/96 & TL Page \\
\hline $\begin{array}{l}\text { 2. Establish routine points of contact and agreed method and } \\
\text { frequency of interface between PNNL and PHMC. }\end{array}$ & $8 / 12 / 96$ & TL Page \\
\hline
\end{tabular}




\subsection{EXTERNAL/INTERNAL COMMUNICATIONS}

\subsubsection{Interface with Site Communications/Public Affairs (RL Lead)} activities.

The PHMC will be responsible for its own public relations and communications

\begin{tabular}{|c|c|c|}
\hline Pre-Award Actions & $\begin{array}{l}\text { Scheduled } \\
\text { Date }\end{array}$ & Actionee(s) \\
\hline Arrange site orientations/tours for PHMC staff. & $7 / 22 / 96$ & $\begin{array}{l}\text { ML Goldie. } \\
\text { DM Conner }\end{array}$ \\
\hline Post-Award Actions & & \\
\hline $\begin{array}{l}\text { 1. Arrange meeting of PHMC public relations/communications } \\
\text { staff with the Hanford Public Affairs Cabinet (all Hanford } \\
\text { Site pubitic affairs/communications managers) and welcome } \\
\text { them to the cabinet. }\end{array}$ & $8 / 12 / 96$ & KK Randolph \\
\hline
\end{tabular}

\subsubsection{Integration of WHC/CF KH/BCSR Program Components Into the PHMC Program}

Coordinate with the PHMC to integrate the existing employee communications, media relations, public involvement and community relations programs into the PHMC's program.

\begin{tabular}{|c|c|c|}
\hline Post-Award Actions & $\begin{array}{l}\text { Scheduled } \\
\text { Date }\end{array}$ & Actionee(s) \\
\hline $\begin{array}{l}\text { 1. Arrange a meeting with } \mathrm{RL} \text { and the PHMC to discuss the } \\
\text { WHC/CF } \mathrm{KH} / \mathrm{BCSR} \text { communications program and potential } \\
\text { integration of the existing program. }\end{array}$ & $8 / 12 / 96$ & $\begin{array}{l}\text { PM Phelps } \\
\text { LE Love } \\
\text { KK Randolph }\end{array}$ \\
\hline $\begin{array}{l}\text { 2. Ensure that WHC/ICF KH/BCSR staff managers discuss } \\
\text { departmental responsibilities and activities with the PHMC. }\end{array}$ & $9 / 03 / 96$ & $\begin{array}{l}\text { PM Phelps } \\
\text { LE Love }\end{array}$ \\
\hline
\end{tabular}

\subsubsection{Tri-Party Agreement Community Relations Plan}

WHC has responsibility for providing direct support to the RL Office of External Affairs, which has responsibility for implementing the Tri-Party Agreement Community Relations Plan (Ecology et al. 1990). The PHMC will be expected to take over these responsibilities. 


\begin{tabular}{|c|c|c|}
\hline Post-Award Actions & $\begin{array}{c}\text { Scheduled } \\
\text { Date }\end{array}$ & Actionee(s) \\
\hline $\begin{array}{l}\text { 1. Use regularly schedule monthly meetings of RL and WHC } \\
\text { Project Managers and Public Information Officers to brief the } \\
\text { PHMC POCs on the Community Relations Plan schedule, } \\
\text { delineate responsibilities, and establish mechanisms for } \\
\text { ongoing support from the PHMC. }\end{array}$ & $8 / 05 / 96$ & $\begin{array}{l}\text { KK Randolph } \\
\text { JK Yerxa } \\
\text { PJ Bengtson }\end{array}$ \\
\hline $\begin{array}{l}\text { 2. Recommend the PHMC submit material to the quarterly } \\
\text { Hanford Update Tri-Party Agreement newsletter and attend } \\
\text { public meetings to introduce key PHMC officials to interested } \\
\text { stakeholders. }\end{array}$ & $8 / 12 / 96$ & PHMC \\
\hline
\end{tabular}

\subsubsection{Introduce the PHMC Personnel to Hanford's External Publics (RL Lead)}

Arrange for the PHMC personnel to meet key RL, Hanford Site contractors; neighbors, business and local government officials; the news media; and special-interest groups.

\begin{tabular}{|l|c|l|}
\hline \multicolumn{1}{|c|}{ Post-Award Actions } & $\begin{array}{l}\text { Scheduled } \\
\text { Date }\end{array}$ & \multicolumn{1}{|c|}{ Actionee(s) } \\
\hline $\begin{array}{l}\text { 1. Arrange a meeting to introduce the PHMC to key RL staff } \\
\text { and contractor principals. }\end{array}$ & $8 / 12 / 96$ & $\begin{array}{l}\text { JD Wagoner } \\
\text { KK Randolph }\end{array}$ \\
\hline $\begin{array}{l}\text { 2. Arrange a meeting to introduce the PHMC to local } \\
\text { government and business officials. }\end{array}$ & $8 / 12 / 96$ & $\begin{array}{l}\text { JD Wagoner } \\
\text { KK Randolph }\end{array}$ \\
\hline $\begin{array}{l}\text { 3. Coordinate with the PHMC to arrange an onsite state } \\
\text { legislator and congressional briefing as directed by RL. }\end{array}$ & $8 / 12 / 96$ & KK Randolph \\
\hline $\begin{array}{l}\text { 4. Recornmend the PHMC schedule a media briefing. } \\
\text { B. Brief the PHMC on the Hanford Advisory Board. }\end{array}$ & $8 / 12 / 96$ & $\begin{array}{l}\text { KK Randolph } \\
\text { PM Phelps } \\
\text { LE Love }\end{array}$ \\
\hline
\end{tabular}

\subsubsection{Provide Employees With Necessary Information During Transition}

Keep WHC/ICF KH/BCSR employees informed about the PHMC and transition activities. 


\begin{tabular}{|c|c|c|}
\hline Pre-Award Actions & $\begin{array}{c}\text { Scheduled } \\
\text { Date }\end{array}$ & Actionee(s) \\
\hline $\begin{array}{l}\text { 1. Continue to address WHC/CF KH/BCSR employees' } \\
\text { concerns and information needs regarding the transition } \\
\text { through Management Network and Hanford Reach articles } \\
\text { addressing key questions and answers. }\end{array}$ & $7 / 31 / 96$ & $\begin{array}{l}\text { TL Cozzens } \\
\text { DL Cresswell } \\
\text { LE Love } \\
\text { ML Jackson } \\
\text { PS Hale } \\
\end{array}$ \\
\hline Post-Award Actions & & \\
\hline $\begin{array}{l}\text { 1. Hold all-manager meeting for WHC/ICF KH/BCSR } \\
\text { managers to discuss transition actions, schedule and } \\
\text { responsibilities. }\end{array}$ & $8 / 12 / 96$ & $\begin{array}{l}\text { TL Cozzens } \\
\text { SA Woody } \\
\text { LE Love } \\
\text { ML Jackson } \\
\end{array}$ \\
\hline $\begin{array}{l}\text { 2. Provide special Hanford Reach and Management Network } \\
\text { coverage for the transition, including coverage of the } \\
\text { turnover meetings/receptions }(6.7 .8) \text { and articles about the } \\
\text { new contractors and their parent firns. }\end{array}$ & $8 / 12 / 96$ & $\begin{array}{l}\text { TL Cozzens } \\
\text { DL Cresswell } \\
\text { PS Hale } \\
\text { ML Jackson }\end{array}$ \\
\hline $\begin{array}{l}\text { 3. Evaluate employee suggestions and concerns regarding the } \\
\text { transition and adjust communications plan, if necessary. }\end{array}$ & $8 / 12 / 96$ & $\begin{array}{l}\text { TL Cozzens } \\
\text { WHC Communications } \\
\text { Council } \\
\text { LE Love } \\
\text { ML Jackson } \\
\end{array}$ \\
\hline $\begin{array}{l}\text { 4. Provide assistance to the PHMC in scheduling and presenting } \\
\text { briefing sessions for employees on benefits package and to } \\
\text { answer questions. Include evening/off-shift sessions which } \\
\text { spouses of affected employees can attend. }\end{array}$ & $8 / 12 / 96$ & $\begin{array}{l}\text { HA Sieber } \\
\text { PHMC }\end{array}$ \\
\hline $\begin{array}{l}\text { 5. Publish a "key contacts" list for employees to use to raise } \\
\text { ongoing or unresolved issues, questions. }\end{array}$ & $\begin{array}{l}8 / 12 / 96 \\
9 / 30 / 96\end{array}$ & $\begin{array}{l}\text { TL Cozens } \\
\text { HA Sieber } \\
\text { PHMC } \\
\text { LE Love } \\
\end{array}$ \\
\hline $\begin{array}{l}\text { 6. Provide a form for submitting written employee questions, } \\
\text { and identify response paths (such as general-interest questions } \\
\text { in Management Network or Hanford Reach, and individual } \\
\text { questions addressed directly to employees). }\end{array}$ & $8 / 12 / 96$ & $\begin{array}{l}\text { HA Sieber } \\
\text { TL Cozzens } \\
\text { PHMC } \\
\text { ML Jackson }\end{array}$ \\
\hline
\end{tabular}

\subsubsection{U.S. Department of Energy "Hanford Reach" and "DOE This Month" Communications}

WHC has responsibility for producing the Hanford Reach, an employee site newspaper, for RL. Since November 1994, WHC also has had responsibility for producing DOE This Month, a monthly publication for DOE Headquarters. The PHMC will be expected to take over these responsibilities. 
WHC-SP-1181 Rev. 1

\begin{tabular}{|l|c|l|}
\hline \multicolumn{1}{|c|}{ Post-Award Actions } & Scheduled & \multicolumn{1}{|c|}{ Actionee(s) } \\
\hline 1. Develop and implement a plan for take-over of the Hanford & $8 / 12 / 96-$ & DL Cresswell \\
Reach weekly site newspaper, so that production continues & $9 / 30 / 96$ & \\
uninterrupted. & & \\
\hline 2. Develop and implement a plan for take-over of DOE This & $8 / 12 / 96-$ & TL Cozzens \\
Month. & $9 / 30 / 96$ & \\
\hline
\end{tabular}

\subsubsection{Hanford Site Emergency Preparedness Communications}

WHC has responsibility for implementing the Hanford Site Emergency Preparedness Plan. Accordingly, WHC Communications has the lead in implementing Emergency Preparedness Communications and staffing for the Joint Information Center (JIC). Communications staff from all Hanford Site prime contractors participate in the JIC and related emergency communications efforts. The PHMC will need to take over this responsibility.

\begin{tabular}{|c|c|c|}
\hline Post-Award Actions & $\begin{array}{c}\text { Scheduled } \\
\text { Date }\end{array}$ & \multicolumn{1}{|c|}{ Actionee(s) } \\
\hline 1. Develop and implement a plan for take-over of Emergency & $8 / 12 / 96-$ & SA Woody \\
Preparedness Communications activities and plans. & $9 / 30 / 96$
\end{tabular}

\subsubsection{WHC/ICF Kaiser/BCSR Farewell Activities}

Arrange activities for WHC/ICF KH/BCSR employees, to bid farewell to coworkers moving to new contractors and be recognized by WHC management for their contributions, in order that the transition and continued teaming will be positive. Arrange farewell activities for external audiences.

\begin{tabular}{|l|c|l|}
\hline \multicolumn{1}{|c|}{ Post-Award Actions } & $\begin{array}{l}\text { Scheduled } \\
\text { Date }\end{array}$ & \multicolumn{1}{|c|}{ Actionee(s) } \\
\hline $\begin{array}{l}\text { 1. Schedule and conduct a series of meetings/receptions for all } \\
\text { employees to introduce them to their new management team, } \\
\text { and recognizing them for past contributions and achievements. }\end{array}$ & $9 / 15 / 96$ & $\begin{array}{l}\text { TL Cozzens } \\
\text { SA Woody } \\
\text { MJ Auckland } \\
\text { LE Love }\end{array}$ \\
\hline $\begin{array}{l}\text { 2. Place an advertisement in the Tri-City Herald and Yakima } \\
\text { Herald-Republic thanking WHC/CF KH/BCSR employees } \\
\text { transferring to the new PHMC for their past contributions and } \\
\text { anticipated continued contributions as a member of the Hanford } \\
\text { Site team under their new employer. }\end{array}$ & $9 / 30 / 96$ & $\begin{array}{l}\text { TL Cozzens } \\
\text { PM Phelps } \\
\text { LE Love }\end{array}$ \\
\hline 3. Schedule a final news media interview for LaMar Trego. & $9 / 30 / 96$ & JC Britton \\
\hline $\begin{array}{l}\text { 4. Mail WHC/ICF KH/BCSR farewell letter to employees and } \\
\text { retirees. }\end{array}$ & $9 / 15 / 96$ & $\begin{array}{l}\text { TL Cozzens } \\
\text { LE Love }\end{array}$ \\
\hline
\end{tabular}




\begin{tabular}{|l|c|c|}
\hline \multicolumn{1}{|c|}{ Post-Award Actions } & $\begin{array}{l}\text { Scheduled } \\
\text { Date }\end{array}$ & Actionee(s) \\
\hline $\begin{array}{l}\text { 5. Mail WHC/ICF KH/BCSR farewell letter to neighbors, } \\
\text { community leaders, activists, school contacts and congressional } \\
\text { representatives. }\end{array}$ & $9 / 15 / 96$ & $\begin{array}{l}\text { PM Phelps } \\
\text { LE Love }\end{array}$ \\
\hline $\begin{array}{l}\text { 6. Recommend the PHMC host a tumover reception for the media } \\
\text { and community. }\end{array}$ & $9 / 30 / 96$ & $\begin{array}{l}\text { KK Randolph } \\
\text { PM Phelps } \\
\text { LE Love }\end{array}$ \\
\hline
\end{tabular}

\subsection{TRAINING}

\begin{tabular}{|c|c|c|}
\hline \multicolumn{1}{|c|}{ Pre-Award Actions } & $\begin{array}{c}\text { Scheduled } \\
\text { Date }\end{array}$ & Actionee(s) \\
\hline $\begin{array}{l}\text { 1. Identify space and equipment within training areas and facilities } \\
\text { for use by the PHMC. }\end{array}$ & $\begin{array}{c}6 / 01 / 96 \\
\text { Completed }\end{array}$ & CL Nansen \\
\hline $\begin{array}{l}\text { 2. In outline form for all facilities/operations to follow, define the } \\
\text { appropriate contents and level of detail to be included in training } \\
\text { briefings to the PHMC (review with RL POC). }\end{array}$ & $\begin{array}{c}5 / 01 / 96 \\
\text { Completed }\end{array}$ & CL Nansen \\
\hline
\end{tabular}

\subsubsection{Training Services}

Training Services (TRS) provides training that facilitates excellence in radiological safety, industrial safety and health, and EM, and contributes to the improvement of technical and administrative performance. It also provides resources and services that assist line management in providing facility-specific training on processes and tools used to improve those processes. TRS provides administrative support to the training process with the Training Matrix (which identifies position-specific training needs for individual employees), training scheduling and registration, and training completion records. Functions of training audit and evaluation are also provided, as well as training partnerships with community educational institutions.

\begin{tabular}{|c|c|c|}
\hline Pre Award Actions & $\begin{array}{l}\text { Scheduied } \\
\text { Date }\end{array}$ & Actionee(s) \\
\hline $\begin{array}{l}\text { 1. Prepare Training Services briefing for the PHMC (coordinate } \\
\text { content with RL POC). }\end{array}$ & $7 / 15 / 96$ & CP Wick \\
\hline $\begin{array}{l}\text { 2. Identify key documents for Training Services. Provide listing } \\
\text { to CL Nansen for consolidation and } \mathrm{RL} \text { review. }\end{array}$ & $\begin{array}{l}5 / 01 / 96 \\
\text { Completed }\end{array}$ & CP Wick \\
\hline $\begin{array}{l}\text { 3. Assemble Training Services documentation, or identify its } \\
\text { location, for use by the PHMC, Includes preparation of a } \\
\text { Training Services "Smart Book," to include information } \\
\text { developed in the following actions. }\end{array}$ & $\begin{array}{l}\text { 6/01/96 } \\
\text { Completed } \\
\text { "Smart Book" } \\
\text { only } \\
\text { See item } \# 13\end{array}$ & CP Wick \\
\hline 4. Identify POCs for Training Services and input to CL Nansen. & $\begin{array}{l}5 / 15 / 96 \\
\text { Completed }\end{array}$ & CP Wick \\
\hline $\begin{array}{l}\text { 5. Develop a listing and description of subcontracts supporting } \\
\text { Training Services and input to CL Nansen. Include any } \\
\text { subcontracts planned to be executed prior to contract takeover. }\end{array}$ & $\begin{array}{c}5 / 15 / 96 \\
\text { Completed }\end{array}$ & CP Wick \\
\hline
\end{tabular}




\begin{tabular}{|c|c|c|}
\hline Pre-Award Actions & $\begin{array}{l}\text { Scheduled } \\
\text { Date }\end{array}$ & Actionee(s) \\
\hline $\begin{array}{l}\text { 6. Using the pre-existing checklist, compile pre-existing condition } \\
\text { information on Training Services facilities and/or major } \\
\text { equipment. Conduct, or schedule as a separate post-award } \\
\text { action(s), any assessments required to adequately address all } \\
\text { relevant items on the checklist. Provide completed checklist to } \\
\text { RL POC for review. }\end{array}$ & $\begin{array}{l}6 / 15 / 96 \\
\text { Completed }\end{array}$ & RG Slocum \\
\hline $\begin{array}{l}\text { 7. Identify applicable milestones and commitments (e.g., Tri-Party } \\
\text { Agreement, DNFSB and consent order milestones), and } \\
\text { determine their status. Describe the form of the deliverable and } \\
\text { what constitutes completion. Provide to RL POC for review. }\end{array}$ & $\begin{array}{c}6 / 01 / 96 \\
\text { Completed }\end{array}$ & CP Wick \\
\hline $\begin{array}{l}\text { 8. Develop a listing of special agreement services provided to RL, } \\
\text { other DOE sites, and other entities. }\end{array}$ & $\begin{array}{c}6 / 01 / 96 \\
\text { Completed }\end{array}$ & CP Wick \\
\hline $\begin{array}{l}\text { 9. Develop a listing and description of TRS databases. Provide } \\
\text { list to CL Nansen for consolidation. }\end{array}$ & $\begin{array}{l}5 / 15 / 96 \\
\text { Completed }\end{array}$ & CP Wick \\
\hline $\begin{array}{l}\text { 10. Develop a list of current work order services or other form of } \\
\text { agreement to provide work for other organizations, on and } \\
\text { offsite. }\end{array}$ & $\begin{array}{l}\text { 6/01/96 } \\
\text { Completed }\end{array}$ & CP Wick \\
\hline $\begin{array}{l}\text { 11. Develop a list of current work order services or other form of } \\
\text { agreement to obtain work from other organizations, on and } \\
\text { offsite. }\end{array}$ & $\begin{array}{l}\text { 6/01/96 } \\
\text { Completed }\end{array}$ & CP Wick \\
\hline $\begin{array}{l}\text { 12. Verify the accuracy of property assignments for the TRS } \\
\text { organization and correct any deficiencies. }\end{array}$ & $\begin{array}{c}6 / 01 / 96 \\
\text { Completed }\end{array}$ & CP Wick \\
\hline 13. Update/submit "Smart Book"/assemble key documents. & $7 / 15 / 96$ & CP Wick \\
\hline $\begin{array}{l}\text { 14. Verify pre-existing condition data is current and supplement as } \\
\text { necessary. }\end{array}$ & $7 / 22 / 96$ & CP Wick \\
\hline । $৯$ Post-Award Actions & & \\
\hline 1. Provide TRS briefing(s) to the PHMC. & $8 / 12 / 96-8 / 30 / 96$ & RG Slocum \\
\hline 2. Provide a TRS tour to the PHMC. & $8 / 12 / 96-8 / 30 / 96$ & RG Slocum \\
\hline $\begin{array}{l}\text { 3. Provide the PHMC access to key documentation, pre-existing } \\
\text { condition information, commitment data, inventory data, etc. (as } \\
\text { established in the pre-award phase) Provide copies upon } \\
\text { request. }\end{array}$ & $8 / 05 / 96$ & CP Wick \\
\hline $\begin{array}{l}\text { 4. Provide any additional documentation or information requested } \\
\text { by the PHMC, if available, that was not identified during the } \\
\text { pre-award phase. }\end{array}$ & by $9 / 01 / 96$ & CP Wick \\
\hline $\begin{array}{l}\text { 5. PHMC identify any recurring reports that they would like to } \\
\text { receive during transition. }\end{array}$ & by $8 / 15 / 96$ & PHMC \\
\hline $\begin{array}{l}\text { 6. Provide an updated status of ongoing work prior to contract } \\
\text { urnover. }\end{array}$ & by $9 / 30 / 96$ & CP Wick \\
\hline $\begin{array}{l}\text { 7. Perform a joint property and equipment inventory with the } \\
\text { PHMC. }\end{array}$ & by $9 / 16 / 96$ & CP Wick \\
\hline $\begin{array}{l}\text { 8. Assist the PHMC in the conduct of a Pre-Existing Condition } \\
\text { Assessment. }\end{array}$ & $8 / 12 / 96-9 / 16 / 96$ & CP Wick \\
\hline $\begin{array}{l}\text { 9. Provide a list of PHMC personnel requiring HGET and other } \\
\text { general training. }\end{array}$ & $8 / 05 / 96$ & PHMC \\
\hline
\end{tabular}


WHC-SP-1181 Rev. 1

\begin{tabular}{|c|c|c|}
\hline \multicolumn{1}{|c|}{ Post-Award Actions } & $\begin{array}{r}\text { Scheduled } \\
\text { Date }\end{array}$ & Actionee(s) \\
\hline $\begin{array}{l}\text { 10. Provide HGET and other general site training to PHMC } \\
\text { personnel as needed for transition. }\end{array}$ & $8 / 05 / 96-9 / 15 / 96$ & CP Wick \\
\hline
\end{tabular}

\subsubsection{Hazardous Materials Management and Emergency Response}

Hazardous Materials Management and Emergency Response (HAMMER) is a worker training and education program for hazardous material, waste management, and emergency response workers. The HAMMER facility is operated as a user center which hosts, brokers, and integrates the capabilities of its partnering organizations to ensure the delivery of state of the art training and education curricula. Its main emphasis is the delivery of its products and services for DOE and DOE contractor workers, managers, and emergency responders.

HAMMER will host training focused on improving safety and health while reducing training costs and establishing a new training industry for the region.

\begin{tabular}{|c|c|c|}
\hline Pre-Award Actions & $\begin{array}{l}\text { Scheduled } \\
\text { Date }\end{array}$ & Actionee(s): \\
\hline $\begin{array}{l}\text { 1. Prepare HAMMER briefing for the PHMC (coordinate } \\
\text { content with RL POC). }\end{array}$ & $\begin{array}{c}\text { 6/01/96 } \\
\text { Completed }\end{array}$ & PJ Vandervert \\
\hline $\begin{array}{l}\text { 2. Identify key documents for the HAMMER } \\
\text { organization. Provide to RL POC for review. }\end{array}$ & $\begin{array}{c}5 / 01 / 96 \\
\text { Completed }\end{array}$ & PJ Vandervert \\
\hline $\begin{array}{l}\text { 3. Assemble key HAMMER documentation, or identify } \\
\text { its location, for use by the PHMC. Includes } \\
\text { preparation of a HAMMER "Smart Book," to inciude } \\
\text { information developed in the following actions. }\end{array}$ & $\begin{array}{c}6 / 01 / 96 \\
\text { Completed "Smart } \\
\text { Book" only } \\
\text { See item } \# 14 \\
\end{array}$ & PJ Vandervert \\
\hline 4. Identify POCs for HAMMER. & $\begin{array}{l}\text { 6/01/96 } \\
\text { Completed }\end{array}$ & PJ Vandervert \\
\hline $\begin{array}{l}\text { 5. Develop a listing and description of subcontracts } \\
\text { supporting HAMMER. Include any subcontracts } \\
\text { planned to be executed prior to contract takeover. }\end{array}$ & $\begin{array}{c}\text { 6/01/96 } \\
\text { Completed }\end{array}$ & PJ Vandervert \\
\hline $\begin{array}{l}\text { 6. Using the pre-existing checklist, compile pre-existing } \\
\text { condition information on HAMMER Facilities and/or } \\
\text { major equipment. Conduct, or schedule as a separate } \\
\text { post-award action(s), any assessments required to } \\
\text { adequately address all relevant iterns on the checklist. } \\
\text { Provide completed checklist to RL POC for review. }\end{array}$ & $\begin{array}{l}\text { 6/15/96 } \\
\text { Completed }\end{array}$ & PJ Vandervert \\
\hline $\begin{array}{l}\text { 7. Identify applicable milestones and commitments (e.g., } \\
\text { Tri-Party Agreement, DNFSB and consent order } \\
\text { milestones), and determine their status. Describe the } \\
\text { form of the deliverable and what constitutes } \\
\text { completion. Provide to RL POC for review. }\end{array}$ & $\begin{array}{l}\text { 6/01/96 } \\
\text { Completed }\end{array}$ & PJ Vandervert \\
\hline $\begin{array}{l}\text { 8. Develop a description of ongoing and planned } \\
\text { construction projects. }\end{array}$ & $\begin{array}{c}6 / 01 / 96 \\
\text { Completed }\end{array}$ & PJ Vandervert \\
\hline $\begin{array}{l}\text { 9. Develop a listing of special agreement services } \\
\text { provided to RL, other DOE sites, and other entities. }\end{array}$ & $\begin{array}{l}\text { 6/01/96 } \\
\text { Completed }\end{array}$ & PJ Vandervert \\
\hline
\end{tabular}


WHC-SP-1181 Rev. 1

\begin{tabular}{|c|c|c|}
\hline Pre-A ward Actions & $\begin{array}{l}\text { Scheduled } \\
\text { Date }\end{array}$ & Actionee(s) \\
\hline $\begin{array}{l}\text { 10. Develop a listing and description of HAMMER } \\
\text { databases. }\end{array}$ & $\begin{array}{l}\text { 6/01/96 } \\
\text { Completed }\end{array}$ & PJ Vandervert \\
\hline $\begin{array}{l}\text { 11. Develop a list of current work order services or other } \\
\text { form of agreement to provide work for other } \\
\text { organizations, on and offsite. }\end{array}$ & $\begin{array}{c}6 / 01 / 96 \\
\text { Completed }\end{array}$ & PJ Vandervert \\
\hline $\begin{array}{l}\text { 12. Develop a list of current work order services or other } \\
\text { form of agreement to obtain work from other } \\
\text { organizations, on and offsite. }\end{array}$ & $\begin{array}{l}6 / 01 / 96 \\
\text { Completed }\end{array}$ & PJ Vandervert \\
\hline $\begin{array}{l}\text { 13. Verify the accuracy of property assignments for the } \\
\text { HAMMER organization and correct any deficiencies. }\end{array}$ & $\begin{array}{l}\text { 6/01/96 } \\
\text { Completed }\end{array}$ & PJ Vandervert \\
\hline 14. Update/submit "Smart Book"/assemble key documents. & $7 / 15 / 96$ & PJ Vandervert \\
\hline 15. Update/finalize briefing to PHMC. & $7 / 15 / 96$ & PJ Vandervert \\
\hline $\begin{array}{l}\text { 16. Update/finalize current inventories of feed, materials, } \\
\text { and/or wastes. }\end{array}$ & $7 / 15 / 96$ & PJ Vandervert \\
\hline $\begin{array}{l}\text { 17. Verify pre-existing condition data is current and } \\
\text { supplement as necessary. }\end{array}$ & $7 / 22 / 96$ & PJ Vandervert \\
\hline 18. Ensure building assignments are correct in RLPS. & $7 / 22 / 96$ & PJ Vandervert \\
\hline Post-Award Actions & & \\
\hline 1. Provide a HAMMER briefing to the PHMC. & $8 / 12 / 96-8 / 30 / 96$ & KA McGinnis \\
\hline 2. Provide a HAMMER tour to the PHMC. & $8 / 12 / 96-8 / 30 / 96$ & KA McGinnis \\
\hline $\begin{array}{l}\text { 3. Provide the PHMC access to key documentation, pre- } \\
\text { existing condition information, cormitment data, } \\
\text { inventory data, etc. (as established in the pre-award } \\
\text { phase). Provide copies upon request. }\end{array}$ & $8 / 05 / 96$ & PJ Vandervert \\
\hline $\begin{array}{l}\text { 4. Provide any additional documentation or information } \\
\text { requested by the PHMC, if available, that was not } \\
\text { identified during the pre-award phase. }\end{array}$ & by $9 / 01 / 96$ & PJ Vandervert \\
\hline $\begin{array}{l}\text { 5. PHMC identify any recurring reports that they would } \\
\text { like to receive during transition. }\end{array}$ & by $8 / 15 / 96$ & PHMC \\
\hline $\begin{array}{l}\text { 6. Provide an updated status of ongoing work prior to } \\
\text { contract turnover. }\end{array}$ & by $9 / 30 / 96$ & PJ Vandervert \\
\hline $\begin{array}{l}\text { 7. Perform a joint property and equipment inventory with } \\
\text { the PHMC. }\end{array}$ & by $9 / 16 / 96$ & J Vandervert \\
\hline $\begin{array}{l}\text { 8. Assist the PHMC in the conduct of a Pre-existing } \\
\text { Condition Assessment. }\end{array}$ & $8 / 12 / 96-9 / 16 / 96$ & PJ Vandervert \\
\hline
\end{tabular}




\subsection{EMERGENCY PREPAREDNESS}

\subsubsection{Emergency Management}

The Emergency Preparedness Program provides an emergency management system including development, coordination, and direction of planning, preparedness, and readiness assurance for response to emergency events on the Hanford Site; and emergency management support to RL.

The Emergency Preparedness Program meets the requirements for an integrated Hanford Site emergency program detailed in DOE/RL-94-02 Hanford Emergency Response Plan. This plan implements the DOE emergency management orders as well as applicable state and Federal regulations to ensure the health and safety of Site employees and the general public.

Responsibilities of the organization include hazards assessments that form the basis of the emergency management program, planning to ensure integrated onsite emergency response, assisting RL with planning with state and local agencies to ensure that the public can be protected, developing and maintaining $R L$ and contractor emergency plans and procedures, staffing and maintaining RL and contractor emergency response facilities, emergency notifications, maintaining the readiness of emergency response organizations through training and drills, and validating the Site Emergency Management program through exercises, evaluation and surveillances.

The Emergency Preparedness Program has the lead for a Hanford Site Emergency Response Organization Re-engineering project. This effort will provide a streamlined emergency response organization, while maintaining a strong response capability for the Site.

\begin{tabular}{|c|c|c|}
\hline Pre-Award Actions & $\begin{array}{c}\text { Scheduled } \\
\text { Date }\end{array}$ & Actionee(s) \\
\hline $\begin{array}{l}\text { 1. Develop list of documentation for the Emergency Preparedness } \\
\text { Program. Provide to RL POC for review. }\end{array}$ & $\begin{array}{c}6 / 01 / 96 \\
\text { Completed }\end{array}$ & DA Marsh \\
\hline $\begin{array}{l}\text { 2. Assemble key program (or project) documentation, or identify } \\
\text { its location, for use by the PHMC. Includes development of a } \\
\text { program/project "Smart Book," including listings and } \\
\text { descriptions developed above. }\end{array}$ & $\begin{array}{l}6 / 01 / 96 \\
\text { Completed } \\
\text { "Smart Book" } \\
\text { only } \\
\text { See item \#13 }\end{array}$ & DA Marsh \\
\hline $\begin{array}{l}\text { 3. Develop an Emergency Preparedness Program Point of Contact } \\
\text { List. }\end{array}$ & $\begin{array}{c}6 / 01 / 96 \\
\text { Completed }\end{array}$ & DA Marsh \\
\hline $\begin{array}{l}\text { 4. Develop Emergency Preparedness Program Review management } \\
\text { presentation (coordinate content with RI POC) } \\
\text { - Technical Program } \\
\text { - Budget } \\
\text { - Organization } \\
\text { - Status of Milestones and Other Commitments. }\end{array}$ & $7 / 15 / 96$ & DA Marsh \\
\hline
\end{tabular}




\begin{tabular}{|c|c|c|}
\hline Pre-Award Actions & Scheduled & Actionee(s) \\
\hline $\begin{array}{l}\text { 5. Develop listing and description of program (or project) } \\
\text { databases, including facility (or operations) databases. }\end{array}$ & $\begin{array}{c}6 / 01 / 96 \\
\text { Completed } \\
\end{array}$ & DA Marsh \\
\hline $\begin{array}{l}\text { 6. Develop a listing of information which constitutes the } \\
\text { "authorization basis" for the program (i.e., SRIDS). }\end{array}$ & $\begin{array}{c}\text { 6/01/96 } \\
\text { Completed } \\
\end{array}$ & DA Marsh \\
\hline $\begin{array}{l}\text { 7. Develop a description of ongoing and planned construction } \\
\text { projects. }\end{array}$ & $\begin{array}{c}6 / 01 / 96 \\
\text { Completed } \\
\end{array}$ & DA Marsh \\
\hline $\begin{array}{l}\text { 8. Develop a listing of special agreement services provided to RL, } \\
\text { other DOE sites, and other entities. }\end{array}$ & $\begin{array}{c}6 / 01 / 96 \\
\text { Completed } \\
\end{array}$ & DA Marsh \\
\hline $\begin{array}{l}\text { 9. Develop a list of current work order services or other form of } \\
\text { agreement to provide work, or obtain work, for other } \\
\text { organizations, on and offsite. }\end{array}$ & $\begin{array}{l}\text { 6/01/96 } \\
\text { Completed }\end{array}$ & DA Marsh \\
\hline $\begin{array}{l}\text { 10. Verify the accuracy of property assignments at the } \\
\text { program/project organizational level and correct any } \\
\text { deficiencies. }\end{array}$ & $\begin{array}{l}\text { 6/01/96 } \\
\text { Completed }\end{array}$ & DA Marsh \\
\hline $\begin{array}{l}\text { 11. Identify space and equipment within program (or project) area } \\
\text { and facilities for use by the PHMC. }\end{array}$ & $\begin{array}{c}\text { 6/01/96 } \\
\text { Completed }\end{array}$ & DA Marsh. \\
\hline 12. Define preexisting conditions. & $\begin{array}{c}6 / 15 / 96 \\
\text { Completed } \\
\end{array}$ & DA Marsh \\
\hline 13. Update/submit "Smart Book"/assemble key documents. & $7 / 15 / 96$ & DA Marsh \\
\hline 40 Post-Award Actions & 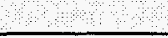 & 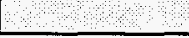 \\
\hline $\begin{array}{l}\text { 1. Provide the PHMC access to the key program (or project) } \\
\text { documentation, any program level generated pre-existing } \\
\text { conditions information, listing of POCs, and listing of } \\
\text { subcontracts. Provide copies upon request. }\end{array}$ & $8 / 05 / 96$ & DA Marsh \\
\hline $\begin{array}{l}\text { 2. Ascertain impact of PHMC contract to current emergency } \\
\text { response organization staffing, and determine need for EP } \\
\text { exercise. }\end{array}$ & $8 / 30 / 96$ & DA Marsh \\
\hline $\begin{array}{l}\text { 3. Provide Emergency Preparedness Program briefings and } \\
\text { emergency response facility tours to PHMC management. }\end{array}$ & $8 / 12 / 96-8 / 30 / 96$ & DA Marsh \\
\hline $\begin{array}{l}\text { 4. Perform a joint program/project level property and equipment } \\
\text { inventory with PHMC personnel. }\end{array}$ & by $9 / 16 / 96$ & DA Marsh \\
\hline $\begin{array}{l}\text { 5. Identify and train new emergency response organization } \\
\text { members as required. Conduct EP exercise, if necessary. }\end{array}$ & $9 / 23 / 96$ & DA Marsh \\
\hline $\begin{array}{l}\text { 6. In concert with the PHMC, develop a plan to revise emergency } \\
\text { management plans and procedures to reflect PHMC contract. }\end{array}$ & 9/23/96 & DA Marsh \\
\hline
\end{tabular}

\subsubsection{Radiological Assistance Program}

The Radiological Assistance Program (RAP) is a direct funded Federal emergency response asset assisting other Federal, tribal, state and local authorities in radiological monitoring and assessment activities associated with radiological incidents or emergencies. Upon request, RAP resources are made available and assistance will be provided until it is 
no longer needed or there are sufficient resources to handle the situation. RL is the Regional Coordinating Office for DOE Region 8 (Alaska, Oregon, Washington).

\begin{tabular}{|c|c|c|}
\hline Pre-Award Actions & $\begin{array}{l}\text { Scheduled } \\
\text { Date }\end{array}$ & Actionee(s) \\
\hline $\begin{array}{l}\text { 1. Develop list of documentation (plans, work authorization, } \\
\text { program review). }\end{array}$ & $\begin{array}{c}\text { 6/01/96 } \\
\text { Completed }\end{array}$ & DE Webb \\
\hline $\begin{array}{l}\text { 2. Assemble key program (or project) documentation, or identify its } \\
\text { location, for use by the PHMC. Includes development of a } \\
\text { program/project "Smart Book," including listings and } \\
\text { descriptions developed above. }\end{array}$ & $\begin{array}{l}\text { 6/01/96 } \\
\text { Completed } \\
\text { "Smart Book" } \\
\text { only } \\
\text { See item } \# 10\end{array}$ & DE Webb \\
\hline 3. Establish Point of Contact. & $\begin{array}{c}6 / 01 / 96 \\
\text { Completed } \\
\end{array}$ & DE Webb \\
\hline $\begin{array}{l}\text { 4. Develop management presentation on RAP } \\
\text { - Program Overview and Direction } \\
\text { - Budget Overview } \\
\text { - Program Status and Planned Activities. }\end{array}$ & $\begin{array}{l}\text { 6/01/96 } \\
\text { Completed }\end{array}$ & DE Webb \\
\hline $\begin{array}{l}\text { 5. Develop a listing of information which constitutes the } \\
\text { "authorization basis" for the program. }\end{array}$ & $\begin{array}{l}\text { 6/01/96 } \\
\text { Completed }\end{array}$ & DE Webb \\
\hline $\begin{array}{l}\text { 6. Develop a listing of special agreement services provided to RL, } \\
\text { other DOE sites, and other regional entities. }\end{array}$ & $\begin{array}{l}6 / 01 / 96 \\
\text { Completed }\end{array}$ & DE Webb \\
\hline $\begin{array}{l}\text { 7. Develop a list of current work order services or other form of } \\
\text { agreernent to provide work, or obtain work, for other } \\
\text { organizations, on and offsite. }\end{array}$ & $\begin{array}{c}6 / 01 / 96 \\
\text { Completed }\end{array}$ & DE Webb \\
\hline $\begin{array}{l}\text { 8. Verify the accuracy of property assignments at the } \\
\text { program/project organizational level and correct any } \\
\text { deficiencies. }\end{array}$ & $\begin{array}{l}6 / 01 / 96 \\
\text { Completed }\end{array}$ & DE Webb \\
\hline 9. Define pre-existing conditions. & $\begin{array}{c}6 / 15 / 96 \\
\text { Completed }\end{array}$ & DE Webb \\
\hline 10. Update/submit "Smart Book"/assemble key documents. & $7 / 15 / 96$ & DE Webb \\
\hline Post-Award Actions & & \\
\hline $\begin{array}{l}\text { 1. Provide the PHMC access to the key program (or project) } \\
\text { documentation, any program level generated pre-existing } \\
\text { conditions information, listing of POCs, and listing of } \\
\text { subcontracts. Provide copies upon request. }\end{array}$ & $8 / 05 / 96$ & DE Webb \\
\hline $\begin{array}{l}\text { 2. Ascertain impact of PHMC contract to the RAP program and } \\
\text { team. }\end{array}$ & $8 / 30 / 96$ & DE Webb \\
\hline 3. Provide RAP briefings to new management. & $8 / 12 / 96-8 / 30 / 96$ & DE Webb \\
\hline 4. Tour RAP facilities. & $8 / 12 / 96-8 / 30 / 96$ & DE Webb \\
\hline $\begin{array}{l}\text { 5. Perform a joint program/project property and equipment } \\
\text { inventory with PHMC personnel. }\end{array}$ & by $9 / 16 / 96$ & DE Webb \\
\hline
\end{tabular}


WHC-SP-1181 Rev. 1

\begin{tabular}{|l|c|l|}
\hline \multicolumn{1}{|c|}{ Post-Avard Actions } & Scheduled & Actionee(s) \\
\hline $\begin{array}{l}\text { 6. Develop plan to revise affected documentation to include } \\
\text { DOE/RL-92-49, RAP Plan and Procedure Document and other } \\
\text { documents and work plans. }\end{array}$ & $9 / 23 / 96$ & DE Webb \\
\hline 7. Identify and train new RAP team members. & $9 / 23 / 96$ & DE Webb \\
\hline
\end{tabular}

\subsubsection{ESH Management Plan}

DOE Order 151.1 Comprehensive Emergency Management System, listed in Section J, Appendix C of the PHMC Request for Proposal has been defined as an ESH order. The PHMC ESH Management Plan must address how the projects will implement the requirements of DOE Order 151.1, as defined in DOE/RL-94-02 Hanford Emergency Response Plan, and support the Site emergency response organization with management and support personnel. WHC Emergency Preparedness performs oversight of the facility emergency management programs and since the transition of these activities is a part of the overall transition, the following pre- and post-award activities are necessary.

\begin{tabular}{|l|c|c|c|}
\hline Post-Award Actions & Scheduled & Date & Actionee(s) \\
\hline $\begin{array}{l}\text { 1. Review project transition plans for emergency preparedness } \\
\text { activities to ensure continuity of emergency response capabilities. }\end{array}$ & $8 / 30 / 96$ & DA Marsh \\
\hline $\begin{array}{l}\text { 2. Review ESH Management Plan for project implementation of } \\
\text { DOE/RL-94-02 Hanford Emergency Response Plan. }\end{array}$ & $9 / 23 / 96$ & DA Marsh \\
\hline
\end{tabular}

\subsection{DIRECT SUPPORT TO RL}

The M\&O Contractor currently provides a range of services (e.g., document control) to RL which are budgeted and costed to RL financial accounts. It is anticipated that this support to RL will continue under the PHMC.

\begin{tabular}{|c|c|c|}
\hline Pre-Award Actions & $\begin{array}{c}\text { Scheduled } \\
\text { Date }\end{array}$ & Actionee(s) \\
\hline $\begin{array}{l}\text { 1. Develop a consolidated list and description of services } \\
\text { and support activities provided to RL by the M\&O } \\
\text { Contractor. }\end{array}$ & $\begin{array}{c}6 / 01 / 96 \\
\text { Completed }\end{array}$ & $\begin{array}{l}\text { JL Waite } \\
\text { RJ Light }\end{array}$ \\
\hline Post-Award Actions & & \\
\hline 1. Provide listing of support to RL to the PHMC. & $8 / 05 / 96$ & RJ Light \\
\hline
\end{tabular}


WHC-SP-1181 Rev. 1

This page intentionally left blank. 


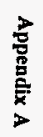

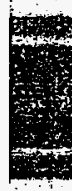

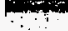




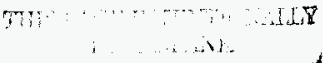




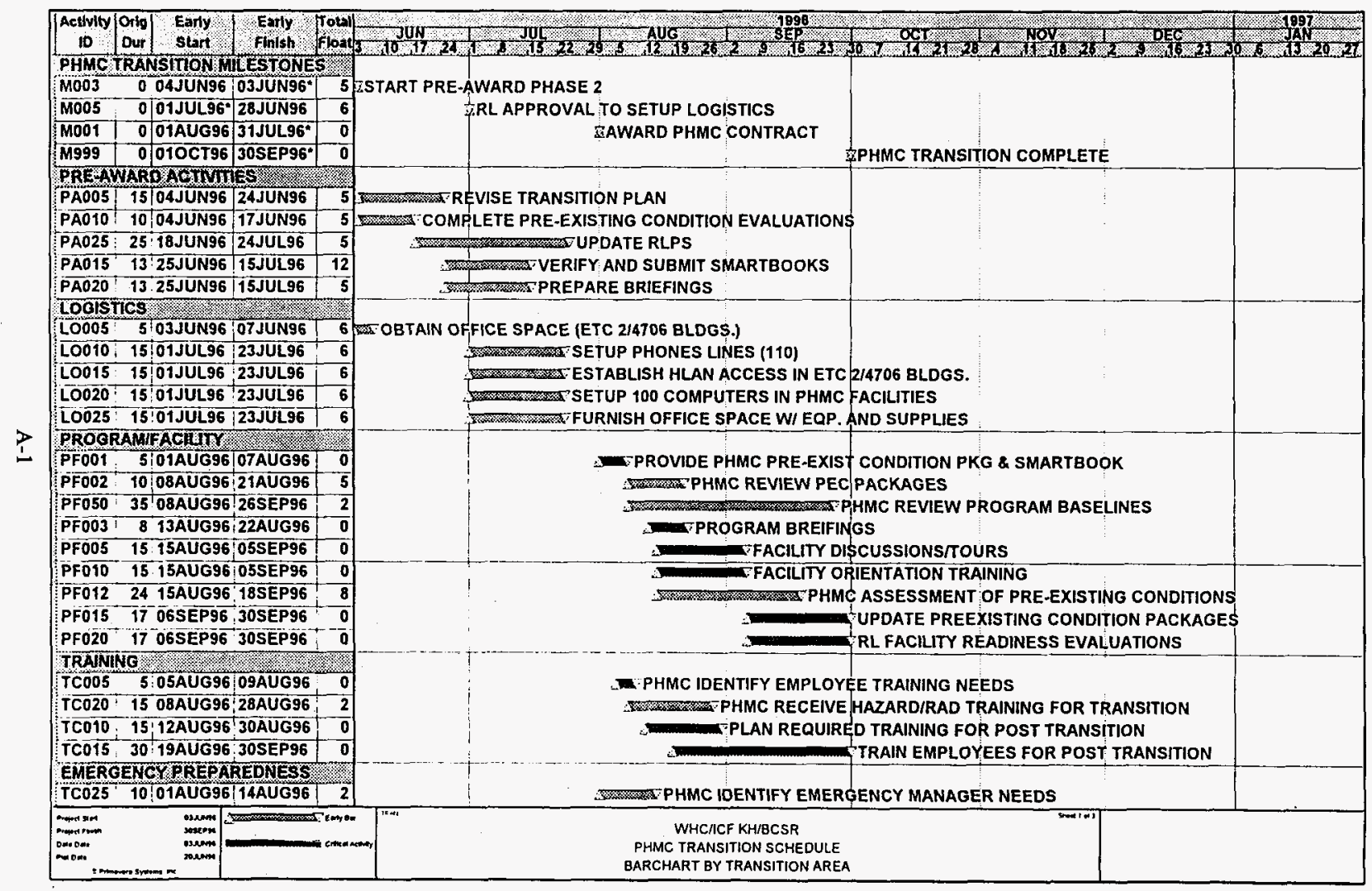




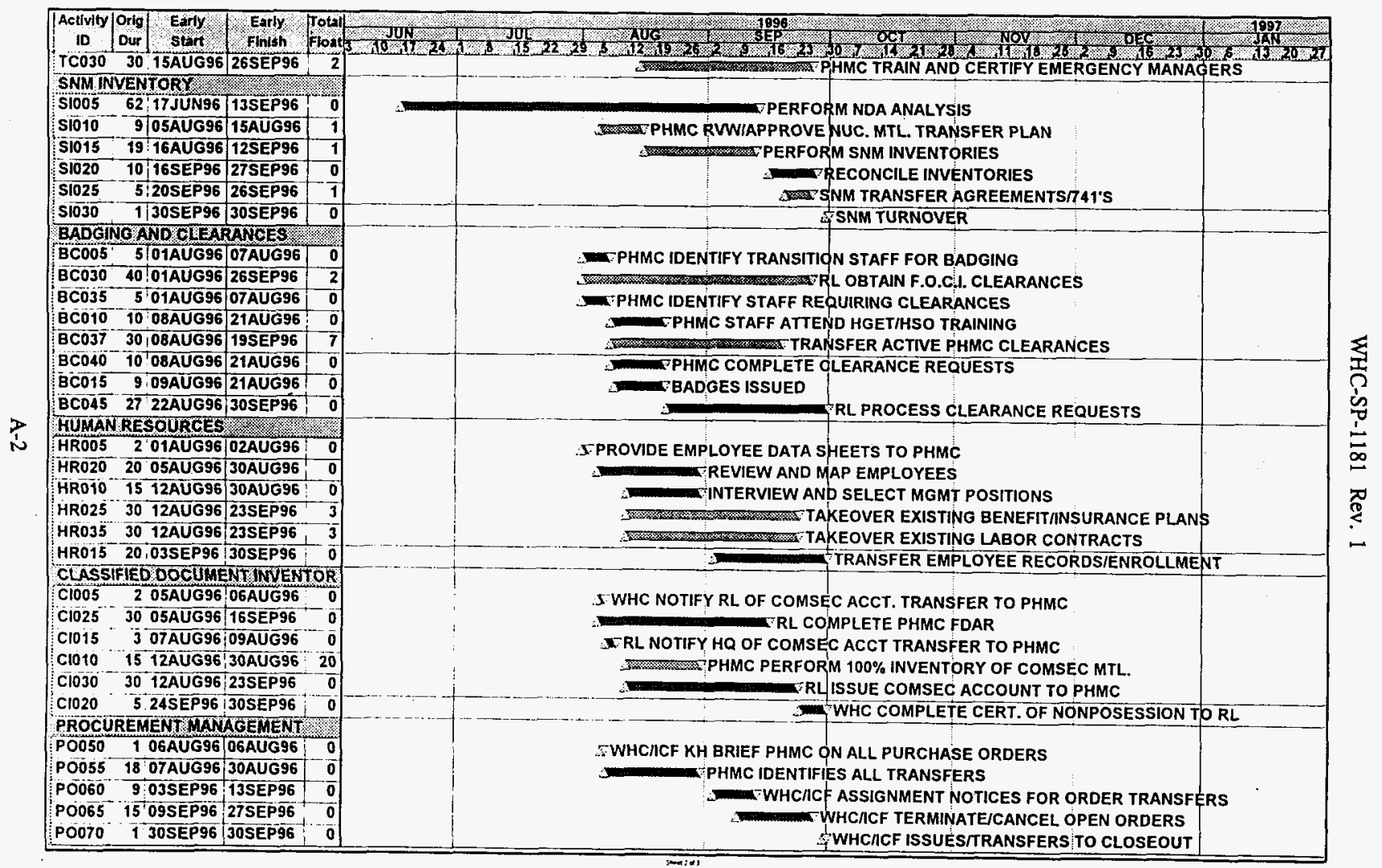




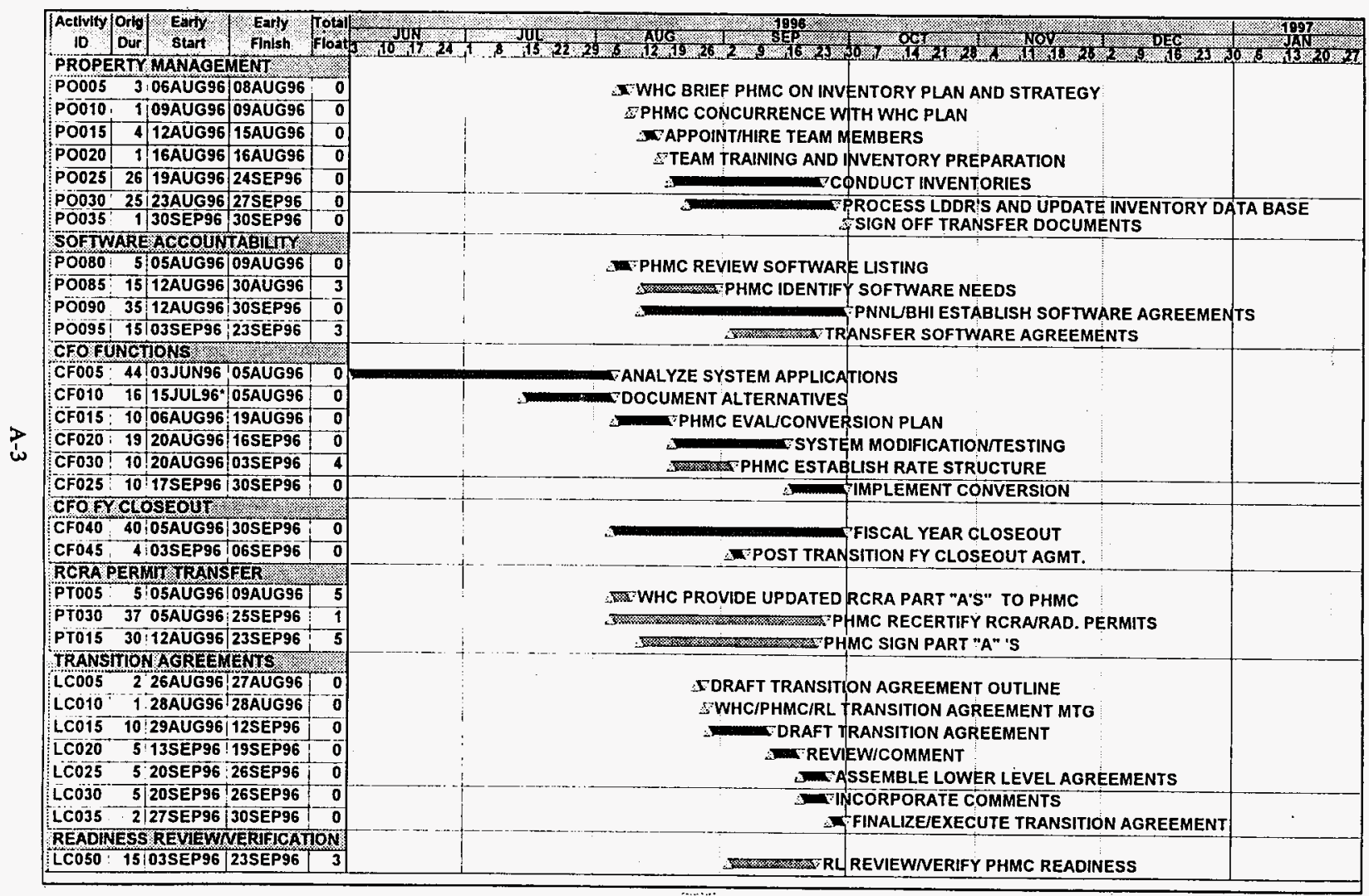


WHC-SP-1181 Rev. 1

This page intentionally left blank. 